Prepared for the U.S. Department of Energy under Contract DE-AC05-76RL01830

\title{
Characterization of Vadose Zone Sediment: Borehole 41-09-39 in the S-SX Waste Management Area
}
RJ Serne
RE Clayton
IV Kutnyakov
GV Last
VL Le Gore
TC Wilson
HT Schaef
MJ O'Hara
KB Wagnon
DC Lanigan
CF Brown
BA Williams
CW Lindenmeier
RD Orr
DS Burke
CC Ainsworth

September 2008 


\title{
DISCLAIMER
}

This report was prepared as an account of work sponsored by an agency of the United States Government. Neither the United States Government nor any agency thereof, nor Battelle Memorial Institute, nor any of their employees, makes any warranty, express or implied, or assumes any legal liability or responsibility for the accuracy, completeness, or usefulness of any information, apparatus, product, or process disclosed, or represents that its use would not infringe privately owned rights. Reference herein to any specific commercial product, process, or service by trade name, trademark, manufacturer, or otherwise does not necessarily constitute or imply its endorsement, recommendation, or favoring by the United States Government or any agency thereof, or Battelle Memorial Institute. The views and opinions of authors expressed herein do not necessarily state or reflect those of the United States Government or any agency thereof.

\author{
PACIFIC NORTHWEST NATIONAL LABORATORY \\ operated by \\ BATTELLE \\ for the \\ UNITED STATES DEPARTMENT OF ENERGY \\ under Contract DE-AC06-76RL01830
}

This document was printed on recycled paper. 


\section{Characterization of Vadose Zone Sediment: Borehole 41-09-39 in the S- SX Waste Management Area}

$\begin{array}{lll}\text { R. J. Serne } & \text { R. E. Clayton } & \text { I. V. Kutnyakov } \\ \text { G. V. Last } & \text { V. L. LeGore } & \text { T. C. Wilson } \\ \text { H. T. Schaef } & \text { M. J. O'Hara } & \text { K. B. Wagnon } \\ \text { D. C. Lanigan } & \text { C. F. Brown } & \text { B. A. Williams } \\ \text { C. W. Lindenmeier } & \text { R. D. Orr } & \text { D. S. Burke } \\ \text { C. C. Ainsworth } & & \end{array}$

September 2008

Prepared for CH2M HILL Hanford Group, Inc., and the U.S. Department of Energy under Contract DEAC06-76RL01830

Pacific Northwest National Laboratory

Richland, Washington 99352 


\section{Executive Summary}

This report was revised in September 2008 to remove acid-extractable sodium data from Table 5.15. The sodium data was removed due to potential contamination introduced during the acid extraction process. The rest of the text remains unchanged from the original report issued in February 2002.

The overall goal of the of the Tank Farm Vadose Zone Project, led by CH2M HILL Hanford Group, Inc., is to define risks from past and future single-shell tank farm activities. To meet this goal, CH2M HILL Hanford Group, Inc., asked scientists from Pacific Northwest National Laboratory to perform detailed analyses on vadose zone sediment from within the S-SX Waste Management Area. This report is one in a series of four reports to present the results of these analyses. Specifically, this report contains all the geologic, geochemical, and selected physical characterization data collected on vadose zone sediment recovered from borehole 41-09-39 installed adjacent to tank SX-109.

This report also presents our interpretation of the data in the context of the sediment lithologies, the vertical extent of contamination, the migration potential of the contaminants, and the correspondence of the contaminant distribution to groundwater. The information presented in this report supports the field investigation report prepared by CH2M HILL Hanford Group, Inc.

The geology under the SX Tank Farm forms the framework through which the contaminants move, and provides the basis with which to interpret and extrapolate the physical and geochemical properties that control the migration and distribution of contaminants. Of particular interest are the interrelationships between the coarserand finer-grained facies and the degree of contrast in their physical and geochemical properties. The vertical distribution of cesium-137, based on borehole gamma logging and the laboratory analysis of the sediment at borehole 41-09-39, suggests that much of the tank fluid that leaked from tanks SX-108, and/or SX-109, traveled within the coarse-grained Hanford formation $\mathrm{H} 1$ unit that is found between 20.4 and 26.8 meters (67 and 88 feet) below ground surface (bgs) at borehole 4109-39.

It is difficult to differentiate natural zones of higher moisture content due to the presence of finer-grained material (finer-grained material retains higher moisture contents) from zones of excess moisture resulting from leaked fluid. Thus, moisture content distribution did not give us a clear indication of the vertical extent of the plume. However, moisture content does help identify intervals that have been recently impacted by drilling operations.

The $\mathrm{pH}$ values are not nearly as high as would be expected for tank liquor completely saturating sediment. Therefore, it would appear that significant $\mathrm{pH}$ reactions occur from the tank bottoms at $\sim 16.8$ meters ( $\sim 55$ feet) to a maximum of 27.4 meters (90 feet) bgs for sediment surrounding the tanks.

The electrical conductivity results suggest that the tank leak fluid dominates the porewater down to a depth of 38.8 meters (127.4 feet) bgs and the deepest (leading edge of plume) is in borehole extension sleeve 3A, at a depth of 41.4 meters (136 feet). For other borehole extension sleeves below 41.4 meters (136 feet) bgs, the electrical conductivity does not show any significant deviations from values found for

(a) Draft Field Investigation Report for Waste Management Area S-SX. RPP-7884, Draft, Volume 2, Appendix D, CH2M HILL Hanford Group, Inc., Richland, Washington. 
vadose zone sediment at nearby uncontaminated Resource Conservation and Recovery Act boreholes. The water obtained at wells near the SX Tank Farm, including the one water sample obtained at the 4109-39 borehole extension, has an electrical conductivity of about $250 \mu \mathrm{S} / \mathrm{cm}$. This suggests that the groundwater beneath the SX Tank Farm still shows the influence of the large volumes of dilute-salt waste liquids disposed to facilities upgradient (north and west). Water extract sodium, nitrate, and technetium99 also indicate the leading edge of the plume is at 41.4 meters (136 feet) bgs. These mobile constituent profiles all suggest that the leading edge of the plume resides about 3.4 meters (11 feet) into the finegrained Plio-Pleistocene mud (PPlz) unit at 41.4 meters (136 feet) bgs. A key finding is that we do not observe a continuous vertical distribution of elevated nitrate or any other tank constituent's concentration from the elevation of the tank bottoms to the water table in this borehole.

Another key finding is that the 1:1 sediment-to-water extracts give a good estimate of the porewater chemistry in the vadose zone sediment. The chemical composition of the actual porewater, obtained by ultracentrifugation, was found to be fairly well estimated by dilution correcting the 1:1 water extracts. The most concentrated porewater was essentially 5 to $6 \mathrm{M}$ sodium nitrate with several tenths molar concentrations of calcium and chromate. Because it is much easier to obtain a water extract of the vadose zone sediment than actually extracting fluid, the finding is important to understanding the porewater chemistry throughout the vadose zone.

The first significant sign of elevated technetium-99 is at 24.2 meters ( 79.5 feet) bgs and a high concentration plume is found from 27.4 to 38.8 meters (90 to 127.4 feet) bgs. Molybdenum distribution is quite similar. The technetium in situ $\mathrm{K}_{\mathrm{d}}$ varies from 0.01 to about $5 \mathrm{~mL} / \mathrm{g}$ over the whole zone of contamination. The most significant chemical contaminant in the sediment is chromium. The leading edge of the chromium plume appears to stop at 34.1 meters (112 feet) bgs, which suggests that it does not migrate as quickly as molybdenum and technetium. The bulk of the water-leachable chromium has been confirmed as chromate by its distinct yellow color and by ion chromatography.

Based on comparing the depth of penetration of various contaminants and comparing the percentages that are water leachable, we determined that chromium migrates faster than cesium-137 but slower than technetium-99 and nitrate. The slight retardation for the chromate may be a reduction process where the ferrous minerals in the sediment react with the tank fluids and cause a portion of the soluble chromate to precipitate.

The major cesium-137 activity is concentrated between the depths 20.1 to 25.6 meters (66 to 84 feet) bgs, moderate amounts of cesium-137 reached 31.1 meters (102 feet) bgs, and the leading edge perhaps reaches 39.9 to 41.5 meters (131 to 136 feet) bgs. However, we believe that the high cesium-137 activity at 40.1 to 40.5 meters (131.7 to 133 feet) bgs is compressed sediment dragged down during the original pile driving of the closed end casing. In traditional batch sorption tests, the cesium $\mathrm{K}_{\mathrm{d}}$ value for several sediment samples taken from the borehole is moderate (4 to $40 \mathrm{~mL} / \mathrm{g})$ for a very high ionic strength but neutral $\mathrm{pH}$ (7.4 $\mathrm{M}$ sodium nitrate) solution. The cesium $\mathrm{K}_{\mathrm{d}}$ increases slightly for the $4 \mathrm{M}$ sodium nitrate solution as would be expected for a cation-exchange-dominated process. The range of 4 to 40 is similar to the in situ desorption $\mathrm{K}_{\mathrm{d}} \mathrm{s}$ (4 to $25 \mathrm{~mL} / \mathrm{g}$ ) calculated from the water extracts and direct counting of the sediment. 
The water-extractable cations suggest that an ion-exchange process dominates the porewater/sediment interactions in the zone where tank fluid passed by or currently exists. The leading edge of the tank leak plume is enriched in alkaline earth cations that were displaced from the native sediment exchange sites. Combining the atypical high nitrate with the sodium-to-calcium ratio data for water extracts suggests that the leading edge of the tank leak plume is at 41.2 meters ( 135 feet) bgs at borehole 41-09-39. One plausible explanation for this depth is that the tank leak plume traveled horizontally and vertically over a relatively short time period through the more permeable Hanford formation sediment and perched on the less permeable Plio-Pleistocene mud (PPlz) unit. Over the next four decades, after the tank lead $\mathrm{k}$, the soluble chemicals/water slowly diffused/percolated into the top of PPlz unit to a depth of 3.4 meters $(\sim 11$ feet).

The matric suction data suggest that the sediment profile at borehole 41-09-39 is draining. We estimate a value somewhat $>5$ millimeters per year. This is lower than expected based on a number of studies at the Hanford Site that show coarse gravel surface covers, as found at the tank farms, cause as much as $50 \%$ of the annual precipitation to recharge to the water table. Another source of localized recharge near tank farms has been hypothesized to be leaking water lines. With the gravel cover alone, one might expect several centimeters of recharge as opposed to 5 millimeters.

Cation exchange measurements show that the fine-grained Plio-Pleistocene mud has a relatively high exchange capacity (13 to $16 \mathrm{meq} / 100 \mathrm{~g}$ ). The coarse-grained Ringold sediment has a very low cation exchange capacity ( $\sim 1$ to $3 \mathrm{meq} / 100 \mathrm{~g}$ ). Although not measured, the Hanford formation sediment would fall in between these two values.

X-ray diffraction analyses of the bulk sidewall core samples from five depths in borehole 41-09-39 indicate that the sediment is mostly quartz ( $\sim 35 \%$ to $50 \%)$ and feldspar ( $25 \%$ to $55 \%$ ), with lesser amounts of mica and chlorite. Plagioclase feldspar is 2 to 10 times more abundant than potassium feldspar. The clay fraction ( $<2$ micron) is dominated by four clay minerals: illite, smectite, chlorite, and kaolinite with minor amounts of quartz, feldspar, and amphibole. Overall, illite was the dominant mineral in the clay fraction with 20 to $35 \mathrm{wt} \%$. The presence of illites as the dominant clay-size mineral is fortuitous because illites are strong adsorbers of cesium and can irreversibly adsorb cesium within interlayer sites.

We conclude that common ion exchange and heterogeneous (solid phase-liquid solute) redox reactions are two mechanisms that influence the distribution of contaminants in the vadose zone sediment within the zone impacted by tank liquor. We did not observe significant indications of $\mathrm{pH}$ alteration of the sediment mineralogy or porosity, but we did observe slightly elevated $\mathrm{pH}$ values between 16.8 to 27.4 meters (55 to 90 feet) bgs. 


\section{Acknowledgments}

This work was conducted as part of the Tank Farm Vadose Zone Project led by CH2M HILL Hanford Group, Inc., in support of the U.S. Department of Energy's Office of River Protection. The authors wish to thank Anthony J. Knepp, Fredrick M. Mann, David A. Myers, Thomas E. Jones, and Harold A. Sydnor with CH2M HILL Hanford Group, Inc., and Marc I. Wood with Fluor Hanford for their support of this work. We would also like to express our gratitude to Robert Yasek with the DOE's Office of River Protection.

We would especially like to thank Kent D. Reynolds (Duratek Federal Services Inc.) for his efforts in the field, and Kevin A. Lindsey (Kennedy Jenkes Consultants, Inc.) for his insights on the geologic nature of the materials penetrated by this borehole.

Finally, we would like to thank Bruce J. Bjornstad and Duane G. Horton for their technical review of this document, Launa F. Morasch, Janet K. Tarantino, and Barbara K. Wilson for their editorial and document production support, and Kathy R. Neiderhiser, Rose M. Watt, JoLynn Draper and Rose Urbina for publication design support. 


\section{Acronyms and Abbreviations}

\begin{tabular}{|c|c|}
\hline ASA & American Society of Agronomy \\
\hline ASTM & American Society for Testing and Materials \\
\hline bgs & below ground surface \\
\hline CEC & cation exchange capacity \\
\hline $\begin{array}{l}\text { DOE } \\
\text { DQO }\end{array}$ & $\begin{array}{l}\text { U.S. Department of Energy } \\
\text { data quality objectives }\end{array}$ \\
\hline $\mathrm{EC}$ & electrical conductivity \\
\hline EMSP & Environmental Management Science Program \\
\hline EPA & U.S. Environmental Protection Agency \\
\hline ERDF & Environmental Restoration Disposal Facility \\
\hline GEA & gamma energy analysis \\
\hline $\mathrm{ICP}$ & inductively coupled plasma \\
\hline ICP-MS & inductively coupled plasma mass spectrometry \\
\hline ICP-OES & inductively coupled plasma optical emission spectroscopy \\
\hline NIST & National Institute of Standards and Technology \\
\hline PNNL & Pacific Northwest National Laboratory \\
\hline PPlc & Plio-Pleistocene carbonate \\
\hline $\mathrm{PPlz}$ & Plio-Pliestocene mud \\
\hline RCRA & Resource Conservation and Recovery Act \\
\hline Rwi(e) & Ringold Formation Wood Island subunit E \\
\hline TEM & transmission electron microscopy \\
\hline UFA & unsaturated flow apparatus \\
\hline $\mathrm{XRD}$ & $\mathrm{x}$-ray diffraction \\
\hline $\mathrm{XRF}$ & x-ray fluorescence \\
\hline
\end{tabular}




\section{Contents}

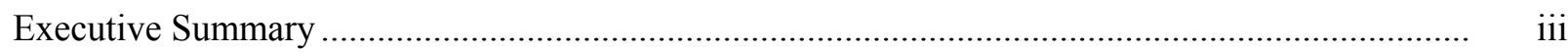

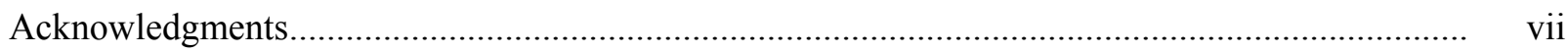

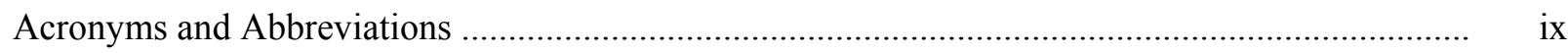

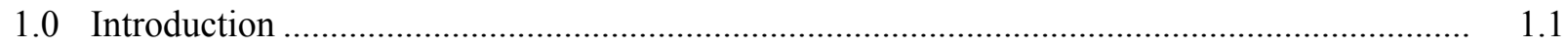

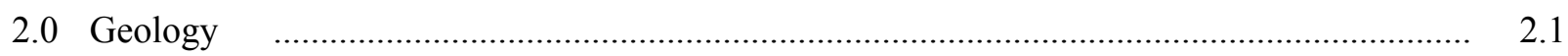

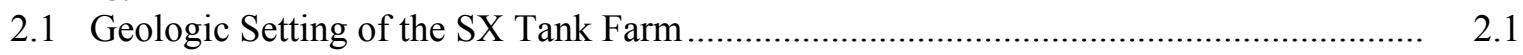

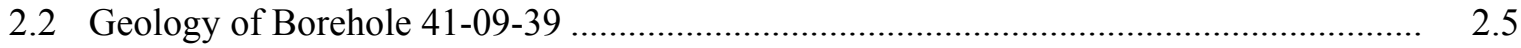

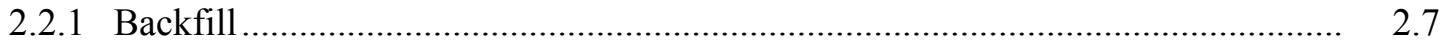

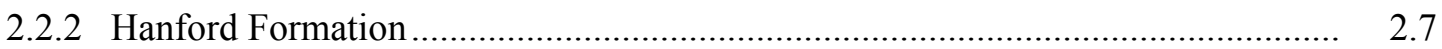

2.2.3 Plio-Pleistocene Unit....................................................................... 2.12

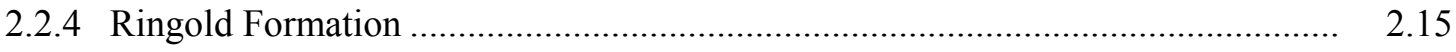

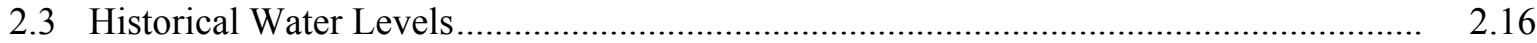

3.0 Geochemical Method and Materials ........................................................................ 3.1

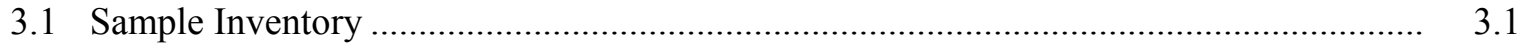

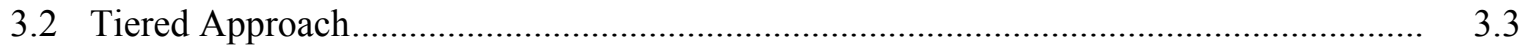

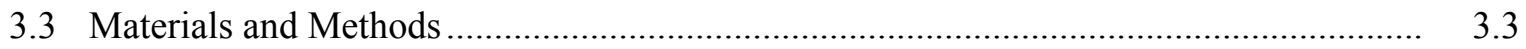

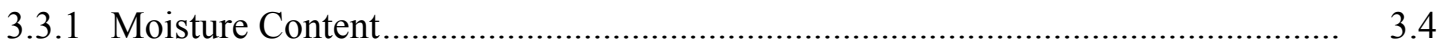

3.3.2 1:1 Sediment-to-Water Extract................................................................ 3.5

3.3.3 Radionuclide Analysis........................................................................ 3.6

3.3.4 Water Potential (Suction) Measurements …..................................................... 3.6

3.3.5 Borehole Extension Composite Samples - Materials and Methods ...................... 3.7

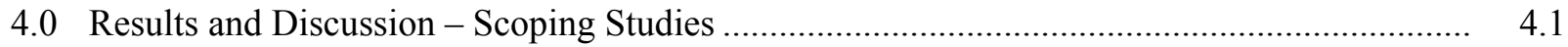

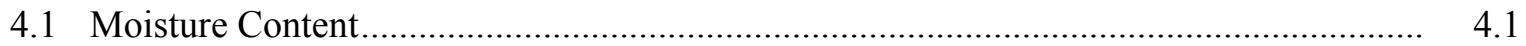

4.2 1:1 Sediment-to-Water Extract Chemistry ............................................................. 4.4

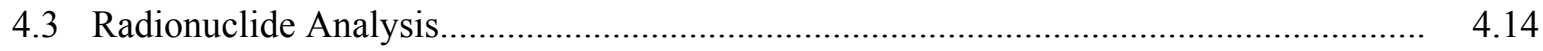

4.4 Soil Suction Measurements - Results and Discussion ............................................... 4.25

5.0 Composite Sample Results and Discussion .................................................................. 5.1

5.1 Moisture Content and 1:1 Sediment-to-Water Extract Electrical

Conductivity and $\mathrm{pH}$ for Borehole Extension Composites ........................................... 5.1

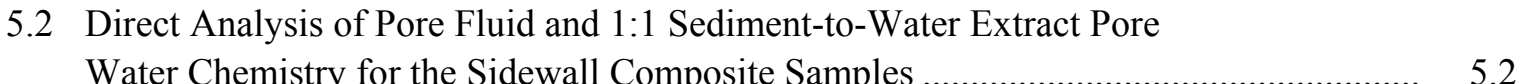

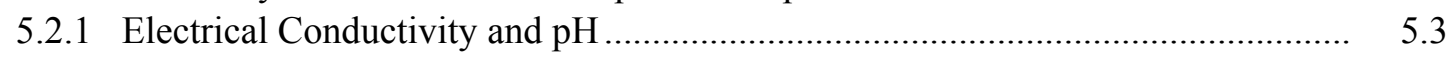

5.2.2 Chemical Composition ................................................................................ 5.3 
5.2.3 Radionuclide Analysis......................................................................... 5.18

5.3 Characterization Results and Discussion for Composite Sediments ............................. $\quad 5.21$

5.3.1 Radionuclide Content of Composite Sidewall Cores and Borehole Extension

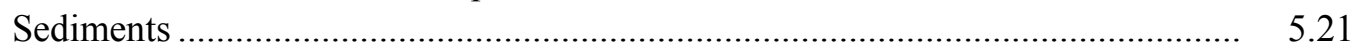

5.3.2 Total Chemical Composition of Composite Sediments ................................... 5.26

5.3.3 Acid-Leachable Cesium-137 versus Direct Sediment Cesium-137 Analysis ........ 5.34

5.3.4 Total Carbon Content of Composite Sediments .................................................. 5.35

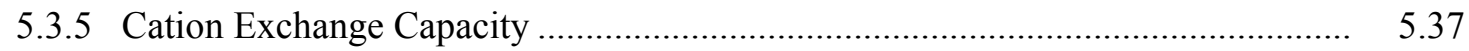

5.3.6 Particle Size Determination............................................................................. 5.38

5.3.7 Mineralogy of Borehole 41-09-39 ..................................................................... 5.43

5.3.8 Cesium-137 Content as a Function of Particle Size ........................................... 5.48

5.3.9 Macro Cation and Trace Metals in Various Particle Size Splits from Borehole Extension Composites ................................................................................. 5.51

5.3.10 Strontium and Cesium $\mathrm{K}_{\mathrm{d}}$ Values - Results and Discussion.............................. 5.55

6.0 Summary and Conclusions ........................................................................................

6.1 Lithologic Model of the Geology at Borehole 41-09-39............................................ 6.1

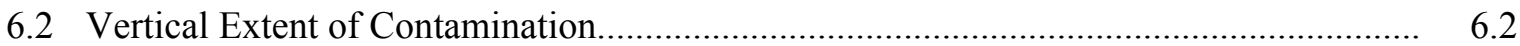

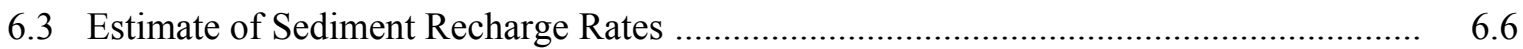

6.4 Detailed Characterization to Elucidate Controlling Geochemical Processes ................... 6.7

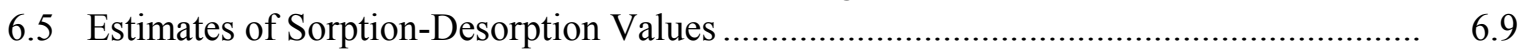

6.6 Other Characterization Observations ..................................................................... 6.11

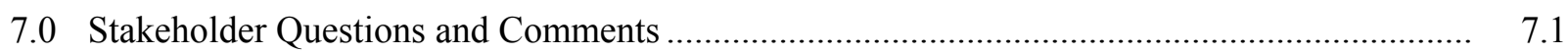

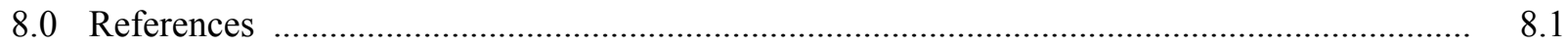

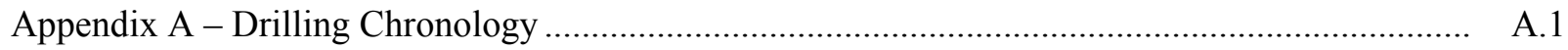

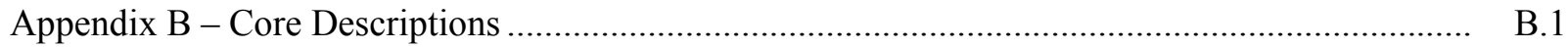

Appendix C - Geologic Descriptions ................................................................................... C.1

Appendix D - Particle Size Data and Folk/Wentworth Classification ........................................... D.1

Appendix E - Details on Mineralogy for Borehole 41-09-39 _....................................................... E. 1 


\section{Figures}

2.1 Generalized, Composite Stratigraphy for the Late Cenozoic Sediments Overlying the Columbia River Basalt Group on the Hanford Site...

2.2 Location of Borehole 41-09-39, Other Pertinent Wells, and Cross Sections .......................... 2.3

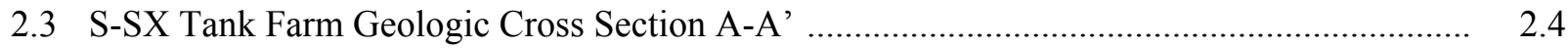

2.4 S-SX Tank Farm Geologic Cross Section B-B' .................................................................. 2.4

2.5 Correlation of Gamma-Ray, Drilling Resistance, and Moisture Logs for the Upper Portion of Borehole 41-09-39

2.6 Detailed Lithology and Geophysical Data from the Lower Portions of Borehole 41-09-39.... $\quad 2.8$

2.7 Lithologic and Analytical Data Correlations for the Lower Portion of Borehole 41-09-39..... 2.9

2.8 Backfill Materials from Composite Sample S9018-14A, B, and C.

2.9 Upper Fine Sand and Mud Sequence of the Hanford Formation H1a Unit from Composite Sample S9018-12A, B, and C.....

2.10 Middle Coarse Sand and Gravel Sequence of the Hanford Formation H1 Unit from Composite Sample S9018-07A, B, and C .

2.11 Lower Fine Sand and Mud Sequence of the Hanford Formation H2 Unit from Composite Sample S9018-03A, B, and C

2.12 Muddy Very Fine Sand of the Plio-Pleistocene Mud (PPlz) Unit.

2.13 Mud of the Plio-Pleistocene Mud (PPlz) Unit from Sample S7061-6C...

2.14 Broken-Up Pedogenic Carbonate Fragments from the Plio-Pleistocene Carbonate (PPlc) Unit.

2.15 Fragmented Ringold Gravels from Sample No. S7061-22A.

2.16 Historic Water Levels Beneath the SX Tank Farm

2.17 Disaggregated Core Sample S0070-17A from the Upper Plio-Pleistocene Mud Unit.....

4.1 Moisture Content Versus Depth for Vadose Sediment from Borehole 41-09-39.

$4.2 \mathrm{pH}$, and Dilution Corrected Electrical Conductivity, and Nitrate Concentrations in Water Extracts

4.3 Dilution-Corrected Cation Concentrations in 1:1 Sediment-to-Water Extracts

4.4 Cesium-137, Technetium-99, and Tritium Activity in Sediment versus Lithology and Depth.

4.5 Steady-State Water Content and Suction Profiles for Representative 200 West Area Sediment above a 67-Meter (219.8 feet)-Deep Water Table

4.6 Matric Suction Profile for Samples Taken from Borehole 41-09-39....

4.7 Water Content of Selected Sleeve Sediment Samples from Borehole 41-09-39 after Equilibrating at 100-Centimeter (39.4-Inch) Matric Suction Compared to Water Contents as Stored in Containers......

5.1 Dilution-Corrected Cations in 1:1 Sediment-to-Water Extracts and Actual Porewaters Versus Lithology and Depth. 
5.2 Dilution-Corrected 1:1 Sediment-to-Water Extract Anions and Actual Porewater Anion

Concentrations versus Lithology and Depth

5.3 Cesium-137, Technitium-99, Strontium-90, and Tritium in Sediments from

Table 5.11 Versus Depth with Geology Breaks

5.4 Particle Size Distribution of Sidewall Core in Hanford Formation Unit H1a.....

5.5 Particle Size Distribution of Sidewall Cores in Hanford Formation Unit H1

5.6 Particle Size Distribution of Sidewall Cores in Hanford Formation Unit H2

5.7 Particle Size Distribution of Borehole Extension Sediment in Plio-Pleistocene

5.8 Particle Size Distribution of Borehole Extension Composites

5.9 Particle Size Distribution of Borehole Extension Sediment in Ringold Formation Unit E

5.10 Typical Illite Particle from Depth 22.7 meters

5.11 Large Chlorite Particle ( $\sim 3$ microns) Surrounded by Smaller Illites and Smectites.

5.12 Large Magnesium-Rich Chlorite....

5.13 Percent of Cesium Found in Each Size Fraction of Composite 2C/D

5.14 Percent of Cesium Found in Each Size Fraction of Composite 2A/B

5.15 Percent of Cesium Found in Each Size Fraction of Composite 3A/B

\section{Tables}

3.1 Nuclides in SX Gamma Library

3.2 Chemical Composition of Hanford Site Groundwater from Well 699-S3-25.

4.1 Moisture Content of Sidewall Cores and Extension Sleeves.

4.2 $\mathrm{pH}$ and EC for 1:1 Water Extracts of Sidewall and Borehole Extension Sleeves

4.3 Anion Concentrations in 1:1 Water Extracts.

4.4 Major Cation Composition of 1:1 Water Extracts from Sidewall Cores and Borehole Extension Sleeves

4.5 Cesium-137 Activity in Sidewall Cores and Borehole Extension Sleeves.

4.6 Cesium-137 Activity in Carefully Sub-Cored Sleeves

4.7 Natural Gamma Emitter Activities in Sidewall Cores and Borehole Extension Sleeves

4.8 Technitium-99 and Strontium-90 Concentrations in Sidewall Cores and Borehole Sleeves at Borehole 41-09-39

4.9 Water Content and Matric Suction Values for Samples from Borehole 41-09-39

5.1 pH, Electrical Conductivity, and Moisture Content of the Composite Samples

5.2 Electrical Conductivity and $\mathrm{pH}$ of Porewater from Sidewall Cores and Water Extracts of Sidewall Cores and Composite Samples from Borehole Extension

5.3 Anion Composition of 1:1 Water Extracts and Porewaters from Sidewall Cores and Borehole Extension Composites 
5.4 Major Cation Composition of 1:1 Water Extracts and Porewaters from Sidewall Cores and Borehole Extension Composites

5.5 Trace Metal Composition of 1:1 Water Extracts and Porewaters from Sidewall Cores and Borehole Extension Composites

5.6 High Z Trace Metal Composition of 1:1 Water Extracts and Porewaters from Composite Sediments

5.7 Dilution-Corrected Anion Composition of 1:1 Water Extracts and Porewaters from Sidewall Cores and Borehole Extension Composites.

5.8 Dilution-Corrected Major Cation Composition of 1:1 Water Extracts and Porewaters from Sidewall Cores and Borehole Extension Composites

5.9 Dilution-Corrected Trace Metal Composition of 1:1 Water Extracts and Porewaters from Sidewall Cores and Borehole Extension Composites

5.10 Radionuclide Content of Water Extracts and Porewater.

5.11 Total Radionuclide Content of Sediment Samples.

5.12 Actinide Content of the Sidewall Cores ....

$5.13 \mathrm{~K}_{\mathrm{d}}$ Estimates from Total Activity in Sediments and Porewaters

5.14 Total Concentration of Selected Constituents in Borehole Extension Composite Sediment ....

5.15 Acid Extractable Elements in Sidewall Cores

5.16 Chromium Mobility Status in the Borehole 41-09-39 Sediments .....

5.17 Cesium-137 Agreement Between Direct Counting and Acid Extraction ...........

5.18 Total Carbon Content of Borehole 41-09-39 Sediment Samples

5.19 Cation Exchange Capacity of the Composite Sediments

5.20 Particle Size Distribution of Sidewall Cores and Composite Sediments.

5.21 Details of Particle Size Distribution of Sidewall Core and Borehole Extension Composite Sediments.

5.24 Semiquantitative Mineral Composition for the Clay Fraction $(<2 \mu \mathrm{m})$ of Sediment Samples

5.26 Chemical Composition of Various Size Fractions of 2CD Composite Sediment.

5.27 Chemical Composition of Various Size Fractions of 2AB Composite Sediment.

5.28 Chemical Composition of Various Size Fractions of 2CD Composite Sediment.

5.29 Adsorption Coefficients $\left(\mathrm{K}_{\mathrm{d}}\right)$ for Strontium-90 for Three Sediment Types of Four Solutions 


\subsection{Introduction}

The overall goal of the of the Tank Farm Vadose Zone Project, led by CH2M HILL Hanford Group, Inc., is to define risks from past and future single-shell tank farm activities, to identify and evaluate the efficacy of interim measures, and to aid, via collection of geotechnical information and data, the future decisions that must be made by the U.S. Department of Energy regarding the near-term operations, future waste retrieval, and final closure activities for the single-shell tank waste management areas. For a more complete discussion of the goals of the Tank Farm Vadose Zone Project, refer to the overall work plan, Phase 1 RCRA Facility Investigation/Corrective Measures Study Work Plan for the Single-Shell Tank Waste Management Areas (DOE/RL-1999). To meet these goals, CH2M HILL Hanford Group, Inc., asked scientists from Pacific Northwest National Laboratory to perform detailed analyses on vadose zone sediment from within the S-SX Waste Management Area.

This report is one in a series of four reports to present recent data collected on vadose zone sediments, both uncontaminated and contaminated, from within the S-SX Waste Management Area. Preliminary interpretations identifying the distribution of key contaminants within the vadose zone and what their future migration potential are also included. The information will be incorporated in the field investigation report ${ }^{(a)}$. This series of documents describe the findings for 1) uncontaminated boreholes surrounding the S-SX Waste Management Area, 2) the 41-09-39 borehole within the SX Tank Farm, 3) the SX-115 borehole (B8809) that has been converted into a Resource Conservation and Recovery Act (RCRA) groundwater monitoring well 299-W23-19, and 4) the SX-108 slant borehole that penetrated below tank SX-108.

Specifically, this report contains all the geologic, geochemical, and selected physical and hydrologic characterization data collected on vadose zone sediments recovered from borehole 41-09-39. We also provide our interpretation of the data in the context of determining the appropriate geologic conceptual model, the vertical extent of contamination, the migration potential of the contaminants that still reside in the vadose zone, and the correspondence of the contaminant distribution in the borehole sediments in relationship to groundwater plumes in the aquifer proximate and downgradient from the SX Tank Farm.

This report is divided into sections that describe the geologic stratification, the geochemical characterization methods employed, the geochemical results, summary and conclusions, a listing of stakeholder questions and comments, references cited, and several appendixes.

(a) Draft Field Investigation Report for Waste Management Area S-SX. RPP-7884, Draft, Volume 2, Appendix D, CH2M HILL Hanford Group, Inc., Richland, Washington. 


\subsection{Geology}

The geology of the vadose zone underlying the SX Tank Farm forms the framework through which the contaminants move and the physical structure that, along with geochemistry properties, controls the migration and distribution of contaminants. Of particular interest are the interrelationships between the coarser- and finer-grained facies, and the degree of contrast in their physical and geochemical properties.

This section presents a brief discussion on the geologic setting of the tank farm. This is followed by brief discussions on the drilling, sampling, and geophysical logging of borehole 41-09-39, and a detailed description of the geologic materials penetrated by borehole 41-09-39 (299-W23-234).

\subsection{Geologic Setting of the SX Tank Farm}

The SX Tank Farm was constructed into the upper Hanford formation sediments underlying the 200 West Area, along the north limb of the Cold Creek syncline. Sedimentary units underlying the tank farm (in descending order), include lower Hanford formation sediments, the Plio-Pleistocene unit, and the Miocene to Pliocene Ringold Formation (Figure 2.1).

The geology beneath this tank farm has been the subject of numerous reports. Price and Fecht (1976a) presented an initial detailed interpretation of the geology. DOE (1996) presented their interpretation of the geology based primarily on groundwater monitoring wells constructed around the perimeter of the tank farm in the early 1990s. Johnson and Chou (1998) updated and refined the geologic interpretation. Myers et al. (1998) presented detailed discussions on the geologic materials penetrated by the extension of borehole 41-09-39, and forms the basis for much of the discussion presented throughout the remainder of this section. Johnson et al. (1999) further described the geology and other subsurface conditions beneath the S and SX Tank Farms relevant to the occurrence and migration of contaminants. Lindsey et al. (2000) provided additional interpretations on the geology, facilitated by the collection of near continuous split-spoon samples from the 299-W22-50 and 299-W23-19 boreholes. Horton and Johnson (2000) compiled a data package on three groundwater-monitoring wells (299-W22-48, -49, and 50) completed near the SX Tank Farm in 1999/2000. Most recently, Socbczyk (2000) presented a reinterpretation on the geology based on gross gamma-ray logs of 98 boreholes within the SX Tank Farm and several published geology reports of the area (e.g., Johnson et al. 1999; and Lindsey et al. 2000). Khaleel et al. (2000) prepared a detailed data package that included geologic and hydrologic descriptions to support numerical simulation of the S and -SX Tank Farms. Serne et al. (2002a, b, and c) present an update of the geologic setting of the SX Tank Farm based on detailed analyses of vadose zone sediment, both uncontaminated and contaminated, from boreholes within the S-SX Waste Management Area. 


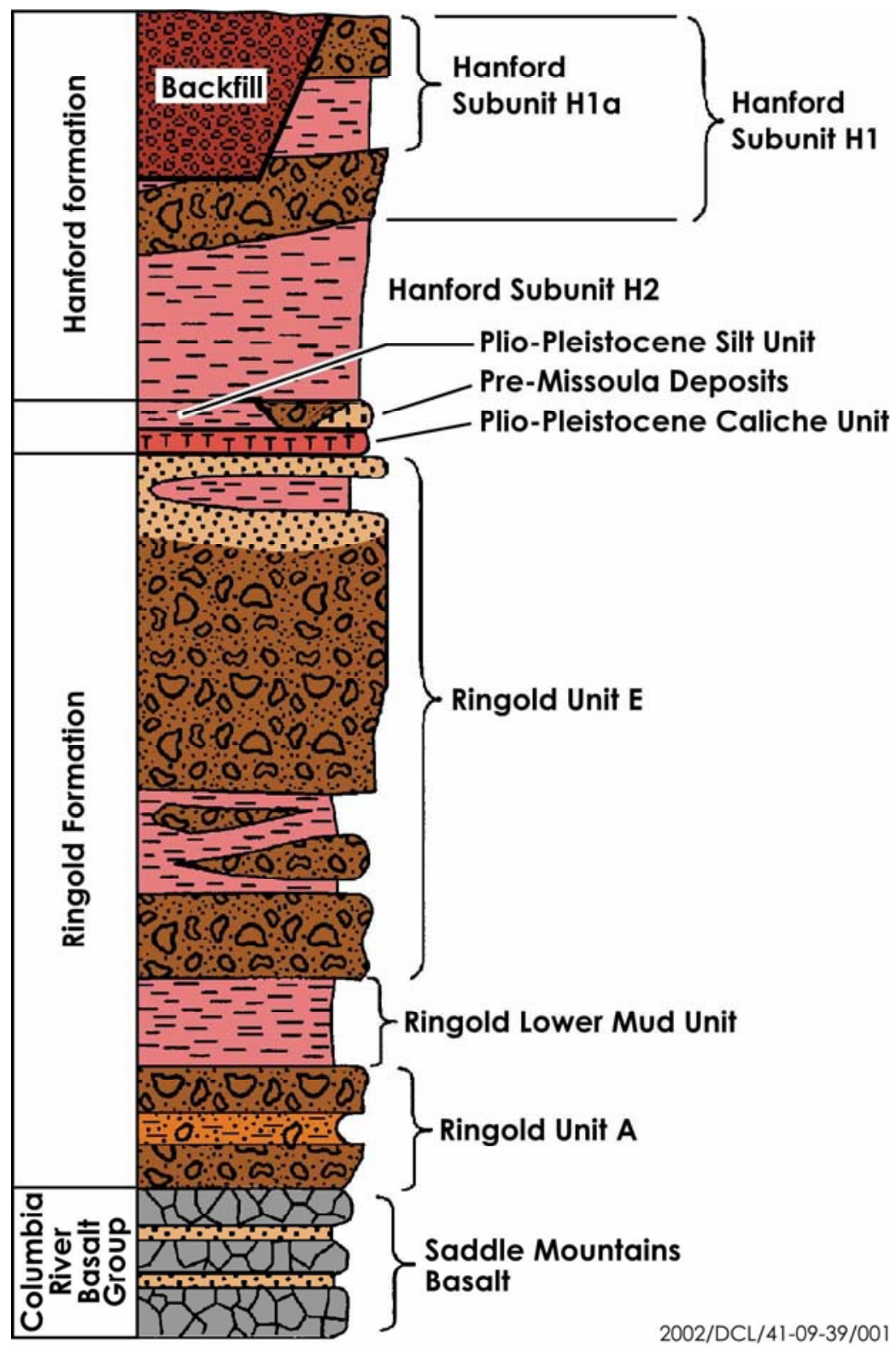

Figure 2.1. Generalized, Composite Stratigraphy for the Late Cenozoic Sediments Overlying the Columbia River Basalt Group on the Hanford Site (after Johnson and Chou 1998, 1999) 
Figure 2.2 is a location map of borehole 41-09-39 and wells used to create the cross sections shown in the following figures. The stratigraphy beneath the SX Tank Farm is illustrated in Figures 2.3 and 2.4. Some slight discrepancies may occur between the depths of the geologic contacts presented here, and those presented by other authors, because of various sources of uncertainty in the geologic data sets and the individual geologist's interpretation. Johnson et al. (1999) described the various sources of uncertainty for locating the stratigraphic contacts within a borehole as well as those uncertainties for drawing correlations between boreholes. They identified the principal sources of uncertainty as related to the drilling and sampling techniques, logging of the boreholes, and uncertainties in the geometric shape of the sedimentary units. They used two different geostatistical techniques to evaluate the stratigraphy/depth uncertainty and found that both techniques indicated that the stratigraphy beneath the S and SX Tank Farms is relatively consistent across the area. They also found that the optimal depths for stratigraphic correlations between different data sets were typically accurate to within 1 to 3 meters (a few feet to 10 feet).

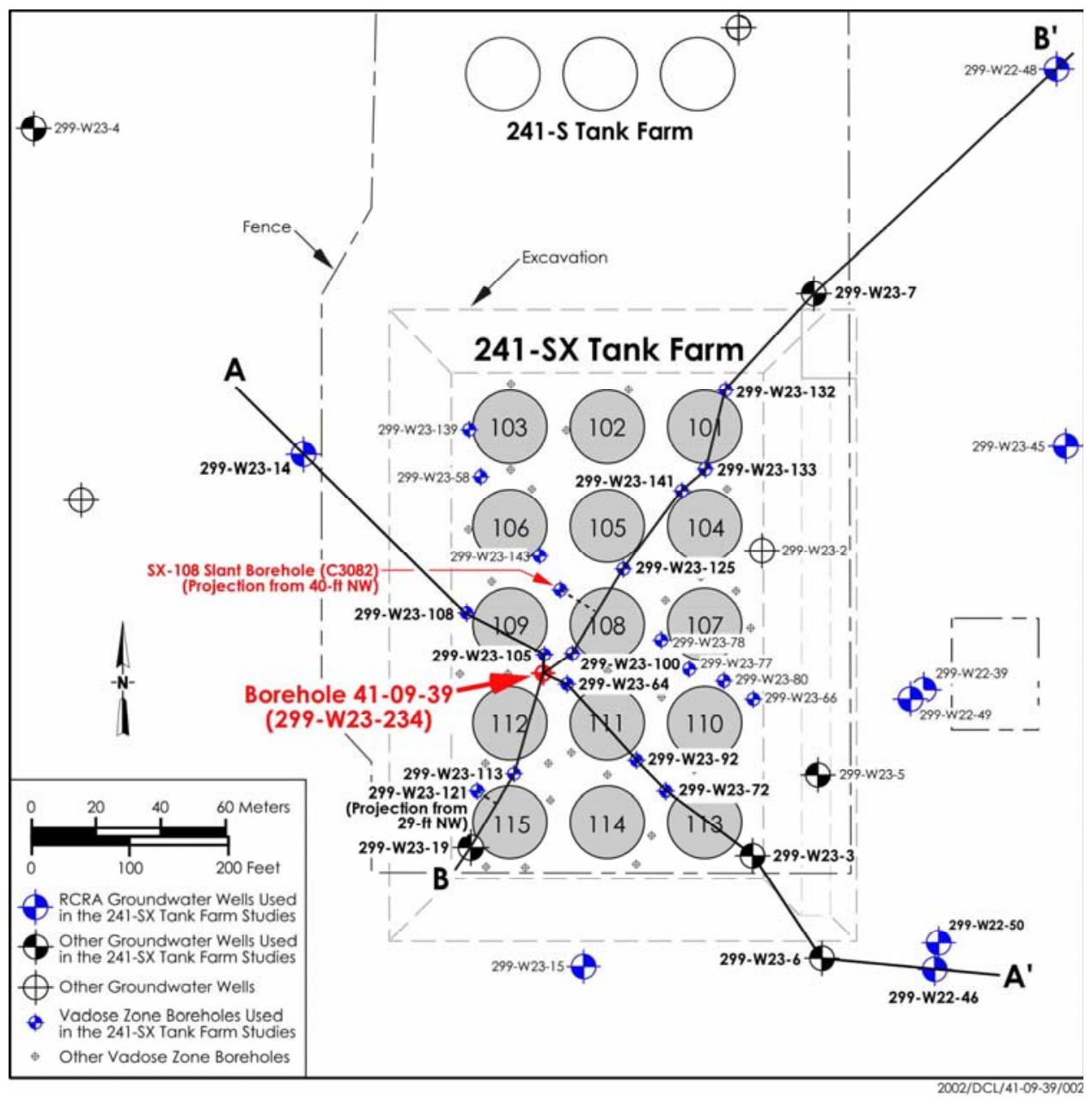

Figure 2.2. Location of Borehole 41-09-39, Other Pertinent Wells, and Cross Sections. A-A' and B-B' are locations of cross sections depicted in Figures 2.3 and 2.4. 

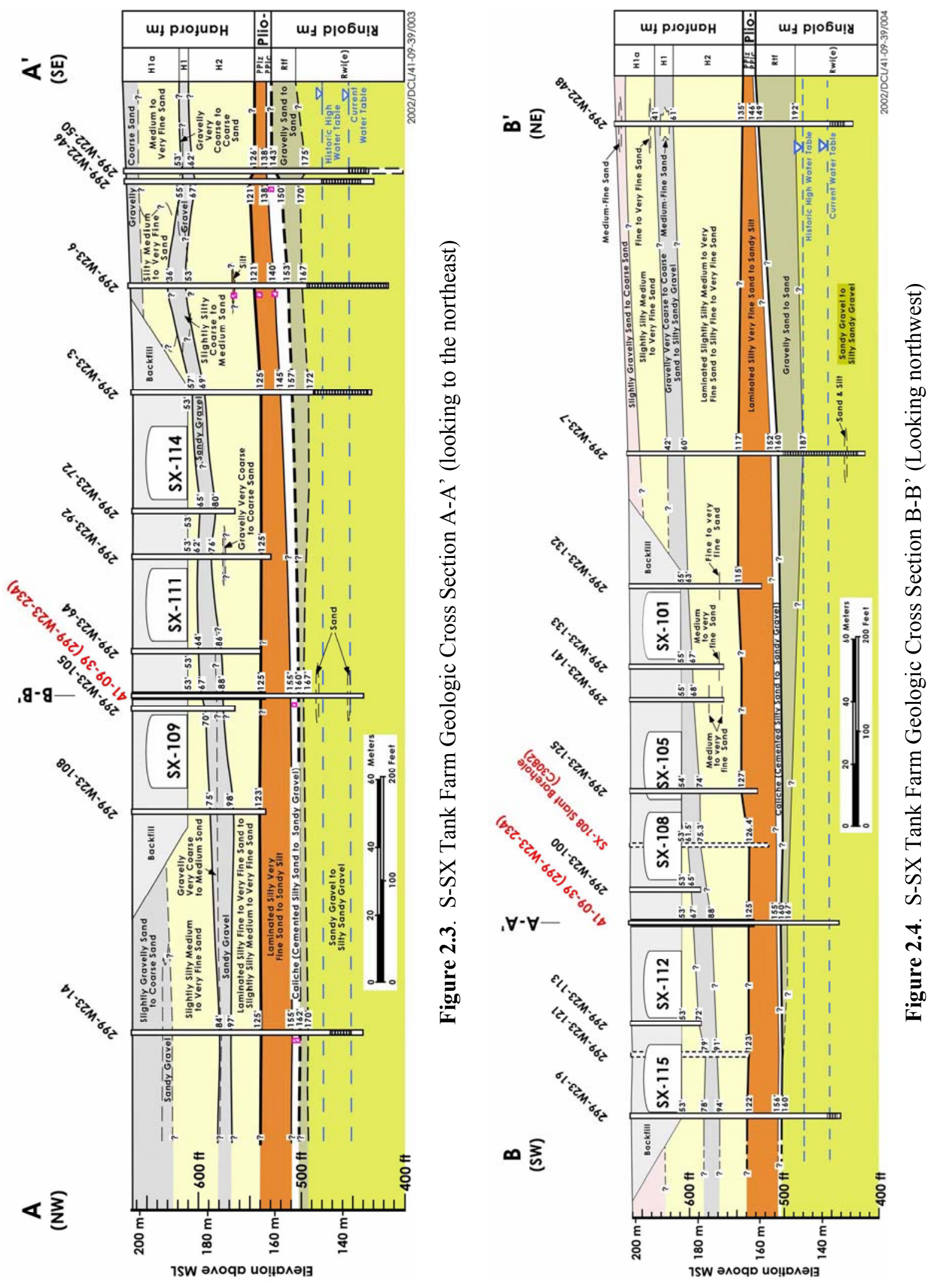


\subsection{Geology of Borehole 41-09-39}

Borehole 41-09-39 (299-W23-234) was originally installed in December 1996. This borehole was installed by driving a 15-centimeter (6-inch)-inside-diameter thick-wall casing and drive point to a depth of 39.8 meters (130.5 feet). The construction of this portion of the borehole was summarized by MACTEC-ERS (1997). The drive point was milled off the end of the casing and the borehole deepened between September 5 and December 19, 1997. A cable-tool drilling rig was used to deepen and collect samples from the borehole to a depth of 68.6 meters (225 feet). Split-spoon samples were collected whenever possible. Myers et al. (1998) provided a summary of the chronology of the drilling and sampling activities and presented the detailed geologic descriptions of the individual split-spoon samples (performed in the laboratory). These are reproduced in Appendix A and B, respectively.

The borehole was decommissioned between May 27 and August 11, 1999. Sidewall core samples were collected at selected intervals throughout the top portion of the borehole (i.e., that portion completed in 1996, and not previously sampled). Three cores were collected from each sample interval at approximately 90 degrees to each other and generally oriented to the north, south, and west. All material from all three sidewall cores at a specific depth was composited in the laboratory into a single sample tray for geologic description and subsampling, with the exception of one depth. For samples from 19.7 meters (65 feet) below ground surface (bgs), it was noted that one sidewall core was reading 10x higher dose than the other two cores and it was treated like a separate sample. A summary of the geologic descriptions made in the laboratory is provided in Appendix C. Note however, that the geologic descriptions of these small ( 2.5 centimeters $\times 2.5$ centimeters $\times 28$ centimeters) sidewall core samples may not be wholly representative of the native geologic materials. These cores likely penetrated (on average) about 23 centimeters ( 9 inches) into the borehole wall and include disturbed material severely compacted around the casing during its pile-driving type installation. The small size also prevented recovery of larger gravel clasts.

Procedures ASTM D2488 (ASTM 1993) and PNL-MA-567-DO-1 (PNL 1990) were followed during visual description and recording of all sidewall and split-spoon samples. The sediment classification scheme used for identification of the soil types is based on the modified Folk/Wentworth classification scheme (Fecht and Price 1977). This sediment classification scheme uses a tertiary diagram to categorize the sediment into one of 19 classes based on the relative proportions of gravel, sand, and mud (silt+clay). The terms mud and silt are used rather interchangeably throughout this document since the mud fraction of these sediments is predominantly silt.

Figure 2.5 presents a generalized stratigraphic section for the upper 40 meters ( 130 feet) of the borehole. Note that very little formation materials (with questionable representativeness) were recovered from the upper 40 meters ( 130 feet) of the borehole. Thus, the stratigraphy was interpreted from a synergistic interpretation of multiple geophysical logs and the drilling resistance log taken from 


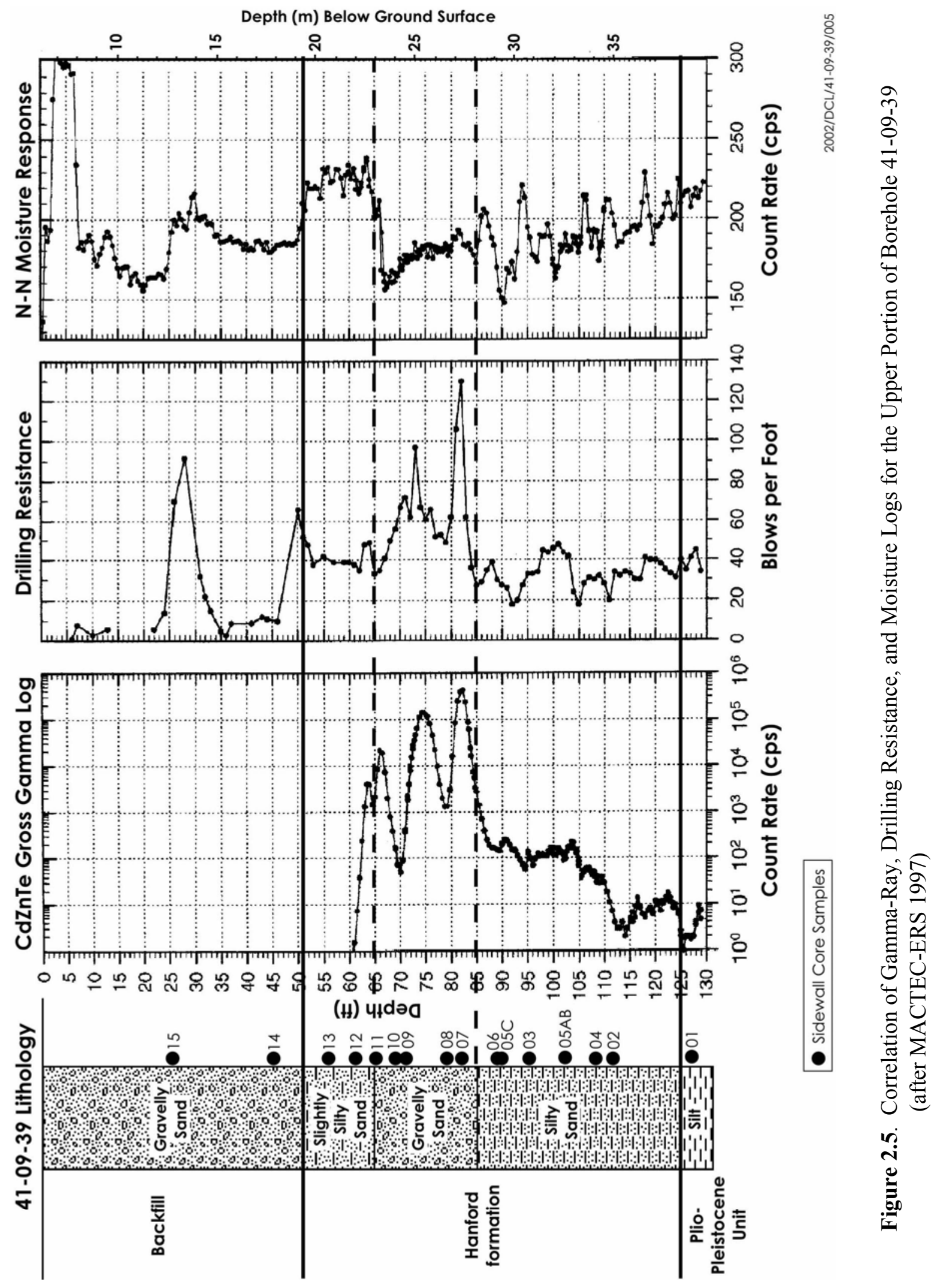


MACTEC-ERS (1997) in conjunction with the laboratory descriptions and analytical results of the sidewall core samples. This portion of the borehole intersected three formations: 1) backfill around the high-level waste tanks, 2) the Hanford formation, and 3) the top of the Plio-Pleistocene unit. The backfill appears to extend to a depth of about 15.9 meters ( 51 feet) where it is in contact with the Hanford formation. The next 4.3 meters (14 feet) is interpreted to correlate with the upper fine sand and mud sequence of the Hanford formation H1a unit. Below this lies the 6-meter (20-foot)-thick middle coarse sand and gravel sequence (Hanford formation $\mathrm{H} 1 \mathrm{unit}$ ). Note that interpretation of the recent sidewall cores and more recent geophysical logs suggests that this unit is $\sim 4$ meters (12 feet) thinner than previously interpreted by Myers et al. (1998). This is underlain by 12.1 meters (40 feet) of the lower fine sand and mud sequence (Hanford formation $\mathrm{H} 2$ unit). The contact between the Hanford formation and the Plio-Pleistocene unit is interpreted to occur at a depth of 38 meters (125 feet).

The lower portion of the borehole (Figures 2.6 and 2.7), from depths of 40 to 69 meters (130 to 225 feet), penetrates the Plio-Pleistocene unit and upper portion of the Ringold Formation. Interpretations are based on visual inspection of split-spoon samples and borehole geophysical logs, as well as $\mathrm{CaCO}_{3}$ and moisture analytical results, drillers' field activity reports, samplers' field notes and blow counts.

Detailed descriptions summarizing the observations and physical soil properties for each of the major stratigraphic units penetrated by this borehole are presented below.

\subsubsection{Backfill}

Only two sets of sidewall cores (S9018-15 and S9018-14) were collected from the backfill region. These samples were described as olive brown to very dark grayish brown very fine to medium or very fine to coarse sand, with some fine to very fine pebble, and variable mud content (Figure 2.8). The samples were moist and exhibited a strong reaction to hydrochloric acid. There were no obvious sedimentary structures observed in the composite samples.

\subsubsection{Hanford Formation}

Sidewall core samples were collected from all three Hanford formation subunits present beneath the backfill.

Upper Fine Sand and Mud Sequence (Hanford formation H1a unit). Samples S9018-13 and S9018-12 were collected from the upper fine sand and mud sequence directly beneath the backfill. These samples were described as olive brown and ranging from very fine to medium sand to slightly muddy very fine to medium sand, with cohesive clumps of mud (Figure 2.9). The samples were moist and exhibited a strong reaction to hydrochloric acid. No sedimentary structures were observed in the composite samples.

Middle Coarse Sand and Gravel Sequence (Hanford formation H1 unit). Six samples (S9018-11 through -07) were collected from the coarse unit at the base of the Hanford formation H1 unit. These samples were generally olive gray and ranged from very fine to coarse sand with some pebbles and/or pebble fragments up to medium pebble observed in each sample (Figure 2.10). Some dark grayish brown 
BOREHOLE 41-09-39 EXTENSION - LITHOLOGY AND GEOPHYSICAL LOG DATA

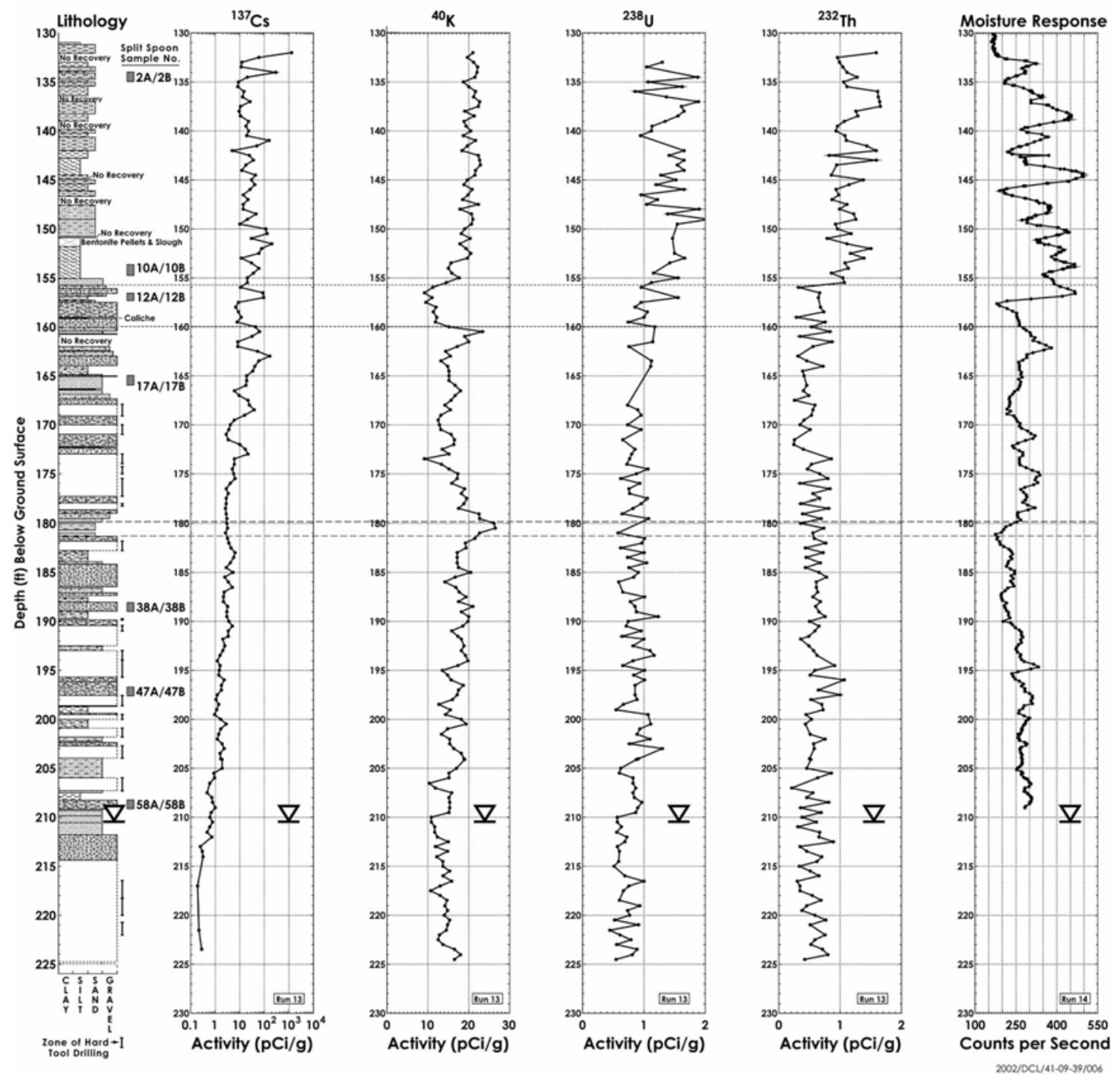

Figure 2.6. Detailed Lithology and Geophysical Data from the Lower Portions of Borehole 41-09-39

cohesive clumps of mud were also present. The samples were moist and generally exhibited a weak reaction to hydrochloric acid except for samples S9018-07 and -08, which exhibited stronger reactions. No sedimentary structures were observed in the composite samples.

Lower Fine Sand and Mud Sequence (Hanford formation H2 unit). Six samples (S9018-06 through -01) were collected from the fine sand and mud sequence correlated with Hanford formation $\mathrm{H} 2$ unit. These materials were described as olive brown to dark yellowish brown and ranging from mud to slightly muddy very fine to medium sand (Figure 2.11). All samples contained at least some weakly cemented mud clumps. The samples were moist and generally exhibited a strong reaction to hydrochloric acid with only weak reactions observed in some mud clumps. No sedimentary structures were observed in the composite samples. 


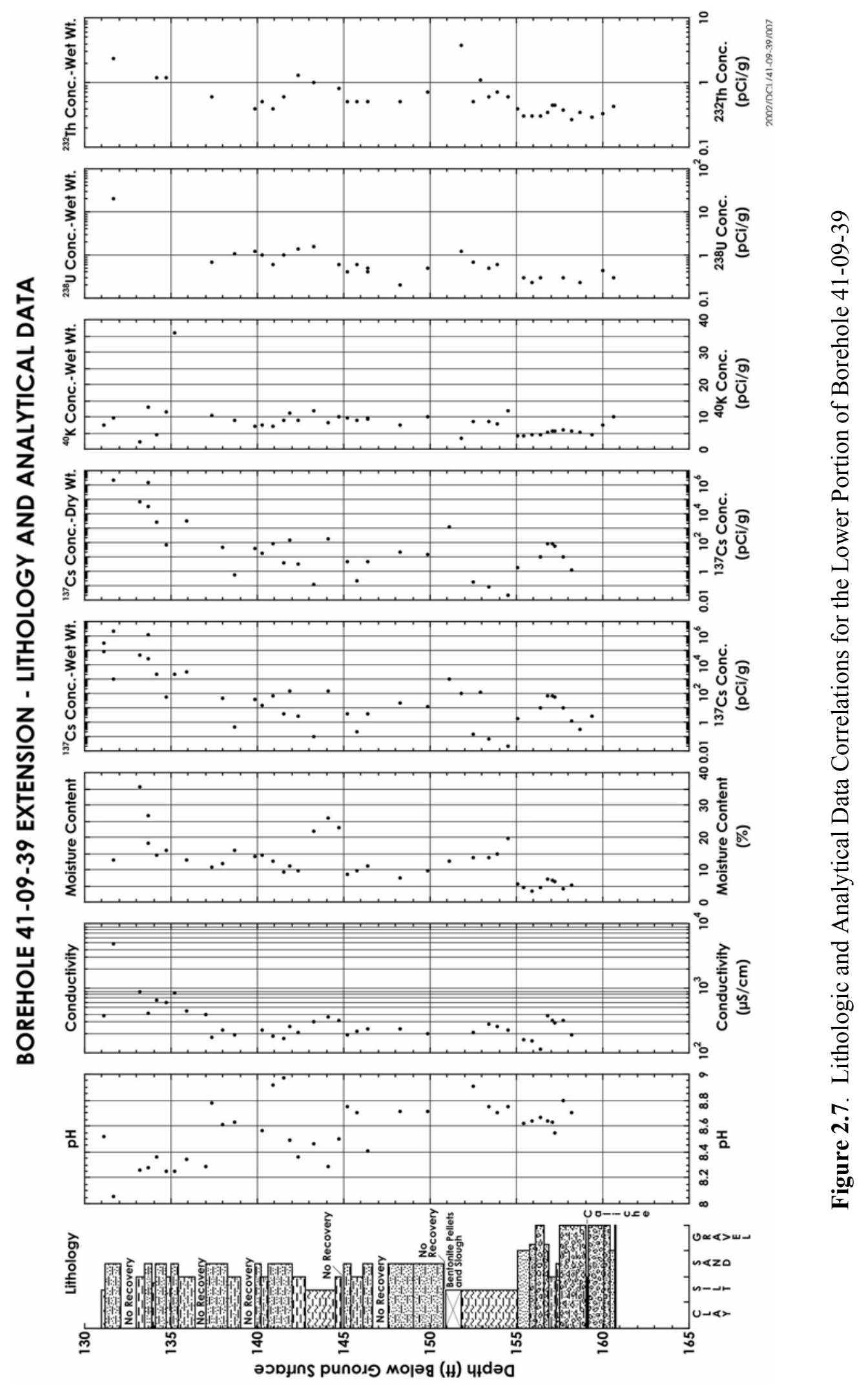




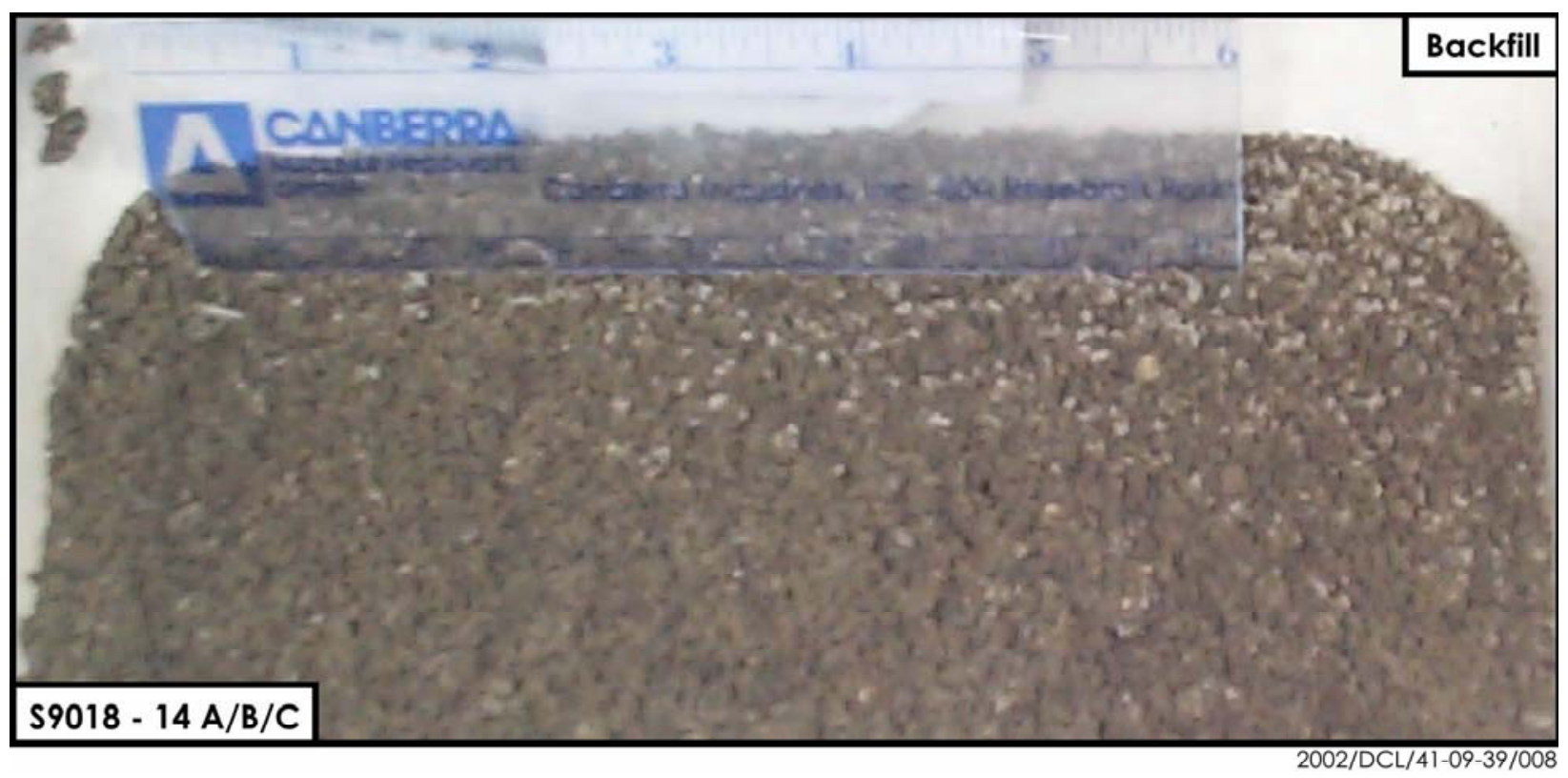

Figure 2.8. Backfill Materials from Composite Sample S9018-14A, B, and C (at a depth of 13.9 meters [45.5 feet])

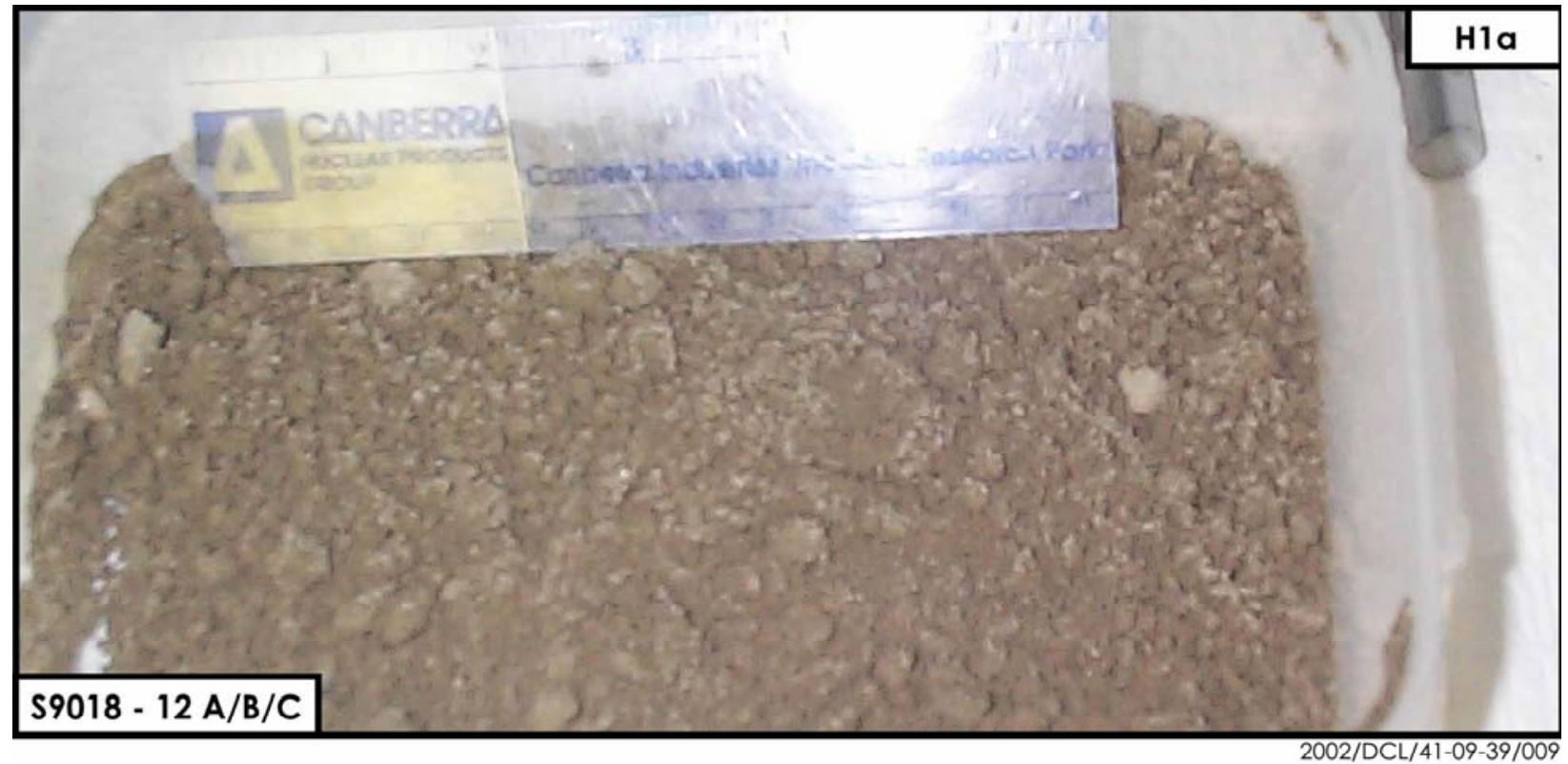

Figure 2.9. Upper Fine Sand and Mud Sequence of the Hanford Formation H1a Unit from Composite Sample S9018-12A, B, and C (at a depth of 18.7 meters [61.5 feet]) 


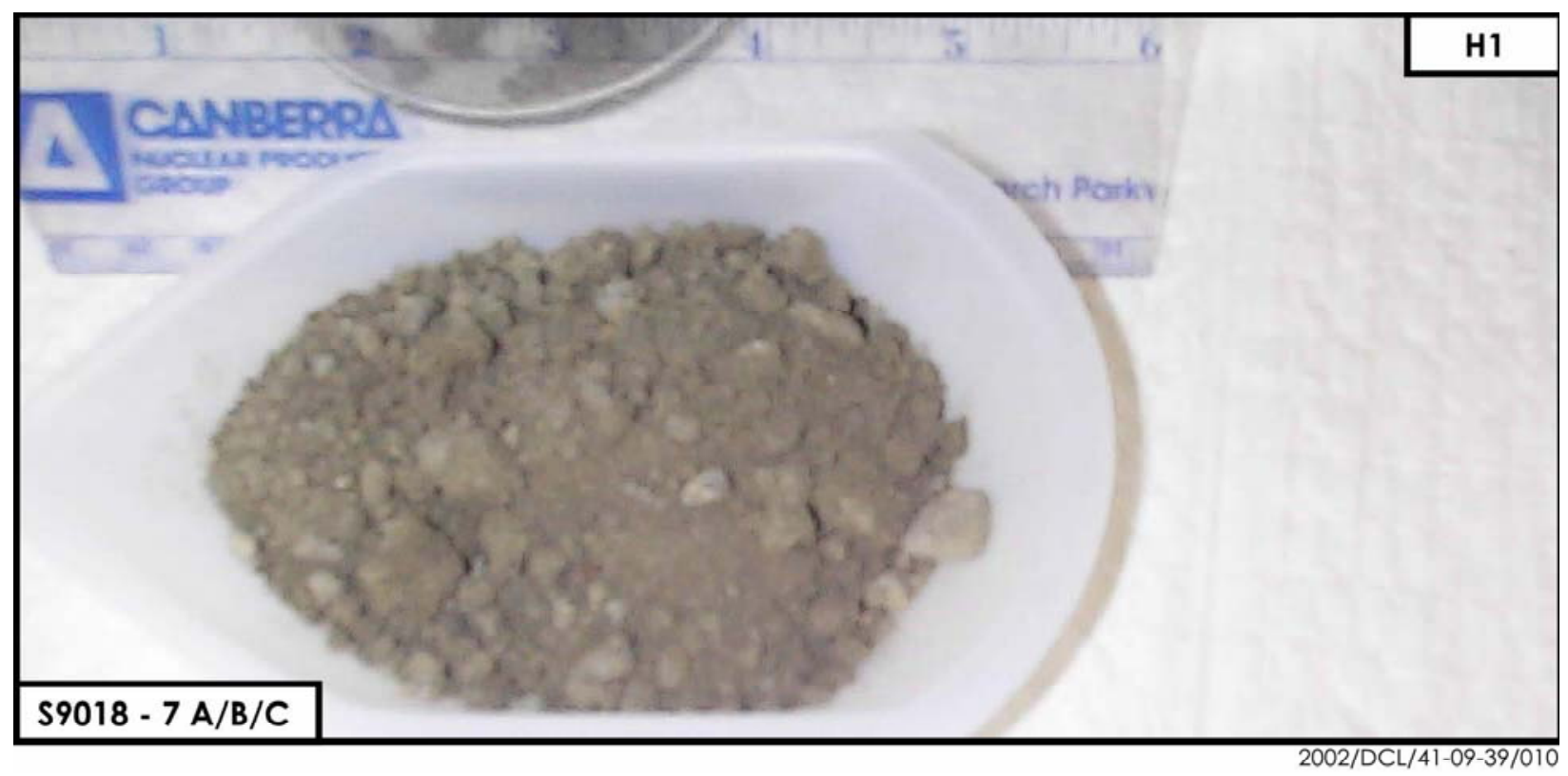

Figure 2.10. Middle Coarse Sand and Gravel Sequence of the Hanford Formation H1 Unit from Composite Sample S9018-07A, B, and C (at a depth of 25.1 meters [82.5 feet])

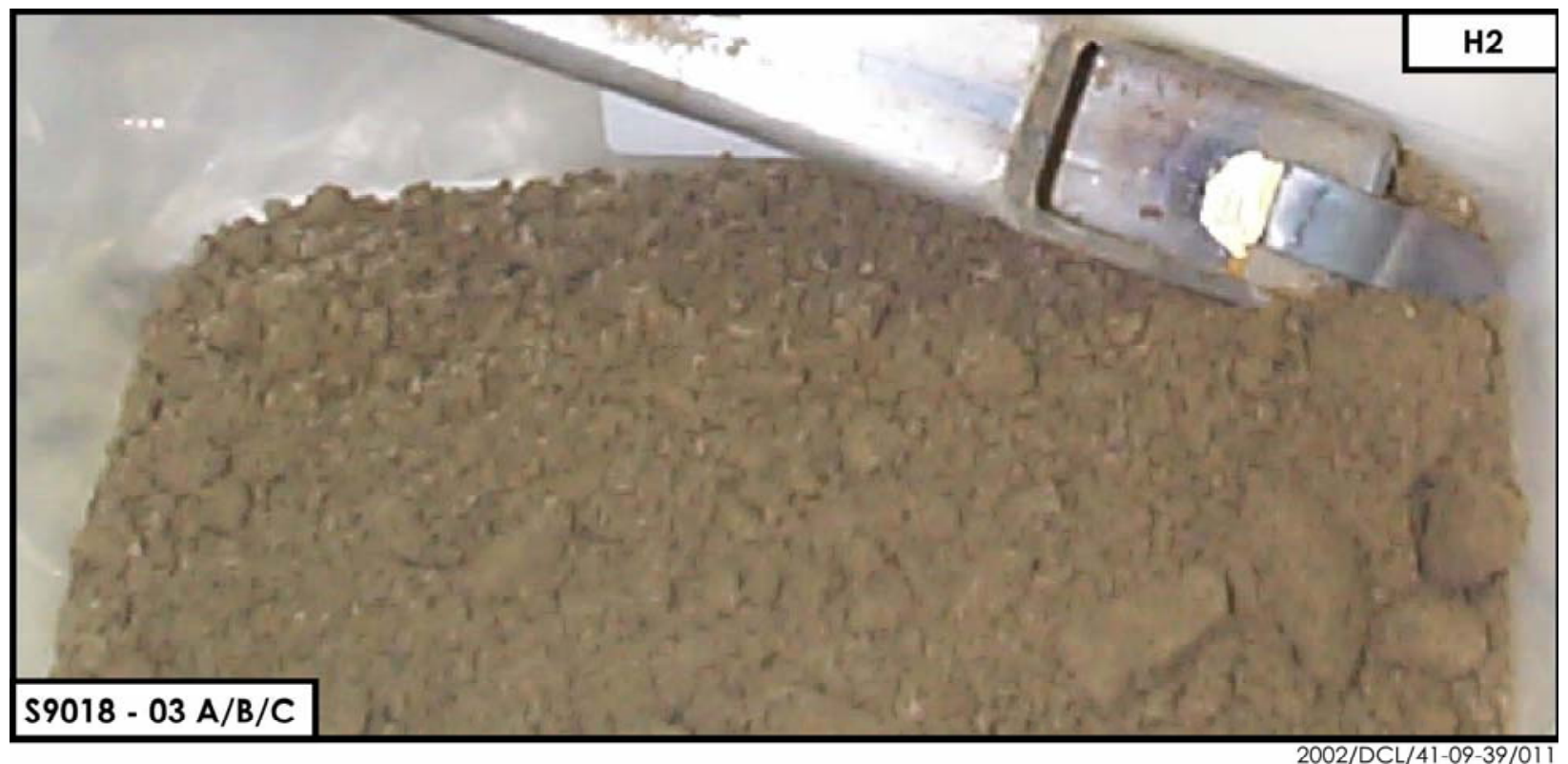

Figure 2.11. Lower Fine Sand and Mud Sequence of the Hanford Formation H2 Unit from Composite Sample S9018-03A, B, and C (at a depth of 29 meters [95.5 feet]). Note tip of sidewall core sampler. 


\subsubsection{Plio-Pleistocene Unit}

The Plio-Pleistocene unit (which includes all material overlying the Ringold Formation and underlying the Hanford formation, including the Early Palouse Soil, the Pre-Missoula Gravels [or equivalent], and the unnamed Hanford formation [?] or Plio-Pleistocene Deposits [?] described by Lindsey et al. 2000) has generally been differentiated from overlying fine-grained Hanford formation sediment by greater calcium carbonate content, more massive structure in core, and high natural gamma response in geophysical logs (DOE 1988; Bjornstad 1990). This unit was penetrated by borehole 41-09-39 between depths of 38.1 to 48.8 meters (125 to 160 feet) and consists of a relatively thick sequence of very fine sand to mud (the Plio-Pleistocene Mud unit), and a caliche zone (the Plio-Pleistocene Carbonate unit).

Very Fine Sand to Mud Sequence (Plio-Pleistocene Mud [PPlz] unit). Ten nearly continuous split-spoon samples (S7061-01 through -10) were collected throughout the lower 7.3 meters (24 feet) of the very fine sand to mud sequence. An eleventh sample (S7061-11) captures this unit's lower contact with the caliche zone. The very fine sand to mud sequence is characterized by relatively higher uranium238 and thorium-232 concentrations, which result in higher natural gamma response.

While this sequence can be distinguished from the overlying Hanford formation $\mathrm{H} 2$ unit by its finergrained texture, higher calcium carbonate content, and higher natural gamma response, its depositional history has come under some question. The higher natural gamma (thorium-232) results are evident in Figures 2.6 and 2.7. Lindsey et al. (2000) described a number of possible stratigraphic correlatives including: an older Touchet Bed-like Hanford formation deposit; an unnamed, locally derived, PlioPleistocene alluvial deposit; or overbank equivalents to the pre-Missoula gravels. Thus, they chose to refer to these materials as unnamed Hanford formation [?] or Plio-Pleistocene Deposits [?].

Visual examination of the split-spoon samples found that the upper portion of this sequence consists of thinly bedded muddy very fine sand to mud. The sands are moist, olive brown (2.5 Y 4/4) in color, and display a strong reaction to hydrochloric acid. These finely laminated sands are friable and often grade upward into sandy mud and/or mud. The mud was more compacted and varied from wet to moist with a slightly darker grayish brown (2.5 Y 3/2) color. These graded beds of muddy sand to mud are on the order of 30 centimeters ( 1 foot).

The bedding appears to get thicker and more massive with depth, with beds on the order of 1 meter ( 3 feet) in the middle portion of the sequence and up to 1.5 meters ( 5 feet) thick at the bottom. The sands in this lower portion of the sequence are dark brown (10 YR 4/3) to olive (5 Y 5/3) with a strong to weak reaction to hydrochloric acid (Figure 2.12). The sands were moist to dry and friable. Some mud stringers on the order of 1 centimeter $(0.39$ inch) thick were also observed.

Two massively bedded mud units are present, one from 43.5 to 44.2 meters (142.8 to 144.9 feet) bgs and one from $\sim 46$ to 47.3 meters (151 to 155.1 feet) bgs. The upper mud unit is olive brown (2.5 Y 4/4) in color and is described as moist with a strong to weak reaction to hydrochloric acid. It is a massively bedded mud at the bottom (Figure 2.13) and grades upward to muddy sand and then back to mud at the top. The lower mud unit is olive in color ( 5 Y 4/4) and is described as very compacted. Some small ( 1 to 2 millimeters or 0.04 to 0.08 inch) stratifications of very fine sand with limonitic staining have been observed. 


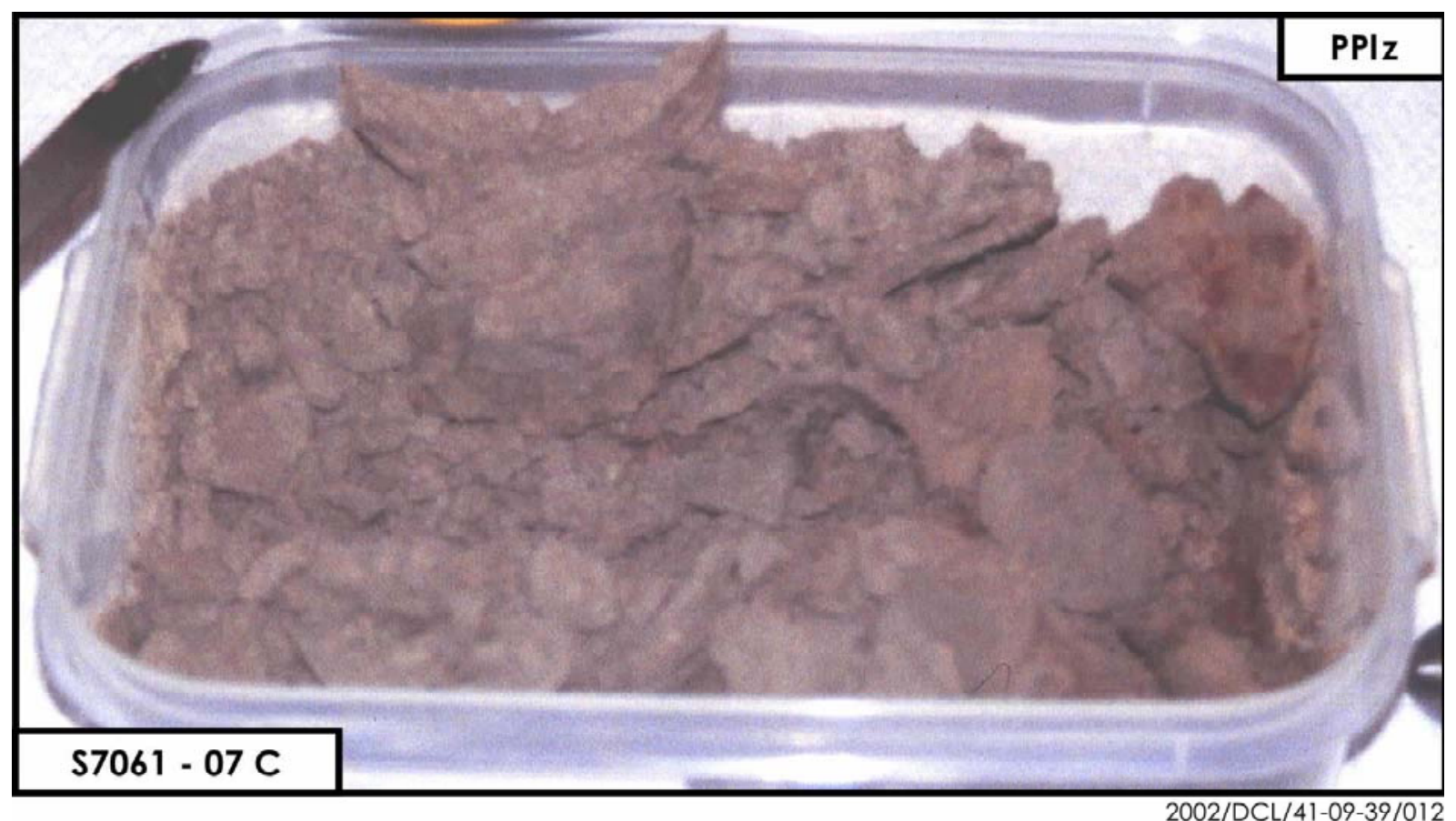

Figure 2.12. Muddy Very Fine Sand of the Plio-Pleistocene Mud (PPlz) Unit (Sample No. S7061-7C at a depth of $\sim 44.6$ meters [146.1 to 146.7 feet])

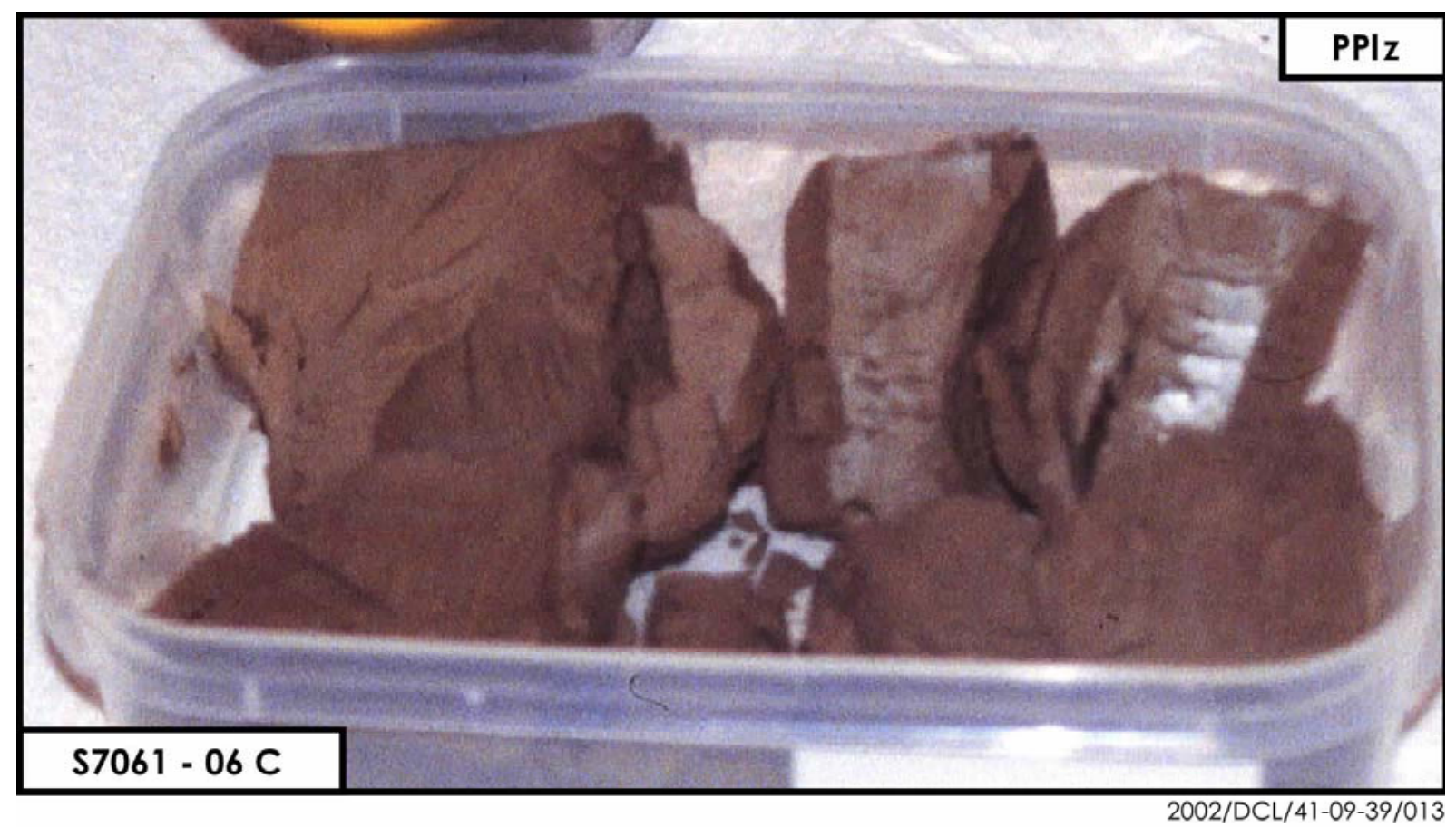

Figure 2.13. Mud of the Plio-Pleistocene Mud (PPlz) Unit from Sample S7061-6C (at a depth of $\sim 43.7$ meters [143.7 to 144.5 feet]) 
The base of this sequence is dominated by a well-sorted 20 -centimeter (8-inch)-thick fine sand. This friable sand is very basaltic with some limonitic staining. It is moist and has a weak reaction to hydrochloric acid.

Caliche (Plio-Pleistocene Carbonate [PPIc] unit). The caliche horizon was penetrated by the lower portion of sample S7061-11, samples S7061-12 and -13, and by the upper portion of sample S7061-14. This horizon is a pedogenic carbonate characterized by relatively low potassium- 40 , uranium-238, and thorium-232 concentrations (see Figures 2.6 to 2.7), a strong reaction to hydrochloric acid, and the visible presence of caliche fragments and/or whitish coloration. This zone is thought to have formed subaerially on the highly weathered paleosurface of the Ringold Formation (Brown 1959, 1960; Slate 1996). This is evidenced by the consistent uranium-238 and thorium-232 signatures with that of the Ringold Formation (see Figures 2.6 and 2.7).

The caliche horizon is $\sim 1.3$ meters ( 4.3 feet) thick and varies in grain size from sandy mud to sandy gravel and is described as moderate to strongly cemented with pinkish to whitish calcium carbonate. In places, the calcium carbonate cement is massive, forming a calcrete layer, which is broken up during drilling and sampling and shows up as calcrete clasts (Figure 2.14). In other places, the calcium carbonate appears disseminated into the sand or mud matrix, or as coatings on the gravel clasts. The calcrete clasts display a very pale brown (10 YR 8/4) to olive yellow $(2.5 \mathrm{Y} 6 / 6)$ coloration.

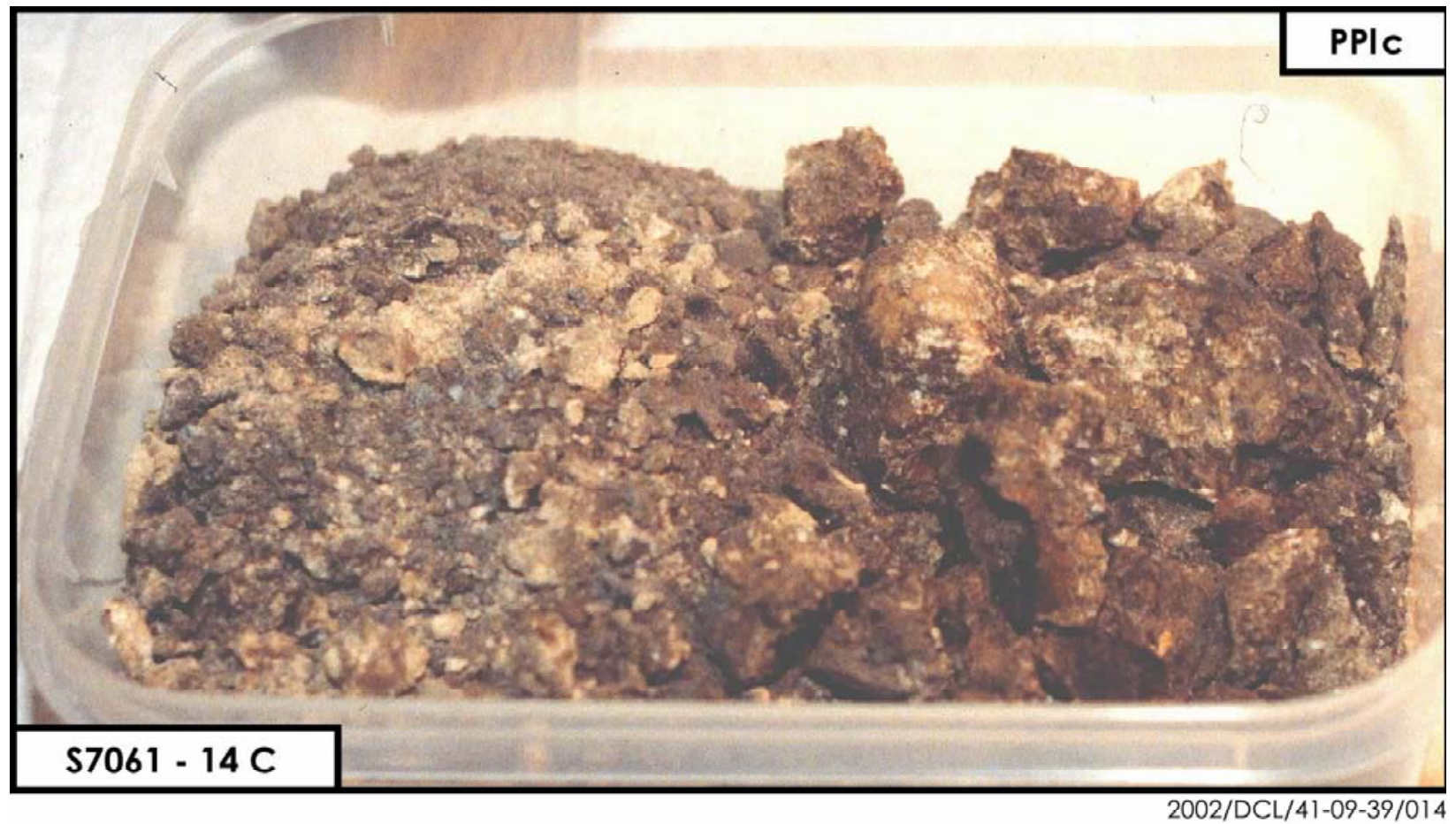

Figure 2.14. Broken-Up Pedogenic Carbonate Fragments from the Plio-Pleistocene Carbonate (PPlc) Unit (Sample No. S7061-14C at a depth of 48.6 meters [159.1 to 159.7 feet]) 
The gravelly facies generally displays a very dark grayish brown $(2.5 \mathrm{Y} 3 / 2)$ coloration, is high in basalt, and is fairly friable. The largest clast size is on the order of 50 millimeters ( 2 inches). The sandy facies is similar in coloration to the gravel facies and is also friable; however, it is dominated by coarse to fine or fine to very fine sand with some small gravel (up to 10 millimeters or $0.39 \mathrm{inch}$ ). The mud facies is also similar in color by is less friable.

\subsubsection{Ringold Formation}

Although the caliche horizon is believed to have formed at the surface of the Ringold Formation, as defined here, the Ringold Formation refers to that material underlying the caliche horizon. Samples of this material were recovered in the lower portion of sample S7061-14, and four of eight additional splitspoon samples. This formation is extremely difficult to drill with a drive barrel and/or to sample with a split-spoon sampler. Thus, the recovery of intact samples was poor as evidenced by the lack of recovery in four of eight samples.

The recovered materials consist predominately of weakly cemented sandy gravel to strongly cemented, matrix-supported conglomerate. This formation is further characterized by relatively moderate to high potassium-40, and low uranium-238 and thorium-232 signatures (see Figures 2.6 and 2.7), a more yellow-brown coloration, and no reaction to hydrochloric acid. The gravel/conglomeratic facies is both clast and matrix supported. The matrix varies from sandy mud, to muddy fine sand, to well-sorted, quartz-rich fine or medium sand, to very coarse sand. The gravel clasts are subrounded to rounded and range up to $<70$ millimeters or less than 2.8 inches (Figure 2.15). The clasts are composed predominantly of quartzite, basalt, and granite. In places, the basalt and granitic clasts are highly weathered, and limonitic staining is common.

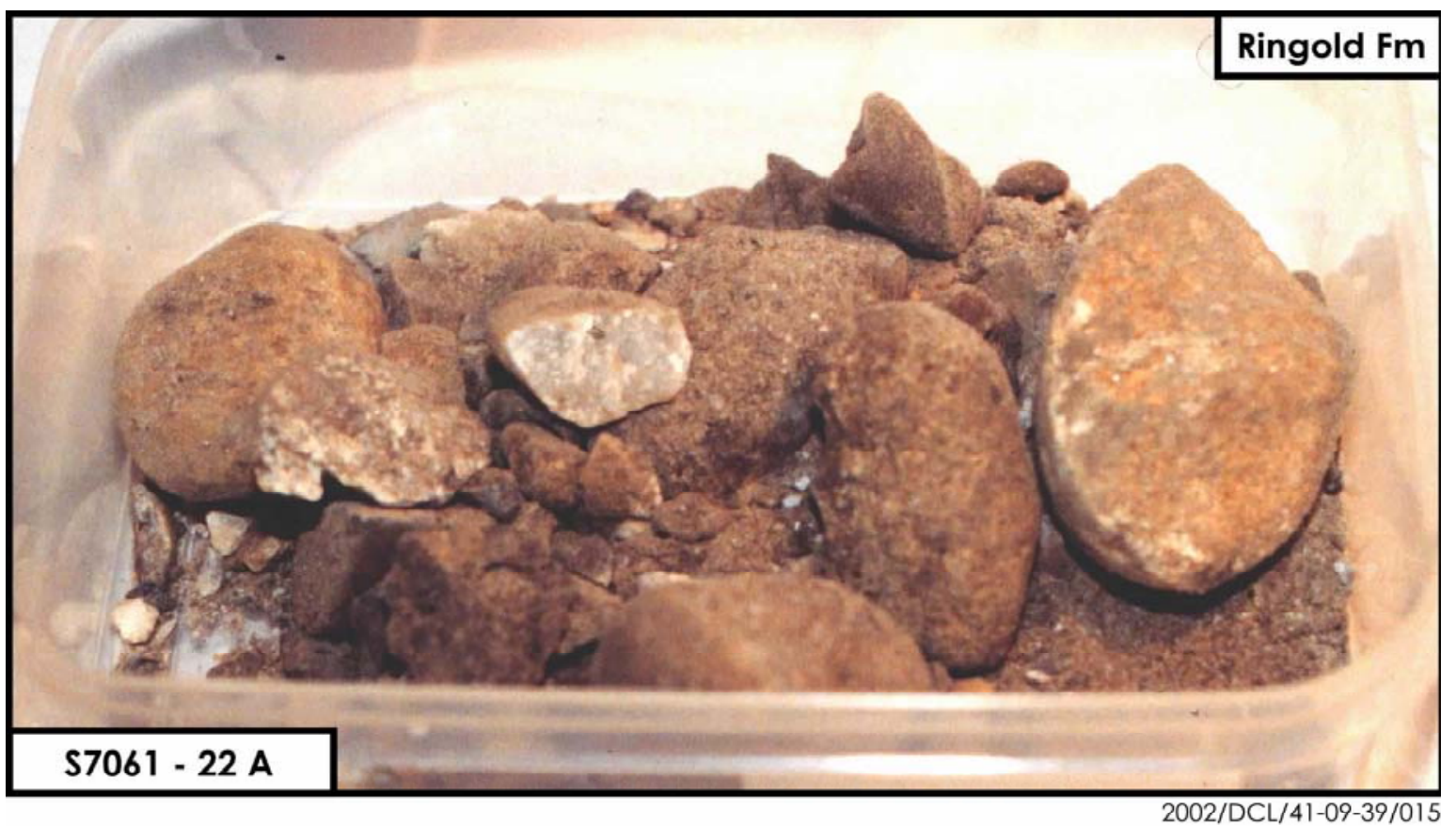

Figure 2.15. Fragmented Ringold Gravels from Sample No. S7061-22A

(at a depth of $\sim 52.6$ meters [172.3 to 173.0 feet]) 
Occasional intercalated sand and mud lenses vary in thickness from a few centimeters to nearly 1 meter and are not likely to be very continuous. The sand units vary from muddy fine to very fine sand, to clean, well-sorted fine or medium quartz sand, to poorly sorted gravelly muddy coarse to fine sand. One prominent sand unit lies at a depth of 54.6 to 55.3 meters (179 to 181 feet). This unit appears to grade upward from a muddy fine to very fine sand, to muddy medium to fine sand, to a clean medium to fine sand, to gravelly medium sand. The lower muddy portions of this unit correlate well with an increased potassium-40 content and a lower moisture response (see Figures 2.6 and 2.7).

The muddy units are generally poorly sorted and consist of slightly gravelly sandy mud to gravelly mud. The muddy units are generally light brownish gray to grayish brown (10 YR 6/2 to 10 YR 5/2), while the muddy (clayey) units are generally dark to very dark grayish brown (10 YR 4/2 to 3/2). Two thin ( 6 to 24 centimeters [ 2 to 9 inches]) clean mud units were observed at depths of 61.6 meters (202.2 feet) and 63.3 meters (207.5 feet). These units are very dark grayish brown (10 YR 3/2) in color.

\subsection{Historical Water Levels}

Figure 2.16 illustrates hydrographs for wells 299-W23-3 and 299-W23-4. Since a complete water level record is unavailable for either well, their water level behavior was extrapolated from each other to complete the record. This is justified by the similar behaviors of their common data sets. Based on this extrapolation between the two data sets and a linear interpolation over the distance between the two wells, it is estimated that the peak water elevation beneath the SX-109 tank was 146.2 meters (479.7 feet).

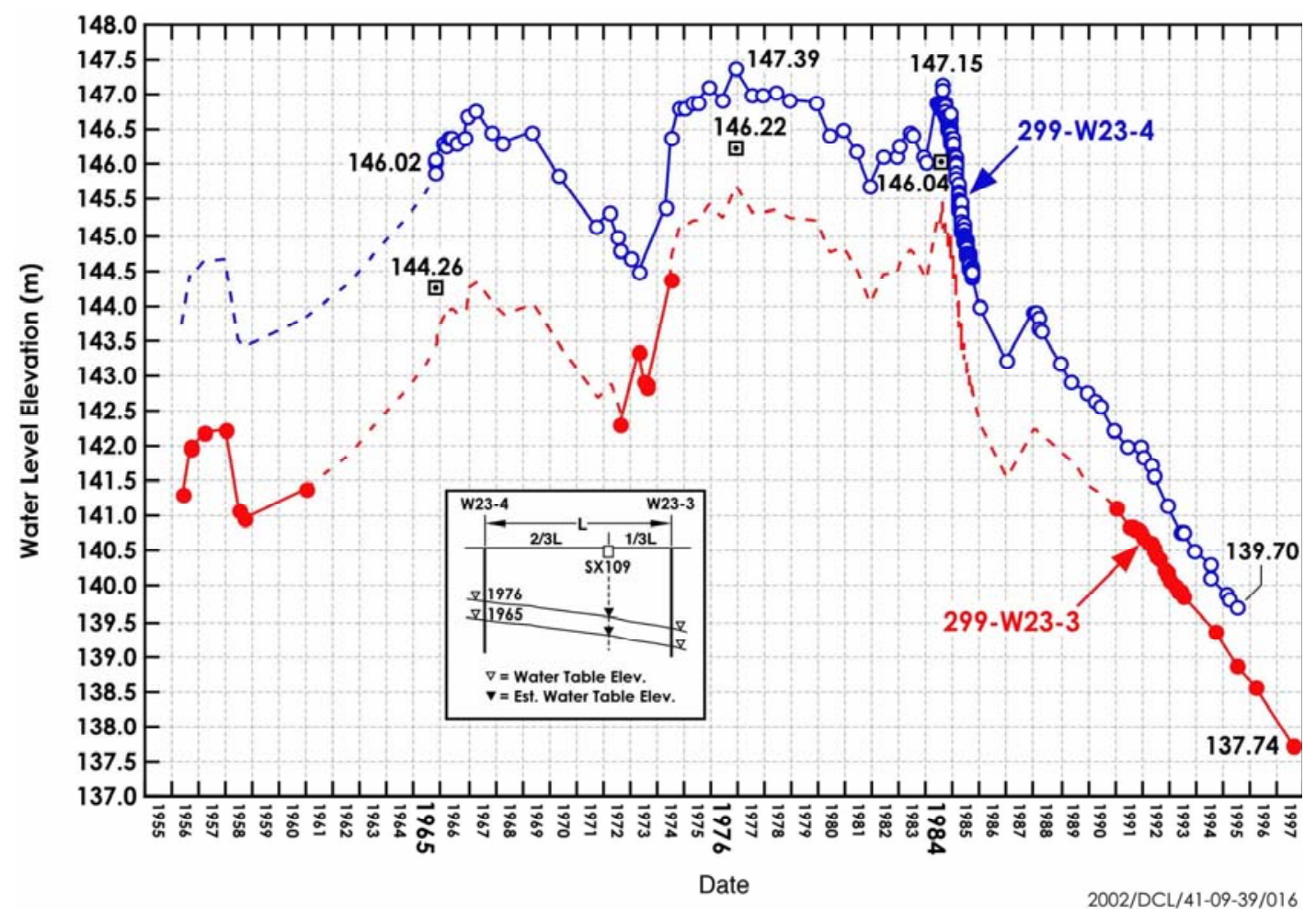

Figure 2.16. Historic Water Levels Beneath the SX Tank Farm 
This occurred in 1976 and places the water table $\sim 55$ to 56 meters ( $\sim 182$ to 184 feet) bgs or 40 meters (132 feet) beneath the bottom of the tank. A secondary maximum occurred in 1984, just before the 216U-10 Pond was decommissioned. At this time, the water table was estimated to have been almost as high as it was in 1976. In December 1997, the water table was encountered at a depth of $\sim 64.5$ meters (211.5 feet) in borehole 41-09-39. Thus, the water table has dropped an estimated 8 to 9 meters ( 28.5 feet) over the last 14 years. An examination of the hydrographs since about 1988 suggests that the water level is dropping at a rate of 0.5 to $0.6 \mathrm{~m} / \mathrm{yr}$ ( 1.5 to 1.9 feet per year).

The water table in 1965, when tank SX-109 was determined to be a leaker and was removed from service (ICF 1996), is estimated to have been about 144.3 meters (473.3 feet), or 57 to 58 meters (189 feet) bgs. 


\subsection{Geochemical Method and Materials}

This section discusses the methods and philosophy used to determine what samples should be characterized and what parameters would be measured.

\subsection{Sample Inventory}

The split-spoon sleeves and sidewall core samples were sent directly to the chemical laboratory after removal from the core barrel and end caps were emplaced to preserve the moisture content and to keep the sample intact. The 41-09-39 borehole extension project (deepening the borehole from 39.9 meters [131 feet] below ground surface [bgs] to groundwater after milling off the end cap) was initiated on September 15, 1997. During the sampling phase of this project, the following were submitted for analysis: 127 split-spoon sleeve samples (each being 15.2 centimeters [6 inches] in length and 10.2 centimeters [4 inches] in diameter), 25 polyethylene bottles containing clean-out soil samples, 3 water samples, 2 samples of sediment brushed off the inside of the casing, 2 plastic-bag samples (used to protect the spectral gamma logging detector), 1 cloth-swab sample (following casing brushing), 1 sediment sample from scraping the hard tool, and 1 sediment sample from scraping the exterior of a piece of core casing retrieved early in the drilling. The first set of samples was sent to PNNL on September 24, 1997, and the final set was received on February 24, 1998. The final depth of the extended borehole was 68.7 meters ( 225.5 feet) below grade or 28.7 meters ( 94 feet) below the depth of the original borehole. In all, 162 samples were received and analyzed for a variety of geochemical, radiochemical, and chemical constituents.

Samples were identified using a project-specific prefix, in this case S7061, followed by a specific sample ID suffix such as -01 , for each split spoon. Individual sleeves within the split spoons were identified by the letters A, B, C, and D, etc., respectively, where the A sleeve was always in the position closest to the drive shoe. The D sleeve would contain the uppermost sediment collected into the sampler and the A sleeve would contain the lowermost sediment collected during each 0.6 meter ( 2 - foot) sampling event. Thus, for any given sampling event, sample $\mathrm{A}$ is the deepest section bgs and sample $\mathrm{D}$ is the shallowest section bgs. In several instances, $>0.6$ meter ( $>2$-foot) sampling events were attempted but after having much less success at obtaining full samples, the majority of the sampling events were 0.6 meter $(2$ feet $)$.

When the borehole was initially extended, water was used as a cooling fluid while milling out the closed-bottom plate of the original 17.8 centimeters (7-inch)-outside-diameter borehole pipe. Approximately 76 liters (20 gallons) of water were assumed to be lost into the surrounding sediments as the drill bit finally penetrated the end shoe. The milling was followed by the retrieval of the shoe, the installation of 11.4-centimeter (4.5-inch) casing, and finally split-spoon sampling using the 0.6 meter ( 2 - foot) sampler containing four 10.2 centimeter (4-inch) stainless steel sleeves. Sampling was an iterative process in which the sampler was lowered into the borehole, pounded into the sediment, removed, split open, and the sleeves removed for shipment to the PNNL analytical laboratory. A new split spoon was then loaded with new stainless steel sleeves. Immediately after the split spoon was 
removed, the outer casing was advanced and a second split spoon containing no sleeves was used to clean out residual sediment to the base of the advanced casing, corresponding to the deepest point that the previous split-spoon sampler had reached.

The formation sediments at the base of the original borehole 39.9 meters (131 feet) were clayey silt and silty sand that combined with the escaped milling water, producing a mud paste that adhered to everything that contacted the sediment. This sticky paste was clearly evident on the surface of the splitspoon sampler used to collect the first set of sleeves. Radiocounting of this first set of sleeves showed cesium-137 contamination levels averaging $40,000 \mathrm{pCi} / \mathrm{g}$, with some thin stringers showing 2 million $\mathrm{pCi} / \mathrm{g}$. Several subsequent split-spoon samplers also were coated with the cesium-137-contaminated sticky paste. Given the nature of this paste, it could be reasonably concluded that much of the inner wall of the extended borehole casing was coated and thus contaminated to some degree. As the casing was pushed deeper, the silty paste was likely covered by deeper sediment or knocked off the walls during subsequent sampling and clean-out activities. In addition, the procedure for borehole logging required wrapping the detector and associated cabling in a plastic sleeve. Because of the tight inner dimension of the extended casing, the plastic sleeve containing the detector would almost act as a brush when moving the detector up and down the borehole. This logging activity occurred 13 times over the duration of the borehole extension project.

The sediment characterization activities performed for the borehole extension required substantial changes from the originally conceived plans. Changes were required when we became aware of the heterogeneity in sample lithology within a 15.2-centimeters (6-inch)-long sample, widely varying contamination levels within a sleeve, and measured sensitivity of cesium-137 activity to drilling and sample collection activities. The most significant change to the plan was the addition of the scoping phase of the laboratory activities to determine the effects of natural lithologic heterogeneity and sensitivity of cesium-137 values to the drilling and sampling activities. The goal of the scoping work was to quantify the extent and influence of the contamination spreading caused by carry down from materials that dropped off the inside of the casing as samplers, clean-out hardware, and the spectral gamma detector were raised and lowered. The scoping analyses allowed sediment samples that were least affected by drag-down and carry-down artifacts to be selected for detailed characterization. In general, we found that the sleeves that contained the sediment farthest from the previous sampling event were least impacted by carry down and drag down. For each sampling event, the sleeves are labeled from A, B, C, D, etc. Sleeve $\mathrm{A}$ is farthest from the original hole (deepest into the uncased formation) and sleeve $\mathrm{D}$ or higher in the alphabet is shallowest (contains the sediment just below the depth of the previous sampling event).

Sidewall samples were collected as the borehole was decommissioned between May 27 and August 11, 1999. Sidewall core samples were collected at selected intervals throughout the top portion of the borehole (i.e., that portion completed in 1996 and not previously sampled) over the time period June 16 to August 10, 1999. Three cores were collected from each sample interval at $\sim 90$ degrees to each other and generally oriented to the north, south, and west. Coring started at the deepest point (38.7 meters [ $\sim 127$ feet] bgs) and proceeded at successively shallower depths until the final coring occurred at 7.6 meters ( $\sim 25$ feet) bgs. In total, 15 depths were sampled between the ground surface and 39.6 meters (130 feet) bgs. All material from three sidewall cores at a specific depth was composited in the laboratory into a single sample tray for geologic description and subsampling, excepting one depth. For samples 
from 19.8 meters (65 feet) bgs, it was noted that one sidewall core was reading 10x higher dose than the other two cores and it was treated as a separate sample. A summary of the geologic descriptions made in the laboratory is provided in Appendix C. Note, however, that the geologic descriptions of these small ( 2.5 centimeters [ $0.98 \mathrm{inch}] \times 2.5$ centimeters [ $0.98 \mathrm{inch}] \times 28$ centimeters [11 inch]) sidewall core samples may not be wholly representative of the native geologic materials. These cores likely penetrated (on average) about 23 centimeters ( 9 inches) into the borehole wall and include disturbed material severely compacted around the casing during its pile-driving-type installation. Their small size also prevented recovery of larger gravel clasts.

\subsection{Tiered Approach}

During the investigation of the first set of sleeves from the borehole extension, changes in sediment type and contaminant concentrations were noted within a distance of a few centimeters (inches) within a given sleeve. It was concluded that a more methodical scoping approach would be necessary to provide the technical justification for selecting samples for detailed characterization as defined in the data quality objectives (DQO) process. Subsequently, a scoping methodology was developed that considered depth, geology (e.g., lithology, grain-size composition, carbonate content, etc.), individual sleeve contaminant concentration (e.g., radionuclides, nitrate, etc.), moisture content, and overall sample quality into account. Inexpensive analyses and certain key parameters (i.e., moisture content, gamma energy analysis) were performed on sediment from each sleeve.

The scoping methodology required each sleeve to be weighed, opened in a radiologically controlled laboratory space, and placed in a sealable plastic container. The sediments were photographed and examined using standard geologic techniques. The geologic examination included the following: fullness of the sleeve, grain size, moisture, color, and qualitative carbonate determination. Drilling records and daily logs were used to assess actual sample depth to compare with the sampling field records. The actual volume of each sleeve also was used to provide a final best estimate of the depth at which the sample was collected. Immediately following the geologic examination, the sleeve contents were subsampled for moisture content (in triplicate), gamma-emission radiocounting (for these samples, effectively cesium137, potassium-40, uranium-238, and thorium-232 data), one-to-one sediment to water extracts (which provide soil $\mathrm{pH}$, electrical conductivity, cation, and nitrate data), and soil tension (in duplicate). The remaining sediment from each sleeve was then sealed and placed in cold storage. The sleeve, bag, tape, and any other associated packaging material were weighed so that sediment wet weight could be estimated. The packaging materials then were disposed. All information was recorded in a laboratory record book.

A similar process was followed for the 16 composite sidewall cores, excepting that only one moisture content measurement was made on each composite and no soil tension samples were taken to preserve the small sample mass for other chemical determinations.

\subsection{Materials and Methods}

During sleeve-sample subsampling, every effort was made to minimize moisture loss and prevent cross contamination between sleeve samples. Depending on the sample matrix, very coarse pebble and 
larger material (>32 millimeters) was avoided during subsampling. Larger substrate was excluded to provide moisture contents representative of counting and 1:1 sediment-to-water extract samples. Results from subsample measurements should then take into consideration a possible bias toward higher concentrations for some analytes that would be considered associated with smaller size sediment fractions. The sediments above the Ringold Formation contain no large pebbles, cobbles, or boulders.

For the 16 sidewall cores, we also attempted to minimize moisture loss and, in most cases, mixed all three samples taken at a particular vertical depth in one composite sample. Because the sidewall cores were only 2.5 -centimeter x 2.5 -centimeter x 28 -centimeter $(0.98$-inch x 0.98 -inch x 11 -inch) samples, they may not be wholly representative of the native geologic materials. These cores likely penetrated (on average) about 23 centimeters ( 9 inches) into the borehole wall and include disturbed material severely compacted around the casing during its pile-driving-type installation. The small size also prevented recovery of larger gravel clasts. In one instance, all three sidewall cores were not mixed together (e.g., $11 \mathrm{~A}$ and $11 \mathrm{~B}$ were mixed but $11 \mathrm{C}$ was kept separate). Sidewall core $5 \mathrm{C}$ was mixed with $5 \mathrm{~A}$ and $5 \mathrm{~B}$ before the driller's log was available and it was learned that core $5 \mathrm{C}$ was at an unknown depth that differed from 5A and 5B. In a few instances, distinctly finer-grain thin stringers were isolated from the sidewall cores and analyzed separately.

Procedures ASTM D2488 (ASTM 1993) and PNL-MA-567-DO-1 (PNL 1990) were followed during visual description and recording of all sidewall and split-spoon samples. The sediment classification scheme used for geologic identification of the sediment types is based on the modified Folk/Wentworth classification scheme described earlier. However, the mineralogic and geochemical characterization relied on further separation of the mud fractions into discrete silt and clay sizes.

\subsubsection{Moisture Content}

Gravimetric water contents of the sediment samples from each sleeve and sidewall core were determined using PNNL Procedure PNL-MA-567-SA-7 (PNL 1990). This procedure is based on the American Society for Testing and Materials (ASTM D2216, ASTM 1986a) procedure, Laboratory Determination of Water (Moisture) Content of Soil, Rock, and Soil-Aggregate Mixtures. Three representative subsamples of at least 60 grams were taken from each sleeve after mixing the sample in the sealable plastic container. Sediment samples were placed in tared containers, weighed, and dried in an oven at $105^{\circ} \mathrm{C}$ until constant weight was achieved, which took at least 24 hours. The containers then were removed from the oven, sealed, cooled, and weighed. All moisture content activities were performed using a calibrated balance. A calibrated weight set was used to verify balance performance before weighing samples. The gravimetric water content was computed as percentage change in soil weight before and after oven drying. The number reported in this report is the average of the three subsamples for each sleeve sample.

Smaller masses (15 to 35 grams) of the sidewall cores were treated in a similar fashion, but to conserve the sample the moisture content was determined on only one subsample. 


\subsubsection{1:1 Sediment-to-Water Extract}

The soluble inorganic constituents were determined using a one-to-one sediment to deionized water extract method described in ASA (1996, part 3 pages 417-422). This method was chosen because the sediments were too dry to easily extract vadose zone porewater. The extracts were prepared by adding an equal weight of deionized plus existing pore water to the weight of the sediment. Approximately 100 grams of sediment were taken from the sleeve samples, while only 50 to 70 grams of sediment were used from the smaller sidewall cores. The amount of deionized water needed was calculated based on the weight of the samples and their previously determined moisture contents. The appropriate amount of deionized water was added to screw-cap jars containing the sediment samples. The jars were sealed and briefly shaken by hand, then placed on a mechanical orbital shaker for 1 hour. The samples were allowed to settle until the supernatant liquid was fairly clear. The supernatant was carefully decanted and separated into unfiltered aliquots for conductivity and $\mathrm{pH}$ determinations, and filtered aliquots (passed through $0.45 \mu \mathrm{m}$ membranes) for anion, cation, carbon, and radionuclide analyses. Carbon analyses were performed only on the composite samples used for detailed characterization (see Section 3.6.2).

\subsubsection{1 pH and Conductivity}

Two $\sim 3$ milliliter aliquots of the unfiltered 1:1 sediment-to-water extract supernatant were used for $\mathrm{pH}$ and conductivity measurements. The $\mathrm{pHs}$ for the extracts were measured with a combination glass electrode for the sleeve samples and a solid-state Ross electrode for the sidewall core extracts and a $\mathrm{pH}$ meter calibrated with buffers 4,7 , and 10 . Conductivity was measured and compared to potassium chloride standards with a range of $0.001 \mathrm{M}$ to $1.0 \mathrm{M}$. More details can be found in Rhoades (1996).

\subsubsection{Anions}

The composite samples selected from the borehole extension sediments, which were used for detailed characterization, and the sidewall core extracts were analyzed for anions using an ion chromatograph. Fluoride, chloride, nitrite, nitrate phosphate, sulfate, and oxalate were separated on a Dionex AS4A column with an eluent of $1.75 \mathrm{mM} \mathrm{NaHCO} / 1.85 \mathrm{mM} \mathrm{Na}_{2} \mathrm{CO}_{3}$ and measured using a conductivity detector following PNL-ALO-212, Rev. 1, which is based on U.S. Environmental Protection Agency (EPA) Method 9056, "Determination of Inorganic Anions by Ion Chromatography" found in SW-846 "Test Methods for Evaluating Solid Waste, Physical/Chemical Methods" that can be accessed online at http://www.epa.gov/epaoswer/hazwaste/test/sw846.htm (EPA 1994).

\subsubsection{Cations}

Cation analysis was performed using an inductively coupled plasma unit. High-purity calibration standards were used to generate calibration curves and to verify continuing calibration during the analysis run. Dilutions of 100x, 50x, 10x, and 5x were made of each sample for analysis to ensure that an analysis would fall within the linear calibration range of the instrument. The analyses on various dilutions also allowed us to check for matrix interference problems. If the results for several sample dilutions gave the same value, the chance that results were biased by unexpected matrix effects were considered remote. 
Details are found in EPA Method 6010B, "Inductively Coupled Plasma-Atomic Emission Spectrometry" in the aforementioned online version of SW-846 (EPA 1996).

\subsubsection{Radionuclide Analysis}

\subsubsection{Gamma Energy Analysis}

Gamma energy analysis (GEA) was typically performed on sediments from every sleeve and composited sidewall core. GEA also was performed on several supernatants from the water and acid extracts of the vadose-zone-contaminated sediments. Results of the GEA measurements of casing scrapings, the logging instrument bags, and the muslin cloth swab and saturated sediments from below the water table and selected water samples were reported in Myers et al. (1998).

All samples for GEA were analyzed using 60\%-efficient intrinsic germanium gamma detectors. All germanium counters were efficiency calibrated for distinct geometries using National Institute of Standards and Technology (NIST)-traceable mixed gamma standards. Samples analyzed were as large as could be allowed without raising detector dead time to $>1 \%$. All spectra were background subtracted. Spectral analysis was conducted using libraries containing most mixed fission products, activation products, and natural decay products. Control samples were run throughout the analysis to ensure correct operation of the detectors. The controls contained isotopes with photo peaks spanning the full detector range and were monitored for peak position, counting rate, and full-width half-maximum. Details are found in PNNL-RRL-01 (PNNL 1997).

\subsubsection{Technetium-99}

Subsamples of sediments from selected sleeves from the borehole extension were measured using the procedures described in Section 3.3.5.6 for the composite samples characterized in detail. For the sidewall cores, all technetium-99 analyses were performed on acid and water extracts using inductively coupled plasma mass spectrometry (ICP-MS).

\subsubsection{Water Potential (Suction) Measurements}

Suction measurements were made on selected sediments from the borehole extension using the filter paper method (PNL-MA-567-FA-2, which is based on ASA 1986; Campbell and Gee 1986). This method relies on the use of filter paper that equilibrates rapidly with the sediment sample. At equilibrium, the matric suction in the filter paper is the same as the matric suction of the sediment sample. The filter paper is weighed and its water content is converted to a matric suction value through its known water retention characteristic. Specifically, dry filter papers are placed in an airtight container with the sediment for at least 1 week to allow sufficient time for the matric suction in the sediment to equilibrate with the matric suction in the filter paper. The mass of the wetted filter paper is subsequently determined and the suction of the sediment is determined from a calibration relationship between filter paper water content and matric suction. In addition to filter paper and sealed containers, the only equipment needed for this test is an analytical balance. 
The relationships used for converting the water content of filter paper to matric suction for Whatman \#42 filter paper have been determined by Deka et al. (1995) and can be expressed as:

$$
\begin{aligned}
& \mathrm{Sm}=10^{(5.144-6.699 \mathrm{w})} / 10 \text { for } \mathrm{w}<0.5 \\
& \mathrm{Sm}=10^{(2.383-1.309 \mathrm{w})} / 10 \text { for } \mathrm{w}>0.5
\end{aligned}
$$

where: $\quad \mathrm{Sm}=$ the matric suction $(\mathrm{m})$

$\mathrm{w}=$ the gravimetric water content $(\mathrm{g} / \mathrm{g})$.

Thirty samples from borehole 41-09-39 were analyzed for water content and soil matric suction. In addition, sleeve samples 16D, 22B, 38C, and 47C were further analyzed for water content and matric suction after several months in storage to ascertain whether the measurements were time sensitive because of unavoidable drying during storage. Subsamples from these four cores were also placed on hanging water columns (Klute 1986). The hanging water columns were constructed by attaching tubing to the end of filter funnels containing porous fritted glass plates, with bubbling pressures in excess of 100 centimeters suction; filling the funnels and the attached tubing with de-aired water; allowing the system to soak for several hours; then flushing the system so that the funnels and tubing were completely filled with water. Subsamples of sediment from these four sleeves were subsequently placed on the fritted plates, saturated, then equilibrated at 100 centimeters matric suction by adjusting the tubing to 100 centimeters below the center of the sediment samples and allowing the samples to drain for 1 week.

\subsubsection{Borehole Extension Composite Samples - Materials and Methods}

Following completion of the scoping phase and identifying the presence of a distinct caliche layer, sediment sleeves from the borehole extension were selected for complete characterization. This selection process was conducted in two phases. The first phase was conducted internally by senior PNNL research staff using the depths defined in the DQO as a guideline, followed by geologic considerations of sample quality and lithologic integrity (e.g., no hard-tooled sediments), and, finally, geochemical and radiochemical representativeness (e.g., no sampling cross contamination). The second phase was completed when, after two meetings, the samples passing the first phase were voted on for further characterization by members of the U.S. Department of Energy (DOE), Washington State Department of Ecology (Ecology), and the primary Hanford Site project contractors.

Seven composite samples were chosen by combining two sleeves each: $2 \mathrm{~A} / \mathrm{B}, 10 \mathrm{~A} / \mathrm{B}, 12 \mathrm{~A} / \mathrm{B}$, $17 \mathrm{~A} / \mathrm{B}, 38 \mathrm{~A} / \mathrm{B}, 47 \mathrm{~A} / \mathrm{B}$, and $58 \mathrm{~A} / \mathrm{B}$. Analyses from composites $2 \mathrm{C} / \mathrm{D}$ and $3 \mathrm{~A} / \mathrm{B}$, which are being used for an independent project on cesium mobility, were also included. The two sleeves of each composite were combined in a sealable plastic container and vigorously agitated for 10 minutes in an attempt to create a homogeneous sample. All 15 of the specific-depth composited sidewall cores and the separate sidewall core $11 \mathrm{C}$ also were completely characterized.

\subsubsection{Moisture Content}

Moisture content determinations were made for each composite sample, as described in Section 3.3.1. 


\subsubsection{1:1 Sediment-to-Water Extract}

Extract porewaters were obtained in the manner described in Section 3.3.2. Unless otherwise described, analyses were performed in the same manner. Dissolved carbon content was determined for each borehole extension and sidewall composite 1:1 sediment-to-water extract.

\subsubsection{Cations}

Major cation analysis was performed using an inductively coupled plasma (ICP) unit using highpurity calibration standards to generate calibration curves and verify continuing calibration during the analysis run. Dilutions of 100x, 50x, 10x, and 5x were made of each sample for analysis to investigate and correct for matrix interferences. The second instrument used to analyze trace metals was an ICP-MS using the PNNL-AGG-415 method (PNNL 1998).

\subsubsection{Carbon}

Inorganic and organic carbon on the water extracts were determined using a carbon analyzer and ASTM Method D4129-80, "Standard Test Method for Total and Organic Carbon in Water by High Temperature Oxidation and by Coulometric Detection." (ASTM 1988)

\subsubsection{Radionuclide Analysis}

For the seven borehole extensions and the 15 sidewall core composite samples, GEA was done as described in Section 3.3.3. A liquid scintillation detector was used to analyze for technetium and strontium. A sodium iodide automatic gamma detector was used to determine recovery for the technetium and strontium analysis. Special 2.5 milliliter Teva Spec and SrSpec ${ }^{(\mathrm{a})}$ extraction columns were used for the wet chemical separation of technetium and strontium, respectively. NEN Life Sciences, a subsidiary of Dow Chemical, supplied the strontium- 85 tracer used to determine the chemical yield for strontium-90. Because if its 6-hour half life, technetium-99 metastable, used as the yield monitor for technetium-99, was purchased locally as needed. Specific details can be found in PNL-ALO-475, "Strontium-90 and Technetium-99 Analysis Using Eichrome Sr-Spec and TEVA Spec Columns" (PNL 1993).

\subsubsection{Technetium and Strontium Analysis}

Sediment aliquots were weighed and spiked with tracers technetium-99 metastable and strontium- 85 . Sediment samples were leached overnight with concentrated nitric acid, then an aliquot of the leachate was diluted $50 \%$ with deionized water. Leachate aliquots were first passed through the SrSpec columns with $8 \mathrm{M}$ nitric acid to capture strontium, then the resins were washed with 10 column volumes of $8 \mathrm{M}$ nitric acid. The strontium was eluted from the SrSpec column using deionized water. The water extract was evaporated to dryness in a liquid scintillation vial and was ready for counting after adding the

(a) Teva Spec and SrSpec are trademarks of EiChrom, Inc. 
cocktail. The effluent from the SrSpec column was diluted to $\mathrm{pH}=1$ and passed through a Teva Spec column to capture technetium. The Teva Spec column then was washed with 20 column volumes of 0.1 $\mathrm{M}$ nitric acid. After the Teva Spec columns were washed, they were broken open and the entire resin bed was discharged into a liquid scintillation vial for analysis. The purified strontium and technetium samples were analyzed by gamma spectroscopy to determine chemical yield from the added tracers and to quantify any contamination from cesium-137. Samples were analyzed by liquid scintillation counting to determine the amount of technetium-99 and strontium-90. A matrix spike, a blank spike, a duplicate, and blanks were run with each sample set to determine the efficiency of the separation procedure as well as the purity of reagents. For the most part, the separation procedure was straightforward. Chemical yields were generally good with some explainable exceptions. Matrix and blank spike yields were good, bias was consistent, and blanks were below detection limits.

\subsubsection{Gamma Energy Analysis}

Intrinsic germanium detectors were calibrated for several constant volume geometries with a NISTtraceable photon standard. These calibrations were then coupled with a library of isotopes, written using the instrument software, and including many isotopes of interest pertinent to Hanford production waste. Data reduction is accomplished by comparing the count rate and energy position of photon lines from the sample spectra to the geometry calibration and software library. Quantitative isotopic determination is made by the instrument software correcting for efficiency, photon yield, and sample mass. Samples of sediment equal in volume to one of the calibrated GEA geometries were placed in an appropriate container and were analyzed for photon emission for time periods necessary to achieve reasonable counting statistics (generally minutes to several hours).

Gamma spectroscopy for the borehole extension campaign was generally straightforward. The samples had only one gamma contaminant of concern, cesium-137. All other radiations were attributed to natural uranium, thorium, and their daughter products, and potassium-40. However, the samples analyzed for GEA were as large as was physically possible, with detector dead time limiting sample size. In some cases, the most radioactive samples showed a distinct sample heterogeneity because of the small aliquots analyzed. This lack of homogeneity was evidenced in technetium data as well. The separation chemistry for the beta emitters (strontium-90 and technetium-99) was somewhat more difficult, but only because of the time constraints imposed by the very short lived ( 6 hours) technetium- 99 metastable tracer used to quantify the technetium yield. Many of the samples were recounted several times to ensure the accuracy and reliability of the separation method.

The sidewall cores were more challenging because of the very high cesium-137 activities. Field moist sediment from sidewall core composites was placed into containers marked to identical volumes to those used for GEA detector calibration. The filled containers were then weighed to determine the mass

of moist sediment. Two calibrated volumes were used for the sidewall core samples; 100 millimeters for those samples judged to be low activity and 5 millimeters for those samples judged to be high activity. The 5-millimeter volume was considered to be the smallest practical sample size to yield accurate measurements based on past homogeneity checks with the borehole extension sediment. Samples were analyzed for 10 to 100 minutes at source-to-detector distances that would minimize detector dead time. Counting times were determined based on the cesium-137 activity of the sample. 
Sidewall core composite 7ABC was so radioactive that it fell outside the instrument capability envelope and required a special calibration of its own. This was accomplished by building a standoff extension 1 meter ( 3.3 feet) long to separate the detector from the sample. The standoff geometry was calibrated using known standards. As a validation of the calibrated standoff geometry, the counting sample of sidewall core $11 \mathrm{C}$ was counted in both the conventional geometry and the standoff geometry. The agreement was acceptable, thus verifying that the standoff geometry could be used for the most radioactive samples.

All data acquisition and reduction was accomplished using Canberra's GENIE2000pc @ spectroscopy software. Photon energies are compared with a user-entered library (ours included the nuclides listed in Table 3.1), to make a qualitative determination of isotopic species. Of course no software is infallible, and all spectra were also manually scrutinized for missed or phantom photon lines.

Quantitative determination of each positively identified isotope was then made by correcting for counter dead time, sample mass (oven dried), geometry efficiency, photon abundance, half-life, and background. The high amount of Compton presented by cesium-137 prevented the possible detection of many isotopes, but this was acceptable because there were no other gamma-emitting isotopes of interest.

\subsubsection{Chemical Analysis of Composite Sediments}

Major cations and selected metals, including uranium, present in the borehole extension composite sediments were detected using ICP and ICP-MS, as described in Section 3.3.2, following total dissolution according to the method described in "Standard Practice for Total Digestion of Sediment Samples for Chemical Analysis of Various Metals," which is procedure ASTM D4698-92 (ASTM 1996).

\subsubsection{Total Organic and Inorganic Carbon Analysis}

The carbon contents of the composite borehole extension and sidewall core sediments were determined using three methods. Total carbon content was determined according to ASTM method D4129-82,

Table 3.1. Nuclides in SX Gamma Library

\begin{tabular}{||l|l|l|l|l|l|l||}
\hline \hline${ }^{40} \mathrm{~K}$ & ${ }^{85} \mathrm{Sr}$ & ${ }^{109} \mathrm{Cd}$ & ${ }^{139} \mathrm{Ce}$ & ${ }^{212} \mathrm{~Pb}$ & ${ }^{230 \mathrm{Th}}$ & ${ }^{239} \mathrm{Pu}$ \\
\hline${ }^{51} \mathrm{Cr}$ & ${ }^{88} \mathrm{Y}$ & ${ }^{110} \mathrm{Ag}$ & ${ }^{144} \mathrm{Ce}$ & ${ }^{214} \mathrm{Bi}$ & ${ }^{231} \mathrm{~Pa}$ & ${ }^{240} \mathrm{Pu}$ \\
\hline${ }^{54} \mathrm{Mn}$ & ${ }^{94} \mathrm{Nb}$ & ${ }^{110 \mathrm{~m}} \mathrm{Ag}$ & ${ }^{152} \mathrm{Eu}$ & ${ }^{214} \mathrm{~Pb}$ & ${ }^{231} \mathrm{Th}$ & ${ }^{241} \mathrm{Pu}$ \\
\hline${ }^{57} \mathrm{Co}$ & ${ }^{95} \mathrm{Nb}$ & ${ }^{113} \mathrm{Sn}$ & ${ }^{154} \mathrm{Eu}$ & ${ }^{223} \mathrm{Ra}$ & ${ }^{233} \mathrm{~Pa}$ & ${ }^{241} \mathrm{Am}$ \\
\hline${ }^{59} \mathrm{Fe}$ & ${ }^{95 \mathrm{~m}} \mathrm{Nb}$ & ${ }^{124} \mathrm{Sb}$ & ${ }^{155} \mathrm{Eu}$ & ${ }^{224} \mathrm{Ra}$ & ${ }^{234} \mathrm{~Pa}$ & ${ }^{242} \mathrm{Am}$ \\
\hline${ }^{60} \mathrm{Co}$ & ${ }^{95 \mathrm{~m}} \mathrm{Tc}$ & ${ }^{125} \mathrm{Sb}$ & ${ }^{203} \mathrm{Hg}$ & ${ }^{226} \mathrm{Ra}$ & ${ }^{234 \mathrm{~m}} \mathrm{~Pa}$ & ${ }^{242} \mathrm{Pu}$ \\
\hline${ }^{65} \mathrm{Zn}$ & ${ }^{95} \mathrm{Zr}$ & ${ }^{131} \mathrm{I}$ & ${ }^{208} \mathrm{Tl}$ & ${ }^{227} \mathrm{Th}$ & ${ }^{234} \mathrm{Th}$ & ${ }^{243} \mathrm{Am}$ \\
\hline${ }^{75} \mathrm{Se}$ & ${ }^{10} \mathrm{Ru}$ & ${ }^{133} \mathrm{Ba}$ & ${ }^{210} \mathrm{~Pb}$ & ${ }^{228} \mathrm{Ac}$ & ${ }^{235} \mathrm{U}$ & ${ }^{243} \mathrm{Cm}$ \\
\hline${ }^{85} \mathrm{Rb}$ & ${ }^{106} \mathrm{Ku}$ & ${ }^{134} \mathrm{Cs}$ & ${ }^{211} \mathrm{Bi}$ & ${ }^{228 \mathrm{Th}}$ & ${ }^{237} \mathrm{~Np}$ & ${ }^{245} \mathrm{Cm}$ \\
\hline
\end{tabular}


A Standard Test Method for Total and Organic Carbon in Water Oxidation by Coulometric Detection (ASTM 1982). The inorganic carbon content of samples 10A/B, 17A/B, 38A/B, 47A/B, and 58A/B was determined using the technique described in the instruction manual for the Shimadzu Solid Sample Module SSM-2000A for the TOC-5000/5050 Total Organic Carbon Analyzer (Shimadzu). Because $2 \mathrm{~A} / \mathrm{B}, 2 \mathrm{C} / \mathrm{D}, 3 \mathrm{~A} / \mathrm{B}$, and $12 \mathrm{~A} / \mathrm{B}$ and all of the sidewall core samples were radioactive, their inorganic carbon contents were determined by the method CAN-3 provided by Coulometrics, Inc., for use with the Carbon Dioxide Coulometer model 5010. Subsamples of previously homogenized soil samples were taken and their dry weights calculated using moisture content data, as described in Section 3.3.1. Organic carbon was calculated by subtracting inorganic carbon from total carbon and using the remainder.

Total carbon in all samples was determined using a Coulometrics, Inc., model 5051 Carbon Dioxide Coulometer with combustion at $\sim 980^{\circ} \mathrm{C}$. Ultrapure oxygen was used to sweep the combustion products through a barium chromate catalyst tube for conversion to carbon dioxide. Evolved carbon dioxide was quantified through coulometric titration following absorption in a solution containing ethanolamine. Equipment output reported carbon content values in micrograms per sample. Soil samples for determining total carbon content were placed into precombusted, tared platinum combustion boats and weighed on a four-place analytical balance. After the combustion boats were placed into the furnace introduction tube, a 1-minute waiting period was allowed so that the ultrapure oxygen carrier gas could remove any carbon dioxide introduced into the system from the atmosphere during sample placement. After this system sparge, the sample was moved into the combustion furnace and titration begun. Sample titration readings were performed at 3 minutes after combustion began and again once stability was reached, usually within the next 2 minutes. The system background was determined by performing the entire process using an empty, precombusted platinum boat. Adequate system performance was confirmed by analyzing known quantities of a calcium carbonate standard.

Inorganic carbon contents of the nonradioactive borehole extension composite samples $10 \mathrm{~A} / \mathrm{B}$, $17 \mathrm{~A} / \mathrm{B}, 38 \mathrm{~A} / \mathrm{B}, 47 \mathrm{~A} / \mathrm{B}$, and 58A/B were determined using a total carbon analyzer in the following manner. The instrument was calibrated using calcium carbonate standards in the range of expected sample values. System background values were determined. Soil samples were weighed into precombusted $\left(900^{\circ} \mathrm{C}\right)$ ceramic combustion boats on a four-place analytical balance. Sample boats were handled with heated forceps to minimize contamination. Inorganic carbon was released through acidassisted evolution ( $25 \%$ phosphoric acid) with heating to $200^{\circ} \mathrm{C}$. Samples were completely covered by the acid to allow full reaction to occur. Ultrapure oxygen gas swept the resultant carbon dioxide through the equipment to determine inorganic carbon content. The computer contained within the apparatus provided weight percent (wt\%) values based on sample weight information input by the user and the standard calibration curve. Samples of known carbon content were analyzed periodically to confirm the equipment was operating properly.

Inorganic carbon contents for borehole extension $2 \mathrm{~A} / \mathrm{B}, 2 \mathrm{C} / \mathrm{D}, 3 \mathrm{~A} / \mathrm{B}$, and $12 \mathrm{~A} / \mathrm{B}$ composites and all of the sidewall core composites were determined using a Coulometrics, Inc., model 5051 Carbon Dioxide Coulometer. Soil samples were weighed on a four-place analytical balance, then placed into acid-treated glass tubes. Following placement of sample tubes into the system, a 1-minute waiting period allowed the ultrapure oxygen carrier gas to remove any carbon dioxide introduced into the system from the atmosphere. Inorganic carbon was released through acid-assisted evolution (50\% hydrochloric acid) with 
heating to $200^{\circ} \mathrm{C}$. Samples were completely covered by the acid to allow full reaction to occur. Ultrapure oxygen gas swept the resultant carbon dioxide through the equipment to determine inorganic carbon content by coulometric titration. Sample titration readings were performed at 5 minutes following acid addition and again once stability was reached, usually within 10 minutes. Known quantities of calcium carbonate standards were analyzed to verify that the equipment was operating properly. Background values were determined. Inorganic carbon content was determined through calculations performed using the microgram-per-sample output data and sample weights.

The total and inorganic carbon contents of all 15 sidewall cores were determined using the Coulometrics, Inc., instrument in a similar fashion.

\subsubsection{Cation Exchange Capacity}

Cation exchange capacity (CEC), usually expressed in milliequivalents per 100 grams of soil, is a measure of the quantity of exchangeable cations available to neutralize the negative charges found in soil. The procedure used for this analysis is found in Methods of Soil Analysis, Part 2 (ASA 1994). It is based on the method of Polemio and Rhoades (1977) and is suited to calcareous soils.

The procedure involved two steps. The first was to saturate the cation exchange sites with sodium by equilibration of the soil sample with a $\mathrm{pH}=8.2,60 \%$ ethanol solution of $0.4 \mathrm{~N}$ sodium acetate- $0.1 \mathrm{~N}$ sodium chloride solution. The second was to extract the cations with a $0.5 \mathrm{~N}$ magnesium nitrate solution. Sodium and chloride analyses were performed on the extracted solutions and on the excess saturating solution. The chloride determination was used to deduce the amount of excess sodium left in the soil pores after saturation by measuring the exchangeable sodium that was adsorbed. The exchangeable sodium is the CEC.

Saturation. A 25-gram sample of $<2$ millimeter-size fraction of each borehole extension composite (the sidewall core samples were not analyzed), was air-dried to a constant weight in the fume hood. Subsamples of the air-dried sample were used to determine the moisture content. The remaining sample was split into 2 samples of $\sim 5$ grams each (air dried) for duplicate cation-exchange analysis. The 5 -gram samples were placed into centrifuge tubes and 33 milliliters aliquots of saturating solution were added to each sample. The samples were placed on a laboratory shaker for 5 minutes, then centrifuged at 1,000 rpm for 5 minutes until the supernatant liquids were clear. The supernatant was decanted and fresh saturating solution was added. The samples were sonified for 30 seconds to disperse the sediment. The shaking, centrifuging, and decanting steps were repeated four times.

Extraction. The sodium extraction was accomplished by adding 33 millimeters of extracting solution to each decanted soil sample. The centrifuge tubes were placed on a shaker for 5 minutes, then centrifuged for 5 minutes. The extracting solution was decanted and placed into a 100 -millimeters volumetric flask. The extraction steps were repeated twice more with fresh solution. After the last extraction, the volumetric flask containing the extraction solutions was brought to volume with fresh extracting solution. Samples were taken for chloride and sodium analysis. Chloride analysis was performed on an ion chromatograph using an AS4A column. Sodium analysis was done using an ICP. 
Appropriate calibration checks were performed on each instrument before and during analysis to verify the validity of the results.

\subsubsection{Particle Size Determination}

The particle size distributions of individual composite samples were determined according to ASTM method D422-63, Standard Method for Particle-Size Analysis of Soils (ASTM 1986b). Subsamples of previously homogenized soil samples were taken and their weights corrected to oven-dry conditions (see Section 3.3.1). Two subsamples of each composite were taken to determine particle size, one for sieve analysis and another for hydrometer analysis. The equivalent dry weight of each subsample was determined by calculation. The subsamples were individually spread on drying trays in a fume hood and air dried to a constant weight in preparation for the following tests.

Sieve Analysis. To determine fractions of gravel, very coarse sand, coarse sand, medium sand, fine sand, and silt plus clay, the air-dried subsamples were wet-sieved using deionized water to disperse the individual particles. The water-soil combinations were shaken for 10 minutes at $150 \mathrm{rpm}$ on an orbit shaker. The total material was then transferred to a 2-millimeter sieve (No. 10 mesh) and wet-sieved to separate materials larger than 2-millimeter. Material passing through the 2-millimeter sieve was further wet-sieved sequentially through 1.00-millimeter (No. 18), 0.5-millimeter (No. 35), 0.25-millimeter (No. 60), 0.106-millimeter (No.140), and 0.053-millimeter (No. 270) sieves. Soil passing through the 0.053millimeter sieve was collected into pans. All fractions smaller than 2-millimeter were placed into an oven at $110^{\circ} \mathrm{C}$ to remove water. After oven-dry material weights were obtained, the samples were dry sieved until not more than 1 mass percent of the residue on the sieve passed through the sieve during 1 minute of sieving, as required by the ASTM procedure. Material remaining on the 2-millimeter sieve was allowed to air dry, then was weighed.

The smaller sieve-size fractions of some subsamples with high silt or clay content were wet-sieved multiple times to ensure all of the clay-size particles had been separated from larger particles. The mass of each size fraction was determined using a calibrated balance.

Hydrometer Analysis. The ASTM method (ASTM D1140-54 ASTM 1986c) allows hydrometer analysis of the entire portion passing through the 2-millimeter sieve, however the actual fraction analyzed by this technique was the fraction passing through the 0.053 -millimeter sieve. This fraction was dispersed with sodium hexametaphosphate. Hydrometer readings indicated the soil sedimentation rates; the rates for each sample then were used to calculate the distribution of materials smaller than 0.053millimeter. Particle density is a needed input for the hydrometer method calculation of particle size. Bulk particle density measurements were performed using the pychnometer method (method 14-3 found in ASA part 1; pages 378-379 [ASA 1986]).

Data obtained through wet-sieving and hydrometer readings were used to calculate the weight percentage of each size fraction for each soil composite. 


\subsubsection{Mineralogy}

Bulk sediment samples were dispersed by mixing 100 grams of sediment with 1 liter of $0.001 \mathrm{M}$ sodium hexametaphosphate. The suspensions were allowed to shake overnight to ensure complete dispersion. The sand fraction was separated from the dispersed sample by wet sieving through a No. 230 sieve. The silt and clay fractions were separated based on Stoke's settling law described in Jackson (1969). The lower limit of the silt fraction was taken at $>2$ microns. Sand and silt fractions were oven dried at $110^{\circ} \mathrm{C}$ and prepared for $\mathrm{x}$-ray diffraction (XRD) and $\mathrm{x}$-ray fluorensence analysis.

Each clay fraction was concentrated to an approximate volume of $10 \mathrm{~mL}$ by adding a few drops of $10 \mathrm{~N}$ magnesium chloride to the dispersed suspension. The weight percent of the clay in the samples was determined by drying known volumes of suspensions and weighing the dried sediment. The density of the suspension was calculated from the volume pipetted and the final weight of dried sediment. Volumes of slurry containing 250 milligrams of clay were transferred into centrifuge tubes and treated to remove carbonates following the procedure given in Jackson (1969). The carbonate-free sample was then saturated with either $\mathrm{Mg}^{2+}$ or $\mathrm{K}^{+}$cations. Samples of the clay fraction were prepared using the Drever (1973) method and placed onto an aluminum slide for XRD analysis. Due to the tendency of the clay film to peel and curl, the $\mathrm{Mg}^{2+}$ saturated slides were solvated with a few drops of a $10 \%$ solution of ethylene glycol in ethanol prior to drying and placed into a desiccator containing excess ethylene glycol for a minimum of 24 hours. After analysis of the $\mathrm{Mg}^{2+}$-saturated, ethylene glycol-solvated specimen, the slides were allowed to air dry overnight before reanalysis. Potassium-saturated slides were air dried and analyzed, then heated to $575^{\circ} \mathrm{C}$ and reanalyzed.

All samples were analyzed on a Scintag XRD unit equipped with a Pelter thermoelectrically cooled detector. Slides of preferentially oriented clay were scanned from 2 to 45 degrees $2 \theta$, and randomly oriented powder mounts of the bulk sediment and silt fraction were scanned from 2 to 75 degrees $2 \theta$. The bulk samples and silt fractions were prepared by crushing $\sim 0.5$ gram of sample to a fine powder that was then packed into a small circular holder.

Semiquantification of mineral phases by XRD was done according to Brindley and Brown (1980). The relationship of intensity and mass absorption to the weight fraction of a mineral in an unknown mixture is expressed as:

$$
\mathrm{I} / \mathrm{I}_{\mathrm{p}}=\mu_{\mathrm{p}} / \mu(\mathrm{wf})
$$

where: $\quad I=$ intensity of the mineral in the mixture

$\mathrm{I}_{\mathrm{p}}=$ intensity of the pure mineral

$\mu_{\mathrm{p}}=$ mass absorption of the pure mineral

$\mu=$ average mass absorption of the mixture

$\mathrm{wf}=$ weight fraction of the mineral in the mixture.

The wf is calculated from measurements of intensities and mass absorption coefficients. Pure mineral samples of illite, smectite, kaolinite, and chlorite were obtained from the Clay Mineral Society's source clays repository (operated from the University of Missouri in Columbia, Missouri), and analyzed under 
the same conditions as the sediment samples. Quartz, feldspars, and calcite were purchased from the Excalibur Mineral Company, Peekskill, New York, ground, and analyzed on the XRD to obtain intensities for pure non-clay phases.

The mass attenuation coefficients of similar, nonradioactive Hanford sediments were measured according to Brindley and Brown (1980). Ground bulk powders or air-dried clay fractions were packed into a 2.4 centimeter (0.94-inch)-thick circular holder with no backing. The holder was placed in front of the detector and positioned to allow the x-ray beam, diffracted from pure quartz, to pass through the sample and into the detector. The scan was analyzed from 26.0 to 27.0 degrees $2 \theta$. The mass attenuation coefficients were measured directly using the following equation:

$$
\mu=(1 / \rho x) \ln \left(\mathrm{I}_{\mathrm{o}} / \mathrm{I}_{\mathrm{x}}\right)
$$

where: $1 / \mathrm{px}=$ mass per unit area as the sample is prepared

$\mathrm{I}_{\mathrm{o}}=$ intensity of the incident beam

$\mathrm{I}_{\mathrm{x}}=$ intensity of the transmitted beam through sample thickness $\mathrm{x}$.

In addition to XRD, transmission electron microscopy (TEM) characterization of selected samples was conducted with a JEOL 1200X electron microscope equipped with a Links detector system. Samples were prepared for TEM by transferring a small aliquot of the dilute clay slurry onto a formvar carboncoated 3-millimeter copper support grid. The clay solution contained $0.15 \%$ tert-butylamine to reduce the surface tension of water.

\subsubsection{Cesium and Strontium $K_{d}$ Tests Using Various Solutions}

Adsorption-desorption tests were performed with the sediments from the 41-09-39 borehole extension project. Batch adsorption tests were conducted using sediments from two nonradioactive composites $(10 \mathrm{~A} / \mathrm{B}$ and $38 \mathrm{~A} / \mathrm{B})$ and one composite $(12 \mathrm{~A} / \mathrm{B})$ minutely contaminated with cesium-137. In addition, one highly contaminated composite $(2 \mathrm{~A} / \mathrm{B})$ was used to perform batch desorption tests. The test matrix design required placing 1 gram of sediment in each of four 30 -milliliter solutions. The solutions were composed to represent the following types of materials found on the Hanford Site:

- a high-ionic-strength, high-soluble-aluminum, high-caustic solution, representing REDOX liquid waste

- a high-ionic-strength sodium nitrite and nitrate solution, representing tank liquor

- a $4 \mathrm{M} \mathrm{NaNO}_{3}$ solution that allowed us to compare these adsorption data with older Hanford Site data for analyses that used solutions up to $4 \mathrm{M}$ sodium

- uncontaminated groundwater from a well located near the 400 Area Fast Flux Test Facility. This water has been used and characterized for over 10 years in various waste-form leaching and adsorption tests (e.g., Serne et al. 1989, 1993). 
Each solution used in the adsorption tests was spiked with enough strontium-85 and cesium-137 tracers to get good counting statistics $(\sim 2,000 \mathrm{cpm} / \mathrm{mL})$. The solutions were allowed to equilibrate for several days. After the spiked solutions were filtered through a 0.45 -micron syringe filter, initial counts were taken. The strontium- 85 and cesium- 137 activities were determined by GEA using the method described in Section 3.3.3. Once the initial activities for each solution were determined, the spiked solutions were added to each composite sediment using a soil-to-solution ratio of $1 \mathrm{~g}: 30$ milliliters. At the completion of the tests (13 days of contact), each tube was centrifuged and 20 to $25 \mathrm{~mL}$ of the supernatant liquid were filtered through a 0.45 -micron syringe filter and counted. The remaining unfiltered effluent was used to measure $\mathrm{pH}$ using a glass electrode. No corrections were made for potentially large junction potentials caused by the high-ionic-strength solutions. (Measured $\mathrm{pH}$ values may vary from the true values by as much as one full unit for the three high-ionic-strength solutions.) Details on the method can be found in Relyea et al. (1980).

For the desorption tests with composite $2 \mathrm{~A} / \mathrm{B}$ sediment, the four solutions were used without tracers and the amount of cesium-137 was measured in the centrifuged and filtered effluent.

All batch tests were run in duplicate with duplicate blanks for each tracer-spiked solution. In all, 32 separate tests were run for adsorption; 8 were run for desorption. None of the sediments were prewashed with unspiked solutions to remove evaporites. The ionic strengths of the three mixed solutions were sufficient to mask any effects that dissolution of evaporites might contribute.

Preparation of Simulated Tank Leak Solutions. For each solution, 500 milliliters were prepared using gravimetric measurement. The solutions were prepared in the following manner.

- Solution 1. Using an oven-dried volumetric flask with calibration marks at 350, 400, and 425 millilters, proceed as follows:

1. Add deionized water by weight to the 350-milliliter mark.

2. Add 40 grams of $\mathrm{NaOH}$ pellets (anhydrous) and allow to dissolve.

3. Add 233.75 grams $\mathrm{NaNO}_{3}$ (anhydrous). Add reagent in portions and check for complete solubility before adding the next portion. Sum weight of total reagent that dissolved.

4. Add additional deionized water by weight to the 425-milliliter mark.

5. Add 10.25 grams $\mathrm{NaAlO}_{2}$ and allow to dissolve.

6. Add deionized water by weight to the 500-milliliter mark.

7. Equilibrate.

8. Weigh flask and calculate solution density.

Solution 1 target concentrations are in molarity (M): $2 \mathrm{M} \mathrm{NaOH}, 5.5 \mathrm{M} \mathrm{NaNO}_{3}, 0.45 \mathrm{M} \mathrm{NaNO}_{2}$, and $0.25 \mathrm{M} \mathrm{NaAlO}_{2}$. 
- Solution 2. Using an oven-dried volumetric flask with calibration marks at 350, 400, and 425 milliliters, proceed as follows:

1. Add deionized water by weight to the 350 -milliliter mark.

2. Add 15.5 grams $\mathrm{NaNO}_{2}$ (anhydrous) and allow to dissolve.

3. Add 329.38 grams $\mathrm{NaNO}_{3}$ (anhydrous). Add reagent in portions, and check for complete solubility before adding the next portion.

4. Add deionized water by weight to the 500-milliliter mark.

5. Equilibrate.

6. Weigh flask and calculate solution density.

Solution 2 target concentrations are in molarity (M): $7.75 \mathrm{M} \mathrm{NaNO}_{3}$ and $0.45 \mathrm{M} \mathrm{NaNO}_{2}$.

- Solution 3, 4 M NaNO . Using an oven-dried volumetric flask with calibration marks at 350, 400, and 425 milliliters, proceed as follows:

1. Add deionized water by weight to the 350-milliliter mark.

2. Add 170 grams $\mathrm{NaNO}_{3}$ (anhydrous) and allow to dissolve.

3. Add deionized water by weight to the 500-milliliter mark.

4. Equilibrate.

5. Weigh flask and calculate solution density.

Solution 3 target concentration is $4 \mathrm{M} \mathrm{NaNO}_{3}$.

- Solution 4, Hanford Site Groundwater. Several liters of groundwater collected at well 699-S3-25 on the Hanford Site were filtered through a 0.45 -micron filter. Based on the most recent analyses of this water, the chemical composition is shown in Table 3.2. The water contains predominately calcium, bicarbonate, and sulfate with a slightly alkaline $\mathrm{pH}$. 
Table 3.2. Chemical Composition of Hanford Site Groundwater from Well 699-S3-25

\begin{tabular}{||l|c|c|c|c||}
\hline \multicolumn{1}{|c|}{ Constituent } & Units & Value & $\mathrm{M}$ & $\mathrm{meq} / \mathrm{L}$ \\
\hline \hline $\mathrm{pH}$ & $\mathrm{none}$ & 7.5 & & \\
\hline $\mathrm{Na}$ & $\mathrm{mg} / \mathrm{L}$ & 24 & $1.04 \mathrm{E}-03$ & 1.043 \\
\hline $\mathrm{K}$ & $\mathrm{mg} / \mathrm{L}$ & 7.6 & $1.94 \mathrm{E}-04$ & 0.194 \\
\hline $\mathrm{Ca}$ & $\mathrm{mg} / \mathrm{L}$ & 69 & $1.72 \mathrm{E}-03$ & 3.443 \\
\hline $\mathrm{Mg}$ & $\mathrm{mg} / \mathrm{L}$ & 15 & $6.17 \mathrm{E}-04$ & 1.234 \\
\hline $\mathrm{Sr}$ & $\mathrm{mg} / \mathrm{L}$ & 0.27 & $3.08 \mathrm{E}-06$ & 0.006 \\
\hline $\mathrm{Ba}$ & $\mathrm{mg} / \mathrm{L}$ & 0.07 & $5.10 \mathrm{E}-07$ & 0.001 \\
\hline $\mathrm{Fe}$ & $\mathrm{mg} / \mathrm{L}$ & 0.002 & $3.58 \mathrm{E}-08$ & 0 \\
\hline $\mathrm{Mn}$ & $\mathrm{mg} / \mathrm{L}$ & 0.08 & $1.46 \mathrm{E}-06$ & 0.003 \\
\hline $\mathrm{Al}$ & $\mathrm{mg} / \mathrm{L}$ & 0.09 & $3.34 \mathrm{E}-06$ & \\
\hline $\mathrm{Si}$ & $\mathrm{mg} / \mathrm{L}$ & 10 & $3.56 \mathrm{E}-04$ & \\
\hline $\mathrm{B}$ & $\mathrm{mg} / \mathrm{L}$ & 0.06 & $5.55 \mathrm{E}-06$ & 0.006 \\
\hline $\mathrm{C}$ & $\mathrm{mg} / \mathrm{L}$ & 32.8 & $2.73 \mathrm{E}-03$ & 2.731 \\
\hline $\mathrm{Cl}$ & $\mathrm{mg} / \mathrm{L}$ & 24 & $6.77 \mathrm{E}-04$ & 0.677 \\
\hline $\mathrm{SO}_{4}$ & $\mathrm{mg} / \mathrm{L}$ & 115 & $1.20 \mathrm{E}-03$ & 2.394 \\
\hline $\mathrm{NO}_{3}$ & $\mathrm{mg} / \mathrm{L}$ & 1 & $1.61 \mathrm{E}-05$ & 0.016 \\
\hline $\mathrm{F}$ & $\mathrm{mg} / \mathrm{L}$ & 0.5 & $2.63 \mathrm{E}-05$ & 0.026 \\
\hline $\mathrm{Cations}$ & & & & 5.925 \\
\hline $\mathrm{Anions}^{2}$ & & & & 5.85 \\
\hline
\end{tabular}




\subsection{Results and Discussion - Scoping Studies}

This section presents the data collected during the scoping phase of the borehole extension project and similar data for the sidewall core samples collected during the decommissioning phase. The scoping phase emphasized tests that were inexpensive or that were key to determining the vertical distribution of contaminants. Our intent was to gather information from the individual sleeves collected in each splitspoon sampling event that would assist in determining which samples were least affected by drilling challenges and, at the same time, represent the sediment underlying the SX Tank Farm. We were particularly interested in avoiding sediment that was impacted by the addition of water to the borehole during milling of the steel end cap and hard tooling through coarse-grained sediment. We also wanted to avoid samples that demonstrated atypical chemical and radionuclide characteristics that could be attributed to smearing or drag-down of sediment from, respectively, the sampling tools or borehole cleanout and shallower depths during the cable-tool drilling. Information on the borehole sediments presented in this report includes moisture content, $\mathrm{pH}$ and electrical conductivity (EC) of 1:1 water extracts, and in some instances, measurements of major cations, anions, and radionuclides. Furthermore, in an attempt to understand whether the sediment's moisture status was wet enough to be actively draining or conversely, dry enough to preclude active water transport, the matric potential of sediment from selected sleeves was measured.

\subsection{Moisture Content}

The moisture content of the sediment from each sleeve/sidewall core is listed in Table 4.1 and presented as a graph in Figure 4.1. Readings obtained from the first two samples collected below the tank farm excavation 17.1 to 18.7 meters (56 to 61.5 feet) below ground surface (bgs) are rather wet at 16.3 and $12.8 \mathrm{wt} \%$. Values then drop over the next 3 meters (10 feet) to near $5 \mathrm{wt} \%$ and are low at the top of the coarse unit, Hanford formation $\mathrm{H} 1$ at 20.4 meters ( 67 feet) bgs. At 24.4 meters ( 80 feet) bgs in the Hanford formation $\mathrm{H} 1$ unit, the moisture content is between 8 and $10 \mathrm{wt} \%$. In the lower laminated sand unit (Hanford formation $\mathrm{H} 2$ unit), the moisture content peaks at 33.2 meters (109 feet) bgs at $12 \mathrm{wt} \%$. At the top of the Plio-Pleistocene mud unit (38.1 meters [125 feet] bgs), the moisture content is $13 \mathrm{wt} \%$, but then sharply increases at 40.5 meters (133 feet) bgs. At 40.5 meters (133 feet) bgs, this wet zone corresponds precisely to the depth where the end cap was milled off during the extension phase of the drilling project. At this depth, about 75 liters of cooling water escaped into the borehole when the end cap was breached. The evidence clearly indicates that excess water in borehole extension sleeves 2D, $2 \mathrm{C}$, and $2 \mathrm{C}-2$ was the direct result of water being used to cool the drill bit during milling of the end drive shoe. Normal amounts of moisture, ranging from 9.4 to $16.1 \mathrm{wt} \%$, were recorded from sleeves down to $\sim 43.4$ meters ( 142.4 feet) bgs (see Appendixes A and B). Abnormally high water readings were observed in sleeves $6 \mathrm{D}, 6 \mathrm{C}$, and 6B. A review of the driller's logs indicated that the sampler was left in the borehole at this depth over several days and that a rain event likely allowed some water to enter the borehole. Overall, the fine-grained sediment in the Plio-Pleistocene unit (between 38.1 and 47.2 meters [125 and 155 feet] bgs) had relatively high moisture contents ranging from 7.4 to $19.6 \mathrm{wt} \%$. We believe that this moisture content range is natural and not an indicator of remnant tank fluids or drilling artifacts (see Serne et al. 2002a). 
Table 4.1. Moisture Content of Sidewall Cores and Extension Sleeves

\begin{tabular}{|c|c|c|c|c|c|c|c|c|}
\hline \begin{tabular}{|c|} 
Depth \\
$(\mathrm{ft} \text { bgs })^{(\mathrm{a})}$
\end{tabular} & $\begin{array}{c}\text { ID } \\
\text { Sample }\end{array}$ & $\begin{array}{c}\text { Content (\%) } \\
\text { Moisture } \\
\end{array}$ & \begin{tabular}{|c|} 
Depth \\
$(\mathrm{ft} \text { bgs })^{(\mathrm{a})}$
\end{tabular} & $\begin{array}{c}\text { ID } \\
\text { Sample }\end{array}$ & $\begin{array}{c}\text { Content (\%) } \\
\text { Moisture }\end{array}$ & \begin{tabular}{|c|}
$\begin{array}{c}\text { Depth } \\
(\mathrm{ft} \text { bgs })^{(\mathrm{a})}\end{array}$ \\
\end{tabular} & $\begin{array}{c}\text { ID } \\
\text { Sample }\end{array}$ & $\begin{array}{c}\begin{array}{c}\text { Content (\%) } \\
\text { Moisture }\end{array} \\
\end{array}$ \\
\hline \multicolumn{3}{|c|}{ Sidewall } & 149.9 & $8 \mathrm{~B}$ & 9.7 & 183.9 & $36 \mathrm{C}$ & 3.37 \\
\hline 25.5 & $15 \mathrm{~A} / \mathrm{B} / \mathrm{C}$ & 8.12 & 151.1 & $9 \mathrm{C}$ & 12.8 & 184.6 & $36 \mathrm{~B}$ & 2.06 \\
\hline 44.5 & $14 \mathrm{~A} / \mathrm{B} / \mathrm{C}$ & 8.57 & 151.8 & 9B & NM & 185.8 & $37 \mathrm{D}$ & 1.39 \\
\hline 56.5 & $13 \mathrm{~A} / \mathrm{B} / \mathrm{C}$ & 16.27 & 152.8 & $9 \mathrm{~A}$ & 13.9 & 186.2 & $37 \mathrm{C}$ & 2.6 \\
\hline 61.5 & $12 \mathrm{~A} / \mathrm{B} / \mathrm{C}$ & 12.84 & 153.4 & $10 \mathrm{C}$ & 13.6 & 186.8 & $37 \mathrm{~B}$ & 2.35 \\
\hline 65.5 & $11 \mathrm{~A} / \mathrm{B}$ & 4.71 & 153.9 & $10 \mathrm{~B}$ & 14.7 & 187.2 & $37 \mathrm{~A}$ & 2.09 \\
\hline 66 & $11 \mathrm{C}$ & 5.29 & 154.5 & $10 \mathrm{~A}$ & 19.6 & 187.8 & $38 \mathrm{C}$ & 2.87 \\
\hline 69.5 & $10 \mathrm{~A} / \mathrm{B} / \mathrm{C}$ & 4.36 & 155.1 & $11 \mathrm{D}$ & 5.4 & 188.3 & $38 \mathrm{~B}$ & 1.9 \\
\hline 74.5 & $9 \mathrm{~A} / \mathrm{B} / \mathrm{C}$ & 5.17 & 155.4 & $11 \mathrm{C}$ & 4.3 & 188.7 & $38 \mathrm{~A}$ & 1.8 \\
\hline 79.5 & $8 \mathrm{~A} / \mathrm{B} / \mathrm{C}$ & 10.71 & 155.9 & $11 \mathrm{~B}$ & 3.5 & 189.2 & $39 \mathrm{~B}$ & 1.54 \\
\hline 82.5 & $7 \mathrm{~A} / \mathrm{B} / \mathrm{C}$ & 8.41 & 156.4 & $11 \mathrm{~A}$ & 4.5 & 189.5 & $39 \mathrm{~A}$ & 1.93 \\
\hline 90 & $6 \mathrm{~A} / \mathrm{B}$ & 10.25 & 156.8 & $12 \mathrm{~B}$ & 6.9 & 190.7 & $40 \mathrm{~A}$ & 5.13 \\
\hline 95.5 & $3 \mathrm{~A} / \mathrm{B} / \mathrm{C}$ & 7.83 & 157.1 & $12 \mathrm{~A}$ & 6.6 & 193.3 & $45 \mathrm{~A}$ & 5.28 \\
\hline 102.5 & $5 \mathrm{~A} / \mathrm{B} / \mathrm{C}$ & 10.4 & 157.2 & $13 \mathrm{D}$ & 6.4 & 195.9 & $47 \mathrm{D}$ & 8.84 \\
\hline 108.5 & $4 \mathrm{~A} / \mathrm{B} / \mathrm{C}$ & 12.01 & 157.7 & $13 \mathrm{C}$ & 4 & 196.4 & $47 \mathrm{C}$ & 6.62 \\
\hline 112 & $2 \mathrm{~B} / \mathrm{C}$ & 8.17 & 158.2 & $13 \mathrm{~B}$ & 5.3 & 196.9 & $47 \mathrm{~B}$ & 5.74 \\
\hline 127.4 & $1 \mathrm{~A} / \mathrm{B} / \mathrm{C}$ & 12.66 & 159.4 & $14 \mathrm{C}$ & 5.9 & 197.4 & $47 \mathrm{~A}$ & 4.94 \\
\hline \multicolumn{3}{|c|}{ Extension } & 160 & 14B & 5.8 & 199.1 & $49 \mathrm{~B}$ & 8.25 \\
\hline 131.1 & $1 \mathrm{C}$ & NM & 160.6 & $14 \mathrm{~A}$ & 7.6 & 199.1 & $49 \mathrm{~A}$ & 9.53 \\
\hline 131.7 & $1 \mathrm{~B}$ & 13 & 162.3 & $16 \mathrm{D}$ & 13.3 & 199.4 & $50 \mathrm{~B}$ & 6.83 \\
\hline 133.2 & $2 \mathrm{D}$ & 35.6 & 162.8 & $16 \mathrm{C}$ & 13.5 & 199.4 & $50 \mathrm{~A}$ & 3.89 \\
\hline 133.7 & $2 \mathrm{C}$ & 18.3 & 163.3 & $16 \mathrm{~B}$ & 4.1 & 200.2 & $52 \mathrm{~B}$ & 5.47 \\
\hline 133.7 & $2 \mathrm{C}-2$ & 26.7 & 163.8 & $16 \mathrm{~A}$ & 4.4 & 200.7 & $52 \mathrm{~A}$ & 8.15 \\
\hline 134.2 & $2 \mathrm{~B}$ & 14.5 & 164.3 & $17 \mathrm{D}$ & 4.91 & 202.5 & $54 \mathrm{~B}$ & 6.58 \\
\hline 134.7 & $2 \mathrm{~A}$ & 16 & 164.8 & $17 \mathrm{C}$ & 3.56 & 202.5 & $54 \mathrm{~A}$ & 5.44 \\
\hline 135.9 & $3 \mathrm{~A}$ & 13.1 & 165.3 & $17 \mathrm{~B}$ & 5.27 & 204.3 & $56 \mathrm{C}$ & 7.74 \\
\hline 137.4 & $4 \mathrm{C}$ & 10.9 & 165.8 & $17 \mathrm{~A}$ & 4.91 & 205 & $56 \mathrm{~B}$ & 6.58 \\
\hline 138 & $4 \mathrm{~B}$ & 11.7 & 166.6 & $18 \mathrm{C}$ & 9.7 & 205.6 & $56 \mathrm{~A}$ & 5.44 \\
\hline 138.7 & $4 \mathrm{~A}$ & 16.1 & 167.1 & $18 \mathrm{~B}$ & 4.7 & 207.6 & $58 \mathrm{D}$ & 9.4 \\
\hline 139.9 & $5 \mathrm{D}$ & 14.1 & 167.7 & $18 \mathrm{~A}$ & 4.7 & 208.1 & $58 \mathrm{C}$ & 10.18 \\
\hline 140.3 & $5 \mathrm{C}$ & 14.5 & 169.9 & $20 \mathrm{~A}$ & 4.3 & 208.6 & $58 \mathrm{~B}$ & 10.57 \\
\hline 140.9 & $5 \mathrm{~B}$ & 12.6 & 171.3 & $22 \mathrm{C}$ & 5.8 & 209 & $58 \mathrm{~A}$ & 8.16 \\
\hline 141.5 & $5 \mathrm{~A}$ & 9.4 & 172 & $22 \mathrm{~B}$ & 5.2 & 209.4 & 59D & 10.1 \\
\hline 141.9 & $6 \mathrm{~F}$ & 11 & 178.2 & $28 \mathrm{~A}$ & 7.94 & 209.8 & $59 \mathrm{C}$ & 12.49 \\
\hline 142.4 & $6 \mathrm{E}$ & 9.6 & 178.8 & $30 \mathrm{C}$ & 6.9 & 210.4 & $59 \mathrm{~B}$ & 17.5 \\
\hline 143.3 & $6 \mathrm{D}$ & 21.8 & 179.2 & $30 \mathrm{~B}$ & 3.35 & 210.9 & $59 \mathrm{~A}$ & 24.97 \\
\hline 144.1 & $6 \mathrm{C}$ & 26 & 179.7 & $30 \mathrm{~A}$ & 3.11 & 211.7 & $62 \mathrm{~B}$ & 8.15 \\
\hline 144.7 & $6 \mathrm{~B}$ & 22.9 & 180.2 & $31 \mathrm{~B}$ & 4.36 & 212.1 & $62 \mathrm{~A}$ & 10.03 \\
\hline 145.2 & $7 \mathrm{E}$ & 8.7 & 180.5 & $31 \mathrm{~A}$ & 3.72 & 212.9 & 64B & 12.58 \\
\hline 145.8 & $7 \mathrm{D}$ & 9.5 & 181.1 & $32 \mathrm{~B}$ & 5.31 & 213.3 & $64 \mathrm{~A}$ & 13.19 \\
\hline 146.4 & $7 \mathrm{C}$ & 11.2 & $\begin{array}{l}181.5 \\
181.5\end{array}$ & $32 \mathrm{~A}$ & 3.15 & 214.1 & $65 \mathrm{~B}$ & 7.76 \\
\hline 146.4 & $7 \mathrm{C}-\mathrm{R}$ & 11.2 & 183 & $35 \mathrm{~B}$ & 3.56 & 214.3 & $65 \mathrm{~A}$ & 8.6 \\
\hline 1483 & $8 \mathrm{C}$ & 7.4 & 183.3 & $35 \mathrm{~A}$ & 284 & & & \\
\hline
\end{tabular}




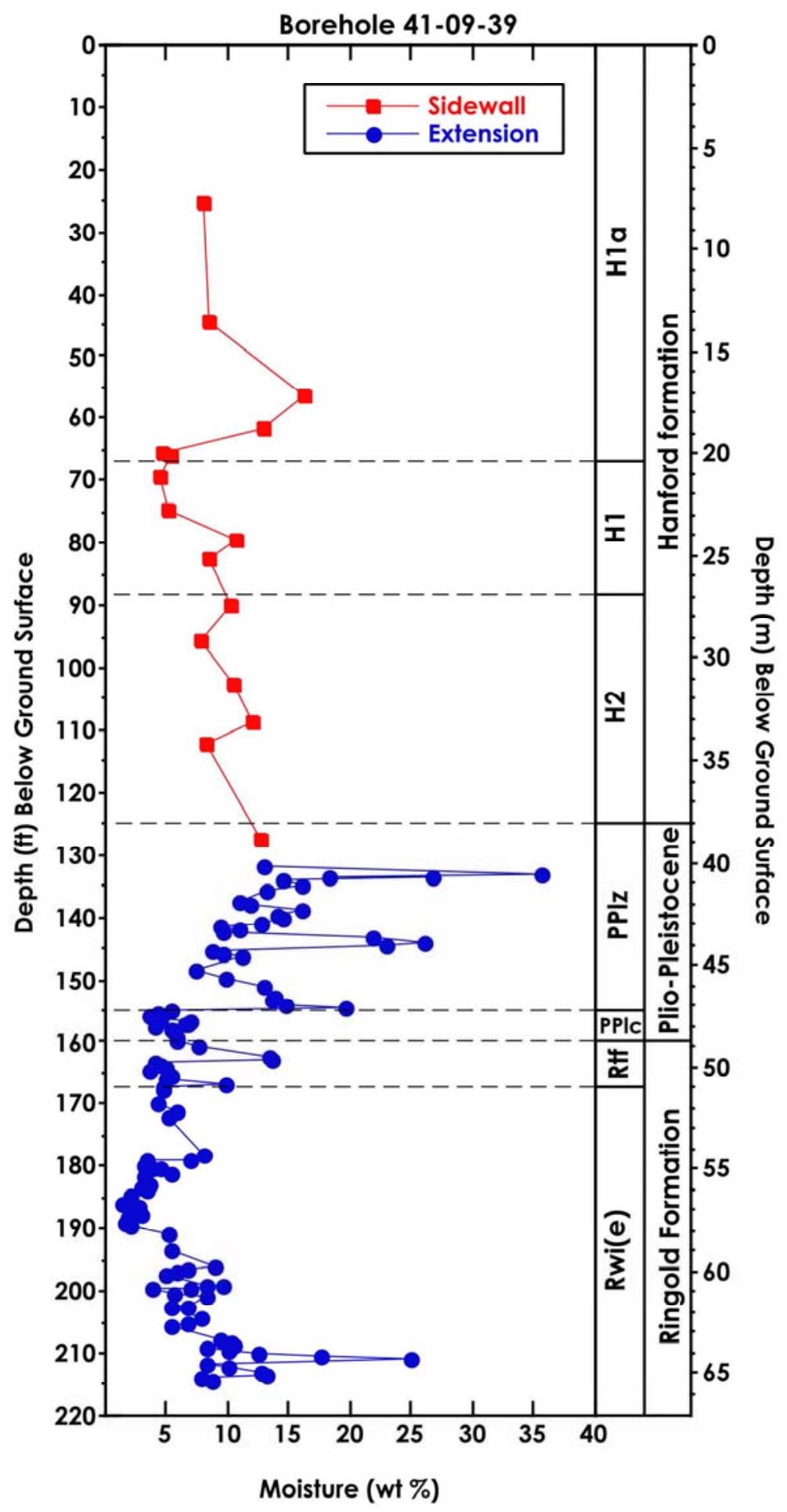

2002/DCL/41-09-39/017

Figure 4.1. Moisture Content Versus Depth for Vadose Sediment from Borehole 41-09-39. 
Hard tool drilling was initiated at 49.1 meters (161 feet) bgs and continued periodically down to 63.4 meters (208 feet) bgs. During 28 hard-tooling events, 58 liters of water were added. Sleeves that showed distinct increases in moisture were 16D, 16C, 18C, 28A, 30C, and perhaps all the sleeves between $40 \mathrm{~A}$ and $47 \mathrm{C}, 49 \mathrm{~B}$ to $50 \mathrm{~B}$, and 52B through 58B; although sleeves 58A and 58B appear to be wet, it is probably due to their position in the capillary fringe right above the water table. Of these potentially suspect samples, only sleeve 58B was used in detailed characterization. Overall, the coarse sediments associated with the Ringold Formation exhibited moisture contents as low as $1.5 \mathrm{wt} \%$ and the upper range on the coarse sediments moisture was about $6 \mathrm{wt} \%$. Although the moisture content itself is not as informative as the matric suction potential (described in Section 4.4), it does help identify intervals, which have been impacted by drilling operations.

\subsection{1:1 Sediment-to-Water Extract Chemistry}

The 1:1 water extracts of the sediments from individual sleeves/sidewall cores were used to measure $\mathrm{pH}$ and EC to give a quick and inexpensive indication of whether measurable effects of highly saline and very alkaline tank-leak liquor were present in the vadose sediments. Other analyses performed included major cations, nitrate, and, in a few cases, major anions. The $\mathrm{pH}$ and $\mathrm{EC}$ data for individual sleeves and sidewall cores are shown in Table 4.2. The $\mathrm{pH}$, of the 1:1 sediment to water extract and the dilution corrected EC, and nitrate data are shown in Figure 4.2.

Table 4.3 shows the major anion data as measured and dilution-corrected. Table 4.4 shows the cation concentrations in the 1:1 water extracts. Dilution corrected values are not shown.

The 1:1 water extract $\mathrm{pH}$ for the shallow sidewall core samples above the tank bottoms 7.8 to 18.7 meters ( 25.5 to 61.5 feet bgs) vary from 8.31 to 8.61 . Below the tanks 19.8 to 24.4 meters ( 65 to 80 feet bgs), the extract $\mathrm{pH}$ varies from 9.18 to 9.76 , which we believe represents interaction of the sediment with the alkaline fluids that leaked from the tanks. However, it is noted that these $\mathrm{pH}$ values are not nearly as high as would be expected for tank liquor completely saturating sediments. As described in Serne et al. (1998), the $\mathrm{pH}$ can reach values of $>13$ when simulated tank liquor reacts with Hanford sediments. One plausible explanation for the lower than expected $\mathrm{pH}$ values is that perhaps the $\mathrm{pH}$ re-neutralizes slowly with time from the slow dissolution of aluminum silicates. Likewise, it is also possible for the tank liquor to react with carbon dioxide in the vadose zone air-filled porosity such that the initial $\mathrm{pH}$ excursion to high values is muted over time.

From 27.4 to 38.7 meters (90 to 127 feet) bgs the sidewall core samples yield 1:1 water extracts that have $\mathrm{pH}$ values between 7.92 and 8.33 , which appears to be close to natural conditions. Therefore, based on information collected from the sidewall cores, it would appear that significant reactions occur that affect the $\mathrm{pH}$ from the tank bottoms at 16.8 meters ( $~ 55$ feet) bgs to a maximum depth of 27.4 meters (90 feet) bgs.

The water extract $\mathrm{pH}$ for borehole extension sleeve samples range from 7.21 to 8.97. These values are similar to Hanford Site background values for sediments that have not been altered by significant chemical reaction. If the sediment was at equilibrium with atmospheric carbon dioxide and the calcium carbonate mineral calcite, the $\mathrm{pH}$ should be fixed at 8.3. Hanford Site sediments generally contain small 
Table 4.2. $\mathrm{pH}$ and EC for 1:1 Water Extracts of Sidewall and Borehole Extension Sleeves

\begin{tabular}{|c|c|c|c|c|c|c|c|c|c|c|c|c|c|c|}
\hline $\begin{array}{c}\text { Depth } \\
\text { (ft bgs) }^{(\mathrm{a})}\end{array}$ & ID & $\begin{array}{c}1 \text { to } 1 \\
\mathrm{pH}\end{array}$ & $\begin{array}{c}1 \text { to } 1 \\
\mu \mathrm{S} / \mathrm{cm} \\
\end{array}$ & $\begin{array}{c}\text { Corrected } \\
\mu \mathrm{S} / \mathrm{cm}\end{array}$ & $\begin{array}{c}\text { Depth } \\
(\mathrm{ft} \text { bgs) }\end{array}$ & ID & $\begin{array}{c}1 \text { to } 1 \\
\mathrm{pH}\end{array}$ & $\begin{array}{l}1 \text { to } 1 \\
\mu \mathrm{S} / \mathrm{cm}\end{array}$ & $\begin{array}{c}\text { Corrected } \\
\mu \mathrm{S} / \mathrm{cm}\end{array}$ & $\begin{array}{c}\text { Depth } \\
(\mathrm{ft} \text { bgs })^{(\mathrm{a})}\end{array}$ & ID & $\begin{array}{c}1 \text { to } 1 \\
\mathrm{pH}\end{array}$ & $\begin{array}{c}1 \text { to } 1 \\
\mu \mathrm{S} / \mathrm{cm} \\
\end{array}$ & $\begin{array}{c}\text { Corrected } \\
\mu \mathrm{S} / \mathrm{cm}\end{array}$ \\
\hline & Sidewall & & & & 140.3 & $5 \mathrm{C}$ & 8.57 & 230 & 1,520 & 162.8 & $16 \mathrm{C}$ & 8.52 & 319 & 2,313 \\
\hline 25.5 & $15 \mathrm{~A} / \mathrm{B} / \mathrm{C}$ & 8.39 & 188 & 2,317 & 140.9 & 5B & 8.92 & 184 & 1,417 & 163.3 & $16 \mathrm{~B}$ & 8.02 & 117 & 2,828 \\
\hline 44.5 & $14 \mathrm{~A} / \mathrm{B} / \mathrm{C}$ & 8.52 & 226 & 2,638 & 141.5 & $5 \mathrm{~A}$ & 8.97 & 167 & 1,737 & 163.8 & $16 \mathrm{~A}$ & 7.79 & 92 & 2,112 \\
\hline 56.5 & $13 \mathrm{~A} / \mathrm{B} / \mathrm{C}$ & 8.31 & 287 & 1,790 & 141.9 & $6 \mathrm{~F}$ & 8.49 & 255 & 4,886 & 164.3 & $17 \mathrm{D}$ & 8.36 & 268 & 5,435 \\
\hline 61.5 & $12 \mathrm{~A} / \mathrm{B} / \mathrm{C}$ & 8.61 & 355 & 2,767 & 142.4 & $6 \mathrm{E}$ & 8.36 & 203 & 4,389 & 164.8 & $17 \mathrm{C}$ & 8.31 & 322 & 9,000 \\
\hline 65.5 & $11 \mathrm{~A} / \mathrm{B}$ & 9.18 & 899 & 19,092 & 143.3 & $6 \mathrm{D}$ & 8.46 & 300 & 3,033 & 165.3 & $17 \mathrm{~B}$ & 7.77 & 149 & 2,828 \\
\hline 66 & $11 \mathrm{C}$ & 9.76 & 504 & 9,535 & 144.1 & $6 \mathrm{C}$ & 8.29 & 353 & 3,057 & 165.8 & $17 \mathrm{~A}$ & 7.78 & 93 & 2,031 \\
\hline 69.5 & $10 \mathrm{~A} / \mathrm{B} / \mathrm{C}$ & 9.18 & 752 & 17,248 & 144.7 & $6 \mathrm{~B}$ & 8.5 & 314 & 3,036 & 166.6 & $18 \mathrm{C}$ & 8.1 & 181 & 1,861 \\
\hline 74.5 & $9 \mathrm{~A} / \mathrm{B} / \mathrm{C}$ & 9.6 & 719 & 13,928 & 145.2 & $7 \mathrm{E}$ & 8.75 & 194 & 2,328 & 167.1 & $18 \mathrm{~B}$ & 7.85 & 99 & 2,100 \\
\hline 79.5 & $8 \mathrm{~A} / \mathrm{B} / \mathrm{C}$ & 9.55 & 1,722 & 16,090 & 145.8 & $7 \mathrm{D}$ & 8.7 & 212 & 2,209 & 167.7 & $18 \mathrm{~A}$ & 7.94 & 105 & 2,246 \\
\hline 82.5 & $7 \mathrm{~A} / \mathrm{B} / \mathrm{C}$ & 8.70 & 8,293 & 98,684 & 146.4 & $7 \mathrm{C}$ & 8.41 & 237 & 2,097 & 169.9 & $20 \mathrm{~A}$ & 8.35 & 216 & 5,033 \\
\hline 90 & $6 \mathrm{~A} / \mathrm{B}$ & 8.33 & 41,820 & 408,017 & 148.3 & $8 \mathrm{C}$ & 8.71 & 236 & 3,160 & 171.3 & $22 \mathrm{C}$ & 8.43 & 250 & 4,295 \\
\hline 95.5 & $3 \mathrm{~A} / \mathrm{B} / \mathrm{C}$ & 7.93 & 41,010 & 521,060 & 149.9 & $8 \mathrm{~B}$ & 8.71 & 200 & 2,032 & 172 & 22B & 8.33 & 180 & 3,433 \\
\hline 102.5 & $5 \mathrm{~A} / \mathrm{B} / \mathrm{C}$ & 8.01 & 41,910 & 402,855 & 152.8 & $9 \mathrm{~A}$ & 8.91 & 210 & 1,489 & 178.2 & $28 \mathrm{~A}$ & 8.46 & 231 & 2,897 \\
\hline 108.5 & $4 \mathrm{~A} / \mathrm{B} / \mathrm{C}$ & 8.07 & 56,480 & 470,722 & 153.4 & $10 \mathrm{C}$ & 8.75 & 277 & 1,997 & 178.8 & $30 \mathrm{C}$ & 8.36 & 183 & 2,637 \\
\hline 112 & $2 B / C$ & 8.12 & 42,770 & 524,413 & 153.9 & $10 \mathrm{~B}$ & 8.7 & 254 & 2,085 & 179.2 & $30 \mathrm{~B}$ & 7.62 & 67 & 2,000 \\
\hline 127.4 & $1 \mathrm{~A} / \mathrm{B} / \mathrm{C}$ & 7.92 & 16,550 & 130,789 & 154.5 & $10 \mathrm{~A}$ & 8.75 & 221 & 1,085 & 179.7 & $30 \mathrm{~A}$ & 7.72 & 74 & 2,375 \\
\hline \multicolumn{3}{|c|}{ Extension } & & & 155.4 & $11 \mathrm{C}$ & 8.62 & 163 & 3,736 & 180.2 & $31 \mathrm{~B}$ & 8.42 & 214 & 4,892 \\
\hline 131.1 & $1 \mathrm{C}$ & 8.52 & 382 & N/A & 155.9 & $11 \mathrm{~B}$ & 8.64 & 155 & 4,431 & 180.5 & $31 \mathrm{~A}$ & 8.39 & 201 & 5,395 \\
\hline 131.7 & $1 \mathrm{~B}$ & 8.06 & 4,858 & 36,192 & 156.4 & $11 \mathrm{~A}$ & 8.67 & 114 & 4,788 & 181.1 & $32 \mathrm{~B}$ & 8.51 & 267 & 5,044 \\
\hline 133.2 & $2 \mathrm{D}$ & 8.26 & 865 & 1,817 & 156.8 & $12 \mathrm{~B}$ & 8.64 & 379 & 5,435 & 181.5 & $32 \mathrm{~A}$ & 8.3 & 151 & 4,793 \\
\hline 133.7 & $2 \mathrm{C}$ & 8.28 & 413 & 1,648 & 157.1 & $12 \mathrm{~A}$ & 8.63 & 317 & 4,761 & 183 & $35 \mathrm{~B}$ & 8.5 & 261 & 7,334 \\
\hline 134.2 & $2 \mathrm{~B}$ & 8.36 & 658 & 4,363 & 157.2 & 13D & 8.55 & 289 & 4,471 & 183.3 & $35 \mathrm{~A}$ & 8.32 & 190 & 6,680 \\
\hline 134.7 & $2 \mathrm{~A}$ & 8.25 & 611 & 3,629 & 157.7 & $13 \mathrm{C}$ & 8.8 & 311 & 7,731 & 183.9 & $36 \mathrm{C}$ & 8.18 & 137 & 4,052 \\
\hline 135.2 & $3 \mathrm{~B}$ & 8.25 & 831 & 5,618 & 158.2 & 13B & 8.7 & 190 & 3,576 & 184.6 & $36 \mathrm{~B}$ & 7.85 & 67 & 3,247 \\
\hline 135.9 & $3 \mathrm{~A}$ & 8.34 & 442 & 3,266 & 158.7 & $13 \mathrm{~A}$ & 7.21 & 207 & 3,912 & 185 & $36 \mathrm{~A}$ & 7.96 & 94 & 5,493 \\
\hline 137 & 4D & 8.29 & 387 & 3,150 & 159.4 & $14 \mathrm{C}$ & 8.5 & 270 & 4,552 & 185.8 & 37D & 7.87 & 68 & 4,891 \\
\hline 137.4 & $4 \mathrm{C}$ & 8.78 & 172 & 1,538 & 160 & $14 \mathrm{~B}$ & 8.46 & 215 & 3,726 & 186.2 & $37 \mathrm{C}$ & 7.48 & 49 & 1,886 \\
\hline 138 & $4 \mathrm{~B}$ & 8.61 & 223 & 1,846 & 160.6 & $14 \mathrm{~A}$ & 8.38 & 189 & 2,482 & 186.8 & $37 \mathrm{~B}$ & 7.66 & 40 & 1,699 \\
\hline 138.7 & $4 \mathrm{~A}$ & 8.63 & 193 & 1,141 & 162.3 & $16 \mathrm{D}$ & 8.28 & 245 & 1,813 & 187.2 & $37 \mathrm{~A}$ & 7.57 & 45 & 2,155 \\
\hline
\end{tabular}


Table 4.2. (contd)

\begin{tabular}{|c|c|c|c|c|c|c|c|c|c|c|c|c|c|c|}
\hline $\begin{array}{c}\text { Depth } \\
(\mathrm{ft} \text { bgs })^{(a)}\end{array}$ & ID & $\begin{array}{c}1 \text { to } 1 \\
\mathrm{pH}\end{array}$ & $\begin{array}{c}1 \text { to } 1 \\
\mu \mathrm{S} / \mathrm{cm} \\
\end{array}$ & $\begin{array}{c}\text { Corrected } \\
\mu \mathrm{S} / \mathrm{cm}\end{array}$ & $\begin{array}{c}\text { Depth } \\
(\mathrm{ft} \text { bgs })^{(\mathrm{a})}\end{array}$ & ID & $\begin{array}{c}1 \text { to } 1 \\
\mathrm{pH}\end{array}$ & $\begin{array}{c}1 \text { to } 1 \\
\mu \mathrm{S} / \mathrm{cm} \\
\end{array}$ & $\begin{array}{c}\text { Corrected } \\
\mu \mathrm{S} / \mathrm{cm}\end{array}$ & $\begin{array}{c}\text { Depth } \\
(\mathrm{ft} \text { bgs })^{(\mathrm{a})}\end{array}$ & ID & $\begin{array}{c}1 \text { to } 1 \\
\mathrm{pH}\end{array}$ & $\begin{array}{c}1 \text { to } 1 \\
\mu \mathrm{S} / \mathrm{cm} \\
\end{array}$ & $\begin{array}{c}\text { Corrected } \\
\mu \mathrm{S} / \mathrm{cm}\end{array}$ \\
\hline "187.8 & $38 \mathrm{C}$ & 8.16 & 2121 & 4,201 & 199.1 & "49A & 8.13 & 10 & 1,605 & 209 & 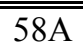 & 7.56 & 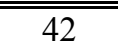 & 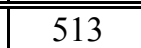 \\
\hline 188.3 & $38 \mathrm{~B}$ & 7.75 & 58 & 3,046 & 199.4 & $50 \mathrm{~B}$ & 8.22 & 165 & 2,412 & 209.4 & 59D & 8.06 & 103 & 1,017 \\
\hline 188.7 & $38 \mathrm{~A}$ & 7.35 & 24 & 1,337 & 199.4 & $50 \mathrm{~A}$ & 7.81 & 102 & 2,631 & 209.8 & $59 \mathrm{C}$ & 7.72 & 56 & 452 \\
\hline 189.2 & 39B & 7.95 & 79 & 5,131 & 200.2 & $52 \mathrm{~B}$ & 8.45 & 160 & 2,928 & 210.4 & 59B & 7.72 & 55 & 316 \\
\hline 189.5 & $39 \mathrm{~A}$ & 8.11 & 123 & 6,379 & 200.7 & $52 \mathrm{~A}$ & 8.33 & 148 & 1,814 & 210.9 & $59 \mathrm{~A}$ & 8.8 & 71 & 284 \\
\hline 190.7 & $40 \mathrm{~A}$ & 7.77 & 59 & 1,147 & 202.5 & $54 \mathrm{~B}$ & 8.08 & 135 & 2,067 & 211.7 & $62 \mathrm{~B}$ & 7.81 & 50 & 610 \\
\hline 193.3 & $45 \mathrm{~A}$ & 8.79 & 275 & 5,239 & 202.5 & $54 \mathrm{~A}$ & 7.96 & 93 & 1,721 & 212.1 & $62 \mathrm{~A}$ & 7.73 & 51 & 512 \\
\hline 195.9 & $47 \mathrm{D}$ & 8.23 & 93 & 1,048 & 204.3 & $56 \mathrm{C}$ & 7.94 & 86 & 1,082 & 212.9 & 64B & 8.04 & 92 & 1,122 \\
\hline 196.4 & $47 \mathrm{C}$ & 8.16 & 101 & 1,523 & 205 & $56 \mathrm{~B}$ & 8.16 & 122 & 1,479 & 213.3 & $64 \mathrm{~A}$ & 7.99 & 76 & 762 \\
\hline 196.9 & $47 \mathrm{~B}$ & 7.4 & 71 & 1,243 & 205.6 & $56 \mathrm{~A}$ & 8.07 & 94 & 1,208 & 214.1 & $65 \mathrm{~B}$ & 7.85 & 87 & 1,124 \\
\hline 197.4 & $47 \mathrm{~A}$ & 7.7 & 76 & 1,546 & 207.6 & $58 \mathrm{D}$ & 8.26 & 191 & 2,034 & 214.3 & $65 \mathrm{~A}$ & 7.93 & 72 & 838 \\
\hline 198.8 & $49 \mathrm{C}$ & 8.22 & 165 & 1,838 & 208.1 & $58 \mathrm{C}$ & 8.3 & 160 & 1,576 & & & & & \\
\hline 199.1 & 49B & 8.17 & 149 & 1,803 & 208.6 & $58 \mathrm{~B}$ & 7.87 & 60 & 569 & & & & & \\
\hline
\end{tabular}




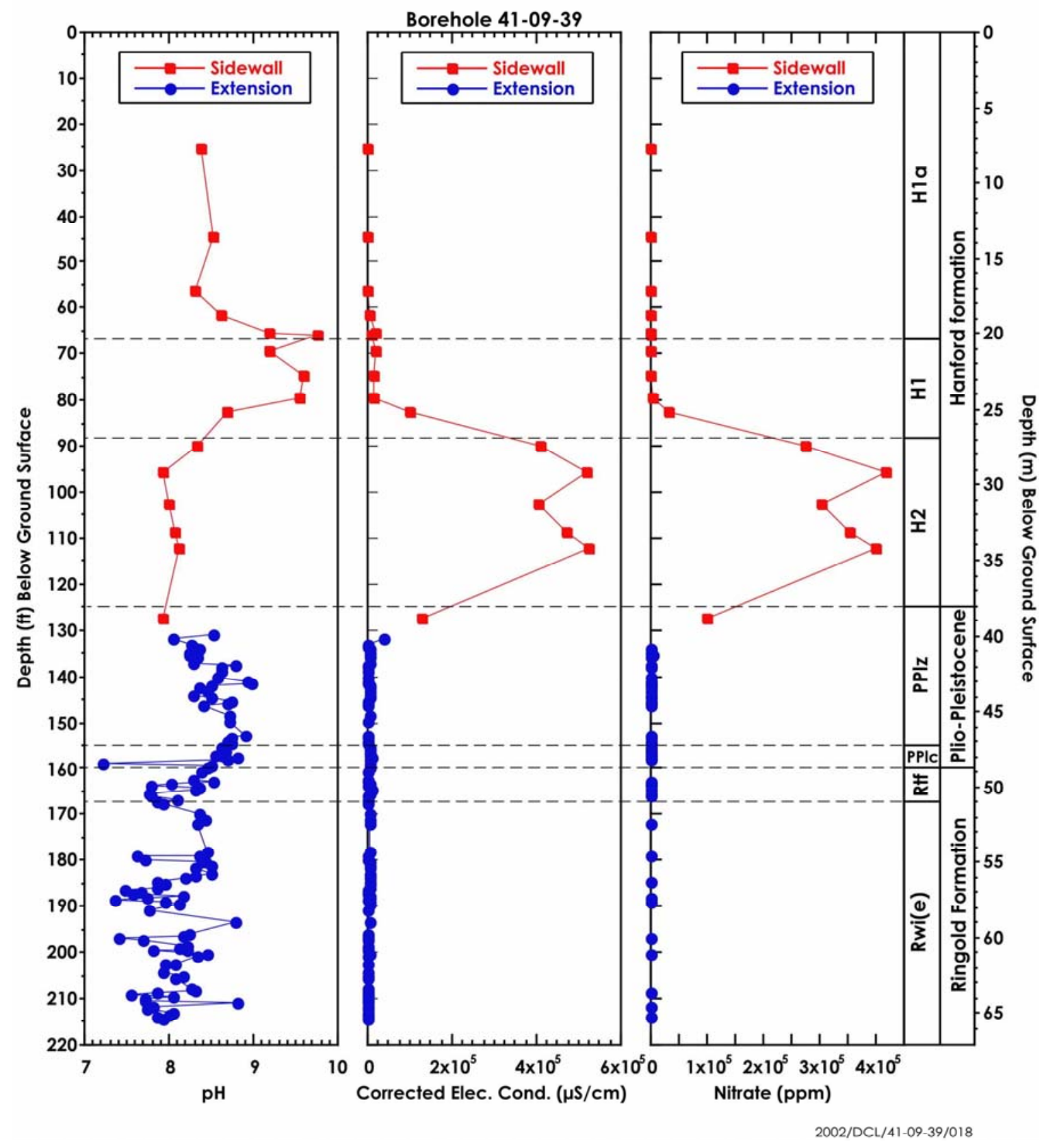

Figure 4.2. $\mathrm{pH}$, and Dilution Corrected Electrical Conductivity, and Nitrate Concentrations in Water Extracts 
Table 4.3. Anion Concentrations in 1:1 Water Extracts

\begin{tabular}{|c|c|c|c|c|c|c|c|c|c|c|c|c|}
\hline \multirow[b]{2}{*}{$\begin{array}{c}\text { Depth } \\
(\mathrm{ft} \text { bgs })^{(\mathrm{a})}\end{array}$} & \multirow[b]{2}{*}{ ID } & \multicolumn{5}{|c|}{ One:One Extracts } & \multirow[b]{2}{*}{$\begin{array}{l}\text { Dilution } \\
\text { Factor }\end{array}$} & \multicolumn{5}{|c|}{ Dilution Ratio Corrected } \\
\hline & & $\begin{array}{l}\mathrm{NO}_{3} \\
(\mathrm{ppm})\end{array}$ & $\begin{array}{c}\mathrm{Cl} \\
(\mathrm{ppm})\end{array}$ & $\begin{array}{c}\mathrm{F} \\
(\mathrm{ppm})\end{array}$ & $\begin{array}{c}\mathrm{NO}_{2} \\
(\mathrm{ppm})\end{array}$ & $\begin{array}{c}\mathrm{SO}_{4} \\
(\mathrm{ppm})\end{array}$ & & $\begin{array}{l}\mathrm{NO}_{3} \\
(\mathrm{ppm})\end{array}$ & $\begin{array}{c}\mathrm{Cl} \\
(\mathrm{ppm})\end{array}$ & $\begin{array}{c}\mathrm{F} \\
(\mathrm{ppm})\end{array}$ & $\begin{array}{c}\mathrm{NO}_{2} \\
(\mathrm{ppm})\end{array}$ & $\begin{array}{c}\mathrm{SO}_{4} \\
(\mathrm{ppm})\end{array}$ \\
\hline \multicolumn{13}{|c|}{ Sidewall } \\
\hline 25.5 & $15 \mathrm{~A} / \mathrm{B} / \mathrm{C}$ & 13.0 & $<0.3$ & NM & 0.26 & 1.70 & 12.3 & 161 & $<4.0$ & NM & 3.18 & 20.9 \\
\hline 44.5 & $14 \mathrm{~A} / \mathrm{B} / \mathrm{C}$ & 13.0 & 0.92 & NM & 0.26 & 16.4 & 11.7 & 152 & 10.7 & NM & 3.01 & 191 \\
\hline 56.5 & $13 \mathrm{~A} / \mathrm{B} / \mathrm{C}$ & 13.0 & 0.84 & NM & 0.26 & $<2.8$ & 6.24 & 81.0 & 5.22 & NM & 1.61 & $<17.2$ \\
\hline 61.5 & $12 \mathrm{~A} / \mathrm{B} / \mathrm{C}$ & 13.0 & 0.81 & NM & 0.26 & 5.13 & 7.79 & 102 & 6.34 & NM & 2.01 & 40.0 \\
\hline 65.5 & $11 \mathrm{~A} / \mathrm{B}$ & 29.1 & 0.52 & NM & 0.26 & 11.5 & 21.2 & 618 & 10.9 & $\mathrm{NM}$ & 5.48 & 244 \\
\hline 66.0 & $11 \mathrm{C}$ & 17.6 & 0.26 & NM & 0.26 & 6.45 & 18.9 & 333 & 4.89 & NM & 4.88 & 122 \\
\hline 69.5 & $10 \mathrm{~A} / \mathrm{B} / \mathrm{C}$ & 33.5 & 0.38 & NM & 0.26 & 8.66 & 22.9 & 767 & 8.80 & NM & 5.91 & 199 \\
\hline 74.5 & $09 \mathrm{~A} / \mathrm{B} / \mathrm{C}$ & 44.3 & 0.54 & NM & 0.26 & 18.1 & 19.4 & 857 & 10.5 & NM & 5.00 & 351 \\
\hline 79.5 & $08 \mathrm{~A} / \mathrm{B} / \mathrm{C}$ & 371 & 3.34 & NM & 1.40 & 51.7 & 9.34 & 3,464 & 31.3 & NM & 13.1 & 483 \\
\hline 82.5 & $07 \mathrm{~A} / \mathrm{B} / \mathrm{C}$ & 2,836 & 30.1 & NM & 82.1 & 336 & 11.9 & 33,746 & 358 & NM & 976 & 3,999 \\
\hline 90.0 & 06A/B & 28,044 & 307 & NM & 139 & 271 & 9.76 & 273,614 & 2992 & NM & 1,355 & 2,642 \\
\hline 95.5 & $03 \mathrm{~A} / \mathrm{B} / \mathrm{C}$ & 32,767 & 321 & NM & 28.6 & 260 & 12.7 & 416,332 & 4074 & NM & 364 & 3,308 \\
\hline 102.5 & $05 \mathrm{~A} / \mathrm{B} / \mathrm{C}$ & 31,666 & 198 & NM & 133 & 198 & 9.61 & 304,385 & 1903 & NM & 1,279 & 1,904 \\
\hline 108.5 & $04 \mathrm{~A} / \mathrm{B} / \mathrm{C}$ & 42,448 & 359 & NM & 130 & 386 & 8.33 & 353,773 & 2990 & NM & 1,084 & 3,215 \\
\hline 112.0 & $02 \mathrm{~B} / \mathrm{C}$ & 32,764 & 344 & NM & 106 & 373 & 12.3 & 401,733 & 4212 & NM & 1,297 & 4,576 \\
\hline 127.4 & $01 \mathrm{~A} / \mathrm{B} / \mathrm{C}$ & 12,808 & 77.5 & NM & 28.6 & 73.3 & 7.90 & 101,213 & 613 & $\mathrm{NM}$ & 226 & 579 \\
\hline \multicolumn{13}{|c|}{ Extension } \\
\hline 134.2 & $2 \mathrm{~B}$ & 291 & NM & NM & NM & $\mathrm{NM}$ & 6.63 & 1,929 & NM & NM & $\mathrm{NM}$ & $\mathrm{NM}$ \\
\hline 134.7 & $2 \mathrm{~A}$ & 268 & $\mathrm{NM}$ & NM & NM & $\mathrm{NM}$ & 5.94 & 1,592 & NM & NM & NM & NM \\
\hline 135.2 & $3 \mathrm{~B}$ & 362 & $\mathrm{NM}$ & NM & $\mathrm{NM}$ & $\mathrm{NM}$ & 6.76 & 2,447 & NM & $\mathrm{NM}$ & $\mathrm{NM}$ & NM \\
\hline 135.9 & $3 \mathrm{~A}$ & 165 & $\mathrm{NM}$ & NM & NM & $\mathrm{NM}$ & 7.39 & 1,219 & NM & NM & $\mathrm{NM}$ & $\mathrm{NM}$ \\
\hline 137.4 & $4 \mathrm{C}$ & 12.0 & $\mathrm{NM}$ & NM & $\mathrm{NM}$ & $\mathrm{NM}$ & 8.94 & 107 & NM & $\mathrm{NM}$ & $\mathrm{NM}$ & NM \\
\hline 138 & $4 \mathrm{~B}$ & 24.0 & NM & NM & NM & $\mathrm{NM}$ & 8.28 & 199 & NM & NM & NM & NM \\
\hline 140.3 & $5 \mathrm{C}$ & 9.90 & $\mathrm{NM}$ & NM & NM & $\mathrm{NM}$ & 6.61 & 65.4 & $\mathrm{NM}$ & NM & NM & NM \\
\hline 140.9 & $5 \mathrm{~B}$ & 3.70 & NM & NM & NM & NM & 7.70 & 28.5 & NM & NM & NM & NM \\
\hline
\end{tabular}


Table 4.3. (contd)

\begin{tabular}{|c|c|c|c|c|c|c|c|c|c|c|c|c|}
\hline \multirow[b]{2}{*}{$\begin{array}{c}\text { Depth } \\
(\mathrm{ft} \text { bgs })^{(\mathrm{a})}\end{array}$} & \multirow[b]{2}{*}{ ID } & \multicolumn{5}{|c|}{ One:One Extracts } & \multirow[b]{2}{*}{$\begin{array}{l}\text { Dilution } \\
\text { Factor }\end{array}$} & \multicolumn{5}{|c|}{ Dilution Ratio Corrected } \\
\hline & & $\begin{array}{l}\mathrm{NO}_{3} \\
(\mathrm{ppm})\end{array}$ & $\begin{array}{c}\mathrm{Cl} \\
(\mathrm{ppm})\end{array}$ & $\begin{array}{c}\mathrm{F} \\
(\mathrm{ppm})\end{array}$ & $\begin{array}{c}\mathrm{NO}_{2} \\
(\mathrm{ppm})\end{array}$ & $\begin{array}{c}\mathrm{SO}_{4} \\
(\mathrm{ppm})\end{array}$ & & $\begin{array}{l}\mathrm{NO}_{3} \\
(\mathrm{ppm})\end{array}$ & $\begin{array}{c}\mathrm{Cl} \\
(\mathrm{ppm})\end{array}$ & $\begin{array}{c}\mathrm{F} \\
(\mathrm{ppm})\end{array}$ & $\begin{array}{c}\mathrm{NO}_{2} \\
(\mathrm{ppm})\end{array}$ & $\begin{array}{c}\mathrm{SO}_{4} \\
(\mathrm{ppm})\end{array}$ \\
\hline 141.5 & $5 \mathrm{~A}$ & 9.80 & NM & NM & NM & NM & 10.4 & 102 & NM & NM & NM & NM \\
\hline 141.9 & $6 \mathrm{~F}$ & 9.10 & NM & NM & NM & NM & 19.2 & 174 & NM & NM & NM & NM \\
\hline 142.4 & $6 \mathrm{E}$ & 14.0 & NM & NM & NM & NM & 21.6 & 303 & NM & NM & NM & NM \\
\hline 143.3 & 6D & 42.0 & NM & NM & NM & NM & 10.1 & 425 & NM & NM & NM & NM \\
\hline 144.1 & $6 \mathrm{C}$ & 58.0 & NM & NM & NM & NM & 8.66 & 502 & NM & NM & NM & NM \\
\hline 144.7 & $6 \mathrm{~B}$ & 48.0 & NM & NM & NM & NM & 9.67 & 464 & NM & NM & NM & NM \\
\hline 145.2 & $7 \mathrm{E}$ & 18.0 & NM & NM & NM & NM & 12.0 & 216 & NM & NM & NM & NM \\
\hline 145.8 & 7D & 32.0 & NM & NM & NM & NM & 10.4 & 333 & NM & NM & NM & NM \\
\hline 146.4 & $7 \mathrm{C}$ & 36.0 & NM & NM & NM & NM & 8.85 & 319 & NM & NM & NM & NM \\
\hline 152.8 & $9 \mathrm{~A}$ & 24.0 & NM & NM & NM & $\mathrm{NM}$ & 7.09 & 170 & NM & $\mathrm{NM}$ & NM & NM \\
\hline 153.4 & $10 \mathrm{C}$ & 48.0 & NM & NM & NM & NM & 7.21 & 346 & NM & NM & NM & NM \\
\hline 153.9 & $10 \mathrm{~B}$ & 49.0 & NM & NM & NM & NM & 8.21 & 402 & NM & NM & NM & NM \\
\hline 154.5 & $10 \mathrm{~A}$ & 33.0 & NM & NM & NM & NM & 4.91 & 162 & NM & NM & NM & NM \\
\hline 155.4 & $11 \mathrm{C}$ & 9.80 & NM & NM & NM & $\mathrm{NM}$ & 22.9 & 225 & NM & $\mathrm{NM}$ & NM & NM \\
\hline 155.9 & $11 \mathrm{~B}$ & 8.40 & NM & NM & NM & NM & 28.6 & 240 & NM & NM & NM & NM \\
\hline 156.4 & $11 \mathrm{~A}$ & 5.30 & NM & NM & NM & NM & 42.0 & 223 & NM & NM & $\mathrm{NM}$ & NM \\
\hline 156.8 & $12 \mathrm{~B}$ & 10.5 & NM & NM & NM & NM & 14.3 & 151 & NM & NM & NM & NM \\
\hline 157.1 & $12 \mathrm{~A}$ & 7.50 & NM & NM & $\mathrm{NM}$ & $\mathrm{NM}$ & 15.0 & 112 & NM & $\mathrm{NM}$ & NM & NM \\
\hline 157.2 & $13 \mathrm{D}$ & 2.00 & NM & NM & NM & NM & 15.5 & 30.9 & NM & NM & $\mathrm{NM}$ & NM \\
\hline 157.7 & $13 \mathrm{C}$ & 6.20 & NM & NM & NM & NM & 24.9 & 154 & NM & NM & NM & NM \\
\hline 158.2 & $13 \mathrm{~B}$ & 6.80 & 1.00 & 0.45 & $<0.06$ & 14.5 & 18.8 & 128 & 18.8 & 8.50 & $<1.1$ & 273 \\
\hline 162.8 & $16 \mathrm{C}$ & 1.90 & NM & NM & NM & NM & 7.25 & 13.8 & NM & NM & NM & NM \\
\hline 163.3 & $16 \mathrm{~B}$ & 4.60 & NM & NM & $\mathrm{NM}$ & $\mathrm{NM}$ & 24.2 & 111 & NM & $\mathrm{NM}$ & NM & NM \\
\hline 163.8 & $16 \mathrm{~A}$ & 6.70 & NM & NM & NM & $\mathrm{NM}$ & 23.0 & 153 & NM & NM & $\mathrm{NM}$ & NM \\
\hline 164.8 & $17 \mathrm{C}$ & 0.30 & NM & NM & NM & $\mathrm{NM}$ & 28.0 & 8.4 & NM & $\mathrm{NM}$ & $\mathrm{NM}$ & NM \\
\hline 165.3 & 17B & 7.60 & NM & NM & NM & NM & 19.0 & 144 & NM & NM & NM & NM \\
\hline
\end{tabular}


Table 4.3. (contd)

\begin{tabular}{|c|c|c|c|c|c|c|c|c|c|c|c|c|}
\hline \multirow[b]{2}{*}{$\begin{array}{c}\text { Depth } \\
(\mathrm{ft} \text { bgs })^{(\mathrm{a})}\end{array}$} & \multirow[b]{2}{*}{ ID } & \multicolumn{5}{|c|}{ One:One Extracts } & \multirow[b]{2}{*}{$\begin{array}{l}\text { Dilution } \\
\text { Factor } \\
\end{array}$} & \multicolumn{5}{|c|}{ Dilution Ratio Corrected } \\
\hline & & $\begin{array}{c}\mathrm{NO}_{3} \\
(\mathrm{ppm})\end{array}$ & $\begin{array}{c}\mathrm{Cl} \\
(\mathrm{ppm})\end{array}$ & $\begin{array}{c}\mathrm{F} \\
(\mathrm{ppm})\end{array}$ & $\begin{array}{c}\mathrm{NO}_{2} \\
(\mathrm{ppm})\end{array}$ & $\begin{array}{c}\mathrm{SO}_{4} \\
(\mathrm{ppm})\end{array}$ & & $\begin{array}{c}\mathrm{NO}_{3} \\
(\mathrm{ppm})\end{array}$ & $\begin{array}{c}\mathrm{Cl} \\
(\mathrm{ppm})\end{array}$ & $\begin{array}{c}\mathrm{F} \\
(\mathrm{ppm})\end{array}$ & $\begin{array}{c}\mathrm{NO}_{2} \\
(\mathrm{ppm})\end{array}$ & $\begin{array}{r}\mathrm{SO}_{4} \\
\text { (ppm) }\end{array}$ \\
\hline 165.8 & $17 \mathrm{~A}$ & 10.8 & NM & NM & NM & NM & 21.8 & 236 & NM & NM & NM & NM \\
\hline 172 & $22 \mathrm{~B}$ & 5.00 & 3.00 & 1.40 & $<0.06$ & 13.0 & 19.1 & 95.4 & 57.2 & 26.7 & 1.10 & 248 \\
\hline 179.2 & $30 \mathrm{~B}$ & 4.70 & 1.30 & 0.38 & $<0.04$ & 4.70 & 29.9 & 140. & 38.8 & 11.3 & 1.20 & 140 \\
\hline 184.6 & $36 \mathrm{~B}$ & 3.00 & 1.60 & 0.52 & $<0.06$ & 3.70 & 48.5 & 145. & 77.5 & 25.2 & $<2.9$ & 179 \\
\hline 189.2 & $39 \mathrm{~B}$ & 0.80 & 2.40 & 0.56 & $<0.06$ & 3.40 & 65.0 & 51.3 & 155 & 36.4 & $<3.9$ & 221 \\
\hline 196.9 & $47 \mathrm{~B}$ & 18.0 & 110 & 0.21 & $<0.06$ & 2.50 & 17.5 & 315. & 1926 & 3.70 & $<0.5$ & 43.8 \\
\hline 200.2 & $52 \mathrm{~B}$ & $<0.10$ & 3.70 & 0.81 & $<0.06$ & 22.0 & 18.3 & $<1.8$ & 67.7 & 14.8 & $<1.1$ & 403 \\
\hline 208.6 & $58 \mathrm{~B}$ & 0.60 & 1.50 & 0.48 & $<0.06$ & 2.50 & 9.49 & 5.20 & 14.2 & 4.6 & $<0.6$ & 23.7 \\
\hline 211.7 & $62 \mathrm{~B}$ & 0.60 & 1.00 & 0.48 & $<0.06$ & 2.70 & 12.2 & 7.70 & 12.2 & 5.9 & $<0.7$ & 32.9 \\
\hline 214.1 & $65 \mathrm{~B}$ & $<0.10$ & 1.50 & 0.45 & $<0.06$ & 5.50 & 12.9 & $<1.29$ & 19.4 & 5.8 & $<0.8$ & 71.1 \\
\hline
\end{tabular}


Table 4.4. Major Cation Composition of 1:1 Water Extracts from Sidewall Cores and Borehole Extension Sleeves

\begin{tabular}{|c|c|c|c|c|c|c|c|c|c|c|c|}
\hline $\begin{array}{c}\text { Depth } \\
(\mathrm{ft} \text { bgs })^{(\mathrm{a})} \\
\end{array}$ & ID & $\begin{array}{c}\mathrm{Ca} \\
(\mathrm{ppm}) \\
\end{array}$ & $\begin{array}{c}\mathrm{K} \\
(\mathrm{ppm}) \\
\end{array}$ & $\begin{array}{c}\mathrm{Mg} \\
(\mathrm{ppm}) \\
\end{array}$ & $\begin{array}{c}\mathrm{Na} \\
(\mathrm{ppm}) \\
\end{array}$ & $\begin{array}{c}\mathrm{Sr} \\
(\mathrm{ppm}) \\
\end{array}$ & $\begin{array}{c}\mathrm{Al} \\
(\mathrm{ppm}) \\
\end{array}$ & $\begin{array}{c}\mathrm{B} \\
(\mathrm{ppm}) \\
\end{array}$ & $\begin{array}{c}\mathrm{Fe} \\
(\mathrm{ppm})\end{array}$ & $\begin{array}{c}\mathrm{Mn} \\
(\mathrm{ppm})\end{array}$ & $\begin{array}{c}\mathrm{Si} \\
(\mathrm{ppm}) \\
\end{array}$ \\
\hline \multicolumn{12}{|c|}{ Sidewall } \\
\hline 25.5 & $15 \mathrm{~A} / \mathrm{B} / \mathrm{C}$ & 9.30 & 6.40 & 1.10 & 29.5 & $(0.053)$ & 0.84 & 5.20 & 0.51 & $(0.01)$ & 21.5 \\
\hline 44.5 & $14 \mathrm{~A} / \mathrm{B} / \mathrm{C}$ & 6.00 & 6.90 & 0.70 & 41.8 & $(0.049)$ & 0.59 & 15.9 & 0.14 & $(0.01)$ & 14.8 \\
\hline 56.5 & $13 \mathrm{~A} / \mathrm{B} / \mathrm{C}$ & 22.4 & 7.40 & 2.00 & 43.2 & $(0.036)$ & 0.51 & 5.30 & $<0.25$ & $<0.05$ & 9.30 \\
\hline 61.5 & $12 \mathrm{~A} / \mathrm{B} / \mathrm{C}$ & 3.60 & 2.60 & 0.30 & 80.7 & $(0.042)$ & 0.56 & 31.0 & $(0.05)$ & $(0.01)$ & 7.50 \\
\hline 65.5 & $11 \mathrm{~A} / \mathrm{B}$ & 1.10 & $(1.70)$ & 0.40 & 121 & $(0.035)$ & 1.48 & 9.00 & 2.03 & $(0.04)$ & 14.2 \\
\hline 66.0 & $11 \mathrm{C}$ & 0.90 & 2.60 & 1.90 & 212 & $(0.051)$ & 5.72 & 10.5 & 8.97 & 0.13 & 37.0 \\
\hline 69.5 & $10 \mathrm{~A} / \mathrm{B} / \mathrm{C}$ & 0.80 & 3.60 & 1.00 & 176 & $(0.035)$ & 3.08 & 4.50 & 4.27 & 0.08 & 24.0 \\
\hline 74.5 & $9 \mathrm{~A} / \mathrm{B} / \mathrm{C}$ & 0.50 & $(2.40)$ & $(0.10)$ & 168 & $(0.047)$ & 0.65 & 10.3 & $(0.11)$ & $<0.05$ & 12.5 \\
\hline 79.5 & $8 \mathrm{~A} / \mathrm{B} / \mathrm{C}$ & 0.70 & 4.20 & $(0.10)$ & 423 & $(0.057)$ & 0.75 & 35.6 & $(0.02)$ & $<0.05$ & 5.30 \\
\hline 82.5 & $7 \mathrm{~A} / \mathrm{B} / \mathrm{C}$ & 22.1 & 16.0 & 2.20 & 2041 & 0.46 & $(0.48)$ & 16.9 & $(0.02)$ & $<0.05$ & 7.00 \\
\hline 90.0 & $6 \mathrm{~A} / \mathrm{B}$ & 142 & 209 & 7.20 & 11270 & 2.68 & $(0.37)$ & 26.6 & $(0.04)$ & $<0.05$ & 3.00 \\
\hline 95.5 & $3 \mathrm{~A} / \mathrm{B} / \mathrm{C}$ & 975 & 129 & 19.8 & 10209 & 15.1 & $(0.22)$ & 12.4 & $(0.06)$ & $<0.05$ & 3.60 \\
\hline 102.5 & $5 \mathrm{~A} / \mathrm{B} / \mathrm{C}$ & 563 & 128 & 24.1 & 10753 & 10.1 & $(0.20)$ & 14.0 & $(0.08)$ & $<0.05$ & 3.70 \\
\hline 108.5 & $4 \mathrm{~A} / \mathrm{B} / \mathrm{C}$ & 449 & 117 & 17.3 & 15256 & 8.76 & $(0.26)$ & 8.30 & $(0.08)$ & $<0.05$ & 4.80 \\
\hline 112.0 & $2 \mathrm{~B} / \mathrm{C}$ & 453 & 81.2 & 23.7 & 11344 & 8.56 & $<0.50$ & 30.2 & $(0.06)$ & $<0.05$ & 4.90 \\
\hline 127.4 & $1 \mathrm{~A} / \mathrm{B} / \mathrm{C}$ & 1705 & 76.7 & 186 & 1921 & 13.6 & $<0.50$ & 15.7 & $(0.04)$ & 0.10 & 5.10 \\
\hline \multicolumn{12}{|c|}{ Extension } \\
\hline 134.2 & $2 \mathrm{~B}$ & 49.3 & 5.38 & 12.9 & 31.3 & 0.22 & $<0.05$ & 1.82 & $<0.05$ & $<0.05$ & 4.60 \\
\hline 134.7 & $2 \mathrm{~A}$ & 57.4 & 4.29 & 13.8 & 22.5 & 0.26 & $<0.05$ & 0.45 & $<0.05$ & $<0.05$ & 6.00 \\
\hline 135.2 & $3 \mathrm{~B}$ & 57.3 & 22.4 & 15.0 & 53.6 & 0.26 & $<0.05$ & 1.86 & $<0.05$ & 0.06 & 7.90 \\
\hline 135.9 & $3 \mathrm{~A}$ & 39.2 & $(10.7)$ & 8.18 & 23.4 & 0.14 & $<0.05$ & 0.77 & $<0.05$ & 0.06 & 4.20 \\
\hline 137.4 & $4 \mathrm{C}$ & 19.7 & $(1.60)$ & 3.13 & 10.8 & 0.06 & $<0.05$ & 0.29 & $<0.05$ & $<0.05$ & 5.00 \\
\hline 138.0 & 4B & 20.0 & $(8.10)$ & 3.98 & 14.8 & 0.06 & $<0.05$ & 0.37 & $<0.05$ & $<0.05$ & 5.40 \\
\hline 140.3 & $5 \mathrm{C}$ & 16.0 & $(3.20)$ & 3.62 & 19.0 & 0.06 & $<0.05$ & 0.50 & $<0.05$ & $<0.05$ & 6.60 \\
\hline 140.9 & $5 B$ & 21.8 & $(6.60)$ & 3.21 & 14.2 & 0.07 & $<0.05$ & 0.41 & $<0.05$ & $<0.05$ & 4.70 \\
\hline 141.5 & $5 \mathrm{~A}$ & 17.8 & $(2.40)$ & 3.24 & $(13.5)$ & 0.07 & $<0.05$ & 0.34 & $<0.05$ & $<0.05$ & 6.30 \\
\hline 141.9 & $6 \mathrm{~F}$ & 19.7 & 4.07 & 6.07 & 19.3 & 0.09 & $<0.05$ & 1.45 & $<0.05$ & $<0.05$ & 9.70 \\
\hline 142.4 & $6 \mathrm{E}$ & 13.4 & 4.83 & 4.18 & 15.0 & 0.07 & -0.06 & 0.99 & $<0.05$ & $<0.05$ & 10.0 \\
\hline
\end{tabular}


Table 4.4. (contd)

\begin{tabular}{|c|c|c|c|c|c|c|c|c|c|c|c|}
\hline $\begin{array}{c}\text { Depth } \\
(\mathrm{ft} \text { bgs) })^{(a)}\end{array}$ & ID & $\begin{array}{c}\mathrm{Ca} \\
(\mathrm{ppm})\end{array}$ & $\begin{array}{c}\mathrm{K} \\
(\mathrm{ppm})\end{array}$ & $\begin{array}{c}\mathrm{Mg} \\
(\mathrm{ppm})\end{array}$ & $\begin{array}{c}\mathrm{Na} \\
(\mathrm{ppm})\end{array}$ & $\begin{array}{c}\mathrm{Sr} \\
(\mathrm{ppm})\end{array}$ & $\begin{array}{c}\mathrm{Al} \\
(\mathrm{ppm})\end{array}$ & $\begin{array}{c}\mathrm{B} \\
(\mathrm{ppm})\end{array}$ & $\begin{array}{c}\mathrm{Fe} \\
(\mathrm{ppm})\end{array}$ & $\begin{array}{c}\mathrm{Mn} \\
(\mathrm{ppm})\end{array}$ & $\begin{array}{c}\mathrm{Si} \\
(\mathrm{ppm})\end{array}$ \\
\hline 143.3 & $6 \mathrm{D}$ & 20.3 & 5.08 & 7.21 & 19.9 & 0.09 & $<0.05$ & 0.54 & $<0.05$ & $<0.05$ & 10.7 \\
\hline 144.1 & $6 \mathrm{C}$ & 25.3 & 7.24 & 9.17 & 25.2 & 0.13 & $(0.07)$ & 0.67 & $<0.05$ & $<0.05$ & 12.3 \\
\hline 144.7 & $6 \mathrm{~B}$ & 22.4 & 5.15 & 8.09 & 22.0 & 0.1 & $<0.05$ & 0.72 & $<0.05$ & $<0.05$ & 11.3 \\
\hline 145.2 & $7 E$ & 12.1 & $(2.50)$ & 3.88 & 17.5 & 0.05 & $(0.08)$ & 1.23 & $<0.05$ & $<0.05$ & 11.2 \\
\hline 145.8 & $7 \mathrm{D}$ & 13.1 & $(2.90)$ & 4.26 & 18.9 & 0.06 & $(0.14)$ & 1.98 & $<0.05$ & $<0.05$ & 13.5 \\
\hline 146.4 & $7 \mathrm{C}$ & 15.3 & $(3.80)$ & 5.53 & 20.1 & 0.08 & $(0.06)$ & 1.26 & $<0.05$ & $<0.05$ & 14.3 \\
\hline 151.1 & $9 \mathrm{C}$ & 15.0 & 5.02 & 4.94 & 16.1 & 0.07 & $(0.07)$ & 1.29 & $<0.05$ & $<0.02$ & 9.90 \\
\hline 151.8 & 9B & 13.2 & 4.63 & 4.45 & 15.6 & 0.06 & $<0.05$ & 0.93 & $<0.05$ & $<0.05$ & 10.4 \\
\hline 152.8 & $9 \mathrm{~A}$ & 10.5 & 4.62 & 3.63 & 16.3 & 0.05 & 0.17 & 1.09 & $<0.05$ & $<0.01$ & 9.80 \\
\hline 153.4 & $10 \mathrm{C}$ & 13.4 & 7.14 & 5.70 & 20.0 & 0.07 & $<0.05$ & 0.84 & $<0.05$ & $<0.05$ & 10.1 \\
\hline 153.9 & $10 \mathrm{~B}$ & 13.7 & 4.22 & 6.36 & 17.6 & 0.06 & 0.18 & 1.42 & $(0.07)$ & $<0.01$ & 16.4 \\
\hline 154.5 & $10 \mathrm{~A}$ & 13.0 & 5.64 & 5.06 & 16.3 & 0.06 & 0.19 & 1.20 & $<0.05$ & $<0.05$ & 14.7 \\
\hline 155.4 & $11 \mathrm{C}$ & 12.5 & $(4.60)$ & 3.60 & 12.1 & 0.04 & $<0.05$ & 0.79 & $(0.04)$ & $<0.01$ & 15.1 \\
\hline 155.9 & $11 \mathrm{~B}$ & 12.9 & $(3.60)$ & 3.5 & 10.9 & 0.05 & $<0.05$ & 0.94 & $<0.05$ & $<0.05$ & 14.9 \\
\hline 156.4 & $11 \mathrm{~A}$ & 11.3 & $(3.40)$ & 2.51 & 6.63 & 0.04 & 0.01 & 0.96 & $<0.05$ & $<0.05$ & 10.9 \\
\hline 156.8 & $12 \mathrm{~B}$ & 12.1 & 8.16 & 4.43 & 49.6 & 0.06 & $<0.05$ & 0.92 & $<0.05$ & $<0.05$ & 14.0 \\
\hline 157.1 & $12 \mathrm{~A}$ & 11.2 & 6.89 & 4.11 & 40.3 & 0.06 & $<0.05$ & 1.12 & $<0.05$ & $<0.05$ & 13.1 \\
\hline 157.2 & $13 \mathrm{D}$ & 13.4 & 11.2 & 4.74 & 50.8 & 0.06 & $<0.05$ & 0.62 & $<0.05$ & $<0.05$ & 12.5 \\
\hline 157.7 & $13 \mathrm{C}$ & 12.6 & 7.34 & 3.73 & 35.1 & 0.06 & $(0.08)$ & 0.99 & $(0.06)$ & $<0.05$ & 13.8 \\
\hline 158.2 & $13 \mathrm{~B}$ & 11.3 & $(4.30)$ & 3.63 & 16.7 & 0.05 & 0.13 & 0.93 & $(0.09)$ & $<0.05$ & 15.9 \\
\hline 162.3 & $16 \mathrm{D}$ & 5.77 & 5.94 & 2.14 & 33.8 & $(0.03)$ & $(0.08)$ & 1.26 & 0.07 & 0.07 & 7.60 \\
\hline 163.3 & $16 \mathrm{~B}$ & 5.40 & 3.14 & 1.60 & 7.58 & $(0.03)$ & 0.08 & 0.81 & 0.08 & $(0.01)$ & 11.1 \\
\hline 163.8 & $16 \mathrm{~A}$ & 2.83 & 1.97 & 0.97 & 7.53 & $(0.01)$ & 0.35 & 1.37 & 0.33 & $(0.03)$ & 15.2 \\
\hline 164.3 & $17 \mathrm{D}$ & 9.52 & 6.00 & 3.02 & 29.3 & 0.05 & $(0.12)$ & 0.93 & 0.14 & $(0.04)$ & 11.3 \\
\hline 165.3 & $17 \mathrm{~B}$ & 2.53 & $(2.00)$ & 0.92 & 9.35 & $(0.01)$ & 0.35 & 1.39 & 0.45 & $(0.02)$ & 16.6 \\
\hline 165.8 & $17 \mathrm{~A}$ & 2.75 & 2.00 & 0.92 & 7.72 & $(0.01)$ & 0.60 & 1.28 & 0.71 & $(0.03)$ & 16.6 \\
\hline
\end{tabular}


amounts of calcite and the aquifer sediments in the upper unconfined aquifer have been shown, based on geochemical modeling, to be in equilibrium with the air and calcite. We can reasonably expect Hanford formation and Plio-Pleistocene vadose zone sediments also to satisfy these equilibria. The data collected support this speculation.

Based on the EC profile (see Figure 4.2), it appears that the tank leak fluid dominates the porewater down to a depth of 38.8 meters (127.4 feet) bgs. For borehole extension sleeves below 40.1 meters (131.7 feet) bgs, the EC does not show any significant deviation from values found for vadose zone sediments at nearby uncontaminated Resource Conservation and Recovery Act (RCRA) boreholes. The dilution-corrected electrical conductivity assumes that no materials leached out of the soil to increase the 1:1 water extract's soluble ion content. This is equivalent to assuming that all the ions measured in the extract were in the pore solution that resided in the vadose zone sediment at the observed moisture content and that the deionized water diluted the existing ions. We know this is an over-simplification and that most sediment types will release ions to solution during extraction. We can assume that the actual EC of porewater in the vadose sediment studied lies between the two values shown in Table 4.2. If we consider the dilution-corrected ECs from sleeves collected in the borehole extension, only sleeve 1B appears to contain dissolved salt that might represent the leading edge of the tank leak fluids that dominate the shallower sediment from the sidewall cores.

Several of the sleeves from the Ringold Formation show rather low ECs, especially where hard tooling had occurred and distilled water was added in small quantities to help retrieve the crushed cobbles. For a point of reference, the EC of uncontaminated Hanford Site groundwater in the 200 Area plateau is about $350 \mu \mathrm{S} / \mathrm{cm}$ and the water obtained at wells near the SX Tank Farm, including the 41-09-39 borehole extension is about $250 \mu \mathrm{S} / \mathrm{cm}$ (see Myers et al. 1998). This suggests that the groundwater beneath the SX Tank Farm still shows the influence of the large volumes of dilute-salt waste liquids disposed to facilities upgradient (north and west).

Nitrate is perhaps the most sensitive chemical marker of tank leaks migrating through the vadose sediments. The tank liquor has up to 6 to $8 \mathrm{M}$ (about 350,000 ppm) nitrate concentrations, but the deep vadose zone sediments in the semiarid region where the Hanford Site is located are not expected to contain more than several parts per million to perhaps a few tens of parts per million. The difference between the background nitrate baseline and the full-strength tank liquor is about $10^{5}$. Therefore, adding about $0.01 \%$ tank liquor into existing porewater should be readily measurable and perhaps even $0.001 \%$ could be discerned above the natural background. Thus, the 1:1 sediment to water extract nitrate data should be quite useful.

Figure 4.2 and Table 4.3 show the dilution-corrected nitrate data versus depth. There are obvious indications of high nitrate concentrations in the sidewall core sediment between depths 24.4 and 38.7 meters ( 80 and 127 feet) bgs. The first indication of elevated nitrate appears at 20 meters (65.5 feet) bgs and the deepest (leading edge of plume) is in sleeve 3A, at depth of 41.5 meters (136 feet).

Further, moderate nitrate concentrations were found in the borehole extension sleeves from the sixth through eleventh split-spoon samplings. Between split-spoon samplings 7 and 9 , the casing was removed and replaced with one containing a drive shoe. The probability is high that sediment from shallow depths 
was knocked into the borehole between samplings 7 and 9, so the nitrate data for sleeves down to the eleventh sampling may be biased high from incorporation of shallow sediment. The nitrate data qualitatively suggest that the leading front of a tank leak may have reached 47.5 meters (156 feet) bgs, which is the bottom of the Plio-Pleistocene mud unit at the contact with the caliche layer. We question whether the nitrate distribution from 41.5 to 47.5 meters (136 to 156 feet) bgs has been adulterated by the original pile driving to 39.9 meters (131 feet), subsequent end cap milling, loss of cooling water, and borehole extension casing insertion and removal prior to final sampling. Thus, with all of these activities related to the borehole placement and casing extension, we cannot determine if the nitrate from tank leaks has actually penetrated down to 41.5 or 47.5 meters (136 or 156 feet) bgs. Sleeve 47B also has a slight increase in nitrate content at 60 meters (196.9 feet) that may be a bathtub ring from horizontal migration of contaminated groundwater when the water table was elevated higher into the Ringold Formation.

The dilution corrected water extract cation concentrations (estimates of actual porewater concentrations), shown in Table 4.4 and Figure 4.3, suggest that tank fluid has impacted the vadose zone sediments to a depth of at least 38.7 to 40.8 meters (127 to 134 feet) bgs based on elevated waterleachable sodium. There appears to be an ion-exchange front where sodium displaces the native calcium, magnesium, and potassium from the sediment surface sites resulting in the elevated calcium, magnesium, and potassium to depths of 41.1 meters (135 feet) bgs. There is obvious low water-leachable calcium, magnesium, and potassium in samples at depths of 18.7 to 24.2 meters (61.5 to 79.5 feet) bgs from the displacement by the high sodium in the tank fluids. There is a hint of elevated water-leachable aluminum and iron in the samples at depths 18.7 to 21.1 meters ( 61.5 to 69.5 feet) bgs, which is at the shallow end of the zone of slightly elevated $\mathrm{pH}$ (found at 20 to 24.2 meters [ 65.5 to 79.5 feet] bgs; see Table 4.2). The borehole extension sleeves 2B (40.9 meters [134.2 feet bgs]), 2A (41.1 meters [134.7 feet bgs]), 3B (41.2 meters [135.2 feet bgs]), and 3A (41.4 meters [135.9 feet bgs]) have significantly higher cation concentrations than sleeves from sediment in the next 10 meters $(32.8$ feet $)$ of the profile. Plausible explanations are presented in Section 5.6.2.1 where extract data for the composite samples are discussed.

Similar 1:1 water extracts from two nearby RCRA monitoring wells are documented in a companion document (Serne et al. 2002a). The comparison to water extracts from the contaminated sediment under and proximate to SX-108/-109 and SX-115 will be discussed in the field investigation report ${ }^{(a)}$ for the entire SX Tank Farm that was published in early 2002.

\subsection{Radionuclide Analysis}

For the most part, the highly radioactive samples were restricted to the sidewall cores between 18.3 and 38.7 meters (60 and 127 feet) bgs. Samples between 20.1 and 25.6 meters (66 and 84 feet) bgs were radioactive enough to require some changes in standard gamma energy analyses counting techniques. The samples from the borehole extension, which started at 39.9 meters (131 feet) bgs and continued to groundwater, were relatively easy to analyze. Most borehole extension samples contained low activity such that large representative aliquots could be taken from each sleeve for gamma counting.

(a) Draft Field Investigation Report for Waste Management Area S-SX. RPP-7884, Draft, Volume 2, Appendix D, CH2M HILL Hanford Group, Inc., Richland, Washington. 


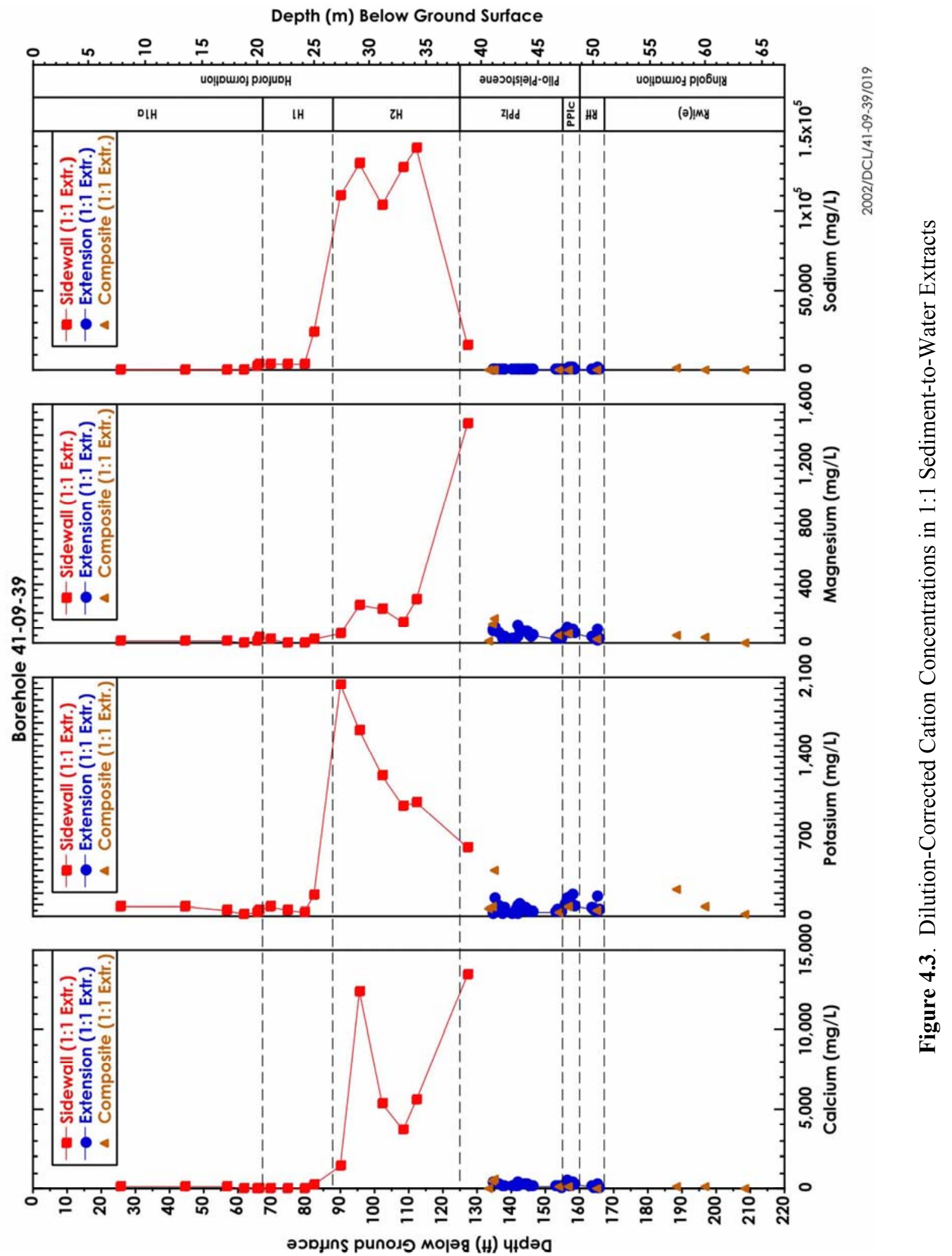

4.15 
Table 4.5. Cesium-137 Activity in Sidewall Cores and Borehole Extension Sleeves (pCi/g)

\begin{tabular}{|c|c|c|c|c|c|c|c|c|c|c|c|c|c|c|}
\hline $\begin{array}{c}\text { Depth } \\
(\mathrm{ft} b g s)^{(a)}\end{array}$ & $\begin{array}{l}\text { Sample } \\
\text { ID }\end{array}$ & $\begin{array}{c}{ }^{137} \mathrm{Cs} \\
\text { (pCi/g wet } \\
\text { wt) }\end{array}$ & $\begin{array}{c}{ }^{137} \mathrm{Cs} \\
( \pm \mathrm{pCi} / \mathrm{g} \\
\text { Error) }\end{array}$ & $\begin{array}{l}{ }^{137} \mathrm{Cs} \\
(\mathrm{pCi} / \mathrm{g} \\
\text { dry wt) }\end{array}$ & $\begin{array}{c}\text { Depth } \\
(\mathrm{ft} b g s)^{(\mathrm{a})}\end{array}$ & $\begin{array}{l}\text { Sample } \\
\text { ID }\end{array}$ & $\begin{array}{c}{ }^{137} \mathrm{Cs} \\
\text { (pCi/g wet } \\
\text { wt) }\end{array}$ & $\begin{array}{c}{ }^{137} \mathrm{Cs} \\
( \pm \mathrm{pCi} / \mathrm{g} \\
\text { Error })\end{array}$ & $\begin{array}{l}{ }^{137} \mathrm{Cs} \\
(\mathrm{pCi} / \mathrm{g} \\
\text { dry wt) }\end{array}$ & $\begin{array}{c}\text { Depth } \\
(\mathrm{ft} b g s)^{(\mathrm{a})}\end{array}$ & $\begin{array}{l}\text { Sample } \\
\text { ID }\end{array}$ & $\begin{array}{c}{ }^{137} \mathrm{Cs} \\
(\mathrm{pCi} / \mathrm{g} \\
\text { wet wt) }\end{array}$ & \begin{tabular}{|c}
${ }^{137} \mathrm{Cs}$ \\
$( \pm \mathrm{pCi} / \mathrm{g}$ \\
Error $)$
\end{tabular} & $\begin{array}{l}{ }^{137} \mathrm{Cs} \\
\text { (pCi/g } \\
\text { dry wt) }\end{array}$ \\
\hline \multicolumn{5}{|c|}{ Sidewall } & 151.8 & $9 \mathrm{~B}$ & $9.54 \mathrm{E}+01$ & $1.83 \mathrm{E}+00$ & NM & 183 & $35 \mathrm{~B}$ & $3.21 \mathrm{E}-01$ & $5.49 \mathrm{E}-02$ & $3.32 \mathrm{E}-01$ \\
\hline 25.5 & $15 \mathrm{~A} / \mathrm{B} / \mathrm{C}$ & $5.50 \mathrm{E}+02$ & $9.70 \mathrm{E}+00$ & $6.06 \mathrm{E}+02$ & 152.8 & $9 \mathrm{~A}$ & $1.67 \mathrm{E}-01$ & $1.24 \mathrm{E}-01$ & $1.82 \mathrm{E}-01$ & 183.3 & $35 \mathrm{~A}$ & $<$ MDA & & $<\mathrm{MDA}$ \\
\hline 44.5 & $14 \mathrm{~A} / \mathrm{B} / \mathrm{C}$ & $1.01 \mathrm{E}+03$ & $1.83 \mathrm{E}+01$ & $1.11 \mathrm{E}+03$ & 152.9 & $10 \mathrm{D}$ & $1.12 \mathrm{E}+02$ & $2.57 \mathrm{E}+00$ & NM & 183.9 & $36 \mathrm{C}$ & $1.75 \mathrm{E}-01$ & $3.95 \mathrm{E}-02$ & $1.81 \mathrm{E}-01$ \\
\hline 56.5 & $13 \mathrm{~A} / \mathrm{B} / \mathrm{C}$ & $2.36 \mathrm{E}+04$ & $5.27 \mathrm{E}+02$ & $2.60 \mathrm{E}+04$ & 153.4 & $10 \mathrm{C}$ & 7.13E-02 & $2.33 \mathrm{E}-02$ & 7.95E-02 & 184.6 & $36 \mathrm{~B}$ & 4.89E-01 & $4.97 \mathrm{E}-02$ & 4.99E-01 \\
\hline 61.5 & $12 \mathrm{~A} / \mathrm{B} / \mathrm{C}$ & $1.13 \mathrm{E}+05$ & $2.19 \mathrm{E}+03$ & $1.25 \mathrm{E}+05$ & 153.9 & 10B & $<\mathrm{MDA}$ & & $<$ MDA & 185 & $36 \mathrm{~A}$ & $<$ MDA & & NM \\
\hline 65.5 & $11 \mathrm{~A} / \mathrm{B}$ & $5.68 \mathrm{E}+05$ & $1.06 \mathrm{E}+04$ & $6.26 \mathrm{E}+05$ & 154.5 & $10 \mathrm{~A}$ & $1.98 \mathrm{E}-02$ & $3.67 \mathrm{E}-02$ & $2.39 \mathrm{E}-02$ & 185.8 & $37 \mathrm{D}$ & $1.57 \mathrm{E}-01$ & $3.51 \mathrm{E}-02$ & $1.59 \mathrm{E}-01$ \\
\hline 66 & $11 \mathrm{C}$ & $3.71 \mathrm{E}+06$ & $6.89 \mathrm{E}+04$ & $4.09 \mathrm{E}+06$ & 155.1 & 11D & $1.75 \mathrm{E}+00$ & $1.70 \mathrm{E}-01$ & $1.84 \mathrm{E}+00$ & 186.2 & $37 \mathrm{C}$ & $<$ MDA & & $<\mathrm{MDA}$ \\
\hline 69.5 & $10 \mathrm{~A} / \mathrm{B} / \mathrm{C}$ & $8.61 \mathrm{E}+03$ & $1.10 \mathrm{E}+02$ & $9.49 \mathrm{E}+03$ & 155.4 & $11 \mathrm{C}$ & $<\mathrm{MDA}$ & & $<\mathrm{MDA}$ & 186.8 & 37B & $<$ MDA & & $<\mathrm{MDA}$ \\
\hline 74.5 & $9 \mathrm{~A} / \mathrm{B} / \mathrm{C}$ & $2.13 \mathrm{E}+06$ & $3.94 \mathrm{E}+04$ & $2.34 \mathrm{E}+06$ & 155.9 & 11B & $<$ MDA & & $<\mathrm{MDA}$ & 187.2 & $37 \mathrm{~A}$ & $<$ MDA & & $<\mathrm{MDA}$ \\
\hline 79.5 & $8 \mathrm{~A} / \mathrm{B} / \mathrm{C}$ & $2.32 \mathrm{E}+06$ & $4.54 \mathrm{E}+04$ & $2.56 \mathrm{E}+06$ & 156.4 & $11 \mathrm{~A}$ & $9.82 \mathrm{E}+00$ & $2.94 \mathrm{E}-01$ & $1.03 \mathrm{E}+01$ & 187.8 & $38 \mathrm{C}$ & $2.91 \mathrm{E}-01$ & $3.23 \mathrm{E}-02$ & $2.99 \mathrm{E}-01$ \\
\hline 82.5 & $7 \mathrm{~A} / \mathrm{B} / \mathrm{C}$ & $1.60 \mathrm{E}+07$ & $4.31 \mathrm{E}+05$ & $1.76 \mathrm{E}+07$ & 156.8 & $12 \mathrm{~B}$ & $7.38 \mathrm{E}+01$ & $1.16 \mathrm{E}-01$ & $7.89 \mathrm{E}+01$ & 188.3 & $38 \mathrm{~B}$ & $<$ MDA & & $<\mathrm{MDA}$ \\
\hline 90 & $6 \mathrm{~A} / \mathrm{B}$ & $3.97 \mathrm{E}+04$ & $5.32 \mathrm{E}+02$ & $4.38 \mathrm{E}+04$ & 157.1 & $12 \mathrm{~A}$ & $7.31 \mathrm{E}+01$ & $9.69 \mathrm{E}-01$ & $7.79 \mathrm{E}+01$ & 188.7 & $38 \mathrm{~A}$ & $<\mathrm{MDA}$ & & $<\mathrm{MDA}$ \\
\hline 95.5 & $3 \mathrm{~A} / \mathrm{B} / \mathrm{C}$ & $3.40 \mathrm{E}+04$ & $4.66 \mathrm{E}+02$ & $3.82 \mathrm{E}+04$ & 157.2 & $13 \mathrm{D}$ & $5.56 \mathrm{E}+01$ & $7.80 \mathrm{E}-01$ & $5.92 \mathrm{E}+01$ & 189.2 & $39 \mathrm{~B}$ & $3.72 \mathrm{E}-01$ & $4.12 \mathrm{E}-02$ & $3.78 \mathrm{E}-01$ \\
\hline 102.5 & $5 \mathrm{~A} / \mathrm{B} / \mathrm{C}$ & $1.44 \mathrm{E}+06$ & $2.83 \mathrm{E}+04$ & $1.62 \mathrm{E}+06$ & 157.7 & $13 \mathrm{C}$ & $1.02 \mathrm{E}+01$ & $2.45 \mathrm{E}-01$ & $1.06 \mathrm{E}+01$ & 189.5 & $39 \mathrm{~A}$ & $2.84 \mathrm{E}-01$ & $3.72 \mathrm{E}-02$ & $2.89 \mathrm{E}-01$ \\
\hline 108.5 & $4 \mathrm{~A} / \mathrm{B} / \mathrm{C}$ & $3.00 \mathrm{E}+05$ & $7.54 \mathrm{E}+03$ & $3.37 \mathrm{E}+05$ & 158.2 & $13 \mathrm{~B}$ & $1.15 \mathrm{E}+00$ & $7.46 \mathrm{E}-02$ & $1.21 \mathrm{E}+00$ & 190.7 & $40 \mathrm{~A}$ & $<$ MDA & & $<\mathrm{MDA}$ \\
\hline 112 & $2 \mathrm{~B} / \mathrm{C}$ & $1.32 \mathrm{E}+03$ & $2.35 \mathrm{E}+01$ & $1.49 \mathrm{E}+03$ & 158.7 & $13 \mathrm{~A}$ & $3.13 \mathrm{E}-01$ & $3.83 \mathrm{E}-02$ & NM & 193.3 & $45 \mathrm{~A}$ & 4.40E-01 & $4.72 \mathrm{E}-02$ & $4.63 \mathrm{E}-01$ \\
\hline 127.4 & $1 \mathrm{~A} / \mathrm{B} / \mathrm{C}$ & $3.73 \mathrm{E}+03$ & $6.40 \mathrm{E}+01$ & $4.20 \mathrm{E}+03$ & 159 & $14 \mathrm{D}$ & NM & & NM & 195.9 & $47 \mathrm{D}$ & $6.34 \mathrm{E}-02$ & $1.87 \mathrm{E}-02$ & $6.90 \mathrm{E}-02$ \\
\hline \multicolumn{5}{|c|}{ Extension } & 159.4 & $14 \mathrm{C}$ & $2.68 \mathrm{E}+00$ & $1.08 \mathrm{E}-01$ & $2.84 \mathrm{E}+00$ & 196.4 & $47 \mathrm{C}$ & $<$ MDA & & $<\mathrm{MDA}$ \\
\hline 131.1 & $1 \mathrm{C}-\mathrm{L}$ & $3.24 \mathrm{E}+05$ & $4.79 \mathrm{E}+03$ & NM & 160 & 14B & $<\mathrm{MDA}$ & & $<\mathrm{MDA}$ & 196.9 & $47 \mathrm{~B}$ & $<\mathrm{MDA}$ & & $<\mathrm{MDA}$ \\
\hline 131.1 & 1C-D & $7.96 \mathrm{E}+04$ & $1.21 \mathrm{E}+03$ & NM & 160.6 & $14 \mathrm{~A}$ & $<\mathrm{MDA}$ & & $<\mathrm{MDA}$ & 197.4 & $47 \mathrm{~A}$ & $1.92 \mathrm{E}-02$ & $6.11 \mathrm{E}-03$ & $2.01 \mathrm{E}-02$ \\
\hline 131.7 & 1B-1 & $2.08 \mathrm{E}+06$ & $3.58 \mathrm{E}+04$ & $2.35 \mathrm{E}+06$ & 162.3 & $16 \mathrm{D}$ & $2.24 \mathrm{E}+00$ & $8.25 \mathrm{E}-02$ & $2.49 \mathrm{E}+00$ & 198.8 & $49 \mathrm{C}$ & $<$ MDA & & NM \\
\hline 131.7 & $1 \mathrm{~B}-2$ & $9.29 \mathrm{E}+02$ & $1.41 \mathrm{E}+01$ & NM & 162.8 & $16 \mathrm{C}$ & $2.28 \mathrm{E}+00$ & $1.04 \mathrm{E}-01$ & $2.61 \mathrm{E}+00$ & 199.1 & 49B & $1.65 \mathrm{E}-01$ & $3.23 \mathrm{E}-02$ & $1.79 \mathrm{E}-01$ \\
\hline 133.2 & $2 \mathrm{D}$ & $4.80 \mathrm{E}+04$ & $6.44 \mathrm{E}+02$ & $6.50 \mathrm{E}+04$ & 163.3 & $16 \mathrm{~B}$ & $4.56 \mathrm{E}+00$ & $3.03 \mathrm{E}-02$ & $4.75 \mathrm{E}+00$ & 199.1 & $49 \mathrm{~A}$ & $9.66 \mathrm{E}-02$ & $2.60 \mathrm{E}-02$ & $1.06 \mathrm{E}-01$ \\
\hline 133.7 & $2 \mathrm{C}$ & $2.66 \mathrm{E}+04$ & $4.09 \mathrm{E}+02$ & $3.15 \mathrm{E}+04$ & 163.8 & $16 \mathrm{~A}$ & $<\mathrm{MDA}$ & & $<\mathrm{MDA}$ & 199.4 & $50 \mathrm{~B}$ & $2.49 \mathrm{E}-01$ & 6.19E-02 & $2.66 \mathrm{E}-01$ \\
\hline 133.7 & $2 \mathrm{C}-2$ & $1.16 \mathrm{E}+06$ & $1.70 \mathrm{E}+04$ & $1.47 \mathrm{E}+06$ & 164.3 & $17 \mathrm{D}$ & $1.86 \mathrm{E}+00$ & $8.63 \mathrm{E}-02$ & $1.95 \mathrm{E}+00$ & 199.4 & $50 \mathrm{~A}$ & $<\mathrm{MDA}$ & & $<\mathrm{MDA}$ \\
\hline 134.2 & $2 \mathrm{~B}$ & $2.27 \mathrm{E}+03$ & $3.25 \mathrm{E}+01$ & $2.60 \mathrm{E}+03$ & 164.8 & $17 \mathrm{C}$ & $9.31 \mathrm{E}-01$ & $6.17 \mathrm{E}-02$ & $9.63 \mathrm{E}-01$ & 200.2 & $52 \mathrm{~B}$ & $<\mathrm{MDA}$ & & $<\mathrm{MDA}$ \\
\hline
\end{tabular}


Table 4.5. (contd)

\begin{tabular}{|c|c|c|c|c|c|c|c|c|c|c|c|c|c|c|}
\hline $\begin{array}{c}\text { Depth } \\
(\mathrm{ft} \mathrm{bgs})^{(\mathrm{a})}\end{array}$ & $\begin{array}{c}\text { Sample } \\
\text { ID }\end{array}$ & $\begin{array}{c}{ }^{137} \mathrm{Cs} \\
\text { (pCi/g wet } \\
\text { wt) }\end{array}$ & $\begin{array}{c}{ }^{137} \mathrm{Cs} \\
( \pm \mathrm{pCi} / \mathrm{g} \\
\text { Error }) \\
\end{array}$ & $\begin{array}{l}{ }^{137} \mathrm{Cs} \\
(\mathrm{pCi} / \mathrm{g} \\
\text { dry wt) }\end{array}$ & $\begin{array}{c}\text { Depth } \\
\left(\mathrm{ft} \mathrm{bgs}^{(\mathrm{a})}\right.\end{array}$ & $\begin{array}{c}\text { Sample } \\
\text { ID }\end{array}$ & $\begin{array}{c}{ }^{137} \mathrm{Cs} \\
\text { (pCi/g wet } \\
\text { wt) }\end{array}$ & $\begin{array}{c}{ }^{137} \mathrm{Cs} \\
( \pm \mathrm{pCi} / \mathrm{g} \\
\text { Error }) \\
\end{array}$ & $\begin{array}{l}{ }^{137} \mathrm{Cs} \\
(\mathrm{pCi} / \mathrm{g} \\
\mathrm{dry} w) \\
\end{array}$ & $\begin{array}{c}\text { Depth } \\
(\mathrm{ft} \text { bgs })^{(\mathrm{a})}\end{array}$ & $\begin{array}{c}\text { Sample } \\
\text { ID }\end{array}$ & $\begin{array}{l}{ }^{137} \mathrm{Cs} \\
(\mathrm{pCi} / \mathrm{g} \\
\text { wet wt) } \\
\end{array}$ & \begin{tabular}{|c|}
${ }^{137} \mathrm{Cs}$ \\
$( \pm \mathrm{pCi} / \mathrm{g}$ \\
Error $)$ \\
\end{tabular} & $\begin{array}{l}{ }^{137} \mathrm{Cs} \\
(\mathrm{pCi} / \mathrm{g} \\
\mathrm{dry} w \mathrm{wt}) \\
\end{array}$ \\
\hline 134.7 & $2 \mathrm{~A}$ & $5.73 \mathrm{E}+01$ & $1.14 \mathrm{E}+00$ & $6.65 \mathrm{E}+01$ & 165.3 & $17 \mathrm{~B}$ & $6.46 \mathrm{E}-02$ & $2.98 \mathrm{E}-02$ & $6.32 \mathrm{E}-02$ & 200.7 & $52 \mathrm{~A}$ & $2.06 \mathrm{E}-01$ & $3.55 \mathrm{E}-02$ & $2.23 \mathrm{E}-01$ \\
\hline 135.2 & $3 \mathrm{~B}$ & $2.29 \mathrm{E}+03$ & $4.04 \mathrm{E}+01$ & NM & 165.8 & $17 \mathrm{~A}$ & $<\mathrm{MDA}$ & & $<\mathrm{MDA}$ & 202.5 & $54 \mathrm{~B}$ & $4.85 \mathrm{E}-02$ & 3.93E-02 & $5.17 \mathrm{E}-02$ \\
\hline 135.9 & $3 \mathrm{~A}$ & $2.93 \mathrm{E}+03$ & $3.79 \mathrm{E}+01$ & $3.31 \mathrm{E}+03$ & 166.1 & $18 \mathrm{D}$ & $3.42 \mathrm{E}-01$ & $8.96 \mathrm{E}-02$ & NM & 202.5 & $54 \mathrm{~A}$ & $2.18 \mathrm{E}-02$ & $1.27 \mathrm{E}-02$ & $2.30 \mathrm{E}-02$ \\
\hline 137.0 & $4 \mathrm{D}$ & NM & & NM & 166.6 & $18 \mathrm{C}$ & $<\mathrm{MDA}$ & & $<\mathrm{MDA}$ & 204.3 & $56 \mathrm{C}$ & $<\mathrm{MDA}$ & & $<\mathrm{MDA}$ \\
\hline 137.4 & $4 \mathrm{C}$ & $<\mathrm{MDA}$ & & $<\mathrm{MDA}$ & 167.1 & $18 \mathrm{~B}$ & $<\mathrm{MDA}$ & & $<\mathrm{MDA}$ & 205 & $56 \mathrm{~B}$ & $1.04 \mathrm{E}-01$ & $5.05 \mathrm{E}-02$ & $1.11 \mathrm{E}-01$ \\
\hline 138.0 & $4 \mathrm{~B}$ & $4.31 \mathrm{E}+01$ & $6.62 \mathrm{E}-01$ & $4.81 \mathrm{E}+01$ & 167.7 & $18 \mathrm{~A}$ & $5.12 \mathrm{E}-02$ & $6.11 \mathrm{E}-02$ & $5.24 \mathrm{E}-01$ & 205.6 & $56 \mathrm{~A}$ & $3.45 \mathrm{E}-02$ & $1.31 \mathrm{E}-02$ & 3.64E-02 \\
\hline 138.7 & $4 \mathrm{~A}$ & 4.76E-01 & $6.19 \mathrm{E}-02$ & $5.86 \mathrm{E}-01$ & 169.2 & 20D & $1.60 \mathrm{E}+00$ & 7.94E-02 & NM & 207.6 & $58 \mathrm{D}$ & $5.27 \mathrm{E}-02$ & $2.07 \mathrm{E}-02$ & 5.77E-02 \\
\hline 139.9 & $5 \mathrm{D}$ & $3.63 \mathrm{E}+01$ & $5.34 \mathrm{E}-01$ & $4.14 \mathrm{E}+01$ & 169.4 & $20 \mathrm{C}$ & $1.42 \mathrm{E}+00$ & $1.14 \mathrm{E}-01$ & NM & 208.1 & $58 \mathrm{C}$ & $1.03 \mathrm{E}-01$ & $3.93 \mathrm{E}-02$ & $1.13 \mathrm{E}-01$ \\
\hline 140.3 & $5 \mathrm{C}$ & $1.54 \mathrm{E}+01$ & $3.11 \mathrm{E}-01$ & $1.76 \mathrm{E}+01$ & 169.7 & $20 \mathrm{~B}$ & $1.75 \mathrm{E}+00$ & $8.73 \mathrm{E}-02$ & NM & 208.6 & $58 \mathrm{~B}$ & $<\mathrm{MDA}$ & & $<\mathrm{MDA}$ \\
\hline 140.9 & $5 \mathrm{~B}$ & $6.93 \mathrm{E}+01$ & $1.06 \mathrm{E}+00$ & $7.81 \mathrm{E}+01$ & 169.9 & $20 \mathrm{~A}$ & 2.94E-01 & $3.37 \mathrm{E}-02$ & $3.02 \mathrm{E}-01$ & 209 & $58 \mathrm{~A}$ & $<\mathrm{MDA}$ & & $<\mathrm{MDA}$ \\
\hline 141.5 & $5 \mathrm{~A}$ & $3.61 \mathrm{E}+00$ & $1.50 \mathrm{E}-01$ & $3.97 \mathrm{E}+00$ & 171.3 & $22 \mathrm{C}$ & 4.81E-01 & $7.19 \mathrm{E}-02$ & $5.08 \mathrm{E}-01$ & 209.4 & 59D & $<\mathrm{MDA}$ & & $<\mathrm{MDA}$ \\
\hline 141.9 & $6 \mathrm{~F}$ & $1.39 \mathrm{E}+02$ & $2.92 \mathrm{E}+00$ & $1.54 \mathrm{E}+02$ & 172 & $22 \mathrm{~B}$ & 4.19E-01 & $3.98 \mathrm{E}-02$ & $4.42 \mathrm{E}-01$ & 209.8 & $59 \mathrm{C}$ & $<\mathrm{MDA}$ & & $<\mathrm{MDA}$ \\
\hline 142.4 & $6 \mathrm{E}$ & $2.69 \mathrm{E}+00$ & $1.57 \mathrm{E}-01$ & $2.96 \mathrm{E}+00$ & 172.7 & $22 \mathrm{~A}$ & $<\mathrm{MDA}$ & & NM & 210.4 & $59 \mathrm{~B}$ & $<\mathrm{MDA}$ & & $<\mathrm{MDA}$ \\
\hline 143.3 & $6 \mathrm{D}$ & $1.10 \mathrm{E}-01$ & $3.69 \mathrm{E}-02$ & $1.34 \mathrm{E}-01$ & 178.2 & $28 \mathrm{~A}$ & $5.78 \mathrm{E}-01$ & $6.25 \mathrm{E}-02$ & $6.24 \mathrm{E}-01$ & 210.9 & $59 \mathrm{~A}$ & $<\mathrm{MDA}$ & & $<\mathrm{MDA}$ \\
\hline 144.1 & $6 \mathrm{C}$ & $1.38 \mathrm{E}+02$ & $2.99 \mathrm{E}+00$ & $1.74 \mathrm{E}+02$ & 178.8 & $30 \mathrm{C}$ & $5.39 \mathrm{E}-01$ & $8.53 \mathrm{E}-02$ & $5.76 \mathrm{E}-01$ & 211.7 & $62 \mathrm{~B}$ & $2.87 \mathrm{E}-02$ & & $3.10 \mathrm{E}-02$ \\
\hline 144.7 & $6 \mathrm{~B}$ & $<\mathrm{MDA}$ & & $<\mathrm{MDA}$ & 179.2 & $30 \mathrm{~B}$ & $<\mathrm{MDA}$ & & $<\mathrm{MDA}$ & 212.1 & $62 \mathrm{~A}$ & $<\mathrm{MDA}$ & & $<\mathrm{MDA}$ \\
\hline 145.2 & $7 \mathrm{E}$ & $4.21 \mathrm{E}+00$ & $1.48 \mathrm{E}-01$ & $4.57 \mathrm{E}+00$ & 179.7 & $30 \mathrm{~A}$ & $<\mathrm{MDA}$ & & $<\mathrm{MDA}$ & 212.9 & $64 \mathrm{~B}$ & $4.01 \mathrm{E}-02$ & $3.37 \mathrm{E}-02$ & $4.51 \mathrm{E}-02$ \\
\hline 145.8 & $7 \mathrm{D}$ & $2.06 \mathrm{E}-01$ & $2.05 \mathrm{E}-01$ & $2.19 \mathrm{E}-01$ & 180.2 & $31 \mathrm{~B}$ & $6.53 \mathrm{E}-01$ & $8.02 \mathrm{E}+00$ & $6.81 \mathrm{E}-01$ & 213.3 & $64 \mathrm{~A}$ & $2.39 \mathrm{E}-02$ & $1.18 \mathrm{E}-02$ & 2.71E-02 \\
\hline 146.4 & $7 \mathrm{C}$ & $<\mathrm{MDA}$ & & $<\mathrm{MDA}$ & 180.5 & $31 \mathrm{~A}$ & $5.18 \mathrm{E}-01$ & $7.34 \mathrm{E}-02$ & $5.37 \mathrm{E}-01$ & 214.1 & $65 \mathrm{~B}$ & $2.31 \mathrm{E}-01$ & $3.52 \mathrm{E}-02$ & $2.49 \mathrm{E}-01$ \\
\hline 148.3 & $8 \mathrm{C}$ & $2.04 \mathrm{E}+01$ & $3.96 \mathrm{E}+00$ & $2.19 \mathrm{E}+01$ & 181.1 & $32 \mathrm{~B}$ & $6.25 \mathrm{E}-01$ & $1.07 \mathrm{E}-01$ & $6.58 \mathrm{E}-01$ & 214.3 & $65 \mathrm{~A}$ & $9.05 \mathrm{E}-02$ & $2.62 \mathrm{E}-02$ & $9.83 \mathrm{E}-02$ \\
\hline 149.9 & $8 \mathrm{~B}$ & $1.27 \mathrm{E}+01$ & $2.99 \mathrm{E}-01$ & $1.39 \mathrm{E}+01$ & 181.5 & $32 \mathrm{~A}$ & $1.53 \mathrm{E}-01$ & $2.87 \mathrm{E}-02$ & $1.58 \mathrm{E}-01$ & 215 & $67 *$ & $1.13 \mathrm{E}-01$ & $9.25 \mathrm{E}-02$ & NM \\
\hline 151.1 & $9 \mathrm{C}$ & $1.09 \mathrm{E}+03$ & $2.17 \mathrm{E}+01$ & $1.23 \mathrm{E}+03$ & & & & & & & & & & \\
\hline \multicolumn{15}{|c|}{$\begin{array}{l}\text { MDA }=\text { Minimum detectable cesium- } 137 \text { activity }<2.00 \mathrm{E}-02 \mathrm{pCi} / \mathrm{g} ; \mathrm{NM}=\text { Not measured. } \\
\text { Note: Sleeves } 1 \mathrm{C} \text { and } 1 \mathrm{~B} \text { were partially filled and were compressed materials from the pile driving of the original casing. } 1 \mathrm{C}-\mathrm{L} \text { and } 1 \mathrm{C}-\mathrm{D} \text { represent light and dark compressed sediment. } 1 \mathrm{~B}- \\
1 \text { and } 1 \mathrm{~B}-2 \text { were two distinctly different grain-sized compressed materials. } 2 \mathrm{C}-2 \text { was a fine-grained lens of silt within a fine sand matrix. } \\
67^{*}=\text { Clean out material in saturated sediments. }\end{array}$} \\
\hline
\end{tabular}


The sidewall cores presented a challenge. Because of high activity, only small aliquots ( 5 grams) could be analyzed, producing some justifiable concern for sample homogeneity. Remarkably, apart from the minor fluctuations of cesium-137 activity between individual sleeves, as shown in Table 4.5, the vast majority of activity is concentrated between the depths 20.1 to 25.6 meters (66 to 84 feet) bgs with the leading edge perhaps reaching 39.9 to 41.5 meters (131 to 136 feet bgs), as shown in Figure 4.4. Figure 4.4 has both a linear and logarithmic scale for cesium-137.

The sidewall cores, obtained about every 1.5 meters ( 5 feet) between 18.2 and 38.7 meters ( 60 and 127 feet), show a highly varying cesium-137 activity between 20.1 and 38.7 meters (66 and 127 feet) bgs. There appears to be three high cesium-137 peaks, at 20.1, 25.1, and 31.1 meters $(66,82.5$ and 102 feet) bgs, that contain $4 \times 10^{6}, 2 \times 10^{7}$, and $2 \times 10^{6} \mathrm{pCi} / \mathrm{g}$, respectively. The peaks appear to correspond to finegrained sand. The peak at 20.1 meters ( 66 feet) bgs represents the contact between the Hanford formation H1a unit and the coarser-grained gravelly sand, Hanford formation H1 unit. There appears to be a large amount of cesium-137 contained in the coarse-grained Hanford formation H1 unit that is found between 20.4 and 26.8 meters (67 and 88 feet) bgs at borehole 41-09-39. The other large peak of cesium-137 is found in the fine laminated sand layer (Hanford formation $\mathrm{H} 2$ unit) that lies between 26.8 and 38.1 meters ( 88 and 125 feet) bgs at this borehole. The high cesium-137 at 40.1 to 40.5 meters (131.7 to 133 feet) bgs is believed to be compressed sediment dragged down during the original pile driving of the closed end casing. It is merely coincidence that the more mobile contaminants also seemed to reach 39.6 to 41.1 meters (130 to 135 feet) bgs, where all the borehole extension activities occurred (see Myers et al. 1998 for discussion of milling the end cap and extending the borehole).

Comparing gamma data from individual borehole extension sleeves from the first two sampling events, 2 and 3, near the beginning of the borehole extension shows that cesium-137 activity drops off many orders of magnitude within just a few inches. This indicates that activity was pushed or dragged down by the closed-end pipe when it was originally installed. This finding, a physical rather than chemical migration, also would tend to explain why technetium and cesium have been found together in the sleeve samples 2C (133.7 ft), 2D (133.2 ft), 3A (135.9 ft), and 3B $(135.2 \mathrm{ft})$, rather than at different depths along the profile that would be expected for these two contaminants with very different migration tendencies. We feel that the first few sampling events for the borehole extension (sleeves $1 \mathrm{C}, 1 \mathrm{~B}, 2 \mathrm{D}$, $2 \mathrm{C}, 2 \mathrm{~B}, 2 \mathrm{~A}, 3 \mathrm{~B}$, and $3 \mathrm{~A}$ [from depths at 131.1 to 135.9 ]) represent materials that were erratically dragged down by the closed-end casing and further mixed by the first few split-spoon sampling events (because the casing was coated with sticky highly contaminated mud from the end cap milling activity where $\sim 20$ gallons of cooling water escaped into the neighboring formation).

The cesium-137 data also show that the split-spoon sampling technique can cause the first sleeve in each sampling event to be contaminated by either sediment falling off the inside of the casing during split-spoon or clean-out tube insertion or removal, or sediment invading from annulus, outside the casing formed by the drive shoe, onto the hole floor. A systematic pattern is evident where the uppermost sleeve, typically the D sleeve, and sometimes even the second sleeve, typically the $\mathrm{C}$ sleeve, show cesium-137 activity two orders of magnitude higher than deeper sleeves, typically B and A. This biased sequence in cesium-137 activity occurred in the split spoon samples 2, 5, 7, 9, 10, 13, 14, 17, 30, 37, and 38. The depth ranges for these sampling events were 40.5 to $41.1,42.7$ to $43.3,41.2$ to $44.8,45.7$ to 46.6 , 


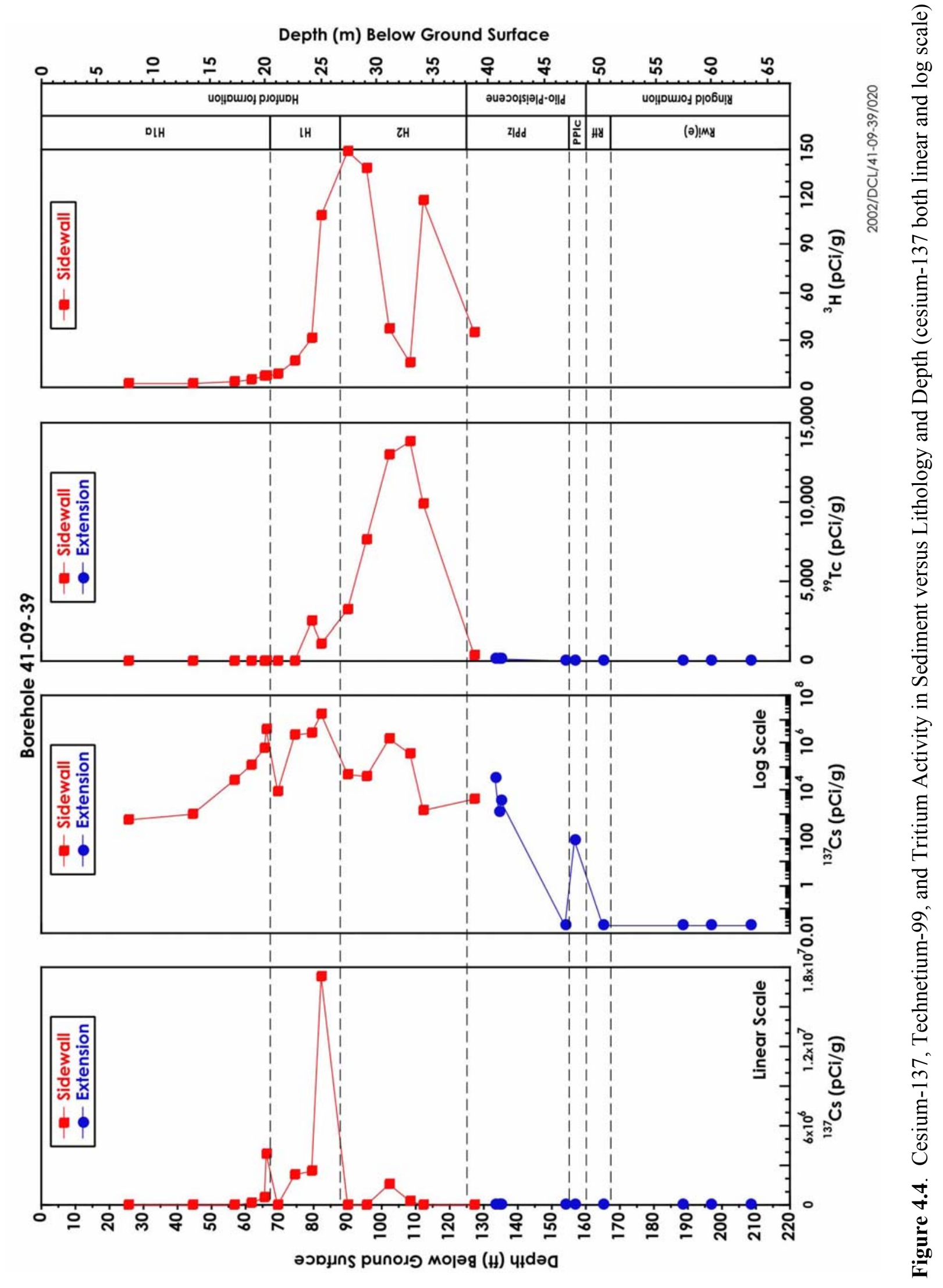

4.19 
$46.647 .2,47.9$ to $48.5,48.5$ to $49.1,50$ to $50.6,54.3$ to $54.9,56.4$ to 57,57 to 58 meters (133 to 135,140 to 142,145 to 147,150 to 153,153 to 155,157 to 159,159 to 161,164 to 166,178 to 180,185 to 187 , and 187 to 190 feet) bgs, respectively.

Sleeve 9C especially shows elevated cesium-137 activity obtained just after the newer casing with the drive shoe was emplaced. The hole had been open between 39.9 and 46.0 meters (131and 151 feet bgs) while the new casing was driven and sediment from above was dropped onto the floor of the original hole.

For a few select cores that remained intact after being removed from the sleeve, an effort was made to sub-core a smaller diameter and shorter cylinder, essentially skinning the sediment to get at more pristine material within the core. Table 4.6 shows the results that confirm some influx of activity onto the floor of the borehole just before the next split-spoon sampling.

The natural gamma emitters (potassium-40, daughters of natural uranium and thorium) were useful in determining changes in lithology and geologic formations when the cesium-137 activity was low (see Section 2.3), but were not useful in elucidating other contaminant distributions. When the cesium-137 activity was high, the natural gamma emitter signals were swamped out. The natural gamma emitter data are shown in Table 4.7 for reference.

Table 4.6. Cesium-137 Activity in Carefully Sub-Cored Sleeves (pCi/g)

\begin{tabular}{|c|c|c|c|}
\hline Sample & $\begin{array}{c}{ }^{137} \mathrm{Cs} \\
(\mathrm{pCi} / \mathrm{g}) \\
\end{array}$ & $\begin{array}{c}\text { Counting } \\
\text { Error } \\
( \pm \mathrm{pCi} / \mathrm{g}) \\
\end{array}$ & Comments \\
\hline $35 \mathrm{~B}$ & 0.33 & 0.06 & $\begin{array}{l}35 \mathrm{~B} \text { was sediment } 0 \text { to } 15.2 \text { centimeters ( } 0 \text { to } 6 \text { inches) into the formation } \\
\text { from the casing }\end{array}$ \\
\hline $35 \mathrm{~A}$ & $<0.02$ & & $\begin{array}{l}35 \mathrm{~A} \text { was sediment between } 15.2 \text { to } 30.5 \text { centimeters ( } 6 \text { to } 12 \text { inches) into the } \\
\text { formation beyond the casing. Thus, } 35 \mathrm{~B} \text { seems contaminated from material } \\
\text { from above falling onto the hole floor prior to sampling. }\end{array}$ \\
\hline $37 \mathrm{D}$ & 0.16 & 0.04 & $\begin{array}{l}37 \mathrm{D} \text { was sediment } 0 \text { to } 15.2 \text { centimeters ( } 0 \text { to } 6 \text { inches) into the formation } \\
\text { from the casing and borehole floor just prior to sampling }\end{array}$ \\
\hline $37 \mathrm{C}, \mathrm{B}, \mathrm{A}$ & $<0.02$ & & $\begin{array}{l}37 \mathrm{C}, \mathrm{B} \text {, and A were sediment between } 15.2 \text { to } 45.7 \text { centimeters ( } 6 \text { to } 18 \\
\text { inches) beyond casing and borehole floor. Thus, } 37 \mathrm{D} \text { seems contaminated } \\
\text { from material from above falling onto the hole floor prior to sampling. }\end{array}$ \\
\hline $39 \mathrm{~B}$ & 0.38 & 0.04 & $\begin{array}{l}\text { 39B was sediment } 0 \text { to } 15.2 \text { centimeters ( } 0 \text { to } 6 \text { inches) out in the formation } \\
\text { from casing and borehole floor. Sediment from the next shallower sampling } \\
\text { event shows no activity for the } 30.5 \text { centimeters ( } 12 \text { inches) directly above } \\
\text { this sleeve. Sediment } 0.3 \text { meter ( } 1 \text { foot) deeper than } 39 \mathrm{~B} \text { in the next } \\
\text { sampling event also shows no activity. Thus, 39B seems contaminated from } \\
\text { material from above falling onto the hole floor prior to sampling. }\end{array}$ \\
\hline $56 \mathrm{~A}$ & 0.04 & 0.02 & $\begin{array}{l}56 \mathrm{~A} \text { was sediment } 30.5 \text { to } 45.7 \text { centimeters ( } 12 \text { to } 18 \text { inches) beyond the } \\
\text { casing and borehole floor. There is little evidence of contamination because } \\
\text { this sample is not near the borehole floor just prior to the sampling event. }\end{array}$ \\
\hline
\end{tabular}


Table 4.7. Natural Gamma Emitter Activities in Sidewall Cores and Borehole Extension Sleeves

\begin{tabular}{|c|c|c|c|c|c|c|c|c|c|c|c|c|c|c|c|c|c|}
\hline $\mid \begin{array}{c}\text { Depth } \\
(\mathrm{ft} b g s)^{(\mathrm{a})}\end{array}$ & $\begin{array}{l}\text { Sample } \\
\text { ID }\end{array}$ & $\begin{array}{c}{ }^{40} \mathrm{~K} \\
\text { Dry Wt } \\
(\mathrm{pCi} / \mathrm{g})\end{array}$ & \begin{tabular}{|l} 
Error \\
Dry Wt \\
$(\mathrm{pCi} / \mathrm{g})$
\end{tabular} & $\begin{array}{l}{ }^{238} \mathrm{U} \\
\text { Dry Wt } \\
\text { (pCi/g) }\end{array}$ & $\begin{array}{l}{ }^{232} \mathrm{Th} \\
\text { Dry Wt } \\
(\mathrm{pCi} / \mathrm{g})\end{array}$ & $\begin{array}{c}\text { Depth } \\
(\mathrm{ft} \text { bgs })^{(\mathrm{a})}\end{array}$ & $\begin{array}{c}\text { Sample } \\
\text { ID }\end{array}$ & $\begin{array}{c}{ }^{40} \mathrm{~K} \\
\text { Dry Wt } \\
\text { (pCi/g) }\end{array}$ & \begin{tabular}{|l} 
Error \\
Dry Wt \\
(pCi/g)
\end{tabular} & \begin{tabular}{|c}
${ }^{238} \mathrm{U}$ \\
Dry Wt \\
(pCi/g)
\end{tabular} & $\begin{array}{l}{ }^{232} \mathrm{Th} \\
\text { Dry Wt } \\
(\mathrm{pCi} / \mathrm{g})\end{array}$ & $\begin{array}{c}\text { Depth } \\
(\mathrm{ft} \mathrm{bgs})^{(\mathrm{a})}\end{array}$ & \begin{tabular}{|c|} 
Sample \\
ID
\end{tabular} & $\begin{array}{c}{ }^{40} \mathrm{~K} \\
\text { Dry Wt } \\
\text { (pCi/g) }\end{array}$ & \begin{tabular}{|l} 
Error \\
Dry Wt \\
(pCi/g)
\end{tabular} & $\begin{array}{c}{ }^{238} \mathrm{U} \\
\mathrm{Dry} \mathrm{Wt} \\
(\mathrm{pCi} / \mathrm{g})\end{array}$ & $\begin{array}{l}{ }^{232} \mathrm{Th} \\
\text { Dry Wt } \\
\text { (pCi/g) }\end{array}$ \\
\hline \multicolumn{6}{|c|}{ Sidewall } & 153.9 & $10 \mathrm{~B}$ & 8.8 & 1.2 & 6.9E-01 & $8.0 \mathrm{E}-01$ & 184.6 & $36 \mathrm{~B}$ & 9.0 & 0.8 & 2.0E-01 & 3.4E-01 \\
\hline 25.5 & $15 \mathrm{~A} / \mathrm{B} / \mathrm{C}$ & $1.9 \mathrm{E}+01$ & 4.3 & $<1.8 \mathrm{E}+00$ & $<4.5 \mathrm{E}+01$ & 154.5 & $10 \mathrm{~A}$ & 14.0 & 1.1 & $<\mathrm{MDA}$ & $7.2 \mathrm{E}-01$ & 185 & $36 \mathrm{~A}$ & 7.0 & 0.6 & $2.5 \mathrm{E}-01$ & $2.8 \mathrm{E}-01$ \\
\hline 44.5 & $14 \mathrm{~A} / \mathrm{B} / \mathrm{C}$ & $1.2 \mathrm{E}+01$ & 1.8 & $<1.6 \mathrm{E}+00$ & $<4.3 \mathrm{E}+01$ & 155.1 & $11 \mathrm{D}$ & 4.3 & 1.1 & $<$ MDA & 4.2E-01 & 185.8 & $37 \mathrm{D}$ & 6.7 & 0.6 & $3.5 \mathrm{E}-01$ & $2.8 \mathrm{E}-01$ \\
\hline 56.5 & $13 \mathrm{~A} / \mathrm{B} / \mathrm{C}$ & $1.4 \mathrm{E}+01$ & 18.4 & $<3.7 \mathrm{E}+01$ & $<1.0 \mathrm{E}+03$ & 155.4 & $11 \mathrm{C}$ & 4.1 & 0.8 & $3.1 \mathrm{E}-01$ & $3.1 \mathrm{E}-01$ & 186.2 & $37 \mathrm{C}$ & 9.1 & 0.7 & $<\mathrm{MDA}$ & $2.9 \mathrm{E}-01$ \\
\hline 61.5 & $12 \mathrm{~A} / \mathrm{B} / \mathrm{C}$ & $<2.1 \mathrm{E}+01$ & & $<4.2 \mathrm{E}+02$ & $<7.6 \mathrm{E}+03$ & 155.9 & $11 \mathrm{~B}$ & 4.6 & 0.8 & 2.4E-01 & $3.1 \mathrm{E}-01$ & 186.8 & $37 \mathrm{~B}$ & 10.1 & 1.0 & $<\mathrm{MDA}$ & $3.6 \mathrm{E}-01$ \\
\hline 65.5 & $11 \mathrm{~A} / \mathrm{B}$ & $<1.6 \mathrm{E}+02$ & & $<2.6 \mathrm{E}+03$ & $<4.6 \mathrm{E}+04$ & 156.4 & $11 \mathrm{~A}$ & 4.7 & 0.6 & 3.1E-01 & $3.1 \mathrm{E}-01$ & 187.2 & $37 \mathrm{~A}$ & 7.1 & 0.6 & $2.5 \mathrm{E}-01$ & $1.7 \mathrm{E}-01$ \\
\hline 66 & $11 \mathrm{C}$ & $<3.2 \mathrm{E}+02$ & & $<8.9 \mathrm{E}+03$ & $<1.6 \mathrm{E}+05$ & 156.8 & $12 \mathrm{~B}$ & 5.5 & 0.9 & $<\mathrm{MDA}$ & $3.6 \mathrm{E}-01$ & 187.8 & $38 \mathrm{C}$ & 6.6 & 0.6 & $2.6 \mathrm{E}-01$ & 3.4E-01 \\
\hline 69.5 & $10 \mathrm{~A} / \mathrm{B} / \mathrm{C}$ & $2.0 \mathrm{E}+01$ & 3.5 & $<6.1 \mathrm{E}+00$ & $<1.3 \mathrm{E}+02$ & 157.1 & $12 \mathrm{~A}$ & 6.1 & 0.7 & $<\mathrm{MDA}$ & $4.8 \mathrm{E}-01$ & 188.3 & $38 \mathrm{~B}$ & 6.2 & 0.7 & $1.8 \mathrm{E}-01$ & $1.9 \mathrm{E}-01$ \\
\hline 74.5 & $09 \mathrm{~A} / \mathrm{B} / \mathrm{C}$ & $<2.0 \mathrm{E}+02$ & & $<5.6 \mathrm{E}+03$ & $<1.0 \mathrm{E}+05$ & 157.2 & $13 \mathrm{D}$ & 6.0 & 0.8 & $<$ MDA & $4.8 \mathrm{E}-01$ & 188.7 & $38 \mathrm{~A}$ & 7.4 & 0.6 & $2.3 \mathrm{E}-01$ & $2.2 \mathrm{E}-01$ \\
\hline 79.5 & 08A/B/C & $<3.2 \mathrm{E}+02$ & & $<7.4 \mathrm{E}+03$ & $<1.3 \mathrm{E}+05$ & 157.7 & $13 \mathrm{C}$ & 6.3 & 1.2 & 3.1E-01 & $4.0 \mathrm{E}-01$ & 189.2 & $39 \mathrm{~B}$ & 8.0 & 0.7 & 2.2E-01 & $2.3 \mathrm{E}-01$ \\
\hline 82.5 & $07 \mathrm{~A} / \mathrm{B} / \mathrm{C}$ & $<5.7 \mathrm{E}+03$ & & $<5.5 \mathrm{E}+04$ & $<1.1 \mathrm{E}+06$ & 158.2 & $13 \mathrm{~B}$ & 5.8 & 1.1 & $<\mathrm{MDA}$ & $2.8 \mathrm{E}-01$ & 189.5 & $39 \mathrm{~A}$ & 8.7 & 0.7 & 2.0E-01 & $2.8 \mathrm{E}-01$ \\
\hline 90 & $06 \mathrm{~A} / \mathrm{B}$ & $2.2 \mathrm{E}+01$ & 5.4 & $<1.1 \mathrm{E}+01$ & $<2.4 \mathrm{E}+02$ & 158.7 & $13 \mathrm{~A}$ & 5.2 & 0.8 & NM & $3.5 \mathrm{E}-01$ & 190.7 & $40 \mathrm{~A}$ & 8.7 & 0.7 & $<\mathrm{MDA}$ & $2.8 \mathrm{E}-01$ \\
\hline 95.5 & $03 \mathrm{~A} / \mathrm{B} / \mathrm{C}$ & $2.3 \mathrm{E}+01$ & 17.2 & $<1.8 \mathrm{E}+01$ & $<5.7 \mathrm{E}+02$ & 159.4 & $14 \mathrm{C}$ & 4.9 & 0.6 & $<\mathrm{MDA}$ & $3.1 \mathrm{E}-01$ & 193.3 & $45 \mathrm{~A}$ & 7.9 & 0.7 & $2.1 \mathrm{E}-01$ & $3.2 \mathrm{E}-01$ \\
\hline 102.5 & $05 \mathrm{~A} / \mathrm{B} / \mathrm{C}$ & $<3.4 \mathrm{E}+02$ & & $<6.4 \mathrm{E}+03$ & $<1.1 \mathrm{E}+05$ & 160 & 14B & 7.8 & 0.8 & 4.8E-01 & $3.5 \mathrm{E}-01$ & 195.9 & $47 \mathrm{D}$ & 9.9 & 0.6 & 3.1E-01 & $5.0 \mathrm{E}-01$ \\
\hline 108.5 & $04 \mathrm{~A} / \mathrm{B} / \mathrm{C}$ & $<2.2 \mathrm{E}+01$ & & $<2.4 \mathrm{E}+02$ & $<5.8 \mathrm{E}+03$ & 160.6 & $14 \mathrm{~A}$ & 10.6 & 1.0 & 3.2E-01 & $4.6 \mathrm{E}-01$ & 196.4 & $47 \mathrm{C}$ & 9.3 & 0.8 & 3.3E-01 & 3.7E-01 \\
\hline 112 & $02 \mathrm{~B} / \mathrm{C}$ & $1.9 \mathrm{E}+01$ & 1.8 & $<1.4 \mathrm{E}+00$ & $<3.9 \mathrm{E}+01$ & 162.3 & $16 \mathrm{D}$ & 9.9 & 0.9 & $3.6 \mathrm{E}-01$ & $4.0 \mathrm{E}+00$ & 196.9 & $47 \mathrm{~B}$ & 7.8 & 0.8 & $<\mathrm{MDA}$ & $3.1 \mathrm{E}-01$ \\
\hline 127.4 & $01 \mathrm{~A} / \mathrm{B} / \mathrm{C}$ & $1.8 \mathrm{E}+01$ & 2.4 & $<2.9 \mathrm{E}+00$ & $<7.6 \mathrm{E}+01$ & 162.8 & $16 \mathrm{C}$ & 9.3 & 0.6 & 3.5E-01 & $4.2 \mathrm{E}+00$ & 197.4 & $47 \mathrm{~A}$ & 8.1 & 0.7 & 3.2E-01 & 4.3E-01 \\
\hline \multicolumn{6}{|c|}{ Extension } & 163.3 & $16 \mathrm{~B}$ & 6.5 & 0.6 & 4.6E-01 & $3.1 \mathrm{E}+00$ & 198.8 & $49 \mathrm{C}$ & 6.6 & 0.6 & $2.5 \mathrm{E}-01$ & $2.6 \mathrm{E}+00$ \\
\hline 131.1 & $1 \mathrm{C}$ & $<1.5 \mathrm{E}+02$ & & $<5.32 \mathrm{E}+03$ & $<\mathrm{MDA}$ & 163.8 & $16 \mathrm{~A}$ & 7.0 & 0.9 & $3.1 \mathrm{E}-01$ & $1.8 \mathrm{E}-01$ & 199.1 & $49 \mathrm{~B}$ & 5.7 & 0.5 & $<\mathrm{MDA}$ & $2.6 \mathrm{E}-01$ \\
\hline 131.7 & $1 \mathrm{~B}$ & $<1.5 \mathrm{E}+02$ & & $<2.00 \mathrm{E}+01$ & $<2.4$ & 164.3 & $17 \mathrm{D}$ & 7.5 & 0.8 & 3.2E-01 & $2.1 \mathrm{E}-01$ & 199.1 & $49 \mathrm{~A}$ & 5.4 & 0.6 & $<\mathrm{MDA}$ & $<\mathrm{MDA}$ \\
\hline 133.2 & $2 \mathrm{D}$ & $<1.5 \mathrm{E}+02$ & & $<2.00 \mathrm{E}+01$ & $<2.4$ & 164.8 & $17 \mathrm{C}$ & 6.2 & 0.9 & 2.3E-01 & $2.1 \mathrm{E}-01$ & 199.4 & $50 \mathrm{~B}$ & 6.1 & 0.7 & $2.1 \mathrm{E}-01$ & $3.5 \mathrm{E}-01$ \\
\hline 133.7 & $2 \mathrm{C}$ & $<9.7 \mathrm{E}+01$ & & $<2.00 \mathrm{E}+01$ & $<2.4$ & 165.3 & 17B & 7.4 & 0.8 & 3.2E-01 & $3.2 \mathrm{E}-01$ & 199.4 & $50 \mathrm{~A}$ & 6.7 & 0.6 & $2.5 \mathrm{E}-01$ & $2.6 \mathrm{E}-01$ \\
\hline 134.2 & $2 \mathrm{~B}$ & $5.0 \mathrm{E}+00$ & 0.6 & $<\mathrm{MDA}$ & $1.4 \mathrm{E}+00$ & 165.8 & $17 \mathrm{~A}$ & 8.1 & 1.1 & $3.2 \mathrm{E}-01$ & $2.3 \mathrm{E}-01$ & 200.2 & $52 \mathrm{~B}$ & 9.1 & 0.7 & $2.3 \mathrm{E}-01$ & $3.0 \mathrm{E}-01$ \\
\hline 134.7 & $2 \mathrm{~A}$ & $1.3 \mathrm{E}+01$ & 2.1 & $<\mathrm{MDA}$ & $1.4 \mathrm{E}+00$ & 166.1 & $18 \mathrm{D}$ & 8.1 & 0.9 & 3.0E-01 & $2.1 \mathrm{E}-01$ & 200.7 & $52 \mathrm{~A}$ & 6.6 & 0.6 & 2.1E-01 & $2.9 \mathrm{E}-01$ \\
\hline 135.2 & $3 \mathrm{~B}$ & $<1.8 \mathrm{E}+01$ & & NM & $<\mathrm{MDA}$ & 166.6 & $18 \mathrm{C}$ & 8.4 & 0.9 & $<\mathrm{MDA}$ & $2.5 \mathrm{E}-01$ & 202.5 & $54 \mathrm{~B}$ & 5.7 & 0.6 & $2.3 \mathrm{E}-01$ & $2.2 \mathrm{E}-01$ \\
\hline 135.9 & $3 \mathrm{~A}$ & $<\mathrm{MDA}$ & & $<\mathrm{MDA}$ & $<\mathrm{MDA}$ & 167.1 & $18 \mathrm{~B}$ & 7.6 & 0.7 & $2.6 \mathrm{E}-01$ & $2.3 \mathrm{E}-01$ & 202.5 & $54 \mathrm{~A}$ & 5.7 & 0.5 & $1.6 \mathrm{E}-01$ & $3.4 \mathrm{E}-01$ \\
\hline
\end{tabular}


Table 4.7. (contd)

\begin{tabular}{|c|c|c|c|c|c|c|c|c|c|c|c|c|c|c|c|c|c|}
\hline \begin{tabular}{|} 
Depth \\
$(\mathrm{ft}$ bgs)
\end{tabular} & $\begin{array}{l}\text { Sample } \\
\text { ID }\end{array}$ & $\begin{array}{c}{ }^{40} \mathrm{~K} \\
\text { Dry Wt } \\
(\mathrm{pCi} / \mathrm{g})\end{array}$ & $\begin{array}{c}\text { Error } \\
\text { Dry Wt } \\
(\mathrm{pCi} / \mathrm{g})\end{array}$ & $\begin{array}{c}{ }^{238} \mathrm{U} \\
\text { Dry Wt } \\
\text { (pCi/g) }\end{array}$ & $\begin{array}{c}{ }^{232} \mathrm{Th} \\
\text { Dry Wt } \\
\text { (pCi/g) }\end{array}$ & $\begin{array}{c}\text { Depth } \\
(\mathrm{ft} b g s)^{(a)}\end{array}$ & $\begin{array}{c}\text { Sample } \\
\text { ID }\end{array}$ & \begin{tabular}{|c|}
${ }^{40} \mathrm{~K}$ \\
$\mathrm{Dry} \mathrm{Wt}$ \\
$(\mathrm{pCi} / \mathrm{g})$
\end{tabular} & $\begin{array}{c}\text { Error } \\
\text { Dry Wt } \\
(\mathrm{pCi} / \mathrm{g})\end{array}$ & $\begin{array}{c}{ }^{238} \mathrm{U} \\
\text { Dry Wt } \\
(\mathrm{pCi} / \mathrm{g})\end{array}$ & $\begin{array}{c}{ }^{232} \mathrm{Th} \\
\text { Dry Wt } \\
\text { (pCi/g) }\end{array}$ & $\begin{array}{c}\text { Depth } \\
(\mathrm{ft} \text { bgs })^{(\mathrm{a})}\end{array}$ & $\begin{array}{c}\text { Sample } \\
\text { ID }\end{array}$ & \begin{tabular}{|c|}
${ }^{40} \mathrm{~K}$ \\
Dry Wt \\
(pCi $/ \mathrm{g}$ )
\end{tabular} & $\begin{array}{c}\text { Error } \\
\text { Dry Wt } \\
(\mathrm{pCi} / \mathrm{g})\end{array}$ & $\begin{array}{c}{ }^{238} \mathrm{U} \\
\text { Dry Wt } \\
\text { (pCi/g) }\end{array}$ & $\begin{array}{l}{ }^{232} \mathrm{Th} \\
\text { Dry Wt } \\
\text { (pCi/g) }\end{array}$ \\
\hline 137.4 & $4 \mathrm{C}$ & 11.6 & 1.4 & $7.8 \mathrm{E}-01$ & $6.7 \mathrm{E}-01$ & 167.7 & $18 \mathrm{~A}$ & 7.5 & 1.0 & $2.3 \mathrm{E}-01$ & $3.5 \mathrm{E}-01$ & 204.3 & $56 \mathrm{C}$ & 8.5 & 0.7 & $2.5 \mathrm{E}-01$ & 3.7E-01 \\
\hline 138.7 & $4 \mathrm{~A}$ & 10.4 & 1.0 & $1.3 \mathrm{E}+00$ & $7.0 \mathrm{E}-02$ & 169.2 & $20 \mathrm{D}$ & 5.7 & 0.6 & $3.0 \mathrm{E}-01$ & $2.1 \mathrm{E}-01$ & 205 & $56 \mathrm{~B}$ & 9.2 & 0.8 & $<\mathrm{MDA}$ & $2.5 \mathrm{E}-01$ \\
\hline 139.9 & $5 \mathrm{D}$ & 8.2 & 0.7 & $1.4 \mathrm{E}+00$ & 4.6E-01 & 169.4 & $20 \mathrm{C}$ & 4.5 & 0.5 & $<\mathrm{MDA}$ & $1.7 \mathrm{E}-01$ & 205.6 & $56 \mathrm{~A}$ & 8.4 & 0.6 & $1.4 \mathrm{E}-01$ & $2.0 \mathrm{E}-01$ \\
\hline 140.3 & $5 \mathrm{C}$ & 8.5 & 0.9 & $1.2 \mathrm{E}+00$ & $5.7 \mathrm{E}-01$ & 169.7 & $20 \mathrm{~B}$ & 4.5 & 0.1 & $4.0 \mathrm{E}-01$ & $2.1 \mathrm{E}-01$ & 207.6 & $58 \mathrm{D}$ & 6.4 & 0.8 & $1.4 \mathrm{E}-01$ & $2.6 \mathrm{E}-01$ \\
\hline 140.9 & $5 \mathrm{~B}$ & 7.9 & 0.6 & $6.8 \mathrm{E}-01$ & $4.5 \mathrm{E}-01$ & 169.9 & $20 \mathrm{~A}$ & 5.2 & 0.6 & $2.1 \mathrm{E}-01$ & $1.9 \mathrm{E}-01$ & 208.1 & $58 \mathrm{C}$ & 7.0 & 0.9 & $3.2 \mathrm{E}-01$ & $3.5 \mathrm{E}-01$ \\
\hline 141.5 & $5 \mathrm{~A}$ & 9.9 & 1.0 & $1.1 \mathrm{E}+00$ & $6.6 \mathrm{E}-01$ & 171.3 & $22 \mathrm{C}$ & 6.9 & 0.7 & $<\mathrm{MDA}$ & $2.1 \mathrm{E}-01$ & 208.6 & $58 \mathrm{~B}$ & 8.0 & 0.6 & $2.7 \mathrm{E}-01$ & $4.5 \mathrm{E}-01$ \\
\hline 141.9 & $6 \mathrm{~F}$ & 12.4 & 2.9 & $<\mathrm{MDA}$ & $<\mathrm{MDA}$ & 172 & $22 \mathrm{~B}$ & 7.6 & 0.6 & $2.1 \mathrm{E}-01$ & $2.1 \mathrm{E}-01$ & 209 & $58 \mathrm{~A}$ & 7.3 & 0.6 & $1.8 \mathrm{E}-01$ & 3.4E-01 \\
\hline 142.4 & $6 \mathrm{E}$ & 9.8 & 1.7 & $1.5 \mathrm{E}+00$ & $1.4 \mathrm{E}+00$ & 172.7 & $22 \mathrm{~A}$ & 6.9 & 0.8 & $3.0 \mathrm{E}-01$ & $<\mathrm{MDA}$ & 209.4 & 59D & 6.0 & 0.7 & $<\mathrm{MDA}$ & $2.8 \mathrm{E}-01$ \\
\hline 143.3 & $6 \mathrm{D}$ & 14.3 & 0.0 & $2.0 \mathrm{E}+00$ & $1.2 \mathrm{E}+00$ & 178.2 & $28 \mathrm{~A}$ & 8.8 & 0.8 & $<\mathrm{MDA}$ & $3.2 \mathrm{E}-01$ & 209.8 & $59 \mathrm{C}$ & 6.4 & 0.9 & $<\mathrm{MDA}$ & $2.2 \mathrm{E}-01$ \\
\hline 144.1 & $6 \mathrm{C}$ & 10.3 & 3.6 & $<\mathrm{MDA}$ & $<\mathrm{MDA}$ & 178.8 & $30 \mathrm{C}$ & 1.3 & 1.2 & $5.1 \mathrm{E}-01$ & $5.4 \mathrm{E}-01$ & 210.4 & $59 \mathrm{~B}$ & 8.7 & 0.6 & $<\mathrm{MDA}$ & $3.6 \mathrm{E}-01$ \\
\hline 144.7 & $6 \mathrm{~B}$ & 12.3 & 1.3 & $7.4 \mathrm{E}-01$ & $9.8 \mathrm{E}-01$ & 179.2 & $30 \mathrm{~B}$ & 1.1 & 0.9 & $2.7 \mathrm{E}-01$ & $<\mathrm{MDA}$ & 210.9 & $59 \mathrm{~A}$ & 6.8 & 0.5 & $1.4 \mathrm{E}-01$ & $2.8 \mathrm{E}-01$ \\
\hline 145.2 & $7 \mathrm{E}$ & 10.5 & 0.9 & 4.4E-01 & $5.4 \mathrm{E}-01$ & 179.7 & $30 \mathrm{~A}$ & 10.3 & 1.0 & $<\mathrm{MDA}$ & $2.2 \mathrm{E}-01$ & 211.7 & $62 \mathrm{~B}$ & 6.9 & 0.7 & $<\mathrm{MDA}$ & $1.5 \mathrm{E}-01$ \\
\hline 145.8 & $7 \mathrm{D}$ & 9.9 & 0.8 & $6.6 \mathrm{E}-01$ & $5.5 \mathrm{E}-01$ & 180.2 & $31 \mathrm{~B}$ & 11.4 & 1.0 & $<\mathrm{MDA}$ & $3.1 \mathrm{E}-01$ & 212.1 & $62 \mathrm{~A}$ & 5.8 & 0.7 & $1.7 \mathrm{E}-01$ & 4.0E-01 \\
\hline 146.4 & $7 \mathrm{C}$ & 10.8 & 1.1 & $4.5 \mathrm{E}-01$ & $5.6 \mathrm{E}-01$ & 180.5 & $31 \mathrm{~A}$ & 12.7 & 1.2 & $<\mathrm{MDA}$ & $1.6 \mathrm{E}-01$ & 212.9 & $64 \mathrm{~B}$ & 5.3 & 0.5 & $2.5 \mathrm{E}-01$ & $1.9 \mathrm{E}-01$ \\
\hline 148.3 & $8 \mathrm{C}$ & 8.1 & 8.5 & $2.2 \mathrm{E}-01$ & $5.4 \mathrm{E}-01$ & 181.1 & $32 \mathrm{~B}$ & 10.6 & 0.8 & $<\mathrm{MDA}$ & $3.5 \mathrm{E}-01$ & 213.3 & $64 \mathrm{~A}$ & 5.8 & 0.5 & $2.5 \mathrm{E}-01$ & $2.9 \mathrm{E}-01$ \\
\hline 149.9 & $8 \mathrm{~B}$ & 11.2 & 1.2 & $5.5 \mathrm{E}-01$ & 7.7E-01 & 181.5 & $32 \mathrm{~A}$ & 10.9 & 0.7 & $3.1 \mathrm{E}-01$ & $5.2 \mathrm{E}-01$ & 214.1 & $65 \mathrm{~B}$ & 5.9 & 0.6 & $2.3 \mathrm{E}-01$ & $2.9 \mathrm{E}-02$ \\
\hline 151.1 & $9 \mathrm{C}$ & $<$ MDA & & $<\mathrm{MDA}$ & $<\mathrm{MDA}$ & 183 & $35 \mathrm{~B}$ & 11.0 & 1.1 & $2.1 \mathrm{E}-01$ & $2.8 \mathrm{E}-01$ & 214.3 & $65 \mathrm{~A}$ & 6.0 & 0.5 & $2.0 \mathrm{E}-01$ & $2.4 \mathrm{E}-01$ \\
\hline 152.8 & $9 \mathrm{~A}$ & 9.9 & 0.9 & $8.0 \mathrm{E}-01$ & $5.7 \mathrm{E}-01$ & 183.3 & $35 \mathrm{~A}$ & 8.4 & 0.7 & $2.5 \mathrm{E}-01$ & $3.5 \mathrm{E}-01$ & 215 & $67 *$ & 7.4 & 0.7 & $2.7 \mathrm{E}-01$ & $2.5 \mathrm{E}-01$ \\
\hline 153.4 & $10 \mathrm{C}$ & 9.8 & 0.9 & $5.7 \mathrm{E}-01$ & $6.8 \mathrm{E}-01$ & 183.9 & $36 \mathrm{C}$ & 9.8 & 0.8 & $2.9 \mathrm{E}-01$ & $3.3 \mathrm{E}-01$ & & & & & & \\
\hline
\end{tabular}


Table 4.8. Technetium- 99 and Strontium- 90 Concentrations in Sidewall Cores and Borehole Sleeves at Borehole 41-09-39

\begin{tabular}{|c|c|c|c|c|c|c|}
\hline $\begin{array}{c}\text { Depth } \\
(\mathrm{ft} \text { bgs })^{(\mathrm{a})}\end{array}$ & $\begin{array}{l}\text { Sample } \\
\text { ID }\end{array}$ & $\begin{array}{c}{ }^{99} \mathrm{Tc} \\
(\mathrm{pCi} / \mathrm{g} \\
\mathrm{ICP} / \mathrm{MS})\end{array}$ & $\begin{array}{c}{ }^{99} \mathrm{Tc} \\
(\mathrm{pCi} / \mathrm{g} \\
\mathrm{Rad})\end{array}$ & $\begin{array}{c}\text { Error }{ }^{99} \mathrm{Tc} \\
( \pm \mathrm{pCi} / \mathrm{g} \\
\mathrm{Rad})\end{array}$ & $\begin{array}{c}{ }^{90} \mathrm{Sr} \\
\text { (pCi/g } \\
\mathrm{Rad})\end{array}$ & $\begin{array}{c}\text { Error }{ }^{90} \mathrm{Sr} \\
( \pm \mathrm{pCi} / \mathrm{g} \\
\mathrm{Rad})\end{array}$ \\
\hline \multicolumn{7}{|c|}{ Sidewall } \\
\hline $25-26$ & $15 \mathrm{~A} / \mathrm{B} / \mathrm{C}$ & $<20.0$ & -50 & 32 & -5.6 & 20.4 \\
\hline $44-45$ & $14 \mathrm{~A} / \mathrm{B} / \mathrm{C}$ & 5.96 & -5 & 33 & -12.6 & 19.5 \\
\hline $56-57$ & $13 \mathrm{~A} / \mathrm{B} / \mathrm{C}$ & 30.3 & 313 & 56 & 0.4 & 21.1 \\
\hline $61-62$ & $12 \mathrm{~A} / \mathrm{B} / \mathrm{C}$ & 10.8 & -17 & 32 & -2.8 & 20.7 \\
\hline $65-66$ & $11 \mathrm{~A} / \mathrm{B}$ & 25.6 & -12 & 34 & -10.2 & 19.8 \\
\hline $65-66$ & $11 \mathrm{C}$ & $<15.8$ & -2 & 27 & -3.4 & 23.2 \\
\hline $69-70$ & $10 \mathrm{~A} / \mathrm{B} / \mathrm{C}$ & $<25.2$ & 95 & 41 & -16.7 & 18.8 \\
\hline $74-75$ & $9 \mathrm{~A} / \mathrm{B} / \mathrm{C}$ & $<35.6$ & 12 & 30 & 13.7 & 22.8 \\
\hline $79-80$ & $8 \mathrm{~A} / \mathrm{B} / \mathrm{C}$ & 2527 & 3164 & 132 & 1.8 & 21.3 \\
\hline $82-83$ & $7 \mathrm{~A} / \mathrm{B} / \mathrm{C}$ & 1325 & 1361 & 77 & -8.9 & 24.4 \\
\hline 90 & $6 \mathrm{~A} / \mathrm{B}$ & 3241 & 3586 & 143 & -15.0 & 19.1 \\
\hline $95-96$ & $3 \mathrm{~A} / \mathrm{B} / \mathrm{C}$ & 7597 & 7448 & 238 & -7.4 & 20.1 \\
\hline $102-103$ & $5 \mathrm{~A} / \mathrm{B} / \mathrm{C}$ & 12979 & 13036 & 384 & -1.2 & 20.9 \\
\hline 108-109 & $4 \mathrm{~A} / \mathrm{B} / \mathrm{C}$ & 13766 & 13877 & 399 & -62.0 & 12.0 \\
\hline 112.0 & $2 \mathrm{~B} / \mathrm{C}$ & 9840 & 9906 & 299 & -10.9 & 19.6 \\
\hline 127.4 & $1 \mathrm{~A} / \mathrm{B} / \mathrm{C}$ & 405 & 405 & 50 & -33.0 & 16.5 \\
\hline \multicolumn{7}{|c|}{ Extension } \\
\hline 133.2 & $2 \mathrm{CD}$ & $\mathrm{NM}^{*}$ & 554 & 71 & -1.4 & 12.0 \\
\hline 134.2 & $2 \mathrm{AB}$ & NM & 70 & 4 & 0.6 & 4.3 \\
\hline 135.2 & $3 \mathrm{AB}$ & NM & 107 & 2 & 0.8 & 8.3 \\
\hline 137.4 & $4 \mathrm{C}$ & NM & 2.8 & 0.6 & 0.1 & 0.4 \\
\hline 138.7 & $4 \mathrm{~A}$ & NM & 1.7 & 7.7 & NM & NM \\
\hline 141.5 & $5 \mathrm{~A}$ & NM & 1.2 & 0.8 & 0.0 & 0.3 \\
\hline 142.4 & $6 \mathrm{E}$ & NM & -6.5 & 7.7 & NM & NM \\
\hline 143.3 & $6 \mathrm{D}$ & NM & 0.2 & 6.9 & NM & NM \\
\hline 144.7 & $6 \mathrm{~B}$ & NM & 16.0 & 10.4 & NM & NM \\
\hline 153.9 & $10 \mathrm{AB}$ & NM & 4.8 & 2.0 & -1.2 & 6.0 \\
\hline 156.8 & $12 \mathrm{AB}$ & NM & 5.9 & 1.9 & -0.8 & 4.0 \\
\hline 158.2 & $13 \mathrm{~B}$ & NM & 1.5 & 4.5 & NM & NM \\
\hline 163.8 & $16 \mathrm{~A}$ & NM & -0.9 & 5.2 & NM & NM \\
\hline
\end{tabular}


Table 4.8. (contd)

\begin{tabular}{|c|c|c|c|c|c|c|}
\hline $\begin{array}{c}\text { Depth } \\
\left(\mathrm{ft} \mathrm{bgs}^{(\mathrm{a})}\right.\end{array}$ & $\begin{array}{l}\text { Sample } \\
\text { ID }\end{array}$ & $\begin{array}{c}{ }^{99} \mathrm{Tc} \\
(\mathrm{pCi} / \mathrm{g} \\
\mathrm{ICP} / \mathrm{MS})\end{array}$ & $\begin{array}{c}{ }^{99} \mathrm{Tc} \\
(\mathrm{pCi} / \mathrm{g} \\
\mathrm{Rad})\end{array}$ & $\begin{array}{c}\text { Error }{ }^{99} \mathrm{Tc} \\
( \pm \mathrm{pCi} / \mathrm{g} \\
\mathrm{Rad})\end{array}$ & $\begin{array}{c}{ }^{90} \mathrm{Sr} \\
(\mathrm{pCi} / \mathrm{g} \\
\mathrm{Rad})\end{array}$ & $\begin{array}{c}\text { Error }{ }^{90} \mathrm{Sr} \\
( \pm \mathrm{pCi} / \mathrm{g} \\
\mathrm{Rad})\end{array}$ \\
\hline 165.3 & $17 \mathrm{AB}$ & NM & -0.3 & 4.0 & -0.8 & 6.5 \\
\hline 167.1 & $18 \mathrm{~B}$ & $\mathrm{NM}$ & 1.9 & 4.5 & NM & NM \\
\hline 169.9 & $20 \mathrm{~A}$ & NM & -0.6 & 5.0 & NM & NM \\
\hline 172.0 & $22 \mathrm{~B}$ & NM & -11.2 & 13.7 & NM & NM \\
\hline 179.2 & $30 \mathrm{~B}$ & $\mathrm{NM}$ & 0.6 & 0.8 & 0.1 & 0.4 \\
\hline 184.6 & $36 \mathrm{~B}$ & NM & 11.4 & 0.4 & -0.2 & 0.4 \\
\hline 188.5 & $38 \mathrm{AB}$ & $\mathrm{NM}$ & 0.1 & 0.4 & 0.2 & 0.5 \\
\hline 197.2 & $47 \mathrm{AB}$ & NM & 1.3 & 1.1 & 0.0 & 0.7 \\
\hline 199.1 & $49 \mathrm{~B}$ & $\mathrm{NM}$ & 1.6 & 1.2 & -0.1 & 0.4 \\
\hline 199.4 & $50 \mathrm{~A}$ & NM & 0.1 & 1.0 & 0.1 & 0.6 \\
\hline 200.2 & $52 \mathrm{~B}$ & $\mathrm{NM}$ & 0.3 & 0.8 & -0.4 & 0.6 \\
\hline 202.2 & $54 \mathrm{AB}$ & $\mathrm{NM}$ & 0.3 & 0.7 & -0.2 & 0.4 \\
\hline 205.3 & $56 \mathrm{AB}$ & $\mathrm{NM}$ & 0.5 & 0.8 & 0.0 & 0.4 \\
\hline 208.8 & $58 \mathrm{AB}$ & $\mathrm{NM}$ & 0.9 & 0.8 & -0.1 & 0.4 \\
\hline 210.6 & $59 \mathrm{AB}$ & $\mathrm{NM}$ & 0.5 & 0.9 & -0.3 & 0.4 \\
\hline 211.8 & $62 \mathrm{~B}$ & NM & 0.0 & 0.8 & 0.0 & 0.3 \\
\hline 214.2 & $65 \mathrm{AB}$ & $\mathrm{NM}$ & 0.7 & 0.9 & 0.0 & 0.4 \\
\hline \multicolumn{7}{|c|}{$\begin{array}{l}\mathrm{NM}=\text { not measured } \\
\text { One of the three cores composited to create sidewall composite } 5 \mathrm{ABC} \text { was taken at a different } \\
\text { depth. Values with beige shadowing are the average of duplicate samples. Values in pink } \\
\text { shadow are the average of triplicate samples. When measuring radionuclides by wet chemical } \\
\text { separations, it is possible to find negative values when the samples contain very little analyte } \\
\text { because of small variations in background and separation efficiencies. Errors in radionuclide } \\
\text { measurements represent one standard deviation in counting uncertainty. } \\
\text { (a) Multiply by } 0.3048 \text { to convert to meters. }\end{array}$} \\
\hline
\end{tabular}

The technetium-99 (see Figure 4.4) and strontium-90 data in Table 4.8 show that there are significant concentrations of technetium-99 between the depths of 24.1 to 41.1 meters ( $\sim 79$ to 135 feet) bgs. For this table, we have added some of the data for borehole extension composite samples (combined sleeves B and C), which are the subject of Section 5.0, to improve the clarity of the discussion. The technetium-99 trailing edge may, in fact, be at 21 meters ( 69 feet) bgs based on a radiologic measurement. Both inductively coupled plasma mass spectrometry (ICP/MS) and traditional wet chemical separation and radiocounting methods were used. The agreement is very good when the technetium-99 concentration exceeds $100 \mathrm{pCi} / \mathrm{g}$ activity (dry weight basis). The leading edge of the technetium-99 plume appears to reach 41.1 meters (135 feet) bgs. Samples below 42 meters (138 feet bgs) are at or near the detection limit, except for sleeves 6B and 36B at 44.1 and 56.3 meters (144.7 and 184.6 feet bgs), respectively. 
Both sleeves have about $15 \mathrm{pCi} / \mathrm{g}$ of technetium- 99 , but sleeve $6 \mathrm{~B}$ has a counting uncertainty almost as large. We consider this the result of insufficient sample volume. However, sleeve 36B also had a low counting uncertainty, so the value obtained, $11.4 \mathrm{pCi} / \mathrm{g}$ (dry weight basis), may be significant.

As presented in Section 2.4, this sleeve is at a depth of 56.3 meters (184.6 feet bgs), which is near the historical high water table of 55.5 meters ( 182 feet bgs). This technetium- 99 value in the vadose zone sediment may reflect the bathtub ring, but, as noted in the anion discussion, this depth does not correspond to the one atypical nitrate and chloride measurement found in the Ringold Formation.

There does not appear to be any consistently positive strontium-90 measurements, which suggests that strontium-90 is not mobile in the REDOX fluid that leaked from the SX tanks. Strontium is not considered to be very soluble in most single-shell tank environments and has been found to reside predominately in precipitates in the sludge at the bottom of the tanks. Little strontium-90 is found in water washes of sludge or in tank supernatant solutions (see Johnson and Chou 1998).

\subsection{Soil Suction Measurements - Results and Discussion}

A key hydrologic measurement in the vadose zone is the soil-water matric potential or suction. Matric potential is defined as the amount of work that must be done per unit of soil solution to transport, reversibly and isothermally, an infinitesimal quantity of water from a pool of soil solution at a given elevation above the water table at atmospheric pressure to the soil pores at the same elevation and pressure (SSSA 1997). When the work (or energy) is expressed on a weight basis, the matric potential is expressed in units of length (i.e., meter or centimeter). Matric potential is always negative (i.e., energy is gained going from a saturated solution to unsaturated soil pores because of adsorptive forces and capillarity of porous material). Matric suction is the absolute value of matric potential and is used to conveniently express the matric forces (potentials) as positive values. By definition, at the water table both matric potential and matric suction are zero.

Unsaturated flow properties include unsaturated hydraulic conductivity and water retention characteristics (the relationship between water content and matric suction values). Analogous to saturated flow where the advective flux is the product of the saturated hydraulic conductivity and the gradient of the hydrostatic head, in unsaturated sediment the advective flow is the product of the unsaturated conductivity and the matric potential (or suction) gradient. The suction gradient defines the direction of flow (from areas of low to high suction). Neither unsaturated conductivity nor water retention characteristics for sediments in the SX Tank Farm have been measured directly. Only water content has been measured. Measurements were taken by neutron logging in dry wells. Unfortunately, these measurements have been qualitative at best and do not provide any direct hydraulic characterization of the vadose zone in the tank farm.

Split-spoon samples taken from borehole 41-09-39 are providing, for the first time, estimates of soil suction and water retention characteristics needed to describe the hydraulic properties of the vadose zone at the SX Tank Farm. 
Sources of recharge giving rise to the suction profiles at the SX Tank Farm are most likely from winter rains and snowmelt and, in certain circumstances, leaking water lines. A number of studies at the Hanford Site (Gee et al. 1992, 1994; Fayer et al. 1996; Ward et al. 1997) have shown that coarse gravel surfaces cause as much as $50 \%$ of the annual precipitation to recharge to the water table. Another source of localized recharge near tank farms has been hypothesized to be leaking water lines (Johnson and Chou 1998; Anderson and Soler 2000). Recharge has not been measured directly at the SX Tank Farm, so the net water flux below the tanks is uncertain. Until direct measurements are made, estimates ranging from $10 \%$ to $50 \%$ of the annual recharge, plus inputs from known water-line leaks, should be reasonable estimates for the range of advective fluxes at the SX Tank Farm.

The SX Tank Farm is located on glaciofluvial sediment (originally deposited by catastrophic flood events more than 10,000 years ago). The bases of the tanks rest on undisturbed sediment about 17 meters (56 feet) bgs and 48 meters ( 157.5 feet) above the water table. Section 2.3 describes the geology of this tank farm. Hydrologic properties (e.g., unsaturated conductivity and water retention characteristics) have been determined on sediment cores taken from these stratigraphic units in the 200 West Area (Connelly et al. 1992).

The suction profile of the underlying sediments is important to the hydrologic description of the tank farm. Under conditions of no drainage (or recharge), the suction profile is linear and equal to the height above the water table. If recharge to the water table is finite, the suction profile depends on the recharge rate and the layering sequence and hydraulic properties of the underlying sediments (Rockhold et al. 1997). As the recharge rates increase, the suction profile shifts toward the zero-suction (saturated) line (the vertical line at the right-hand side of Figure 4.5 at 0 centimeter of pressure head). Figure 4.5 shows profiles of soil matric potential (the same as suction when the negative sign is ignored) under conditions of steady-state recharge, assuming typical hydrologic characteristics for the 200 West Area sediment.

The calculated profiles, shown in Figure 4.5, can be used to estimate expected suction values under the SX Tank Farm and provide a basis for estimating the advective flux (recharge) beneath leaking tanks. With direct measures of suction and unsaturated hydraulic conductivity on the SX borehole extension samples, the recharge rates could be computed and the advective flux rates of mobile contaminants predicted. These types of direct measurements have not been attempted on the contaminated sediment, but some preliminary work has been funded by the Science and Technology Program using uncontaminated sediment cores from 299-W22-48. The results will be discussed in the field investigation report. $^{(a)}$

Thirty sleeve sediment samples from the borehole extension of borehole 41-09-39 were analyzed for matric suction. The data, tabulated in Table 4.9 and shown in Figure 4.6, indicate that, of the 30 samples, 18 are at matric suctions lower (wetter) than the elevation of the sample above the water table. As shown in Figure 4.6, when the suction for a sample is less than the sample's elevation above the water table, the sample is draining.

(a) Draft Field Investigation Report for Waste Management Area S-SX. RPP-7884, Draft, Volume 2, Appendix D, CH2M HILL Hanford Group, Inc., Richland, Washington. 
For one-dimensional, steady-state flow conditions, if the unsaturated hydraulic conductivity can be approximated by the values used by Rockhold et al. (1997) for 200 West Area sediment, the matric suction profile provides a means to estimate the recharge rate from the suction profile data. Figure 4.6 shows the suction profile plotted against the height of the samples above the water table. The range of suctions is relatively wide, from near zero to above 20 meters ( $65.6 \mathrm{feet})$. If samples that lie below the 1:1 line are excluded, the average suction value is $<5$ meters (16.4 feet). Based on the analysis of Rockhold et al. (1997), as shown in the right-hand portion of Figure 4.5, this suggests that the average recharge flux for the analyzed samples at the 41-09-39 borehole is somewhat $>5 \mathrm{~mm} / \mathrm{yr}$. However, given the wide range of suction values observed in the 30 samples and the lack of unsaturated hydraulic properties for the specific SX sediment, providing a rigorous and quantitative discussion is not possible at this time.

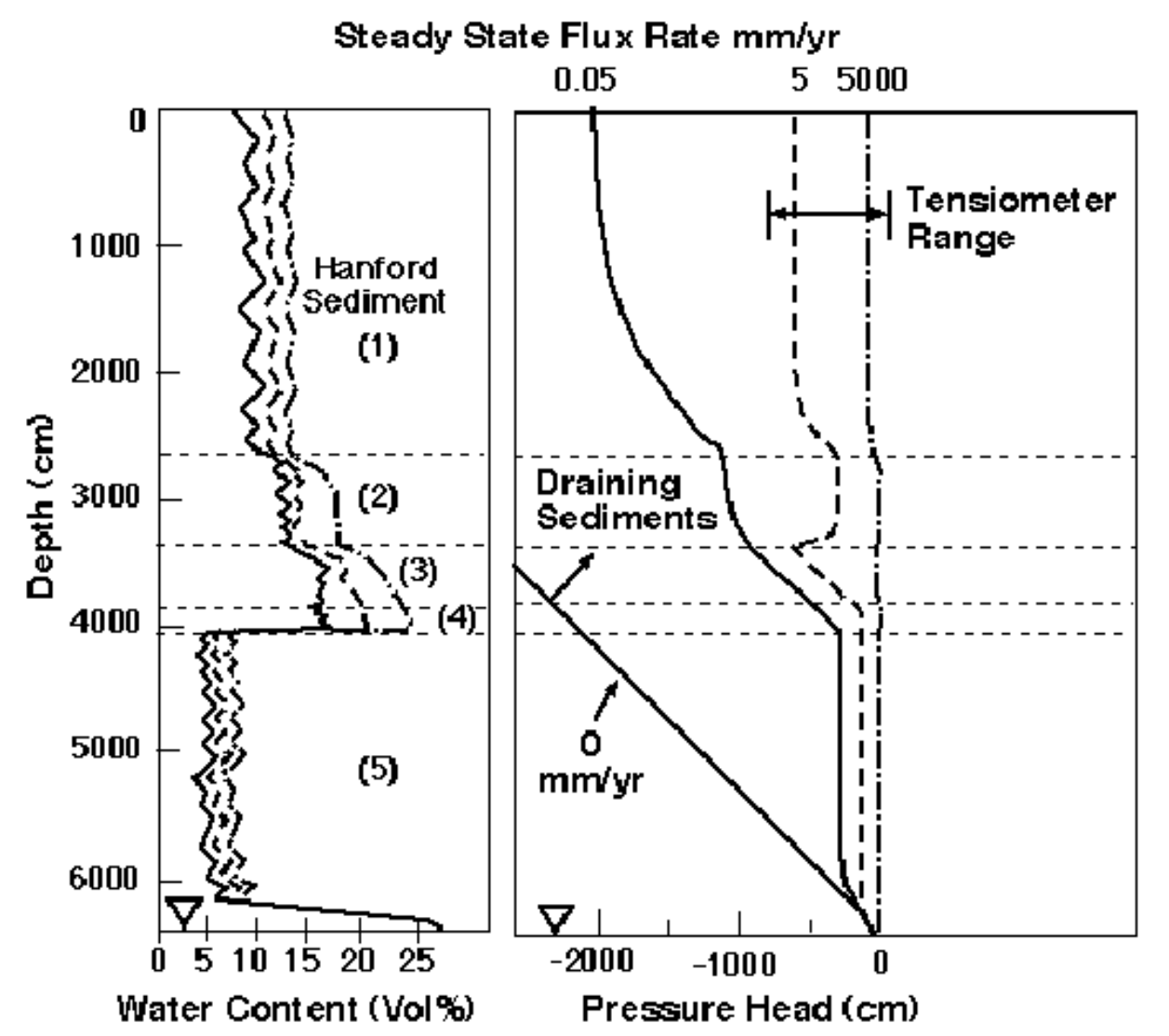

Figure 4.5. Steady-State Water Content and Suction Profiles for Representative 200 West Area Sediment above a 67-Meter (219.8 feet)-Deep Water Table. Soil stratigraphy corresponds to 1) Hanford formation coarse-grained sequence, 2) Hanford formation fine-grained sequence, 3) early Palouse soil, 4) Plio-Pleistocene soil, and 5) middle Ringold unit. No flow condition ( $0 \mathrm{~mm} / \mathrm{yr}$ ) shows suction to be equal to the height above the water table. Depth and suction units are in centimeters $(\mathrm{cm})$. 
Table 4.9. Water Content and Matric Suction Values for Samples from Borehole 41-09-39

\begin{tabular}{||c|c|c|c|c|c|c|c|}
\hline \hline Sample & $\begin{array}{c}\text { Depth } \\
(\mathrm{ft} \mathrm{bgs})^{(\mathrm{a})}\end{array}$ & $\begin{array}{c}\text { Water } \\
\text { Content } \\
(\mathrm{g} / \mathrm{g})\end{array}$ & $\begin{array}{c}\text { Suction } \\
(\mathrm{m})\end{array}$ & Sample & $\begin{array}{c}\text { Depth } \\
(\mathrm{ft} \mathrm{bgs})\end{array}$ & $\begin{array}{c}\text { Water } \\
\text { Content } \\
(\mathrm{g} / \mathrm{g})\end{array}$ & $\begin{array}{c}\text { Suction } \\
(\mathrm{m})\end{array}$ \\
\hline \hline 6F & 141.9 & 0.11 & 3.93 & $37 \mathrm{C}$ & 186.2 & 0.026 & 21.08 \\
\hline 6E & 142.4 & 0.096 & 5.38 & $38 \mathrm{C}$ & 187.8 & 0.029 & 8.42 \\
\hline 6D & 143.3 & 0.218 & 12.74 & $38 \mathrm{~B}$ & 188.3 & 0.019 & 4.65 \\
\hline 6C & 144.1 & 0.26 & 3.3 & $39 \mathrm{~A}$ & 189.5 & 0.018 & 50.16 \\
\hline 6B & 144.7 & 0.229 & 5.03 & $40 \mathrm{~A}$ & 190.7 & 0.051 & 12.83 \\
\hline $7 \mathrm{C}$ & 146.4 & 0.112 & 26.81 & $47 \mathrm{C}$ & 196.4 & 0.066 & 3.53 \\
\hline 8C & 148.3 & 0.074 & 14.99 & $50 \mathrm{~A}$ & 199.4 & 0.039 & 96.42 \\
\hline 8B & 149.9 & 0.097 & 9.6 & $56 \mathrm{C}$ & 204.3 & 0.077 & 5.34 \\
\hline 9A & 152.8 & 0.139 & 6.04 & $56 \mathrm{~B}$ & 205.0 & 0.066 & 27.75 \\
\hline 10C & 153.4 & 0.136 & 24.86 & $56 \mathrm{~A}$ & 205.6 & 0.054 & 30.77 \\
\hline 10B & 153.9 & 0.147 & 2 & $58 \mathrm{C}$ & 208.1 & 0.102 & 2.97 \\
\hline 10A & 154.5 & 0.196 & 4.8 & $58 \mathrm{~B}$ & 208.6 & 0.106 & 4.34 \\
\hline 16D & 162.3 & 0.133 & 0.25 & $59 \mathrm{C}$ & 209.8 & 0.125 & 0.33 \\
\hline 16C & 162.8 & 0.135 & 2.21 & $59 \mathrm{~A}$ & 210.9 & 0.25 & 0.02 \\
\hline 22B & 172.0 & 0.052 & 5.54 & $64 \mathrm{~A}$ & 213.3 & 0.132 & 0.03 \\
\hline
\end{tabular}

(a) Multiply by 0.3048 to convert to meters.

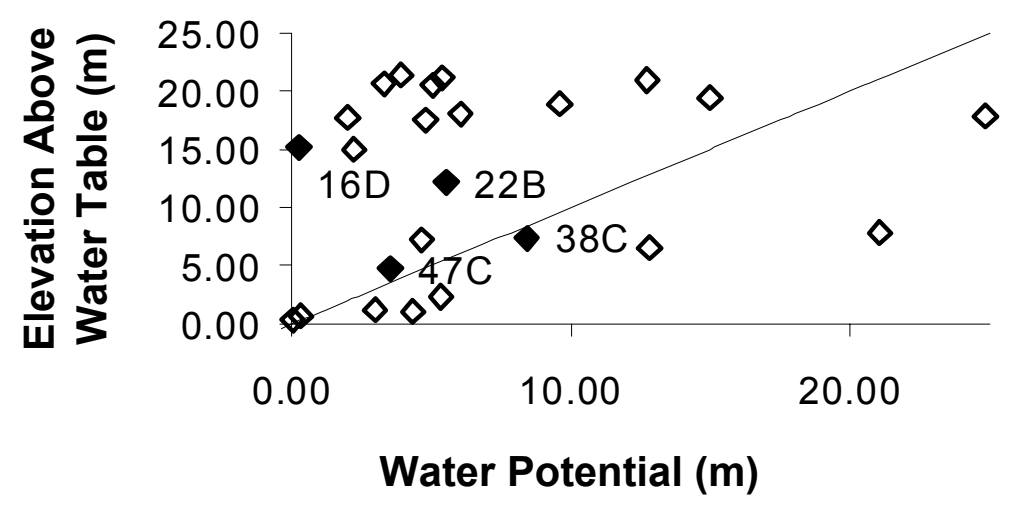

Figure 4.6. Matric Suction Profile for Samples Taken from Borehole 41-09-39 
One explanation for the observed variation in suction values for the borehole 41-09-39 samples is core disturbance. Changes in compaction or water content or both during sampling can affect the soil matric suction. Only two of the samples below the 1:1 line (i.e., samples with higher suction values than their elevation above the water table) have water contents above $0.11(11 \mathrm{wt} \%)$. At low water contents, slight variations in sample handling (i.e., drying during sampling, manipulating the split spoons in the field, and opening the sleeves in the laboratory to perform geologic interpretations and aliquoting for chemical analyses) can affect the measured suction values. This is particularly true for sediment samples with $<0.06(<6 \mathrm{wt} \%)$ water content. Over half the samples in question have water content values of $<6 \mathrm{wt} \%$.

To test the stability of the SX sediment water contents and independently assess the matric suction values of selected samples, four SX sediment samples were taken from refrigerated storage and reanalyzed for water content. Further aliquots of the same stored samples were tested for water content after being equilibrated at 100-centimeter (39.4-inch) matric suction using hanging water columns (Klute 1986).

Table 4.10 compares the water content of stored samples to the initial water contents measured soon after these cores were received from the field. The reanalysis of water contents indicates that the four samples tested lost little water in storage. The sample with the lowest water content (water content 0.029) did not lose any water and the one with the highest apparently lost about $1 \%$ (absolute) (from 0.133 to 0.121 ). These data suggest that refrigerated storage in leak-proof plastic containers does not significantly desiccate the sediment samples.

Figure 4.7 shows the water content of the four samples after equilibrating at 100 -centimeter (39.4 inch) matric suction on a hanging water column for 1 week. The data from the hanging water columns suggest that the observed water contents (assumed field water contents), with one exception (sample 16D), were lower than the water contents at 100-centimeter (39.4-inch) matric suction. From the Rockhold et al. (1997) analysis and observations from lysimeters at the Hanford Site (Gee 1987), the borehole 41-09-39 sediment sample suction values appear to be higher than expected (the sediments are dryer than expected). Suctions ranging from 0.3 to 0.6 meter ( 1 to 2 feet) were observed in Gee's 7.6meter (24.9 feet) deep lysimeter (Gee 1987) that has been known to drain (recharge) at rates of $50 \mathrm{~mm} / \mathrm{yr}$ to $100 \mathrm{~mm} / \mathrm{yr}$.

Table 4.10. Water Content of Stored Samples Compared to the Field-Measured Values

\begin{tabular}{|c|c|c|c||}
\hline Sample & $\begin{array}{c}\text { Depth } \\
(\mathrm{ft} \mathrm{bgs})^{(\mathrm{a})}\end{array}$ & $\begin{array}{c}\text { Initial Water Content } \\
(\mathrm{g} / \mathrm{g})\end{array}$ & $\begin{array}{c}\text { Verified Water Content } \\
(\mathrm{g} / \mathrm{g})\end{array}$ \\
\hline \hline 16D & 162.3 & 0.133 & 0.121 \\
\hline 22B & 172.0 & 0.052 & 0.059 \\
\hline 38C & 187.8 & 0.029 & 0.029 \\
\hline 47C & 196.4 & 0.066 & 0.057 \\
\hline (a) Multiply by 0.3048 to convert to meters. \\
\hline
\end{tabular}




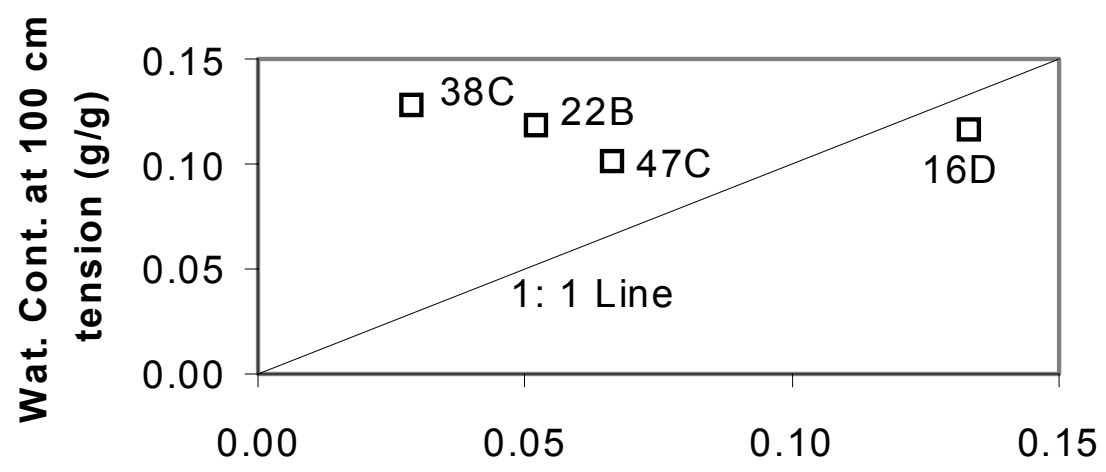

Field Water Content $(g / g)$

Figure 4.7. Water Content of Selected Sleeve Sediment Samples from Borehole 41-09-39 after Equilibrating at 100-Centimeter (39.4-Inch) Matric Suction Compared to Water Contents as Stored in Containers

The matric suction values from the borehole 41-09-39 cores were expected to be in a similar range to Gee's (1987) lysimeter sediment. Three possibilities present themselves for the core samples having higher matric suction. The first is that much less drainage (recharge) is occurring at this borehole location than was observed during the lysimeter test where $50 \mathrm{~mm} / \mathrm{yr}$ to $100 \mathrm{~mm} / \mathrm{yr}$ occurs. The second is that the 41-09-39 samples were disturbed enough during coring that the water content and, possibly, density were altered enough to increase the matric suction values. The third is that large thermal gradients inferred from past casing air temperature measurements (Conaway et al. 1997) and the observed high temperature on the casing removed (see Myers et al. 1998) have driven moisture out of the sediment.

Analyses of steady-state profiles from 200 West Area sites and elsewhere on the Hanford Site indicate that, at 100-centimeter (39.4 inch) suction, the drainage or recharge rate is typically $<70 \mathrm{~mm} / \mathrm{yr}$. The data shown in Figure 4.7 suggest that the recharge rates in the vicinity of borehole 41-09-39 are likely to be $<70 \mathrm{~mm} / \mathrm{yr}$. Additional in situ field sampling of matric suction using tensiometers could provide needed confirmation of the suction gradients and help determine the recharge flux at this location and elsewhere in the vadose zone of the Hanford Site tank farms. Until direct measures of the matric suction are obtained, this issue will not be resolved.

Tensiometers could be installed into the vadose zone at depth to measure the matric suction directly. Such efforts would help resolve uncertainty in the present measurements. In the meantime, based on the data obtained, recharge appears to be occurring at the borehole 41-09-39 location, but at considerably lower rates than expected. 


\subsection{Composite Sample Results and Discussion}

In this section, we present the results of the detailed characterization on the seven borehole extension composite samples and integrate the information with detailed characterization data obtained on 15 sidewall core composites. Recall that the seven composite samples from the borehole extension were chosen from all the individual sleeves as most likely to represent the formation sediments. As discussed in Myers et al. (1998), choosing sediments not altered by the drilling and sampling process required careful assimilation of all the drillers' and field samplers' observations, geologists' interpretations, and laboratory measurements performed during the scoping investigations (just discussed in Section 4.0).

Essentially, we took two individual B and A sleeve samples from selected split-spoon sampling events out of refrigerated storage and thoroughly mixed them to create a larger sample that could be parsed into numerous subsamples for various analyses. We also included a composite of sleeves 2D and $2 \mathrm{C}$ because this was the first material obtained after removing the original borehole's closed-end drive shoe. We consider this composite to represent sediment with substantial artifacts from drilling and sampling, but the sample was characterized in detail anyway because it was used in some separately funded basic science work that will be merged into the SX Tank Farm field investigation report ${ }^{(\mathrm{a})}$.

\subsection{Moisture Content and 1:1 Sediment-to-Water Extract Electrical Conductivity and pH for Borehole Extension Composites}

The composite sample data from the borehole extension are shown in Table 5.1. In general, the moisture content of the composites is slightly less than the individual sleeves. We attribute the water loss to exposure of the moist sediment to dry air during mixing. More time was taken to mix the sediments for the composites than was taken during subsampling of the individual sleeve sediments during the

Table 5.1. pH, Electrical Conductivity, and Moisture Content of the Composite Samples

\begin{tabular}{|c|c|c|c|c|}
\hline $\begin{array}{c}\text { Composites } \\
\text { Depth (ft bgs) }\end{array}$ & $\begin{array}{l}\text { 1:1 Extract } \\
\text { ID }\end{array}$ & $\begin{array}{c}\text { Moisture } \\
\text { Content (\%) }\end{array}$ & $\mathrm{pH}$ & $\begin{array}{c}\text { Electrical Conductivity } \\
(\mu \mathrm{S} / \mathrm{cm})\end{array}$ \\
\hline "133.2 & $2 \mathrm{C} / \mathrm{D}$ & 19.0 & 8.79 & 294 \\
\hline 134.2 & $2 \mathrm{~A} / \mathrm{B}$ & 11.2 & 8.57 & 763 \\
\hline 135.2 & $3 \mathrm{~A} / \mathrm{B}$ & 9.47 & 8.58 & 835 \\
\hline 153.9 & $10 \mathrm{~A} / \mathrm{B}$ & 15.0 & 8.68 & 296 \\
\hline 156.8 & $12 \mathrm{~A} / \mathrm{B}$ & 5.12 & 8.88 & 305 \\
\hline 165.3 & $17 \mathrm{~A} / \mathrm{B}$ & 3.81 & 8.45 & 87 \\
\hline 188.3 & $38 \mathrm{~A} / \mathrm{B}$ & 0.81 & 8.87 & 82 \\
\hline 196.9 & $47 \mathrm{~A} / \mathrm{B}$ & 2.15 & 8.78 & 99 \\
\hline 208.6 & $58 \mathrm{~A} / \mathrm{B}$ & 4.42 & 8.73 & 56 \\
\hline
\end{tabular}

(a) Draft Field Investigation Report for Waste Management Area S-SX. RPP-7884, Draft, Volume 2, Appendix D, CH2M HILL Hanford Group, Inc., Richland, Washington. 
scoping study. The composite samples may have lost about $1 \%$ to $3 \%$ water by weight during the mixing. Four individual sleeves that were resampled quickly during the matric suction activities discussed in Section 4.4 did not show much moisture loss, so we concluded that refrigerated storage was adequate for at least 3 months.

Comparing the $\mathrm{pH}$ for these composites with the original values on the individual sleeves (see Tables 4.1 and 4.2), we find that the composite $\mathrm{pH}$ values seem to be higher than the values for the individual sleeves by about one-half to a full unit. We have no explanation other than the possibility of carbon dioxide degassing or measurement calibration differences between the two data sets that were performed approximately 6 months apart.

The electrical conductivity (EC) data for the composites show no differing trends from the data for the individual sleeves. As discussed for the individual sleeves, the $\mathrm{pH}$ and EC results for the borehole extension composites do not indicate that significant amounts of tank liquor have reacted with the vadose zone sediments from the depths sampled. These borehole extension composite results are not easily distinguishable from natural Hanford Site sediments, however at the shallower depths sampled during the sidewall coring there are significant amounts of highly saline tank fluid.

\subsection{Direct Analysis of Pore Fluid and 1:1 Sediment-to-Water Extract Pore Water Chemistry for the Sidewall Composite Samples}

We extracted pore fluid from five of the sidewall core composites using ultracentrifuge techniques. Several hundred grams of each sediment sample were placed in special cells that had drains in the bottom that allowed liquid to be collected in cups attached to the bottom of the cell. The sediment-filled cells were spun at several thousand rpm overnight to extract native pore fluids out. The five samples, including two replicates for one, are shown in Table 5.2.

Table 5.2. Electrical Conductivity and $\mathrm{pH}$ of Porewater from Sidewall Cores and Water Extracts of Sidewall Cores and Composite Samples from Borehole Extension

\begin{tabular}{|c|c|c|c|c|}
\hline $\begin{array}{c}\text { Depth } \\
(\mathrm{ft} \text { bgs) }\end{array}$ & $\begin{array}{c}\text { Sample } \\
\text { ID } \\
\end{array}$ & $\begin{array}{c}\text { Sample } \\
\text { Type } \\
\end{array}$ & $\mathrm{pH}$ & $\begin{array}{c}\begin{array}{l}E C^{(b)} \\
(\mu \mathrm{S} / \mathrm{cm})\end{array} \\
\end{array}$ \\
\hline 56.5 & $13 \mathrm{~A} / \mathrm{B} / \mathrm{C}$ & $\begin{array}{c}1: 1 \\
u^{\left({ }^{(c)}\right.}\end{array}$ & $\begin{array}{l}8.31 \\
8.25\end{array}$ & $\begin{array}{l}1,790 \\
2,870\end{array}$ \\
\hline 79.5 & $8 \mathrm{~A} / \mathrm{B} / \mathrm{C}$ & $\begin{array}{c}1: 1 \\
\mathrm{ufa}^{(\mathrm{c})}\end{array}$ & $\begin{array}{l}9.55 \\
9.10\end{array}$ & $\begin{array}{l}16,090 \\
10,900\end{array}$ \\
\hline 90 & $6 \mathrm{~A} / \mathrm{B}$ & $\begin{array}{c}1: 1 \\
\mathrm{ufa}^{(\mathrm{c})}\end{array}$ & $\begin{array}{l}8.33 \\
7.33\end{array}$ & $\begin{array}{l}408,017 \\
398,630\end{array}$ \\
\hline 108.5 & $4 \mathrm{~A} / \mathrm{B} / \mathrm{C}$ & $\begin{array}{c}1: 1 \\
\mathrm{ufa}^{\text {(c) }}\end{array}$ & $\begin{array}{l}8.07 \\
7.04\end{array}$ & $\begin{array}{l}470,722 \\
469,500\end{array}$ \\
\hline 112 & $2 \mathrm{~B} / \mathrm{C}$ & $\begin{array}{c}1: 1 \\
\mathrm{ufa}^{(\mathrm{c})}\end{array}$ & $\begin{array}{l}8.12 \\
6.88\end{array}$ & $\begin{array}{l}524,413 \\
528,380\end{array}$ \\
\hline \multicolumn{5}{|c|}{$\begin{array}{l}\text { (a) Multiply by } 0.3048 \text { to convert to meters. } \\
\text { (b) } 1: 1 \text { EC measurements are dilution-corrected. } \\
\text { (c) ufa represents the actual porewater obtained by ultracentrifugation. }\end{array}$} \\
\hline
\end{tabular}




\subsubsection{Electrical Conductivity and $\mathrm{pH}$}

The five unsaturated flow apparatus- (UFA)-extracted porewaters can be compared to the 1:1 water extracts (see Table 5.2). The $\mathrm{pH}$ values for the shallower two samples agree quite well, but the UFAextracted porewater $\mathrm{pH}$ values for the three deeper samples are at least one unit lower than the 1:1 water extracts. We do not have an explanation for this discrepancy because we do not believe that there needs to be any dilution correction for the water extracts $\mathrm{pH}$ and, thus we thought a direct comparison would be a correct approach. Note that the $\mathrm{pH}$ of both the 24.2 meters ( $79.5 \mathrm{feet}$ ) below ground surface (bgs) waters is elevated from natural conditions. That is, the observed $\mathrm{pH}$ ranges from 9.1 to 9.55 , in contrast to the range of $\mathrm{pH}$ values for natural vadose zone fluids (7.5 to 8.3). Therefore, in agreement with the data shown in Table 4.2, we suggest that the sediments between 20 and 24.2 meters (65.5 and 79.5 feet) bgs exhibit some $\mathrm{pH}$ alteration.

Conversely, the EC of the deeper three samples shows excellent agreement between the actual porewater and the dilution-corrected water extracts. All three samples show very high conductivity caused by the highly saline tank fluids. The UFA-extracted porewaters for the two shallowest samples have significantly lower EC than the dilution-corrected water extracts. The sample at 17.2 meters (56.5 feet) bgs has no tank fluid signature. The porewater at 24.2 meters ( 79.5 feet) bgs has a lower EC than the dilutioncorrected water extract and both show evidence of some tank fluid. Based on the EC measurements shown in Table 5.2, there is tank fluid in the sediment between 24.2 and 34.1 meters (79.5 and 112 feet) bgs. The lower EC at 24.2 meters ( 79.5 feet) bgs may reflect some drainage to deeper depths or simple dilution of the tank fluid with subsequent natural recharge waters.

\subsubsection{Chemical Composition}

The chemical composition of the 1:1 sediment to deionized water extracts for the composite sediments are shown in Tables 5.3 through 5.6 for the major anions, major cations, and trace metals. We also include the data for the UFA-squeezed porewaters highlighted with its comparable water extract. Additional data on 1:1 water extracts of other individual borehole sleeves and the sidewall cores were discussed in Section 4.2. The sidewall core information is discussed again to allow more detailed interpretations.

Tables 5.3 through 5.6 show the actual results and Tables 5.7 through 5.9 show calculations of what the porewater in the sediment might be if all the dissolved salts were originally in solution and the added water is strictly a diluent. We expect that some solids were leached from the unsaturated sediment during the extraction. Thus, the calculated (dilution-corrected) values shown in Tables 5.7 to 5.9 are likely, on average, to be biased high when considered as representative of the porewater in the sediment, until the contribution from tank liquor overwhelms the amount potentially dissolved from the solid phase. That is, when the pore fluid is totally dominated by the high ionic strength tank liquors, the agreement between the UFA squeezings and dilution-corrected 1:1 water extracts will be excellent.

The water extract and porewater anion data in Tables 5.3 and 5.7 show that there is elevated chloride, fluoride, nitrate, nitrite, sulfate, and inorganic carbon (carbonate) that is leachable in the sediment between 24.2 meters ( 79.5 feet) bgs and, in most cases, 39 meters (128 feet) bgs. There is some 
Table 5.3. Anion Composition of 1:1 Water Extracts and Porewaters from Sidewall Cores and Borehole Extension Composites

\begin{tabular}{|c|c|c|c|c|c|c|c|c|}
\hline \multirow[b]{2}{*}{$\begin{array}{c}\text { Composite } \\
\text { Depth (ft bgs) } \\
\end{array}$} & \multirow[b]{2}{*}{$\begin{array}{c}\text { Sample } \\
\text { ID }\end{array}$} & \multicolumn{6}{|c|}{ IC Analysis } & \multirow{2}{*}{$\begin{array}{c}\text { Carbon } \\
\text { Inorganic C } \\
(\mathrm{ppm})\end{array}$} \\
\hline & & $\begin{array}{c}\mathrm{Cl} \\
(\mathrm{ppm}) \\
\end{array}$ & $\begin{array}{c}\mathrm{F} \\
(\mathrm{ppm}) \\
\end{array}$ & $\begin{array}{c}\mathrm{NO}_{3} \\
(\mathrm{ppm})\end{array}$ & $\begin{array}{c}\mathrm{NO}_{2} \\
(\mathrm{ppm}) \\
\end{array}$ & $\begin{array}{c}\mathrm{PO}_{4} \\
(\mathrm{ppm}) \\
\end{array}$ & $\begin{array}{c}\mathrm{SO}_{4} \\
(\mathrm{ppm}) \\
\end{array}$ & \\
\hline \multicolumn{9}{|c|}{ Sidewall } \\
\hline 25.5 & $15 \mathrm{~A} / \mathrm{B} / \mathrm{C}$ & $<0.3$ & NA & 13.0 & $<0.3$ & NA & 1.70 & 19.0 \\
\hline 44.5 & $14 \mathrm{~A} / \mathrm{B} / \mathrm{C}$ & 0.90 & NA & 13.0 & $<0.3$ & NA & 16.4 & 20.8 \\
\hline \multirow[t]{2}{*}{56.5} & $13 \mathrm{~A} / \mathrm{B} / \mathrm{C}$ & 0.80 & NA & 13.0 & $<0.3$ & NA & 2.8 & 29.9 \\
\hline & 13 UFA & 57.9 & $<33$ & 1264 & $<33.3$ & $<167$ & 444 & 19.2 \\
\hline 61.5 & $12 \mathrm{~A} / \mathrm{B} / \mathrm{C}$ & 0.80 & NA & 13.0 & $<0.3$ & NA & 5.10 & 29.6 \\
\hline 65.5 & $11 \mathrm{~A} / \mathrm{B}$ & 0.50 & NA & 29.0 & $<0.3$ & NA & 11.5 & 42.3 \\
\hline 66 & $11 \mathrm{C}$ & 0.30 & NA & 18.0 & $<0.3$ & NA & 6.5 & 62.9 \\
\hline 69.5 & $10 \mathrm{~A} / \mathrm{B} / \mathrm{C}$ & 0.40 & NA & 33.0 & $<0.3$ & $\mathrm{NA}$ & 8.7 & 53.5 \\
\hline 74.5 & 09A/B/C & 0.50 & NA & 44.0 & $<0.3$ & $\mathrm{NA}$ & 18.1 & 47.5 \\
\hline \multirow[t]{2}{*}{79.5} & $08 \mathrm{~A} / \mathrm{B} / \mathrm{C}$ & 3.30 & NA & 371 & 1.40 & NA & 51.7 & 84.1 \\
\hline & 8 UFA & 122 & 532 & 4,065 & $<62.5$ & $<313$ & 1,161 & 230 \\
\hline 82.5 & $07 \mathrm{~A} / \mathrm{B} / \mathrm{C}$ & 30.0 & NA & 2,836 & 82.0 & NA & 336 & 22.3 \\
\hline \multirow[t]{2}{*}{90} & 06A/B & 307 & NA & 28,044 & 139 & $\mathrm{NA}$ & 271 & 16.0 \\
\hline & 6 UFA & 1,336 & 1,203 & 258,114 & $<125$ & $<625$ & 3,365 & 37.8 \\
\hline 95.5 & $03 \mathrm{~A} / \mathrm{B} / \mathrm{C}$ & 321 & NA & 32,767 & 28.6 & NA & 260 & 7.10 \\
\hline 102.5 & $05 \mathrm{~A} / \mathrm{B} / \mathrm{C}$ & 198 & NA & 31,666 & 133 & NA & 198 & 11.0 \\
\hline \multirow[t]{2}{*}{108.5} & $04 \mathrm{~A} / \mathrm{B} / \mathrm{C}$ & 359 & NA & 42,448 & 130 & NA & 386 & 11.9 \\
\hline & 4 UFA & 3,984 & 1,236 & 323,940 & $<125$ & $<625$ & 4,520 & 30.3 \\
\hline \multirow[t]{2}{*}{112} & $02 \mathrm{~B} / \mathrm{C}$ & 344 & NA & 32,764 & 106 & NA & 373 & 8.30 \\
\hline & 2 UFA & 4,489 & $<125$ & 375,601 & $<125$ & $<625$ & 3,883 & 38.6 \\
\hline 127.4 & $01 \mathrm{~A} / \mathrm{B} / \mathrm{C}$ & 77.5 & NA & 12,808 & 28.6 & NA & 73.3 & 6.10 \\
\hline
\end{tabular}


Table 5.3. (contd)

\begin{tabular}{|c|c|c|c|c|c|c|c|c|}
\hline \multirow[b]{2}{*}{$\begin{array}{c}\text { Composite } \\
\text { Depth (ft bgs) }\end{array}$} & \multirow[b]{2}{*}{$\begin{array}{l}\text { Sample } \\
\text { ID }\end{array}$} & \multicolumn{6}{|c|}{ IC Analysis } & \multirow{2}{*}{$\begin{array}{c}\text { Carbon } \\
\text { Inorganic C } \\
(\mathrm{ppm})\end{array}$} \\
\hline & & $\begin{array}{c}\mathrm{Cl} \\
(\mathrm{ppm})\end{array}$ & $\begin{array}{c}\mathrm{F} \\
(\mathrm{ppm}) \\
\end{array}$ & $\begin{array}{c}\mathrm{NO}_{3} \\
(\mathrm{ppm})\end{array}$ & $\begin{array}{c}\mathrm{NO}_{2} \\
(\mathrm{ppm})\end{array}$ & $\begin{array}{c}\mathrm{PO}_{4} \\
(\mathrm{ppm})\end{array}$ & $\begin{array}{c}\mathrm{SO}_{4} \\
(\mathrm{ppm})\end{array}$ & \\
\hline \multicolumn{9}{|c|}{ Extension } \\
\hline 133.2 & $2 \mathrm{C} / \mathrm{D}$ & 19.1 & 1.39 & 1.10 & $<0.1$ & $<0.2$ & 5.00 & 30.2 \\
\hline 134.2 & $2 \mathrm{~A} / \mathrm{B}$ & 10.5 & 0.51 & 297 & 0.24 & $<0.2$ & 7.50 & 12.1 \\
\hline 135.2 & $3 \mathrm{~A} / \mathrm{B}$ & 33.3 & $<33$ & 306 & 0.13 & $<0.2$ & 14.3 & 17.2 \\
\hline 153.9 & $10 \mathrm{~A} / \mathrm{B}$ & 4.80 & 0.61 & 52.8 & 0.27 & 0.45 & 21.1 & 15.1 \\
\hline 156.8 & $12 \mathrm{~A} / \mathrm{B}$ & 4.20 & 0.24 & 6.30 & $<0.1$ & 0.37 & 40.7 & 18.6 \\
\hline 165.3 & $17 \mathrm{~A} / \mathrm{B}$ & 1.10 & 0.74 & 7.70 & $<0.1$ & 0.28 & 6.50 & 2.40 \\
\hline 188.3 & $38 \mathrm{~A} / \mathrm{B}$ & 1.30 & 0.43 & 2.60 & $<0.1$ & 0.27 & 1.70 & 3.30 \\
\hline 196.9 & $47 \mathrm{~A} / \mathrm{B}$ & 2.30 & 0.53 & 17.2 & $<0.1$ & $<0.2$ & 3.50 & 1.90 \\
\hline 208.6 & $58 \mathrm{~A} / \mathrm{B}$ & 1.00 & 0.37 & 0.30 & $<0.1$ & 0.30 & 1.40 & 1.40 \\
\hline
\end{tabular}


Table 5.4. Major Cation Composition of $1: 1$ Water Extracts and Porewaters from Sidewall Cores and Borehole Extension Composites

\begin{tabular}{|c|c|c|c|c|c|c|c|c|c|c|c|}
\hline \multirow{2}{*}{$\begin{array}{c}\text { Composite } \\
\text { Depth } \\
(\mathrm{ft} \text { bgs })^{(\mathrm{a})}\end{array}$} & \multirow[b]{2}{*}{$\begin{array}{l}\text { 1:1 Extract } \\
\text { ID }\end{array}$} & \multicolumn{4}{|c|}{ ICP } & \multirow{2}{*}{$\frac{\text { ICP-MS }}{\begin{array}{c}\mathrm{Sr} \\
(\mathrm{ppb})\end{array}}$} & \multicolumn{3}{|c|}{ ICP } & \multirow{2}{*}{ 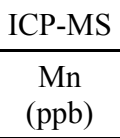 } & \multirow{2}{*}{ 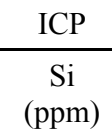 } \\
\hline & & $\begin{array}{c}\mathrm{Ca} \\
(\mathrm{ppm})\end{array}$ & $\begin{array}{c}\mathrm{K} \\
(\mathrm{ppm})\end{array}$ & $\begin{array}{c}\mathrm{Mg} \\
(\mathrm{ppm})\end{array}$ & $\begin{array}{c}\mathrm{Na} \\
(\mathrm{ppm})\end{array}$ & & $\begin{array}{c}\mathrm{Al} \\
(\mathrm{ppm})\end{array}$ & $\begin{array}{c}\mathrm{B} \\
(\mathrm{ppm})\end{array}$ & $\begin{array}{c}\mathrm{Fe} \\
(\mathrm{ppm})\end{array}$ & & \\
\hline \multicolumn{12}{|c|}{ Sidewall } \\
\hline 25.5 & $15 \mathrm{~A} / \mathrm{B} / \mathrm{C}$ & 9.30 & 6.40 & 1.10 & 29.5 & (53) & 0.84 & 5.2 & 0.51 & $(4.0)$ & 21.5 \\
\hline 44.5 & $14 \mathrm{~A} / \mathrm{B} / \mathrm{C}$ & 6.00 & 6.90 & 0.70 & 41.8 & (49) & 0.59 & 15.9 & 0.14 & $(1.0)$ & 14.8 \\
\hline \multirow[t]{2}{*}{56.5} & $13 \mathrm{~A} / \mathrm{B} / \mathrm{C}$ & 22.4 & 7.40 & 2.00 & 43.2 & (36) & 0.51 & 5.30 & $<0.25$ & $<50$ & 9.30 \\
\hline & 13 UFA & 50.3 & 15.8 & 3.90 & 561 & 545 & 0.90 & 27.0 & $(0.07)$ & (73.0) & 19.8 \\
\hline 61.5 & $12 \mathrm{~A} / \mathrm{B} / \mathrm{C}$ & 3.60 & 2.60 & 0.30 & 80.7 & (42) & 0.56 & 31.0 & $(0.05)$ & (6.0) & 7.50 \\
\hline 65.5 & $11 \mathrm{~A} / \mathrm{B}$ & 1.10 & $(1.70)$ & 0.40 & 121 & (35) & 1.48 & 9.00 & 2.03 & $(41.0)$ & 14.2 \\
\hline 66 & $11 \mathrm{C}$ & 0.90 & 2.60 & 1.90 & 212 & (51) & 5.72 & 10.5 & 8.97 & (125) & 37.0 \\
\hline 69.5 & $10 \mathrm{~A} / \mathrm{B} / \mathrm{C}$ & 0.80 & 3.60 & 1.00 & 176 & (35) & 3.08 & 4.50 & 4.27 & $(78.0)$ & 24.0 \\
\hline 74.5 & $09 \mathrm{~A} / \mathrm{B} / \mathrm{C}$ & 0.50 & $(2.40)$ & $(0.1)$ & 168.3 & $(47.0)$ & 0.65 & 10.3 & $(0.11)$ & $<50$ & 12.5 \\
\hline \multirow[t]{2}{*}{79.5} & $08 \mathrm{~A} / \mathrm{B} / \mathrm{C}$ & 0.70 & 4.20 & $(0.10)$ & 423 & $(57.0)$ & 0.75 & 35.6 & $(0.02)$ & $<50$ & 5.30 \\
\hline & 8 UFA & 10.1 & 41.9 & 1.50 & 2438 & 468 & 1.70 & 111 & $(0.06)$ & (625) & 18.1 \\
\hline 82.5 & $07 \mathrm{~A} / \mathrm{B} / \mathrm{C}$ & 22.1 & 16.0 & 2.20 & 2041 & 460 & $(0.48)$ & 16.9 & $(0.02)$ & $<50$ & 7.00 \\
\hline \multirow[t]{2}{*}{90} & $06 \mathrm{~A} / \mathrm{B}$ & 142 & 209 & 7.20 & 11270 & 2,680 & $(0.37)$ & 26.6 & $(0.04)$ & $<50$ & 3.00 \\
\hline & 6 UFA & 1190 & 2156 & 63.4 & 91415 & 44,740 & 1.10 & 138 & $(0.10)$ & $<1250$ & 20.3 \\
\hline 95.5 & $03 \mathrm{~A} / \mathrm{B} / \mathrm{C}$ & 975 & 129 & 19.8 & 10209 & 15,080 & $(0.22)$ & 12.4 & $(0.06)$ & $<50$ & 3.60 \\
\hline 102.5 & $05 \mathrm{~A} / \mathrm{B} / \mathrm{C}$ & 563 & 128 & 24.1 & 10753 & 10,130 & $(0.20)$ & 14.0 & $(0.08)$ & $<50$ & 3.70 \\
\hline \multirow[t]{2}{*}{108.5} & $04 \mathrm{~A} / \mathrm{B} / \mathrm{C}$ & 449 & 117 & 17.3 & 15256 & 8,760 & $(0.26)$ & 8.30 & $(0.08)$ & $<50$ & 4.80 \\
\hline & 4 UFA & $3,828 \pm 22$ & $942 \pm 21$ & $123 \pm 1$ & $109,258 \pm 101$ & $95,644 \pm 3,854$ & $1 \pm 0.2$ & $80.8 \pm 1.3$ & $0.096 \pm 0.01$ & $<1250$ & $23.6 \pm 3$ \\
\hline \multirow[t]{2}{*}{112} & $02 \mathrm{~B} / \mathrm{C}$ & 453 & 81.2 & 23.7 & 11342 & 8560 & $<0.50$ & 30.2 & $(0.06)$ & $<50$ & 4.90 \\
\hline & 2 UFA & 6388 & 1103 & 255 & 124505 & 141099 & 1.10 & 380 & $(0.08)$ & $<1250$ & 18.3 \\
\hline 127.4 & $01 \mathrm{~A} / \mathrm{B} / \mathrm{C}$ & 1706 & 76.7 & 186 & 1921 & 13570 & $<0.50$ & 15.7 & $(0.04)$ & (103) & 5.10 \\
\hline
\end{tabular}


Table 5.4. (contd)

\begin{tabular}{|c|c|c|c|c|c|c|c|c|c|c|c|}
\hline \multirow{2}{*}{$\begin{array}{c}\text { Composite } \\
\text { Depth } \\
(\mathrm{ft} b g s)^{(\mathrm{a})}\end{array}$} & \multirow[b]{2}{*}{$\begin{array}{l}\text { 1:1 Extract } \\
\text { ID }\end{array}$} & \multicolumn{4}{|c|}{$\mathrm{ICP}$} & \multirow{2}{*}{$\begin{array}{c}\text { ICP-MS } \\
\begin{array}{c}\mathrm{Sr} \\
(\mathrm{ppb})\end{array}\end{array}$} & \multicolumn{3}{|c|}{$\mathrm{ICP}$} & \multirow{2}{*}{$\begin{array}{c}\text { ICP-MS } \\
\mathrm{Mn} \\
(\mathrm{ppb})\end{array}$} & \multirow{2}{*}{$\begin{array}{c}\mathrm{ICP} \\
\mathrm{Si} \\
(\mathrm{ppm})\end{array}$} \\
\hline & & $\begin{array}{c}\mathrm{Ca} \\
(\mathrm{ppm})\end{array}$ & $\begin{array}{c}\mathrm{K} \\
(\mathrm{ppm}) \\
\end{array}$ & $\begin{array}{c}\mathrm{Mg} \\
(\mathrm{ppm})\end{array}$ & $\begin{array}{c}\mathrm{Na} \\
(\mathrm{ppm})\end{array}$ & & $\begin{array}{c}\mathrm{Al} \\
(\mathrm{ppm})\end{array}$ & $\begin{array}{c}\mathrm{B} \\
(\mathrm{ppm}) \\
\end{array}$ & $\begin{array}{c}\mathrm{Fe} \\
(\mathrm{ppm})\end{array}$ & & \\
\hline \multicolumn{12}{|c|}{ Extension } \\
\hline 133.2 & $2 \mathrm{C} / \mathrm{D}$ & 7.17 & 13.9 & 1.38 & 38.4 & 40.0 & 1.05 & 5.19 & -0.67 & 40.9 & 5.81 \\
\hline 134.2 & $2 \mathrm{~A} / \mathrm{B}$ & 54.5 & 10.2 & 14.1 & 34.1 & 270 & $<0.05$ & 3.38 & $<0.05$ & 5.48 & 11.2 \\
\hline 135.2 & $3 \mathrm{~A} / \mathrm{B}$ & 56.5 & 37.4 & 16.0 & 46.7 & 292 & $<0.05$ & 5.34 & $<0.05$ & 37.2 & 10.2 \\
\hline 153.9 & $10 \mathrm{~A} / \mathrm{B}$ & 16.5 & $(4.70)$ & 7.17 & 15.2 & 87.2 & 0.27 & 2.5 & 0.09 & 2.90 & 15.0 \\
\hline 156.8 & $12 \mathrm{~A} / \mathrm{B}$ & 8.99 & $(4.60)$ & 3.27 & 32.4 & 47.4 & 0.18 & 1.65 & 0.28 & 6.39 & 10.4 \\
\hline 165.3 & $17 \mathrm{~A} / \mathrm{B}$ & 2.46 & 1.85 & 0.93 & 7.22 & 13.0 & 0.92 & 1.38 & 1.06 & 50.2 & 13.0 \\
\hline 188.3 & $38 \mathrm{~A} / \mathrm{B}$ & 1.11 & $(1.9)$ & 0.37 & 7.35 & 4.97 & 0.30 & 1.33 & 0.20 & 4.60 & 8.05 \\
\hline 196.9 & $47 \mathrm{~A} / \mathrm{B}$ & 2.40 & 1.74 & 0.79 & 7.76 & 11.5 & 0.13 & 1.68 & 0.12 & 5.60 & 8.86 \\
\hline 208.6 & $58 \mathrm{~A} / \mathrm{B}$ & 0.54 & $<1.00$ & 0.21 & 4.48 & 1.80 & 0.14 & 1.38 & 0.21 & $<0.5$ & 8.53 \\
\hline $\begin{array}{l}\text { Numbers in p } \\
\text { Two UFA san } \\
\text { (a) Multiply }\end{array}$ & $\begin{array}{l}\text { es are belc } \\
\text { re squeez } \\
8 \text { to conve }\end{array}$ & $\begin{array}{l}\text { uantific } \\
\text { lewall } \\
\text { ters. }\end{array}$ & $\begin{array}{l}\text { lue but } \\
\text { 3C. Me }\end{array}$ & $\begin{array}{l}\text { look } \\
\text { stan }\end{array}$ & $\begin{array}{l}\text { so a te } \\
\text { ation }\end{array}$ & $\begin{array}{l}\text { alue is } \mathrm{r} \\
\text { ted. }\end{array}$ & & & & & \\
\hline
\end{tabular}


Table 5.5. Trace Metal Composition of $1: 1$ Water Extracts and Porewaters from Sidewall Cores and Borehole Extension Composites

\begin{tabular}{|c|c|c|c|c|c|c|c|c|c|}
\hline \multirow[b]{2}{*}{$\begin{array}{l}\text { Composite Depth } \\
\text { (ft bgs })^{(a)}\end{array}$} & \multirow[b]{2}{*}{$\begin{array}{l}\text { 1:1 Extract } \\
\text { ID }\end{array}$} & \multicolumn{8}{|c|}{ ICP-MS } \\
\hline & & $\begin{array}{l}{ }^{99} \mathrm{Tc} \\
(\mathrm{pCi} / \mathrm{L})\end{array}$ & $\begin{array}{c}\mathrm{Cr} \\
(\mathrm{ppb})\end{array}$ & $\begin{array}{c}\mathrm{Co} \\
(\mathrm{ppb})\end{array}$ & $\begin{array}{c}\mathrm{Ni} \\
(\mathrm{ppb})\end{array}$ & $\begin{array}{c}\mathrm{Zn} \\
(\mathrm{ppb})\end{array}$ & $\begin{array}{c}\text { As } \\
(\mathrm{ppb})\end{array}$ & $\begin{array}{c}\mathrm{Se} \\
(\mathrm{ppb})\end{array}$ & $\begin{array}{c}\text { Mo } \\
(\mathrm{ppb})\end{array}$ \\
\hline \multicolumn{10}{|c|}{ Sidewall } \\
\hline 25.5 & $15 \mathrm{~A} / \mathrm{B} / \mathrm{C}$ & $<1.0 \mathrm{E}+03$ & $<0.5$ & $(3.00)$ & $<100$ & $(68.0)$ & 26.0 & $<5$ & 14.0 \\
\hline 44.5 & $14 \mathrm{~A} / \mathrm{B} / \mathrm{C}$ & $<1.0 \mathrm{E}+03$ & (2.89) & $(1.00)$ & $<100$ & $(65.0)$ & 14.9 & $<5$ & 74.6 \\
\hline \multirow[t]{2}{*}{56.5} & $13 \mathrm{~A} / \mathrm{B} / \mathrm{C}$ & $<1.0 \mathrm{E}+03$ & (3.47) & $<50$ & $<100$ & $(53.0)$ & 0.77 & $<5$ & 62.6 \\
\hline & 13 UFA & $2.88 \mathrm{E}+05$ & 6,463 & $<83$ & $(9.00)$ & 109 & $(4.33)$ & 44.7 & 104 \\
\hline 61.5 & $12 \mathrm{~A} / \mathrm{B} / \mathrm{C}$ & $7.0 \mathrm{E}+02$ & $(8.15)$ & $(3.00)$ & $<100$ & $(56.0)$ & 4.00 & $<5$ & 24.9 \\
\hline 65.5 & $11 \mathrm{~A} / \mathrm{B}$ & $4.1 \mathrm{E}+03$ & 344 & $(2.00)$ & $(4.00)$ & (104) & 24.3 & $<5$ & 304 \\
\hline 66 & $11 \mathrm{C}$ & $7.8 \mathrm{E}+03$ & 342 & $(1.00)$ & $(2.00)$ & (102) & 15.8 & $<5$ & 93.4 \\
\hline 69.5 & $10 \mathrm{~A} / \mathrm{B} / \mathrm{C}$ & $5.5 \mathrm{E}+03$ & 5070 & $(7.00)$ & $<100$ & $(86.0)$ & 30.0 & $<5$ & 516 \\
\hline 74.5 & $09 \mathrm{~A} / \mathrm{B} / \mathrm{C}$ & $7.7 \mathrm{E}+03$ & 4091 & $(5.00)$ & $(4.00)$ & (51.0) & 24.3 & $<5$ & 552 \\
\hline \multirow[t]{2}{*}{79.5} & $08 \mathrm{~A} / \mathrm{B} / \mathrm{C}$ & $1.8 \mathrm{E}+04$ & 718 & $(1.00)$ & $(2.00)$ & $(49.0)$ & 7.84 & $(4.86)$ & 1,003 \\
\hline & 8 UFA & $5.17 \mathrm{E}+05$ & 20,850 & $<156$ & $(11.0)$ & 1,848 & $(8.13)$ & $(74.2)$ & 2,596 \\
\hline 82.5 & $07 \mathrm{~A} / \mathrm{B} / \mathrm{C}$ & $3.9 \mathrm{E}+05$ & 745,753 & 96.0 & (6.0) & $(66.0)$ & 7.63 & 85.7 & 6,378 \\
\hline \multirow[t]{2}{*}{90} & $06 \mathrm{~A} / \mathrm{B}$ & $2.7 \mathrm{E}+06$ & 711,595 & 86.0 & (6.0) & $(88.0)$ & $<5$ & 135 & 2,911 \\
\hline & 6 UFA & $2.48 \mathrm{E}+07$ & $5,627,500$ & $<453$ & $(41.0)$ & 944 & 264 & 1,218 & 5,032 \\
\hline 95.5 & $03 \mathrm{~A} / \mathrm{B} / \mathrm{C}$ & $7.1 \mathrm{E}+06$ & 260,333 & $(30.0)$ & $<100$ & (83) & $<5$ & 219 & 427 \\
\hline 102.5 & $05 \mathrm{~A} / \mathrm{B} / \mathrm{C}$ & $6.1 \mathrm{E}+06$ & 527,799 & 60.0 & $<100$ & (112) & $<5$ & 228 & 1,575 \\
\hline \multirow[t]{2}{*}{108.5} & $04 \mathrm{~A} / \mathrm{B} / \mathrm{C}$ & $1.2 \mathrm{E}+07$ & 481,344 & 63.0 & $(5.00)$ & (60) & $<5$ & 388 & 604 \\
\hline & 4 UFA & $9.40 \mathrm{E}+07$ & $3,183,229 \pm 23,128$ & $259 \pm 17$ & $29 \pm 13$ & $235 \pm 9$ & $628 \pm 19$ & $2,836 \pm 62$ & $943 \pm 27$ \\
\hline \multirow[t]{2}{*}{112} & $02 \mathrm{~B} / \mathrm{C}$ & $8.6 \mathrm{E}+06$ & 175,630 & 25.0 & $<100$ & $(50)$ & $<5$ & 288 & 17.0 \\
\hline & 2 UFA & $1.23 \mathrm{E}+08$ & $1,795,521$ & (157) & $(41.0)$ & (332) & 685 & 3,074 & (102) \\
\hline 127.4 & $01 \mathrm{~A} / \mathrm{B} / \mathrm{C}$ & $3.3 \mathrm{E}+05$ & 11.47 & (0) & $<100$ & (89) & 1.05 & 31.5 & 167 \\
\hline
\end{tabular}


Table 5.5. (contd)

\begin{tabular}{|c|c|c|c|c|c|c|c|c|c|}
\hline \multirow[b]{2}{*}{$\begin{array}{l}\text { Composite Depth } \\
{\text { (ft bgs })^{(a)}}^{(a)}\end{array}$} & \multirow[b]{2}{*}{$\begin{array}{l}\text { 1:1 Extract } \\
\text { ID }\end{array}$} & \multicolumn{8}{|c|}{ ICP-MS } \\
\hline & & $\begin{array}{c}{ }^{99} \mathrm{Tc} \\
(\mathrm{pCi} / \mathrm{L})\end{array}$ & $\begin{array}{c}\mathrm{Cr} \\
(\mathrm{ppb})\end{array}$ & $\begin{array}{c}\mathrm{Co} \\
(\mathrm{ppb})\end{array}$ & $\begin{array}{c}\mathrm{Ni} \\
(\mathrm{ppb})\end{array}$ & $\begin{array}{c}\mathrm{Zn} \\
(\mathrm{ppb})\end{array}$ & $\begin{array}{c}\text { As } \\
(\mathrm{ppb})\end{array}$ & $\begin{array}{c}\mathrm{Se} \\
(\mathrm{ppb})\end{array}$ & $\begin{array}{l}\text { Mo } \\
(\mathrm{ppb})\end{array}$ \\
\hline \multicolumn{10}{|c|}{ Extension } \\
\hline 133.2 & $2 \mathrm{C} / \mathrm{D}$ & $1,000 \pm 2,500$ & 3.00 & 6.86 & $<5$ & 32.0 & NA & $<10$ & 121 \\
\hline 134.2 & $2 \mathrm{~A} / \mathrm{B}$ & $5,650 \pm 2,200$ & $<0.5$ & $<0.5$ & $<5$ & 7.00 & NA & $<10$ & 15.2 \\
\hline 135.2 & $3 \mathrm{~A} / \mathrm{B}$ & $2,720 \pm 2,500$ & $<0.5$ & $<0.5$ & $<5$ & 22.0 & NA & $<10$ & 58.0 \\
\hline 153.9 & $10 \mathrm{~A} / \mathrm{B}$ & $3,900 \pm 5,200$ & $<0.5$ & $<0.5$ & $<5$ & 11.0 & NA & $<10$ & 31.1 \\
\hline 156.8 & $12 \mathrm{~A} / \mathrm{B}$ & $380 \pm 2,900$ & $<0.5$ & $<0.5$ & $<5$ & 7.00 & NA & $<10$ & 87.1 \\
\hline 165.3 & $17 \mathrm{~A} / \mathrm{B}$ & $9,320 \pm 12,800$ & $<0.5$ & $<0.5$ & $<5$ & 14.0 & NA & $<10$ & 13.0 \\
\hline 188.3 & $38 \mathrm{~A} / \mathrm{B}$ & $50 \pm 2,800$ & $<0.5$ & $<0.5$ & $<5$ & 6.00 & NA & $<10$ & 22.0 \\
\hline 196.9 & $47 \mathrm{~A} / \mathrm{B}$ & $(-190) \pm 4,700$ & $<0.5$ & $<0.5$ & $<5$ & 13.5 & NA & $<10$ & 11.7 \\
\hline 208.6 & $58 \mathrm{~A} / \mathrm{B}$ & $(-470) \pm 3,800$ & $<0.5$ & $<0.5$ & $<5$ & 10.2 & NA & $<10$ & 9.80 \\
\hline \multicolumn{10}{|c|}{$\begin{array}{l}\text { NA = Not analyzed. } \\
\text { For }{ }^{99} \mathrm{Tc} \text {, the borehole extension samples were analyzed by wet chemical separations and liquid scintillation counting. Samples were analyzed in duplicate and mean and } \\
\text { uncertainty are presented. The deepest two composites yielded negative values. The technique is not as sensitive as the ICP-MS data. Note units for }{ }^{99} \mathrm{Tc} \text { are pCi/L not } \\
\text { mass units. } \\
\text { (a) Multiply by } 0.3048 \text { to convert to meters. }\end{array}$} \\
\hline
\end{tabular}


Table 5.6. High Z Trace Metal Composition of 1:1 Water Extracts and Porewaters from Composite Sediments

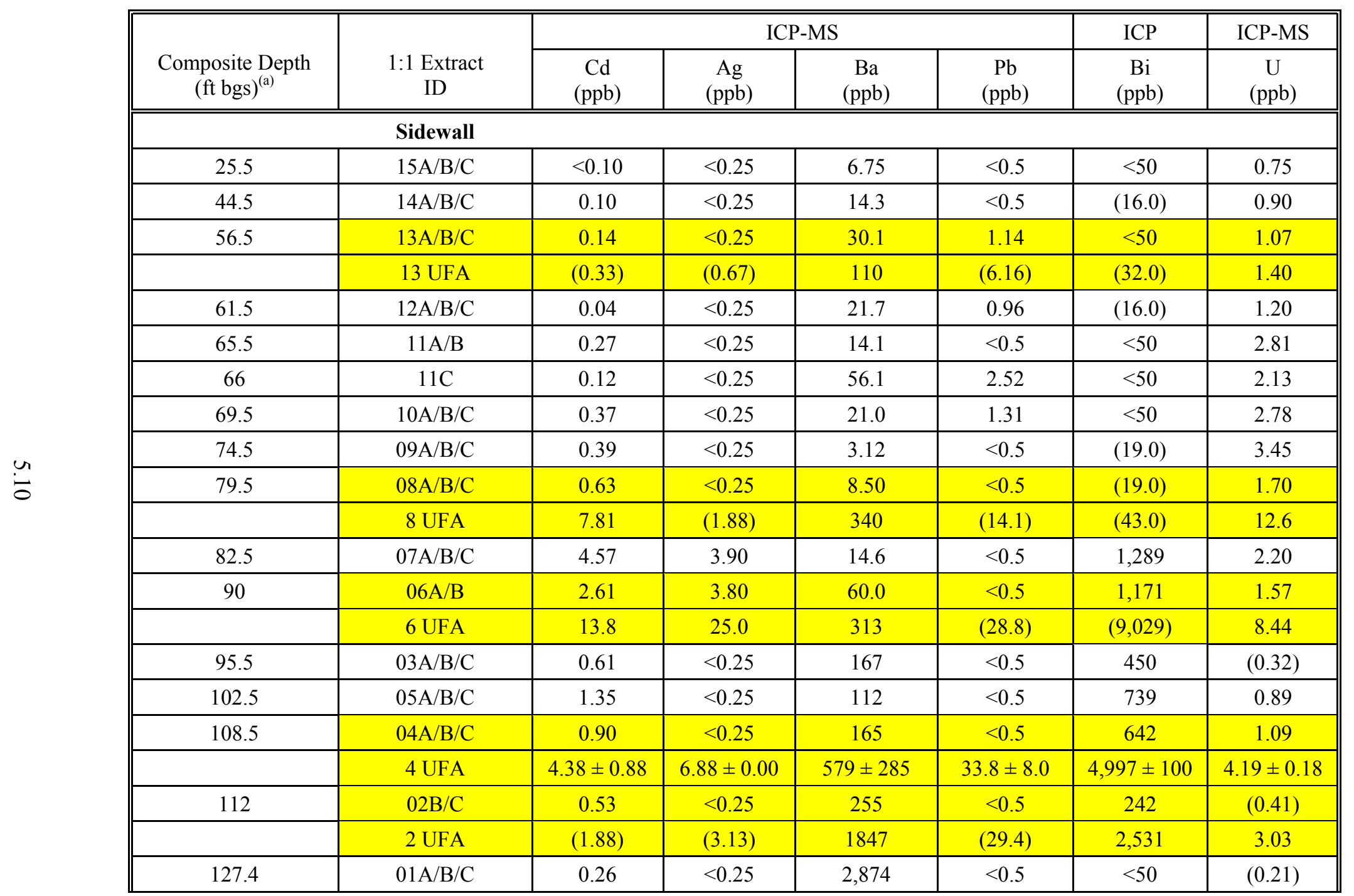


Table 5.6. (contd)

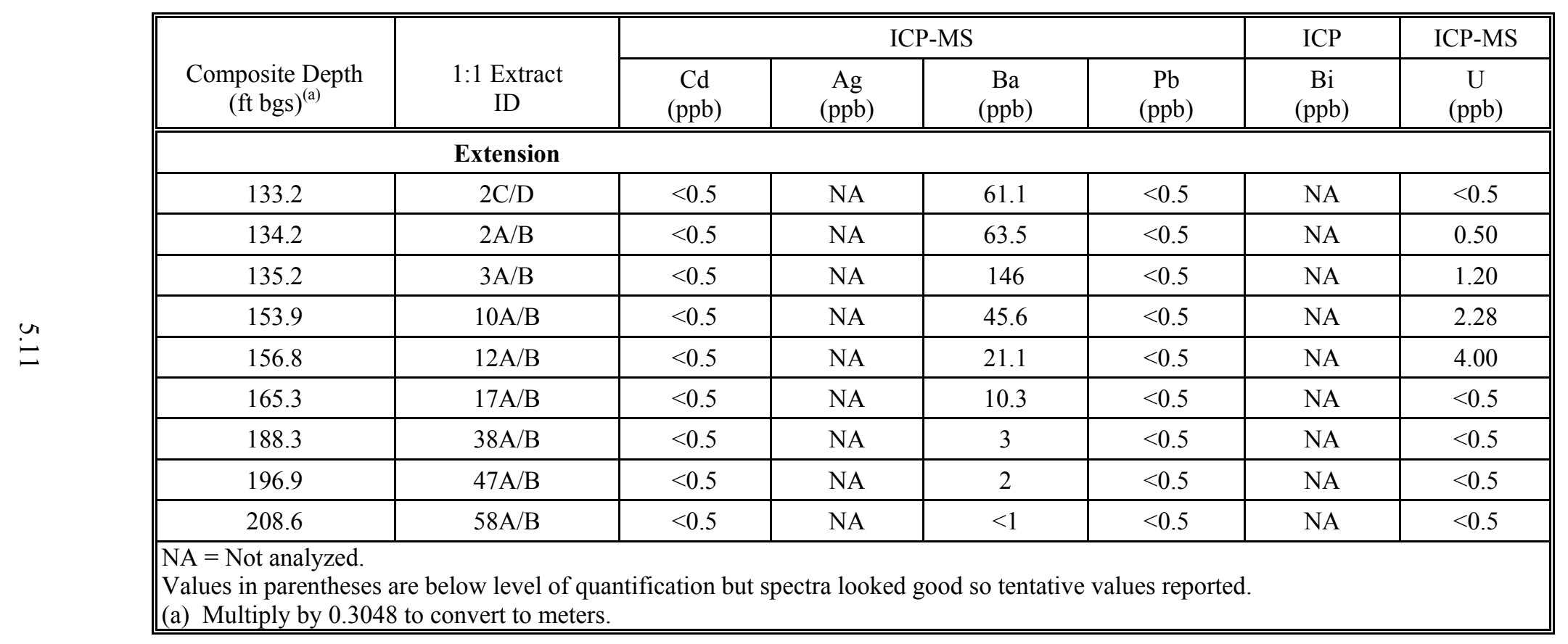


Table 5.7. Dilution-Corrected Anion Composition of 1:1 Water Extracts and Porewaters from Sidewall Cores and Borehole Extension Composites

\begin{tabular}{|c|c|c|c|c|c|c|c|c|c|}
\hline \multirow{2}{*}{$\begin{array}{c}\text { Composite } \\
\text { Depth } \\
(\mathrm{ft} \mathrm{bgs})^{(\mathrm{a})} \\
\end{array}$} & \multirow[b]{2}{*}{$\begin{array}{l}\text { 1:1 Extract } \\
\text { ID }\end{array}$} & \multirow[b]{2}{*}{$\begin{array}{c}\text { Dilution } \\
\text { Factor } \\
\end{array}$} & \multicolumn{7}{|c|}{ Dilution Corrected } \\
\hline & & & $\begin{array}{c}\mathrm{Cl} \\
(\mathrm{ppm})\end{array}$ & $\begin{array}{c}\mathrm{F} \\
(\mathrm{ppm}) \\
\end{array}$ & $\begin{array}{c}\mathrm{NO}_{3} \\
(\mathrm{ppm})\end{array}$ & $\begin{array}{c}\mathrm{NO}_{2} \\
(\mathrm{ppm})\end{array}$ & $\begin{array}{r}\mathrm{PO}_{4} \\
(\mathrm{ppm}) \\
\end{array}$ & $\begin{array}{c}\mathrm{SO}_{4} \\
(\mathrm{ppm}) \\
\end{array}$ & \begin{tabular}{|c}
$\begin{array}{c}\text { Inorganic } \\
(\mathrm{ppm})\end{array}$ \\
\end{tabular} \\
\hline \multicolumn{10}{|c|}{ Sidewall } \\
\hline \multirow[t]{2}{*}{56.5} & $13 \mathrm{~A} / \mathrm{B} / \mathrm{C}$ & 6.24 & 5.2 & $\mathrm{NA}$ & 81.2 & 1.6 & $\mathrm{NA}$ & 17.2 & 186.5 \\
\hline & 13 UFA & & 57.9 & $<33$ & 1,264 & $<33.3$ & $<167$ & 443.6 & 19.2 \\
\hline & & & & & & & & & \\
\hline \multirow[t]{2}{*}{79.5} & $08 \mathrm{~A} / \mathrm{B} / \mathrm{C}$ & 9.34 & 31.2 & $\mathrm{NA}$ & 3,464 & 13.1 & $\mathrm{NA}$ & 483.3 & 786.1 \\
\hline & 8 UFA & & 122.1 & $<532$ & 4,065 & $<62.5$ & $<313$ & $1,161.0$ & 230.4 \\
\hline & & & & & & & & & \\
\hline \multirow[t]{2}{*}{90} & $06 \mathrm{~A} / \mathrm{B}$ & 9.76 & $2,992.2$ & $\mathrm{NA}$ & $2.74 \mathrm{E}+05$ & $1,355.0$ & $\mathrm{NA}$ & $2,641.7$ & 156.5 \\
\hline & 6 UFA & & $1,336.4$ & $<1,203$ & $2.58 \mathrm{E}+05$ & $<125$ & $<625$ & $3,364.8$ & 37.8 \\
\hline & & & & & & & & & \\
\hline 108.5 & $04 \mathrm{~A} / \mathrm{B} / \mathrm{C}$ & 8.33 & $2,990.4$ & NA & $3.54 \mathrm{E}+05$ & $1,084.2$ & $\mathrm{NA}$ & $3,214.9$ & 99.0 \\
\hline ur & 4 UFA & & $3,984.4$ & $<1,236$ & $3.24 \mathrm{E}+05$ & $<125$ & $<625$ & $4,519.8$ & 30.3 \\
\hline & & & & & & & & & \\
\hline \multirow[t]{2}{*}{112} & $02 \mathrm{~B} / \mathrm{C}$ & 12.26 & $4,212.0$ & $\mathrm{NA}$ & $4.02 \mathrm{E}+05$ & $1,297.2$ & $\mathrm{NA}$ & $4,576.0$ & 101.2 \\
\hline & 2 UFA & & $4,488.6$ & $<125$ & $3.76 \mathrm{E}+05$ & $<125$ & $<625$ & $3,882.6$ & 38.6 \\
\hline \multicolumn{10}{|c|}{ Extension } \\
\hline 133.2 & $2 \mathrm{C} / \mathrm{D}$ & 5.25 & 100.38 & 7.29 & 5.98 & $<0.52$ & $<1.05$ & 26.45 & 158.47 \\
\hline 134.2 & $2 \mathrm{~A} / \mathrm{B}$ & 8.98 & 93.85 & 4.58 & $2,667.26$ & 2.16 & $<1.80$ & 67.18 & 108.4 \\
\hline 135.2 & $3 \mathrm{~A} / \mathrm{B}$ & 10.65 & 354.9 & 0 & $3,263.65$ & 1.39 & $<2.13$ & 152.68 & 182.94 \\
\hline 153.9 & $10 \mathrm{~A} / \mathrm{B}$ & 6.65 & 31.87 & 4.06 & 351.02 & 1.8 & 2.99 & 140.65 & 100.33 \\
\hline 156.8 & $12 \mathrm{~A} / \mathrm{B}$ & 19.44 & 81.84 & 4.67 & 121.68 & $<1.94$ & 7.19 & 790.76 & 361.36 \\
\hline 165.3 & $17 \mathrm{~A} / \mathrm{B}$ & 26.3 & 27.88 & 19.46 & 203.06 & $<2.63$ & 7.37 & 170.19 & 62.87 \\
\hline 188.3 & $38 \mathrm{~A} / \mathrm{B}$ & 123.64 & 157.03 & 53.17 & 322.71 & $<12.36$ & 33.38 & 205.25 & 403.08 \\
\hline 196.9 & $47 \mathrm{~A} / \mathrm{B}$ & 46.48 & 108.75 & 24.63 & 797.07 & $<4.65$ & $<9.30$ & 163.6 & 86.91 \\
\hline 208.6 & $58 \mathrm{~A} / \mathrm{B}$ & 22.69 & 22.92 & 8.4 & 6.58 & $<2.27$ & 6.81 & 31.54 & 32.67 \\
\hline
\end{tabular}


Table 5.8. Dilution-Corrected Major Cation Composition of 1:1 Water Extracts and Porewaters from Sidewall Cores and Borehole Extension Composites

\begin{tabular}{|c|c|c|c|c|c|c|c|c|c|c|c|c|}
\hline \multirow[b]{2}{*}{$\begin{array}{c}\text { Composite Depth } \\
(\mathrm{ft} \text { bgs })^{(\mathrm{a})}\end{array}$} & \multirow[b]{2}{*}{$\begin{array}{c}\text { 1:1 Extract } \\
\text { ID }\end{array}$} & \multirow[b]{2}{*}{\begin{tabular}{|c} 
Dilution \\
Factor
\end{tabular}} & \multicolumn{4}{|c|}{ ICP } & \multirow{2}{*}{$\begin{array}{c}\text { ICP-MS } \\
\begin{array}{c}\mathrm{Sr} \\
(\mathrm{ppm})\end{array}\end{array}$} & \multicolumn{3}{|c|}{ ICP } & \multirow{2}{*}{\begin{tabular}{|c|}
$\mathrm{ICP}-\mathrm{MS}$ \\
$\begin{array}{c}\mathrm{Mn} \\
(\mathrm{ppm})\end{array}$ \\
\end{tabular}} & \multirow{2}{*}{$\begin{array}{c}\mathrm{ICP} \\
\mathrm{Si} \\
(\mathrm{ppm}) \\
\end{array}$} \\
\hline & & & $\begin{array}{c}\mathrm{Ca} \\
(\mathrm{ppm})\end{array}$ & $\begin{array}{c}\mathrm{K} \\
(\mathrm{ppm}) \\
\end{array}$ & \begin{tabular}{|c|}
$\mathrm{Mg}$ \\
$(\mathrm{ppm})$
\end{tabular} & $\begin{array}{c}\mathrm{Na} \\
(\mathrm{ppm})\end{array}$ & & \begin{tabular}{|c|}
$\mathrm{Al}$ \\
$(\mathrm{ppm})$ \\
\end{tabular} & $\begin{array}{c}\mathrm{B} \\
(\mathrm{ppm}) \\
\end{array}$ & $\begin{array}{c}\mathrm{Fe} \\
(\mathrm{ppm}) \\
\end{array}$ & & \\
\hline \multicolumn{13}{|c|}{ Sidewall } \\
\hline \multirow[t]{2}{*}{56.5} & $13 \mathrm{~A} / \mathrm{B} / \mathrm{C}$ & 6.24 & 140 & 46.3 & 12.6 & 270 & 0.22 & 3.20 & 33.1 & 1.60 & 0.31 & 58.2 \\
\hline & 13 UFA & & 50.3 & 15.8 & 3.9 & 561 & 0.55 & 0.90 & 27 & $(0.07)$ & $(0.07)$ & 19.8 \\
\hline & & & & & & & & & & & & \\
\hline \multirow{2}{*}{79.5} & $08 \mathrm{~A} / \mathrm{B} / \mathrm{C}$ & 9.34 & 6.60 & 38.9 & 0.7 & 3953 & 0.53 & 7.00 & 333 & 0.20 & 0.47 & 49.4 \\
\hline & 8 UFA & & 10.1 & 41.9 & 1.5 & 2438 & 0.47 & 1.70 & 111 & $(0.06)$ & $(0.63)$ & 18.1 \\
\hline \multirow[t]{2}{*}{90} & 06A/B & 9.76 & 1,385 & 2,039 & 70.5 & $1.10 \mathrm{E}+05$ & 26.2 & 3.60 & 259 & 0.40 & 0.49 & 29.0 \\
\hline & 6 UFA & & 1,190 & 2,156 & 63.4 & $9.14 \mathrm{E}+04$ & 44.7 & 1.10 & 138 & $(0.10)$ & $<1.3$ & 20.3 \\
\hline \multirow[t]{2}{*}{108.5} & $04 \mathrm{~A} / \mathrm{B} / \mathrm{C}$ & 8.33 & 3,744 & 971 & 144 & $1.27 \mathrm{E}+05$ & 73.0 & 2.20 & 68.9 & 0.60 & 0.42 & 40.1 \\
\hline & 4 UFA & & $3,828 \pm 22$ & $942 \pm 21$ & $123 \pm 1$ & $1.09 \mathrm{E}+05 \pm 101$ & $95.64 \pm 3.8$ & $1 \pm 0.2$ & $80.8 \pm 1.3$ & $0.10 \pm 0.01$ & $<1.3$ & $23.6 \pm 3$ \\
\hline & & & & & & & & & & & & \\
\hline \multirow[t]{3}{*}{112} & $02 \mathrm{~B} / \mathrm{C}$ & 12.3 & 5,557 & 996 & 291 & $1.39 \mathrm{E}+05$ & 105 & 6.10 & 370 & 0.80 & 0.61 & 59.7 \\
\hline & 2 UFA & & 6,388 & 1,103 & 255 & $1.25 \mathrm{E}+05$ & 141 & 1.10 & 380 & $(0.08)$ & $<1.3$ & 18.3 \\
\hline & Extension & & & & & & & & & & & \\
\hline 133.2 & \begin{tabular}{|l|}
$2 \mathrm{C} / \mathrm{D}$ \\
\end{tabular} & 5.25 & 37.6 & 72.7 & 7.25 & 201 & $<0.26$ & 5.49 & 27.3 & 3.52 & $<0.26$ & 30.5 \\
\hline 134.2 & $2 \mathrm{~A} / \mathrm{B}$ & 8.98 & 490 & 91.9 & 126 & 306 & 2.42 & \begin{tabular}{|c|}
$<0.45$ \\
\end{tabular} & 30.4 & $<0.45$ & $<0.45$ & 101 \\
\hline 135.2 & $3 \mathrm{~A} / \mathrm{B}$ & 10.7 & 602 & 399 & 170 & 498 & 3.11 & 0.53 & 56.9 & $<0.53$ & $<0.40$ & 109 \\
\hline 153.9 & $10 \mathrm{~A} / \mathrm{B}$ & 6.65 & 110 & 31.1 & 47.7 & 101 & 0.58 & $<0.53$ & 16.7 & 0.58 & $<0.33$ & 99.8 \\
\hline 156.8 & $12 \mathrm{~A} / \mathrm{B}$ & 19.4 & 175 & 88.4 & 63.5 & 630 & $<0.97$ & 3.49 & 32.0 & 5.36 & $<0.97$ & 202 \\
\hline 165.3 & $17 \mathrm{~A} / \mathrm{B}$ & 26.3 & 64.7 & 48.7 & 24.5 & 190 & $<1.32$ & 24.2 & 36.3 & 27.9 & $<1.32$ & 342 \\
\hline 188.3 & $38 \mathrm{~A} / \mathrm{B}$ & 124 & 137 & 238 & 45.3 & 909 & $<6.18$ & 36.8 & 165 & 24.4 & $<6.18$ & 996 \\
\hline 196.9 & $47 \mathrm{~A} / \mathrm{B}$ & 46.5 & 112 & 81.0 & 36.8 & 361 & $<2.32$ & 5.82 & 77.9 & 5.52 & $<2.32$ & 412 \\
\hline 208.6 & $58 \mathrm{~A} / \mathrm{B}$ & 22.7 & 12.2 & $<22.69$ & 4.87 & 102 & $<1.13$ & 3.26 & 31.4 & 4.74 & $<1.13$ & 194 \\
\hline
\end{tabular}


Table 5.9. Dilution-Corrected Trace Metal Composition of 1:1 Water Extracts and Porewaters from Sidewall Cores and Borehole Extension Composites

\begin{tabular}{|c|c|c|c|c|c|c|}
\hline \multirow[b]{2}{*}{$\begin{array}{l}\text { Composite Depth } \\
(\mathrm{ft} \text { bgs })^{(\mathrm{a})}\end{array}$} & \multirow[b]{2}{*}{$\begin{array}{l}\text { 1:1 Extract } \\
\text { ID }\end{array}$} & \multirow[b]{2}{*}{ Dilution Factor } & \multicolumn{4}{|c|}{ Dilution-Corrected } \\
\hline & & & $\begin{array}{c}{ }^{99} \mathrm{Tc} \\
(\mathrm{pCi} / \mathrm{L})\end{array}$ & $\begin{array}{c}\mathrm{Cr} \\
(\mathrm{ppb})\end{array}$ & $\begin{array}{c}\mathrm{Se} \\
(\mathrm{ppb})\end{array}$ & $\begin{array}{l}\text { Mo } \\
(\mathrm{ppb})\end{array}$ \\
\hline \multicolumn{7}{|c|}{ Sidewall } \\
\hline \multirow[t]{2}{*}{56.5} & $13 \mathrm{~A} / \mathrm{B} / \mathrm{C}$ & 6.24 & $<6.2 \mathrm{E}+03$ & $(2.17 \mathrm{E}+01)$ & $<31.19$ & $3.90 \mathrm{E}+02$ \\
\hline & 13 UFA & & $2.88 \mathrm{E}+05$ & 6,463 & 44.7 & $1.04 \mathrm{E}+02$ \\
\hline \multirow[t]{2}{*}{79.5} & $08 \mathrm{~A} / \mathrm{B} / \mathrm{C}$ & 9.34 & $1.70 \mathrm{E}+05$ & $6.71 E+03$ & $(4.54 \mathrm{E}+01)$ & $9.37 \mathrm{E}+03$ \\
\hline & 8 UFA & & $5.17 \mathrm{E}+05$ & 2,0850 & $\overline{(74.2)}$ & $2.60 \mathrm{E}+03$ \\
\hline \multirow[t]{2}{*}{90} & 06A/B & 9.76 & $2.68 \mathrm{E}+07$ & $6.94 \mathrm{E}+06$ & $1.32 \mathrm{E}+03$ & $2.84 \mathrm{E}+04$ \\
\hline & 6 UFA & & $2.48 \mathrm{E}+07$ & $5.67 \mathrm{E}+06$ & 1,218 & $5.03 \mathrm{E}+03$ \\
\hline \multirow[t]{2}{*}{108.5} & $04 \mathrm{~A} / \mathrm{B} / \mathrm{C}$ & 8.33 & $9.92 \mathrm{E}+07$ & $4.01 \mathrm{E}+06$ & $3.24 \mathrm{E}+03$ & $5.03 \mathrm{E}+03$ \\
\hline & 4 UFA & & $9.40 \mathrm{E}+07$ & $3.18 \mathrm{E}+06 \pm 2.3 \mathrm{E}+04$ & $2,836 \pm 62$ & $943 \pm 27$ \\
\hline \multirow[t]{2}{*}{112} & $02 \mathrm{~B} / \mathrm{C}$ & 12.3 & $1.05 \mathrm{E}+08$ & $2.15 \mathrm{E}+06$ & $3.53 \mathrm{E}+03$ & $2.04 \mathrm{E}+02$ \\
\hline & 2 UFA & & $1.23 \mathrm{E}+08$ & $1.80 \mathrm{E}+06$ & 3,074 & $(102)$ \\
\hline \multicolumn{7}{|c|}{ Extension } \\
\hline 133.2 & $2 \mathrm{C} / \mathrm{D}$ & 5.25 & -- & 16 & $<53$ & 635 \\
\hline 134.2 & $2 \mathrm{~A} / \mathrm{B}$ & 8.98 & -- & $<4$ & $<90$ & 136 \\
\hline 135.2 & $3 \mathrm{~A} / \mathrm{B}$ & 10.7 & -- & $<5$ & $<107$ & 618 \\
\hline 153.9 & $10 \mathrm{~A} / \mathrm{B}$ & 6.65 & -- & $<3$ & $<67$ & 207 \\
\hline 156.8 & $12 \mathrm{~A} / \mathrm{B}$ & 19.4 & -- & $<10$ & $<194$ & 1,693 \\
\hline 165.3 & $17 \mathrm{~A} / \mathrm{B}$ & 26.3 & -- & $<13$ & $<263$ & 342 \\
\hline 188.3 & $38 \mathrm{~A} / \mathrm{B}$ & 124 & -- & $<62$ & $<1,236$ & 2,720 \\
\hline 196.9 & $47 \mathrm{~A} / \mathrm{B}$ & 46.5 & -- & $<23$ & $<465$ & 544 \\
\hline 208.6 & $58 \mathrm{~A} / \mathrm{B}$ & 22.7 & -- & $<11$ & $<227$ & 222 \\
\hline \multicolumn{7}{|c|}{$\begin{array}{l}\text { Values in parentheses are below level of quantification but spectra looked good so values are recorded. } \\
--={ }^{99} \mathrm{Tc} \text { values in the borehole extension studies were not reliable and are not reported. } \\
\text { (a) Multiply by } 0.3048 \text { to convert to meters. }\end{array}$} \\
\hline
\end{tabular}

indication that elevated chloride might reach 40.5 meters (133 feet) bgs and the nitrate concentrations might be continuously elevated as deep as 46.9 meters (154 feet) bgs. The nitrate value at 60 meters (197 feet) bgs is also high but we do not believe that the value is related to the leaked tank plume. The nitrite distribution suggests that once leaked into the sediments, oxidation converts the nitrite to nitrate because the ratio of nitrite to nitrate is much lower than the values within the tanks.

Data in Table 5.4 and Figure 5.1 show that the major cations are involved in an ion exchange reaction wherein the massive amounts of sodium in the tank liquor replace and push the alkaline earth cations into the leading edge of the sodium plume. The water-extractable calcium and magnesium from the sediments 


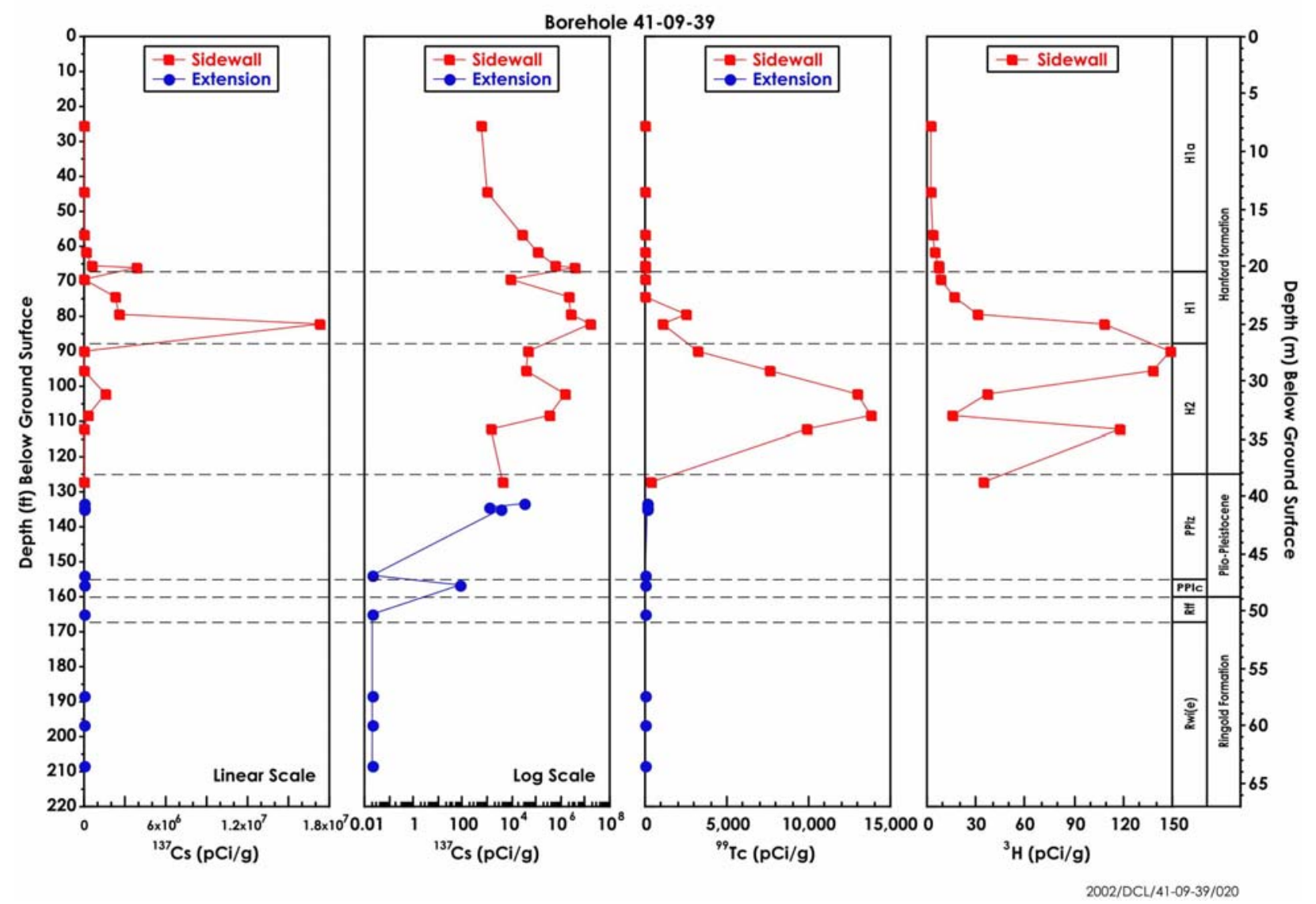

Figure 5.1. Dilution-Corrected Cations in 1:1 Sediment-to-Water Extracts and Actual Porewaters Versus Lithology and Depth

at depths between 18.6 and 25 meters (61 and 82 feet) bgs are very low. The trace strontium does not show the trend as well. Potassium may be depleted between the depths of 18.3 to 22.6 meters (60 to 74 feet) bgs. These cations, including strontium, are found at elevated water-extractable concentrations at the leading edge of the sodium plume at depths between 29 to 38.7 meters (95 to 127 feet) bgs. The water extract data for silicon, iron, manganese, and aluminum do not show any obvious differences from similar extracts of natural sediments from the same Hanford and Plio-Pleistocene formations (Serne et al. 2002a).

Data in Tables 5.5 and 5.6 for trace metals show that there is elevated technetium-99, chromium, molybdenum and, to a lesser extent, selenium in the region where EC, sodium, and nitrate show the presence of tank fluids. The first significant sign of elevated technetium-99 is at 24.2 meters (79.5 feet) bgs and a high concentration plume is found from 27.4 to 38.8 meters (90 to 127.4 feet) bgs. The molybdenum distribution is quite similar. The leading edge of the chromium and selenium appears to stop at 34.1 meters (112 feet) bgs, which suggests that they do not migrate as quickly as molybdenum and technetium-99. There are at least two plausible mechanisms for the slight retardation of chromium and selenium. The first is that there could be some redox mediated reduction of the highly mobile oxyanion forms of these two metals and the second mechanism is precipitation of some of the oxyanion into perhaps alkaline earth co-precipitates such as calcium chromate or calcium/barium-mixed 
chromates/selenate/sulfates. Recall that the alkaline earth cations show higher concentrations in the water extracts between 29 and 38.7 meters ( 95 and 127 feet) bgs and that the zone between 29 and 34.1 meters (95 and 112 feet) bgs may be a zone of active reaction of the tank fluids and sediments. This differing technetium-99 and molybdenum versus chromium and selenium migration observation is being studied by several investigators within the Environmental Management Science Program (EMSP) and Science and Technology Programs. It was also observed during the inductively coupled plasma mass spectrometry (ICP-MS) analyses that molybdenum and perhaps other elements exhibit a fission product isotope ratio instead of natural ratios. The elements zinc, arsenic, cobalt, nickel, cadmium, silver, lead, uranium, and ruthenium did not leach into water at high enough concentrations to show any tankenhanced concentrations. There does appear to be enhanced concentrations of bismuth present and its vertical distribution suggests that it is less mobile than the chromium and selenium.

Table 5.7 and Figure 5.2 show the dilution-corrected 1:1 extracts comparison with the actual porewater. The agreement for porewaters that contain significant amounts of tank fluid to the dilutioncorrected extracts is acceptable (agreement is within 10\% or better) for nitrate but not very good for chloride and sulfate. We have improved agreement by using a different laboratory with a newer ion chromatograph for future work on contaminated sediments. It is clear that porewaters contain less inorganic carbon than the theoretical dilution-corrected samples likely because calcite is moderately soluble and ubiquitous in the sediments. The process of water extraction dissolves some carbonate that is not originally part of the vadose zone porewater.

It is clear from the anion data in Figure 5.2 that the vertical distribution of the tank fluid that leaked currently resides between 24.4 and 38.7 meters (80 and 127 feet) bgs; however, the current leading edge of the plume is difficult to pinpoint. The nitrate composition of the extracts for composite sediments 2A/B (40.9 meters [134.2 feet] bgs), 3A/B (41.2 meters [135.2 feet] bgs), and 47A/B (60 meters [196.9 feet] bgs) accounts for more than $70 \%, 60 \%$, and $40 \%$, respectively, of the anion charge in these solutions. For natural Hanford Site groundwater, the anion distribution is dominated by bicarbonate and sulfate with minor amounts of chloride and borate. The other composite extracts have anion distributions similar to natural groundwater. The composite $2 \mathrm{C} / \mathrm{D}$ extract has a natural anion distribution, probably because it is dominated by the drilling fluid lost during milling the end cap. The cooling water was Columbia River water that has an anion distribution similar to the groundwater. Composites $2 \mathrm{~A} / \mathrm{B}$ and $3 \mathrm{~A} / \mathrm{B}$ suggest that the leading edge of the nitrate-rich fluid has reached this depth. The 47A/B composite, located at 60 meters (197 feet bgs) deep may represent a zone influenced by water with a high nitrate composition, relative to naturally occurring water, that was disposed to upgradient ponds. The sediment in this zone may show residual water from Hanford Site operations when the water table was at this depth. As liquid discharges from Site operations slowed and finally ceased in 1995, residual water could have been captured in the vadose zone pores by capillary forces. The nitrate would have reached the sediment by horizontal travel and not vertical migration from beneath the tanks. The deep nitrate could also signify diluted tank fluid that reached the water table at an upgradient location that traveled back to the borehole location. We do not observe a continuous vertical distribution of elevated nitrate concentrations, or other tank constituents, from the tank bottom to the water table at this borehole. 


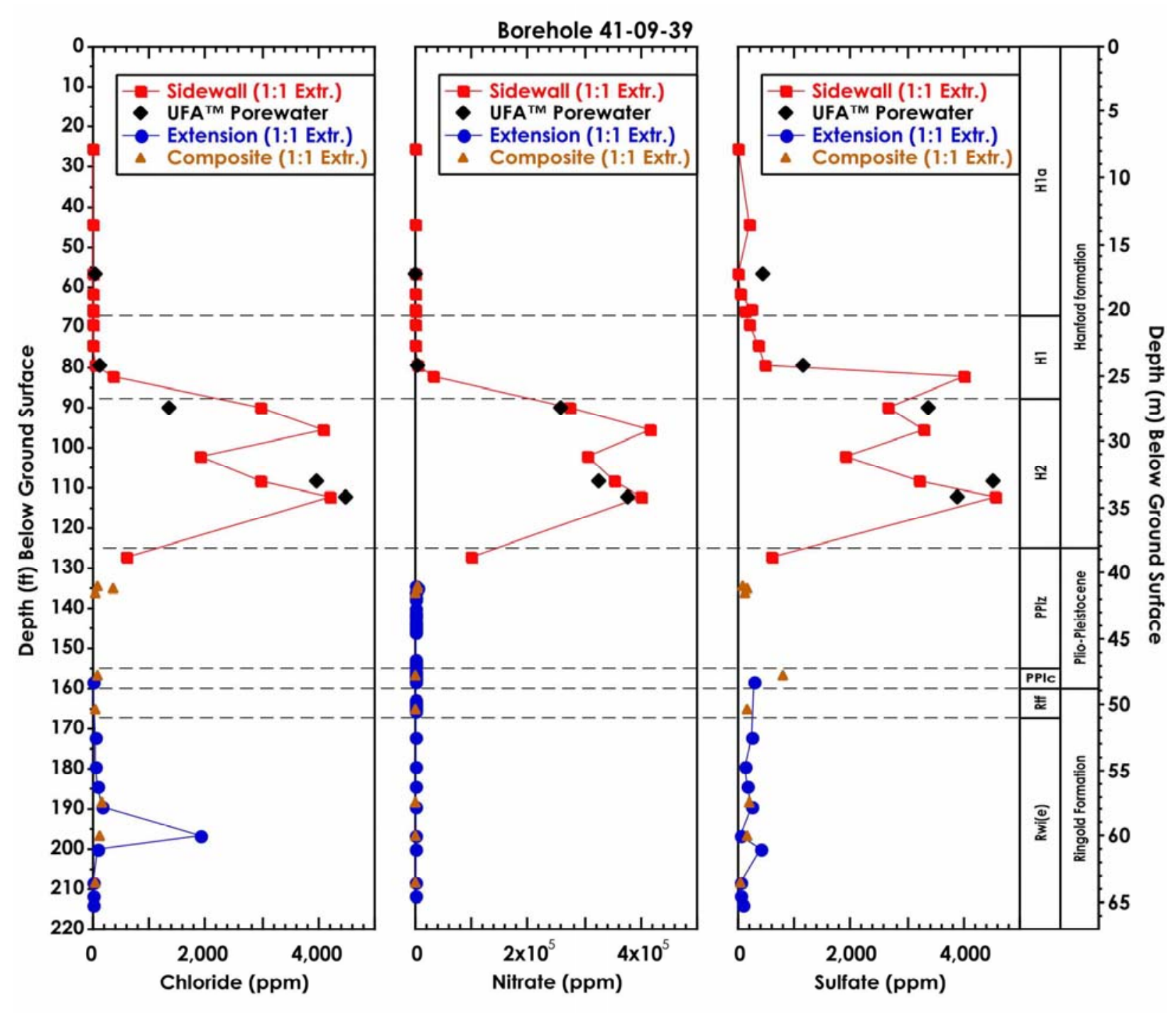

2002/DCL/41-09-39/022

Figure 5.2. Dilution-Corrected 1:1 Sediment-to-Water Extract Anions and Actual Porewater Anion Concentrations versus Lithology and Depth

Table 5.8 and Figure 5.1 suggest that the ion-exchangeable cations in porewaters in the zone where there are significant concentrations of tank fluid are accurately estimated by making the dilution corrections for the 1:1 water extracts. The iron, silicon, manganese, and aluminum concentrations in actual porewater are lower than the dilution-corrected concentrations likely because of minor dissolution reactions that occur during water extraction. The data in Table 5.8 suggest that the tank fluids have not significantly penetrated beyond 39.9 meters (131 feet) bgs and the leading edge is an ion exchange front that is enriched in alkaline earth cations. On an electrical-charge-equivalents basis, the water extracts of the composite sediments show a sodium-to-calcium ratio that varies between 0.5 to 7.25 . Three extracts have this ratio at a value below $1(2 \mathrm{~A} / \mathrm{B}, 3 \mathrm{~A} / \mathrm{B}$, and $10 \mathrm{~A} / \mathrm{B})$. The groundwater taken at the bottom of the borehole (see Myers et al. 1998) had a sodium-to-calcium ratio of 1.11 on an equivalents (charge) basis. 
The three composite extract samples with a ratio of less than 1 may reflect the ion-exchange process whereby the high-sodium-bearing tank liquor interacts with the natural Hanford Site sediments that have their exchange sites saturated with calcium. The sodium exchanges for calcium and a pulse of calcium is present in the leading edge of the solution plume that percolates into the deeper vadose zone. As more tank liquor with high concentrations of dissolved sodium percolate through the system, the pore fluid becomes dominated by sodium and the sodium-to-calcium ratio increases significantly. The ratio for composites $2 \mathrm{~A} / \mathrm{B}, 3 \mathrm{~A} / \mathrm{B}$, and $10 \mathrm{~A} / \mathrm{B}$ suggest that the leading edge of a sodium-enriched plume may be at shallower depths than these composite samples at 40.8 to 46.9 meters (134 to 154 feet bgs). Combining the atypical high nitrate with the sodium-to-calcium ratio data suggests that the leading edge of a tank leak plume may be reaching the 41.1-meter (135-foot) bgs depth, but more vadose zone sediment extracts need to be studied from natural sediment profiles to develop a database that can be used to corroborate these hypotheses.

The other chemical results presented in Table 5.9 reveal a few noteworthy items. The data show little indication that the sediments contain leachable technetium-99 or chromium below 38.8 meters (127.4 feet) bgs. As mentioned, the chromium does not migrate as fast as the technetium-99 and its leading edge is found nearer to 34.1 meters (112 feet) bgs than 38.7 meters (127 feet) bgs. The agreement between the porewaters that were extracted from the vadose zone sediments and the dilution-corrected water extracts are good for the three deeper samples. For the two shallower samples (13 and 8), the extracted porewaters appear to have been contaminated or have higher concentrations of technetium-99 and chromium than the dilution-corrected water extracts.

The bulk of the water-leachable chromium has been confirmed as chromium(VI)(chromate) by its distinct yellow color and by ion chromatography (data not shown). There are no elevated concentrations of water-leachable uranium in any of the borehole sediments. The uranium concentrations found are similar to natural background levels in Hanford Site groundwater.

The chemical data show that borehole extension composite $2 \mathrm{C} / \mathrm{D}$ is influenced strongly by the drill bit cooling water that escaped from the casing when the drive shoe was breached. The water extracted from the sediment is dilute compared to the next composite (sediments immediately below [composites $2 \mathrm{~A} / \mathrm{B}$ and $3 \mathrm{~A} / \mathrm{B}$ ] in the Plio-Pleistocene strata). The extracts of composites from the Ringold Formation show the least dissolved salts. When the dilution correction calculation is made, the trends remain similar except that the composite with the largest dilution factor, 38A/B, the sample with the least moisture, shows as high a dissolved salt content as $2 \mathrm{~A} / \mathrm{B}$ and $3 \mathrm{~A} / \mathrm{B}$. This observation suggests that the sediments are leaching material into the extracts that are not present in the existing porewater.

\subsubsection{Radionuclide Analysis}

Table 5.10 lists the radionuclide analyses performed on the 1:1 water extracts and centrifuged porewaters. There is measurable water-leachable cesium-137 and technetium- 99 in samples between the depths of 18.3 and 40.5 meters (60 and 133 feet) bgs. The peak concentrations are found in the vicinity of 27.5 to 34.1 meters (90 to 112 feet) bgs for technetium-99 and there appears to be a bimodal cesium-137 peak with highs at 25 and 31.1 to 32.4 meters ( 82 and 102 to 108 feet) bgs, respectively. Very little 
Table 5.10. Radionuclide Content of Water Extracts and Porewater

\begin{tabular}{|c|c|c|c|c|c|c|c|c|}
\hline \multirow[b]{2}{*}{$\begin{array}{l}\text { Composite Depth } \\
\left(\mathrm{ft} \mathrm{bgs}^{(\mathrm{a})}\right.\end{array}$} & \multirow[b]{2}{*}{$\begin{array}{l}\text { 1:1 Extract } \\
\text { ID }\end{array}$} & \multirow[b]{2}{*}{$\begin{array}{c}\text { ICP-MS } \\
{ }^{99} \mathrm{Tc} \\
(\mathrm{pCi} / \mathrm{L})\end{array}$} & \multirow[b]{2}{*}{$\begin{array}{c}\text { GEA } \\
{ }^{137} \mathrm{Cs} \\
(\mathrm{pCi} / \mathrm{L})\end{array}$} & \multirow[b]{2}{*}{$\begin{array}{c}{ }^{137} \mathrm{Cs} \\
\text { Uncertainty } \\
(\mathrm{pCi} / \mathrm{L})\end{array}$} & \multirow[b]{2}{*}{$\begin{array}{l}\text { Dilution } \\
\text { Factor }\end{array}$} & \multicolumn{3}{|c|}{ Dilution Corrected } \\
\hline & & & & & & $\begin{array}{l}\text { ICP-MS } \\
{ }^{99} \mathrm{Tc} \\
(\mathrm{pCi} / \mathrm{L})\end{array}$ & $\begin{array}{c}\text { GEA } \\
{ }_{137} \mathrm{Cs} \\
(\mathrm{pCi} / \mathrm{L})\end{array}$ & $\begin{array}{l}{ }^{137} \mathrm{Cs} \\
\text { Uncertainty } \\
\text { (pCi/L) }\end{array}$ \\
\hline \multicolumn{9}{|c|}{ Sidewall } \\
\hline 25.5 & $15 \mathrm{~A} / \mathrm{B} / \mathrm{C}$ & $<1.0 \mathrm{E}+03$ & ND & & 12.33 & $<1.2 \mathrm{E}+04$ & ND & \\
\hline 44.5 & $14 \mathrm{~A} / \mathrm{B} / \mathrm{C}$ & $<1.0 \mathrm{E}+03$ & ND & & 11.67 & $<1.2 \mathrm{E}+04$ & ND & \\
\hline \multirow[t]{2}{*}{56.5} & $13 \mathrm{~A} / \mathrm{B} / \mathrm{C}$ & $<1.0 \mathrm{E}+03$ & $6.89 \mathrm{E}+03$ & $4.20 \mathrm{E}+03$ & 6.24 & $<6.2 \mathrm{E}+03$ & $4.30 \mathrm{E}+04$ & $2.62 \mathrm{E}+04$ \\
\hline & 13 UFA & & & & & $2.88 \mathrm{E}+05$ & ND & \\
\hline 61.5 & $12 \mathrm{~A} / \mathrm{B} / \mathrm{C}$ & $7.0 \mathrm{E}+02$ & $9.57 \mathrm{E}+03$ & $6.20 \mathrm{E}+03$ & 7.79 & $5.43 \mathrm{E}+03$ & $7.46 \mathrm{E}+04$ & $4.83 \mathrm{E}+04$ \\
\hline 65.5 & $11 \mathrm{~A} / \mathrm{B}$ & $4.1 \mathrm{E}+03$ & $2.45 \mathrm{E}+04$ & $1.20 \mathrm{E}+04$ & 21.24 & $8.63 \mathrm{E}+04$ & $5.20 \mathrm{E}+05$ & $2.55 \mathrm{E}+05$ \\
\hline 66 & $11 \mathrm{C}$ & $7.8 \mathrm{E}+03$ & $3.97 \mathrm{E}+06$ & $2.06 \mathrm{E}+05$ & 18.92 & $1.48 \mathrm{E}+05$ & $7.52 \mathrm{E}+07$ & $3.89 \mathrm{E}+06$ \\
\hline 69.5 & $10 \mathrm{~A} / \mathrm{B} / \mathrm{C}$ & $5.5 \mathrm{E}+03$ & $7.49 \mathrm{E}+04$ & $8.34 \mathrm{E}+03$ & 22.94 & $1.27 \mathrm{E}+05$ & $1.72 \mathrm{E}+06$ & $1.91 \mathrm{E}+05$ \\
\hline \multirow[t]{2}{*}{74.5} & $09 \mathrm{~A} / \mathrm{B} / \mathrm{C}$ & $7.7 \mathrm{E}+03$ & $2.61 \mathrm{E}+05$ & $1.15 \mathrm{E}+04$ & 19.37 & $1.49 \mathrm{E}+05$ & $5.05 \mathrm{E}+06$ & $2.22 \mathrm{E}+05$ \\
\hline & 9 UFA & & & & & - & $9.79 \mathrm{E}+04$ & $1.44 \mathrm{E}+04$ \\
\hline \multirow[t]{2}{*}{79.5} & $08 \mathrm{~A} / \mathrm{B} / \mathrm{C}$ & $1.8 \mathrm{E}+04$ & $2.67 \mathrm{E}+05$ & $1.30 \mathrm{E}+06$ & 9.34 & $1.70 \mathrm{E}+05$ & $2.49 \mathrm{E}+06$ & $1.21 \mathrm{E}+07$ \\
\hline & 8 UFA & & & & & $5.17 \mathrm{E}+05$ & $8.71 \mathrm{E}+05$ & $2.08 \mathrm{E}+04$ \\
\hline 82.5 & $07 \mathrm{~A} / \mathrm{B} / \mathrm{C}$ & $3.9 \mathrm{E}+05$ & $3.82 \mathrm{E}+07$ & $1.41 \mathrm{E}+05$ & 11.90 & $4.67 \mathrm{E}+06$ & $4.54 \mathrm{E}+08$ & $1.68 \mathrm{E}+06$ \\
\hline \multirow[t]{2}{*}{90} & 06A/B & $2.7 \mathrm{E}+06$ & $2.21 \mathrm{E}+05$ & $3.36 \mathrm{E}+04$ & 9.76 & $2.68 \mathrm{E}+07$ & $2.15 \mathrm{E}+06$ & $3.28 \mathrm{E}+05$ \\
\hline & 6 UFA & & & & & $2.48 \mathrm{E}+07$ & $6.09 \mathrm{E}+06$ & $1.15 \mathrm{E}+05$ \\
\hline 95.5 & $03 \mathrm{~A} / \mathrm{B} / \mathrm{C}$ & $7.1 \mathrm{E}+06$ & $7.47 \mathrm{E}+05$ & $2.28 \mathrm{E}+04$ & 12.71 & $8.99 \mathrm{E}+07$ & $9.49 \mathrm{E}+06$ & $2.90 \mathrm{E}+05$ \\
\hline 102.5 & $05 \mathrm{~A} / \mathrm{B} / \mathrm{C}$ & $6.1 \mathrm{E}+06$ & $9.67 \mathrm{E}+06$ & $1.34 \mathrm{E}+05$ & 9.61 & $5.90 \mathrm{E}+07$ & $9.29 \mathrm{E}+07$ & $1.29 \mathrm{E}+06$ \\
\hline \multirow[t]{2}{*}{108.5} & $04 \mathrm{~A} / \mathrm{B} / \mathrm{C}$ & $1.2 \mathrm{E}+07$ & $1.64 \mathrm{E}+06$ & $3.19 \mathrm{E}+04$ & 8.33 & $9.92 \mathrm{E}+07$ & $1.36 \mathrm{E}+07$ & $2.66 \mathrm{E}+05$ \\
\hline & 4 UFA & & & & & $9.40 \mathrm{E}+07$ & $3.27 \mathrm{E}+07$ & $4.58 \mathrm{E}+05$ \\
\hline
\end{tabular}


Table 5.10. (contd)

\begin{tabular}{|c|c|c|c|c|c|c|c|c|}
\hline \multirow[b]{2}{*}{$\begin{array}{l}\text { Composite Depth } \\
(\mathrm{ft} \text { bgs) })^{(\mathrm{a})}\end{array}$} & \multirow[b]{2}{*}{$\begin{array}{l}\text { 1:1 Extract } \\
\text { ID }\end{array}$} & \multirow[b]{2}{*}{$\begin{array}{l}\text { ICP-MS } \\
{ }_{99} \mathrm{Tc} \\
(\mathrm{pCi} / \mathrm{L})\end{array}$} & \multirow[b]{2}{*}{$\begin{array}{c}\text { GEA } \\
{ }^{137} \mathrm{Cs} \\
(\mathrm{pCi} / \mathrm{L})\end{array}$} & \multirow[b]{2}{*}{$\begin{array}{l}{ }^{137} \mathrm{Cs} \\
\text { Uncertainty } \\
(\mathrm{pCi} / \mathrm{L})\end{array}$} & \multirow[b]{2}{*}{$\begin{array}{l}\text { Dilution } \\
\text { Factor }\end{array}$} & \multicolumn{3}{|c|}{ Dilution Corrected } \\
\hline & & & & & & $\begin{array}{l}\text { ICP-MS } \\
{ }_{99} \mathrm{Tc} \\
(\mathrm{pCi} / \mathrm{L})\end{array}$ & $\begin{array}{c}\text { GEA } \\
{ }^{137} \mathrm{Cs} \\
(\mathrm{pCi} / \mathrm{L})\end{array}$ & $\begin{array}{c}{ }^{137} \mathrm{Cs} \\
\text { Uncertainty } \\
\text { (pCi/L) }\end{array}$ \\
\hline \multirow[t]{2}{*}{112} & $02 \mathrm{~B} / \mathrm{C}$ & $8.6 \mathrm{E}+06$ & $6.30 \mathrm{E}+03$ & $6.05 \mathrm{E}+03$ & 12.26 & $1.05 \mathrm{E}+08$ & $7.73 \mathrm{E}+04$ & $7.42 \mathrm{E}+04$ \\
\hline & 2 UFA & & & & & $1.23 \mathrm{E}+08$ & $1.11 \mathrm{E}+05$ & $1.43 \mathrm{E}+04$ \\
\hline 127.4 & $01 \mathrm{~A} / \mathrm{B} / \mathrm{C}$ & $3.3 \mathrm{E}+05$ & $6.34 \mathrm{E}+03$ & $4.73 \mathrm{E}+03$ & 7.90 & $2.64 \mathrm{E}+06$ & $5.01 \mathrm{E}+04$ & $3.73 \mathrm{E}+04$ \\
\hline \multicolumn{4}{|c|}{ Extension } & \multicolumn{5}{|c|}{ Rad } \\
\hline 133.2 & $2 \mathrm{C} / \mathrm{D}$ & $1,000 \pm 2,500$ & 4,110 & & 5.25 & -- & $2.16 \mathrm{E}+04$ & \\
\hline 134.2 & $2 \mathrm{~A} / \mathrm{B}$ & $5,650 \pm 2,200$ & 1,500 & & 8.98 & -- & $1.35 \mathrm{E}+04$ & \\
\hline 135.2 & $3 \mathrm{~A} / \mathrm{B}$ & $2,720 \pm 2,500$ & $<600$ & & 10.7 & -- & -- & \\
\hline 153.9 & $10 \mathrm{~A} / \mathrm{B}$ & $3,900 \pm 5,200$ & $<600$ & & 6.65 & -- & -- & \\
\hline 156.8 & $12 \mathrm{~A} / \mathrm{B}$ & $380 \pm 2,900$ & 550 & & 19.4 & -- & $1.07 \mathrm{E}+04$ & \\
\hline 165.3 & $17 \mathrm{~A} / \mathrm{B}$ & $9,320 \pm 12,800$ & $<600$ & & 26.3 & -- & -- & \\
\hline 188.3 & $38 \mathrm{~A} / \mathrm{B}$ & $50 \pm 2,800$ & $<600$ & & 124 & -- & -- & \\
\hline 196.9 & $47 \mathrm{~A} / \mathrm{B}$ & $(-190) \pm 4,700$ & $<600$ & & 46.5 & -- & -- & \\
\hline 208.6 & $58 \mathrm{~A} / \mathrm{B}$ & $(-470) \pm 3,800$ & $<600$ & & 22.7 & -- & -- & \\
\hline \multicolumn{9}{|c|}{$\begin{array}{l}\text { ND = Not detected (below detection limit). } \\
-\quad=\text { Analytical data is poor quality because low concentrations are present. } \\
\text { Values within parentheses are below limit of quantification but spectra was good enough to assign tentative value. } \\
\text { Values in red are averages of duplicate samples. } \\
\text { (a) Multiply by } 0.3048 \text { to convert to meters. }\end{array}$} \\
\hline
\end{tabular}


cesium-137 or technetium-99 activity was found in the water extracts of the sediments below 38.7 meters (127 feet) bgs. Strontium-90 analyses were not performed on water extracts because no detectable strontium-90 was found in most of the sediment samples that were measured (data to be discussed later).

The technetium-99 analyses performed using wet chemical separations and radiocounting for the borehole extension water extracts showed a wide variability in the duplicate analyses, as indicated in the footnotes to Table 5.10. Given the small volume of extract available and the high detection limit, the technetium-99 data are not useful. The agreement between the dilution-corrected water extracts and the actual porewaters from the sidewall cores is fair to excellent for technetium-99. For cesium-137, the porewaters appear to have lower activities than the water extracts. Possible explanations are that the water extract process releases some sorbed cesium-137 during the vigorous shaking that occurs during the mixing process and the cesium does not re-equilibrate before the fluid is separated. The extract data may also contain colloidal cesium-137 that passes through the filtering step and is analyzed with the truly soluble phase. During centrifugation, the centrifugal forces may not be large enough to flocculate colloids. A final possibility for the differing cesium-137 concentrations is that the sediment samples used to obtain the water extract fluids and the porewater were not the same aliquot. Thus, sample heterogeneity may be the cause for less than ideal agreement. Sample heterogeneity would be expected to affect cesium-137 more than technetium-99 because cesium adsorbs strongly to certain clay particles and colloids that may not be homogeneously distributed in the sidewall core and sleeve samples that were only partially mixed prior to subsampling.

\subsection{Characterization Results and Discussion for Composite Sediments}

Characterization included analyses of the dried solids for several radionuclides, total chemical composition, cation exchange capacity, and particle size analyses. The cesium-137 distribution on individual particle size separates for selected composite sediments also was measured.

\subsubsection{Radionuclide Content of Composite Sidewall Cores and Borehole Extension Sediments}

The activity of cesium-137, strontium-90, technetium-99, and tritium in the composite sediments is reported in Table 5.11, along with the counting uncertainty, or one standard deviation of the mean when two or three replicates were measured. In almost all cases, the standard deviation of the mean of the replicates was larger than the instrument counting error, suggesting that the samples were not as well mixed as we had hoped. The radionuclide data as a function of depth are shown in Figure 5.3. It can be seen that there appears to be two peaks in the cesium-137 activity at 20.1 and 25.1 meters (66 and 82.5 feet) bgs. The former is a bit lower activity $\left(4 \times 10^{5} \mathrm{pCi} / \mathrm{g}\right)$ and the deeper peak is $\sim 2 \times 10^{7} \mathrm{pCi} / \mathrm{g}$. The composites $2 \mathrm{C} / \mathrm{D}, 2 \mathrm{~A} / \mathrm{B}$, and $3 \mathrm{~A} / \mathrm{B}$ contain elevated levels of cesium-137 but it is difficult to determine whether this is drag down of the compressed plug from pile driving the casing to 39.9 meters (131 feet) bgs or if the activity is truly in the formation. The GEA of individual sleeves was reported in Table.4.5. Sleeve 2A (at 41.1 meters [134.7 feet] bgs) has rather low cesium-137 activity but sleeves 3A and 3B show 100 times higher activity. We suspect that there is a compression ring of disturbed sediment in this area from the pile driving and end cap milling operations. We can say that the cesium-137 does drop off 
Table 5.11. Total Radionuclide Content of Sediment Samples (pCi/g dry wt)

\begin{tabular}{|c|c|c|c|c|c|c|c|c|c|}
\hline $\begin{array}{c}\text { Composite Depth } \\
\text { (ft bgs) }^{\text {(a) }}\end{array}$ & ID & $\begin{array}{c}{ }^{137} \mathrm{Cs} \\
\text { (pCi/g) } \\
\text { GEA }\end{array}$ & $\begin{array}{l}( \pm) \mathrm{pCi} / \mathrm{g} \\
\text { Uncertainty }\end{array}$ & $\begin{array}{c}{ }^{90} \mathrm{Sr} \\
(\mathrm{pCi} / \mathrm{g}) \\
\mathrm{Rad}\end{array}$ & $\begin{array}{l}( \pm) \mathrm{pCi} / \mathrm{g} \\
\text { Uncertainty }\end{array}$ & $\begin{array}{c}{ }^{99} \mathrm{Tc} \\
(\mathrm{pCi} / \mathrm{g}) \\
\text { ICP/MS }\end{array}$ & $\begin{array}{c}{ }^{99} \mathrm{Tc} \\
(\mathrm{pCi} / \mathrm{g}) \\
\mathrm{Rad}\end{array}$ & $\begin{array}{l}( \pm) \mathrm{pCi} / \mathrm{g} \\
\text { Uncertainty }\end{array}$ & $\begin{array}{c}{ }^{3} \mathrm{H} \\
\text { (pCi/g) } \\
\mathrm{Rad} \\
\end{array}$ \\
\hline \multicolumn{10}{|c|}{ Sidewall } \\
\hline 25.5 & $15 \mathrm{~A} / \mathrm{B} / \mathrm{C}$ & $5.943 \mathrm{E}+02$ & $9.699 \mathrm{E}+00$ & -4.8 & 20.4 & $<19.95$ & -49.9 & 32 & 2.9 \\
\hline 44.5 & $14 \mathrm{~A} / \mathrm{B} / \mathrm{C}$ & $1.097 \mathrm{E}+03$ & $1.834 \mathrm{E}+01$ & -3.6 & 19.5 & (5.96) & -4.7 & 33 & 2.4 \\
\hline 56.5 & $13 \mathrm{~A} / \mathrm{B} / \mathrm{C}$ & $2.743 \mathrm{E}+04$ & $5.273 \mathrm{E}+02$ & -1.7 & 21.1 & 30.32 & 313.2 & 56 & 3.9 \\
\hline 61.5 & $12 \mathrm{~A} / \mathrm{B} / \mathrm{C}$ & $1.275 \mathrm{E}+05$ & $2.192 \mathrm{E}+03$ & 0.0 & 20.7 & 10.44 & -17.5 & 32 & 5.4 \\
\hline 65.5 & $11 \mathrm{~A} / \mathrm{B}$ & $5.944 \mathrm{E}+05$ & $1.059 \mathrm{E}+04$ & 3.4 & 19.8 & 25.59 & -11.8 & 34 & 7.5 \\
\hline 66 & $11 \mathrm{C}$ & $3.908 \mathrm{E}+06$ & $6.889 \mathrm{E}+04$ & $3.9 \pm 13.3$ & & $(13 \pm 4.3)$ & $0 \pm 30$ & & 7.3 \\
\hline 69.5 & $10 \mathrm{~A} / \mathrm{B} / \mathrm{C}$ & $8.988 \mathrm{E}+03$ & $1.096 \mathrm{E}+02$ & -0.2 & 18.8 & $(25.24)$ & 95.3 & 41 & 8.2 \\
\hline 74.5 & 09A/B/C & $2.235 \mathrm{E}+06$ & $3.937 \mathrm{E}+04$ & 18.4 & 22.8 & $(35.61)$ & 12.2 & 30 & 16.2 \\
\hline 79.5 & 08A/B/C & $2.569 \mathrm{E}+06$ & $4.539 \mathrm{E}+04$ & 6.9 & 21.3 & 2,527 & $3,164.3$ & 132 & 31.4 \\
\hline 82.5 & $07 \mathrm{~A} / \mathrm{B} / \mathrm{C}$ & $1.730 \mathrm{E}+07$ & $4.307 \mathrm{E}+05$ & $4.8 \pm 12$ & & $1,079 \pm 350$ & $1,150 \pm 300$ & & 108.0 \\
\hline 90 & 06A/B & $4.379 \mathrm{E}+04$ & $5.319 \mathrm{E}+02$ & -7.9 & 19.1 & 3,241 & $3,586.3$ & 143 & 149.2 \\
\hline 95.5 & $03 \mathrm{~A} / \mathrm{B} / \mathrm{C}$ & $3.661 \mathrm{E}+04$ & $4.656 \mathrm{E}+02$ & 6.4 & 20.1 & 7,597 & $7,447.9$ & 238 & 138.6 \\
\hline 102.5 & $05 \mathrm{~A} / \mathrm{B} / \mathrm{C}$ & $1.586 \mathrm{E}+06$ & $2.825 \mathrm{E}+04$ & -6.6 & 20.9 & 12,979 & $13,036.0$ & 384 & 37.1 \\
\hline 108.5 & $04 \mathrm{~A} / \mathrm{B} / \mathrm{C}$ & $3.355 \mathrm{E}+05$ & $7.544 \mathrm{E}+03$ & -49.2 & 12.0 & 13,766 & $13,877.2$ & 399 & 15.7 \\
\hline 112 & $02 \mathrm{~B} / \mathrm{C}$ & $1.432 \mathrm{E}+03$ & $2.349 \mathrm{E}+01$ & 0.9 & 19.6 & 9,840 & $9,906.5$ & 299 & 118.2 \\
\hline \multirow[t]{2}{*}{127.4} & $01 \mathrm{~A} / \mathrm{B} / \mathrm{C}$ & $4.199 \mathrm{E}+03$ & $6.400 \mathrm{E}+01$ & -16.0 & 16.5 & 405 & $4,04.7$ & 50 & 34.0 \\
\hline & Extension & & & & & & & & \\
\hline 133.2 & $2 \mathrm{C} / \mathrm{D}$ & $3.42 \mathrm{E}+04$ & $5.76 \mathrm{E}+02$ & 1.0 & 2.5 & $(172 \pm 22)$ & 333.0 & $10^{*}$ & NA \\
\hline 134.2 & $2 \mathrm{~A} / \mathrm{B}$ & $1.29 \mathrm{E}+03$ & $5.72 \mathrm{E}+01$ & 5.7 & 2.2 & NA & 134.0 & $87^{*}$ & NA \\
\hline 135.2 & $3 \mathrm{~A} / \mathrm{B}$ & $3.90 \mathrm{E}+03$ & $1.04 \mathrm{E}+02$ & 3.4 & 2.5 & NA & 158.0 & $89 *$ & NA \\
\hline 153.9 & $10 \mathrm{~A} / \mathrm{B}$ & $<0.02$ & & $3.9 \pm 5.2$ & & NA & 2.3 & $4.6^{*}$ & NA \\
\hline
\end{tabular}


Table 5.11. (contd)

\begin{tabular}{|c|c|c|c|c|c|c|c|c|c|}
\hline $\begin{array}{c}\text { Composite Depth } \\
\text { (ft bgs) }^{(\mathrm{a})}\end{array}$ & ID & $\begin{array}{c}{ }^{137} \mathrm{Cs} \\
(\mathrm{pCi} / \mathrm{g}) \\
\mathrm{GEA} \\
\end{array}$ & $\begin{array}{l}( \pm) \mathrm{pCi} / \mathrm{g} \\
\text { Uncertainty }\end{array}$ & $\begin{array}{c}{ }^{90} \mathrm{Sr} \\
(\mathrm{pCi} / \mathrm{g}) \\
\mathrm{Rad} \\
\end{array}$ & $\begin{array}{c}( \pm) \mathrm{pCi} / \mathrm{g} \\
\text { Uncertainty }\end{array}$ & $\begin{array}{c}{ }^{99} \mathrm{Tc} \\
(\mathrm{pCi} / \mathrm{g}) \\
\mathrm{ICP} / \mathrm{MS} \\
\end{array}$ & $\begin{array}{c}{ }^{99} \mathrm{Tc} \\
(\mathrm{pCi} / \mathrm{g}) \\
\mathrm{Rad} \\
\end{array}$ & $\begin{array}{l}( \pm) \mathrm{pCi} / \mathrm{g} \\
\text { Uncertainty }\end{array}$ & $\begin{array}{c}{ }^{3} \mathrm{H} \\
(\mathrm{pCi} / \mathrm{g}) \\
\mathrm{Rad} \\
\end{array}$ \\
\hline 156.8 & $12 \mathrm{~A} / \mathrm{B}$ & $7.52 \mathrm{E}+01$ & $1.23 \mathrm{E}+00$ & 0.4 & 2.9 & NA & 2.9 & $2.5^{*}$ & NA \\
\hline 165.3 & $17 \mathrm{~A} / \mathrm{B}$ & $<0.02$ & & $9.32 \pm 12.8$ & & NA & $(-3.1 \pm 4.6)$ & & NA \\
\hline 188.3 & $38 \mathrm{~A} / \mathrm{B}$ & $<0.02$ & & 0.1 & 3.0 & NA & $0.11 \pm 0.05$ & & NA \\
\hline 196.9 & $47 \mathrm{~A} / \mathrm{B}$ & $<0.02$ & & -0.2 & 4.3 & NA & $1.3 \pm 1.5$ & & NA \\
\hline 208.6 & $58 \mathrm{~A} / \mathrm{B} / \mathrm{C}$ & $<0.02$ & & -0.5 & 3.7 & NA & 0.9 & $0.87 *$ & NA \\
\hline \multicolumn{10}{|c|}{$\begin{array}{l}\text { NA }=\text { Not analyzed. * To uncertainty based on b } \\
\text { Values within parentheses are below limit of qua } \\
\text { Values in red are averages of duplicate samples. } \\
\text { (a) Multiply by } 0.3048 \text { to convert to meters. }\end{array}$} \\
\hline
\end{tabular}




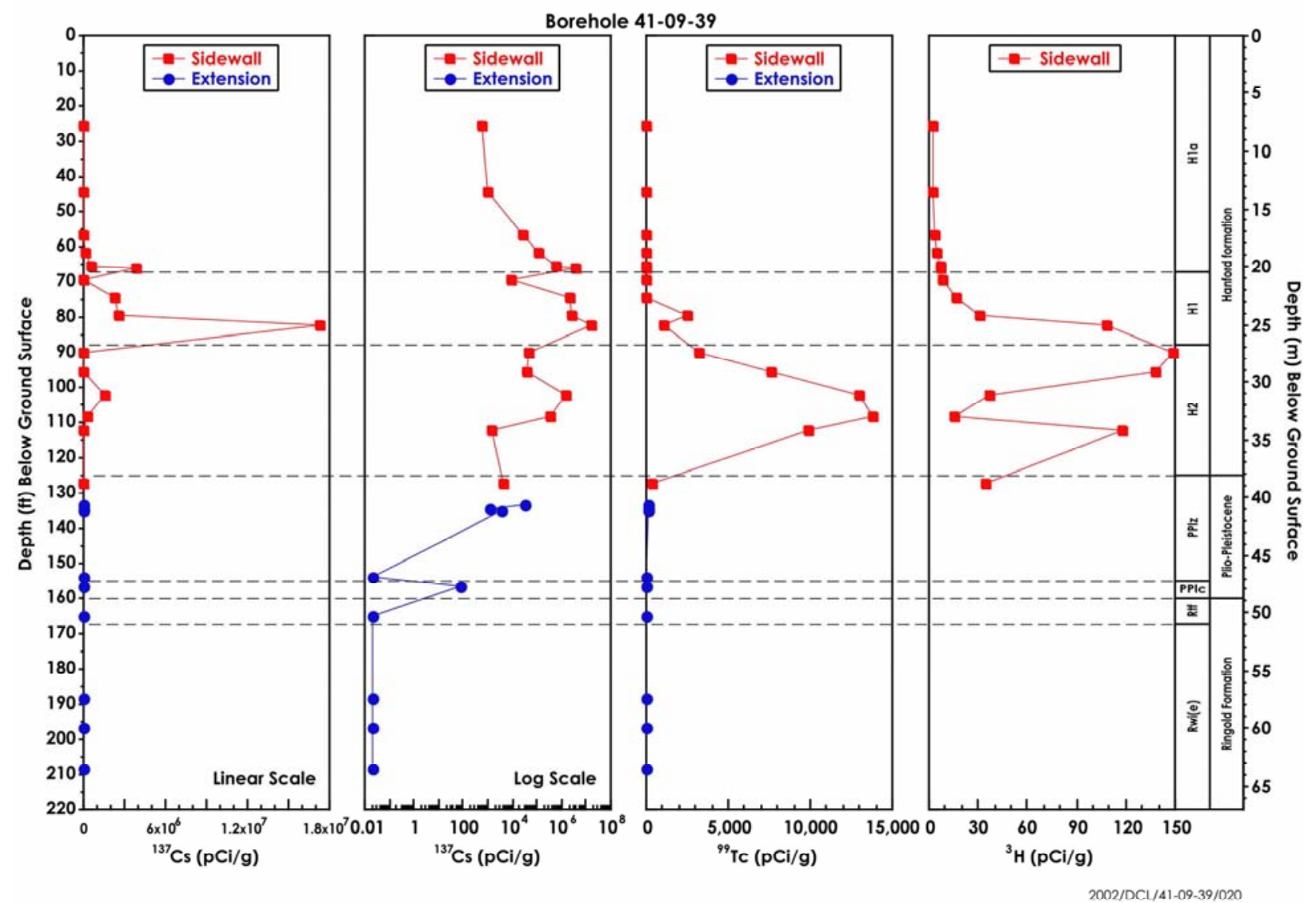

Figure 5.3. Cesium-137, Technetium-99, Strontium-90, and Tritium in Sediments from Table 5.11 Versus Depth with Geology Breaks

beyond 42.1 meters (138 feet) bgs except in sleeves that were obtained after the whole casing had been removed to insert a drive shoe on the casing. Technetium-99 activities appear to be in the formation down to a depth of 41.1 meters (135 feet) bgs. None of the composites show elevated levels of strontium90. The tritium distribution appears to be bimodal with the main peak between 25.1 and 29.1 meters ( 82.5 and 95.5 feet) bgs with a second slightly lower peak at 34.1 meters (112 feet) bgs. It is not clear whether the tritium is traveling along with the technetium- 99 and nitrate and our detection limit is not sensitive enough to follow the leading edge of the tritium or the distribution is not the same as these two mobile constituents.

The actinide content of the sidewall cores was determined by acid extraction, wet chemical separations, electrodeposition, and alpha energy analysis. The results are shown in Table 5.12 and suggest that actinide levels are very low. We believe that the plutonium value for sample 13ABC is accurate, but the neptunium values that appear to be above our counting/separation uncertainty (marked in shading) may not be accurate. 
Table 5.12. Actinide Content of the Sidewall Cores (pCi/g)

\begin{tabular}{|c|c|c|c|c|c|c|c|c|c|c|}
\hline $\begin{array}{c}\text { Depth } \\
\text { (ft bgs) }^{(a)}\end{array}$ & Sample & $\begin{array}{c}\mathrm{Pu}^{239} \\
(\mathrm{pCi} / \mathrm{g}) \\
\end{array}$ & $\begin{array}{c}\text { Uncertainty } \\
(\mathrm{pCi} / \mathrm{g})\end{array}$ & $\begin{array}{c}\text { Min. Detect } \\
(\mathrm{pCi} / \mathrm{g})\end{array}$ & $\begin{array}{c}\mathrm{Am}^{241} \\
(\mathrm{pCi} / \mathrm{g}) \\
\end{array}$ & $\begin{array}{c}\text { Uncertainty } \\
(\mathrm{pCi} / \mathrm{g})\end{array}$ & $\begin{array}{c}\text { Min. Detect } \\
(\mathrm{pCi} / \mathrm{g})\end{array}$ & $\begin{array}{c}\mathrm{Np}^{237} \\
(\mathrm{pCi} / \mathrm{g}) \\
\end{array}$ & $\begin{array}{c}\text { Uncertainty } \\
(\mathrm{pCi} / \mathrm{g})\end{array}$ & $\begin{array}{c}\text { Min. Detect } \\
(\mathrm{pCi} / \mathrm{g}) \\
\end{array}$ \\
\hline $25-26$ & $15 \mathrm{~A} / \mathrm{B} / \mathrm{C}$ & -0.113 & 0.019 & 0.005 & -0.220 & 0.032 & 0.004 & 0.016 & 0.005 & 0.008 \\
\hline 44-45 & $14 \mathrm{~A} / \mathrm{B} / \mathrm{C}$ & -0.119 & 0.019 & 0.012 & -0.035 & 0.035 & 0.003 & 0.167 & 0.049 & 0.051 \\
\hline $56-57$ & $13 \mathrm{~A} / \mathrm{B} / \mathrm{C}$ & 3.960 & 0.171 & 0.005 & 0.088 & 0.038 & 0.004 & 0.014 & 0.004 & 0.007 \\
\hline $61-62$ & $12 \mathrm{~A} / \mathrm{B} / \mathrm{C}$ & -0.109 & 0.019 & 0.006 & -0.216 & 0.033 & 0.009 & 0.033 & 0.009 & 0.013 \\
\hline $65-66$ & $11 \mathrm{~A} / \mathrm{B}$ & -0.076 & 0.022 & 0.016 & -0.200 & 0.032 & 0.003 & -0.002 & 0.002 & 0.018 \\
\hline $65-66$ & $11 \mathrm{C}$ & -0.112 & 0.018 & 0.012 & -0.198 & 0.033 & 0.004 & 0.001 & 0.004 & 0.023 \\
\hline $69-70$ & $10 \mathrm{~A} / \mathrm{B} / \mathrm{C}$ & -0.115 & 0.019 & 0.005 & -0.161 & 0.033 & 0.003 & 0.003 & 0.003 & 0.012 \\
\hline $74-75$ & $9 \mathrm{~A} / \mathrm{B} / \mathrm{C}$ & -0.111 & 0.019 & 0.012 & -0.032 & 0.036 & 0.003 & 0.031 & 0.014 & 0.039 \\
\hline $79-80$ & $8 \mathrm{~A} / \mathrm{B} / \mathrm{C}$ & -0.118 & 0.019 & 0.007 & -0.025 & 0.038 & 0.004 & 0.004 & 0.002 & 0.006 \\
\hline $82-83$ & $7 \mathrm{~A} / \mathrm{B} / \mathrm{C}$ & -0.090 & 0.020 & 0.006 & -0.213 & 0.035 & 0.017 & 0.048 & 0.010 & 0.025 \\
\hline 90 & $6 \mathrm{~A} / \mathrm{B}$ & -0.111 & 0.018 & 0.013 & -0.238 & 0.034 & 0.005 & 0.038 & 0.242 & 1.260 \\
\hline $95-96$ & $3 \mathrm{~A} / \mathrm{B} / \mathrm{C}$ & 0.012 & 0.024 & 0.010 & 0.085 & 0.045 & 0.006 & 0.900 & 0.704 & 2.370 \\
\hline $102-103$ & $5 \mathrm{~A} / \mathrm{B} / \mathrm{C}$ & -0.085 & 0.020 & 0.011 & -0.214 & 0.040 & 0.009 & 0.217 & 0.294 & 1.180 \\
\hline 108-109 & $4 \mathrm{~A} / \mathrm{B} / \mathrm{C}$ & -0.053 & 0.021 & 0.004 & 0.783 & 1.405 & 3.406 & -0.224 & 0.129 & 0.890 \\
\hline 112 & $2 \mathrm{~B} / \mathrm{C}$ & -0.081 & 0.020 & 0.005 & 0.709 & 0.658 & 0.796 & -0.052 & 0.052 & 0.433 \\
\hline 127.4 & $1 \mathrm{~A} / \mathrm{B} / \mathrm{C}$ & -0.019 & 0.023 & 0.005 & -0.217 & 0.041 & 0.023 & 0.946 & 0.368 & 0.284 \\
\hline
\end{tabular}


The ratio of cesium-137 to technetium-99 activity in these sediments from 18.3 to 25 meters (60 to 82 feet) bgs is $>10,000$. Johnson and Chou (1998) suggested that the ratio in SX tank liquors would be 10,000. The observed ratios suggest that cesium and technetium-99 may be traveling at the same rate through these shallow sediments within 7.6 meters ( 25 feet) of the tank bottom and within 3 to 4.6 meters (10 to 15 feet) from the side of tank SX-109 and 10.7 meters ( 35 lateral feet) from the side of tank SX108. However, between the depths of 27.4 and 40.8 meters (90 and 134 feet) bgs, the cesium-137 to technetium-99 ratio ranges from $<1$ to $\sim 100$, which suggests that cesium gets removed from the fluid and technetium-99 continues to migrate deeper into the sediments. Both nuclides drop to very low concentrations after 41.1 meters (135 feet) bgs. The hypothesis that the two nuclides have the same $\mathrm{K}_{\mathrm{d}}$ value can be addressed using the results of the 1:1 sediment-to-water extracts with the total activities in the sediments.

By combining the data from the dilution-corrected 1:1 sediment-to-water extracts that represent the porewater (see Table 5.10) with the activities measured on the sediments (see Table 5.11), we can get a semiquantitative sense of what the desorption $\mathrm{K}_{\mathrm{d}}$ is. For a contaminant that has very little water-soluble mass, such as cesium-137, the $\mathrm{K}_{\mathrm{d}}$ can be approximated as the amount of mass on the solid per gram of dry sediment divided by the amount of mass in the porewater per milliliter. For a contaminant that is quite soluble in the water extract ( equivalent to saying that the contaminant resides mainly in the porewater within the sediment), one needs to subtract the amount that was water extractable from the total amount present in the sediment to obtain a value for the amount that would remain on the solid at equilibrium with the pore fluid. This value for the amount left on the sediment becomes the numerator, and the concentration in the porewater (water extract multiplied by the dilution factor) becomes the denominator in the $K_{d}$ calculation. Table 5.13 shows the calculated $K_{d}$ values for cesium-137 and technetium- 99 for the sidewall cores and a few of the composites from the borehole extension.

The data in Table 5.13 show that the in situ $\mathrm{K}_{\mathrm{d}}$ for cesium varies from 4 to $25 \mathrm{~mL} / \mathrm{g}$ in the sediments between 27.4 and 34.1 meters (90 and 112 feet) bgs where the bulk of the tank fluid with high salinity resides. Above and below this depth range, cesium in situ $\mathrm{K}_{\mathrm{d}}$ is larger because the pore fluids do not contain high sodium concentrations that compete for adsorption sites.

The technetium-99 in situ $\mathrm{K}_{\mathrm{d}}$ varies from 0.01 to about $5 \mathrm{~mL} / \mathrm{g}$ over the whole zone of contamination. The agreement between in situ $K_{d}$ values calculated using the water extract versus the actual porewater is good for all data sets except sample 13. These cesium and technetium in situ $\mathrm{K}_{\mathrm{d}}$ values suggest that cesium is held more strongly to the sediment than technetium. The technetium data are consistent with a wealth of literature that finds essentially no technetium adsorption onto Hanford Site sediments from less saline waters (Kaplan et al. 1995, 1996, 1998). Therefore, despite the fact that the ratio of cesium-137 to technetium-99 in the sediment between 18.3 and 25 meters ( 60 and 82 feet) bgs is >10,000 and similar to the ratio expected in the tank supernatant fluids, there is no reason to believe that the two nuclides would migrate together through the sediments today based on the in situ $\mathrm{K}_{\mathrm{d}}$ construct.

\subsubsection{Total Chemical Composition of Composite Sediments}

Table 5.14 lists the total concentration of selected constituents in the composite borehole extension sediments obtained by completely dissolving an aliquot of each sediment. The total chemical analysis of 
Table 5.13. $K_{d}$ Estimates from Total Activity in Sediments and Porewaters (mL/g)

\begin{tabular}{|c|c|c|c|c|c|c|c|c|c|c|c|}
\hline $\begin{array}{c}\text { Depth } \\
(\mathrm{ft} \text { bgs) } \\
\end{array}$ & $\begin{array}{c}\text { Sample } \\
\text { ID } \\
\end{array}$ & $\begin{array}{l}{ }^{137} \mathrm{Cs} \\
\mathrm{K}_{\mathrm{d}} \\
\end{array}$ & $\begin{array}{c}{ }^{137} \mathrm{Cs} \\
\% \text { Water } \\
\text { Leachable } \\
\end{array}$ & $\begin{array}{c}{ }^{99} \mathrm{Tc} \\
\mathrm{K}_{\mathrm{d}} \\
\end{array}$ & $\begin{array}{c}{ }^{99} \mathrm{Tc} \\
\% \text { Water } \\
\text { Leachable } \\
\end{array}$ & $\begin{array}{c}\text { Depth } \\
\text { (ft bgs) }^{(\mathrm{a})} \\
\end{array}$ & $\begin{array}{c}\text { Sample } \\
\text { ID }\end{array}$ & $\begin{array}{c}{ }^{137} \mathrm{Cs} \\
\mathrm{K}_{\mathrm{d}} \\
\end{array}$ & $\begin{array}{c}{ }^{137} \mathrm{Cs} \\
\% \text { Water } \\
\text { Leachable } \\
\end{array}$ & $\begin{array}{c}{ }^{99} \mathrm{Tc} \\
\mathrm{K}_{\mathrm{d}} \\
\end{array}$ & $\begin{array}{c}{ }^{99} \mathrm{Tc} \\
\% \text { Water } \\
\text { Leachable } \\
\end{array}$ \\
\hline & Sidewall & & & & & & Sidewall & & & & \\
\hline 25.5 & $15 \mathrm{~A} / \mathrm{B} / \mathrm{C}$ & -- & -- & -- & $>81 \%$ & 102.5 & $05 \mathrm{~A} / \mathrm{B} / \mathrm{C}$ & 17 & $0.60 \%$ & 0.12 & $47 \%$ \\
\hline 44.5 & $14 \mathrm{~A} / \mathrm{B} / \mathrm{C}$ & -- & -- & 0.43 & $>16 \%$ & 108.5 & $04 \mathrm{~A} / \mathrm{B} / \mathrm{C}$ & 25 & $0.48 \%$ & 0.02 & $86 \%$ \\
\hline \multirow{2}{*}{56.5} & $13 \mathrm{~A} / \mathrm{B} / \mathrm{C}$ & 638 & $0.03 \%$ & 4.70 & $2 \%$ & & 4 UFA & 10 & -- & 0.02 & -- \\
\hline & 13 UFA & -- & -- & 95.26 & -- & 112 & $02 \mathrm{~B} / \mathrm{C}$ & 19 & $0.42 \%$ & 0.01 & $87 \%$ \\
\hline 61.5 & $12 \mathrm{~A} / \mathrm{B} / \mathrm{C}$ & 1,709 & $0.01 \%$ & 1.79 & $6 \%$ & & 2 UFA & 13 & -- & 0.01 & -- \\
\hline 65.5 & $11 \mathrm{~A} / \mathrm{B}$ & 1,143 & $0.04 \%$ & 0.25 & $16 \%$ & 127.4 & $01 \mathrm{~A} / \mathrm{B} / \mathrm{C}$ & 84 & $0.15 \%$ & 0.03 & $82 \%$ \\
\hline 66 & $11 \mathrm{C}$ & 52 & $0.10 \%$ & 0.04 & $60 \%$ & & Extension & & & & \\
\hline 69.5 & $10 \mathrm{~A} / \mathrm{B} / \mathrm{C}$ & 5.2 & $0.79 \%$ & 0.16 & $>22 \%$ & 133.2 & $2 \mathrm{C} / \mathrm{D}$ & 1585 & -- & -- & -- \\
\hline \multirow[t]{2}{*}{74.5} & $09 \mathrm{~A} / \mathrm{B} / \mathrm{C}$ & 443 & $0.01 \%$ & 0.19 & $>22 \%$ & 134.2 & $2 \mathrm{~A} / \mathrm{B}$ & 96 & -- & -- & -- \\
\hline & 9 UFA & 22,831 & -- & -- & -- & 135.2 & $3 \mathrm{~A} / \mathrm{B}$ & -- & -- & -- & -- \\
\hline \multirow[t]{2}{*}{79.5} & $08 \mathrm{~A} / \mathrm{B} / \mathrm{C}$ & 1,031 & $0.01 \%$ & 14.79 & $1 \%$ & 153.9 & $10 \mathrm{~A} / \mathrm{B}$ & -- & -- & -- & -- \\
\hline & 8 UFA & 2,949 & -- & 4.85 & -- & 156.8 & $12 \mathrm{~A} / \mathrm{B}$ & 7.0 & -- & -- & -- \\
\hline 82.5 & $07 \mathrm{~A} / \mathrm{B} / \mathrm{C}$ & 38 & $0.22 \%$ & 0.15 & $36 \%$ & 165.3 & $17 \mathrm{~A} / \mathrm{B}$ & -- & -- & -- & -- \\
\hline \multirow[t]{2}{*}{90} & 06A/B & 20 & $0.50 \%$ & 0.02 & $85 \%$ & 188.3 & $38 \mathrm{~A} / \mathrm{B}$ & -- & -- & -- & -- \\
\hline & 6 UFA & 7.2 & -- & 0.02 & -- & 196.9 & $47 \mathrm{~A} / \mathrm{B}$ & -- & -- & -- & -- \\
\hline 95.5 & $03 \mathrm{~A} / \mathrm{B} / \mathrm{C}$ & 3.9 & $1.95 \%$ & 0.01 & $93 \%$ & 208.6 & $58 \mathrm{~A} / \mathrm{B}$ & -- & -- & -- & -- \\
\hline
\end{tabular}


Table 5.14. Total Concentration of Selected Constituents in Borehole Extension Composite Sediment

\begin{tabular}{|c|c|c|c|c|c|c|c|c|c|c|}
\hline \multicolumn{2}{|c|}{ Total Chemical Composition } & \multirow[b]{2}{*}{$2 \mathrm{C} / 2 \mathrm{D}$} & \multirow[b]{2}{*}{$2 \mathrm{~A} / 2 \mathrm{~B}$} & \multirow[b]{2}{*}{$3 \mathrm{~A} / 3 \mathrm{~B}$} & \multirow[b]{2}{*}{$10 \mathrm{~A} / 10 \mathrm{~B}$} & \multirow[b]{2}{*}{$12 \mathrm{~A} / 12 \mathrm{~B}$} & \multirow[b]{2}{*}{$17 \mathrm{~A} / 17 \mathrm{~B}$} & \multirow[b]{2}{*}{$38 \mathrm{~A} / 38 \mathrm{~B}$} & \multirow[b]{2}{*}{$47 \mathrm{~A} / 47 \mathrm{~B}$} & \multirow[b]{2}{*}{$58 \mathrm{~A} / 58 \mathrm{~B}$} \\
\hline Element & Units & & & & & & & & & \\
\hline $\mathrm{Na}$ & $\%$ & 1.39 & 2.18 & -- & 2.91 & 3.09 & 1.98 & 2.82 & 2.44 & 2.40 \\
\hline $\mathrm{Mg}$ & $\%$ & 0.84 & 1.39 & -- & 2.20 & 2.92 & 0.67 & 1.02 & 1.12 & 0.71 \\
\hline $\mathrm{Al}$ & $\%$ & 5.29 & 7.61 & -- & 10.7 & 9.83 & 4.32 & 7.73 & 7.18 & 6.86 \\
\hline $\mathrm{K}$ & $\%$ & 1.77 & 2.62 & -- & 1.82 & 1.49 & 1.26 & 1.89 & 1.71 & 1.41 \\
\hline $\mathrm{Ca}$ & $\%$ & 2.9 & 1.85 & -- & 1.63 & 3.72 & 0.92 & 0.95 & 0.85 & 0.93 \\
\hline $\mathrm{Ti}$ & $\%$ & 0.48 & 0.60 & -- & 1.93 & 2.48 & 0.28 & 0.92 & 0.46 & 0.72 \\
\hline $\mathrm{Fe}$ & $\%$ & 4.82 & 3.38 & -- & 5.28 & 9.85 & 2.45 & 2.9 & 2.84 & 2.65 \\
\hline $\mathrm{Cr}$ & $\mu \mathrm{g} / \mathrm{g}$ & 252 & 151 & -- & 205 & 177 & 201 & 173 & 167 & 158 \\
\hline $\mathrm{Zn}$ & $\mu \mathrm{g} / \mathrm{g}$ & -- & 133 & -- & 172 & 229 & 49 & 123 & 123 & 117 \\
\hline $\mathrm{Sr}$ & $\mu \mathrm{g} / \mathrm{g}$ & 343 & 299 & -- & 218 & 279 & 232 & 438 & 259 & 279 \\
\hline $\mathrm{Cd}$ & $\mu \mathrm{g} / \mathrm{g}$ & -- & $<5.56$ & -- & $<5.75$ & $<5.26$ & $<5.19$ & $<5.04$ & $<5.11$ & $<5.22$ \\
\hline $\mathrm{Ba}$ & $\mu \mathrm{g} / \mathrm{g}$ & 692 & 801 & -- & 641 & 603 & 515 & 811 & 591 & 548 \\
\hline $\mathrm{La}$ & $\mu \mathrm{g} / \mathrm{g}$ & -- & 30.7 & -- & 23.1 & 20.71 & 7.27 & 9.07 & 18.39 & 7.31 \\
\hline $\mathrm{Ce}$ & $\mu \mathrm{g} / \mathrm{g}$ & -- & 6.67 & -- & 11.5 & $<5.26$ & $<5.19$ & $<5.04$ & $<5.11$ & $<5.22$ \\
\hline $\mathrm{Nd}$ & $\mu \mathrm{g} / \mathrm{g}$ & -- & $<5.56$ & -- & $<5.75$ & $<5.26$ & $<5.19$ & $<5.04$ & $<5.11$ & $<5.22$ \\
\hline $\mathrm{Pb}$ & $\mu \mathrm{g} / \mathrm{g}$ & -- & 113 & -- & 131 & 121 & 29.5 & 108 & 123 & 123 \\
\hline $\mathrm{U}$ & $\mu \mathrm{g} / \mathrm{g}$ & 237 & 3.33 & -- & 3.22 & 3.15 & $<2.08$ & 2.02 & 3.06 & 3.13 \\
\hline
\end{tabular}


the composite sediments was performed to determine the uranium content of the sediments and, if possible, the uranium-235 to uranium-238 ratio. Too little uranium was present in the sediments to determine the ratio, and the total uranium concentration is no greater than that found in natural sediments. The data from the composite sediments, as corroborated by the total GEA on individual sleeves (see Table 4.7), suggest that no excess uranium is present from a tank leak. Comparable total fusions were not consistently performed on the sidewall cores because of difficulty in getting total dissolution. Because the major component in the sediments, silicon, was not measured, we cannot perform an oxide mass balance to see if the fusion successfully measures the total mass of elements in the sediment.

The fusion digestion chemical composition of the composite sediments suggests that sample 12A/B (47.8 meters [ 156.8 feet] bgs) has calcite and high iron content, as evidenced by the high calcium, magnesium, and iron content. Composite 17A/B (50.4 meters [165.3 feet] bgs), in the upper Ringold Formation, has low aluminum, calcium, iron, and trace metals, suggesting that it is high in silica and perhaps dominated by quartz. All the composites below 12A/B have very low inorganic carbon, an indicator of calcite, and the data agree with the qualitative geologic acid treatment test that indicated no carbonates were present. The composites in the Plio-Pleistocene Formation above 12A/B contain some calcite.

For the sidewall cores, a strong acid extract was performed, similar to the procedure used by the U.S. Environmental Protection Agency (EPA) to determine the total concentration of manmade metals in contaminated sediments. Table 5.15 shows the composition of the acid extract in terms of mass of each element leached per gram of dry sediment. It is obvious from comparing Table 5.14 and Table 5.15 that the $8 \mathrm{M}$ nitric acid digestion is not removing more than about $10 \%$ as much of the major and several minor elements (sodium, aluminum, potassium, titanium, strontium, barium, lead, and uranium) as the fusion digestion. For calcium, iron, and zinc, the acid digestion appears to remove about $30 \%$ as much as total fusion. The acid extract shows very high levels of chromium in the sediments between 24.1 and 33.2 meters ( 79 and 109 feet) bgs. It is obvious that there are significant amounts of chromium from the leaking tank fluid but it is difficult to determine whether the acid, in fact, removes all of the tank-released chromium or what percentage is natural chromium from the sediments. We now realize that a more productive method to determine the total elemental concentration of all major and minor elements (including manmade contributions) in vadose zone sediments is $\mathrm{x}$-ray fluorescence (XRF). For the uncontaminated sediments from nearby RCRA groundwater monitoring boreholes, XRF analyses gave excellent total chemical composition data (i.e., 100\% oxide recoveries) as presented in Serne et al. (2002a).

The most significant chemical contaminant in the sediments is chromium. Table 5.16 shows data on the in situ $K_{d}$ value calculated in the same fashion as described for the technetium-99 and cesium-137 shown in Section 5.3.1 (see also Table 5.13). It can be seen in the last column of Table 5.16 that there is significantly elevated chromium concentrations between 24.1 and 33.2 meters ( 79 and 109 feet) bgs. The sidewall core sediment total concentrations are based on strong acid extraction whereas the borehole extension sediment total concentrations are based on total fusion of the sample. It is clear that the acid extraction does not remove all the chromium from the sediment. For nearby uncontaminated boreholes, the strong acid extract removes $20 \%$ to $40 \%$ of the chromium from sediment based on XRF measurement of the total chromium concentration (Serne et al. 2002a). There must be chromium present in mineral 
Table 5.15. Acid Extractable Elements in Sidewall Cores ( $\mu \mathrm{g} / \mathrm{g}$ dry sediment)

\begin{tabular}{|c|c|c|c|c|c|c|c|c|c|c|c|}
\hline $\begin{array}{c}\text { Depth } \\
(\mathrm{ft} b g s)^{(\mathrm{a})}\end{array}$ & $\begin{array}{c}\text { Sample } \\
\text { ID }\end{array}$ & $\begin{array}{c}\mathrm{Na} \\
(\mu \mathrm{g} / \mathrm{g}) \\
\end{array}$ & $\begin{array}{c}\mathrm{Mg} \\
(\mu \mathrm{g} / \mathrm{g}) \\
\end{array}$ & $\begin{array}{c}\mathrm{Al} \\
(\mu \mathrm{g} / \mathrm{g}) \\
\end{array}$ & $\begin{array}{c}\mathrm{Si} \\
(\mu \mathrm{g} / \mathrm{g}) \\
\end{array}$ & $\begin{array}{c}\mathrm{P} \\
(\mu \mathrm{g} / \mathrm{g}) \\
\end{array}$ & $\begin{array}{c}\mathrm{K} \\
(\mu \mathrm{g} / \mathrm{g}) \\
\end{array}$ & $\begin{array}{c}\mathrm{Ca} \\
(\mu \mathrm{g} / \mathrm{g}) \\
\end{array}$ & $\begin{array}{c}\mathrm{Ti} \\
(\mu \mathrm{g} / \mathrm{g}) \\
\end{array}$ & $\begin{array}{c}\mathrm{Mn} \\
(\mu \mathrm{g} / \mathrm{g}) \\
\end{array}$ & $\begin{array}{c}\mathrm{Fe} \\
(\mu \mathrm{g} / \mathrm{g})\end{array}$ \\
\hline $25-26$ & $15 \mathrm{~A} / \mathrm{B} / \mathrm{C}$ & $\ldots$ & 4,397 & 5,339 & $<25$ & 846 & 1,209 & 7,605 & 363 & 313 & 16,419 \\
\hline 44-45 & $14 \mathrm{~A} / \mathrm{B} / \mathrm{C}$ & $-\cdots$ & 4,776 & 4,997 & $<25$ & 1,085 & 1,073 & 9,123 & 468 & 303 & 18,484 \\
\hline $56-57$ & $13 \mathrm{~A} / \mathrm{B} / \mathrm{C}$ & -- & 6,416 & 10,872 & $<25$ & 820 & 2,139 & 10,278 & 951 & 545 & 29,824 \\
\hline $61-62$ & $12 \mathrm{~A} / \mathrm{B} / \mathrm{C}$ & --- & 5,063 & 7,677 & $<25$ & 560 & 2,183 & 8,839 & 405 & 394 & 16,475 \\
\hline $65-66$ & $11 \mathrm{~A} / \mathrm{B}$ & -- & 3,244 & 4,307 & $<25$ & 896 & 775.3 & 5,772 & 457 & 231 & 13,870 \\
\hline \multirow[t]{2}{*}{$65-66$} & $11 \mathrm{C}$ & $\ldots$ & 3,447 & 4,673 & $<25$ & 663 & 999.9 & 8,051 & 352 & 225 & 13,051 \\
\hline & 11C DUP & $\ldots$ & 3,550 & 5,316 & $<25$ & 716 & 1,019 & 6,019 & 505 & 233 & 15,334 \\
\hline 69-70 & $10 \mathrm{~A} / \mathrm{B} / \mathrm{C}$ & $\ldots$ & 4,356 & 5,967 & $<25$ & 751 & 1,039 & 7,310 & 781 & 248 & 16,659 \\
\hline 74-75 & $9 \mathrm{~A} / \mathrm{B} / \mathrm{C}$ & $\cdots$ & 3,973 & 5,632 & $<25$ & 667 & 995.2 & 6,750 & 636 & 226 & 15,622 \\
\hline $79-80$ & $8 \mathrm{~A} / \mathrm{B} / \mathrm{C}$ & $\ldots$ & 4,634 & 6,780 & $<25$ & 785 & 1,314 & 8,591 & 687 & 318 & 21,500 \\
\hline \multirow[t]{2}{*}{$82-83$} & $7 \mathrm{~A} / \mathrm{B} / \mathrm{C}$ & $\ldots$ & 4,724 & 6,698 & $<25$ & 1,000 & 1,127 & 12,731 & 1,207 & 273 & 22,668 \\
\hline & 7 DUP & $\cdots$ & 3,508 & 5,744 & $<25$ & 926 & 898 & 10,591 & 865 & 239 & 17,520 \\
\hline 90 & $6 \mathrm{~A} / \mathrm{B}$ & $-\cdots$ & 5,117 & 6,608 & $<25$ & 527 & 2,241 & 7,729 & 353 & 306 & 13,274 \\
\hline $95-96$ & $3 \mathrm{~A} / \mathrm{B} / \mathrm{C}$ & $-\cdots$ & 4,508 & 6,147 & $<25$ & 471 & 1,852 & 9,548 & 319 & 302 & 12,949 \\
\hline $102-103$ & $5 \mathrm{~A} / \mathrm{B} / \mathrm{C}$ & $-\cdots$ & 5,113 & 6,743 & $<25$ & 478 & 1,674 & 9,384 & 369 & 323 & 16,689 \\
\hline 108-109 & $4 \mathrm{~A} / \mathrm{B} / \mathrm{C}$ & $-\cdots$ & 6,164 & 8,467 & $<25$ & 550 & 2,960 & 9,568 & 522 & 333 & 18,655 \\
\hline 112 & $2 \mathrm{~B} / \mathrm{C}$ & $-\cdots$ & 4,304 & 6,091 & $<25$ & 434 & 1,677 & 8,405 & 371 & 333 & 13,115 \\
\hline 127.4 & $1 \mathrm{~A} / \mathrm{B} / \mathrm{C}$ & - - - & 5,472 & 6,770 & $<25$ & 571 & 1,689 & 13,308 & 294 & 324 & 16,859 \\
\hline
\end{tabular}


Table 5.15. (contd)

\begin{tabular}{|c|c|c|c|c|c|c|c|c|c|c|c|}
\hline $\begin{array}{c}\text { Depth } \\
(\mathrm{ft} \text { bgs })^{(\mathrm{a})}\end{array}$ & $\begin{array}{l}\text { Sample } \\
\text { ID } \\
\end{array}$ & $\begin{array}{c}\mathrm{Cr} \\
(\mu \mathrm{g} / \mathrm{g}) \\
\end{array}$ & $\begin{array}{c}\mathrm{Cr}^{*} \\
(\mu \mathrm{g} / \mathrm{g}) \\
\end{array}$ & $\begin{array}{c}\mathrm{Cu} \\
(\mu \mathrm{g} / \mathrm{g}) \\
\end{array}$ & $\begin{array}{c}\mathrm{Cu}^{*} \\
(\mu \mathrm{g} / \mathrm{g}) \\
\end{array}$ & $\begin{array}{c}\mathrm{Zn} \\
(\mu \mathrm{g} / \mathrm{g}) \\
\end{array}$ & $\begin{array}{c}\mathrm{As}^{*} \\
(\mu \mathrm{g} / \mathrm{g}) \\
\end{array}$ & $\begin{array}{c}\mathrm{Se}^{*} \\
(\mu \mathrm{g} / \mathrm{g}) \\
\end{array}$ & $\begin{array}{c}\mathrm{Rb}^{*} \\
(\mu \mathrm{g} / \mathrm{g}) \\
\end{array}$ & $\begin{array}{c}\mathrm{Sr} \\
(\mu \mathrm{g} / \mathrm{g}) \\
\end{array}$ & $\begin{array}{c}\mathrm{Y}^{*} \\
(\mu \mathrm{g} / \mathrm{g}) \\
\end{array}$ \\
\hline $25-26$ & $15 \mathrm{~A} / \mathrm{B} / \mathrm{C}$ & $<6.0$ & 7 & 12 & 12 & 39 & 3.2 & 0.10 & 8.2 & 26.3 & 9.9 \\
\hline $44-45$ & $14 \mathrm{~A} / \mathrm{B} / \mathrm{C}$ & $<6.0$ & 6 & 16 & 15 & 45 & 4.1 & 0.12 & 8.0 & 27.1 & 12.1 \\
\hline $56-57$ & $13 \mathrm{~A} / \mathrm{B} / \mathrm{C}$ & 26 & 26 & 21 & 19 & 61 & 3.0 & 0.10 & 15.6 & 38.4 & 11.5 \\
\hline $61-62$ & $12 \mathrm{~A} / \mathrm{B} / \mathrm{C}$ & 105 & 126 & 12 & 11 & 49 & 2.1 & $(0.07)$ & 13.7 & 28.8 & 6.5 \\
\hline $65-66$ & $11 \mathrm{~A} / \mathrm{B}$ & 59 & 66 & 14 & 13 & 33 & 1.4 & 0.10 & 4.6 & 18.4 & 10.9 \\
\hline \multirow[t]{2}{*}{$65-66$} & $11 \mathrm{C}$ & 77 & 75 & 13 & 12 & 32 & 2.0 & 0.05 & 6.0 & 24.8 & 7.5 \\
\hline & $11 \mathrm{C}$ DUP & 72 & 72 & 13 & 12 & 34 & 3.7 & 0.11 & 6.1 & 22.4 & 8.4 \\
\hline $69-70$ & $10 \mathrm{~A} / \mathrm{B} / \mathrm{C}$ & 41 & 42 & 13 & 13 & 39 & 1.9 & 0.11 & 5.8 & 23.7 & 8.3 \\
\hline $74-75$ & $9 \mathrm{~A} / \mathrm{B} / \mathrm{C}$ & 103 & 123 & 15 & 14 & 38 & 1.8 & 0.08 & 5.8 & 19.2 & 7.9 \\
\hline $79-80$ & $8 \mathrm{~A} / \mathrm{B} / \mathrm{C}$ & 567 & 629 & 16 & 14 & 37 & 1.9 & 0.09 & 7.6 & 33.7 & 8.9 \\
\hline \multirow[t]{2}{*}{$82-83$} & $7 \mathrm{~A} / \mathrm{B} / \mathrm{C}$ & 1,344 & 1,528 & 120 & 109 & 112 & 3.1 & $(0.19)$ & 6.7 & 44.0 & 12.1 \\
\hline & 7 DUP & 1,192 & 1,360 & 18 & 15 & 38 & 2.8 & 0.14 & 5.5 & 36.6 & 10.2 \\
\hline 90 & $6 \mathrm{~A} / \mathrm{B}$ & 1,198 & 1,278 & 12 & 11 & 44 & 3.1 & 0.11 & 12.9 & 26.1 & 4.9 \\
\hline $95-96$ & $3 \mathrm{~A} / \mathrm{B} / \mathrm{C}$ & 635 & 711 & 13 & 12 & 53 & 2.9 & 0.16 & 12.2 & 39.1 & 5.1 \\
\hline $102-103$ & $5 \mathrm{~A} / \mathrm{B} / \mathrm{C}$ & 1,017 & 1,170 & 17 & 16 & 45 & 3.3 & 0.16 & 11.2 & 36.0 & 5.6 \\
\hline 108-109 & $4 \mathrm{~A} / \mathrm{B} / \mathrm{C}$ & 750 & 784 & 18 & 16 & 60 & 4.8 & 0.26 & 19.8 & 39.3 & 6.0 \\
\hline 112 & $2 \mathrm{~B} / \mathrm{C}$ & 269 & 299 & 14 & 12 & 52 & 2.2 & 0.22 & 11.4 & 33.7 & 4.7 \\
\hline 127.4 & $1 \mathrm{~A} / \mathrm{B} / \mathrm{C}$ & 16 & 16 & 27 & 25 & 63 & 2.7 & $(0.05)$ & 13.6 & 43.6 & 6.5 \\
\hline
\end{tabular}


Table 5.15. (contd)

\begin{tabular}{|c|c|c|c|c|c|c|c|c|c|c|}
\hline $\begin{array}{c}\text { Depth } \\
(\mathrm{ft} \text { bgs })^{(\mathrm{a})}\end{array}$ & $\begin{array}{c}\text { Sample } \\
\text { ID } \\
\end{array}$ & $\begin{array}{c}\mathrm{Zr*} \\
(\mu \mathrm{g} / \mathrm{g}) \\
\end{array}$ & $\begin{array}{c}\mathrm{Mo}^{*} \\
(\mu \mathrm{g} / \mathrm{g}) \\
\end{array}$ & $\begin{array}{c}\mathrm{Ru}^{*} \\
(\mu \mathrm{g} / \mathrm{g}) \\
\end{array}$ & $\begin{array}{c}\mathrm{Pd}^{*} \\
(\mu \mathrm{g} / \mathrm{g}) \\
\end{array}$ & $\begin{array}{c}\mathrm{Ag}^{*} \\
(\mu \mathrm{g} / \mathrm{g}) \\
\end{array}$ & $\begin{array}{c}\mathrm{Cd}^{*} \\
(\mu \mathrm{g} / \mathrm{g}) \\
\end{array}$ & $\begin{array}{c}\mathrm{Ba} \\
(\mu \mathrm{g} / \mathrm{g}) \\
\end{array}$ & $\begin{array}{c}\mathrm{Pb}^{*} \\
(\mu \mathrm{g} / \mathrm{g}) \\
\end{array}$ & $\begin{array}{c}U^{*} \\
(\mu \mathrm{g} / \mathrm{g}) \\
\end{array}$ \\
\hline $25-26$ & $15 \mathrm{~A} / \mathrm{B} / \mathrm{C}$ & 16.1 & 0.1 & $<0.03$ & 0.07 & 0.02 & 0.07 & 101 & 4.5 & 0.44 \\
\hline $44-45$ & $14 \mathrm{~A} / \mathrm{B} / \mathrm{C}$ & 17.8 & 0.4 & $<0.01$ & 0.07 & 0.02 & 0.07 & 87 & 4.3 & 0.68 \\
\hline $56-57$ & $13 \mathrm{~A} / \mathrm{B} / \mathrm{C}$ & 23.0 & 0.6 & $<0.01$ & 0.09 & 0.04 & 0.10 & 146 & 5.0 & 0.65 \\
\hline $61-62$ & $12 \mathrm{~A} / \mathrm{B} / \mathrm{C}$ & 8.9 & 0.2 & $<0.01$ & 0.04 & 0.04 & 0.07 & 117 & 3.9 & 0.61 \\
\hline $65-66$ & $11 \mathrm{~A} / \mathrm{B}$ & 12.7 & 0.8 & $<0.01$ & 0.05 & 0.02 & 0.06 & 62 & 2.4 & 0.39 \\
\hline \multirow[t]{2}{*}{$65-66$} & $11 \mathrm{C}$ & 12.3 & 0.3 & $<0.01$ & 0.06 & 0.03 & 0.19 & 112 & 2.9 & 0.45 \\
\hline & 11C DUP & 12.1 & 0.5 & $<0.02$ & 0.05 & 0.04 & 0.09 & 69 & 3.1 & 0.35 \\
\hline $69-70$ & $10 \mathrm{~A} / \mathrm{B} / \mathrm{C}$ & 12.8 & 1.0 & $<0.01$ & 0.05 & 0.03 & 0.06 & 66 & 2.5 & 0.44 \\
\hline $74-75$ & $9 \mathrm{~A} / \mathrm{B} / \mathrm{C}$ & 11.7 & 1.5 & $<0.01$ & 0.05 & 0.02 & 0.05 & 62 & $(3.3)$ & 0.44 \\
\hline $79-80$ & $8 \mathrm{~A} / \mathrm{B} / \mathrm{C}$ & 14.8 & 11.4 & $<0.01$ & 0.06 & 0.06 & 0.08 & 81 & 3.5 & 0.48 \\
\hline \multirow[t]{2}{*}{$82-83$} & $7 \mathrm{~A} / \mathrm{B} / \mathrm{C}$ & 18.2 & 14.6 & $<0.01$ & 0.07 & 0.04 & 0.08 & 96 & $(5.9)$ & 0.63 \\
\hline & 7 DUP & 16.9 & 10.8 & $<0.02$ & 0.07 & 0.03 & 0.06 & 74 & 2.6 & 0.62 \\
\hline 90 & $6 \mathrm{~A} / \mathrm{B}$ & 4.7 & 5.2 & $<0.01$ & 0.03 & 0.05 & 0.07 & 80 & 4.9 & 0.45 \\
\hline $95-96$ & $3 \mathrm{~A} / \mathrm{B} / \mathrm{C}$ & 5.1 & 1.7 & $<0.01$ & 0.02 & 0.04 & 0.08 & 89 & 4.5 & 0.47 \\
\hline $102-103$ & $5 \mathrm{~A} / \mathrm{B} / \mathrm{C}$ & 6.2 & 4.4 & $<0.01$ & 0.03 & 0.18 & 0.10 & 88 & 6.2 & 0.62 \\
\hline 108-109 & $4 \mathrm{~A} / \mathrm{B} / \mathrm{C}$ & 7.4 & 2.3 & $<0.02$ & 0.03 & 0.08 & 0.11 & 135 & $(8.0)$ & 0.55 \\
\hline 112 & $2 \mathrm{~B} / \mathrm{C}$ & 6.3 & 0.4 & $<0.01$ & 0.03 & 0.03 & 0.07 & 93 & $(3.9)$ & 0.36 \\
\hline 127.4 & $1 \mathrm{~A} / \mathrm{B} / \mathrm{C}$ & 7.3 & 2.3 & $<0.02$ & 0.06 & 0.03 & 0.10 & 88 & 6.3 & 0.47 \\
\hline
\end{tabular}


Table 5.16. Chromium Mobility Status in the Borehole 41-09-39 Sediments

\begin{tabular}{|c|c|c|c|c|c|}
\hline $\begin{array}{c}\text { Depth } \\
(\mathrm{ft} \text { bgs })^{(\mathrm{a})}\end{array}$ & $\begin{array}{l}\text { Sample } \\
\text { ID }\end{array}$ & $\begin{array}{c}\mathrm{K}_{\mathrm{d}}(\mathrm{Cr}) \\
\text { Extracts }(\mathrm{mL} / \mathrm{g})\end{array}$ & $\begin{array}{c}\mathrm{K}_{\mathrm{d}}(\mathrm{Cr}) \\
\text { UFA }(\mathrm{mL} / \mathrm{g})\end{array}$ & $\begin{array}{c}\% \text { Water } \\
\text { Leachable }\end{array}$ & $\begin{array}{c}\text { Total Sediment } \\
\mu \mathrm{g} / \mathrm{g}\end{array}$ \\
\hline \multicolumn{6}{|c|}{ Sidewall } \\
\hline $25-26$ & $15 \mathrm{~A} / \mathrm{B} / \mathrm{C}$ & 973 & & $>0.01$ & $<6$ \\
\hline $44-45$ & $14 \mathrm{~A} / \mathrm{B} / \mathrm{C}$ & 177 & & $>0.05$ & $<6$ \\
\hline $56-57$ & $13 \mathrm{~A} / \mathrm{B} / \mathrm{C}$ & 1,197 & 3.0 & 0.01 & 26 \\
\hline $61-62$ & $12 \mathrm{~A} / \mathrm{B} / \mathrm{C}$ & 1,653 & & 0.01 & 105 \\
\hline $65-66$ & $11 \mathrm{~A} / \mathrm{B}$ & 7.1 & & 0.58 & 59 \\
\hline $65-66$ & $11 \mathrm{C}$ & 10.5 & & 0.46 & 74.5 \\
\hline $69-70$ & $10 \mathrm{~A} / \mathrm{B} / \mathrm{C}$ & -0.6 & & 12.38 & 41 \\
\hline $74-75$ & $9 \mathrm{~A} / \mathrm{B} / \mathrm{C}$ & 0.3 & & 3.97 & 103 \\
\hline $79-80$ & $8 \mathrm{~A} / \mathrm{B} / \mathrm{C}$ & 83.5 & 26.3 & 0.13 & 567 \\
\hline $82-83$ & $7 \mathrm{~A} / \mathrm{B} / \mathrm{C}$ & -0.9 & & 58.86 & 1268 \\
\hline 90 & $6 \mathrm{~A} / \mathrm{B}$ & -0.8 & -0.8 & 59.38 & 1198 \\
\hline $95-96$ & $3 \mathrm{~A} / \mathrm{B} / \mathrm{C}$ & -0.8 & & 41.00 & 635 \\
\hline $102-103$ & $5 \mathrm{~A} / \mathrm{B} / \mathrm{C}$ & -0.8 & & 51.88 & 1017 \\
\hline 108-109 & $4 \mathrm{~A} / \mathrm{B} / \mathrm{C}$ & -0.8 & -0.8 & 64.24 & 750 \\
\hline 112 & $2 \mathrm{~B} / \mathrm{C}$ & -0.9 & -0.9 & 65.41 & 269 \\
\hline 127.4 & $1 \mathrm{~A} / \mathrm{B} / \mathrm{C}$ & 176 & & 0.07 & 16 \\
\hline \multicolumn{6}{|c|}{ Extension } \\
\hline 133.2 & $2 \mathrm{CD}$ & 15,749 & & 0.01 & 252 \\
\hline 134.2 & $2 \mathrm{AB}$ & 37,789 & & 0.00 & 151 \\
\hline 135.2 & $3 \mathrm{AB}$ & -- & & -- & -- \\
\hline 153.9 & $10 \mathrm{AB}$ & 68,209 & & 0.00 & 205 \\
\hline 156.8 & $12 \mathrm{AB}$ & 17,659 & & 0.01 & 177 \\
\hline 165.3 & $17 \mathrm{AB}$ & 15,491 & & 0.01 & 201 \\
\hline 188.3 & $38 \mathrm{AB}$ & 2,796 & & 0.04 & 173 \\
\hline 196.9 & $47 \mathrm{AB}$ & 7,238 & & 0.01 & 167 \\
\hline 208.6 & $58 \mathrm{AB}$ & 14,333 & & 0.01 & 158 \\
\hline
\end{tabular}

matrices that is not acid-extractable. However, the tank leak portion of the chromium in the sediments may readily dissolve in the strong acid treatment. We are in the process of obtaining an XRF instrument that can be used on radioactive samples and in the future we will be able to measure the total concentration of chromium in contaminated sediments. 
From the calculated $\mathrm{K}_{\mathrm{d}}$ values and percentage of the chromium that is water-extractable versus acidextractable (sidewall cores) or water-leachable versus total chromium (borehole extension samples), it is clear that the majority of the tank leak chromium is water soluble $\mathrm{Cr}(\mathrm{VI})$ (chromate). The in situ $\mathrm{K}_{\mathrm{d}}$ values for the most contaminated depths all are slightly negative, suggesting some systematic error in our measurements. In general, the low $\mathrm{K}_{\mathrm{d}}$ values signify mobile chromate. Above the tank bottom $(<15.2$ meters $[<50$ feet] bgs) and below the leading edge of the tank leak chromium (38.7 meters [127 feet] bgs), the chromium $\mathrm{K}_{\mathrm{d}}$ value is $>100$ and the percentage of water soluble chromium is $<0.1 \%$ because the native chromium is present as $\mathrm{Cr}(\mathrm{III})$. One sample from the highly contaminated zone (sidewall core $8 \mathrm{ABC} 24.1$ to 24.4 meters [79 to 80 feet]) shows a peculiarly low water-leachable fraction and thus, high $\mathrm{K}_{\mathrm{d}}$. This sample is being studied in a separately funded basic science project to evaluate the cause. It would appear that a portion of the tank leaked $\mathrm{Cr}(\mathrm{VI})$ has either been reduced to insoluble $\mathrm{Cr}$ (III) or that it has formed a water insoluble precipitate. Based on comparing the depth of penetration of various contaminants and comparing the percentages that are water-leachable, we can state that chromium migrates faster than cesium-137 but slower than technetium-99 and nitrate. In other waste disposal situations at Hanford, oxidized chromium, in reactor cooling water (low ionic strength and neutral $\mathrm{pH}$ ), appears to migrate similarly to technetium-99 and nitrate.

\subsubsection{Acid-Leachable Cesium-137 versus Direct Sediment Cesium-137 Analysis}

Table 5.17 shows the comparison of cesium-137 concentrations in the sediments determined from direct GEA counting of an aliquot of the sediment and GEA counting of an $8 \mathrm{M}$ nitric acid extraction of a separate aliquot. The data show that there is good agreement (based on assuming that $\pm 30 \%$ covers counting and sampling heterogeneity) for 8 of the 15 pairs of data. For 5 pairs of data, the direct counting values are larger than the acid extract values. We have observed that cesium-137 is very strongly

Table 5.17. Cesium-137 Agreement Between Direct Counting and Acid Extraction

\begin{tabular}{|c|c|c|c|c|}
\hline $\begin{array}{c}\text { Depth } \\
(\mathrm{ft} \mathrm{bgs})^{(\mathrm{a})}\end{array}$ & $\begin{array}{l}\text { Sample } \\
\text { ID }\end{array}$ & $\begin{array}{c}{ }^{137} \mathrm{Cs}(\mathrm{pCi} / \mathrm{g}) \\
\text { Direct }\end{array}$ & $\begin{array}{c}{ }^{137} \mathrm{Cs}(\mathrm{pCi} / \mathrm{g}) \\
\text { Acid }\end{array}$ & $($ dir-acid $) / \operatorname{dir}$ \\
\hline $25-26$ & $15 \mathrm{~A} / \mathrm{B} / \mathrm{C}$ & $\begin{array}{c}5.94 \mathrm{E}+02 \\
\end{array}$ & $2.81 \mathrm{E}+04$ & $-4,630 \%$ \\
\hline $44-45$ & $14 \mathrm{~A} / \mathrm{B} / \mathrm{C}$ & $1.10 \mathrm{E}+03$ & $1.18 \mathrm{E}+03$ & $-8 \%$ \\
\hline $56-57$ & $13 \mathrm{~A} / \mathrm{B} / \mathrm{C}$ & $2.74 \mathrm{E}+04$ & $7.11 \mathrm{E}+03$ & $74 \%$ \\
\hline $61-62$ & $12 \mathrm{~A} / \mathrm{B} / \mathrm{C}$ & $1.28 \mathrm{E}+05$ & $1.21 \mathrm{E}+05$ & $5 \%$ \\
\hline $65-66$ & $11 \mathrm{~A} / \mathrm{B}$ & $5.94 \mathrm{E}+05$ & $5.44 \mathrm{E}+05$ & $8 \%$ \\
\hline $65-66$ & $11 \mathrm{C}$ & $3.91 \mathrm{E}+06$ & $3.79 \mathrm{E}+06$ & $3 \%$ \\
\hline $69-70$ & $10 \mathrm{~A} / \mathrm{B} / \mathrm{C}$ & $8.99 \mathrm{E}+03$ & $4.48 \mathrm{E}+03$ & $50 \%$ \\
\hline $74-75$ & $9 \mathrm{~A} / \mathrm{B} / \mathrm{C}$ & $2.23 \mathrm{E}+06$ & $1.65 \mathrm{E}+06$ & $26 \%$ \\
\hline $79-80$ & $8 \mathrm{~A} / \mathrm{B} / \mathrm{C}$ & $2.57 \mathrm{E}+06$ & $2.64 \mathrm{E}+06$ & $-3 \%$ \\
\hline $82-83$ & $7 \mathrm{~A} / \mathrm{B} / \mathrm{C}$ & $1.73 \mathrm{E}+07$ & $1.70 \mathrm{E}+07$ & $1 \%$ \\
\hline 90 & $6 \mathrm{~A} / \mathrm{B}$ & $4.38 \mathrm{E}+04$ & $1.46 \mathrm{E}+04$ & $67 \%$ \\
\hline $95-96$ & $3 \mathrm{~A} / \mathrm{B} / \mathrm{C}$ & $3.66 \mathrm{E}+04$ & $7.47 \mathrm{E}+04$ & $-104 \%$ \\
\hline $102-103$ & $5 \mathrm{~A} / \mathrm{B} / \mathrm{C}$ & $1.59 \mathrm{E}+06$ & $7.50 \mathrm{E}+05$ & $53 \%$ \\
\hline $108-109$ & $4 \mathrm{~A} / \mathrm{B} / \mathrm{C}$ & $3.35 \mathrm{E}+05$ & $2.28 \mathrm{E}+05$ & $32 \%$ \\
\hline 112 & $2 \mathrm{~B} / \mathrm{C}$ & $1.43 \mathrm{E}+03$ & $1.74 \mathrm{E}+02$ & $88 \%$ \\
\hline 127.4 & $1 \mathrm{~A} / \mathrm{B} / \mathrm{C}$ & $4.20 \mathrm{E}+03$ & Not analyzed & -- \\
\hline
\end{tabular}


adsorbed to the mica grains in the sediments and that even strong acid does not remove all of the cesium-137 from sediment, thus some disagreement in values is expected. In two cases, the acid extract values are larger than the direct counting, which is not expected. The only explanation is heterogeneity in cesium-137 content in the two aliquots used to make the separate analyses.

The shallowest sediment sample shows the worst agreement. We are certain that the contamination in the two shallower samples is indicative of contamination that was pulled into the shallow sediments during the extraction of the contaminated casing. The fact that the sidewall coring did not penetrate very deeply into the formation no doubt accentuated the impact of smearing contamination off the casing. The material that sticks to the casing has been visually observed to be very fine grained (silts and clays). As shown in Table 5.25, the silts and clays contain the highest concentrations of cesium-137. It would, thus, appear that the mixing of some of the samples prior to aliquoting was not successful in getting a homogeneous mixture. This lack of homogeneity likely led to the differences in cesium-137 concentrations shown in Table 5.17. The most contaminated sediments between 18.6 and 33.2 meters (61 and 109 feet) bgs show fairly good agreement except sample $3 \mathrm{ABC}$ at 29 meters ( 95 feet) bgs. Because direct counting is feasible for gamma emitters, acid extracts are not necessary or preferred when determining total concentrations in vadose zone sediments.

\subsubsection{Total Carbon Content of Composite Sediments}

The total carbon, inorganic carbon, and organic carbon content of the sidewall and borehole extension composite sediments are shown in Table 5.18. The calculated equivalent weight percent (wt\%) of calcium carbonate is also shown based on the inorganic carbon measurement.

The samples measured do not show that high levels of calcium carbonate are present. Even the calcrete sample shown in Figure 2.20 showed only $3 \mathrm{wt} \%$. This is within the range of carbonate contents found in a nearby uncontaminated caliche layer in borehole 299-W22-50 that has 2 to $8 \mathrm{wt} \%$ calcium carbonate. However, farther north at borehole 299-W22-48, the caliche layer contains 35 to $40 \mathrm{wt} \%$ calcium carbonate. The lower values for this cementing agent in the southern portion of the tank farm may be important in that it could signify a less cemented zone at the Ringold-Plio-Pliestocene contact at the southern edge of the SX Tank Farm. On the other hand, there appears to be significant amounts of ferric oxides present in the caliche that is another cementing agent. No attempt was made to compare permeabilities of cores from the caliche zones of different boreholes. In fact, a common method for forming high concentrations of caliche requires highly permeable sediments so that large quantities of carbonate-bearing waters can flow through and deposit calcite.

The Plio-Pliestocene sediments that start 38.1 meters (125 feet) bgs have higher concentrations of calcium carbonate than the overlying Hanford formation sediments, and the Ringold sediments below the caliche zone at $>47.8$ meters ( $>160$ feet) bgs have virtually no calcium carbonate present. 
Table 5.18. Total Carbon Content of Borehole 41-09-39 Sediment Samples

\begin{tabular}{|c|c|c|c|c|c|}
\hline $\begin{array}{c}\text { Depth } \\
(\mathrm{ft} \text { bgs })^{(\mathrm{a})}\end{array}$ & $\begin{array}{l}\text { Sample } \\
\text { ID }\end{array}$ & $\begin{array}{c}\text { Total C } \\
(\%)\end{array}$ & $\begin{array}{c}\text { Inorganic } C \\
(\%)\end{array}$ & $\begin{array}{c}\text { Organic C } \\
(\%)\end{array}$ & $\begin{array}{c}\text { Inorganic C } \\
\text { as } \mathrm{CaCO}_{3} \\
(\%)\end{array}$ \\
\hline \multicolumn{6}{|c|}{ Sidewall } \\
\hline $25-26$ & $15 \mathrm{~A} / \mathrm{B} / \mathrm{C}$ & 0.13 & 0.12 & 0.01 & 0.98 \\
\hline $44-45$ & $14 \mathrm{~A} / \mathrm{B} / \mathrm{C}$ & 0.09 & 0.11 & -0.02 & 0.95 \\
\hline $56-57$ & $13 \mathrm{~A} / \mathrm{B} / \mathrm{C}$ & 0.15 & 0.17 & -0.02 & 1.44 \\
\hline $61-62$ & $12 \mathrm{~A} / \mathrm{B} / \mathrm{C}$ & 0.23 & 0.12 & 0.11 & 1.00 \\
\hline $65-66$ & $11 \mathrm{~A} / \mathrm{B}$ & 0.25 & 0.15 & 0.09 & 1.26 \\
\hline $65-66$ & $11 \mathrm{C}$ & 0.16 & 0.12 & 0.04 & 1.01 \\
\hline $69-70$ & $10 \mathrm{~A} / \mathrm{B} / \mathrm{C}$ & 0.20 & 0.18 & 0.03 & 1.49 \\
\hline $74-75$ & $9 \mathrm{~A} / \mathrm{B} / \mathrm{C}$ & 0.23 & 0.18 & 0.04 & 1.52 \\
\hline $79-80$ & $8 \mathrm{~A} / \mathrm{B} / \mathrm{C}$ & 0.29 & 0.27 & 0.02 & 2.26 \\
\hline $82-83$ & $7 \mathrm{~A} / \mathrm{B} / \mathrm{C}$ & 0.30 & 0.32 & -0.02 & 2.67 \\
\hline 90 & $6 \mathrm{~A} / \mathrm{B}$ & 0.25 & 0.25 & -0.01 & 2.10 \\
\hline $95-96$ & $3 \mathrm{~A} / \mathrm{B} / \mathrm{C}$ & 0.26 & 0.24 & 0.02 & 2.03 \\
\hline $102-103$ & $5 \mathrm{~A} / \mathrm{B} / \mathrm{C}$ & 0.28 & 0.20 & 0.09 & 1.63 \\
\hline 108-109 & $4 \mathrm{~A} / \mathrm{B} / \mathrm{C}$ & 0.45 & 0.19 & 0.27 & 1.55 \\
\hline 112 & $2 \mathrm{~B} / \mathrm{C}$ & 0.25 & 0.25 & 0.00 & 2.07 \\
\hline 127.4 & $1 \mathrm{~A} / \mathrm{B} / \mathrm{C}$ & 0.44 & 0.40 & 0.04 & 3.34 \\
\hline \multicolumn{6}{|c|}{ Extension } \\
\hline 133.2 & $2 \mathrm{C} / 2 \mathrm{D}$ & 0.50 & $1.51^{(\mathrm{b})}$ & (c) & 4.13 \\
\hline 134.2 & $2 \mathrm{~A} / 2 \mathrm{~B}$ & 0.47 & $2.37^{(\mathrm{b})}$ & (c) & 3.93 \\
\hline 135.2 & $3 \mathrm{~A} / 3 \mathrm{~B}$ & 0.48 & $1.86^{(\mathrm{b})}$ & (c) & 4.03 \\
\hline 153.9 & $10 \mathrm{~A} / 10 \mathrm{~B}$ & 0.53 & 0.32 & 0.21 & 2.67 \\
\hline 156.8 & $12 \mathrm{~A} / 12 \mathrm{~B}$ & 0.30 & 0.34 & (c) & 2.82 \\
\hline 165.3 & $17 \mathrm{~A} / 17 \mathrm{~B}$ & 0.08 & 0.00 & 0.08 & 0.01 \\
\hline 188.3 & $38 \mathrm{~A} / 38 \mathrm{~B}$ & 0.07 & 0.00 & 0.07 & 0.03 \\
\hline 196.9 & $47 \mathrm{~A} / 47 \mathrm{~B}$ & 0.03 & 0.00 & 0.03 & 0.01 \\
\hline 208.6 & $58 \mathrm{~A} / 58 \mathrm{~B}$ & 0.01 & 0.00 & 0.01 & 0.01 \\
\hline \multicolumn{6}{|c|}{ (a) Multiply by 0.3048 to convert to } \\
\hline \multicolumn{6}{|c|}{$\begin{array}{l}\text { (b) Inorganic carbon was run on a different carbon analyzer than total carbon and } \\
\text { results do not seem correct. } \\
\text { (c) Organic carbon obtained by difference but cannot be negative; suspect inorganic } \\
\text { carbon value in error. } \\
\text { Values in red are computed from total carbon whereas all others are calculated from the } \\
\text { inorganic carbon. }\end{array}$} \\
\hline
\end{tabular}


Table 5.19. Cation Exchange Capacity of the Composite Sediments $(<2 \mathrm{~mm})$

\begin{tabular}{|c|c|c|c|c|c|}
\hline $\begin{array}{c}\text { Depth } \\
(\mathrm{ft} \text { bgs })^{(\mathrm{a})}\end{array}$ & $\begin{array}{l}\text { Sample } \\
\text { ID }\end{array}$ & $\begin{array}{c}\text { Geologic } \\
\text { Unit }\end{array}$ & $\begin{array}{l}\mathrm{meq} / 100 \mathrm{~g} \\
\text { Average }\end{array}$ & $\begin{array}{l}\text { Standard } \\
\text { Deviation } \\
(\%)\end{array}$ & $\begin{array}{l}\text { CEC for } \\
\text { Bulk } \\
\text { Sediments }\end{array}$ \\
\hline 133.2 & $2 \mathrm{C} / \mathrm{D}$ & $\mathrm{PPlz}$ & 13.42 & 8.56 & 13.1 \\
\hline 134.2 & $2 \mathrm{~A} / \mathrm{B}$ & $\mathrm{PPlz}$ & 14.73 & 3.42 & 14.7 \\
\hline 135.2 & $3 \mathrm{~A} / \mathrm{B}$ & $\mathrm{PPlz}$ & 15.68 & 21.73 & 15.6 \\
\hline 153.9 & $10 \mathrm{~A} / \mathrm{B}$ & $\mathrm{PPlz}$ & 15.27 & 1.80 & 15.3 \\
\hline 156.8 & $12 \mathrm{~A} / \mathrm{B}$ & PPlc & 10.9 & 8.27 & 9.4 \\
\hline 165.3 & $17 \mathrm{~A} / \mathrm{B}$ & Rtf & 14.97 & 3.30 & 14.7 \\
\hline 188.3 & $38 \mathrm{~A} / \mathrm{B}$ & Rwi(e) & 7.51 & 1.74 & 1.0 \\
\hline 196.9 & $47 \mathrm{~A} / \mathrm{B}$ & Rwi(e) & 12.74 & 1.60 & 2.9 \\
\hline 208.6 & $58 \mathrm{~A} / \mathrm{B}$ & Rwi(e) & 14.06 & 0.31 & 3.0 \\
\hline \multicolumn{6}{|c|}{$\begin{array}{l}\text { Note: Last column are values adjusted to bulk size, assuming no sites for the }>2 \mathrm{~mm} \\
\text { sizes. } \\
\text { PPlz = Plio-Pliestocene very fine sand to clayey silt. } \\
\text { PPlc = Plio-Pliestocene calcrete (caliche) or carbonate facies. } \\
\text { Rtf = Ringold Taylor Flat member (interstratified fluvial sand and overbank- } \\
\quad \text { paleosol deposits. } \\
\text { Rwi(e) = Ringold Wooded Island Unit subunit E (moderate to strongly cemented well } \\
\quad \text { rounded fluvial gravel and sand deposits). } \\
\text { (a) Multiply by } 0.3048 \text { to convert to meters. }\end{array}$} \\
\hline
\end{tabular}

\subsubsection{Cation Exchange Capacity}

The cation exchange capacity of the $<2$ millimeter fraction of the composite sediments from the borehole extension was measured as described in Section 3.3.5.11. The results are shown in Table 5.19. The values are remarkably similar except for $12 \mathrm{~A} / \mathrm{B}$, the caliche sample, and 38A/B from the Ringold Formation. These two composites have lower cation exchange capacity (CEC) values. If we account for the particle size of the samples and acknowledge that all the material $>2$ millimeters was not used, the CEC values might not be so similar. One simple method to compensate for particle size is to hypothesize that the larger than 2-millimeter material has no exchange sites such that the $<2$ millimeter material constitutes all the exchange capacity. Using this assumption, we can estimate the cation exchange capacity of the bulk sediment. As shown in the last column in Table 5.19, the adjusted CECs show that the sediments deeper in the Ringold Formation (composites 38A/B through 58A/B) have low adsorption capacity. However, because of its fine-grained size, the uppermost Ringold composite (17A/B) exhibits good adsorption capacity, similar to the fine-grained Plio-Pleistocene sediments found above the caliche. No cation exchange analyses were performed on the highly radioactive sidewall core samples but the CECs for similar uncontaminated sediments from outside the SX Tank Farm are found in Serne et al. (2002a). The values in Table 5.19 are considerably larger than values shown in Serne et al. (2002a) likely because the Polemio and Rhodes (1977) CEC methodology yields larger values ( a factor of 3 ) than the Amrhein and Suarez (1990) method that was chosen for the CEC measurements of uncontaminated sediments. 


\subsubsection{Particle Size Determination}

The particle size distribution data for sidewall core and borehole extension composite samples are shown in Tables 5.20 and 5.21 and in Figures 5.4 through 5.9. The sidewall cores were very small

Table 5.20. Particle Size Distribution of Sidewall Cores and Composite Sediments

\begin{tabular}{|c|c|c|c|c|c|c|c|c|c|}
\hline \multirow[b]{2}{*}{$\begin{array}{c}\text { Depth } \\
(\mathrm{ft} b g s)^{(a)}\end{array}$} & \multirow[b]{2}{*}{$\begin{array}{l}\text { Sample } \\
\text { ID }\end{array}$} & \multirow{2}{*}{$\begin{array}{c}\text { Gravel } \\
(2 \mathrm{~mm}) \\
\% \text { Retain } \\
\end{array}$} & \multirow{2}{*}{$\begin{array}{c}\text { Very Coarse } \\
\text { Sand }(1 \mathrm{~mm}) \\
\% \text { Retain }\end{array}$} & \multirow{2}{*}{$\begin{array}{c}\text { Coarse Sand } \\
(500 \mu \mathrm{m}) \\
\% \text { Retain }\end{array}$} & \multirow{2}{*}{$\begin{array}{c}\text { Medium Sand } \\
(250 \mu \mathrm{m}) \\
\% \text { Retain } \\
\end{array}$} & \multirow{2}{*}{$\begin{array}{c}\text { Fine Sand } \\
(106 \mu \mathrm{m}) \\
\% \text { Retain } \\
\end{array}$} & \multicolumn{2}{|c|}{ Very Fine Sand } & \multirow[b]{2}{*}{$\begin{array}{l}\text { Silt and Clay } \\
(<53 \mu \mathrm{m})\end{array}$} \\
\hline & & & & & & & $\begin{array}{l}(75 \mu \mathrm{m}) \\
\% \text { Retain }\end{array}$ & $\begin{array}{l}(53 \mu \mathrm{m}) \\
\% \text { Retain }\end{array}$ & \\
\hline \multicolumn{10}{|c|}{ Sidewall } \\
\hline 56.5 & $13 \mathrm{ABC}$ & 0.09 & 0.36 & 3.32 & 13.2 & 16.6 & 7.49 & 5.81 & 53.1 \\
\hline 74.5 & $9 \mathrm{ABC}$ & 21.8 & 17.7 & 21.5 & 12.7 & 8.88 & 1.61 & 0.56 & 15.3 \\
\hline 79.5 & $8 \mathrm{ABC}$ & 7.60 & 11.5 & 11.1 & 7.63 & 9.69 & 4.10 & 3.71 & 44.7 \\
\hline 90 & $6 \mathrm{AB}$ & 0.34 & 0.66 & 3.45 & 5.71 & 34.4 & 14.2 & 9.61 & 31.6 \\
\hline 108.5 & $4 \mathrm{ABC}$ & 0.05 & 0.37 & 1.31 & 4.19 & 34.9 & 15.7 & 9.01 & 34.5 \\
\hline \multicolumn{10}{|c|}{ Extension } \\
\hline 133.2 & $2 \mathrm{CD}$ & 2.18 & 2.61 & 3.52 & 1.94 & 12.9 & -- & 28.3 & 48.5 \\
\hline 134.2 & $2 \mathrm{AB}$ & 0.20 & 0.92 & 0.68 & 0.64 & 8.98 & -- & 37.9 & 50.7 \\
\hline 135.2 & $3 \mathrm{AB}$ & 0.36 & 0.98 & 1.67 & 0.69 & 8.72 & -- & 30.9 & 56.7 \\
\hline 153.9 & $10 \mathrm{AB}$ & 0.12 & 0.08 & 0.35 & 0.81 & 5.44 & -- & 13.4 & 79.8 \\
\hline 156.8 & $12 \mathrm{AB}$ & 13.5 & 6.50 & 6.69 & 9.54 & 20.7 & -- & 9.48 & 33.6 \\
\hline 165.3 & $17 \mathrm{AB}$ & 1.49 & 1.24 & 22.5 & 46.7 & 12.9 & -- & 5.24 & 9.93 \\
\hline 188.3 & $38 \mathrm{AB}$ & 86.7 & 1.07 & 0.78 & 4.99 & 2.78 & -- & 0.99 & 2.68 \\
\hline 196.9 & $47 \mathrm{AB}$ & 77.3 & 2.25 & 1.38 & 2.92 & 4.25 & -- & 5.15 & 6.78 \\
\hline 208.6 & $58 \mathrm{AB}$ & 78.4 & 1.59 & 1.39 & 8.33 & 4.64 & -- & 1.58 & 4.11 \\
\hline
\end{tabular}

Table 5.21. Details of Particle Size Distribution of Sidewall Core and Borehole Extension Composite Sediments

\begin{tabular}{|c|c|c|c|c|c|}
\hline $\begin{array}{c}\text { Depth } \\
\text { (ft bgs) }^{(a)}\end{array}$ & $\begin{array}{c}\text { Sample } \\
\text { ID }\end{array}$ & $\begin{array}{c}\text { Gravel } \\
(\%)\end{array}$ & $\begin{array}{c}\text { Sand } \\
(\%)\end{array}$ & $\begin{array}{l}\text { Silt } \\
(\%)\end{array}$ & $\begin{array}{c}\text { Clay } \\
(\%)\end{array}$ \\
\hline \multicolumn{6}{|c|}{ Sidewall } \\
\hline 56.5 & $13 \mathrm{ABC}$ & 0.09 & 46.77 & 48.04 & 5.10 \\
\hline 74.5 & $9 \mathrm{ABC}$ & 21.76 & 62.99 & 12.15 & 3.10 \\
\hline 79.5 & $8 \mathrm{ABC}$ & 7.60 & 47.66 & 39.94 & 4.80 \\
\hline 90 & $\overline{6 \mathrm{AB}}$ & 0.34 & 68.04 & 26.22 & 5.40 \\
\hline 108.5 & $4 \mathrm{ABC}$ & 0.05 & 65.47 & 29.48 & 5.00 \\
\hline \multicolumn{6}{|c|}{ Extension } \\
\hline 133.2 & $2 \mathrm{CD}$ & 2.18 & 49.33 & 39.71 & 8.79 \\
\hline 134.2 & $2 \mathrm{AB}$ & 0.20 & 49.09 & 42.34 & 8.37 \\
\hline 135.2 & $3 \mathrm{AB}$ & 0.36 & 42.96 & 37.16 & 19.52 \\
\hline 153.9 & $10 \mathrm{AB}$ & 0.12 & 20.12 & 62.56 & 17.20 \\
\hline 156.8 & $12 \mathrm{AB}$ & 13.52 & 52.85 & 24.57 & 9.05 \\
\hline 165.3 & $17 \mathrm{AB}$ & 1.49 & 88.58 & 8.08 & 1.85 \\
\hline 188.3 & $38 \mathrm{AB}$ & 86.70 & 10.62 & 2.28 & 0.40 \\
\hline 196.9 & $47 \mathrm{AB}$ & 77.27 & 15.95 & 5.71 & 1.07 \\
\hline 208.6 & $58 \mathrm{AB}$ & 78.35 & 17.53 & 2.39 & 1.72 \\
\hline \multicolumn{6}{|c|}{ Multiply by 0.3048 to convert to meters } \\
\hline
\end{tabular}




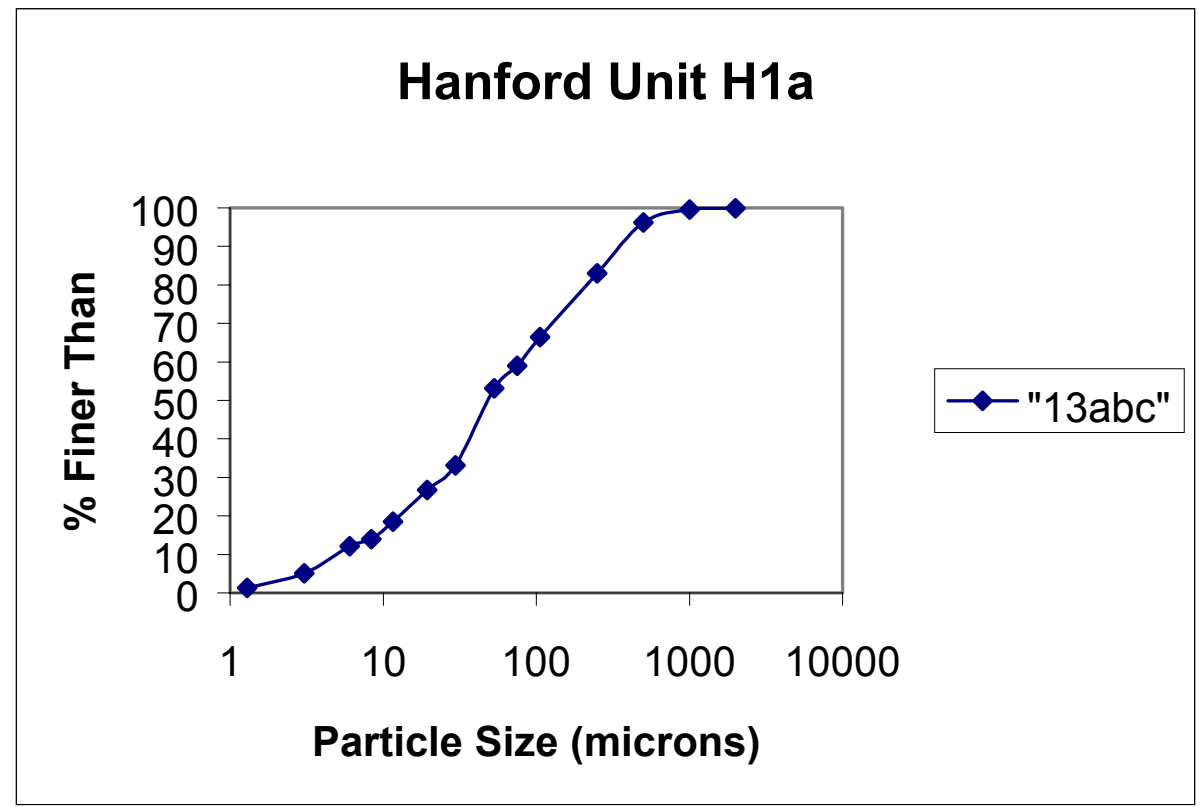

Figure 5.4. Particle Size Distribution of Sidewall Core in Hanford Formation Unit H1a

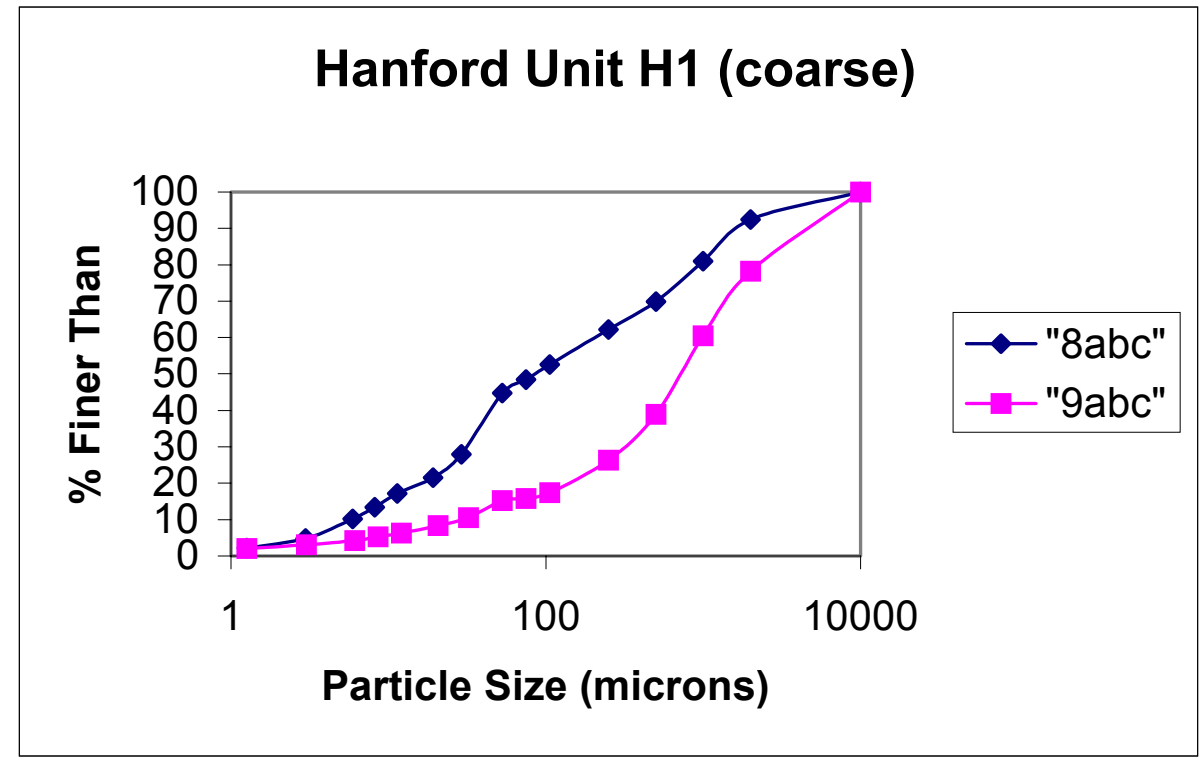

Figure 5.5. Particle Size Distribution of Sidewall Cores in Hanford Formation Unit H1 


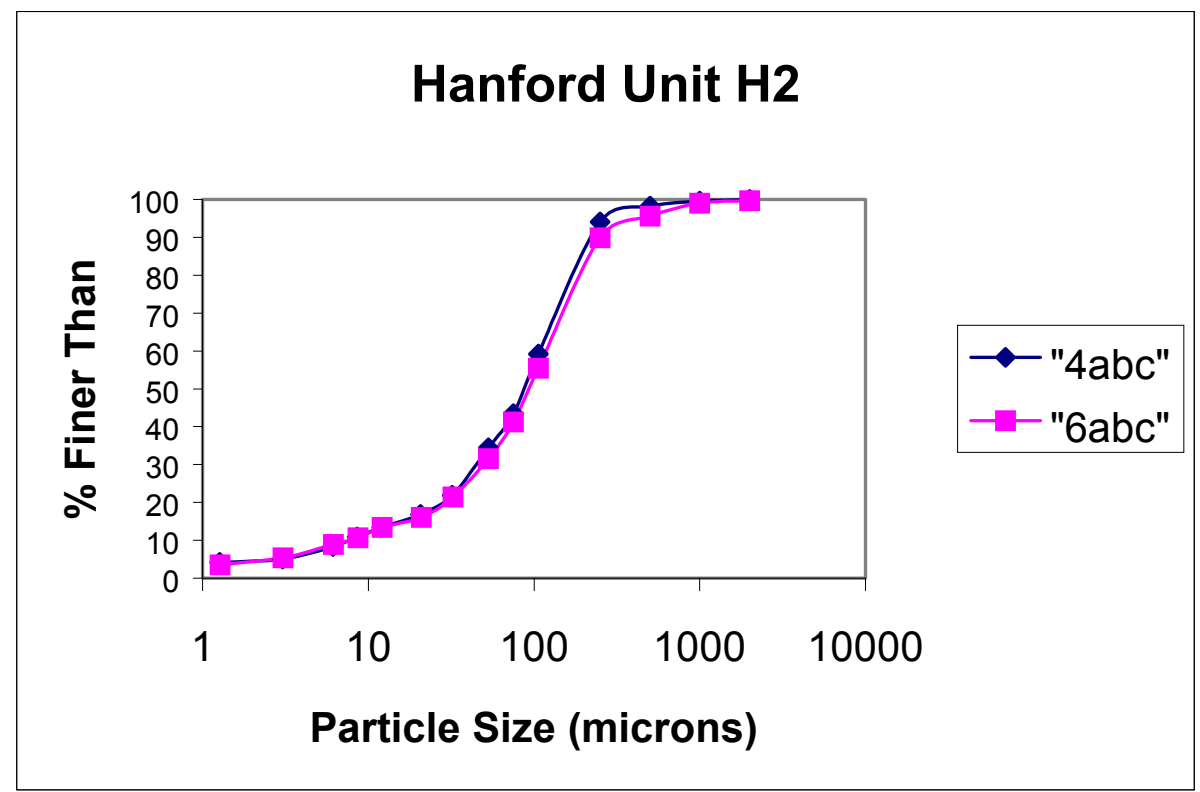

Figure 5.6. Particle Size Distribution for Sidewall Cores in Hanford Formation Unit H2 (laminated sands)

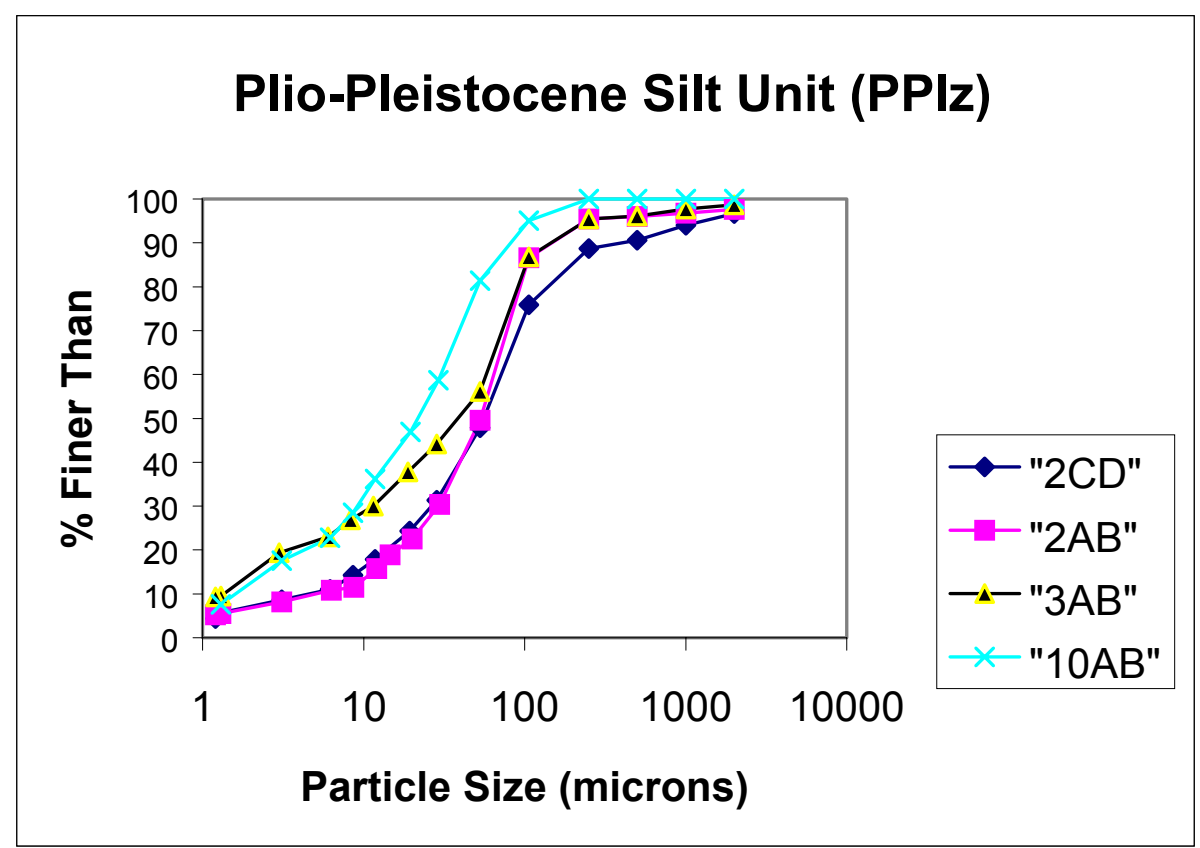

Figure 5.7. Particle Size Distribution for Borehole Extension Sediment in Plio-Pleistocene 


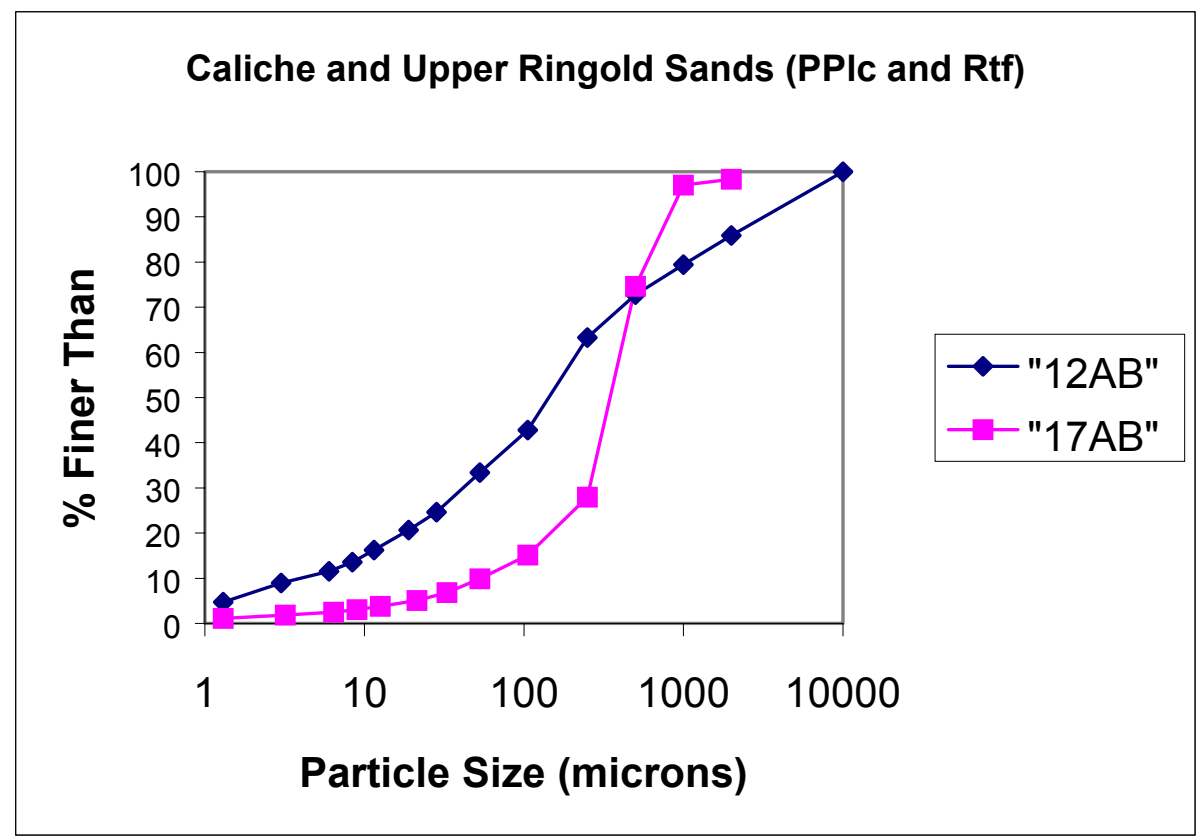

Figure 5.8. Particle Size Distribution for Borehole Extension Composites ([12-AB] caliche and [17-AB] Ringold Taylor Flat fluvial sand)

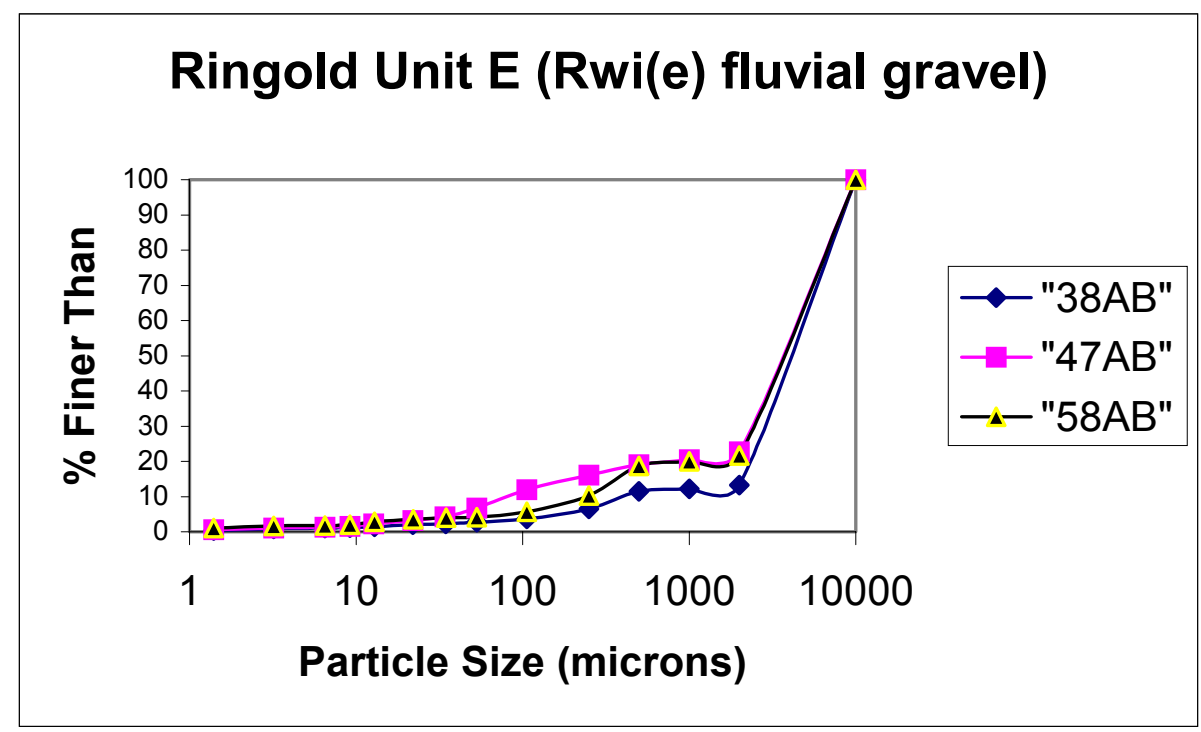

Figure 5.9. Particle Size Distribution for Borehole Extension Composites in Ringold Formation Unit E 
samples (25 millimeters x 25 millimeters x 28 centimeters [0.99 inch x 0.99 inch x 11 inches]) and may not retain larger gravels. Thus, the reported particle sizes may be biased to a finer distribution than is actually present. The relative amounts of gravel, several sand sizes, and the sum of silt and clay are listed in Tables 5.20 and 5.21, which include wet sieving and hydrometer data to separate the silt and clay. Each figure presents the particle size distributions for one distinct stratigraphic unit, to give a visual estimate of variability, when more than one data set is available for a unit. The samples may be grouped into the following seven units:

- Predominately medium to fine sand + silt + clay (Hanford formation unit H1a) (sidewall 13ABC)

- Coarse sand and gravel with little fine sand and silt (Hanford formation unit H1) (sidewall 8 and 9 $\mathrm{ABC})$

- Predominately medium to fine sand + silt + clay (Hanford formation unit $\mathrm{H} 2$ ) (sidewall cores $6 \mathrm{AB}$ and $4 \mathrm{ABC})$

- Very fine sand + silt + clay grained PPlz (borehole extension composites $2 \mathrm{C} / \mathrm{D}, 2 \mathrm{~A} / \mathrm{B}, 3 \mathrm{~A} / \mathrm{B}$, and $10 \mathrm{~A} / \mathrm{B})$

- Mixed gravel + fine sand + silt and clay PPlc carbonate facies (borehole extension composite 12A/B)

- Predominately sand with some silt + clay Ringold Rtf (member of Ringold Taylor Flat facies also called Upper Ringold unit [borehole extension composite 17A/B])

- Predominately gravel with some fines Ringold Rwi(e) (member of Ringold Wooded Island member facies also called Ringold Formation unit $\mathrm{E}$ [borehole extension composites 38A/B, 47A/B, and $58 \mathrm{~A} / \mathrm{B}])$.

The fourth group is the slack-water Plio-Pleistocene sediments, the fifth is the weakly cemented caliche, the sixth is an interstratified fluvial sand and overbank-paleosol deposits unit above the Ringold Formation unit E (member of Taylor Flat), and the seventh is the Ringold Formation unit E (also known as the member of Wooded Island) moderate to strongly cemented well rounded fluvial gravel and sand deposits, with interstratified overbank-paleosol and lacustrine deposits. Serne et al. (2002a) more thoroughly describes the geology and presents some additional particle size information from dry sieving of archived samples for each unit. Care must be taken when comparing past particle size information based on dry sieving with the wet sieving and dispersed particle hydrometer measurements of silt and clay. Wet sieving and hydrometer tests using dispersed material yields a finer particle size distribution than dry sieving, which does not break up as many agglomerates and does not remove all the fines that cling to larger particles.

Table 5.22 gives the particle densities of the bulk sediments from several of the sidewall core composites. In general, the data are comparable to other measurements of Hanford formation sediments (see Serne et al. 1993) and somewhat more dense than quartz $\left(2.65 \mathrm{~g} / \mathrm{cm}^{3}\right)$. The particle densities are slightly heavier than measurements recently performed on uncontaminated sediments from neighboring 
Table 5.22. Particle Density of Bulk Sediment

\begin{tabular}{||c|c|c||}
\hline $\begin{array}{c}\text { Depth } \\
(\mathrm{ft} \mathrm{bgs})^{(\mathrm{a})}\end{array}$ & $\begin{array}{c}\text { Sample } \\
\text { ID (Unit) }\end{array}$ & $\begin{array}{c}\text { Particle Density } \\
\left(\mathrm{g} / \mathrm{cm}^{3}\right)\end{array}$ \\
\hline \hline \multicolumn{3}{|c||}{ Sidewall } \\
\hline 56.5 & 13ABC (H1a) & 2.829 \\
\hline 74.5 & $9 \mathrm{ABC}(\mathrm{H} 1)$ & 2.801 \\
\hline 79.5 & $8 \mathrm{ABC}(\mathrm{H} 1)$ & 2.808 \\
\hline 90 & $6 \mathrm{AB}(\mathrm{H} 2)$ & 2.758 \\
\hline 108.5 & 4ABC (H2) & 2.774 \\
\hline (a) Multiply by 0.3048 to convert to meters. \\
\hline
\end{tabular}

boreholes (Serne et al. 2002a). The differences are likely not real suggesting that the measurements in Serne et al. (2002a) are slightly biased to lighter densities. Slight differences in analyst's techniques are suspected as the cause.

The particle density of the sample from the Hanford formation H1a unit (upper fine sand and silt sequence) is larger than the density of the Hanford formation H1 unit (middle coarse sand and gravel sequence), which in turn is slightly denser than the Hanford formation H2 unit (lower fine sand and silt and sequence) samples. There is good agreement between the pairs of measurements for the Hanford formation $\mathrm{H} 1$ unit and Hanford formation $\mathrm{H} 2$ unit samples.

\subsubsection{Mineralogy of Borehole 41-09-39}

X-ray diffraction (XRD) analyses of the bulk sidewall core samples from five depths (Table 5.23) in borehole 41-09-39 indicate that the sediments are mostly quartz ( $\sim 35 \%$ to $50 \%)$ and feldspar ( $\sim 25 \%$ to $55 \%$ ), with lesser amounts of mica and chlorite. Plagioclase feldspar is 2 to 10 times more abundant than potassium feldspar. Minor amounts of amphibole and calcite were also detected in the sediments. Examples of x-ray diffractograms are presented in Appendix E.

The clay fraction $(<2$ micron) is dominated by four clay minerals: illite $(10 \AA)$, smectite $(15 \AA)$, chlorite (14.1 $\AA$ ), and kaolinite (7 $\AA$ ) with minor amounts of quartz $(3.34 \AA)$, feldspar $(3.18 \AA)$, and amphibole $(8.4 \AA)$. The smectites, when saturated with $\mathrm{Mg}^{2+}$, gave a basal reflection of $15.0 \AA$, overlapping the chlorite $14.1 \AA$ peak (see Appendix E). When solvated with ethylene glycol, the smectite reflection expanded up to $17 \AA$, leaving the chlorite $(14.1 \AA)$ and illite $(10 \AA)$ reflections unchanged. An additional analysis with $\mathrm{K}^{+}$as the interlayer cation shifted the smectite peak to approximately $12 \AA$ and again the chlorite $(14.4 \AA)$ and illite $(10 \AA)$ remain unaffected. Heating the $\mathrm{K}^{+}$-saturated slide to $575^{\circ} \mathrm{C}$, collapsed the smectite structure from $12.0 \AA$ to $10.0 \AA$ and the $7.01 \AA$ and $3.58 \AA$ kaolinite peaks disappeared. The chlorite reflection (14.1 $\AA$ ) remained constant whereas the $7.1 \AA$ and $3.54 \AA$ reflections disappeared. 
Table 5.23. Semiquantitative Mineral Composition for Bulk Samples (wt $\%)$

\begin{tabular}{|c|c|c|c|c|c|c|}
\hline Sample ID & Depth $\mathrm{ft}^{(\mathrm{a})}$ & Geologic Unit & Quartz & K-Feldspar & Plagioclase & Calcite \\
\hline $13 \mathrm{ABC}$ & $56-57$ & H1a & 50 & 5 & 50 & ND \\
\hline $9 \mathrm{ABC}$ & $74-75$ & H1 & 50 & 5 & 25 & ND \\
\hline $8 \mathrm{ABC}$ & $79-80$ & H1 & 35 & 5 & 55 & ND \\
\hline $6 \mathrm{AB}$ & 90 & $\mathrm{H} 2$ & 50 & 15 & 25 & $<5$ \\
\hline $4 \mathrm{ABC}$ & 108-109 & $\mathrm{H} 2$ & 45 & 5 & 40 & $<5$ \\
\hline
\end{tabular}

The identification of each clay mineral was based on the following criteria:

- Smectites are the fraction of the $\mathrm{Mg}^{2+}$ saturated subsample that gives a $\sim 12$ to $15 \AA$ peak (air-dried) which expands to $\sim 17 \AA$ after saturation with ethylene glycol. Upon saturation with $\mathrm{K}^{+}$, the peak shifts to $\sim 12 \AA$ and collapses to $\sim 10 \AA$ after heating to $575^{\circ} \mathrm{C}$ for 1 hour.

- Illites are the fraction of the subsample that gives a $10 \AA$ reflection and remains unchanged during saturation with $\mathrm{Mg}^{2+}, \mathrm{K}^{+}$, or ethylene glycol. Furthermore, microprobe analysis showed the illites containing $\sim 0.75 \mathrm{~K}^{+}$atoms per $\mathrm{O}_{10}(\mathrm{OH})_{2}$, which is the definition of illite given by Reynolds and Reynolds (1989).

- Kaolinites are the fraction of the $\mathrm{Mg}^{2+}$ saturated subsample that gives a $7.1 \AA$ and $3.58 \AA$ reflection that does not change when saturated with ethylene glycol. Heating either the $\mathrm{Mg}^{2+}$ or $\mathrm{K}^{+}$saturated slide to $575^{\circ} \mathrm{C}$ destroys the kaolinite structure and the $7.1 \AA$ and $3.58 \AA$ kaolinite reflections disappear. Additionally, electron microprobe analysis was used to confirm the presence of kaolinite.

- Chlorites are the fraction of the $\mathrm{Mg}^{2+}$ saturated subsample that gives $14.1 \AA$, $7.1 \AA$, and $3.54 \AA$ reflections that remain unchanged either by cation saturation $\left(\mathrm{Mg}^{2+}, \mathrm{K}^{+}\right)$or ethylene glycol treatment. Heating the sample to $575^{\circ} \mathrm{C}$ leaves the $14.1 \AA$ unchanged or slightly decreased; the $7.1 \AA$ and $3.54 \AA$ reflections are greatly weakened, but not totally eliminated.

The semiquantitative analysis of minerals in the clay fraction is given in Table 5.24. Overall, illite was the dominant mineral in the clay fraction with 20 to $35 \mathrm{wt} \%$. Smectites ranged in concentrations from as high as $20 \mathrm{wt} \%$ (samples $13 \mathrm{ABC}$ and $4 \mathrm{ABC}$ at depths 17.1 and 32.9 meters (56 and 108 feet), respectively) to as low as $5 \mathrm{wt} \%$; chlorite occurred between $\sim 10$ and $30 \mathrm{wt} \%$. Minor amounts of kaolinite $(<10 \mathrm{wt} \%)$ were detected at all depths. Quartz and feldspar made up $\sim 5$ to $20 \mathrm{wt} \%$ of the clay fraction. Amphibole was identified in the clay fraction in minor amounts; however, it was not quantified. Total mass balance for the clay fractions ranged from a low of $65 \%$ to a high of $105 \%$. Recoveries ranging from $80 \%$ to $120 \%$ are deemed acceptable for XRD semiquantitative analysis. 
Table 5.24. Semiquantitative Mineral Composition for the Clay Fraction $(<2 \mu \mathrm{m})$ of Sediment Samples (wt $\%)$

\begin{tabular}{||l|l|c|c|c|c|c|c|c||}
\hline \hline Sample ID & Depth $\mathrm{ft}^{(\mathrm{a})}$ & Geologic Unit & Quartz & Feldspar & Smectite & Illite & Chlorite & Kaolinite \\
\hline \hline 13ABC & $56-57$ & $\mathrm{H} 1 \mathrm{a}$ & 5 & $<5$ & 20 & 35 & 30 & 10 \\
\hline $9 \mathrm{ABC}$ & $74-75$ & $\mathrm{H} 1$ & 10 & 10 & 5 & 30 & 20 & 10 \\
\hline $8 \mathrm{ABC}$ & $79-80$ & $\mathrm{H} 1$ & 10 & $<5$ & 10 & 20 & 15 & 5 \\
\hline $6 \mathrm{AB}$ & 90 & $\mathrm{H} 2$ & $<5$ & $<5$ & 15 & 20 & 10 & 10 \\
\hline 4ABC & $108-109$ & $\mathrm{H} 2$ & 5 & $<5$ & 20 & 20 & 10 & 10 \\
\hline (a) Multiply by 0.3048 to convert to meters.
\end{tabular}

Transmission electron microscopy (TEM) analysis of the illite from 22.7-, 27.4-, and 33.1-meter (74.5-, 90-, and 108.5-foot) depths show large, angular, platy particles typical of weathered muscovite and, in lesser amounts, weathered biotite. The iron content of the illite particles (produced from weathered muscovite) was dependent on size and thickness. Larger illite particles ( $>1$ micron) tended to have less iron than the more abundant, smaller and thinner particles. Figure 5.10 is a typical illite from 22.7-meter (74.5-foot) depth. Using data from the TEM analysis and assuming all iron as $\mathrm{Fe}^{3+}$, the following structural formula was calculated for the illite in Figure 5.10:

$$
\left[\mathrm{K}_{0.69}^{+}\right]^{+0.69}\left[\left(\mathrm{Al}^{3+}{ }_{1.69} \mathrm{Mg}^{2+}{ }_{0.22} \mathrm{Fe}^{3+}{ }_{0.11} \mathrm{Ti}^{4+}{ }_{0.02}\right)^{-0.08}\left(\mathrm{Si}^{4+}{ }_{3.39} \mathrm{Al}^{3+}{ }_{0.61}\right)^{-0.61}\right]^{-0.69} \mathrm{O}_{10}(\mathrm{OH})_{2}
$$

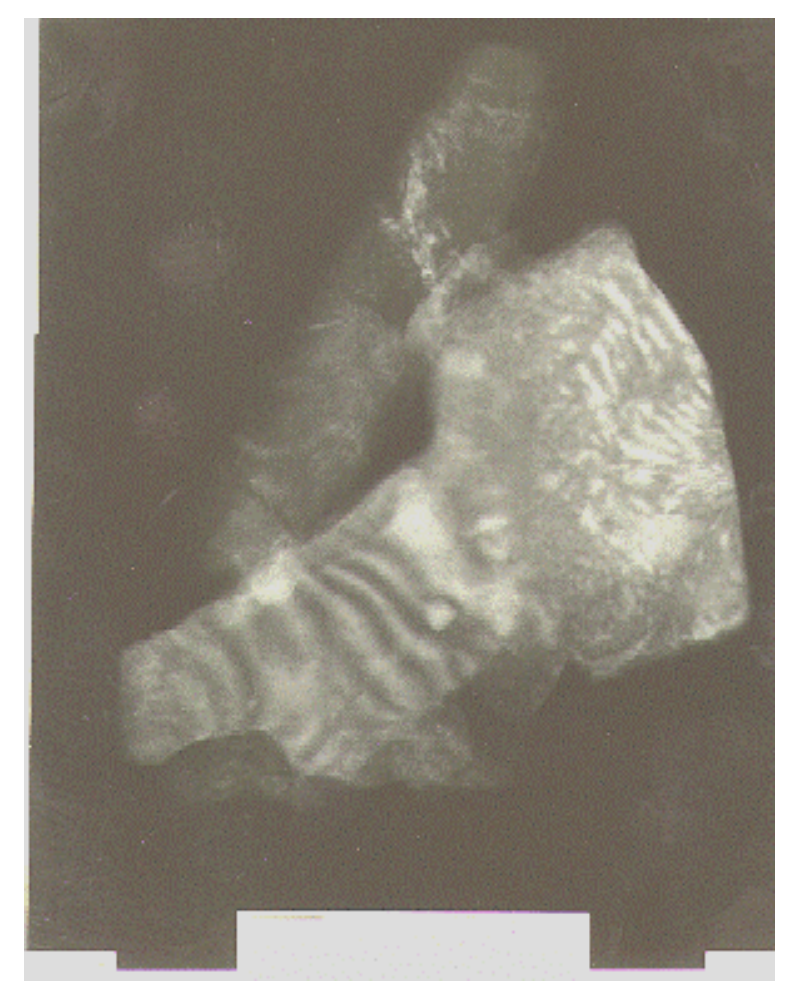

Figure 5.10. Typical Illite Particle from Depth 22.7 meters ( 74.5 feet) ( $\sim 1$ micron) 
As in muscovite, most of the layer charge for the illite originates in the tetrahedral sheet (-0.61), with some contribution from the octahedral sheet $(-0.08)$, resulting in a 2:1 layer charge of -0.69 . The interlayer charge of +0.69 balances the charge on the $2: 1$ silicate structure. Iron content of six illites examined ranged between 0.05 and 0.33 atoms per $\mathrm{O}_{10}(\mathrm{OH})_{2}$ and $\mathrm{Mg}^{2+}$ ranged between 0.11 and 0.35 atoms per $\mathrm{O}_{10}(\mathrm{OH})_{2}$. Traces of $\mathrm{Ti}^{+4}$ were detected in most of the illites examined.

Weathered biotite particles were large and denser than other platy clay minerals examined in the clay fraction. TEM analysis of two weathered biotites from a depth of 33.1 meters ( 108.5 feet) produced an average structural formula with almost equal amounts of $\mathrm{Mg}^{2+}$ and $\mathrm{Fe}^{3+}$ :

$$
\left[\mathrm{K}_{0.73}^{+} \mathrm{Ca}_{0.02}\right]^{+0.77}\left[\left(\mathrm{Al}^{3+}{ }_{0.74} \mathrm{Mg}^{2+}{ }_{0.78} \mathrm{Fe}^{3+}{ }_{1.00} \mathrm{Ti}^{4+}{ }_{0.12}\right)^{+0.26}\left(\mathrm{Si}^{4+}{ }_{2.93} \mathrm{Al}^{3+}{ }_{1.07}\right)^{-1.07}\right]^{-0.81} \mathrm{O}_{10}(\mathrm{OH})_{2}
$$

Substitution of $\mathrm{Al}^{3+}$ for $\mathrm{Si}^{4+}$ results in a tetrahedral charge of -1.07. The principal octahedral cations $\left(\mathrm{Al}^{3+}, \mathrm{Fe}^{2+}\right.$, and $\left.\mathrm{Mg}^{2+}\right)$ along with minor amounts of $\mathrm{Ti}^{4+}$ contribute a layer charge of +0.26 . The sum of the charges of the interlayer cations balances the layer charge from the octahedral sheet $(+0.26)$ and the tetrahedral sheet (-1.07). Octahedral cations total 2.64 atoms per $\mathrm{O}_{10}(\mathrm{OH})_{2}$, which is less than the ideal 3 atoms per $\mathrm{O}_{10}(\mathrm{OH})_{2}$ for a typical trioctahedral biotite mineral. The chemistry of these particles closely resembles a ferroan biotite analyzed by Newman (1987), which had $\mathrm{Mg}^{2+}$ and $\mathrm{Fe}^{2+}$ concentrations of 1.01 and $1.1\left(\right.$ per $\mathrm{O}_{10}(\mathrm{OH})_{2}$, respectively.

The presence of illites as the dominant clay sized mineral is fortuitous because illites are strong adsorbers of cesium-137 and can irreversibly adsorb cesium-137 within interlayer sites. More discussion of cesium-137 adsorption on illites is found in documents prepared for publication under companion EMSP and Science and Technology Program (see Zachara et al. 1999, 2002).

Chlorite had a similar morphology to the illite in TEM images, with most being thin and platy (Figure 5.11). Examination of chlorites by TEM showed a significant variability in the concentrations of $\mathrm{Mg}^{2+}$ and $\mathrm{Fe}^{2+}$. The chlorites ranged between a magnesium-rich chamosite to an iron-rich chlinoclore. Figure 5.11 is an example of a platy chlorite containing almost equal amounts of $\mathrm{Mg}^{2+}$ and $\mathrm{Fe}^{2+}$, and Figure 5.12 is an example of a magnesium-rich chlorite.

Developing a structural formula from an average of the $\mathrm{Mg}^{2+}$-rich chlorites analyzed by TEM from the three depths gives the following:

$$
\left[\mathrm{K}_{0.01} \mathrm{Ca}_{0.04}\right]^{+0.05}\left[\left(\mathrm{Al}_{1.21} \mathrm{Mg}_{2.86} \mathrm{Fe}_{1.82}\right)\right]^{+0.99}\left[\left(\mathrm{Si}_{2.93} \mathrm{Al}_{1.07}\right)\right]^{-1.07} \mathrm{O}_{10}(\mathrm{OH})_{8}
$$

The structural formula shows the negative charge in the tetrahedral sheet, originating from the substitution of $\mathrm{Al}^{3+}$ for $\mathrm{Si}^{4+}$, is balanced by the inclusion of the trivalent cation, $\mathrm{Al}^{3+}$, and divalent cations, $\mathrm{Mg}^{2+}$ and $\mathrm{Fe}^{2+}$, into the octahedral sheets. The total number of cations occupying octahedral sites is 5.89 per $\mathrm{O}_{10}(\mathrm{OH})_{8}$, making it a trioctahedral ferroan chamasite. By contrast, the structural formula developed for the $\mathrm{Fe}^{2+}$ rich chlorites analyzed by TEM from three depths gives the following:

$$
\left[\mathrm{K}_{0.13} \mathrm{Ca}_{0.08}\right]^{+0.29}\left[\left(\mathrm{Al}_{1.74} \mathrm{Mg}_{0.91} \mathrm{Fe}_{2.34} \mathrm{Ti}_{0.01}\right)\right]^{-0.24}\left[\left(\mathrm{Si}_{3.96} \mathrm{Al}_{0.04}\right)\right]^{-0.04} \mathrm{O}_{10}(\mathrm{OH})_{8}
$$




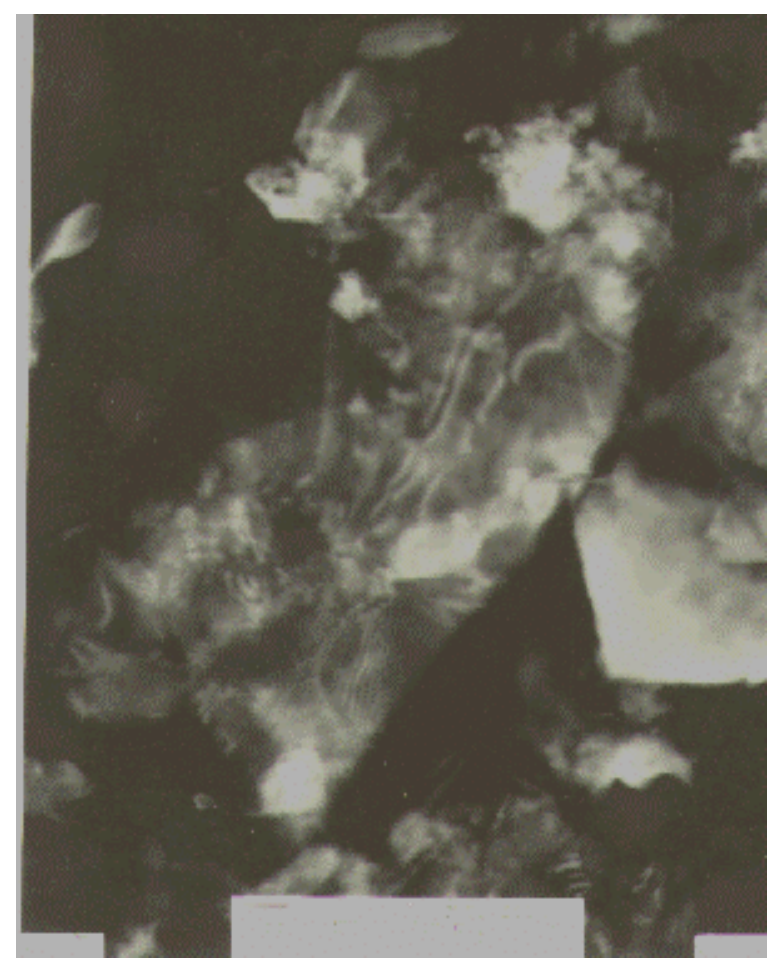

Figure 5.11. Large Chlorite Particle ( $\sim 3$ microns) Surrounded by Smaller Illites and Smectites

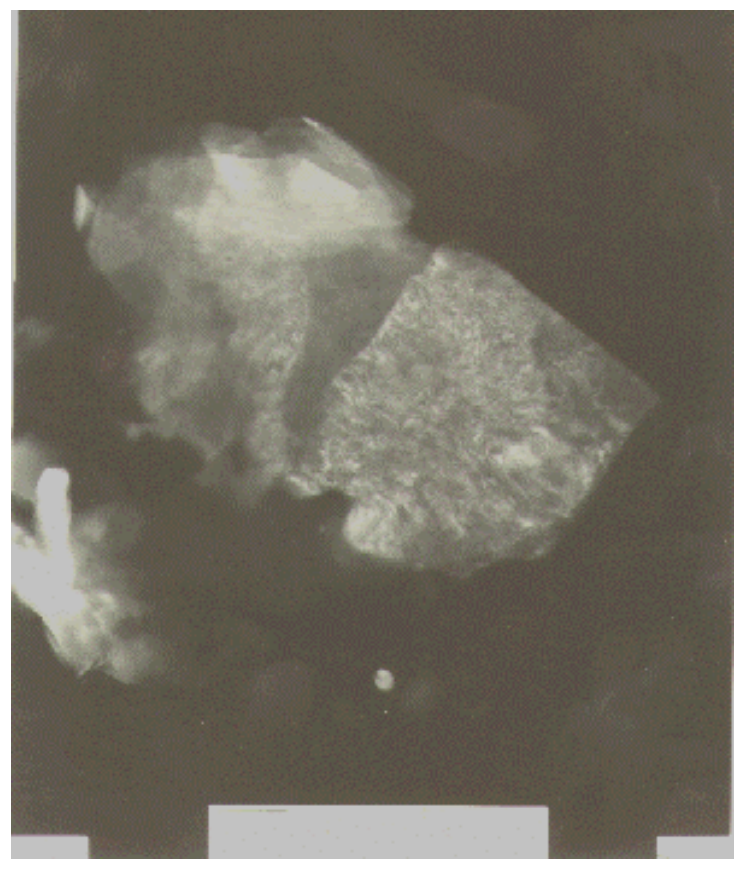

Figure 5.12. Large Magnesium-Rich Chlorite ( $\sim 4$ microns) 
With only $0.04 \mathrm{Al}^{3+}$ atoms per $\mathrm{O}_{10}(\mathrm{OH})_{8}$ substituting for $\mathrm{Si}^{4+}$ in the tetrahedral sheet, a -0.04 charge is produced. Charges derived from the octahedral sheet of $(-0.24)$ are balanced by small amounts of $\mathrm{K}^{+}$and $\mathrm{Ca}^{2+}$, which are possibly included in structure of the chlorite. Cations for the octahedral sheets total five per $\mathrm{O}_{10}(\mathrm{OH})_{8}$, making this chlorite a di-trioctahedral (one dioctahedral sheet and one trioctahedral sheet).

Smectite particles were very small ( $<0.5$ microns) and difficult to isolate from other clay minerals for characterization by TEM. The average composition of several smectite aggregates shows $\sim 0.53$ atoms of $\mathrm{Fe}^{3+}$, per $\mathrm{O}_{10}(\mathrm{OH})_{4}$ in the octahedral site with the remaining sites occupied by $\mathrm{Al}^{3+}, \mathrm{Mg}^{2+}$, and trace amounts of $\mathrm{Ti}^{4+}$. During $\mathrm{Mg}^{2+}$-saturation, prior to the TEM analysis, the smectites exchanged natural $\mathrm{Na}^{+}$ and $\mathrm{Ca}^{2+}$ interlayer cations for $\mathrm{Mg}^{2+}$. Therefore, $\mathrm{Mg}^{2+}$ occurs as the interlayer cation and in the octahedral sheet and could not be partitioned between the two sites.

Kaolinite particles were platy in habit, but lacked the typical hexagonal morphology found in many samples. Analysis showed a slight substitution of $\mathrm{Fe}^{3+}$ for $\mathrm{Al}^{3+}$ in the octahedral sites for $\mathrm{Al}^{3+}$, as shown in the structural formula:

$$
\left(\mathrm{Al}_{3.90} \mathrm{Fe}_{0.09}\right) \mathrm{Si}_{4.00} \mathrm{O}_{10}(\mathrm{OH})_{8}
$$

Additionally, minerals such as sepeolite, apatite, Fe-oxide, and anatase were detected in trace amounts during TEM analysis.

Three intervals from the borehole extension project were selected for limited mineralogical characterization. The samples studied $(2 \mathrm{C} / \mathrm{D}, 2 \mathrm{~A} / \mathrm{B}$, and 3A/B) were from depths 40.6 to 41.2 meters (133.2 to 135.2 feet) bgs, which showed elevated radioactivity compared to the deeper sediments. However, these samples were also from the region that was disturbed by the original pile driving of the casing and end cap milling. As discussed below, we determined later that all three samples have abnormally high iron contamination from the milling of the end cap. Optical examination of the sediments revealed quartz, muscovite, and biotite. Additionally, abundant limonite cement was observed in sample $2 \mathrm{C} / \mathrm{D}$, which probably resulted from the corrosion of the steel from cutting through the casing end cap and destruction of several drill bits during drilling of the end cap. No crystalline iron phases were detected during the $\mathrm{XRD}$ analysis of these sediments.

After sieving and drying sediment from sleeve 2C/D, XRD analysis was conducted on the individual size fractions. Quartz, plagioclase, and mica were identified in all seven fractions. Analysis of the clay fraction from $2 \mathrm{C} / \mathrm{D}, 2 \mathrm{AB}$, and $3 \mathrm{~A} / \mathrm{B}$ indicated the presence of smectite, illite, chlorite, and kaolinite, along with minor amounts of non-clay minerals such as quartz and plagioclase feldspar. Examples of the $\mathrm{XRD}$ tracings from the sieved sample $2 \mathrm{C} / \mathrm{D}$ and from the magnesium-saturated clay fractions of $2 \mathrm{C} / \mathrm{D}$, $2 \mathrm{~A} / \mathrm{B}$, and $3 \mathrm{~A} / \mathrm{B}$ are presented in Appendix E.

\subsubsection{Cesium-137 Content as a Function of Particle Size}

Finding that trace constituents, such as cesium-137, adsorb onto the fine-grained portion of a bulk sediment is quite common. The size separations for composites $2 \mathrm{C} / \mathrm{D}, 2 \mathrm{~A} / \mathrm{B}, 3 \mathrm{~A} / \mathrm{B}$, and $12 \mathrm{~A} / \mathrm{B}$ shown in 
Table 5.20 were gamma counted to determine where the cesium-137 was adsorbed. The data for the individual size fractions are shown in Table 5.25.

In general, the data support the hypothesis that the fine-grained material (silt and clay) contains most of the mass of radioactive cesium. It is, however, surprising that the concentrations ( $\mathrm{pCi} / \mathrm{g}$ ) of cesium-137 in each size fraction do not show a wider range of values with the finer-grained sizes showing much higher values than the coarse-grained sizes. The silt and clay fractions contain $67 \%, 27 \%, 81 \%$, and $69 \%$ of the cesium- 137 for composites $2 \mathrm{C} / \mathrm{D}, 2 \mathrm{~A} / \mathrm{B}, 3 \mathrm{~A} / \mathrm{B}$, and $12 \mathrm{~A} / \mathrm{B}$, respectively. Figures 5.13 through 5.16 are bar charts showing the percentage of the total cesium-137 in each size separation. The composite sediments show $12 \%$ to $15 \%$ of the cesium-137 in the gravel and coarse sand fraction except

Table 5.25. Cesium-137 Distribution as a Function of Particle Size

\begin{tabular}{|c|c|c|c|c|c|c|c|c|}
\hline $\begin{array}{c}\text { Depth } \\
(\mathrm{ft} \mathrm{bgs})^{(\mathrm{a})}\end{array}$ & ID & $\begin{array}{l}\text { Gravel } \\
(2 \mathrm{~mm}) \\
{ }_{137} \mathrm{Cs} \\
(\mathrm{pCi} / \mathrm{g})\end{array}$ & $\begin{array}{c}\text { Very Coarse } \\
\text { Sand } \\
(1 \mathrm{~mm}) \\
{ }_{137} \mathrm{Cs} \\
(\mathrm{pCi} / \mathrm{g})\end{array}$ & $\begin{array}{c}\text { Coarse Sand } \\
(500 \mu \mathrm{m}) \\
{ }_{137} \mathrm{Cs} \\
(\mathrm{pCi} / \mathrm{g})\end{array}$ & $\begin{array}{c}\text { Medium Sand } \\
(250 \mu \mathrm{m}) \\
{ }_{137} \mathrm{Cs} \\
(\mathrm{pCi} / \mathrm{g})\end{array}$ & $\begin{array}{c}\text { Fine Sand } \\
(106 \mu \mathrm{m}) \\
{ }^{137} \mathrm{Cs} \\
(\mathrm{pCi} / \mathrm{g})\end{array}$ & $\begin{array}{c}\text { Very Fine Sand } \\
(53 \mu \mathrm{m}) \\
{ }_{137} \mathrm{Cs} \\
(\mathrm{pCi} / \mathrm{g})\end{array}$ & $\begin{array}{c}\text { Silt and Clay } \\
(<53 \mu \mathrm{m}) \\
{ }_{137} \mathrm{Cs} \\
(\mathrm{pCi} / \mathrm{g})\end{array}$ \\
\hline \multicolumn{9}{|c|}{ Extension } \\
\hline 133.2 & $2 \mathrm{CD}$ & $6.22 \mathrm{E}+04$ & $6.80 \mathrm{E}+04$ & $5.13 E+04$ & $7.22 \mathrm{E}+04$ & $8.01 \mathrm{E}+03$ & $1.07 \mathrm{E}+04$ & $4.28 \mathrm{E}+04$ \\
\hline 134.2 & $2 \mathrm{AB}$ & $5.16 \mathrm{E}+04$ & $7.05 \mathrm{E}+04$ & $6.13 \mathrm{E}+04$ & $3.76 \mathrm{E}+04$ & $1.21 \mathrm{E}+03$ & $1.91 \mathrm{E}+02$ & $1.15 \mathrm{E}+03$ \\
\hline 135.2 & $3 \mathrm{AB}$ & $2.72 \mathrm{E}+04$ & $2.71 \mathrm{E}+04$ & $2.22 \mathrm{E}+04$ & $1.98 \mathrm{E}+04$ & $1.11 \mathrm{E}+03$ & $5.79 \mathrm{E}+02$ & $8.71 \mathrm{E}+03$ \\
\hline 156.8 & $12 \mathrm{AB}$ & $4.36 \mathrm{E}+00$ & $1.40 \mathrm{E}+02$ & $9.75 \mathrm{E}+01$ & $9.96 \mathrm{E}+01$ & $6.79 \mathrm{E}+01$ & $3.39 \mathrm{E}+01$ & $2.85 \mathrm{E}+02$ \\
\hline & & \multicolumn{7}{|c|}{$\%{ }^{137} \mathrm{Cs}$} \\
\hline 133.2 & $2 \mathrm{CD}$ & 4.35 & 5.70 & 5.80 & 4.49 & 3.32 & 9.71 & 66.63 \\
\hline 134.2 & $2 \mathrm{AB}$ & 4.74 & 29.81 & 19.16 & 11.08 & 4.99 & 3.32 & 26.89 \\
\hline 135.2 & $3 \mathrm{AB}$ & 1.61 & 4.37 & 6.09 & 2.25 & 1.59 & 2.94 & 81.16 \\
\hline 156.8 & $12 \mathrm{AB}$ & 0.42 & 6.56 & 4.70 & 6.85 & 10.11 & 2.31 & 69.04 \\
\hline
\end{tabular}

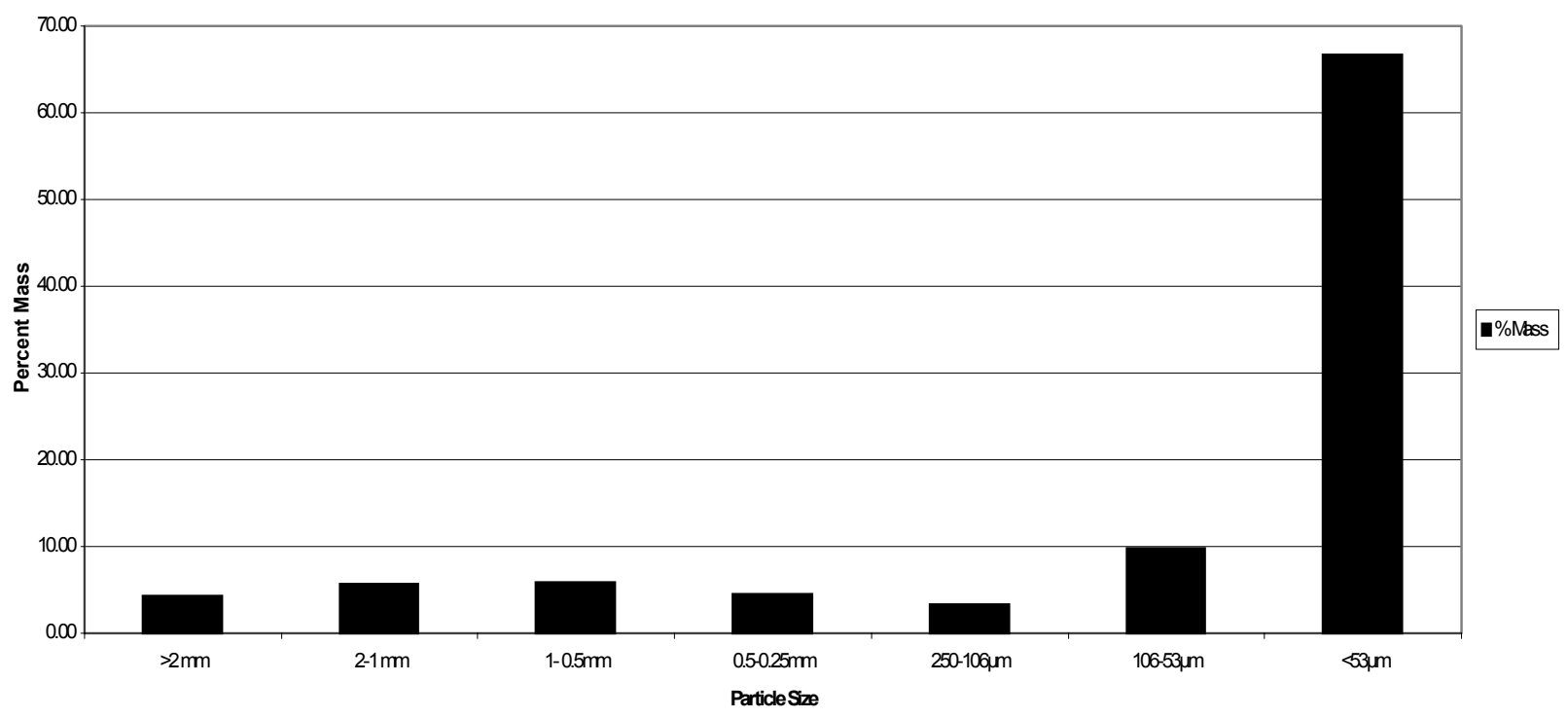

Figure 5.13. Percent of Cesium Found in each Size Fraction of Composite 2C/D 


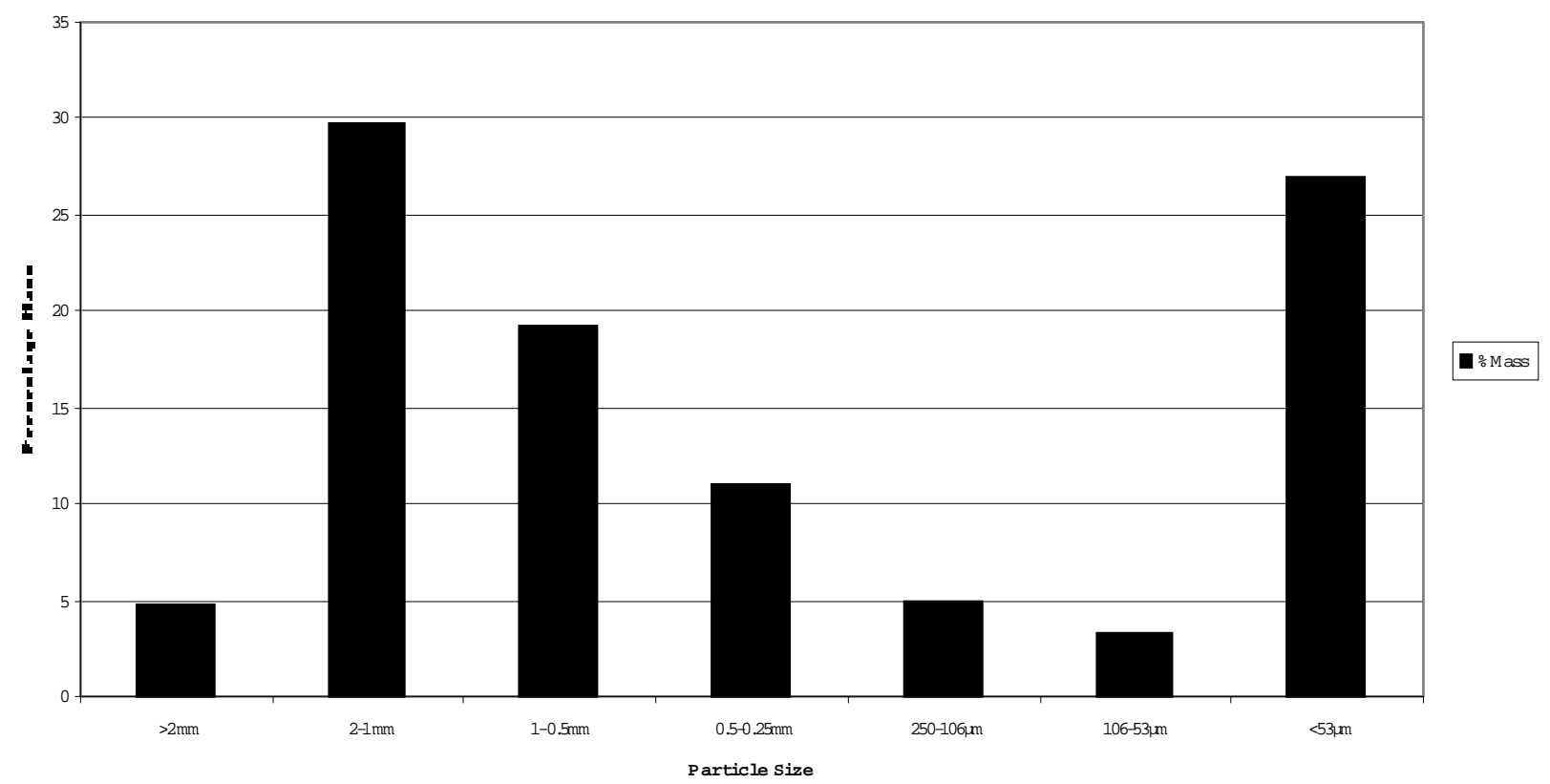

Figure 5.14. Percent of Cesium Found in Each Size Fraction of Composite 2A/B

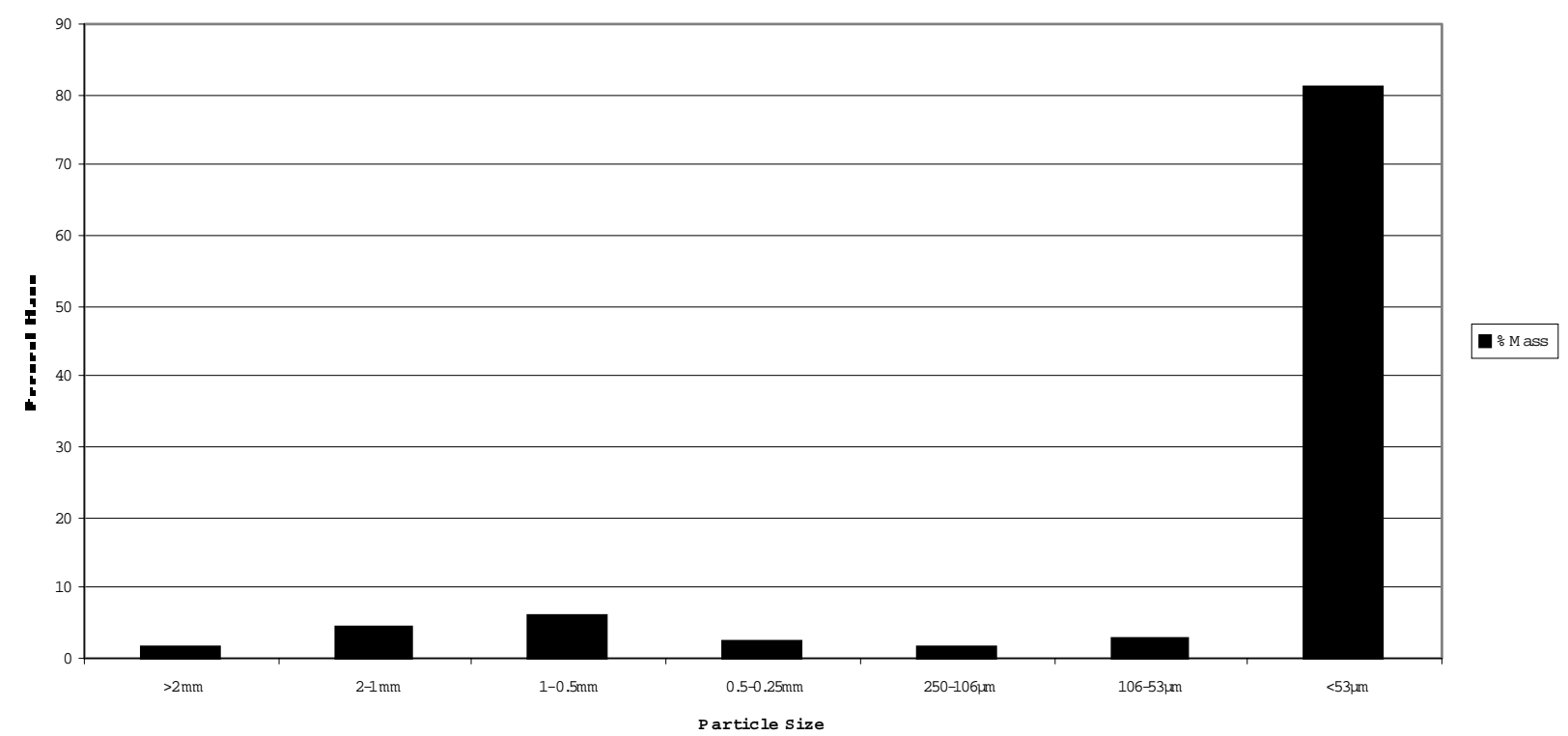

Figure 5.15. Percent of Cesium Found in Each Size Fraction of Composite 3A/B

for sample $2 \mathrm{~A} / \mathrm{B}$, which shows $54 \%$ of the cesium- 137 mass in the coarse fractions. These rather unexpected findings that coarse materials have tens of percent of the activity may be caused by finer material being cemented to larger-grained material or agglomerated together.

Caliche, the common name attributed to composite $12 \mathrm{~A} / \mathrm{B}$, is enriched in calcium carbonate, a known cementing agent. The fine-grained Plio-Pleistocene sediments (composites $2 \mathrm{C} / \mathrm{D}, 2 \mathrm{~A} / \mathrm{B}$, and $3 \mathrm{~A} / \mathrm{B}$ ) also contain a few percent carbonates. The mineralogy of the fine-grained sediments obtained from this 


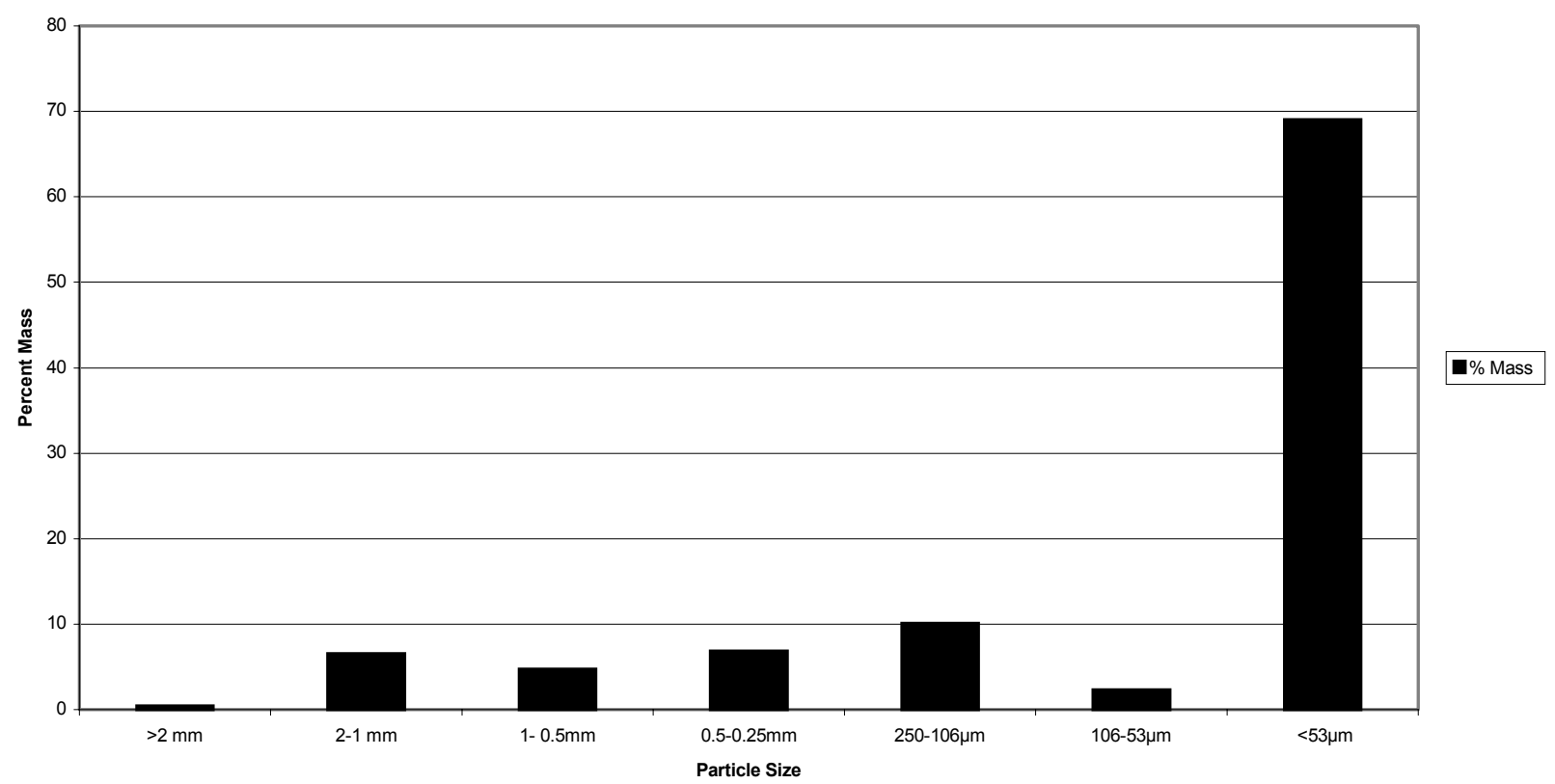

Figure 5.16. Percent of Cesium Found in Each Size Fraction of Composite 12A/B

borehole showed significant amounts of illite and mica minerals, known to be highly selective to adsorbing cesium cations. Cesium adsorption onto selected fine-grained purified minerals and finegrained sediments obtained from nearby 200 West Area Resource Conservation and Recovery Act (RCRA) boreholes, taken from the archive library, is being studied. Results of these basic science studies will be published in the field investigation report ${ }^{(a)}$.

\subsubsection{Macro Cation and Trace Metals in Various Particle Size Splits from Borehole Extension Composites}

Besides measuring the distribution of cesium-137 as a function of particle size, the size separates from composites $2 \mathrm{CD}, 2 \mathrm{AB}$, and $3 \mathrm{AB}$ were also dissolved by lithium metaborate fusion and dissolution in acid. The total concentration of selected major cations and trace metals was determined by ICP. The results are shown in Tables 5.26 through 5.28. The data for the bulk sediment is also repeated from Table 5.14 for convenience.

During the wet sieving to obtain particle size measurements on the borehole extension composite samples that were obtained right below the depth at which the closed end casing had been milled off, we found evidence of enriched ferric oxides. This prompted us to determine the chemical composition of the

(a) Draft Field Investigation Report for Waste Management Area S-SX. RPP-7884, Draft, Volume 2, Appendix D, CH2M HILL Hanford Group, Inc., Richland, Washington. 
Table 5.26. Chemical Composition of Various Size Fractions of 2CD Composite Sediment

\begin{tabular}{|c|c|c|c|c|c|c|c|c|c|}
\hline \multicolumn{2}{|c|}{$\mathrm{Wt} \%$ in Each Split } & \multirow{2}{*}{$\begin{array}{c}\begin{array}{c}\% \\
\text { (bulk) }\end{array} \\
1.39 \\
\end{array}$} & \multirow{2}{*}{$\begin{array}{c}\begin{array}{c}2.18 \\
(>2 \mathrm{~mm})\end{array} \\
0.898 \\
\end{array}$} & \multirow{2}{*}{$\begin{array}{c}\begin{array}{c}2.61 \\
\text { (2 to } 1 \mathrm{~mm} \text { ) }\end{array} \\
0.819\end{array}$} & \multirow{2}{*}{$\begin{array}{c}3.52 \\
\text { (1 to } 0.5 \mathrm{~mm}) \\
0.842\end{array}$} & \multirow{2}{*}{$\begin{array}{c}1.94 \\
(500 \text { to } 250 \mu \mathrm{m}) \\
1.18 \\
\end{array}$} & \multirow{2}{*}{$\begin{array}{c}12.93 \\
(250 \text { to } 106 \mu \mathrm{m}) \\
1.58\end{array}$} & \multirow{2}{*}{$\begin{array}{c}28.32 \\
(106 \text { to } 53 \mu \mathrm{m}) \\
1.52\end{array}$} & \multirow{2}{*}{$\begin{array}{c}\begin{array}{c}48.5 \\
(<53 \mu \mathrm{m})\end{array} \\
1.36\end{array}$} \\
\hline $\mathrm{Na}$ & $\%$ & & & & & & & & \\
\hline $\mathrm{Mg}$ & $\%$ & 0.84 & 0.72 & 0.63 & 0.61 & 0.71 & 0.42 & 0.63 & 1.12 \\
\hline $\mathrm{Si}$ & $\%$ & 26.5 & 18.4 & 17.8 & 18.6 & 25.3 & 26.6 & 29.8 & 26 \\
\hline $\mathrm{Al}$ & $\%$ & 5.29 & 3.55 & 3.33 & 3.4 & 4.34 & 5.05 & 5.02 & 5.88 \\
\hline $\mathrm{K}$ & $\%$ & 1.77 & 1.25 & 1.14 & 1.14 & 1.41 & 1.93 & 1.69 & 1.9 \\
\hline $\mathrm{Ca}$ & $\%$ & 2.9 & 2.58 & 2.48 & 2.35 & 2.42 & 2.53 & 2.85 & 3.1 \\
\hline $\mathrm{Ti}$ & $\%$ & 0.48 & 0.35 & 0.3 & 0.27 & 0.34 & 0.17 & 0.31 & 0.52 \\
\hline $\mathrm{Fe}$ & $\%$ & 4.82 & 26.2 & 24.6 & 20.8 & 17.6 & 2.47 & 2.13 & 3.32 \\
\hline $\mathrm{Cr}$ & $\mu \mathrm{g} / \mathrm{g}$ & 252 & 1,840 & 2,020 & 1,640 & 1,390 & 158 & 70 & 70 \\
\hline $\mathrm{Sr}$ & $\mu \mathrm{g} / \mathrm{g}$ & 343 & 258 & 247 & 238 & 283 & 404 & 372 & 329 \\
\hline $\mathrm{Ba}$ & $\mu \mathrm{g} / \mathrm{g}$ & 692 & 498 & 489 & 545 & 634 & 750 & 662 & 728 \\
\hline $\mathrm{U}$ & $\mu \mathrm{g} / \mathrm{g}$ & 237 & 14.2 & 24.4 & 56.9 & 148 & 620 & 520 & 7.2 \\
\hline
\end{tabular}


Table 5.27. Chemical Composition of Various Size Fractions of $2 \mathrm{AB}$ Composite Sediment

\begin{tabular}{|c|c|c|c|c|c|c|c|c|c|}
\hline \multicolumn{2}{|c|}{$\mathrm{Wt} \%$ in Each Split } & \multirow{2}{*}{$\begin{array}{c}\begin{array}{c}\% \\
\text { (bulk) }\end{array} \\
2.18 \\
\end{array}$} & \multirow{2}{*}{$\begin{array}{c}0.2 \\
(>2 \mathrm{~mm})\end{array}$} & \multirow[t]{2}{*}{$\begin{array}{c}0.92 \\
\text { (2 to } 1 \mathrm{~mm} \text { ) } \\
\end{array}$} & \multirow{2}{*}{$\begin{array}{c}0.68 \\
(1 \text { to } 0.5 \mathrm{~mm}) \\
1.94\end{array}$} & \multirow{2}{*}{$\begin{array}{c}0.64 \\
(500 \text { to } 250 \mu \mathrm{m}) \\
1.87 \\
\end{array}$} & \multirow{2}{*}{$\begin{array}{c}8.98 \\
(250 \text { to } 106 \mu \mathrm{m}) \\
2.14 \\
\end{array}$} & \multirow{2}{*}{$\begin{array}{c}37.85 \\
(106 \text { to } 53 \mu \mathrm{m}) \\
2\end{array}$} & \multirow{2}{*}{$\begin{array}{c}\begin{array}{c}50.71 \\
(<53 \mu \mathrm{m})\end{array} \\
1.29\end{array}$} \\
\hline $\mathrm{Na}$ & $\%$ & & & & & & & & \\
\hline $\mathrm{Mg}$ & $\%$ & 1.39 & 1.18 & & 1.2 & 1.33 & 0.791 & 0.92 & 1.06 \\
\hline $\mathrm{Si}$ & $\%$ & NA & 29.6 & & 30.8 & 35.5 & 34 & 37.2 & 27 \\
\hline $\mathrm{Al}$ & $\%$ & 7.61 & 6.05 & & 4.67 & 5.9 & 4.56 & 4.71 & 2.87 \\
\hline $\mathrm{K}$ & $\%$ & 2.62 & 3.35 & & 3.25 & 3.62 & 3.15 & 3.03 & 2.63 \\
\hline $\mathrm{Ca}$ & $\%$ & 1.85 & 2.48 & & 3.25 & 2.72 & 2.38 & 2.565 & 2.02 \\
\hline $\mathrm{Ti}$ & $\%$ & 0.6 & 0.374 & & 0.401 & 0.419 & 0.198 & 0.279 & 0.35 \\
\hline $\mathrm{Fe}$ & $\%$ & 3.38 & 9.96 & & 16 & 6.49 & 1.81 & 1.6 & 2.11 \\
\hline $\mathrm{Mn}$ & $\mu \mathrm{g} / \mathrm{g}$ & NA & 1,040 & & 1,670 & 881 & 332 & 367 & 463 \\
\hline $\mathrm{Cr}$ & $\mu \mathrm{g} / \mathrm{g}$ & 151.16 & 620 & & 1,320 & 379 & 71.7 & 54 & 62 \\
\hline $\mathrm{Zn}$ & $\mu \mathrm{g} / \mathrm{g}$ & 133.38 & 298 & & 160 & 140 & 52.2 & 42.4 & 45 \\
\hline $\mathrm{Sr}$ & $\mu \mathrm{g} / \mathrm{g}$ & 298.99 & 334 & & 309 & 323 & 386 & 338 & 204 \\
\hline $\mathrm{Ba}$ & $\mu \mathrm{g} / \mathrm{g}$ & 801.39 & 700 & & 622 & 834 & 769 & 600 & 469 \\
\hline $\mathrm{U}$ & $\mu \mathrm{g} / \mathrm{g}$ & 3.33 & 1.1 & & 0.99 & 7,380 & 0.5 & 0.75 & 1 \\
\hline $\mathrm{Ni}$ & $\mu \mathrm{g} / \mathrm{g}$ & NA & 145 & & 219 & 106 & 37.4 & 71 & 72.9 \\
\hline $\mathrm{Cu}$ & $\mu \mathrm{g} / \mathrm{g}$ & NA & 402 & & 732 & 529 & 173 & 218 & 148 \\
\hline $\mathrm{Zr}$ & $\mu \mathrm{g} / \mathrm{g}$ & NA & 251 & & 241 & 194 & 83.1 & 122 & 239 \\
\hline Mo & $\mu \mathrm{g} / \mathrm{g}$ & NA & 41.3 & & 77.5 & 20.1 & 2.2 & 3.85 & 1.22 \\
\hline $\mathrm{Sn}$ & $\mu \mathrm{g} / \mathrm{g}$ & NA & 15.7 & & 30.7 & 66.9 & 10.8 & 8.3 & 7 \\
\hline $\mathrm{W}$ & $\mu \mathrm{g} / \mathrm{g}$ & NA & 2,060 & & 3,580 & 834 & 200 & 38 & 67 \\
\hline \multicolumn{10}{|c|}{$\mathrm{NA}=$ Not analyzed } \\
\hline
\end{tabular}


Table 5.28. Chemical Composition of Various Size Fractions of 2CD Composite Sediment

\begin{tabular}{|c|c|c|c|c|c|c|c|c|c|}
\hline \multicolumn{2}{|c|}{$\mathrm{Wt} \%$ in Each Split } & \multirow{2}{*}{$\begin{array}{c}\begin{array}{c}\% \\
\text { (bulk) }\end{array} \\
\text { NA }\end{array}$} & \multirow{2}{*}{$\begin{array}{c}\begin{array}{c}0.36 \\
(>2 \mathrm{~mm})\end{array} \\
1.55\end{array}$} & \multirow{2}{*}{$\begin{array}{c}0.98 \\
\text { (2 to } 1 \mathrm{~mm}) \\
1.92\end{array}$} & \multirow{2}{*}{$\begin{array}{c}1.67 \\
\text { (1 to } 0.5 \mathrm{~mm}) \\
1.83\end{array}$} & \multirow{2}{*}{$\begin{array}{c}0.69 \\
(500 \text { to } 250 \mu \mathrm{m}) \\
2.02\end{array}$} & \multirow{2}{*}{$\begin{array}{c}8.72 \\
(250 \text { to } 106 \mu \mathrm{m}) \\
2.31\end{array}$} & \multirow{2}{*}{$\begin{array}{c}\begin{array}{c}30.91 \\
(106 \text { to } 53 \mu \mathrm{m})\end{array} \\
2.18\end{array}$} & \multirow{2}{*}{$\begin{array}{c}\begin{array}{c}56.69 \\
(<53 \mu \mathrm{m})\end{array} \\
1.48\end{array}$} \\
\hline $\mathrm{Na}$ & $\%$ & & & & & & & & \\
\hline $\mathrm{Mg}$ & $\%$ & NA & 0.963 & 1.14 & 1.12 & 1.05 & 0.526 & 0.814 & 1.88 \\
\hline $\mathrm{Si}$ & $\%$ & NA & 31 & 29.3 & 36.2 & 33.5 & 34.9 & 42.5 & 34.1 \\
\hline $\mathrm{Al}$ & $\%$ & NA & 4.18 & 5.73 & 4.9 & 5.57 & 5.68 & 3.84 & 7.88 \\
\hline $\mathrm{K}$ & $\%$ & NA & 2.59 & 3.24 & 2.95 & 3.47 & 3.33 & 3.31 & 3.975 \\
\hline $\mathrm{Ca}$ & $\%$ & NA & 3.1 & 2.5 & 3.28 & 2.72 & 2.27 & 3.25 & 3.31 \\
\hline $\mathrm{Ti}$ & $\%$ & NA & 0.324 & 0.37 & 0.386 & 0.343 & 0.156 & 0.279 & 0.535 \\
\hline $\mathrm{Fe}$ & $\%$ & NA & 22.5 & 9.56 & 10.4 & 8.55 & 1.33 & 1.78 & 5.34 \\
\hline $\mathrm{Mn}$ & $\mu \mathrm{g} / \mathrm{g}$ & NA & 2,100 & 1,090 & 1,120 & 928 & 238 & 387 & 924 \\
\hline $\mathrm{Cr}$ & $\mu \mathrm{g} / \mathrm{g}$ & NA & 1,850 & 672 & 713 & 601 & 126 & 74 & 111 \\
\hline $\mathrm{Zn}$ & $\mu \mathrm{g} / \mathrm{g}$ & NA & 381 & 405 & 430 & 1,055 & 60 & 35 & 151 \\
\hline $\mathrm{Sr}$ & $\mu \mathrm{g} / \mathrm{g}$ & NA & 287 & 317 & 324 & 351 & 399 & 348 & 246 \\
\hline $\mathrm{Ba}$ & $\mu \mathrm{g} / \mathrm{g}$ & NA & 551 & 702 & 686 & 794 & 664 & 582 & 801 \\
\hline $\mathrm{U}$ & $\mu \mathrm{g} / \mathrm{g}$ & NA & 0.74 & 0.9 & 1.2 & 1.1 & 0.5 & 0.74 & 1.6 \\
\hline $\mathrm{Ni}$ & $\mu \mathrm{g} / \mathrm{g}$ & NA & 327 & 176 & 141 & 120 & 191 & 80.3 & 46 \\
\hline $\mathrm{Cu}$ & $\mu \mathrm{g} / \mathrm{g}$ & NA & 779 & 480 & 382 & 1,160 & 131 & 59 & 70 \\
\hline $\mathrm{Zr}$ & $\mu \mathrm{g} / \mathrm{g}$ & NA & 172 & 564 & 266 & 184 & 94.4 & 186 & 192 \\
\hline Mo & $\mu \mathrm{g} / \mathrm{g}$ & NA & 131 & 47 & 45 & 39.5 & 1.3 & 0.9 & 4.8 \\
\hline Sn & $\mu \mathrm{g} / \mathrm{g}$ & NA & 36.4 & 24.6 & 21.2 & 31.4 & 4.3 & 15 & 6.6 \\
\hline W & $\mu \mathrm{g} / \mathrm{g}$ & NA & 3,720 & 293 & 2,580 & 2,355 & 138 & 180 & 162 \\
\hline \multicolumn{10}{|c|}{$\mathrm{NA}=$ Not analyzed $\quad$ Shaded text $=$ Values that are not natural } \\
\hline
\end{tabular}


various size fractions obtained. The values highlighted in red seem extraordinarily high for natural sediments. We assumed that the very high iron must have come from milling off the thick casing end plate and from the grinding of several drill bits. In addition, there were high concentrations of chromium, manganese, zinc, molybdenum, copper, tin, and tungsten that could have come from the drill bits based on conversations with technical representatives from the manufacturer of the very hard alloy drill bits. The largest particle sizes of these three samples, all within a few feet of where the casing end plate was milled, contained up to $25 \%$ iron. It was later determined that these large particles were actually agglomerates that were cemented together with ferric oxides. Optical and scanning electron microscopy did not show any metallic iron or material that was obviously shavings of cast iron plate or drill bits (McKinley et al. 2001). The hydrous ferric oxides appeared similar to iron oxide-rich sediments that formed naturally. We speculate that the ferric oxides formed both naturally in the sediments during storage and the wet sieving activities to create the size separates. The sediments, thus, contained unnaturally high ferric oxide concentrations during subsequent testing. Unfortunately, they were the only contaminated sediments available from borehole 41-09-39 for the first 2 years of the tank farm studies. The sidewall cores obtained when the borehole 41-09-39 casing was removed and the slant borehole under tank SX-108 during 2000 have allowed us to refocus studies on highly cesium-137-contaminated sediments that are not of questionable representativeness.

\subsubsection{Strontium and Cesium $K_{d}$ Values - Results and Discussion}

Batch adsorption tests were performed with sediments from two nonradioactive composites (10A/B and $38 \mathrm{~A} / \mathrm{B})$ and one composite $(12 \mathrm{~A} / \mathrm{B})$ slightly contaminated with cesium-137. Composite $10 \mathrm{~A} / \mathrm{B}$ is fine-grained sediment from the Plio-Pleistocene very fine sand to clayey silt (PPlz unit) (sampled just above the caliche layer), 12A/B is from within the caliche layer (PPlc), and 38A/B is a coarse-grained sediment from within the Ringold Formation (Rwi[e]). In addition, one highly contaminated composite, $2 \mathrm{~A} / \mathrm{B}$, was used to perform batch desorption tests. The composite $2 \mathrm{~A} / \mathrm{B}$ also is from the fine-grained $\mathrm{PPlz}$ sediments. As discussed in Section 3.3.5.13, four aqueous solutions were selected to represent the wide range of fluids that the Hanford sediments might encounter after a tank leak. Solution 1 represents a high ionic strength, high $\mathrm{pH}$, and high soluble aluminum REDOX tank liquor; solution 2 was to represent the high ionic strength tank liquor without caustic and soluble aluminum. Solution 3 was a simple $4 \mathrm{M}$ sodium nitrate solution that conceptually represents a more dilute tank waste with no complicating soluble aluminum or high $\mathrm{pH}$. $4 \mathrm{M}$ sodium nitrate solutions were used in the 1970s as the surrogate tank liquor and a relatively large database of contaminant $\mathrm{K}_{\mathrm{d}}$ values are available on other sediments. The fourth solution used was uncontaminated Hanford groundwater that represents the dilute end member for when the tank leak liquor has been completely diluted back to near natural conditions.

Solution 2 was targeted to be $7.75 \mathrm{M}$ sodium nitrate and $0.45 \mathrm{M}$ sodium nitrite, but the salts would not dissolve completely. The final concentration of dissolved salts was $7.43 \mathrm{M}$ sodium nitrate and $0.45 \mathrm{M}$ sodium nitrite. This solution was 0.34 molal in sodium nitrite and 10.16 molal in sodium nitrate, and was determined to be very close to a saturated binary solution of these two salts based on extrapolating solubility data presented as a function of temperature in Felmy et al. (1997). Because we made the solution carefully and found that all the sodium nitrate would not dissolve, we expect this solution to be at saturation at room temperature. In a sense, we are corroborating Felmy et al. (1997) solubility predictions. This work puts reported results on supernate solution from tanks SX-108 and SX-111 in question, unless 
the elevated temperatures in the tanks were somehow preserved or accounted for during the analyses. As reported in Serne et al. (1998), historical data in the Tank Waste Characterization Database suggest SX tanks can contain up to $10 \mathrm{M}$ sodium, $8 \mathrm{M}$ nitrate, and $0.45 \mathrm{M}$ nitrite in solution at an unspecified temperature. At room temperature, we could not get more than $7.43 \mathrm{M}$ nitrate in solution before precipitation occurred. Perhaps the presence of other salts in actual tank liquor suppresses the thermodynamic activity of sodium, nitrate, or nitrite to make it more soluble, but we also consider the old analytical data to be suspect.

The densities of the three simulated solutions were $1.336 \mathrm{~g} / \mathrm{cm}^{3}, 1.362 \mathrm{~g} / \mathrm{cm}^{3}$, and $1.213 \mathrm{~g} / \mathrm{cm}^{3}$ for the caustic tank liquor, the $7.43 \mathrm{M}$ nitrate, and the $4 \mathrm{M}$ nitrate-bearing solutions, respectively. These values are of interest to transport modelers and could be compared to values used in past and current modeling of the SX Tank Farm leaks that address the impacts of high-density solutions on contaminant and water migration (see Ward et al. 1997 for past work). The field investigation report ${ }^{(a)}$ will contain discussions on recent transport modeling performed under the Science and Technology Program. In general, the measured densities are slightly lower than the upper bounds used in the Ward et al. (1997) modeling.

The $K_{d}$ values obtained for strontium and cesium on the three sediments for the simulated tank liquor and brines are shown in Tables 5.29 and 5.30, respectively. The highly caustic simulated tank liquor (Solution 1) shows very high strontium $\mathrm{K}_{\mathrm{d}}$ values for the sediments compared to the other three solutions. The fine-grained Plio-Pleistocene sediment (composite 10A/B) shows the highest adsorption, while the caliche sediment adsorbs strontium almost as strongly, and the Ringold Formation sediment adsorbs strontium moderately. The slurry $\mathrm{pH}$ values at the end of the contact period also are shown in the tables. The $\mathrm{pH}$ for Solution 1 is about 14, approximating $1 \mathrm{M}$ free hydroxide. As mentioned in Serne et al. (1998), strontium is insoluble in highly alkaline solutions and this forces the apparent $\mathrm{K}_{\mathrm{d}}$ to be high. The strontium $\mathrm{K}_{\mathrm{d}}$ varies with the particle size of the sediments, with the finer grained sediment exhibiting the highest adsorption. The effects of both $\mathrm{pH}$ and particle size are as expected.

Table 5.29. Adsorption Coefficients $\left(K_{d}\right)$ for Strontium- 90 for Three Sediment Types and Four Solutions

\begin{tabular}{|c|c|c|c|c|c|c|c|}
\hline \multirow[b]{3}{*}{ Solutions } & \multicolumn{6}{|c|}{ Sediment } & \multirow[b]{3}{*}{$\mathrm{pH}$ Values } \\
\hline & \multicolumn{2}{|c|}{$\begin{array}{c}\text { Extension \#10A/B } \\
(\mathrm{PPlz})\end{array}$} & \multicolumn{2}{|c|}{$\begin{array}{c}\text { Extension \#12A/B } \\
\text { Caliche (PPlc) }\end{array}$} & \multicolumn{2}{|c|}{$\begin{array}{c}\text { Extension \#38A/B } \\
\text { Ringold }\end{array}$} & \\
\hline & Rep \#1 & Rep \#2 & Rep \#1 & Rep \#2 & Rep \#1 & Rep \#2 & \\
\hline $4 \mathrm{M} \mathrm{NaNO}_{3}$ & 4 & 29 & 1 & 5 & 3 & 1 & $8.5-8.8$ \\
\hline Tank Liquor \#1 & 2,713 & 1,519 & 1,646 & 997 & 57 & 39 & $13.9-14.0$ \\
\hline Tank Liquor \#2 & 2 & 1 & 3 & 1 & 2 & 3 & $7.8-8.4$ \\
\hline Hanford Groundwater & 21 & 21 & 39 & 18 & 0 & 2 & $7.5-8.5$ \\
\hline
\end{tabular}

(a) Draft Field Investigation Report for Waste Management Area S-SX. RPP-7884, Draft, Volume 2, Appendix D, CH2M HILL Hanford Group, Inc., Richland, Washington. 
Table 5.30. Adsorption and Desorption Coefficients $\left(\mathrm{K}_{\mathrm{d}}\right)$ for Cesium-137 for Three Sediment Types and Four Solutions

\begin{tabular}{|c|c|c|c|c|c|c|c|c|c|}
\hline Cs $\mathrm{K}_{\mathrm{d}}$ (Adsorption) & \multicolumn{6}{|c|}{ Sediment } & \multirow[b]{3}{*}{ pH Values } & \multirow{2}{*}{\multicolumn{2}{|c|}{$\begin{array}{c}\mathrm{Cs} \mathrm{K}_{\mathrm{d}} \text { (Desorption) } \\
\text { Extension \#2A/B } \\
(\mathrm{PPlz})\end{array}$}} \\
\hline \multirow[b]{2}{*}{ Solutions } & \multicolumn{2}{|c|}{$\begin{array}{c}\text { Extension \#10A/B } \\
(\mathrm{PPlz})\end{array}$} & \multicolumn{2}{|c|}{$\begin{array}{c}\text { Extension \#12A/B } \\
\text { Caliche (PPlc) }\end{array}$} & \multicolumn{2}{|c|}{$\begin{array}{c}\text { Extension \#38A/B } \\
\text { Ringold }\end{array}$} & & & \\
\hline & Rep \#1 & Rep \#2 & Rep \#1 & Rep \#2 & Rep \#1 & Rep \#2 & & Rep \#1 & Rep \#2 \\
\hline $4 \mathrm{M} \mathrm{NaNO}_{3}$ & 52 & 208 & 168 & 198 & 2,098 & 1,447 & $8.5-8.8$ & $>1,280$ & $>1,310$ \\
\hline Tank Liquor \#1 & 210 & 149 & 253 & 238 & 23 & 16 & $13.9-14.0$ & $>1,260$ & $>1,280$ \\
\hline Tank Liquor \#2 & 38 & 32 & 61 & 55 & 5 & 8 & $7.8-8.4$ & $>1,360$ & $>1,310$ \\
\hline Hanford Groundwater & 63,534 & 59,023 & 8,430 & 6,344 & 7 & 9 & $7.5-8.5$ & $>1,290$ & $>1,330$ \\
\hline
\end{tabular}

Simulated tank brine (Solution 2) is a very high-ionic-strength solution of sodium, nitrate, and nitrite with a near-neutral $\mathrm{pH}$. The strontium $\mathrm{K}_{\mathrm{d}}$ values for this solution are, in general, the lowest observed for the four solutions, as would be expected if cation competition is the process that determines $\mathrm{K}_{\mathrm{d}}$. The three sediment types show little difference in strontium adsorption suggesting that the high $\mathrm{Na}^{+}$competition dominates over differing particle sizes and surface areas.

The $4 \mathrm{M}$ sodium nitrate solution shows slightly higher strontium $\mathrm{K}_{\mathrm{d}}$ values in accordance with the lower ionic strength (sodium nitrate dropped from 7.4 to $4 \mathrm{M}$ ). The scatter in the replicates is large, but the finer-grained Plio-Pleistocene sediment (composite 10A/B) appears to exhibit more adsorption than the other two sediments, which would be expected if ion exchange determined $\mathrm{K}_{\mathrm{d}}$.

The groundwater interactions with the sediments yield higher strontium $\mathrm{K}_{\mathrm{d}}$ values than the neutral $\mathrm{pH}$ brines if we discount the abnormally high value for the replicate \#2 in the $4 \mathrm{M}$ nitrate test. The effect of particle size is particularly evident when comparing the strontium $\mathrm{K}_{d}$ values for the fine-grained PlioPleistocene fine sand/silt to the Ringold gravelly sand. The strontium $\mathrm{K}_{\mathrm{d}}$ values for groundwater contacting the caliche are the highest observed for the neutral $\mathrm{pH}$ solutions, suggesting strontium incorporation into carbonate mineral matrices. The observed strontium $\mathrm{K}_{\mathrm{d}}$ values for the groundwater are in the same range as other Hanford sediments (Serne and LeGore 1996; Serne et al. 1993; Kaplan et al. 1998). Strontium is considered to be moderately adsorbed by most sediments at the Hanford Site and the strontium $\mathrm{K}_{\mathrm{d}}$ values have been shown to be quite sensitive to high $\mathrm{pH}$ conditions and high ionic strength. It is well known that calcium and other divalent alkaline earth cations strongly compete with trace amounts of strontium for adsorption sites. Sodium is a much weaker competitor (see Routson et al. 1981a, b; Routson and Serne 1972 for further discussion).

For the SX Tank Farm environment, we would expect strontium to be bound tightly in high-pHimpacted sediments near the base of the tank. This fact, coupled with the low solubility of strontium inside the tank, may explain our not finding strontium-90 in the borehole sediments.

The cesium $\mathrm{K}_{\mathrm{d}}$ values are shown in Table 5.30. The cesium $\mathrm{K}_{\mathrm{d}}$ data also show high values for the highly alkaline simulated tank liquor (Solution 1) especially for the Plio-Pleistocene sediment (composite 10A/B) and caliche sediment. In earlier work reported in Serne et al. (1998), a highly alkaline and high 
aluminum/ionic strength solution contacting a 200 East Area coarse sand yielded a cesium $\mathrm{K}_{\mathrm{d}}$ value of $26 \mathrm{~mL} / \mathrm{g}$. This earlier testing on the coarse-grained sand agrees remarkably well with the current data for the coarse Ringold composite sample (38A/B) whose cesium $\mathrm{K}_{\mathrm{d}}$ ranged from 16 to $23 \mathrm{~mL} / \mathrm{g}$.

The cesium $\mathrm{K}_{\mathrm{d}}$ value for all the sediments is moderate for the very high ionic strength but neutral $\mathrm{pH}$ (7.4 M sodium nitrate) solution. The cesium $\mathrm{K}_{\mathrm{d}}$ increases slightly for the $4 \mathrm{M}$ sodium nitrate solution as would be expected for a cation exchange-dominated process. The rise in cesium $\mathrm{K}_{\mathrm{d}}$ is especially large for the Ringold sediments.

Most noteworthy is the cesium $\mathrm{K}_{\mathrm{d}}$ in Hanford groundwater contacting Plio-Pleistocene sediment (composite 10A/B) and caliche sediment (composite 12A/B). The cesium $\mathrm{K}_{\mathrm{d}}$ for the fine-grained PlioPleistocene sediment is $>50,000 \mathrm{~mL} / \mathrm{g}$. This is undoubtedly caused by selective cesium fixation/ adsorption onto mica and mica-like clay minerals. In essence, there is very little competition for adsorption sites on the Plio-Pleistocene and caliche sediments until the ionic strength increases to molar levels. The cesium $\mathrm{K}_{\mathrm{d}}$ values for other Hanford sediments with a wide range of solutions is reviewed in Serne et al. (1998).

The desorption tests using the composite $2 \mathrm{~A} / \mathrm{B}$ sediment that contained 1,290 $\mathrm{pCi} / \mathrm{g}$ cesium- $137 \mathrm{did}$ not yield very interesting results. Upon contacting the sediment with each of the four solutions (without added radiotracers), no detectable cesium-137 was found in the leachates after 13 days of contact. The desorption $\mathrm{K}_{\mathrm{d}}$ values calculated using our detection limit for cesium-137 in the leachate $(10.4 \mathrm{pCi} / \mathrm{mL})$ were all $>1,200 \mathrm{~mL} / \mathrm{g}$. The initial amount of cesium-137 adsorbed on the sediment was constant, our detection limit for cesium-137 in leachates was constant, and the solid-to-solution ratio used in all tests was essentially constant. With no detectable cesium- 137 in any leachate, the desorption $\mathrm{K}_{\mathrm{d}}$ calculation yields the same value regardless of the chemical composition of the solution and the resultant $\mathrm{pH}$ of the solution, which was in the same range as shown in Table 5.30.

None of the data collected during this SX Borehole Extension Project can be interpreted to suggest that cesium-137 can travel through Hanford sediments without adsorbing to a considerable degree. Albeit, the data and tests performed to date do not address elevated temperature or colloidal transport. These two drivers are not expected to significantly change the conclusions. The temperature effect on adsorption reactions is not well studied but the few available studies suggest only small effects (see Ames et al. 1982 for cesium adsorption onto basalt and smectite clay-weathering products).

The overall effect of temperature on the reactions of highly caustic simulated tank liquor on Hanford sediments in the presence of trace amounts of cesium that was recently adsorbed onto the sediment is under way in the Science and Technology Program and discussions will be presented in the field investigation report $^{(a)}$.

(a) Draft Field Investigation Report for Waste Management Area S-SX. RPP-7884, Draft, Volume 2, Appendix D, CH2M HILL Hanford Group, Inc., Richland, Washington. 


\subsection{Summary and Conclusions}

In this section, we present summary statements on our interpretation of characterization data and our conclusions.

\subsection{Lithologic Model of the Geology at Borehole 41-09-39}

Borehole 41-09-39 intersects seven near-horizontal stratigraphic layers. Several of the layers dip toward the southwest. The layers, in ascending order, include two Ringold facies (member of Wood Island subunit E [Rwi(e)] and member of Taylor Flat [Rtf]), two Plio-Pleistocene facies (carbonate [PPlc] and $\mathrm{mud} / \mathrm{silt}$ [PPlz]) and three Hanford facies (fine sand [Hanford formation $\mathrm{H} 2$ unit], coarse sand/gravel [Hanford formation H1unit], and fine sand [Hanford formation H1a unit]). The shallowest Hanford facies, the Hanford formation H1a unit, is sporadic at the SX Tank Farm because it was removed during excavation and replaced with backfill. The backfill is relatively non-cohesive, friable, massive sand with minor variable mud and pebble content. The backfill has a hardened zone at its base, generally 15.9 meters (51 feet) below ground surface (bgs).

Slate $(1996,2000)$ interpreted the surface of the Ringold Formation to be erosional in this area giving the surface of the Ringold Formation under the tank farm a southwesterly dip.

The upper surface of the Plio-Pleistocene unit also dips to the southwest toward the Cold Creek depression, resembling the Ringold Formation. The fine-grained facies of the Plio-Pleistocene unit (PPlz) beneath the tank farm consists mainly of interstratified mud to muddy very-fine sand deposits.

Quaternary age sediments of the Hanford formation overlie the Plio-Pleistocene unit and represent the main vadose zone materials beneath the tank farm. The Hanford formation beneath the SX Tank Farm consists of interstatified fine-grained sands intercalated with coarse sand and gravel and thinner lenses of mud. The basal portion of the unit consists of a muddy fine sand facies, designated the Hanford formation $\mathrm{H} 2$ unit. A sandy gravel to coarse sand facies dominates the middle portion of the Hanford formation, which is then overlain by a slightly muddy medium sand facies and finally by a slightly gravelly coarse sand facies. The middle coarse sand to gravel facies is called the Hanford formation H1 unit. The slightly silty medium sand and overlying slightly gravelly coarse sand facies is called the Hanford formation H1a unit. The upper coarse-grained facies of Hanford formation H1a unit is completely missing beneath the tank farm because it was removed during construction.

The backfill appears to extend to a depth of about 15.9 meters (51 feet) where it contacts the Hanford formation. The next 4.3 to 4.9 meters (14 to 16 feet) is interpreted to correlate with the upper fine sand and mud sequence of the Hanford formation H1a unit. Below this lies the 6.4- to 7-meter- (21- to 23-foot)- thick middle coarse sand and gravel sequence (Hanford formation H1 unit). Note that interpretation of the sidewall cores and more recent geophysical logs suggests that the Hanford formation H1 unit is $\sim 4$ meters (12 feet) thinner than previously interpreted by Myers et al. (1998). This is underlain by 12.1 meters ( 37 feet) of the fine sand and silt sequence (the Hanford formation $\mathrm{H} 2$ unit). 
The contact between the Hanford formation and the Plio-Pleistocene unit is interpreted to occur at a depth of 38 meters (125 feet). The lower portion of the borehole from depths of 40 to 69 meters (130 to 225 feet) penetrates the Plio-Pleistocene unit and upper portion of the Ringold Formation.

In general, the near-horizontal layers likely cause anisotropy in water flow. The vertical distribution of cesium-137, based on borehole gamma logging within the SX Tank Farm, suggests that much of the tank fluid that leaked from tanks SX-108, SX-109, SX-111, and SX-112 traveled within the coarsegrained Hanford formation $\mathrm{H} 1$ unit that is found between 20.4 and 26.8 meters (67 and 88 feet) bgs at borehole 41-09-39.

Clastic dikes are also known to be present and may impact contaminant transport through the vadose zone based on their near-vertical orientation versus the traditional horizontal strata common in the Hanford formation.

We estimate, based on historical well level measurements, that the peak water elevation beneath the SX-109 tank was 146.2 meters (479.7 feet mean sea level). This occurred in 1976 and places the water table $\sim 55$ to 56 meters (182 feet) bgs or 40 meters (132 feet) beneath the bottom of the tank. A secondary maximum occurred in 1984, just before the 216-U-10 Pond was decommissioned. At this time, the water table was estimated to have been almost as high as it was in 1976. In December 1997, the water table was encountered at a depth of $\sim 64.5$ meters (211.5 feet) in borehole 41-09-39. Thus, the water table has dropped an estimated 8 to 9 meters (28.5 feet) since 1984. An examination of the hydrographs since about 1988 suggests that the water level is dropping at a rate of 0.5 to 0.6 meters (1.5 to 1.9 feet) per year.

\subsection{Vertical Extent of Contamination}

We used several parameters including moisture content, $\mathrm{pH}$, electrical conductivity (EC), nitrate, sodium, chromium, and technetium concentrations in water extracts for our main indicators of the leading edge of the tank leak plume. The acid-extractable and direct measurements of several of these constituents in the sediments were used to delineate the total inventory of the plume distribution. In many cases, the water-extractable and acid/total measurements were similar, signifying that the most mobile constituents do, in fact, remain in the vadose zone porewater and hardly interact with the sediments. The vertical distribution of all these parameters was evaluated so that we could distinguish sediment that had been impacted/altered by drilling and sampling artifacts as opposed to actual tank liquor. Based on evaluating all these measurements, we are confident that we did establish the vertical extent of tank leak contamination at this location. We do not claim that the information from one borehole can be extrapolated across the entire tank farm. However, when we couple the data from borehole 41-09-39 with the historical gamma logging of the dry wells, detailed lithologic models, and the two newer boreholes at SX Tank Farm along with the predictive unsaturated flow and transport modeling that is being performed under the auspices of the Groundwater/Vadose Zone/Science and Technology Program, a defensible picture is anticipated.

The moisture content is a direct measure of the mass of fluid in the vadose zone sediments. One would logically assume that wetter than normal conditions would represent the existence of leaked tank liquor but there are numerous other causes of higher than anticipated moisture. Water line leaks, episodic 
rapid snow melts, and continual slow drainage over time are some complicating factors that have not been entirely addressed. The current moisture content distribution does not give a clear indication of the vertical extent of the plume. However, moisture content does help identify intervals that have been impacted by drilling operations. For example, moisture readings obtained from the first two samples collected below the tank bottom ( 17.1 to 18.7 meters [56 to 61.5 feet] bgs) are rather wet at 16.3 and 12.8 $\mathrm{wt} \%$. Values then drop over the next 3 meters (10 feet) to near $5 \mathrm{wt} \%$ and are low at the top of the coarse unit, Hanford formation $\mathrm{H} 1$ unit at 19.8 to 20.4 meters (65 to 67 feet) bgs. At 24.4 meters ( 80 feet) bgs (at the base of the Hanford formation $\mathrm{H} 1 \mathrm{unit}$ ), the moisture content is between 8 and $10 \mathrm{wt} \%$. In the lower laminated sand unit (Hanford formation $\mathrm{H} 2$ unit), the moisture content peaks at $12 \mathrm{wt} \%$ at 33.2 meters (109 feet) bgs. At the top of the Plio-Pleistocene unit fine-grained muddy sand unit (PPlz) ( 38.1 meters [125 feet] bgs), the moisture content is $13 \mathrm{wt} \%$, but then sharply increases at 40.5 meters (133 feet) bgs. At 40.5 meters (133 feet) bgs, this wet zone corresponds precisely to the depth where the end cap drive point was milled off the casing prior to the extension phase of the drilling project. At this depth, about 75 liters of cooling water escaped into the borehole when the end cap was breached. The evidence clearly indicates that excess water in borehole extension sleeves $2 \mathrm{D}, 2 \mathrm{C}$, and $2 \mathrm{C}-2$ was the direct result of water being used to cool the drill bit during milling of the end cap. Normal amounts of moisture, ranging from 9.4 to $16.1 \mathrm{wt} \%$, were recorded down to a depth of $\sim 43.4$ meters ( 142.4 feet) bgs. Abnormally high water readings were observed in sleeves 6D, 6C, and 6B. A review of the drillers' logs indicated that the sampler was left in the borehole at this depth over several days and that a rain event likely allowed some water to enter the borehole. Overall, the fine-grained sediments in the PlioPleistocene unit (between 38.1 and 47.2 meters [125 and 155 feet] bgs) had relatively high moisture contents ranging from 7.4 to $19.6 \mathrm{wt} \%$. We believe that this moisture content range is natural and not an indicator of remnant tank fluids or drilling artifacts. Hard-tool drilling was initiated at 49.1 meters (161 feet) bgs and continued periodically down to 63.4 meters (208 feet) bgs. During 28 hard-tooling events, 58 liters of water were added. Sleeves that showed distinct increases in moisture were 16D, 16C, $18 \mathrm{C}, 28 \mathrm{~A}, 30 \mathrm{C}$, and perhaps all the sleeves between $40 \mathrm{~A}$ and $47 \mathrm{C}, 49 \mathrm{~B}$ to $50 \mathrm{~B}$, and $52 \mathrm{~B}$ through $58 \mathrm{~B}$; although sleeves 58A and 58B appear to be wet, it is probably due to their position in the capillary fringe right above the water table. Overall, the coarse sediments associated with the Ringold Formation exhibited moisture contents as low as $1.5 \mathrm{wt} \%$ and the upper range on the coarse sediments moisture was about $6 \mathrm{wt} \%$.

The second parameter measured was the $\mathrm{pH}$ of water extracts of the vadose zone sediments. We anticipated that the highly caustic tank liquor would alter the sediment $\mathrm{pH}$ dramatically. The 1:1 sediment-to-water extract $\mathrm{pH}$ for the shallow sidewall core samples above and along the side of SX-109 tank varies from 8.3 to 8.5 , suggesting natural conditions. Below the tank bottom from a 19.8- to 24.4meter (65- to 80-foot) depth, the extract $\mathrm{pH}$ varies from 9.2 to 9.8 , which we believe represents interaction with the alkaline fluids that leaked from the tanks. The $\mathrm{pH}$ values are not nearly as high as would be expected for tank liquor completely saturating sediments. As described in Serne et al. (1998), the $\mathrm{pH}$ can reach values of $>13$ when simulated tank liquor reacts with Hanford sediments. One plausible explanation for the lower than expected $\mathrm{pH}$ values is perhaps that the $\mathrm{pH}$ reneutralizes slowly with time from the slow dissolution of alumino-silicates. Likewise, it is also possible for the tank liquor to react with carbon dioxide in the vadose zone air-filled porosity such that the initial $\mathrm{pH}$ excursion to high values is muted over time. Finally, the borehole is between 3 and 10.7 meters (10 and 35 feet) from the perimeter of tanks SX-109 and SX-108, respectively, and no one is certain where the leak or leaks 
occurred. Perhaps the sediment between the leak(s) and the borehole was adequate to neutralize most of the tank leak caustic before the fluid reached the borehole position.

From 27.4 to 38.7 meters (90 to 127 feet) bgs, the sidewall core samples yield 1:1 sediment to water extracts that have $\mathrm{pH}$ values between 7.92 and 8.33 , which appears to be close to natural conditions. Therefore, it would appear that the significant $\mathrm{pH}$ reactions occur from the tank bottoms at $\sim 15.5$ meters ( $\sim 51$ feet) to a maximum of 27.4 meters ( 90 feet) bgs for sediment surrounding the tanks. The slant borehole under SX-108 addresses conditions directly under a REDOX tank (Serne et al. 2002c).

The water extract $\mathrm{pH}$ for borehole extension sleeve samples (obtained from 40 to 67.1 meters [131 to 220 feet] bgs) ranges from 7.21 to 8.97 with an arithmetic mean of 8.22 and standard deviation of 0.38 . The geometric mean is 8.21 and the median value is 8.28 . These values are similar to Hanford Site background values for sediments that have not been altered by significant chemical reaction. If the sediment was at equilibrium with atmospheric carbon dioxide and the calcium carbonate mineral calcite, the $\mathrm{pH}$ should be fixed at 8.3. Hanford Site sediments generally contain small amounts of calcite and the aquifer sediments in the upper unconfined aquifer have been shown, based on geochemical modeling, to be in equilibrium with the air and calcite. We can reasonably expect Hanford Formation and PlioPleistocene unit vadose zone sediments also to satisfy these equilibria. The data collected support this speculation.

The third parameter that was assessed to estimate the vertical extent of the leak plume was dilutioncorrected EC for water extracts. The EC results suggest that the tank leak fluid dominates the porewater down to a depth of 38.8 meters (127.4 feet) bgs. For sleeves collected in the borehole extension, only sleeve 1B at 40.1 meters (131.7 feet) bgs appears to contain dissolved salt that might represent the leading edge of the tank leak fluids that dominate the shallower sediment from the sidewall cores. For other borehole extension sleeves below 40.1 meters (131.7 feet) bgs, the EC does not show any significant deviations from values found for vadose zone sediments at nearby uncontaminated Resource Conservation and Recovery Act (RCRA) boreholes (Serne et al. 2002a). For a point of reference, the EC of uncontaminated Hanford Site groundwater in the 200 Area plateau is about $350 \mu \mathrm{S} / \mathrm{cm}$ and the water obtained at wells near the SX Tank Farm, including the one water sample obtained at the 41-09-39 borehole extension, is about $250 \mu \mathrm{S} / \mathrm{cm}$. This suggests that the groundwater beneath the SX Tank Farm still shows the influence of the large volumes of dilute-salt waste liquids disposed to facilities upgradient (north and west).

Nitrate is perhaps the most sensitive chemical marker of tank leaks migrating through the vadose sediments. The tank liquor has up to 6 to $8 \mathrm{M}$ (about 350,000 ppm) nitrate concentrations and there are obvious indications of high nitrate concentrations in the sidewall core sediments between depths 24.4 and 38.7 meters ( 80 and 127 feet) bgs. The first indication of elevated nitrate appears at 20 meters ( 65.5 feet) bgs and the deepest (leading edge of plume) is in borehole extension sleeve 3A, at a depth of 41.4 meters (136 feet). However, moderate nitrate concentrations were found in the borehole extension sleeves from the sixth through eleventh split-spoon samplings. Between split-spoon samplings 7 and 9, the casing was removed and replaced with one containing a drive shoe. The probability is high that sediment from shallow depths was knocked into the borehole between samplings 7 and 9, so the nitrate data for sleeves down to the eleventh sampling may be biased high from incorporation of shallower sediment. The nitrate 
data qualitatively suggest that the leading front of a tank leak may have reached 47.5 meters (156 feet) bgs, which is the bottom of the PPlz unit at the contact with the caliche layer. But we cannot ascertain whether the slightly elevated nitrate between 41.5 and 47.5 meters (136 and 156 feet) bgs has been artificially increased by the original casing pile driving to 39.9 meters (131 feet), subsequent end cap milling, loss of cooling water, and borehole extension casing insertion and removal prior to obtaining samples below 44.2 meters (145 feet) bgs. Sleeve 47B also has a slight increase in nitrate content at 60.0 meters (196.9 feet) that may reflect fluid that migrated horizontally from upgradient when the water table was higher in the profile.

The fifth indicator species used was sodium in the water extract. Sodium is the dominant cation in leaking tank liquor and, like nitrate, is present at molar concentrations. Water extract sodium data suggest that tank fluid has impacted the vadose zone sediments to a depth of at least 38.7 to 40.8 meters (127 to 134 feet) bgs. There also appears to be an ion-exchange front where sodium displaces the native calcium, magnesium, and potassium from the sediment surface sites resulting in the elevated calcium, magnesium, and potassium to depths of 41.1 meters (135 feet) bgs. There is obvious low water-leachable calcium, magnesium, and potassium in samples at depths of 18.7 to 24.2 meters (61.5 to 79.5 feet) bgs from the displacement by the high sodium in the tank fluids.

Finally, there is a hint of elevated water-leachable aluminum and iron in the samples at depths of 18.7 to 21.2 meters (61.5 to 69.5 feet) bgs, which is at the shallow end of the zone of slightly elevated $\mathrm{pH}$ (found at 20 to 24.2 meters [ 65.5 to 79.5 feet] bgs). We expected to find more significant changes in $\mathrm{pH}$, aluminum, and iron in both the water extracts and sediments themselves, but the zone of caustic alteration is either small and closer to the tank, perhaps completely underneath the tanks, where sampling is difficult or the caustic zone has been muted by continued weathering since the leaks.

The traditional sampling of Hanford sediments concentrates on determining the total concentration of radionuclides in the sediment. For the most part, the highly radioactive samples were restricted to the sidewall cores between 18.2 and 38.7 meters (60 and 127 feet) bgs. Samples between 20.1 and 25.6 meters ( 66 and 84 feet) bgs were radioactive enough to require some changes in standard gamma energy analyses (GEA) counting techniques. Because of high activity in the sidewall cores, only small aliquots (5 grams) could be analyzed, producing some justifiable concern for sample homogeneity. Remarkably, apart from the minor fluctuations of cesium-137 activity between individual sleeves, the vast majority of activity is concentrated between the depths of 20.1 to 25.6 meters (66 to 84 feet) bgs with the leading edge perhaps reaching 39.9 to 41.5 meters (131 to 136 feet bgs). The samples from the borehole extension, which started at 39.9 meters (131 feet) bgs and continued to groundwater, were relatively easy to analyze and contained little radioactivity.

There appears to be three high cesium-137 peaks; at 20.1, 25.1, and 31.1 meters $(66,82.5$, and 102 feet) bgs that contain $4 \times 10^{6}, 2 \times 10^{7}$, and $2 \times 10^{6} \mathrm{pCi} / \mathrm{g}$, respectively. The peak at 20.1 meters (66 feet) bgs represents the contact between the Hanford formation H1a unit (fine sand) and the coarsergrained gravelly sand Hanford formation $\mathrm{H} 1$ unit. There appears to be a large amount of cesium contained in the coarse-grained Hanford formation H1 unit that is found between 20.4 and 26.8 meters (67 and 88 feet) bgs at borehole 41-09-39. The other large peak of cesium is found in the fine laminated sand layer (Hanford formation $\mathrm{H} 2$ unit) that lies between 26.8 and 38.1 meters (88 and 125 feet) bgs at 
this borehole. The high cesium-137 at 40.1 to 40.5 meters (131.7 to 133 feet) bgs is believed to be compressed sediment dragged down during the original pile driving of the closed end casing.

The technetium-99 data show that there are significant concentrations of technetium between the depths of $\sim 24.1$ to 41.1 meters ( $\sim 79$ to 135 feet) bgs. The leading edge of the technetium plume appears to reach 41.1 meters (135 feet) bgs. Samples below 42 meters (138 feet) are at or near the detection limit with two exceptions. There does not appear to be readily measured strontium- 90 activities $(>10 \mathrm{pCi} / \mathrm{g})$ in the sediments, thus suggesting that strontium-90 is not mobile in the REDOX fluid that leaked from the SX tanks. Strontium is not considered to be very soluble in most single-shell tank environments and has been found to reside predominately in precipitates in the sludge at the bottom of the tanks.

In summary, the moisture content, $\mathrm{pH}, \mathrm{Cr}(\mathrm{VI})$ (to be discussed below), and cesium-137 profiles do not identify the leading edge of the plume. More mobile constituent profiles such as EC, sodium, nitrate, and technetium-99 all suggest that the leading edge of the plume resides about 3 meters (10 feet) into the fine-grained PPlz unit at $\sim 41.1$ meters $(\sim 135$ feet $)$ bgs.

\subsection{Estimate of Sediment Recharge Rates (Matric Potential)}

To assess the water status in the sediment, we measured the soil-water matric suction on 30 sleeves from the Plio-Pleistocene units and Ringold Formation. Matric suction is the absolute value of matric potential and is used to conveniently express the matric forces (potentials) as positive values. Water flow in unsaturated sediments is the product of the unsaturated conductivity and the matric suction gradient. The suction gradient defines the direction of flow (from areas of low to high suction). Under conditions of no drainage/recharge, the suction profile for a sediment column is linear and equal to the height above the water table. If recharge to the water table is occurring, the suction profile depends on the recharge rate and the layering sequence and hydraulic properties of the underlying sediments. As the recharge rates increase, the suction profile shifts toward zero-suction (i.e., saturation). The data on the 30 sleeves indicate that 18 are at matric suctions lower (wetter) than the elevation of the sample above the water table, suggesting that the sediment profile at borehole 41-09-39 is draining. Using simple onedimensional, steady-state flow conditions and generic unsaturated hydraulic conductivity estimates from Rockhold et al. (1997) for 200 West Area sediments, our observed matric suction profile provides a means to estimate the recharge rate. We estimate a value somewhat $>5 \mathrm{~mm} / \mathrm{yr}$. This is lower than we expected based on a number of studies at the Hanford Site that have shown coarse gravel surfaces to cause as much as $50 \%$ of the annual precipitation to recharge to the water table. Another source of localized recharge near tank farms has been hypothesized to be leaking water lines. With the gravel cover allowing up to $50 \%$ of annual precipitation of 8 centimeters to recharge, we would have expected much higher than $5 \mathrm{~mm} / \mathrm{yr}$. Recharge has not been measured directly at the SX Tank Farm, so this simple analysis based on measuring matric suction of 30 samples may be too simple and uncertain.

Two possibilities may explain the higher matric suction (dryer condition) found for our profile. The first is that the samples were disturbed enough during coring that the water content and, possibly, density were altered enough to increase the matric suction values. The second is that large thermal gradients inferred from past casing air temperature measurements (Conaway et al. 1997) and the observed high temperature on the casing removed (see Myers et al. 1998) have driven moisture out of the sediments. 
The latter situation would allow our unexpected results to be realistic. It would also require that nonisothermal flow modeling and more moisture retention data as a function of temperature would be needed to better predict flow and transport in the vadose zone.

\subsection{Detailed Characterization to Elucidate Controlling Geochemical Processes}

The more detailed characterization activities of the sidewall cores and the seven borehole extension composites added some insight on the processes that control the observed vertical distribution of contaminants and on their migration potential into the future. The first key finding was that the 1:1 sediment-towater extracts for sediments, influenced strongly by high ionic strength tank liquors, were shown to give a good estimate of the porewater chemistry in the vadose zone sediments. We were able to extract porewater from selected vadose zone sediments using high-speed centrifugation. The chemical composition of the actual porewater was found to be fairly well estimated by dilution correcting the 1:1 sediment-towater extracts. Because it is much easier to obtain a water extract of the vadose zone sediments, this finding is important to understanding the porewater chemistry throughout the vadose zone plumes under disposal facilities and leaking tanks. Constituents that showed the best agreement include EC, nitrate, and sodium. Technetium would be another constituent that should be monitored but was not present in significant concentrations (based on our detection limit using small samples of sediment) at borehole 4109-39. The porewaters in the sediments from the sidewall cores in the three Hanford formation units (H1a, H1, and H2) were dominated by sodium and nitrate. The most concentrated porewater was essentially 5 to $6 \mathrm{M}$ sodium nitrate with several tenths molar concentrations of calcium and chromate.

The nitrite distribution suggests that once leaked into the sediments, oxidation converts the nitrite to nitrate because the ratio of nitrite to nitrate is much lower than the values within the tanks. Other waterleachable parameters such as sodium, EC, and technetium-99 suggest that the leading edge of the plume is currently near 39.6 to 41.1 meters (130 to 135 feet) bgs, 3 meters ( 10 feet) into the PPlz mud facies.

Both water and strong acid extracts for trace metals show that there is elevated technetium-99, chromium, molybdenum, and to a lesser extent, selenium in the region where EC, sodium, and nitrate show the presence of tank fluids. The first significant sign of elevated technetium is at 24.2 meters (79.5 feet) bgs and a high concentration plume is found from 27.4 to 38.8 meters (90 to 127.4 feet) bgs. Molybdenum distribution is quite similar. The leading edge of the chromium and selenium appears to stop at 34.1 meters (112 feet) bgs, thus, suggesting that they do not migrate as quickly as molybdenum and technetium. It was also observed during the inductively coupled plasma mass spectrometry (ICPMS) analyses that molybdenum and perhaps other elements exhibit a fission product isotope ratio instead of natural ratios. More discussion of this fingerprint showing that the fluid in the vadose zone is tankrelated will occur in the field investigation report. ${ }^{\text {(a) }}$ The elements zinc, arsenic, cobalt, nickel, cadmium, silver, lead, uranium, and ruthenium did not leach into water at high enough concentrations to show any tank-enhanced concentrations. There does appear to be some elevated levels of some of these metals (not

(a) Draft Field Investigation Report for Waste Management Area S-SX. RPP-7884, Draft, Volume 2, Appendix D, CH2M HILL Hanford Group, Inc., Richland, Washington. 
uranium) in the acid extracts of the sediments. Enhanced concentrations of bismuth are present and its vertical distribution suggests that it is less mobile than the chromium and selenium.

A key finding is that we do not observe a continuous vertical distribution of elevated nitrate or any other tank constituent concentrations from the tank bottom to the water table at this borehole. There is one indication of nitrate-rich porewater at 60 meters (197 feet) bgs (sleeve 47B) that may represent a zone influenced by water with a high nitrate that flowed horizontally into the sediments when the water table was elevated or perhaps the extract represents a local natural enrichment.

The water-extractable cations suggest that an ion-exchange process dominates the porewater/sediment interactions in the zone where tank fluid passed by or currently exists. The leading edge of the tank leak plume is enriched in alkaline earth cations that were displaced from the native sediment exchange sites.

The water and acid leach data show little indication that the sediments contain leachable technetium99 or chromium below 38.8 meters ( 127.4 feet), just 0.6 meters ( 2 feet) into the top of the PPlc unit. The bulk of the technetium mass is found in the vicinity of 27.4 to 34.1 meters (90 to 112 feet) bgs. Very little technetium-99 activity was found in the water extracts of the sediment below 38.7 meters (127 feet) bgs. As mentioned, the chromium does not migrate as fast as the technetium and its leading edge is found nearer to 34.1 meters (112 feet) bgs than 38.7 meters (127 feet) bgs. The bulk of the water-leachable chromium has been confirmed as $\mathrm{Cr}(\mathrm{VI})$ (chromate) by its distinct yellow color and by ion chromatography. There are at least two plausible mechanisms for the slight retardation of chromium (and selenium) compared to technetium and molybdenum. The first, which has more probability, is that there could be some redox-mediated reduction of the highly mobile oxyanion forms of these two metals and the second mechanism is precipitation of some of the oxyanion into alkaline earth co-precipitates such as calcium chromate or calcium/barium-mixed chromates/selenate/sulfates. Recall that the alkaline earth cations show higher concentrations in the water extracts between 29 and 38.7 meters (95 and 127 feet) bgs and that the zone between 29 and 34.1 meters ( 95 and 112 feet) bgs may be a zone of active reaction of the tank fluids and sediments. This differing technetium and molybdenum versus chromium and selenium migration observation is being studied by several investigators within the Environmental Management Science Program (EMSP) and Science and Technology Program and some results will be discussed in the field investigation report. ${ }^{(a)}$

Combining the atypical high nitrate with the sodium-to-calcium ratio data for water extracts suggests that the leading edge of a tank leak plume is currently at 41.1 meters (135 feet) bgs at borehole 41-09-39. One simple but plausible explanation for this depth is that the tank leak plume traveled horizontally and vertically over a relatively short time period through the more permeable Hanford formation sediments and perched on the less permeable Plio-Pleistocene unit. Over the next three to four decades, the water slowly percolated into the top of PPlz unit to a depth of $\sim 3$ meters $(\sim 10$ feet).

Another more detailed conceptualization of the leak event history is that the initial leak was rapid and tank liquor percolated through the $\sim 4.6$ meters ( $\sim 15$ feet) of fine-grained Hanford formation H1a unit and

(a) Draft Field Investigation Report for Waste Management Area S-SX. RPP-7884, Draft, Volume 2, Appendix D, CH2M HILL Hanford Group, Inc., Richland, Washington. 
settled into the $\sim 6.7$ meter ( $\sim 22$-foot)-thick coarser Hanford formation H1 unit. The bulk of the reactive material, including cesium-137, slightly elevated $\mathrm{pH}$, and the highest concentrations of sodium, was originally left in the Hanford formation $\mathrm{H} 1$ unit when the leak driving force dissipated. The stratigraphic break between the Hanford formation $\mathrm{H} 1$ unit and finer-grained Hanford formation $\mathrm{H} 2$ unit acted as the initial horizontal spreading surface. After the initial rapid flux, slow vertical advection under natural recharge conditions has allowed the more mobile species (nitrate, technetium-99 and displaced calcium and magnesium ion-exchangeable cations, and some of the residual soluble sodium) to migrate through the fine-grained Hanford formation $\mathrm{H} 2$ unit. These slowly moving constituents have ultimately reached the contact between the Hanford formation $\mathrm{H} 2$ unit and the Plio-Pleistocene unit. Whether the PPlz unit will act as another barrier to further vertical migration under natural recharge rates will take several tens to hundreds of years to determine.

In summary, we have identified common ion-exchange and heterogeneous (solid phase-liquid solute) redox reactions as two mechanisms that are influencing the distribution of contaminants in the vadose zone sediments. Two conceptual leak models are offered. One suggests that the PPlz unit acted as the confining layer while the other suggests that the original leak-driving force dissipated in the Hanford formation $\mathrm{H} 1$ unit and that subsequent slow vertical migration has moved the more mobile contaminants through the Hanford formation $\mathrm{H} 2$ unit laminated sand into the top of the PPlz unit. The latter conceptual model does not require the PPlz unit to act as a confining layer to past or future slow vertical migration under natural recharge forces.

\subsection{Estimates of Sorption-Desorption Values}

In this section, we discuss our measurement and data synthesis used to quantify the adsorptiondesorption values for the major contaminants found in the sediments at borehole 41-09-39. We estimated the $\mathrm{K}_{\mathrm{d}}$ for various contaminants using two methods. First, by combining the data from the dilutioncorrected 1:1 sediment-to-water extracts, which represent the porewater with the activities measured on the sediments, we can get our first semiquantitative sense of what the desorption $\mathrm{K}_{\mathrm{d}}$ is. For a contaminant that has very little water-soluble mass, such as cesium-137, the $\mathrm{K}_{\mathrm{d}}$ can be approximated as the amount of mass on the solid per gram of dry sediment divided by the amount of mass in the porewater per milliliter. For a contaminant that is quite soluble in the water extract (equivalent to saying that the contaminant resides mainly in the porewater within the sediment), one needs to subtract the amount that was water extractable from the total amount present in the sediment sample to obtain a value for the amount that would remain on the solid at equilibrium with the pore fluid. Using these measured distributions, the in situ desorption $\mathrm{K}_{\mathrm{d}}$ for cesium varies from 4 to $25 \mathrm{~mL} / \mathrm{g}$ in the sediments between 27.1 and 34.1 meters (90 and 112 feet) bgs where the bulk of the tank fluid with high salinity resides. Above and below this depth range, cesium in situ desorption $\mathrm{K}_{\mathrm{d}}$ is larger because the pore fluids do not contain high sodium concentrations that compete for adsorption sites.

The second method of determining $\mathrm{K}_{\mathrm{d}}$ values was laboratory batch tests. Batch adsorption tests were performed with sediments from three geologic units. Four solutions ranging from highly saline and caustic simulated tank liquor to low ionic strength groundwater were used. 
The simulated tank liquor (Solution 1) shows very high strontium $K_{d}$ values for the sediments compared to the other three solutions. The fine-grained Plio-Pleistocene sediment (composite 10A/B) shows the highest adsorption, while the caliche sediment adsorbs strontium almost as strongly, and the Ringold Formation sediment adsorbs strontium moderately. The $\mathrm{pH}$ for Solution 1 is about 14, approximating $1 \mathrm{M}$ free hydroxide. As mentioned in Serne et al. (1998), strontium is insoluble in highly alkaline solutions and this forces the apparent $\mathrm{K}_{\mathrm{d}}$ to be high. The strontium $\mathrm{K}_{\mathrm{d}}$ varies with the particle size of the sediments, with the finer-grained sediment exhibiting the highest adsorption. The effects of both $\mathrm{pH}$ and particle size are as expected. For the SX Tank Farm environment, we would expect strontium to be bound tightly in high-pH-impacted sediments near the base of the tank. This fact, coupled with the low solubility of strontium inside the tank, may explain our not finding strontium-90 in the borehole sediments.

The cesium $\mathrm{K}_{\mathrm{d}}$ data also show high values for the highly alkaline simulated tank liquor (Solution 1) especially for the Plio-Pleistocene (composite 10A/B) and caliche sediments. In earlier work reported in Serne et al. (1998), a highly alkaline and high aluminum/ionic strength solution contacting a 200 East Area coarse sand yielded a cesium $\mathrm{K}_{\mathrm{d}}$ value of $26 \mathrm{~mL} / \mathrm{g}$. This earlier testing on the coarse-grained sand agrees remarkably well with the current data for the coarse Ringold composite sample (38A/B) whose cesium $\mathrm{K}_{\mathrm{d}}$ ranged from 16 to $23 \mathrm{~mL} / \mathrm{g}$.

The cesium $\mathrm{K}_{\mathrm{d}}$ value for all the sediments is moderate (4 to $40 \mathrm{~mL} / \mathrm{g}$ ) for Solution 2, the very high ionic strength (7.4 M sodium nitrate) but neutral $\mathrm{pH}$ solution, similar to the composition of the actual pore fluids obtain via centrifugation. The cesium $\mathrm{K}_{\mathrm{d}}$ increases slightly for the $4 \mathrm{M}$ sodium nitrate solution (Solution 3) as would be expected for a cation-exchange-dominated process. The range of 4 to 40 is similar to the in situ desorption $\mathrm{K}_{\mathrm{d}} \mathrm{S}$ (4 to $25 \mathrm{~mL} / \mathrm{g}$ ) calculated from comparing the water extracts and direct counting of the sediment.

None of the data collected during this SX Borehole Extension project can be interpreted to suggest that cesium-137 can travel through Hanford sediments without adsorbing to a considerable degree. However, the data and tests performed to date do not address elevated temperature or colloidal transport. These two drivers are not expected to significantly change the conclusions. The temperature effect on adsorption reactions is not well studied but the few available studies suggest only small effects (see Ames et al. [1982] for cesium adsorption onto basalt and smectite clay-weathering products).

The technetium in situ $\mathrm{K}_{\mathrm{d}}$ varies from 0.01 to about $5 \mathrm{~mL} / \mathrm{g}$ over the whole zone of contamination. The agreement between in situ $\mathrm{K}_{\mathrm{d}}$ values calculated using the 1:1 sediment-to-water extract versus the actual porewater is good for all data sets except sidewall core sample 13. These cesium and technetium in situ $\mathrm{K}_{\mathrm{d}}$ values suggest that cesium is held more strongly to the sediment than technetium.

Another technique to estimate mobility is to compare the ratio of cesium-137 to technetium-99 activity in the borehole sediments. Sediments from 18.3 to 25 meters (60 to 82 feet) bgs have a cesium137 to technetium-99 activity ratio $>10,000$. Johnson and Chou (1998) suggested that the ratio in SX tank liquors would be 10,000. Between the depths of 27.4 and 40.8 meters ( 90 and 134 feet) bgs, the ratio ranges from $<1$ to $\sim 100$, which suggests that cesium gets removed from the fluid and technetium continues to migrate deeper into the sediments. 
These technetium data are consistent with a wealth of literature that finds essentially no technetium adsorption onto Hanford Site sediments from less saline waters (Kaplan et al. 1995; Kaplan and Serne 2000).

The most significant chemical contaminant in the sediments is chromium. The in situ desorption $\mathrm{K}_{\mathrm{d}}$ value, calculated in the same fashion as described for technetium-99 and cesium-137, is slightly negative for sediments between 24.1 and 33.2 meters (79 and 109 feet) bgs where the elevated chromium concentrations are found. Above the tank bottom $(<15.2$ meters $[<50$ feet] bgs $)$ and below the leading edge of the tank leak ( 38.7 meters [127 feet] bgs), the chromium $\mathrm{K}_{\mathrm{d}}$ value is $>100 \mathrm{~mL} / \mathrm{g}$ and the percentage of water soluble chromium is $<0.1 \%$ because the source of chromium is native $\mathrm{Cr}(\mathrm{III})$. One sample from the highly contaminated zone (sidewall core 8ABC) shows a peculiarly low water-leachable fraction and, thus, high $\mathrm{K}_{\mathrm{d}}$. This sample is being studied within an independently funded basic science project.

Based on comparing the depth of penetration of various contaminants and comparing the percentages that are water-leachable, we can state that chromium migrates faster than cesium-137 but slower than technetium-99 and nitrate. In other waste disposal situations at Hanford, oxidized chromium, released with reactor cooling water (low ionic strength and neutral $\mathrm{pH}$ ), appears to migrate similarly to technetium-99 and nitrate. All of these observations, based on directly measuring sediments or their water and acid extracts or performing traditional laboratory batch tests suggest that nitrate, technetium-99, and molybdenum migrate with no measurable retardation; while sodium, chromium, and selenium migrate with a small amount of retardation; and cesium-137 and bismuth migrate with moderate retardation.

\subsection{Other Characterization Observations}

It is obvious from comparing $8 \mathrm{M}$ nitric acid digestions to total fusions that the $8 \mathrm{M}$ nitric acid is not removing more than about $10 \%$ as much of the major and several minor elements (sodium, aluminum, potassium, titanium, strontium, barium, lead, and uranium) as the fusion digestion. We now realize that a more productive method to determine the total elemental concentration of all major and minor elements (including manmade contributions) in vadose zone sediments is $\mathrm{x}$-ray fluorescence.

As part of our characterization of the contaminated sediments, parameters that can control contaminant migration were measured. Key parameters that were measured include the cation exchange capacity (CEC) of the sediments, the calcium carbonate content, particle size distribution, and bulk and clay size mineralogy. The CEC of the bulk sediments was estimated for samples from the borehole extension by performing tests on the $<2$ millimeters fraction, the typical size used by all soil chemists, and calculating the bulk exchange capacity by assuming all material greater than 2 millimeters has no exchange capacity. The calculated CECs show that the fine-grained Plio-Pleistocene unit have a relatively high exchange capacity (13 to $16 \mathrm{meq} / 100 \mathrm{~g})$. The coarse-grained Ringold sediments have a very low CEC ( $\sim 1$ to 3 meq/100 g). Although not measured, the Hanford formation sediments would fall in between these two values. The CEC for Hanford formation sediments from nearby clean boreholes confirm this statement (see Serne et al. 2002a for details). 
The particle size distributions for the various geologic layers encountered at borehole 41-09-39 show that right under the tanks there is a thin layer ( $\sim 4.3$ meters $[\sim 14$ feet]) of fine sand (Hanford formation H1a unit) with little gravel and up to $50 \%$ by weight mud. Below the Hanford formation H1a unit is the coarser-grained sand/gravel Hanford formation $\mathrm{H} 1$ unit with $10 \%$ to $30 \%$ gravel and $15 \%$ to $45 \%$ mud. The Hanford formation H1 unit is $\sim 6.4$ to 7 meters ( $\sim 21$ to 23 feet) thick at borehole 41-09-39. Below the Hanford formation $\mathrm{H} 1$ unit is the lower fine sand Hanford formation $\mathrm{H} 2$ unit that is $\sim 11.3$ meters ( $\sim 37$ feet) thick with $<1 \%$ gravel and $30 \%$ to $40 \%$ mud. Below the Hanford formation $\mathrm{H} 2$ unit is the $\sim 11.3$-meter ( $\sim 37$-foot)-thick fine-grained PPlz with $<2 \%$ gravel and $50 \%$ to $80 \%$ mud. Below the PPlz unit is the 1.5-meter (5-foot)-thick caliche layer (PPlc) with a highly variable particle size including tens of percent gravel and up to $30 \%$ silt and clay. Below the caliche is a thin layer $(\sim 2.1$ meters $[\sim 7$ feet]) of Ringold sand [Rtf] with $<10 \%$ gravel and $<10 \%$ silt plus clay. The final layer extending 13.4 meters (44 feet) to the water table is the coarse gravel Ringold unit $\mathrm{E}$ (Rwi[e]) that on average is $80 \%$ gravel and $<10 \%$ silt and clay.

X-ray diffraction analyses of the bulk sidewall core samples from five depths in borehole 41-09-39 indicate that the sediments are mostly quartz ( $\sim 35 \%$ to $50 \%)$ and feldspar ( $\sim 25 \%$ to $55 \%)$, with lesser amounts of mica and chlorite. Plagioclase feldspar is 2 to 10 times more abundant than potassium feldspar. Minor amounts of amphibole and calcite were also detected in the sediments. The clay fraction ( $<2$ micron) is dominated by four clay minerals: illite, smectite, chlorite, and kaolinite with minor amounts of quartz, feldspar, and amphibole. Overall, illite was the dominate mineral in the clay fraction with 20 to $35 \mathrm{wt} \%$. Smectites, ranged in concentrations up to a high of $20 \mathrm{wt} \%$ (samples $13 \mathrm{ABC}$ and $4 \mathrm{ABC}$ at depths of (17.1 and 32.9 meters [56 and 108 feet], respectively) to as low as $5 \mathrm{wt} \%$; chlorite occurred between $\sim 10$ and $30 \mathrm{wt} \%$. Minor amounts of kaolinite $(<10 \mathrm{wt} \%)$ were detected at all depths. Quartz and feldspar made up $\sim 5$ to $20 \mathrm{wt} \%$ of the clay fraction. Smectite particles were very small $(<0.5$ microns). The presence of illites as the dominant clay-size mineral is fortuitous because illites are strong adsorbers of cesium and can irreversibly adsorb cesium within interlayer sites. More discussion of cesium-137 adsorption on illites is found in documents being prepared for publication under companion EMSP and Science and Technology Programs.

A few of the contaminated sediments in the PPlz unit were wet sieved into several particle size ranges and the cesium-137 concentration of each particle size was determined. Finding that trace constituents, such as cesium-137, adsorb onto the fine-grained portion of a bulk sediment is quite common. In general, the data from the PPlz material support the hypothesis that the fine-grained material (silt and clay) contains most of the mass of radioactive cesium. It is, however, surprising that the concentrations (pCi/g) of cesium-137 in each size fraction do not show a wider range of values with the finer-grained sizes showing much higher values than the coarse-grained sizes. The silt and clay fractions contain $67 \%, 27 \%$, and $81 \%$ of the cesium- 137 for composites $2 \mathrm{C} / \mathrm{D}, 2 \mathrm{~A} / \mathrm{B}$, and $3 \mathrm{~A} / \mathrm{B}$. 
Much of the detailed characterization performed on the contaminated sediments was performed on the $2 \mathrm{C} / \mathrm{D}, 2 \mathrm{~A} / \mathrm{B}$, and $3 \mathrm{~A} / \mathrm{B}$ borehole extension composites obtained very near the depth where the end cap was milled off the original pile driven closed end casing. We are still puzzled as to the source of the high iron in these sediments and whether these three composites are useful for studying the fate of cesium-137 in the Hanford sediment. Unfortunately, they were the only contaminated sediments that were available from borehole 41-09-39 for the first 2 years of the tank farm studies. The sidewall cores obtained when the borehole 41-09-39 casings were removed and the slant borehole under tank SX-108 during 2000 are now being used in similar studies to be certain that preliminary conclusions stated in this report remain accurate. 


\subsection{Stakeholder Questions and Comments}

The following section presents some specific questions that have been raised by stakeholders and our conclusions on what statements can be made based on the study of the data generated on characterizing the vadose zone sediments from borehole 41-09-39.

What is the role(s) of co-contaminants such as aluminum on the transport mechanisms for contaminants in the tank leak plume?

There were a few objectives that were not adequately addressed in the borehole 41-09-39 studies reported in this report because highly altered sediments (from reaction with highly caustic high soluble aluminum redox fluids) were not found. We could not address this particular issue using actual samples from borehole 41-09-39. However, numerous basic science studies are under way that include using simulated redox liquid wastes and Hanford formation sediments, both contaminated and uncontaminated. Preliminary results are found in the field investigation report. ${ }^{\text {(a) }}$

Is there any indication of reduced porosity due to formation of a gel, precipitate, or crystalline solid?

We did not observe measurable quantities of aluminum precipitates, obvious changes in sediment mineralogy, or visual signs of highly altered sediment that would result in dramatic changes in physical/ permeability properties. We did not observe large deviations in sediment $\mathrm{pH}$ conditions from those in the natural sediments.

Are there differences in waste liquor-sediment reaction with depth (i.e., leading edge versus trailing edge of the soil plume)?

There are very subtle signs that there has been some tank plume caustic neutralization closer to the tank bottoms where $\mathrm{pH}$ values are slightly elevated (9 to 10). This fact may also argue for horizontal flow early in the tank leak. If the flow was mainly vertical and the neutralization process occurs within a short distance of the tanks, then we would not expect the $\mathrm{pH}$-altered sediment to be at shallow depths at boreholes off to the side of tanks. Instead, the altered zone should remain under the leaking tanks if the plume migrates with mostly a vertical direction.

How much of the total plutonium, americium, cesium-137, strontium-90, iodine-129 if found, technetium-99, and chromium is water-extractable (mobility indicator)?

We did not find measurable quantities of long-lived non-gamma-emitting radionuclides such as strontium-90, and actinides or regulated Resource Conservation and Recovery Act metals. We did not do detailed analyses for iodine-129, selenium-79, or carbon-14. We did, however, find small concentrations

(a) Draft Field Investigation Report for Waste Management Area S-SX. RPP-7884, Draft, Volume 2, Appendix D, CH2M HILL Hanford Group, Inc., Richland, Washington. 
of stable selenium from the tank leak that appears to travel with the chromium (VI) in the tank fluids. As mentioned, there is a small interaction of both the chromium and selenium with the sediments and they are found at shallower depths than the technetium and molybdenum.

\section{Was the direction that the contaminants moved vertical or horizontal?}

It is not possible to state whether the contamination found in the vadose zone sediments at borehole 41-09-39 came from tank SX-109 or the upgradient but farther away tank SX-108. If the latter is the source, then there appears to be a large horizontal component to the migration of the contaminants. The sediments at borehole 41-09-39 at 19.8 meters ( 65 feet) (as shallow as 4.6 meters [ 15 feet] below the bottom of the nearby SX tanks) are highly contaminated. If the plume traveled mostly as a vertical plume, the shallower sediments would not show as much activity. See also the observation on the $\mathrm{pH}$ distribution in the sediments that also suggests a large horizontal component to the plume migration. We will revisit this question when the data from the slant borehole beneath SX-108 has been assimilated and reported in a companion report.

\section{Are there obvious signs of preferred vertical migration such as via clastic dikes?}

The sediments actively adsorb cesium-137 and the macro cations show an obvious ion-exchange front where the high sodium in the tank leak plume replaces the native alkaline earth cations from the sediment exchange sites. The development of the ion-exchange front can be used to confirm that the plume must be traveling through the sediments via massive porous media flow as opposed to traveling through highly selective preferred pathways. If the latter flow conditions were controlling the plume, one would not see the well developed ion-exchange front throughout the borehole profile.

\section{What is the long-term capability of the vadose zone to retain contaminants?}

The sediments actively adsorb cesium-137. The sediments, especially the fine-grained silt in PlioPleistocene mud contain significant quantities of illite and mica clays with high affinity for adsorbing cesium-137, especially when the cesium mass is in micromolar concentrations.

\section{What information on what factors contributed to mobility were generated?}

The only contaminant with a large inventory that shows significant adsorption is cesium-137. It would appear that surface adsorption, including classical ion exchange, controls the fate of cesium-137 in the vadose zone sediments. As the plume dilutes from interaction with less salty recharge water and existing vadose zone porewater, the $\mathrm{K}_{\mathrm{d}}$ for cesium should increase and its migration potential should decrease. The cesium-137 exhibits a very nonlinear adsorption $\mathrm{K}_{\mathrm{d}}$ because of the low abundance of the very specific adsorption sites on the illite/mica clay minerals that can withstand the competition of the massive amounts of sodium in the pore fluids. These rare, highly selective sites quickly fill and then the cesium-137 molecules must compete for less selective sites along with the high sodium concentrations. Besides cesium-137, the fission process creates long-lived cesium-135 and stable cesium-133 at roughly equal atom ratios (that is, on a mass basis, there was three times as many cesium atoms present as reflected by the activity of cesium-137). Assuming that the cesium-137 has decayed one half life, there is 
now six times as many cesium atoms in the porewater as measurable by converting the cesium-137 activity to mass. Zachara et al. $(1999,2002)$ have shown that in high sodium nitrate solutions and $10^{-3} \mathrm{M}$ total cesium (or greater), that the $\mathrm{K}_{\mathrm{d}}$ can be quite small ( $<1$ to $3 \mathrm{~mL} / \mathrm{g}$ ). Conversely, as the total sodium and cesium mass concentrations in the porewater decreases, because of dilution, the $K_{d}$ can increase dramatically. This nonlinear $K_{d}$ attribute should make future migration of cesium- 137 very slow. The historical gross gamma analyses versus time show few places in the SX Tank Farm where there is vertical migration of cesium-137. There are a few more instances where there has been horizontal influxes of gamma activity at dry wells around the tanks.

As discussed previously, there is some interaction between the sediments and the chromium (VI) in the porewaters within the leak plume. The most plausible mechanism is reduction of chromium (VI) by a heterogeneous reaction with the reduced iron within several of the minerals in the sediment. More detailed basic science studies are under way to further investigate this hypothesis.

\section{Are there indications on contaminants moving downward?}

There is little data (gamma logging as a function of time) suggesting that the cesium-137 within the contaminated sediments is migrating downward toward the water table. We do not have any means to currently investigate whether there is ongoing migration of the more mobile contaminants technetium- 99 and chromium (VI). At this time, the only mechanism would be to wait for many years and then drill another borehole near the existing one to compare the vertical distribution of the mobile contaminants. Based on general geochemical knowledge, it is reasonable to assume that there is migration at the same velocity as the recharging water or at slightly lower velocities for these mobile contaminants. As described in Section 4.4, our matric potential data suggest that the recharge rate at borehole 41-09-039 through the Plio-Pleistocene and Ringold Formations may be lower than previous estimates, but there is downward movement in this profile.

\section{Is there separation of highly mobile and less mobile contaminants in the vadose zone profile?}

Yes, there is vertical separation of cesium-137 from chromium (VI) and technetium-99. The leading edge of the cesium occurs near 31.1 meters (102 feet) bgs (but the center of mass is even shallower), while the chromium (VI) leading edge is at 34.1 meters (112 feet) bgs and the technetium-99 and nitrate leading edge is near 41.1 meters ( 135 feet) bgs.

\section{Are there identifiable characteristics that have controlled the placement of the contaminants?}

It would appear that commonly understood solute/sediment interactions such as ion exchange and redox-mediated reduction are active in the sediments at borehole 41-09-39. Closer to the tanks and within the tanks, $\mathrm{pH}$-mediated reactions likely are controlling and limiting the migration of uranium, actinides, and strontium-90 in the tank fluids. 


\section{Is there evidence for colloidal transuranics in the water-extractable fraction? If so, what percentage of the total? Does the extractable fraction vary with ionic strength of the extractant?}

We found only one positive hit for plutonium in the borehole sediments at a very low concentration of $4 \mathrm{pCi} / \mathrm{g}$. There was not enough activity to warrant studies on actinide colloids. We also did not find evidence that colloids are responsible for the enhanced (over past simplistic predictions using linear $\mathrm{K}_{\mathrm{d}}$ relationships) migration of cesium-137. We also suggest that the transport of contaminants within the tank leak plume is dominated by slow porous flow (with a significant horizontal component) through the sedimentary layers. This type of flow system has not been shown to be optimum for colloid transport. There are several Environmental Management Science Program-funded studies ongoing that are investigating the influence of colloid generation during highly alkaline waste fluids with Hanford sediments and the subsequent transport properties of the colloids. None of the contaminants that are observed in groundwater wells surrounding single-shell tank waste management areas are suspected of being colloids. The contaminants found in the groundwater are generally mobile oxyanions (pertechnetate, chromate, nitrate), anions (iodine-129), or tritiated water. 


\subsection{References}

ASA - American Society of Agronomy. 1986. Methods of soil analysis-part 1, $2^{\text {nd }}$ edition physical and mineralogical methods. A Klute (ed.), SSSA Book Series 5, Soil Science Society of America, Madison, Wisconsin.

ASA - American Society of Agronomy. 1994. Methods of Soil Analysis-Part 2, $2^{\text {nd }}$ Edition Chemical and Microbiological Properties. AL Page et al. (eds.), SSSA Book Series 5, Soil Science Society of America, Madison, Wisconsin.

ASA - American Society of Agronomy. 1996. Methods of soil analysis-part 3 chemical methods. DL Sparks (ed.), pp. 417-422, SSSA Book Series 5, Soil Science Society of America, Madison, Wisconsin.

ASTM. 1982. A Standard Test Method for Total and Organic Carbon in Water Oxidation by coulometric detection. D4129-82. American Society for Testing and Materials, West Conshohocken, Pennsylvania.

ASTM. 1986a. "Laboratory determination of water (moisture) content of soil, rock, and soil-aggregate mixtures.” Annual Book of ASTM Standards, Vol. 4.08. ASTM D2216. American Society for Testing and Materials, Philadelphia, Pennsylvania.

ASTM. 1986b. "Standard method for particle size analysis soils." Annual Book of ASTM Standards, Vol 4.08. ASTM D422-63 (Reapproved 1972), American Society for Testing and Materials, Philadelphia, Pennsylvania.

ASTM. 1986c. "Standard test methods for amount of material in soils finer than the No. $200(75-\mu \mathrm{m})$ sieve." Annual Book of ASTM Standards, Vol 4.08. ASTM D1140-54, American Society for Testing and Materials, Philadelphia, Pennsylvania.

ASTM. 1988. "Standard test method for total and organic carbon in water by high temperature oxidation and by coulometric detection." Annual Book of ASTM Standards Vol 11.01.. ASTM D4129-88, American Society for Testing and Materials, Philadelphia, Pennsylvania.

ASTM. 1993. "Standard practice for description and identification of soils (visual-manual procedure)." ASTM D 2488-93, American Society for Testing and Materials, West Conshohocken, Pennsylvania.

ASTM. 1996. "Standard Practice for Total Digestion of Sediment Samples for Chemical Analysis of Various Metals," which is procedure ASTM D4698-92. American Society for Testing and Materials, West Conshohocken, Pennsylvania.

Ames LL, JE McGarrah, BA Walker, and PF Salter. 1982. "Absorption of uranium and cesium by Hanford basalts and associated secondary smectite." Chem. Geol. 35:205-225. 
Amrhein C and DL Suarez. 1990. "Procedure for determining sodium-calcium selectivity in calcareous and gypsiferous soils." Soil Sci. Soc. Am. J. 54:999-1007.

Anderson FJ and LJ Soler. 2000. Interim measures to limit the migration of radioactive contaminants through the vadose zone at Hanford's single shell tank farms. Poster H61A-16, 2000 Fall Meeting American Geophysical Union, December 15-19, 2000, in San Francisco, California, EOS:Vol. 81, No. 48, pp. F413.

Bjornstad BN. 1990. Geohydrology of the 218-W-5 Burial Ground, 200 West Area, Hanford Site. PNL-7336, Pacific Northwest Laboratory, Richland, Washington.

Brindley GW and G Brown (eds.). 1980. Crystal structures of clay minerals and their x-ray identification: Monograph 5, Mineralogical Society, London.

Brown DJ. 1959. Subsurface geology of the Hanford separations areas. HW-61780, General Electric Company, Richland, Washington.

Brown DJ. 1960. An eolian deposit beneath 200-West Area. HW-67549, General Electric Company, Richland, Washington.

Campbell GS and GW Gee. 1986. "Water potential: Miscellaneous methods." In Methods of Soil Analysis Part 1, A Klute (ed.), pp. 619-632. American Society of Agronomy, Madison, Wisconsin.

Conaway JG, RJ Luxmoore, JM Matusek, and RO Patt. 1997. Tank waste remediation system vadose zone contamination issue: Independent expert panel status report. DOE/RL-97-49, Rev. 0, U.S. Department of Energy, Richland, Washington.

Connelly MP, BH Ford, and JU Borghese. 1992. Hydrogeologic model for the 200 West groundwater area. WHC-SD-EN-T-014 (Rev. 0), Westinghouse Hanford Company, Richland Washington.

Deka RN, M Wairiu, PW Mtakwa, CE Mullins, EM Veenendaal, and J Townend. 1995. "Use and accuracy of the filter-paper technique for measurement of soil matric potential." European Journal of Soil Sci. 16:233-238.

DOE. 1988. Consultation draft site characterization plan. DOE/RW-0164, 9 volumes. U.S. Department of Energy, Richland, Washington.

DOE. 1996. Vadose zone characterization project at the Hanford tank farms, SX Tank Farm report. Final Draft, July 1996, U.S. Department of Energy, Richland, Washington.

DOE/RL. 1999. Phase I RCRA facility investigation/corrective measures study work plan for the SST Waste Management Areas. DOE/RL-99-36, Rev. 0, U.S. Department of Energy, Richland Operations Office, Richland, Washington. 
Drever JI. 1973. "The preparation of oriented clay mineral specimens for X-ray diffraction analysis by a filter-membrane peel technique." Amer. Minerl. 58:553-554.

EPA. Method 6010B. 1996. "Inductively Coupled Plasma-Atomic Emission Spectrometry." Test methods for evaluating solid waste, physical/chemical methods. EPA publication SW-846, available online http://www.epa.gov/epaoswer/hazwaste/test/sw846.htm

EPA. Method 9056. 1994. Determination of Inorganic Anions by Ion Chromatography, Washington, D.C.

Fayer MJ, GW Gee, ML Rockhold, MD Freshley, and TB Walters. 1996. "Estimating recharge rates for a groundwater model using a GIS.” J. Environ. Qual. 25:510-518.

Felmy AR, JR Rustad, MJ Mason, and R De la Bretonne. 1997. A chemical model for the major electrolyte components of the Hanford waste tanks: The binary electrolytes in the system: $\mathrm{Na}-\mathrm{NO}_{3}-\mathrm{NO}_{2}-$ $\mathrm{SO}_{4}-\mathrm{CO}_{3}-\mathrm{F}-\mathrm{PO}_{4}-\mathrm{OH}-\mathrm{Al}(\mathrm{OH})_{4}-\mathrm{H}_{2} \mathrm{O}$. Letter Report to Westinghouse Hanford Company, Richland, Washington.

Gee GW. 1987. Recharge at the Hanford Site: Status report. PNL-6403, Pacific Northwest Laboratory, Richland, Washington.

Gee GW, MJ Fayer, ML Rockhold, and MD Campbell. 1992. "Variations in recharge at the Hanford Site." Northwest Sci. 60(4):237-250.

Gee GW, PJ Wierenga, BJ Andraski, MH Young, MJ Fayer, and ML Rockhold. 1994. "Variations in water balance and recharge potential at three western desert sites." Soil Sci. Soc. Am. J. 58:63-72.

Horton DG and VG Johnson. 2000. Borehole data package for wells 299-W22-48, 299-W22-49, and 299-W22-50 at Single-Shell Tank Waste Management Area S-SX. PNNL-13200, Pacific Northwest National Laboratory, Richland, Washington.

ICF - ICF Kaiser Hanford Company. 1996. Historical vadose zone contamination of S and SX Tank Farms. Letter Report prepared for Westinghouse Hanford Company by ICF Kaiser Hanford Company, Richland, Washington.

Jackson ML. 1969. Soil chemical analysis - Advanced course $-2^{\text {nd }}$ edition. Department of Soil Science, University of Wisconsin, Madison, Wisconsin.

Johnson V and CJ Chou. 1998. Results of Phase I groundwater quality assessment for Single-Shell Tank Waste Management Areas S-SX at the Hanford Site. PNNL-11810, Pacific Northwest National Laboratory, Richland, Washington. 
Johnson V and CJ Chou. 1999. Addendum to the RCRA assessment report for Single-Shell Tank Waste Management Areas $S$-SX at the Hanford Site. PNNL-11810 ADD. 1, Pacific Northwest National Laboratory, Richland, Washington.

Johnson VG, TE Jones, SP Reidel, and MI Wood. 1999. Subsurface conditions description for the S-SX Waste Management Area. HNF-4936, Rev. 0, Lockheed Martin Hanford Corporation, Richland, Washington.

Kaplan DI, RJ Serne, and MG Piepho. 1995. Geochemical factors affecting radionuclide transport through near and far field at a low-level waste disposal site. PNL-10379, Pacific Northwest Laboratory, Richland, Washington.

Kaplan DI, RJ Serne, AT Owen, JA Conca, TW Wietsma, and TL Gervais. 1996. Radionuclide Adsorption Distribution Coefficients Measured in Hanford Sediments for the Low Level Waste Performance Assessment Project. PNNL-11385, Pacific Northwest National Laboratory, Richland, Washington.

Kaplan DI, KE Parker, and IV Kutnyakov. 1998. Radionuclide Distribution Coefficients for Sediments Collected from Borehole 299-E17-21: Final Report for Subtask 1a. PNNL-11996. Pacific Northwest National Laboratory, Richland, Washington.

Kaplan DI and RJ Serne. 2000. Geochemical data package for the Hanford immobilized low-activity tank waste performance assessment (ILAW-PA). PNNL-13037, Rev.1, Pacific Northwest National Laboratory, Richland, Washington.

Khaleel R, TE Jones, AJ Knepp, FM Mann, DA Myers, PM Rogers, RJ Serne, and MI Wood. 2000. Modeling data package for S-SX field investigation report (FIR). RPP-6296, Rev. 0, CH2M HILL Hanford Group, Inc., Richland, Washington.

Klute A. 1986. "Water retention: Laboratory methods." In Methods of soil analysis, Part 1, A Klute (ed.), Agronomy Monograph 9, American Society Agronomy, Madison, Wisconsin.

Lindsey KA, SE Kos, and KD Reynolds. 2000. Vadose zone geology of Boreholes 299-W22-50 and 299W23-19 S-SX Waste Management Area Hanford Site, south-central Washington. RPP-6149, Rev. 0, Daniel B. Stephens \& Associates, Richland, Washington.

MACTEC-ERS. 1997. Assessment of log data for Borehole 41-09-39 and correlation with Borehole 41-09-04 in the SX Tank Farm. GJO-97-4-TAR, MACTEC-ERS, Grand Junction Office, Grand Junction, Colorado.

McKinley JP, RJ Serne, JM Zachara, CJ Zeissler, and RM Lindstrom. 2001. "The distribution and retention of ${ }^{137} \mathrm{Cs}$ in sediments beneath leaked waste tanks at the Hanford Site, Washington." Environ. Sci. and Technol. 35:3433-3441. 
Myers DA, DL Parker, G Gee, VG Johnson, GV Last, RJ Serne, and DJ Moak. 1998. Findings of the extension of Borehole 41-09-39, 241-SX Tank Farm. HNF-2855, Lockheed Martin Hanford Company, Richland, Washington.

Newman ACD (ed.). 1987. Chemistry of clays and clay minerals: Monograph No 6, Mineralogical Society, London.

PNL - Pacific Northwest Laboratory. 1990. Procedures for groundwater investigations. PNL-MA-567DO-1, Pacific Northwest Laboratory, Richland, Washington.

PNL - Pacific Northwest Laboratory. 1993. Technetium-99 analysis using EIChroM Sr-Spec and TEVASpec columns. PNL-ALO-475, Pacific Northwest Laboratory, Richland, Washington.

PNNL - Pacific Northwest National Laboratory. 1997. Gamma energy analysis operation and instrument verification using the Genie2000 ${ }^{\mathrm{TM}}$ support software. PNNL-RRL-01, Pacific Northwest National Laboratory, Richland, Washington.

PNNL - Pacific Northwest National Laboratory. 1998. Inductively coupled plasma mass spectrometric (ICP-MS) analysis. PNNL-AGG-415, Pacific Northwest National Laboratory, Richland, Washington.

Polemio M and JD Rhoades. 1977. "Determining cation exchange capacity: A new procedure for calcareous and gypsiferous soils." Soil Sci. Soc. Am. J. 41:524-528.

Price WH and KR Fecht. 1976a. Geology of the 241-SX Tank Farm. ARH-LD-134, Atlantic Richfield Hanford Company, Richland, Washington.

Relyea JF, RJ Serne and D Rai. 1980. Methods for determining radionuclide retardation factors: Status report. PNL-3349, Pacific Northwest Laboratory, Richland, Washington.

Reynolds DM and RC Reynolds Jr. 1989. X-ray diffraction and the identification and analysis of clay minerals. Oxford University Press, New York.

Rhoades JD. 1996. "Salinity: Electrical conductivity and total dissolved solids." In Methods of soil analysis Part 3. JM Bigham (ed.), pp. 417-435. American Society of Agronomy, Madison, Wisconsin.

Rockhold ML, CS Simmons, and MJ Fayer. 1997. "An analytical solution technique for onedimensional, steady vertical water flow in layered soils." Water Resour. Res. 33(4):897-902.

Routson RC and RJ Serne. 1972. Experimental support studies for the PERCOL and transport models. USAEC Report BNWL-1719, Pacific Northwest Laboratory, Richland, Washington.

Routson RC, GS Barney, and RM Smith. 1981a. "Hanford Site sorption studies for the control of radioactive wastes: A review." Nucl. Technol.54:100-106. 
Routson RC, GS Barney, RM Smith, CH Delegard, and L Jensen. 1981b. Fission product sorption parameters for Hanford 200-Area sediment types. RHO-35, Rockwell Hanford Operations, Richland, Washington.

Serne RJ, WJ Martin, VL LeGore, CW Lindenmeier, SB McLaurine, PFC Martin, and RO Lokken, 1989. Leach tests on grouts made with actual and trace metal-spiked synthetic phosphate/sulfate waste. PNL-7121, Pacific Northwest Laboratory, Richland, Washington.

Serne RJ, CW Lindenmeier, JL Conca, JA Campbell, VL LeGore, JE Amonette, KJ Cantrell, and MI Wood. 1993. Solid-waste leach characteristics and contaminant-sediment interactions. Vol. 1: Batch leach and adsorption tests and sediment characterization. PNL-8889, Pacific Northwest Laboratory, Richland, Washington.

Serne RJ and VL LeGore. 1996. ${ }^{90}$ Sr adsorption-desorption properties and sediment characterization at 100 N-Area. PNL-10899, Pacific Northwest Laboratory, Richland, Washington.

Serne RJ, JM Zachara, and DS Burke. 1998. Chemical information on tank supernatants, Cs adsorption from tank liquids onto Hanford sediments, and field observations of Cs migration from past tank leaks. PNNL-11495, Pacific Northwest National Laboratory, Richland, Washington.

Serne RJ, HT Schaef, BN Bjornstad, BA Williams, DC Lanigan, DG Horton, RE Clayton, VL LeGore, MJ O’Hara, CF Brown, KE Parker, IV Kutnyakov, JN Serne, AV Mitroshkov, GV Last, SC Smith, CW Lindenmeier, JM Zachara, and DB Burke. 2002a. Characterization of vadose zone sediment, Part 1: Uncontaminated RCRA borehole core samples and composite samples. PNNL-13757-1, Pacific Northwest National Laboratory, Richland, Washington.

Serne RJ, HT Schaef, BN Bjornstad, DC Lanigan, GW Gee, CW Lindenmeier, RE Clayton, VL LeGore, MJ O'Hara, CF Brown, RD Orr, GV Last, IV Kutnyakov, DS Burke, TC Wilson, and BA Williams. 2002b. Characterization of vadose zone sediment, Part 2: Borehole 299 W23-19 [SX-115] in the S-SX Waste Management Area. PNNL-13757-2, Pacific Northwest National Laboratory, Richland, Washington.

Serne RJ, GV Last, HT Schaef, DC Lanigan, CW Lindenmeier, CC Ainsworth, RE Clayton, VL LeGore, MJ O'Hara, CF Brown, RD Orr, IV Kutnyakov, TC Wilson, KB Wagnon, BA Williams, and DB Burke. 2002c. Characterization of vadose zone sediment, Part 4: Slant borehole SX-108 in the S-SX Waste Management Area. PNNL-13757-4, Pacific Northwest National Laboratory, Richland, Washington.

Slate JL. 1996. "Buried carbonate paleosols developed in Pliocene-Pleistocene deposits of the Pasco Basin, south-central Washington.” USA: Quaternary International 34-36:191-196.

Slate JL. 2000. Nature and variability of the Plio-Pleistocene unit in the 200 West Area of the Hanford Site. BHI-01203, Rev. 0, Bechtel Hanford, Inc., Richland, Washington. 
Sobcyzk SM. 2000. Subsurface interpretation of the SX Tank Farm, Hanford Site, Washington based on gamma-ray logging. Nez Perce Tribe ERWM Program, Lapwai, Idaho.

SSSA - Soil Science Society of America. 1997. Glossary of soil science terms. 134 pp., Soil Science Society of America, Madison, Wisconsin.

Ward AL, GW Gee, and MD White. 1997. A comprehensive analysis of contaminant transport in the vadose zone beneath Tank SX-109. PNNL-11463, Pacific Northwest National Laboratory, Richland, Washington.

Zachara JM, RJ Serne, and JP McKinley. 1999. "Mineral surface processes responsible for the decreased retardation (or enhanced mobilization of ${ }^{137} \mathrm{Cs}$ from HLW tank discharges." In Science to support DOE site cleanup: The Pacific Northwest National Laboratory Environmental Management Science Program awards. PNNL-12208, Pacific Northwest National Laboratory, Richland, Washington.

Zachara JM, SC Smith, C Lui, JP McKinley, RJ Serne, and PL Gassman. 2002. "Sorption of $\mathrm{Cs}^{+}$to micaceous subsurface sediments from the Hanford Site." Geochemica et Cosmochemica ActaI. 66:193211. (submitted). 


\section{Appendix A}

\section{Drilling Chronology}




\begin{tabular}{|c|c|c|c|c|c|c|}
\hline Date & $\begin{array}{l}\text { Casing } \\
\text { Depth } \\
(\mathrm{ft})\end{array}$ & $\begin{array}{c}\text { Depth to } \\
\text { Bottom (ft) }\end{array}$ & $\begin{array}{l}\text { Open hole } \\
\text { during } \\
\text { sampling } \\
\text { (ft)* }\end{array}$ & Drilling/Sampling Activity & Sample & Rad. Readings \\
\hline \multicolumn{7}{|c|}{ Inside 8 inch casing } \\
\hline 9/5/97 & 130.6 & 130.6 & & Start milling drive point. Using water. & & \\
\hline 9/6-7/97 & 130.6 & 130.6 & & No Activity & & \\
\hline 9/8/97 & 130.6 & 130.6 & & $\begin{array}{l}\text { Pumped out water. Trip out of hole. Looks like mud } \\
\text { (soil) on bit. Trip in to drill dry. }\end{array}$ & & \\
\hline 9/9-11/97 & 130.6 & 130.6 & & $\begin{array}{l}\text { Continued milling. Using water. } \sim 155 \text { galllons } \\
\text { pumped from well. No water used in last run }(\# 10) \text {, } \\
\text { however brown tint seen in water return. }\end{array}$ & & \\
\hline 9/12/97 & 130.6 & 130.6 & & $\begin{array}{l}\text { Magnet run in, and came up with } 20-30 \mathrm{~K} \text { counts. } \\
\text { Water pumped down to } 124.8^{\prime} \text {. Bit replaced - had a lot } \\
\text { of loose rad. contam. on the surface. }\end{array}$ & magnet & $20-30 \mathrm{~K}$ counts \\
\hline $9 / 13-15 / 97$ & 130.6 & 130.6 & & No Activity & & \\
\hline 9/16/97 & 130.6 & 130.6 & & $\begin{array}{l}\text { Water now at } 129.4 \text { '. An estimated water loss was } \\
\text { calculated at } 20 \text { gallons. }\end{array}$ & & \\
\hline 9/17-18/97 & 130.6 & 130.6 & & No Activitiy & & \\
\hline $9 / 19 / 97$ & 130.6 & 130.6 & & Drive point removed from hole. & & \\
\hline 9/20/97 & 130.6 & 130.6 & & $\begin{array}{l}\text { Set up Cable Tool Rig. Installed } 6 \text { inch casing to } \\
130 \mathrm{ft} \text {. }\end{array}$ & & \\
\hline \multicolumn{7}{|c|}{ Inside 6 inch casing } \\
\hline 9/21/97 & 130 & & & No Activity & & \\
\hline 9/22/97 & 130 & & & Install 4.5 inch casing to $130^{\prime}$. & & \\
\hline \multicolumn{7}{|c|}{ Inside 4.5 inch casing } \\
\hline 9/22/97 & 130 & 131 & & $<\mathbf{1} \mathrm{ft}$ of water in hole. & & \\
\hline & 130 & $131-133$ & 1 & $\begin{array}{l}\text { Drive } \mathrm{S} / \mathrm{S} \text { sampler from } 131-133 \text {. Collect samples. } \\
\text { Collect } \sim 20 \mathrm{ml} \text { water sample from glove box } \\
\text { (S7061-1E). }\end{array}$ & S7061-01 & $\begin{array}{l}\text { 50,000 dpm (on outside). } \\
25-12 \mathrm{mR} / \mathrm{hr} \text { in liners B \& C. }\end{array}$ \\
\hline
\end{tabular}




\begin{tabular}{|c|c|c|c|c|c|c|}
\hline Date & $\begin{array}{l}\text { Casing } \\
\text { Depth } \\
(\mathrm{ft})\end{array}$ & $\begin{array}{c}\text { Depth to } \\
\text { Bottom (ft) }\end{array}$ & $\begin{array}{l}\text { Open hole } \\
\text { during } \\
\text { sampling } \\
\text { (ft)* }\end{array}$ & Drilling/Sampling Activity & Sample & Rad. Readings \\
\hline & 133 & 133 & & Drive casing to 133? Clean out to133. & Clean Out & $30,000 \mathrm{dpm}$ \\
\hline & 133 & $133-135$ & 0 & Drive $\mathrm{S} / \mathrm{S}$ sampler from $133-135$. & S7061-02 & $\begin{array}{c}3 \mathrm{mR} / \mathrm{hr} .0 .5-2 \mathrm{mR} / \mathrm{hr} \text { in } \\
\text { liners A-D. }\end{array}$ \\
\hline \multirow[t]{7}{*}{ 9/23/97 } & 133 & 135 & & No water in hole. & & \\
\hline & 135 & 135 & & $\begin{array}{l}\text { Decon. drilling string \& tools. Drive casing to } 135 \text {. } \\
\text { Clean out to } 135 \text {. }\end{array}$ & Clean Out & $40,000 \mathrm{dpm}$ \\
\hline & 135 & $135-136.4$ & 0 & Drive $\mathrm{S} / \mathrm{S}$ sampler from $135-136.4$ & S7061-03 & $\begin{array}{c}250,000-150,000 \mathrm{dpm} \text { in liners } \\
\text { A and B. }\end{array}$ \\
\hline & 136.4 & 137 & & Drive casing to 136.4 . Clean out to 136.4 (137) & Clean Out & \\
\hline & 136.4 & $137-139$ & 0.6 & Drive S/S sampler from 137-139. & S7061-04 & All liners $<$ bkgd. \\
\hline & 139 & 140 & & Drive casing to 139 . Clean out to 140 . & Clean Out & \\
\hline & 140 & 140 & & Drive casing to 140 . Prepare for logging. & & \\
\hline \multirow[t]{2}{*}{$9 / 24 / 97$} & 140 & 139.9-141.9 & -0.1 & Drive S/S sampler from 139.9-141.9. & S7061-05 & All liners < bkgd. \\
\hline & 140 & 141 & & $\begin{array}{l}\text { Tried to drive casing, but stuck at } 140 \text {. Cleaned out to } \\
\text { 141. Continued efforts to drive casing. }\end{array}$ & Clean Out & $\begin{array}{c}\text { 300/400 dpm (most likely from } \\
\text { previously contaminated } \\
\text { casing) }\end{array}$ \\
\hline $9 / 25-10 / 6 / 97$ & & & & No Activity. & & \\
\hline \multirow[t]{3}{*}{$10 / 7 / 97$} & 140 & 141.63 & & $\begin{array}{l}\text { Clean out to } 141.7 \text { (141.9). Collected sample (S7061- } \\
\text { 5E). }\end{array}$ & $\begin{array}{l}\text { Clean Out. } \\
\text { S7061-05E }\end{array}$ & 200/300 dpm. \\
\hline & 140 & 141.63 & & Decon. tools (some rad. found). & Drilling tools. & Some rad. found. \\
\hline & 140 & $141.7-145.7$ & 1.7 & $\begin{array}{l}\text { Drive S/S sampler from } 141.7-145.7 \text {. Left sampler in } \\
\text { borehole, covered hole with plastic. }\end{array}$ & S7061-06 & \\
\hline 10/8/97 & & & & $\begin{array}{l}\text { Removed plastic and not recovered. Some rain } \\
\text { entered borehole. No Activity. }\end{array}$ & & \\
\hline 10/9/97 & 140 & 145.7 & & $\begin{array}{l}\text { Removed sampler from hole. Some water on } \mathrm{S} / \mathrm{S} \text { from } \\
\text { rain. Bottom of hole muddy. }\end{array}$ & & $\begin{array}{l}\text { 300/400 cpm. All liners } \\
\text { <bkgd. }\end{array}$ \\
\hline
\end{tabular}




\begin{tabular}{|c|c|c|c|c|c|c|}
\hline Date & $\begin{array}{c}\text { Casing } \\
\text { Depth } \\
\text { (ft) }\end{array}$ & $\begin{array}{c}\text { Depth to } \\
\text { Bottom }(\mathrm{ft})\end{array}$ & $\begin{array}{l}\text { Open hole } \\
\text { during } \\
\text { sampling } \\
(\mathrm{ft})^{*}\end{array}$ & Drilling/Sampling Activity & Sample & Rad. Readings \\
\hline & 140 & 145 & & Reamed hole from 142 to 145 . Cleaned out to 145 . & Clean out & \\
\hline & 140 & $145-148$ & 5 & $\begin{array}{l}\text { Drive S/S sampler from } 145-148 \text {. Sampler left in } \\
\text { borehole and borehole covered with plastic. }\end{array}$ & S7061-07 & \\
\hline \multirow{4}{*}{$10 / 10 / 97$} & 140 & 148 & & Removed sampler from borehole. & & All liners $<$ bkgd. \\
\hline & 140 & 147.6 & & $\begin{array}{l}\text { Attempted to drive casing. Depth to bottom measured } \\
\text { 146.5. Cleaned out to 147.6. No Rad. }\end{array}$ & Cleanout & \\
\hline & 140 & $147.6-152.6$ & 7.6 & Drive S/S sampler from 147.6-152.6. & S7061-08 & All liners $<$ bkgd. \\
\hline & 140 & 151.5 & & Check bottom at 151.5. Set up to backpull casing. & & \\
\hline $10 / 11-12 / 97$ & & & & No Activity & & \\
\hline $10 / 13 / 97$ & 140 & 151.5 & & Spectral Gamma Logging. & & \\
\hline \multirow[t]{3}{*}{$10 / 14 / 97$} & 140 & 151.5 & & $\begin{array}{l}\text { Installed } \sim 1 \mathrm{ft} \text { plug of bentonite ( } 1 \text { coffee can of } \\
\text { pellets). }\end{array}$ & & \\
\hline & 0 & & & $\begin{array}{l}\text { Pulled } 4.5 " \text { casing all the way out. Pipe was hot to } \\
\text { touch and last } 10 \text { feet heavily encrusted with soil and } \\
\text { sand; rad reading }=2 \mathrm{mR} \text {. Double bagged last } 20 \mathrm{ft} \\
\text { joint. }\end{array}$ & $\begin{array}{l}\text { bottom } 10 \mathrm{ft} \\
\text { of casing }\end{array}$ & $2 \mathrm{mR} / \mathrm{hr}$ \\
\hline & 139 & & & $\begin{array}{l}\text { Installed new shoe and } 20 \mathrm{ft} \text { section of pipe. Installed } \\
\text { casing to } 139 .\end{array}$ & & \\
\hline \multicolumn{7}{|c|}{ Inside new/reinstalled 4.5 inch casing } \\
\hline \multirow[t]{2}{*}{$10 / 15 / 97$} & 145 & 145 & & $\begin{array}{l}\text { Installed casing to } 145^{\prime} \text {. Began clean out slough and } \\
\text { driving casing. }\end{array}$ & $\begin{array}{l}\text { Clean out at } \\
148.5 \text {. }\end{array}$ & $25,000 \mathrm{dpm}$ \\
\hline & $\begin{array}{c}150 \\
(151)\end{array}$ & 150.8 & & Drove casing to $150^{\prime}\left(151^{\prime}\right)$. Cleaned out hole to 150.8 . & Clean out & \\
\hline $10 / 16 / 97$ & $\begin{array}{c}150 \\
(151)\end{array}$ & $150.8-152.8$ & $0.8(-0.2)$ & $\begin{array}{l}\text { Drove } \mathrm{S} / \mathrm{S} \text { sampler from } 150.8 \text { to } 152.8 \text { (151-153). } \\
\text { Note, this covers a previously sampled depth. }\end{array}$ & S7061-09 & $\begin{array}{l}\text { 15,000 dpm in liners C \& D, } \\
<\text { bkgd in A and B. }\end{array}$ \\
\hline
\end{tabular}




\begin{tabular}{|c|c|c|c|c|c|c|}
\hline Date & $\begin{array}{l}\text { Casing } \\
\text { Depth } \\
\text { (ft) }\end{array}$ & $\begin{array}{c}\text { Depth to } \\
\text { Bottom }(\mathrm{ft})\end{array}$ & $\begin{array}{l}\text { Open hole } \\
\text { during } \\
\text { sampling } \\
(\mathrm{ft})^{*}\end{array}$ & Drilling/Sampling Activity & Sample & Rad. Readings \\
\hline & & 152.8 & & Clean out to 152.8 (153). Tools read 5,000 dpm. & Drilling Tools & $5,000 \mathrm{dpm}$ \\
\hline & $151 ?$ & $152.8-154.8$ & 1.8 & Drove S/S sampler from 152.8 to 154.8 (153-155). & S7061-10 & $\begin{array}{c}\text { 4,000 dpm in D liner; all others } \\
<\text { bkgd. }\end{array}$ \\
\hline & & 155 & & Clean out to $155^{\prime}$. & Clean out & \\
\hline & $151 ?$ & $155-156.6$ & 4 & Drove S/S sampler form 155-156.6. & S7061-11 & $\begin{array}{c}\text { 3,000 dpm in D liner; all others } \\
<\text { bkgd }\end{array}$ \\
\hline & 151 & 157 & & Clean out to 157 . & & \\
\hline & 154 & & & Drive casing to 154 . & & \\
\hline $10 / 17-19 / 97$ & & & & No Activity & & \\
\hline \multirow[t]{8}{*}{$10 / 20 / 97$} & 154 & 155 & & Clean out to 155 & Clean out & $4,000 \mathrm{dpm}$ \\
\hline & 154 & 156 & & Clean out to 156. Moisture on cleanout. & Clean out & $50,000 \mathrm{dpm}$ \\
\hline & 156 & 156 & & Drive casing to 156 . & & \\
\hline & 156 & 156.6 & & Clean out to 157 . Bottom of hole at 156.6. & & \\
\hline & 156 & $156.6-157.3$ & 0.6 & Drove S/S sampler from 156.6-157.3. & S7061-12 & $<$ bkgd \\
\hline & 157.3 & 157.3 & & Drive casing and clean out to 157.3 & & \\
\hline & 157.3 & $157-159$ & -0.3 & Drove S/S sampler from 157-159. & S7061-13 & $<$ bkgd \\
\hline & 158.5 & 158.5 & & Drive casing and clean out to 158.5 . & & \\
\hline \multirow[t]{4}{*}{$10 / 21 / 97$} & 158.5 & 158.5 & & Logging hole (spectral gamma) & & \\
\hline & 158.5 & 159 & & Clean out to 159. & & \\
\hline & 158.5 & $159-161$ & & Drove S/S sampler from 159-161. & S7061-14 & \\
\hline & 160.8 & 160.8 & & $\begin{array}{l}\text { Drive casing and clean out to } 160.8 \text { (160.5). Unable to } \\
\text { advance further. }\end{array}$ & & \\
\hline \multirow[t]{2}{*}{$10 / 22 / 97$} & 160.8 & 161 & & $\begin{array}{l}\text { Used wedge to break cobble into powder. Cleaned out } \\
161 .\end{array}$ & & \\
\hline & 160.8 & 161 & 0.2 & Tried S/S sampler. No advancement. & S7061-15 & \\
\hline
\end{tabular}




\begin{tabular}{|c|c|c|c|c|c|c|}
\hline Date & $\begin{array}{l}\text { Casing } \\
\text { Depth } \\
(\mathrm{ft})\end{array}$ & $\begin{array}{c}\text { Depth to } \\
\text { Bottom }(\mathrm{ft})\end{array}$ & $\begin{array}{l}\text { Open hole } \\
\text { during } \\
\text { sampling } \\
(\mathrm{ft})^{*}\end{array}$ & Drilling/Sampling Activity & Sample & Rad. Readings \\
\hline & 160.8 & 161 & & $\begin{array}{l}\text { Used wedge to break up cobble. Attempted to clean } \\
\text { out with core barrel; recovered only } \sim 1 \text { cup of fine } \\
\text { gray powder. }\end{array}$ & & \\
\hline $10 / 23 / 97$ & & & & No Activity & & \\
\hline \multirow[t]{6}{*}{$10 / 24 / 97$} & 160.8 & 161 & & Added 2 liters of water. & & \\
\hline & & $161-162$ & & $\begin{array}{l}\text { Hardtool drilling. Collected } 2 \mathrm{~L} \text { jug of mud from } \\
\text { hard tool (S7061-15). Bottom of hole at } 162 .\end{array}$ & & $<$ bkgd \\
\hline & 160.8 & $162-164$ & 1.2 & Drove S/S sampler from 162-164. & S7061-16 & $<$ bkgd \\
\hline & 164 & 164 & & Drive casing and clean out to 164 . & & \\
\hline & 164 & $164-166$ & 0 & Drove S/S sampler from 164-166. & S7061-17 & $<$ bkgd \\
\hline & 165.5 & 165.5 & & Drive casing and clean out to 165.5 & & \\
\hline $10 / 25-26 / 1997$ & & & & No Activity & & \\
\hline \multirow[t]{3}{*}{$10 / 27 / 97$} & $\begin{array}{l}165.5 \\
(166)\end{array}$ & 166 & & $\begin{array}{l}\text { Clean out hole from } 165 \text { to } 166 \text {. Clean out slough was } \\
\text { muddy \& wet (condensation?) }\end{array}$ & & \\
\hline & $166 ?$ & $166-168$ & 0 & Drive S/S sampler 166-168. & S7061-18 & $<$ bkgd \\
\hline & 167 & 167 & & Drive casing to 167 . Begin clean out. & & \\
\hline \multirow[t]{5}{*}{$10 / 28 / 97$} & 168 & 168 & & Drive casing and clean out to 168 . & & \\
\hline & 168 & $168-169$ & 0 & $\begin{array}{l}\text { Drive S/S sampler 168-169. No Recovery; shoe } \\
\text { collapsed. }\end{array}$ & S7061-19 & \\
\hline & 168 & $168-169$ & & $\begin{array}{l}\text { Hardtool drilling. Added } 2 \text { liters of water (R/O-DI } \\
\text { Type II). }\end{array}$ & & \\
\hline & 169 & & & Drove casing to 169 . & & \\
\hline & 169 & 169.1 & 0 & $\begin{array}{l}\text { Hardtool drilling. Collected } 2 \mathrm{~L} \text { jug of mud from } \\
\text { hard tool (S7061-19). Tagged bottom at } 169.1 .\end{array}$ & S7061-19 & \\
\hline
\end{tabular}




\begin{tabular}{|c|c|c|c|c|c|c|}
\hline Date & $\begin{array}{l}\text { Casing } \\
\text { Depth } \\
(\mathrm{ft})\end{array}$ & $\begin{array}{l}\text { Depth to } \\
\text { Bottom (ft) }\end{array}$ & $\begin{array}{l}\text { Open hole } \\
\text { during } \\
\text { sampling } \\
(\mathrm{ft})^{*}\end{array}$ & Drilling/Sampling Activity & Sample & Rad. Readings \\
\hline & 169 & $169.1-170$ & 0 & $\begin{array}{l}\text { Drive S/S sampler 169.1-170. Driven } 10 \text { " but recoved } \\
24 \text { " of core. Core of large granite cobble lodged in } \\
\text { shoe. }\end{array}$ & S7061-20 & $<$ bkgd \\
\hline & 169 & $169-171$ & & $\begin{array}{l}\text { Hardtool drilling. Added } 2 \text { liters of water (R/O-DI } \\
\text { Type II). Bottom of hole at } 171 .\end{array}$ & & \\
\hline & 169 & 171 & & Spectral gamma logging. & & \\
\hline \multirow[t]{4}{*}{$10 / 29 / 97$} & 169 & 171 & 2 & $\begin{array}{l}\text { Drive casing to } 170 \text {. Collected sample from core } \\
\text { barrel (S7061-21) in to } 2 \text { L jug (169-171). }\end{array}$ & S7061-21 & \\
\hline & 171 & 171 & & Drive casing and clean out to 171 . & & \\
\hline & 171 & $171-173$ & 0 & Drive $\mathrm{S} / \mathrm{S}$ sampler from $171-173$ & S7061-22 & $<$ bkgd \\
\hline & 172 & 172 & & Drive casing and clean out to 172 . & & \\
\hline \multicolumn{7}{|l|}{$10 / 30 / 97$} \\
\hline \multirow[t]{6}{*}{$10 / 31 / 97$} & 172 & & & Cleaning out. Shoe broke off in hole. & & \\
\hline & 172 & & & Driving casing. & & \\
\hline & 172 & $172-173$ & & $\begin{array}{l}\text { Hardtool drilling. Added } 2 \text { liters of water (R/O-DI } \\
\text { Type II). }\end{array}$ & & \\
\hline & 173 & 173 & & Drove casing to 173 , and cleaned out to 173 . & & \\
\hline & 173 & 173 & 0 & Drove S/S samper. Refusal. S/S empty. & S7061-23 & \\
\hline & 173 & 173 & & $\begin{array}{l}\text { Hardtool drilling. Added } 3 \text { liters of water (R/O-DI } \\
\text { Type II). }\end{array}$ & & \\
\hline \multicolumn{7}{|l|}{$11 / 1-4 / 97$} \\
\hline $11 / 4 / 97$ & & & & $\begin{array}{l}\text { Blank water sample of the RO/DII Type II water; } \\
\text { given no. S7061-24 }\end{array}$ & $\begin{array}{l}\text { S7061-22 } \\
(\mathrm{S} 7061-24)\end{array}$ & \\
\hline $11 / 5 / 97$ & 173 & 173.9 & & $\begin{array}{l}\text { Hardtool drilling from 173.9-174.4. Added } 1 \text { liter } \\
\text { of DI water. }\end{array}$ & & $\begin{array}{l}\text { Posible contamination from } \\
\text { above. }\end{array}$ \\
\hline
\end{tabular}




\begin{tabular}{|c|c|c|c|c|c|c|}
\hline Date & $\begin{array}{l}\text { Casing } \\
\text { Depth } \\
(\mathrm{ft})\end{array}$ & $\begin{array}{c}\text { Depth to } \\
\text { Bottom (ft) }\end{array}$ & $\begin{array}{l}\text { Open hole } \\
\text { during } \\
\text { sampling } \\
(\mathrm{ft})^{*}\end{array}$ & Drilling/Sampling Activity & Sample & Rad. Readings \\
\hline & & & & $\begin{array}{l}\text { Using core barrel for clean out. Half of shoe is } \\
\text { missing. }\end{array}$ & & $<$ bkgd \\
\hline & $173 ?$ & $173.3-174.1$ & $0.3 ?$ & $\begin{array}{l}\text { Collected full core barrel. Some broken metal. } \\
\text { Collected sample in } 2 \text { L poly jug (S7061-23) } 173.3 \text { - } \\
174.1\end{array}$ & S7061-23 & $<$ bkgd \\
\hline & 173.8 & 174.1 & & Cleaned out to 174.1 Casing at 173.8 . & & \\
\hline \multirow[t]{7}{*}{$11 / 6 / 97$} & 173.8 & & & Using small core barrel to spear broken shoe. No luck. & & \\
\hline & & $174.7-174.8$ & & $\begin{array}{l}\text { Hardtool drilling. Added } 2 \text { liters of water (R/O-DI } \\
\text { Type II). 0.8' advance. }\end{array}$ & & \\
\hline & 174.5 & $174.1-174.6$ & & $\begin{array}{l}\text { Drove core barrel. Collected sample in in } 2 \text { liter poly } \\
\text { jug. (174.1-174.6) Muddy. Drove casing to } 174.5 \text {. }\end{array}$ & S706125 & \\
\hline & & $174.1-175$ & & $\begin{array}{l}\text { Drove core barrel to } 175 \text {. Clean out new material. } \\
\text { Discarded sample from } 174.1-174.6 \text {. Collected sample } \\
\text { from } 174.1-175 \text { in } 2 \text { L poly jug. }\end{array}$ & S7061-25 & \\
\hline & & $175-175.5$ & & Drove S/S sampler from 175-175.5. No recovery. & & \\
\hline & 175 & & & Drove casing and cleaned out. Broke shoe off in hole. & & \\
\hline & & & & $\begin{array}{l}\text { Made up Hardtools. } \\
\text { DI Type II). }\end{array}$ & & \\
\hline $11 / 7 / 97$ & 175 & $175.5-176$ & & $\begin{array}{l}\text { Hardtool drilling using star bit. Drilled approx. } 0.5 \\
\text { ft. }\end{array}$ & & \\
\hline \multirow[t]{3}{*}{$11 / 10 / 97$} & 175 & $175.5-176.5$ & & $\begin{array}{l}\text { Hardtool drilling. Added } 2 \text { liters of water (R/O-DI } \\
\text { Type II). Drilled } \sim 1 \text { ' to } 176.5^{\prime} \text {. }\end{array}$ & & \\
\hline & 176 & & & Drove casing to 176 . & & \\
\hline & & & & $\frac{\text { Hardtool drilling. Added } 2 \text { liters of water (R/O-DI }}{\text { Type II). }}$ & & \\
\hline
\end{tabular}




\begin{tabular}{|c|c|c|c|c|c|c|}
\hline Date & $\begin{array}{l}\text { Casing } \\
\text { Depth } \\
(\mathrm{ft})\end{array}$ & $\begin{array}{c}\text { Depth to } \\
\text { Bottom (ft) }\end{array}$ & $\begin{array}{l}\text { Open hole } \\
\text { during } \\
\text { sampling } \\
\text { (ft)* }\end{array}$ & Drilling/Sampling Activity & Sample & Rad. Readings \\
\hline & 176 & & & Drove casing to 176 . & & \\
\hline & & $175.6-176$ & & $\begin{array}{l}\text { Clean out with core barrel. Collected sample in } 2 \mathrm{~L} \\
\text { poly jug (S7061-26). Contained metal from broken } \\
\text { shoes. }\end{array}$ & S7061-26 & \\
\hline & & $176-176.5$ & & Hardtool drilling (dry). & & \\
\hline $11 / 11 / 97$ & 176 & $176.5-177.3$ & & $\begin{array}{l}\text { Hardtool drilling. Added } 2 \text { liters of water (R/O-DI } \\
\text { Type II). Advanced } 1 \text { foot to } 177.3^{\prime} \text {. }\end{array}$ & & \\
\hline & 177 & & & Drive casing to 177 . & & \\
\hline & & $176-177.3$ & & $\begin{array}{l}\text { Cleaned out to } 177.3^{\prime} \text {. Collected sample in } 2 \mathrm{~L} \text { poly } \\
\text { jug (S7061-27) }\end{array}$ & S7061-27 & \\
\hline & & $177.3-177.9$ & 0.3 & Drove S/S sampler from 177.3 - 177.9. & S7061-28 & \\
\hline & 177.2 & & & Drove casing to $177.2^{\prime}$. & & \\
\hline & & & & Hardtool drilling (dry). & & \\
\hline $11 / 12 / 97$ & & & & & & \\
\hline $11 / 13 / 97$ & 177 & 177.8 & & $\begin{array}{l}\text { Added } 2 \text { liters of DI water (RO/DI TYPE II). } \\
\text { Hardtool drilling to 178'. }\end{array}$ & & \\
\hline & 178 & & & Drove casing to $178^{\prime}$. & & \\
\hline & & 178 & & $\begin{array}{l}\text { Cleaned out to } 178 \text { '. Collected cleanout in } 2 \text { L Poly } \\
\text { Jug (177.9-178.6) }\end{array}$ & S7061-29 & \\
\hline & & $178.6-180$ & 0.6 & $\begin{array}{l}\text { Drove S/S sampler from } 178-180(178.6-180.6) \text {. } \\
\text { First attempt came up empty. Second attempt } \\
\text { successfull. }\end{array}$ & S7061-30 & \\
\hline & 180 & 180 & & Drove casing to 180 (179.5), and cleaned out to 180 . & & \\
\hline & & $180-181$ & 0 & Drove S/S sampler from 180-181. Shoe bent. & S7061-31 & \\
\hline & 180.5 & & & Drove casing to $180.5^{\prime}$. (Cleaned out) & & \\
\hline
\end{tabular}




\begin{tabular}{|c|c|c|c|c|c|c|}
\hline Date & $\begin{array}{c}\text { Casing } \\
\text { Depth } \\
(\mathrm{ft})\end{array}$ & $\begin{array}{c}\text { Depth to } \\
\text { Bottom (ft) }\end{array}$ & $\begin{array}{l}\text { Open hole } \\
\text { during } \\
\text { sampling } \\
(\mathrm{ft})^{*}\end{array}$ & Drilling/Sampling Activity & Sample & Rad. Readings \\
\hline \multirow[t]{4}{*}{$11 / 14 / 97$} & 180.5 & 180.5 & & $\begin{array}{l}\text { Standby for Spectral-Logging. Collected plastic wrap } \\
\text { for counting. }\end{array}$ & S7061-33 & \\
\hline & & 181 & & Clean out to $181^{\prime}$ & & \\
\hline & & $181-181.8$ & 0 & Drove S/S sampler from 181-181.6' (181.8'). & S7061-32 & \\
\hline & & 182 & & $\begin{array}{l}\text { Added } 2 \text { liters of DI water (RO/DI Type II). } \\
\text { Hardtool drilling to } 182^{\prime} \text {. }\end{array}$ & & \\
\hline \multicolumn{7}{|l|}{$11 / 15-26 / 97$} \\
\hline \multirow[t]{7}{*}{$11 / 17 / 97$} & 180.5 & 182 & & Continued to hardtool. & & \\
\hline & & & & $\begin{array}{l}\text { Clean out to } 182.8^{\prime} \text { Collected } 2 \text { L Poly Jug from core } \\
\text { barrel (181.8-182.8) }\end{array}$ & S7061-34 & \\
\hline & 182.2 & 182.7 & & $\begin{array}{l}\text { Removing and adding casing (iron slag?). Drove } \\
\text { casing to } 182.2 \text {. Cleaned out to 182.7'. }\end{array}$ & & \\
\hline & & $182.8-183.6$ & 0.6 & Drove $\mathrm{S} / \mathrm{S}$ from $182.7-183.2^{\prime}\left(182.8-183.6^{\prime}\right)$. & S7061-35 & \\
\hline & 183 & 183 & & Drove casing to $183^{\prime}$ (?). Cleaned out to $183^{\prime}$. & & \\
\hline & & 183.6-185.6 & 0.6 & Drove S/S from 183-185' (183.6-185.6'). & S7061-36 & \\
\hline & 184 & 184.5 & & Drove casing to 184 '. Cleaned out to $184.5^{\prime}$ (approx.) & & \\
\hline \multirow[t]{3}{*}{$11 / 18 / 97$} & 184 & 184.5 & & Clean out from 184-185.6' & & \\
\hline & 184.5 & 185.6 & & Drove casing to $184.5^{\prime}$. Taped hole at $185.6^{\prime}$. & & \\
\hline & & $185.6-187.3$ & 1.1 & Drove $\mathrm{S} / \mathrm{S}$ to $187.2^{\prime}$ (185.6-187.3'). & & \\
\hline \multicolumn{7}{|l|}{$11 / 19 / 97$} \\
\hline \multirow[t]{4}{*}{$11 / 20 / 97$} & 184.5 & 187.3-187.6 & NA & Finished driving S/S to187-187.6' (187.3-187.6). & S7061-37 & \\
\hline & 187.6 & 187.6 & & $\begin{array}{l}\text { Added casing. Drove casing and cleaned out to } 187.6 \\
(186.3) \text {. }\end{array}$ & & \\
\hline & & $187.6-189$ & 0 & Drove S/S from187.6-189'. & S7061-38 & \\
\hline & 188.5 & 188.5 & & Drove casing to 188.5 . Cleaned out to 188.5 & & \\
\hline
\end{tabular}




\begin{tabular}{|c|c|c|c|c|c|c|}
\hline Date & $\begin{array}{c}\text { Casing } \\
\text { Depth } \\
\text { (ft) }\end{array}$ & $\begin{array}{c}\text { Depth to } \\
\text { Bottom (ft) }\end{array}$ & $\begin{array}{l}\text { Open hole } \\
\text { during } \\
\text { sampling } \\
(\mathrm{ft})^{*}\end{array}$ & Drilling/Sampling Activity & Sample & Rad. Readings \\
\hline & 189 & 189 & & Clean out and drive casing to $189^{\prime}$ & & \\
\hline & & 189-189.9 & 0 & Drove S/S 189-189.9'. & S7061-39 & \\
\hline & & 190 & & $\begin{array}{l}\text { Added } 2 \text { liters of DI water (RO/DI Type II). } \\
\text { Hardtool drilling to } \sim 190^{\prime} \text {. }\end{array}$ & & \\
\hline $11 / 21 / 97$ & 189 & 190 & & Clean out to 189.9 . & & \\
\hline & 189.6 & 189.5 & & $\begin{array}{l}\text { Drove casing to } 189.6^{\prime}(189.2) \text {. Cleaned out to } 189.5 \\
(189.9) .\end{array}$ & & \\
\hline & & 189.9-190.4 & & Drove S/S to 190.2 (189.9-190.4'). & S7061-40 & \\
\hline & & & & $\begin{array}{l}\text { Drove cleanout barrel } 0.7 \text {. Broke barrel. Standyby for } \\
\text { Spectral Logging. }\end{array}$ & & \\
\hline $11 / 22-23 / 97$ & & & & & & \\
\hline $11 / 24 / 97$ & 189.6 & 190 & & $\begin{array}{l}\text { Pulled and drove casing several times. Added } 3 \text { liters } \\
\text { of DI water. Tried several attempts at fishing. }\end{array}$ & & \\
\hline $11 / 25 / 97$ & 189.6 & 190 & & Fished out broken splitspoon (core barrel). & & \\
\hline & 189.9 & 190 & & Drove casing to 189.9 & & \\
\hline & & 191 & & $\begin{array}{l}\text { Added } 2 \text { liters of DI water. Hardtool drilling to } \\
\sim 191^{\prime} \text {. }\end{array}$ & & \\
\hline $11 / 26-30 / 1997$ & & & & & & \\
\hline $12 / 1 / 97$ & 189.9 & 191 & & Cleaned out hardtool cuttings to $190.7^{\prime}$. & & \\
\hline & & & & $\begin{array}{l}\text { Brushed casing with wire brush (bagged up material } \\
\text { on top of brush). }\end{array}$ & S7061-41 & \\
\hline & & 190.7 & & Swabbed with Masolin. Bagged up for counting. & S7061-42 & \\
\hline & & $190.7-191.3$ & & $\begin{array}{l}\text { Drove sampler from 190.7-191.3'. (Sampler broke } \\
\text { off). }\end{array}$ & & \\
\hline
\end{tabular}




\begin{tabular}{|c|c|c|c|c|c|c|}
\hline Date & $\begin{array}{l}\text { Casing } \\
\text { Depth } \\
(\mathrm{ft})\end{array}$ & $\begin{array}{c}\text { Depth to } \\
\text { Bottom (ft) }\end{array}$ & $\begin{array}{l}\text { Open hole } \\
\text { during } \\
\text { sampling } \\
(\mathrm{ft})^{*}\end{array}$ & Drilling/Sampling Activity & Sample & Rad. Readings \\
\hline & & & & $\begin{array}{l}\text { Collected cleanout from retrieval tool in 2L Poly Jug } \\
(190.7-191.3)\end{array}$ & S7061-43 & \\
\hline & & & & Added 2 liters of DI water. & & \\
\hline & & & & Drove casing to 190.2 & & \\
\hline & & & & $\begin{array}{l}\text { Made several attempts to fish out sampler. Advance } \\
\text { hole from } 191.3 \text { to } 191.9^{\prime} \text { below splitspoon. }\end{array}$ & & \\
\hline $12 / 2 / 97$ & $\begin{array}{l}190.2- \\
190.3\end{array}$ & $192-192.5$ & & $\begin{array}{l}\text { Attempted to fish out splitspoon. Deepend hole } 0.5^{\prime} \text {. } \\
\text { Attempted to drive casing. }\end{array}$ & & \\
\hline $12 / 3 / 97$ & 190.3 & 192.5 & & $\begin{array}{l}\text { Made video inspection. Made several attempts to fish } \\
\text { out splitspoon. }\end{array}$ & & \\
\hline $12 / 4 / 97$ & 190.3 & 192.5 & & $\begin{array}{l}\text { Attempted to fish out splitspoon. Pulled casing back } \\
\sim 0.1 \mathrm{ft} \text { and drove back. Added } 2 \text { liters of DI Water. } \\
\text { Finally retrieved sampler with fishing grapple. }\end{array}$ & & \\
\hline & 191.6 & 191.6 & & $\begin{array}{l}\text { Removed and added casing (slag?). Drove casing to } \\
191.6 \text { ' (191.5) and cleaned out to same depth. } \\
\text { Collected cleanout, but due to depth inaccuracies will } \\
\text { not use sample. }\end{array}$ & & \\
\hline $12 / 5 / 97$ & 191.6 & 192.5 & & Cleaned out hole from $191.4-192$. & & \\
\hline & 191.8 & & & $\begin{array}{l}\text { Drove casing to } 192.5 \text { (191.8). Cleaned out to } 192.5 \text {. } \\
\text { Collected cleanouts from 191.4-192.5, placed in } 2 \text { L } \\
\text { Ploy Jug. }\end{array}$ & S7061-44 & \\
\hline & & $192.5-193$ & 0.7 & Attempted to drive S/S (192.5-193). & S7061-45 & \\
\hline & 192 & & & $\begin{array}{l}\text { Cleaning out. Corebarrel broke at shoe. Drive casing } \\
\text { to } 192 \text {. }\end{array}$ & & \\
\hline & & & & Added 1 liter of DI water. Began Hardtool drilling. & & \\
\hline
\end{tabular}




\begin{tabular}{|c|c|c|c|c|c|c|}
\hline Date & $\begin{array}{c}\text { Casing } \\
\text { Depth } \\
(\mathrm{ft})\end{array}$ & $\begin{array}{c}\text { Depth to } \\
\text { Bottom }(\mathrm{ft})\end{array}$ & $\begin{array}{l}\text { Open hole } \\
\text { during } \\
\text { sampling } \\
(\mathrm{ft})^{*}\end{array}$ & Drilling/Sampling Activity & Sample & Rad. Readings \\
\hline $12 / 6-7 / 97$ & & & & & & \\
\hline $12 / 8 / 97$ & & & & Hardtooling. & & \\
\hline & & & & Drive casing to 193. & & \\
\hline & 192.5 & 193-194 & & Added 4 liters of DI water. Hardtool drill to 194'. & & \\
\hline & 194 & 194 & & $\begin{array}{l}\text { Drive casing to } 194 \text { (193.8). Clean out to } 194 . \\
\text { Crumpled shoe on corebarrel. }\end{array}$ & & \\
\hline & 194.3 & & & $\begin{array}{l}\text { Added } 2 \text { liters of DI water. Hardtool drill and } \\
\text { drive casing to 194.3'. }\end{array}$ & & \\
\hline & 195.5 & & & $\begin{array}{l}\text { Added } 2 \text { liters of DI water. Hardtool drill and } \\
\text { drive casing to 195.6'. }\end{array}$ & & \\
\hline $12 / 9 / 97$ & 195.5 & $195.5-195.7$ & & $\begin{array}{l}\text { Clean out mud and cuttings to } 195.7 \text { '. Collected } \\
\text { cleanout in } 2 \text { L Poly Jug (194.3-195.7). }\end{array}$ & S7061-46 & \\
\hline & & $195.7-197.7$ & 0.2 & Drove S/S from 195.7-197.7. & S7061-47 & \\
\hline & 197 & 197 & & Add casing. Drove casing to 197 . Cleaned out to 197 . & & \\
\hline & & 197.8 & & Cleaned out to 197.8 (taped reading). & & \\
\hline & 197.4 & & & Drove casing to 197.4 . & & \\
\hline & & 198.5 & & $\begin{array}{l}\text { Added } 2 \text { liters of DI water. Hardtool drill to } \\
\sim 198.5^{\prime} \text {. }\end{array}$ & & \\
\hline $12 / 10 / 97$ & 198.5 & 198.5 & & $\begin{array}{l}\text { Drove casing to 198.5. Clean out to 198.6. Collectd } \\
\text { cuttings from 197.7-198.6 in } 2 \text { L Poly Jug. }\end{array}$ & S7061-48 & \\
\hline & & $198.6-199.3$ & 0.1 & Drove S/S 197.7 to 198.6 (198.6-199.3) & S7061-49 & \\
\hline & 198.6 & 198.6 & & Drove casing and cleaned out to $198.6^{\prime}$ & & \\
\hline & & 199.3-199.6 & 0.7 & Drove S/S 198.6-199.3 (199.3-199.6) & S7061-50 & \\
\hline
\end{tabular}




\begin{tabular}{|c|c|c|c|c|c|c|}
\hline Date & $\begin{array}{l}\text { Casing } \\
\text { Depth } \\
(\mathrm{ft})\end{array}$ & $\begin{array}{c}\text { Depth to } \\
\text { Bottom (ft) }\end{array}$ & $\begin{array}{l}\text { Open hole } \\
\text { during } \\
\text { sampling } \\
(\mathrm{ft})^{*}\end{array}$ & Drilling/Sampling Activity & Sample & Rad. Readings \\
\hline & & 200 & & $\begin{array}{l}\text { Added } 2 \text { liters of DI water. Hardtool drill from } \\
199.3-200^{\circ} \text {. }\end{array}$ & & \\
\hline & 199.8 & 200 & & $\begin{array}{l}\text { Drove casing to } 199.8 \text { (199.7). Cleaned out to } 200 . \\
\text { Collected cleanout from 199.6-200 in } 2 \text { L Poly Jug } \\
\text { Prepared for logging. }\end{array}$ & S7061-51 & \\
\hline $12 / 11 / 97$ & 199.8 & 200 & & Spectral logging. & & \\
\hline & & $200-200.9$ & 0.2 & Drove S/S from 200 ' to $200.9^{\prime}$ & S7061-52 & \\
\hline & 200.1 & 201 & & Drove casing to 200.1. Cleaned out to 201 (200.8). & & \\
\hline & & 201 & & Added 2 liters of DI water. Hardtool drill to 201'. & & \\
\hline & 201 & & & Remove and add casing (slag?). Drove casing to 201. & & \\
\hline & & 201.8 & & $\begin{array}{l}\text { Added } 2 \text { liters of DI water. Hardtool drill 201'- } \\
\text { 201.8. }\end{array}$ & & \\
\hline & & & & Drive casing to 201.4 . & & \\
\hline & & & & Continue hardtooling. & & \\
\hline & & & & $\begin{array}{l}\text { Clean out from 200.9-201.8. Collected cleanout in } 2 \mathrm{~L} \\
\text { Jug. }\end{array}$ & S7061-53 & \\
\hline & & & 0.8 & Drove $\mathrm{S} / \mathrm{S}$ from $201.8-202.8$ & S7061-54 & \\
\hline & & & & $\begin{array}{l}\text { Added } 2 \text { liters of DI water. Hardtool drill 202.8'- } \\
204 .\end{array}$ & & \\
\hline & 204 & 203.7 & & $\begin{array}{l}\text { Drove casing to } 204 \text { (203.8). Cleaned out to } \sim 203.7 \text {. } \\
\text { collected cleanout from 202.8-204 in } 2 \text { L jug. }\end{array}$ & S7061-55 & \\
\hline $12 / 12 / 97$ & 204 & 204 & & Clean out to 204. Added to previous sample. & & \\
\hline & & 204-206 & 0 & Drove $\mathrm{S} / \mathrm{S}$ from $204-206$ & S7061-56 & \\
\hline
\end{tabular}




\begin{tabular}{|c|c|c|c|c|c|c|}
\hline Date & $\begin{array}{l}\text { Casing } \\
\text { Depth } \\
(\mathrm{ft})\end{array}$ & $\begin{array}{c}\text { Depth to } \\
\text { Bottom (ft) }\end{array}$ & $\begin{array}{l}\text { Open hole } \\
\text { during } \\
\text { sampling } \\
\text { (ft)* }\end{array}$ & Drilling/Sampling Activity & Sample & Rad. Readings \\
\hline & 205 & 205 & & Drove casing to 205 . Cleaned out to 205 . & & \\
\hline & & 206 & & Cleaned out to 206. & & \\
\hline & 205.1 & & & Drove casing to 205.1. Attempt to clean out to 206 . & & \\
\hline & & 207 & & Added 2 liters of DI water. Hardtool drill to 207. & & \\
\hline & 207 & 207.3 & & $\begin{array}{l}\text { Drove casing to 207. Clean out to 205-207. Collected } \\
\text { clean out in } 2 \text { L Poly Jug. Cleaned out to 207.3. }\end{array}$ & S7061-57 & \\
\hline & & 207.3-209.3 & 0.3 & Drove S/S from 207.3 to $209.3^{\prime}$ & S7061-58 & \\
\hline & 208 & & & Drove casing from 207-208. & & \\
\hline & & & & Clean out $\sim 0.33^{\prime}$. Broke of splitspoon body. & & \\
\hline & 208 & 209 & & $\begin{array}{l}\text { Removed shoe and splitspoon body. Continued clean } \\
\text { out. }\end{array}$ & & \\
\hline $12 / 13-15 / 97$ & & & & & & \\
\hline $12 / 15 / 97$ & 208 & 208.5 & & $\begin{array}{l}\text { No water in hole. Cleaned out hole to } 209.3 \text { '. Cuttings } \\
\text { appeared damp. Raining. First cleanout appears } \\
\text { damp. Could be condensation in borehole. }\end{array}$ & & \\
\hline & & 209.3-211.2 & 1.3 & $\begin{array}{l}\text { Drove } \mathrm{S} / \mathrm{S} \text { from } 209.4(209.3) \text { to } 211.2^{\prime} \text {. Bottom } 2 \\
\text { liners appeared to be saturated silty sand. Water in } \\
\text { hole 210.9'. }\end{array}$ & S7061-59 & Hit Water Table \\
\hline & 210 & 210.9 & & $\begin{array}{l}\text { Drove casing to } 210^{\prime} \text {. Cleaned out to } 210.9^{\prime} \text {. Detected } \\
\text { rad. activity on core barrel. }\end{array}$ & & $\begin{array}{l}2500 \text { dpm on cleanout to } \\
210.9 \text {. }\end{array}$ \\
\hline & & & & $\begin{array}{l}\text { Specral gamma logging. Plastic sleeve collected as } \\
\text { sample. }\end{array}$ & S7061-60 & $\begin{array}{l}5000 \text { dpm Beta/gamma; } 700 \\
\text { dpm alpha. }\end{array}$ \\
\hline
\end{tabular}




\begin{tabular}{|c|c|c|c|c|c|c|}
\hline Date & $\begin{array}{l}\text { Casing } \\
\text { Depth } \\
\text { (ft) }\end{array}$ & $\begin{array}{l}\text { Depth to } \\
\text { Bottom }(\mathrm{ft})\end{array}$ & $\begin{array}{l}\text { Open hole } \\
\text { during } \\
\text { sampling } \\
\text { (ft)* }\end{array}$ & Drilling/Sampling Activity & Sample & Rad. Readings \\
\hline & 211 & 211.5 & & $\begin{array}{l}\text { No water in hole. Drove casing to } 211^{\prime} \text {. Cleaned out } \\
\text { to } 211.5 \text { '. Mud and silt showed close to } 1000 \text { counts. } \\
\text { Collected samples from mud showing counts of } 7000 \\
\text { dpm B/G at } 210-211^{\prime} \text { in } 2 \text { L Poly Jug. Still no water in } \\
\text { hole. }\end{array}$ & S7061-61 & 1000 counts \\
\hline & & $211.5-212.5$ & 0.5 & Drove S/S from 211.5 to $212.5^{\prime}$. No water. & S7061-62 & \\
\hline & 212 & 212 & & $\begin{array}{l}\text { Added casing and drove to } 211.8 \text { (212). Cleaned out } \\
\text { to } 212 \text { (but penetrated to } 212.5^{\prime} \text { ). Broke core barrel. } \\
\text { No water. }\end{array}$ & & \\
\hline $12 / 16 / 97$ & & & & & & \\
\hline $12 / 17 / 97$ & 212 & 212 & & Static water level at 211.35. Bottom at 112.14. & & \\
\hline & & & & $\begin{array}{l}\text { Collected } \sim 500 \mathrm{ml} \text { of water using } 1 / 2 \text { sample bottle } \\
\text { attached to E-tape and nylon rope. Transferred to } 2 \mathrm{~L} \\
\text { Poly Jug. }\end{array}$ & S7061-63 & No rad. \\
\hline & & 212.5 & & Cleaned out hole to $212.5^{\prime}$. & & \\
\hline & & $212.5-213.5$ & 0.5 & Drove $\mathrm{S} / \mathrm{S}$ from $212.5-213.5^{\prime}$. & S7061-64 & \\
\hline & 213.2 & 213.9 & & Drove casing to 213.2 and cleaned out to $213.9^{\prime}$. & & \\
\hline & & $213.9-214.5$ & 0.7 & $\begin{array}{l}\text { Drove } \mathrm{S} / \mathrm{S} \text { from 213.9-214.5. Static water level at } \\
\mathbf{2 1 3 . 5} \text { (slow recharge rate). }\end{array}$ & S7061-65 & \\
\hline & 214 & 214.6 & & $\begin{array}{l}\text { Static water level at } 212.9^{\prime} \text {. Drove casing to } 214.1 \\
\text { (214). Cleaned out to } 214.6 \text {. }\end{array}$ & & \\
\hline & & $214.6-216.6$ & 0.6 & $\begin{array}{l}\text { Drove } \mathrm{S} / \mathrm{S} \text { from } 214.6 \text { to } 216.6 \text {. Large cobble in shoe, } \\
\text { possibly blocked recovery. }\end{array}$ & S7061-66 & \\
\hline & 215.8 & & & $\begin{array}{l}\text { Drove casing to } 215.8 \text {. Collected } 2 \text { nd and 3rd } \\
\text { cleanouts }(214.6-216.6) \text { in } 2 \text { L Poly Jug. Drove } \\
\text { cleanout barrel to } 216.8 \text {, but no recovery. Broke } \\
\text { corebarrel on large cobbles. }\end{array}$ & S7061-67 & \\
\hline
\end{tabular}




\begin{tabular}{|c|c|c|c|c|c|c|}
\hline Date & $\begin{array}{l}\text { Casing } \\
\text { Depth } \\
(\mathrm{ft})\end{array}$ & $\begin{array}{c}\text { Depth to } \\
\text { Bottom (ft) }\end{array}$ & $\begin{array}{l}\text { Open hole } \\
\text { during } \\
\text { sampling } \\
(\mathrm{ft})^{*}\end{array}$ & Drilling/Sampling Activity & Sample & Rad. Readings \\
\hline & & 216.3 & & $\begin{array}{l}\text { Changed to Hardtool. Static at 211.18; bottom at } \\
216.3 \text {. }\end{array}$ & & \\
\hline & 216.5 & & & $\begin{array}{l}\text { Added casing and drove to } 216.5^{\prime} \text {. Hardtool from } \\
216.6 \text { to } 218.3\end{array}$ & & \\
\hline & 218.3 & 218.7 & & $\begin{array}{l}\text { Drove casing to } 218.3^{\prime} \text { (218). Cleaned out with barrel. } \\
\text { Collected cleanout from 216.6-218.7 in } 2 \text { L Poly Jug. } \\
\text { Attempted S/S sampling - refusal and no recovery. }\end{array}$ & S7061-68 & \\
\hline & & & & Hardtool drilled from $218.3-218.7$ & & \\
\hline & 218.7 & & & Drove casing to 218.7. & & \\
\hline $12 / 18 / 97$ & 218.7 & 218.8 & & Water level at 211.95. Bottom $218.8^{\prime}$. & & \\
\hline & & & & $\begin{array}{l}\text { Hardtool drilled from 218.7-220. Used bailer to } \\
\text { recover slurry. }\end{array}$ & & \\
\hline & 220 & 220 & & $\begin{array}{l}\text { Drove casing to } 219.8 \text { (220). Drove core barrel to } 220 \text {. } \\
\text { Collected cleanout from } 218.6 \text { to } 220 \text { in } 2 \text { L Ploy Jug. } \\
\text { Bailed out mudy water. }\end{array}$ & S7061-69 & \\
\hline & & & 0 & Drove S/S from 220-220.8. & S7061-70 & \\
\hline & & & & Water level at 211.5. & & \\
\hline & & 222 & & $\begin{array}{l}\text { Hardtool drilled to } 222 \text {. Using bailer to recover } \\
\text { slurry/muddy water. }\end{array}$ & & \\
\hline & 222 & & & $\begin{array}{l}\text { Drove casing to 222. Bailed well - no water (only two } \\
\text { liters of heavy mud). }\end{array}$ & & \\
\hline & & $222-222.7$ & & $\begin{array}{l}\text { Switched to corebarrel. Collected cleanout in } 2 \mathrm{~L} \\
\text { Poly Jug. }\end{array}$ & S7061-71 & \\
\hline & & $222.7-223.3$ & & Collected clean out in 2 L Poly Jug. & S7061-72 & \\
\hline & 223.1 & $223.3-223.7$ & & $\begin{array}{l}\text { Drove casing to 223.1'. Collected clean out in } 2 \mathrm{~L} \\
\text { Poly Jug. }\end{array}$ & S7061-73 & \\
\hline
\end{tabular}




\begin{tabular}{|c|c|c|c|c|c|c|}
\hline Date & $\begin{array}{c}\text { Casing } \\
\text { Depth } \\
\text { (ft) }\end{array}$ & $\begin{array}{c}\text { Depth to } \\
\text { Bottom }(\mathrm{ft})\end{array}$ & $\begin{array}{l}\text { Open hole } \\
\text { during } \\
\text { sampling } \\
(\mathrm{ft})^{*} \\
\end{array}$ & Drilling/Sampling Activity & Sample & Rad. Readings \\
\hline & 223.4 & $223.7-224.0$ & & $\begin{array}{l}\text { Drove casing to } 223.4 \text {. Collected clean out in } 2 \mathrm{~L} \\
\text { Poly Jug. }\end{array}$ & S7061-74 & \\
\hline & 224 & 224.5 & & Drove casing to 224 . Cleaned out to 224.5 . & & \\
\hline & & 224.5 & & Prepared for logging. Static 212.9; Bottom 224.5. & & \\
\hline \multirow[t]{4}{*}{$12 / 19 / 97$} & 224 & 223.5 & & $\begin{array}{l}\text { Water level at 211.55. Bottom of hole at 223.5' ( } 1 \mathrm{ft} \\
\text { of sediment hole). }\end{array}$ & & \\
\hline & & $224-225$ & & $\begin{array}{l}\text { Cleaned out. Collected cleanout in } 2 \text { L Poly Jug. } \\
\text { Drove corebarrel to } 224.5^{\prime} \text {. }\end{array}$ & S7061-75 & \\
\hline & & $\begin{array}{l}224.65- \\
225.2\end{array}$ & 0.5 & Drove S/S from 224.5-225.3' (224.65-225.2). & S7061-76 & \\
\hline & & & & $\begin{array}{l}\text { Drove casing to } 225 \text {. Drove corebarrel to cleanout. } \\
\text { Unable to recover clean out. Bottom of hole } 225.3^{\prime} \text {. }\end{array}$ & & \\
\hline \multicolumn{7}{|l|}{$12 / 20-21 / 97$} \\
\hline $12 / 22 / 97$ & 225 & 225.3 & & Standby for spectral logging & & \\
\hline $12 / 23 / 97$ & & & & & & \\
\hline * Open ho & calcul & by subtra & the depth & o bottom (prior to sampling) from the casing depth. & & \\
\hline
\end{tabular}


Appendix B

Core Descriptions 


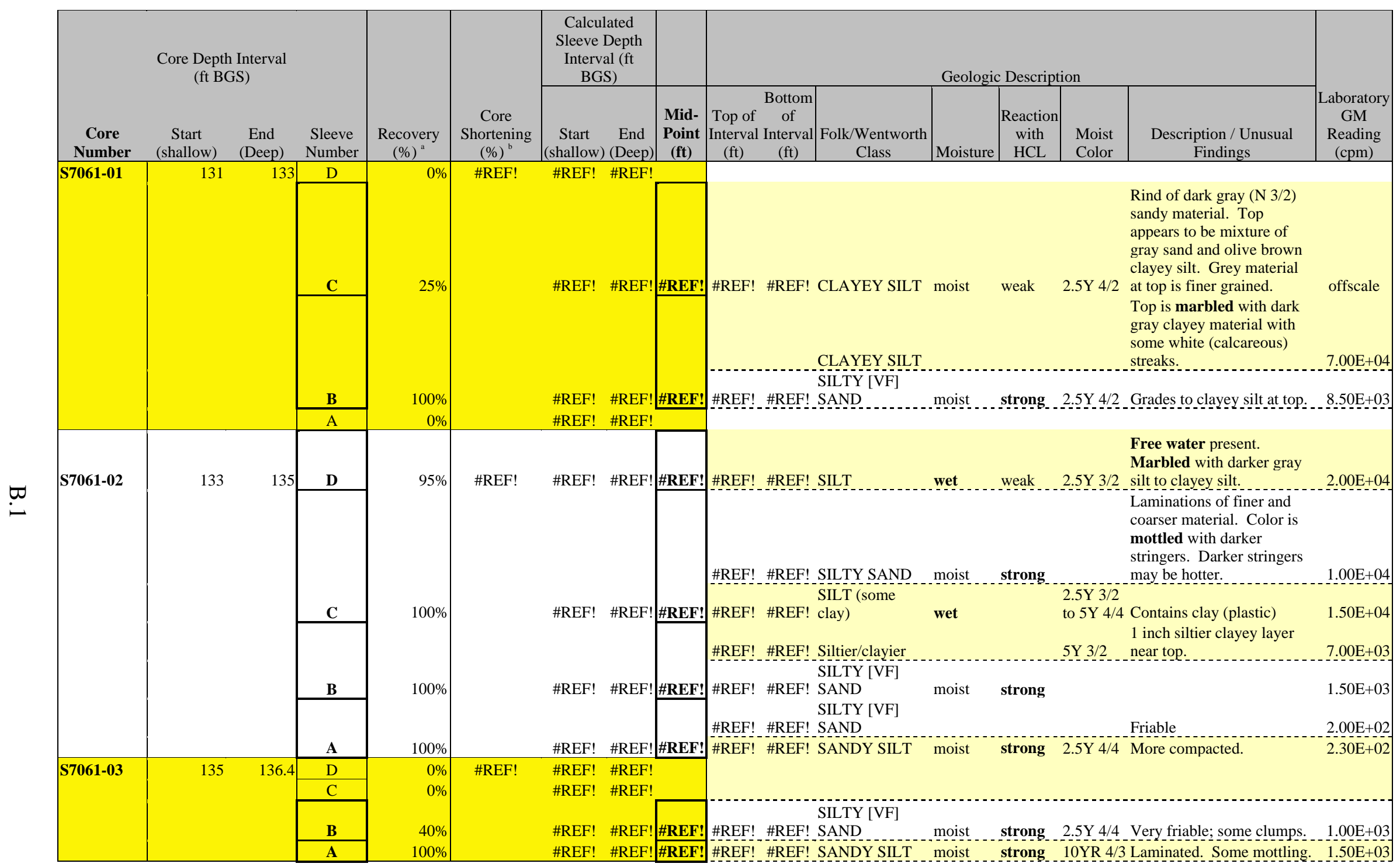




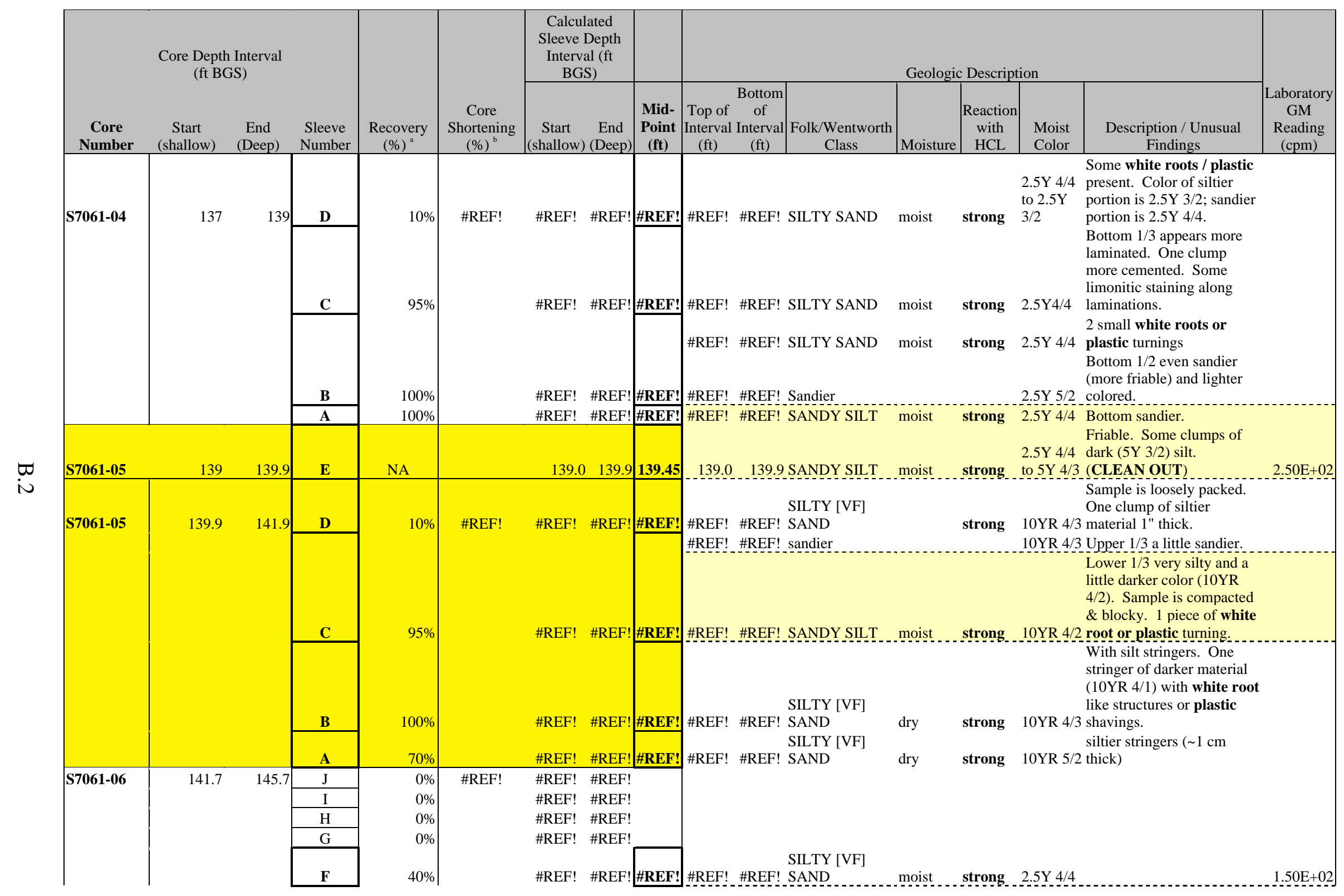




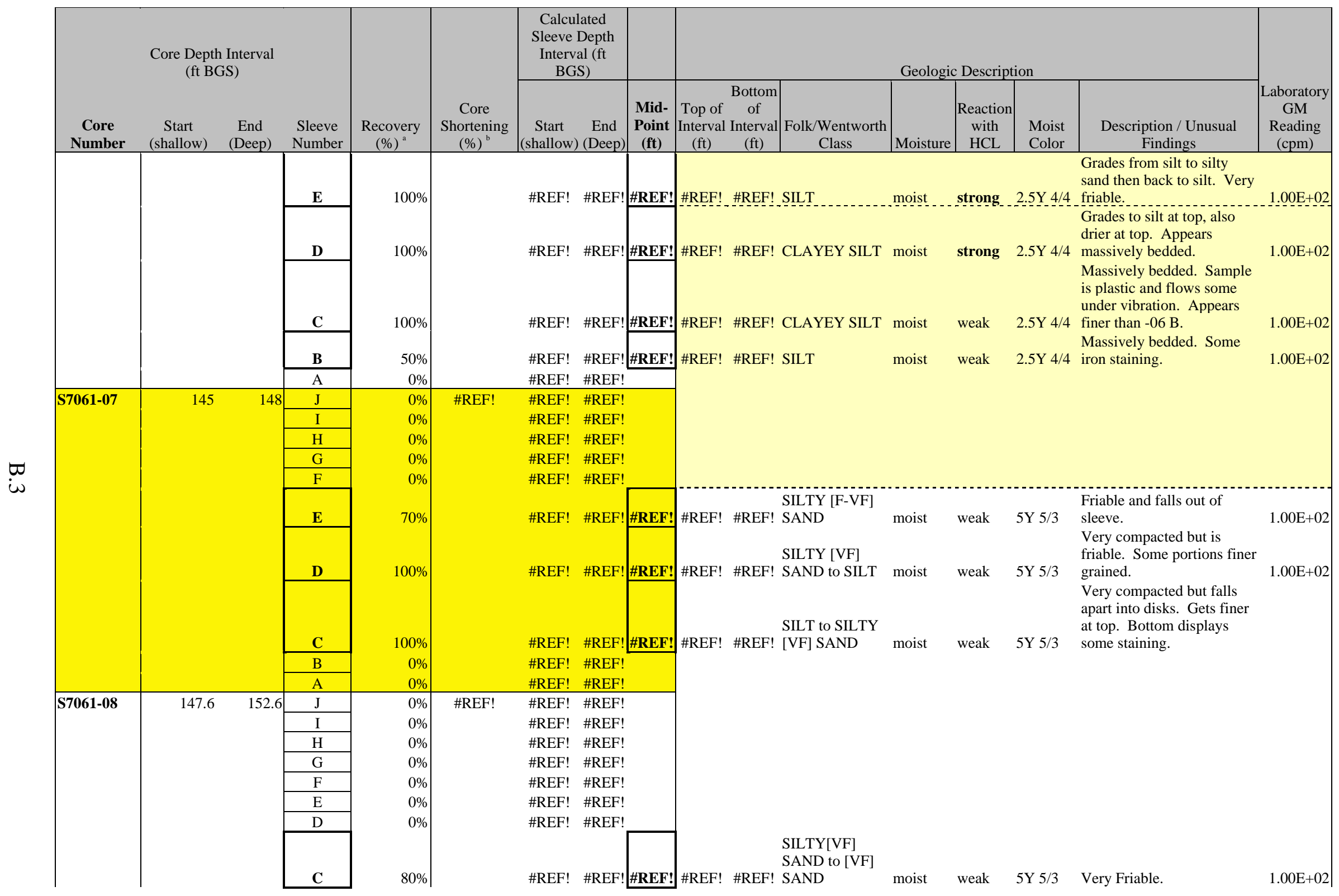




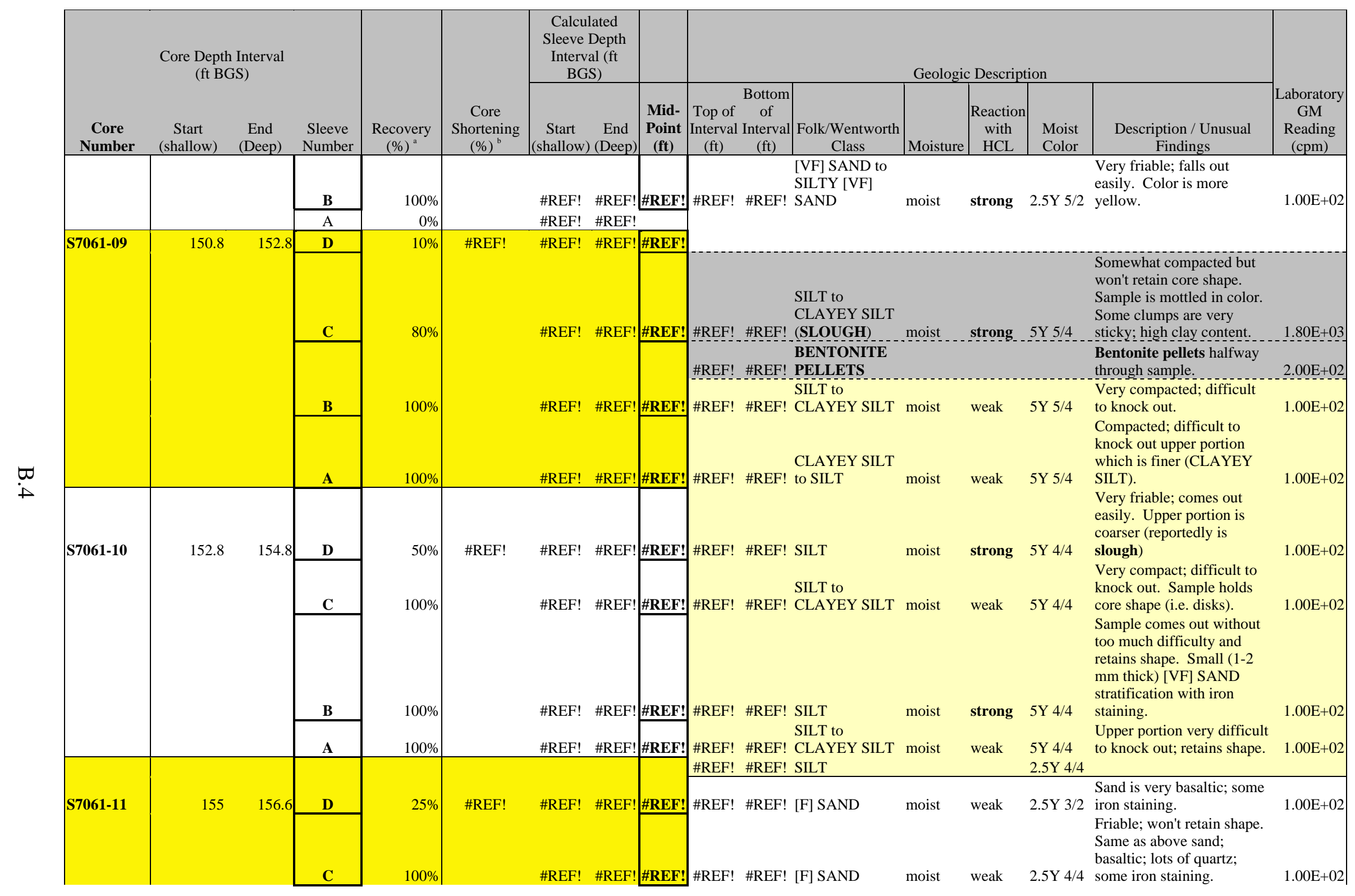




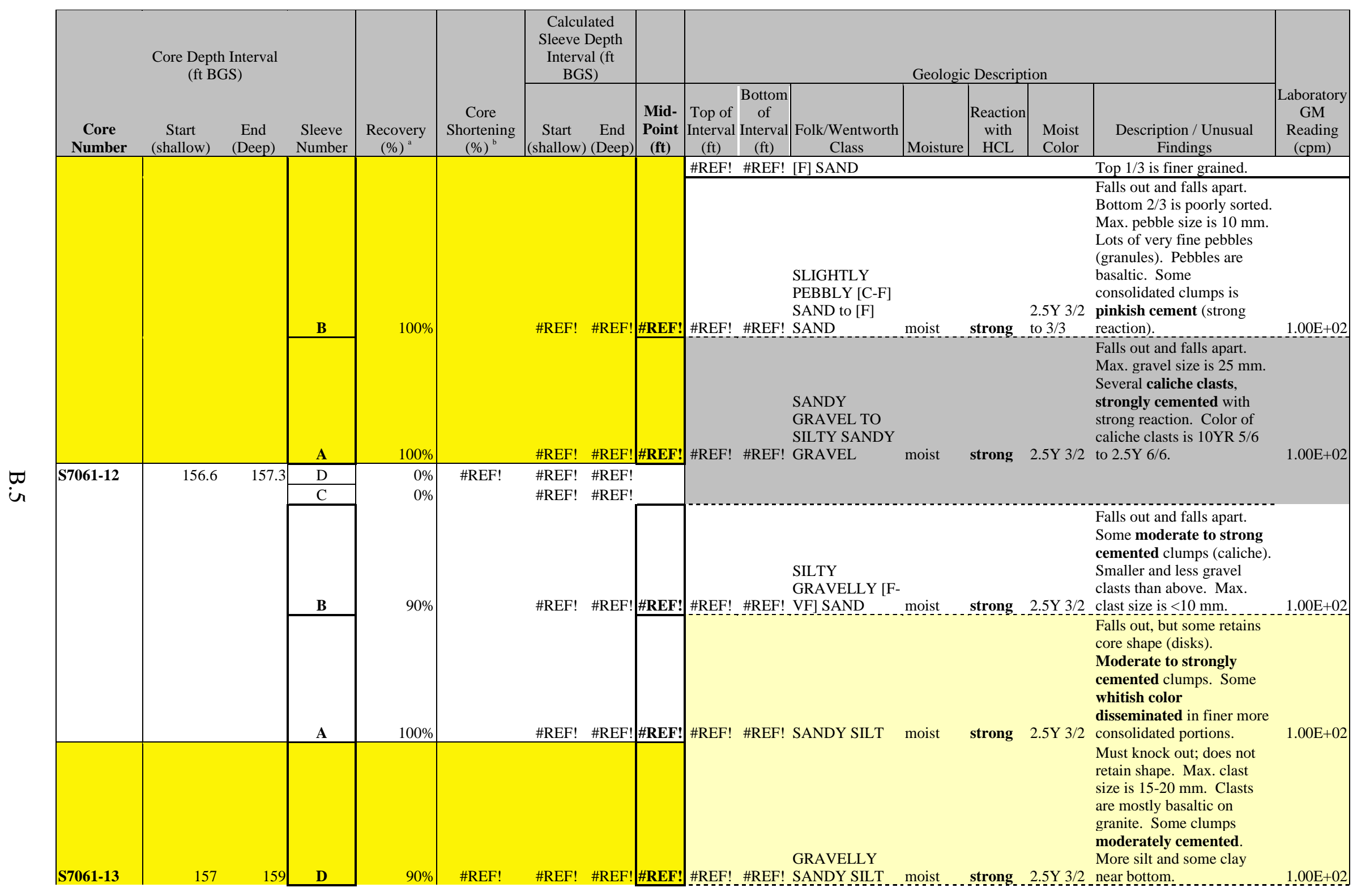




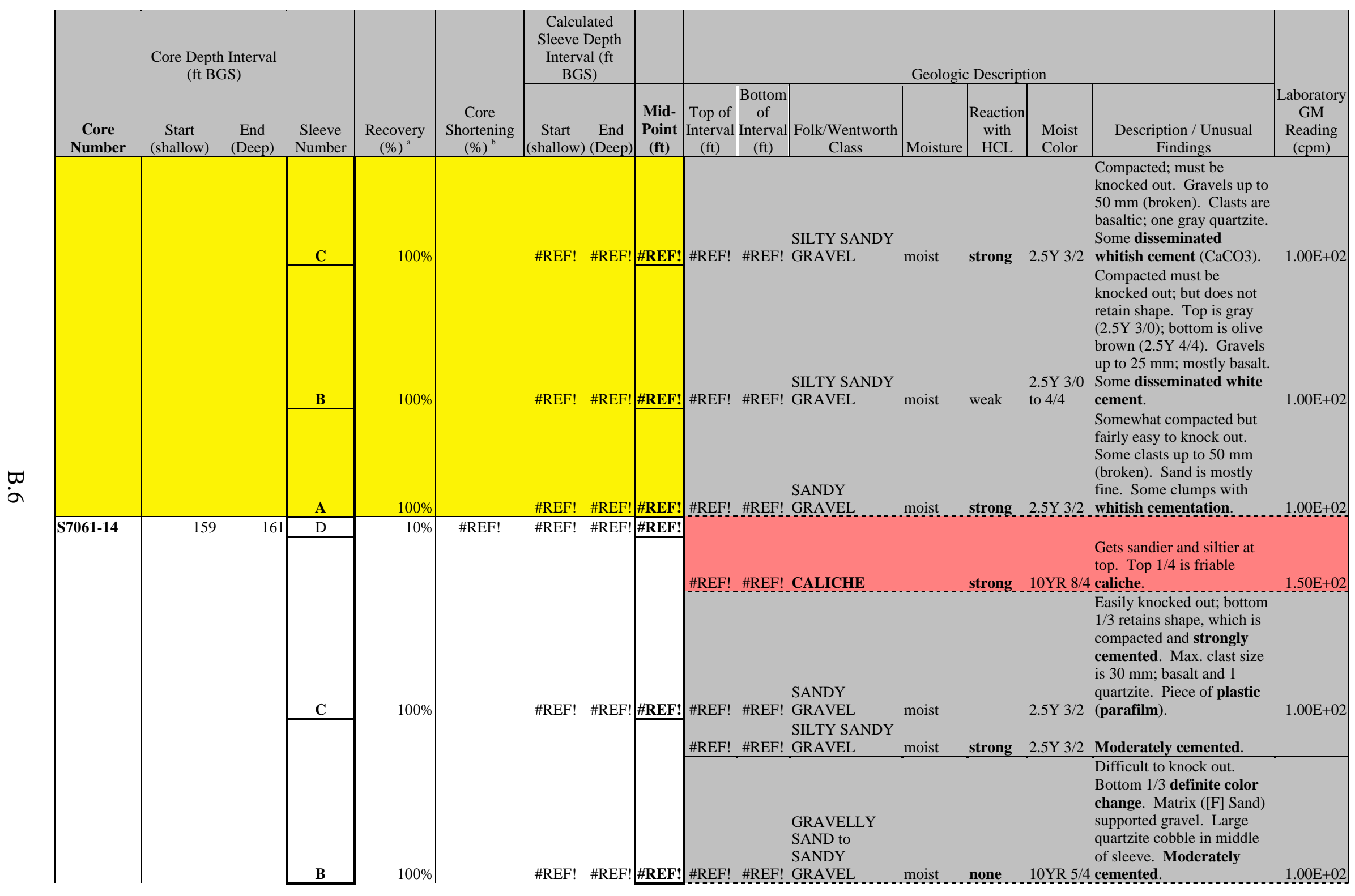




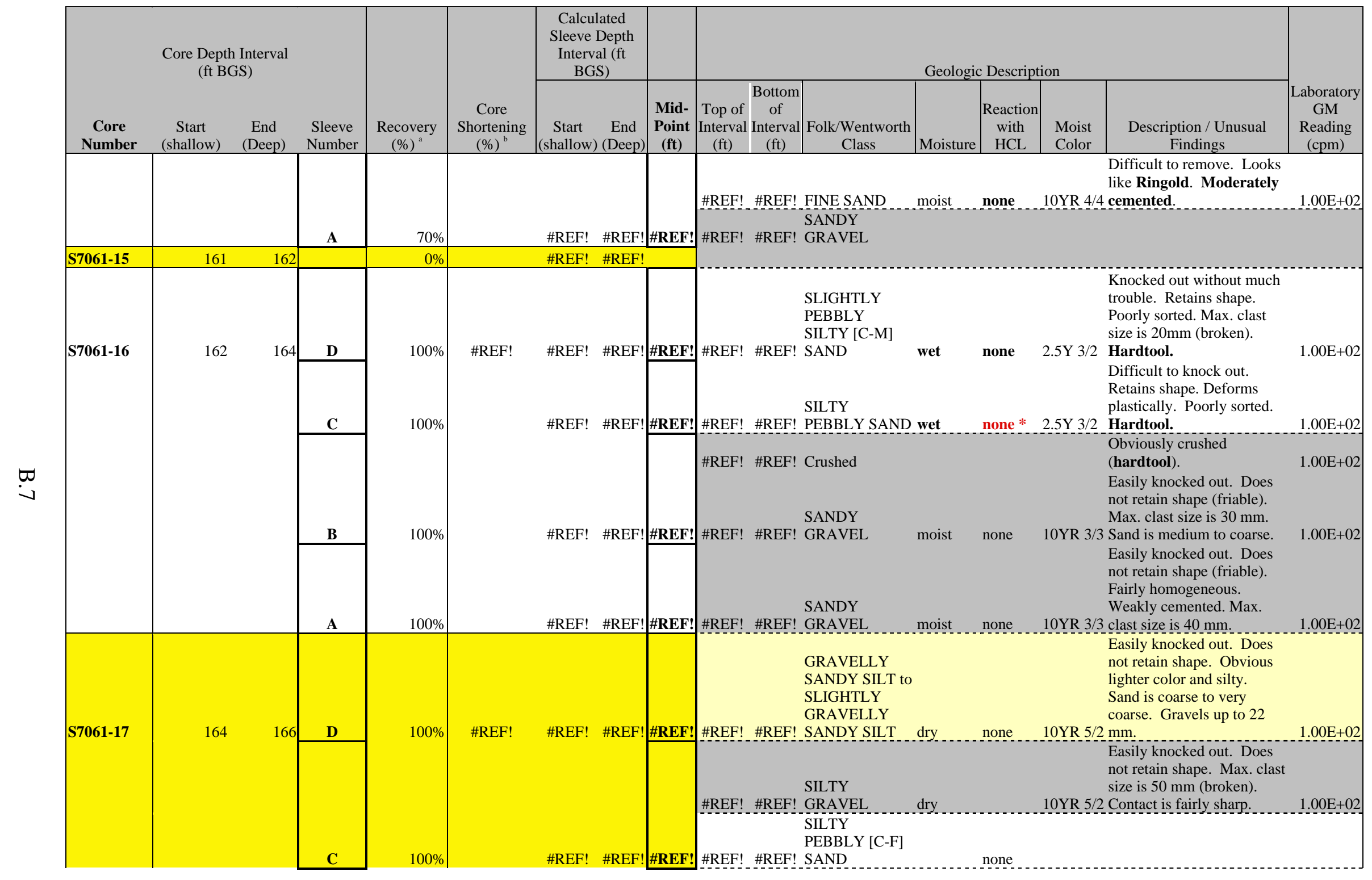




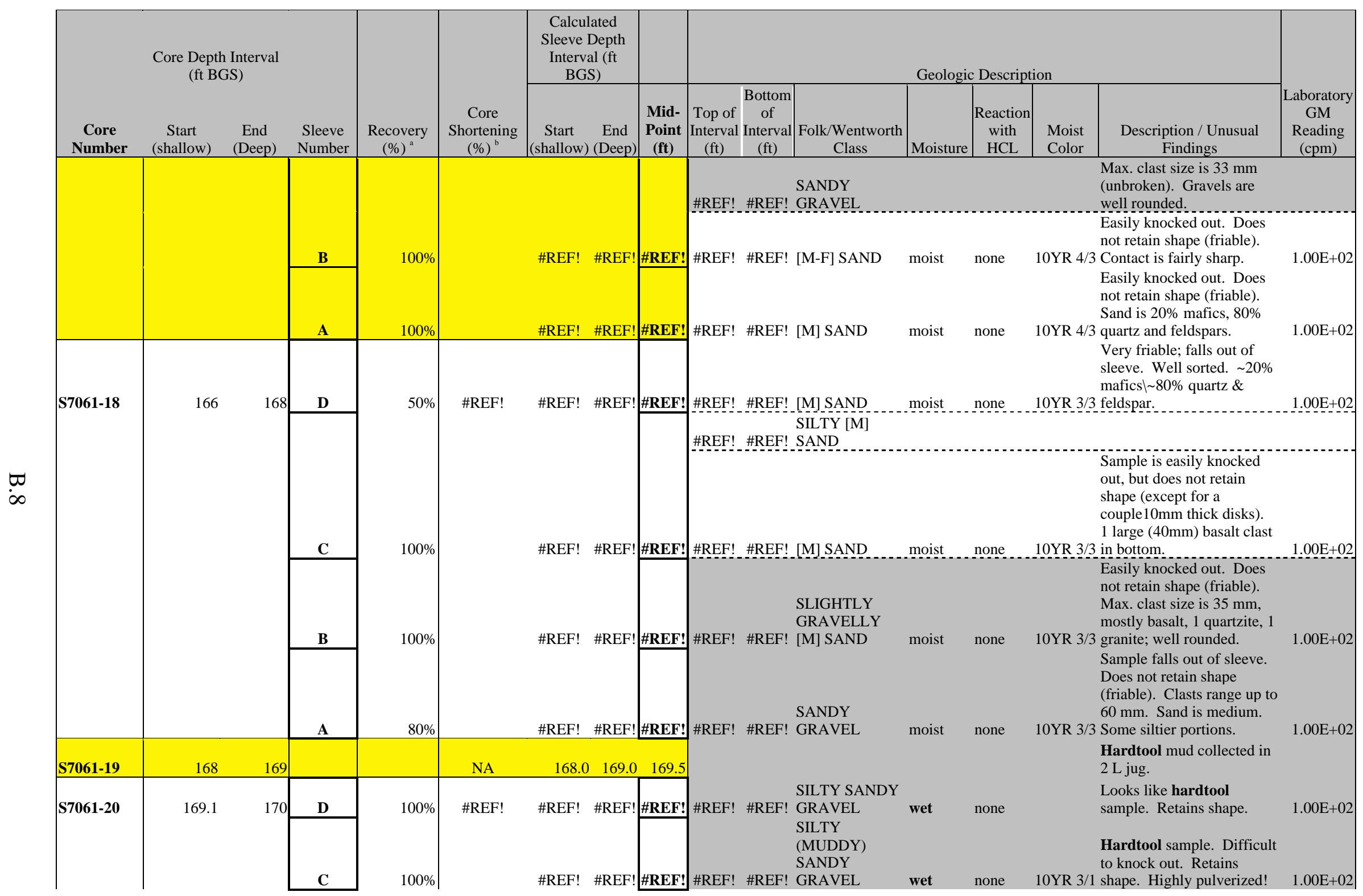




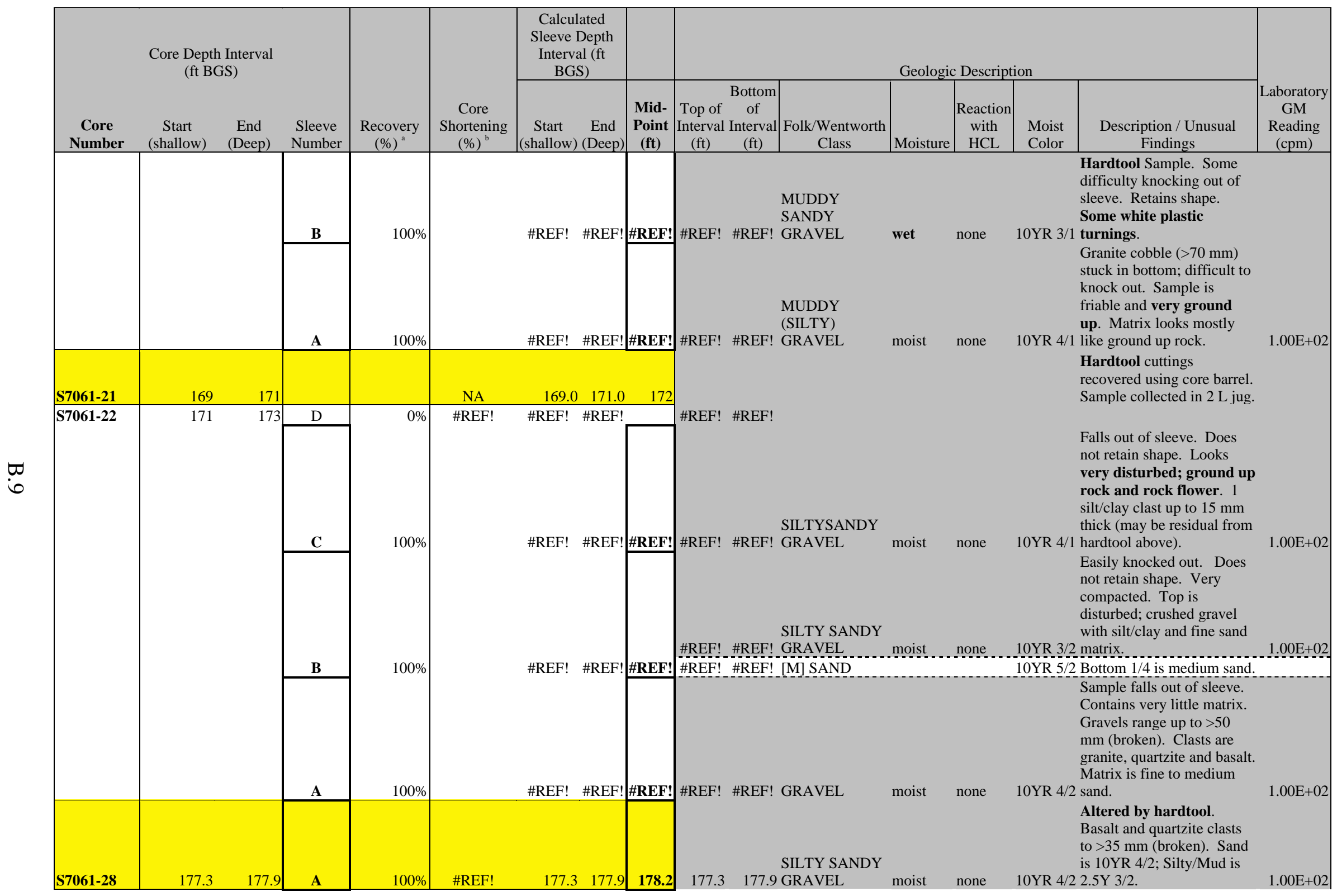




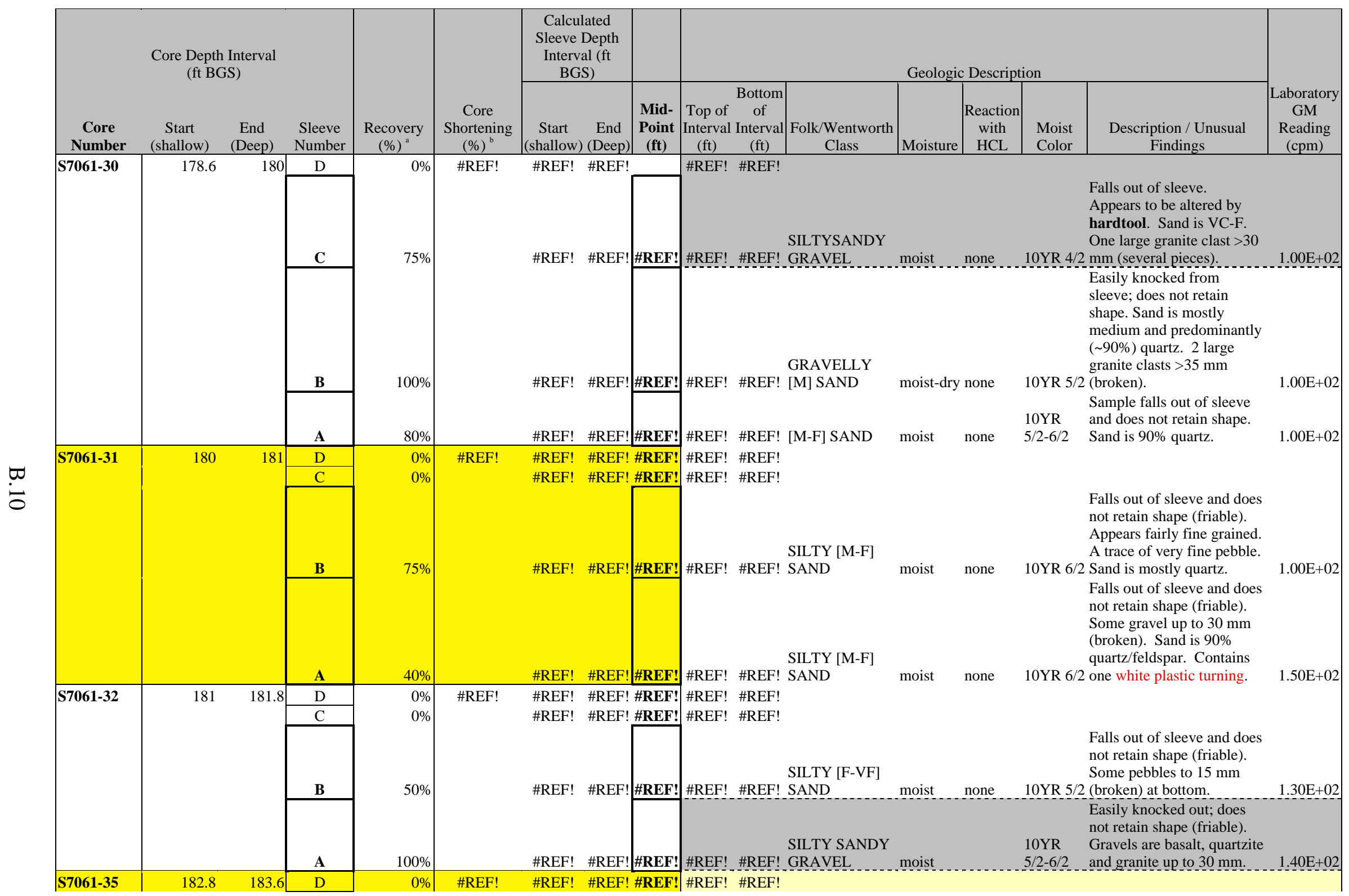




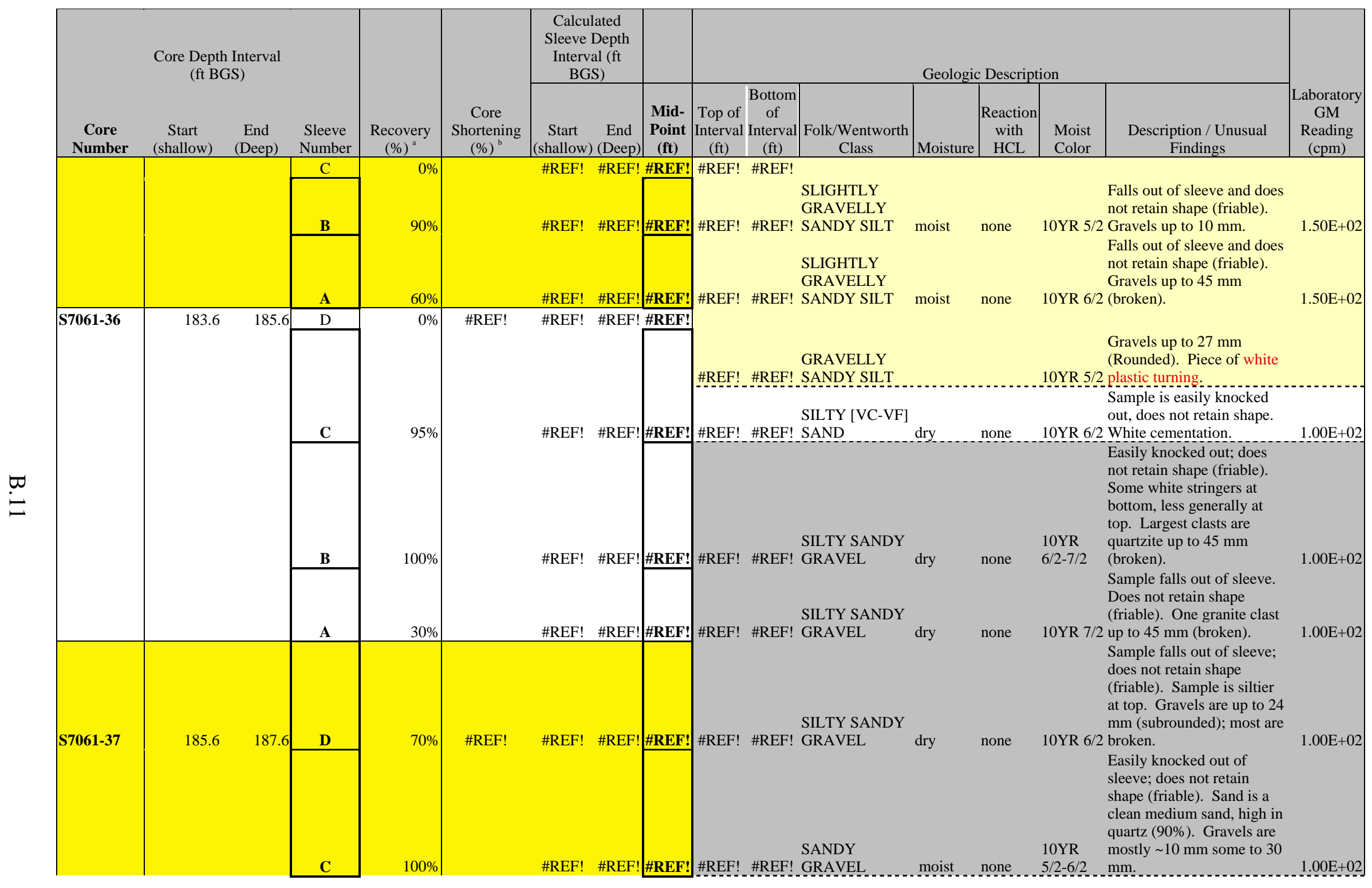




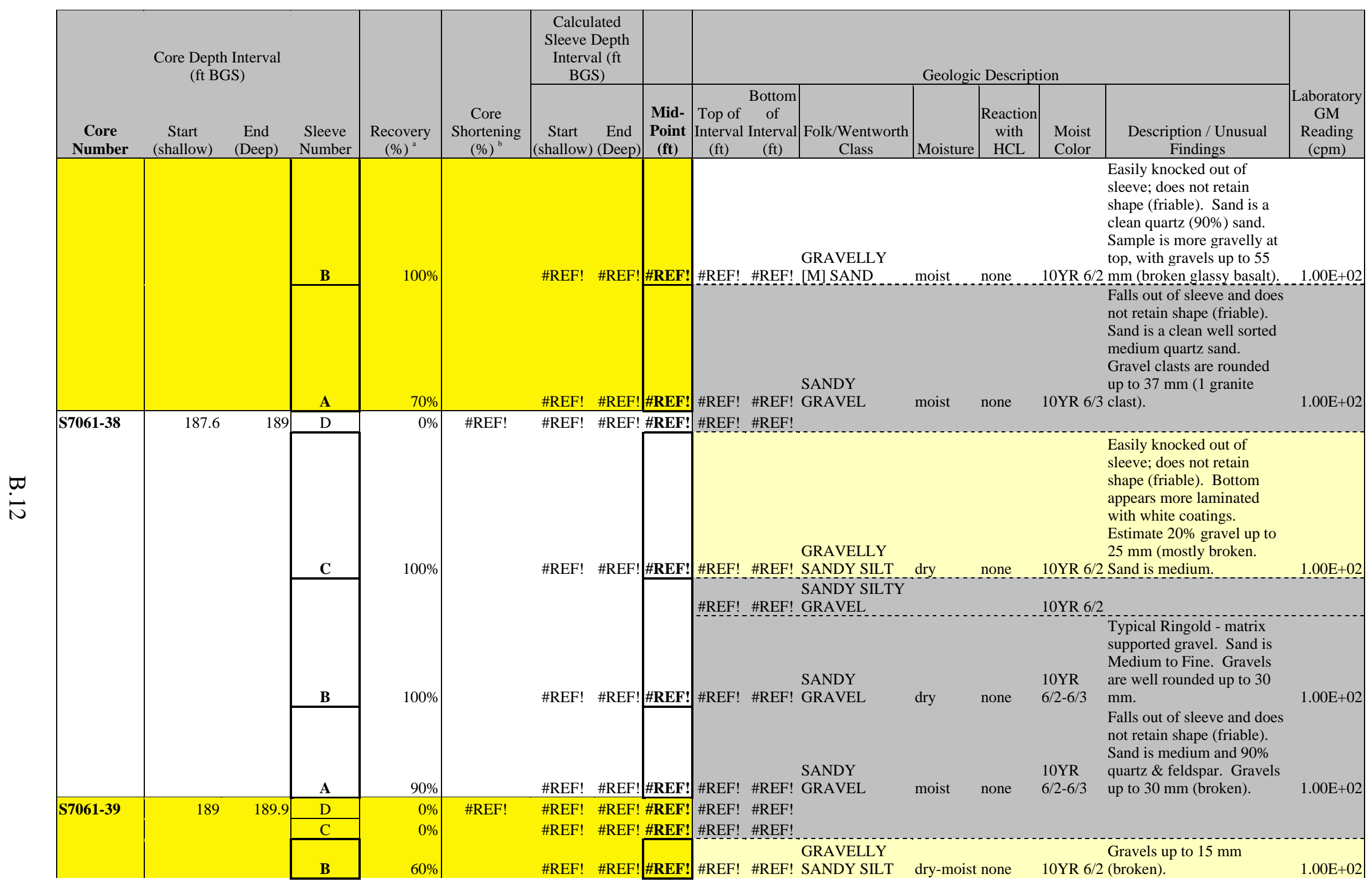




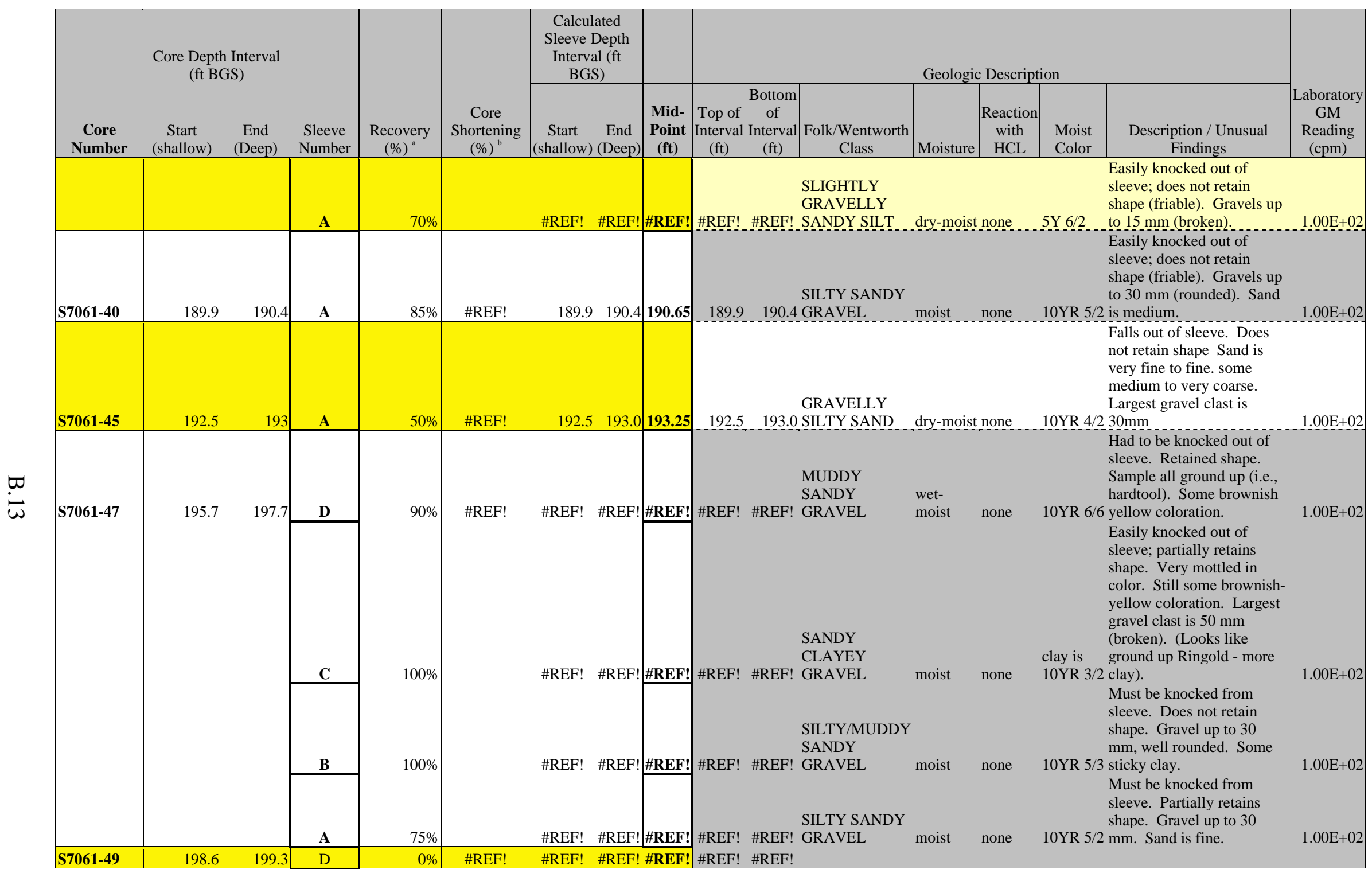




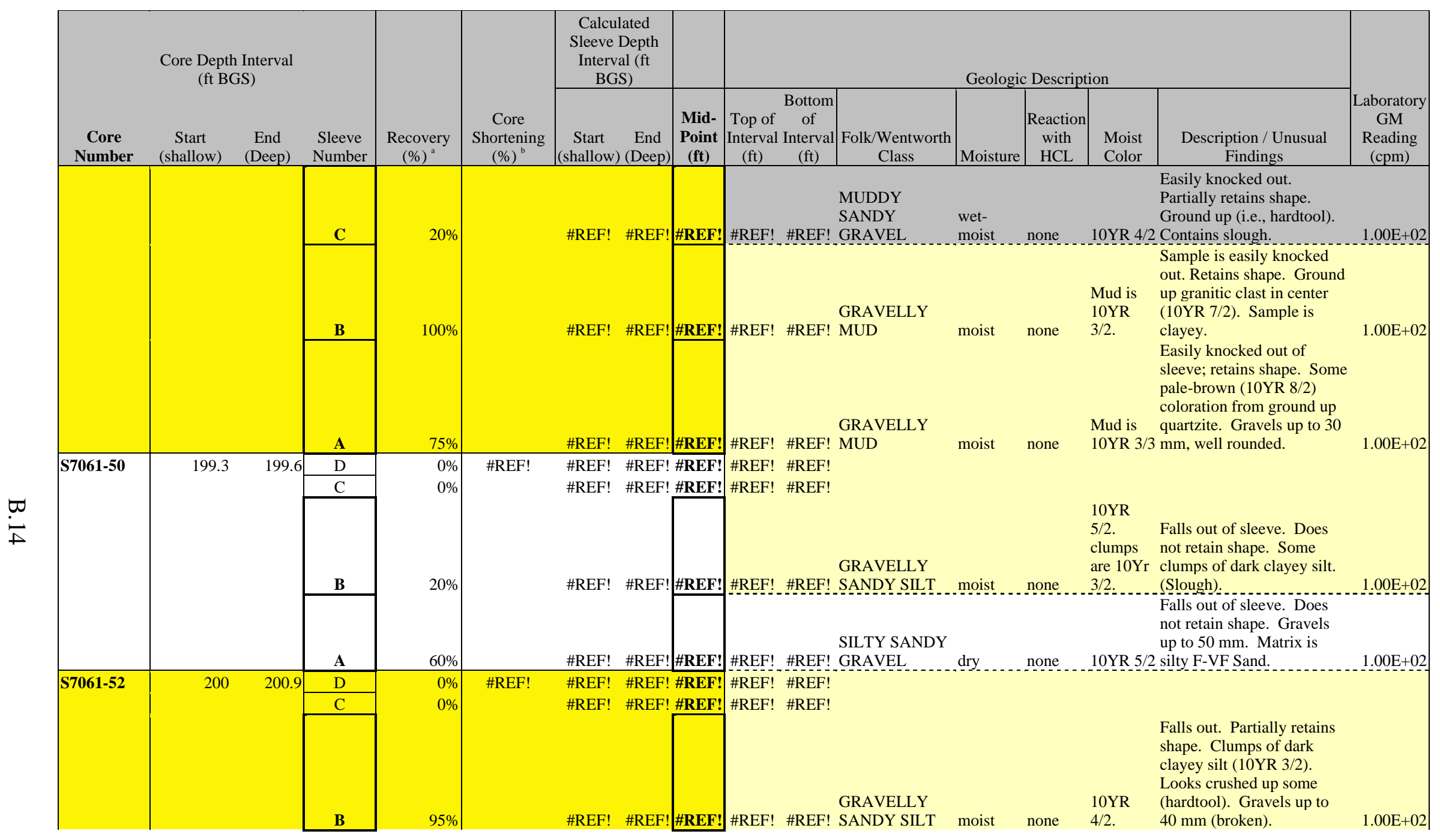




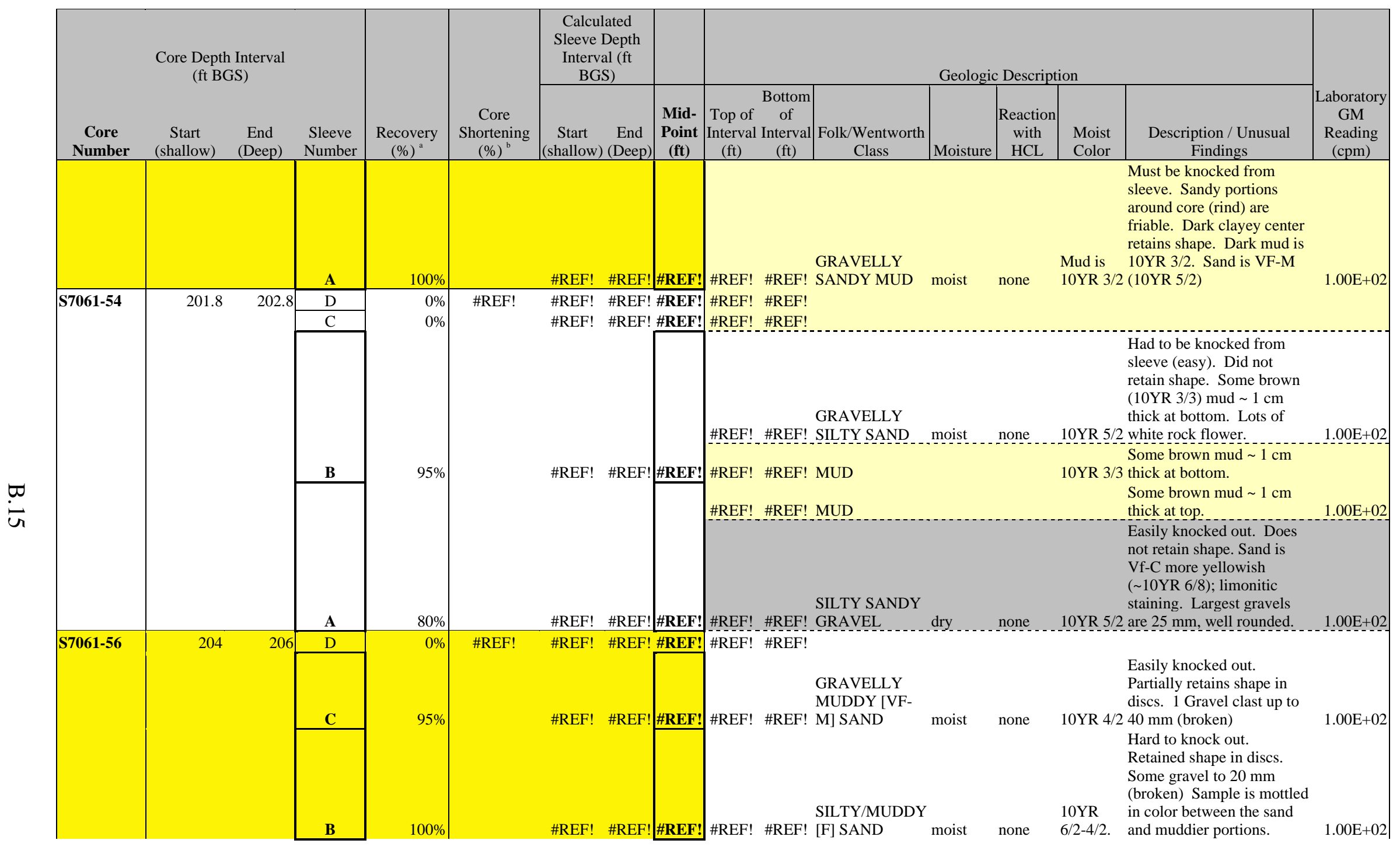




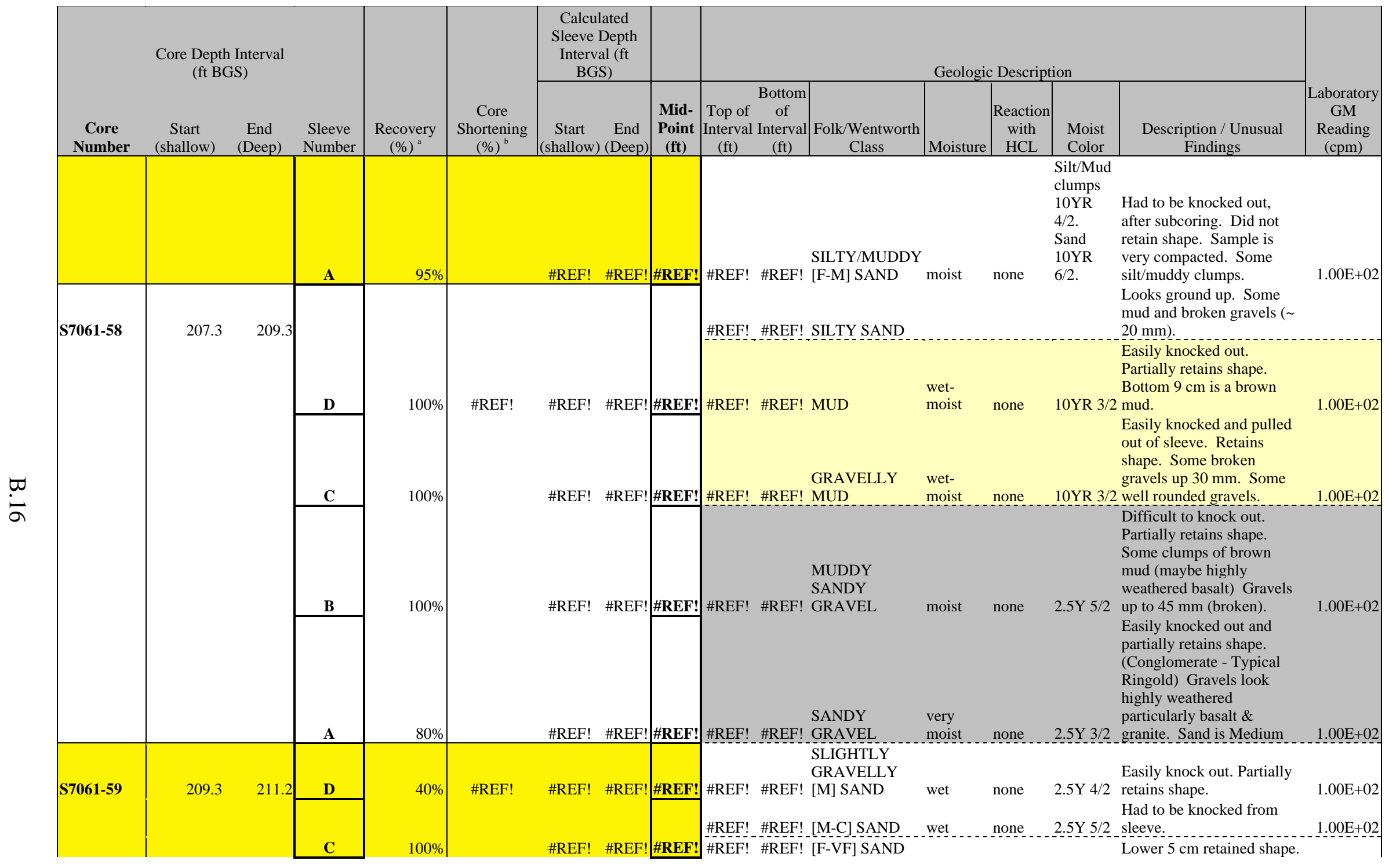




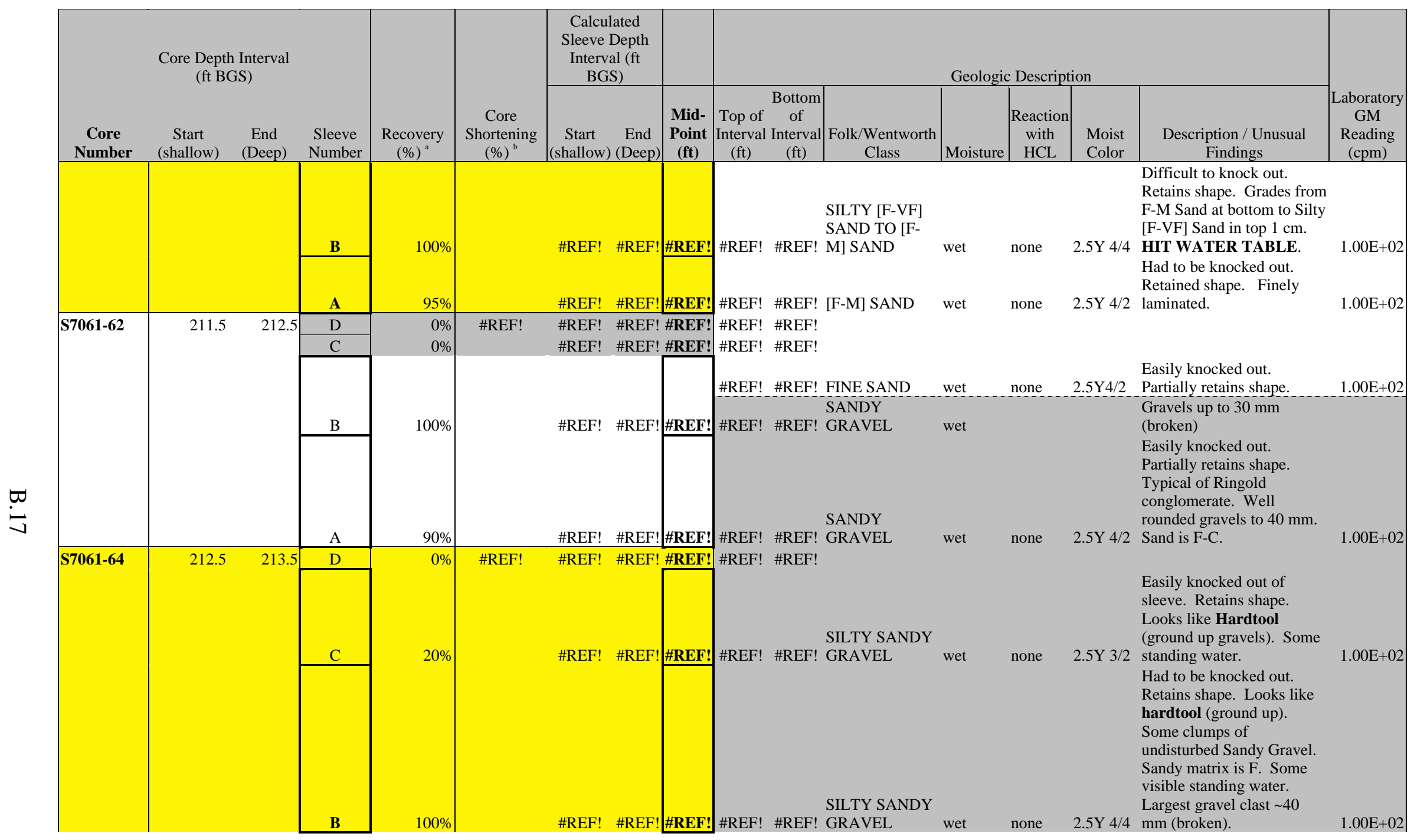




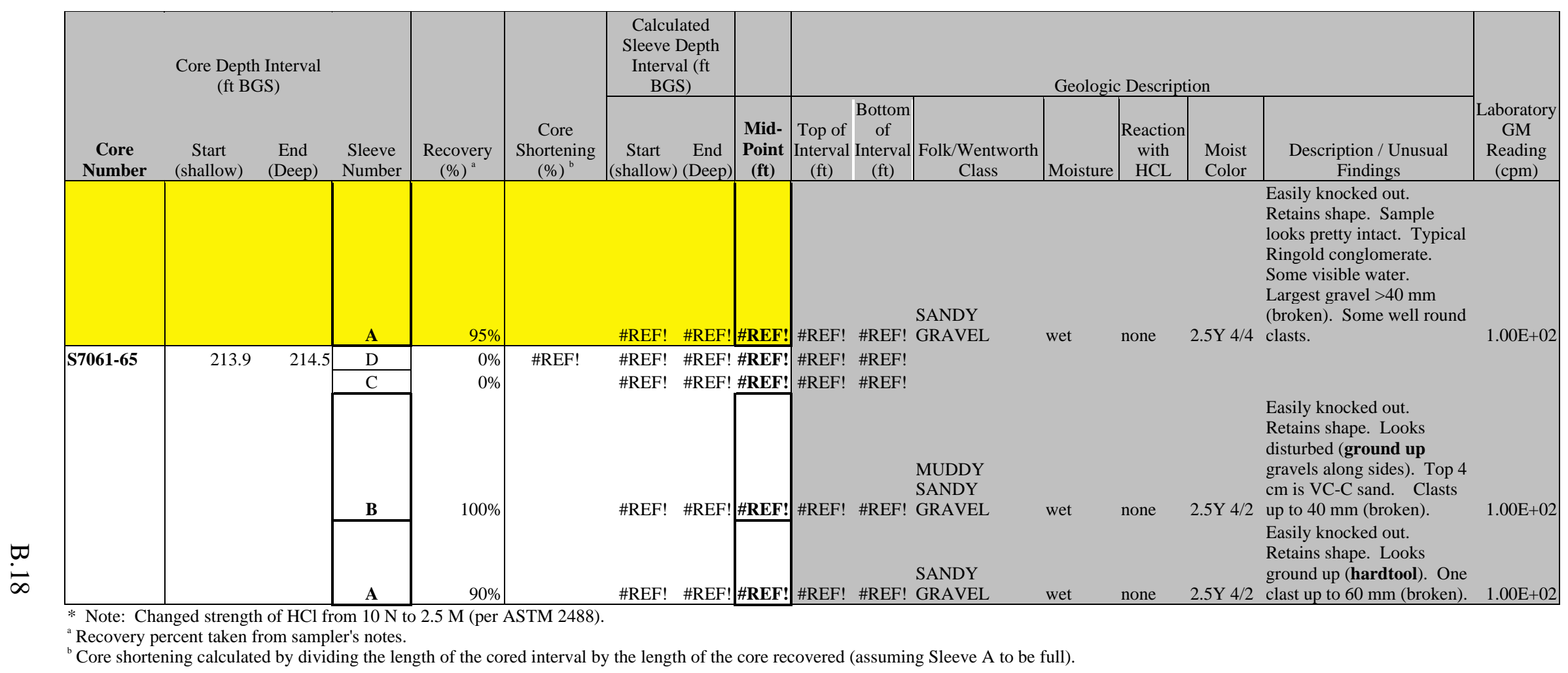




\section{Appendix C}

\section{Geologic Descriptions}




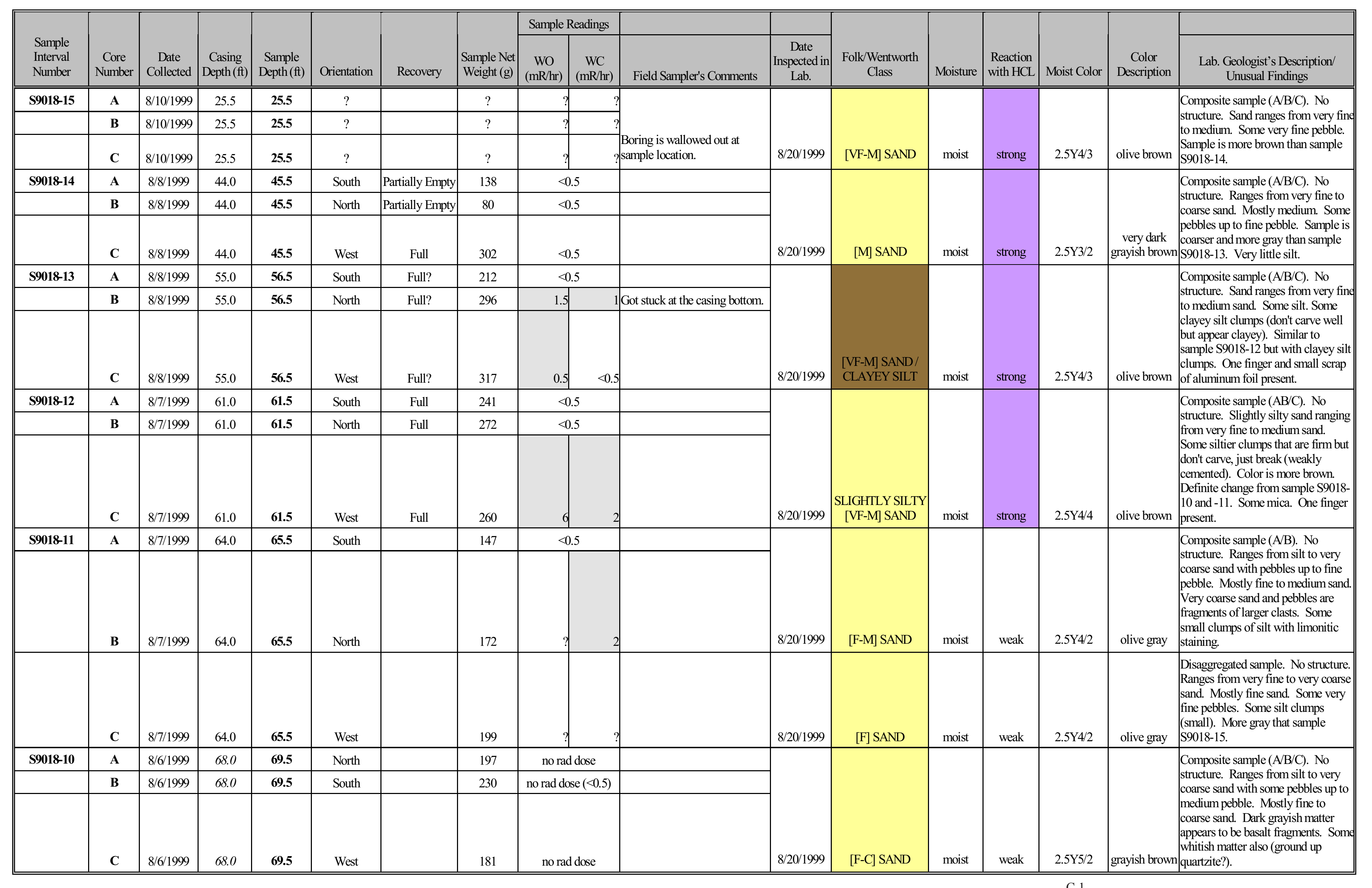




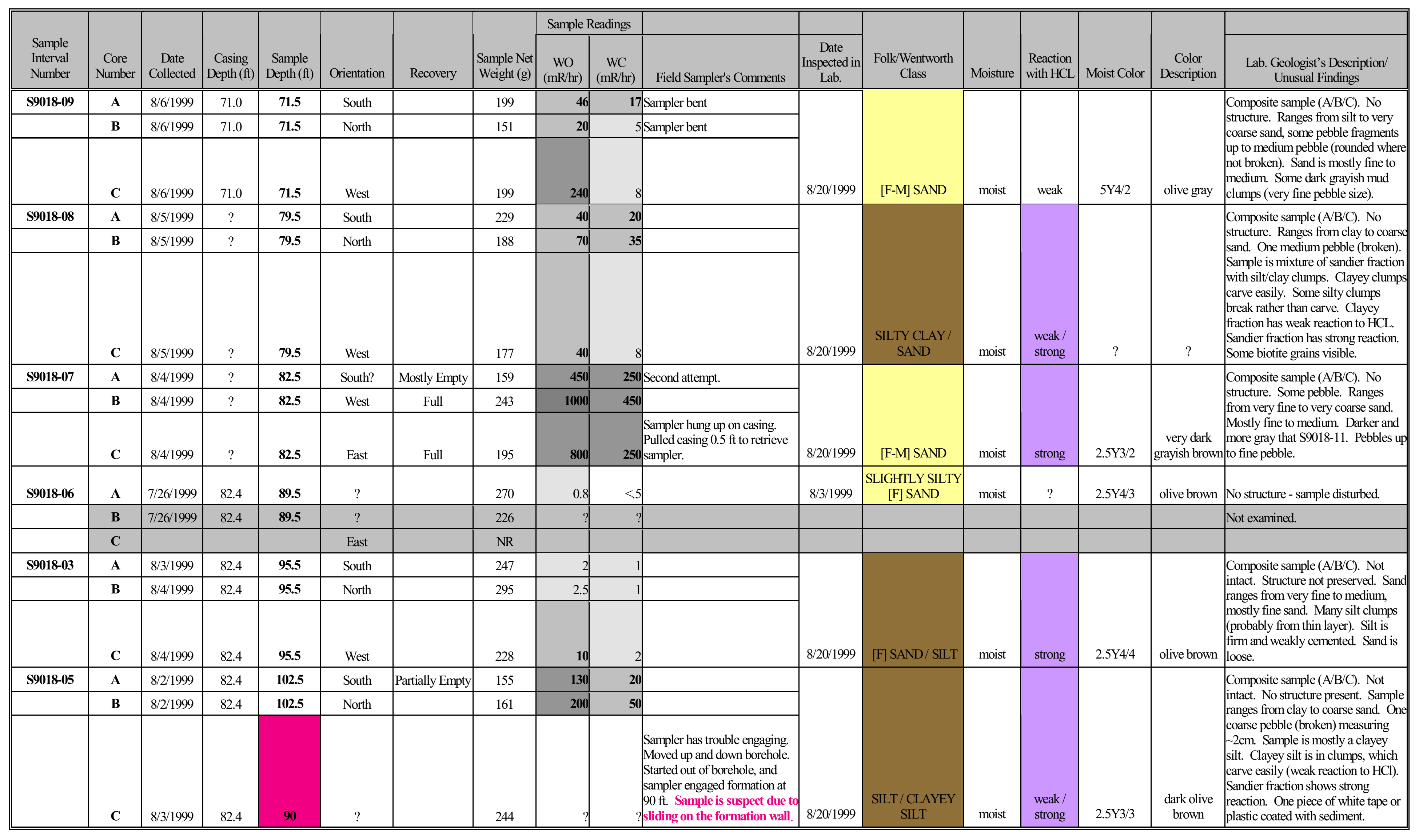




\begin{tabular}{|c|c|c|c|c|c|c|c|c|c|c|c|c|c|c|c|c|c|}
\hline \multirow[b]{2}{*}{$\begin{array}{l}\text { Sample } \\
\text { Interval } \\
\text { Number }\end{array}$} & \multirow[b]{2}{*}{$\begin{array}{c}\text { Core } \\
\text { Number }\end{array}$} & \multirow[b]{2}{*}{$\begin{array}{c}\text { Date } \\
\text { Collected }\end{array}$} & \multirow[b]{2}{*}{$\begin{array}{c}\text { Casing } \\
\text { Depth (ft) }\end{array}$} & \multirow[b]{2}{*}{$\begin{array}{c}\text { Sample } \\
\text { Depth (ft) }\end{array}$} & \multirow[b]{2}{*}{ Orientation } & \multirow[b]{2}{*}{ Recovery } & \multirow[b]{2}{*}{$\left|\begin{array}{l}\text { Sample Net } \\
\text { Weight }(\mathrm{g})\end{array}\right|$} & \multicolumn{2}{|c|}{ Sample Readings } & \multirow[b]{2}{*}{ Field Sampler's Comments } & \multirow[b]{2}{*}{$\begin{array}{c}\text { Date } \\
\text { Inspected in } \\
\text { Lab. } \\
\end{array}$} & \multirow[b]{2}{*}{$\begin{array}{l}\text { Folk/Wentworth } \\
\text { Class }\end{array}$} & \multirow[b]{2}{*}{ Moisture } & \multirow[b]{2}{*}{\begin{tabular}{|} 
Reaction \\
with HCL \\
\end{tabular}} & \multirow[b]{2}{*}{ Moist Color } & \multirow[b]{2}{*}{$\begin{array}{c}\text { Color } \\
\text { Description } \\
\end{array}$} & \multirow[b]{2}{*}{$\begin{array}{l}\text { Lab. Geologist's Description/ } \\
\text { Unusual Findings }\end{array}$} \\
\hline & & & & & & & & $\begin{array}{c}\text { WO } \\
(\mathrm{mR} / \mathrm{hr})\end{array}$ & $\begin{array}{c}\text { WC } \\
(\mathrm{mR} / \mathrm{hr})\end{array}$ & & & & & & & & \\
\hline \multirow[t]{3}{*}{ S9018-04 } & $\mathbf{A}$ & 7/31/1999 & $?$ & 108.5 & South & & 264 & $?$ & $?$ & ? & & & & & & & Composite sample $(\mathrm{A} / \mathrm{B} / \mathrm{C})$ \\
\hline & B & 7/31/1999 & ? & 108.5 & North & Empty & 120 & & & ? & & & & & & & $\begin{array}{l}\text { Structure not retained. Sand ranges } \\
\text { from very fine to medium, mostly }\end{array}$ \\
\hline & C & 8/2/1999 & ? & 108.5 & ? & Mostly Empty & 163 & & 3 ? & & $8 / 20 / 1999$ & $\begin{array}{l}\text { [VF-F] SAND / } \\
\text { SILT }\end{array}$ & moist & strong & $\begin{array}{c}10 \mathrm{YR} 4 / 4- \\
10 \mathrm{YR} 3 / 4\end{array}$ & $\begin{array}{c}\text { dark yellowish } \\
\text { brown }\end{array}$ & $\begin{array}{l}\text { - very firm and slightly more gray } \\
\text { (10YR3/4). Sand is more reddish } \\
\text { than sample S9018-03, perhaps more } \\
\text { limonitic staining. }\end{array}$ \\
\hline \multirow[t]{3}{*}{ S9018-02 } & $\mathbf{A}$ & 7/21/1999 & ? & 111.9 & West & Empty & ? & & & ? Water Present. & & & & & & & \\
\hline & B & 7/21/1999 & ? & 111.9 & Northeast & $80 \%$ & ? & & & $80 \%$ Recovery. & 8/20/1999 & [VF-M] SAND & moist & strong & $2.5 \mathrm{Y} 4 / 3$ & olive brown & $\begin{array}{l}\text { Composite sample. No observable } \\
\text { structure. Sample ranges from very } \\
\text { fine to medium sand. Mostly fine to } \\
\text { medium. Some silt. Some clumps of } \\
\text { silt with firm consistency/weak } \\
\text { cementation. }\end{array}$ \\
\hline & C & 7/21/1999 & ? & 111.9 & South & $50 \%$ & ? & ר & & ?50\% Recovery. & & & & & & & \\
\hline \multirow[t]{3}{*}{ S9018-01 } & $\mathbf{A}$ & 6/16/1999 & ? & 127.4 & ? & $43 \%$ & ? & & & $\begin{array}{l}\text { Recovered 3" of sample inside } \\
? \begin{array}{l}\text { 7" sampler. } \\
\end{array}\end{array}$ & & & & & & & \multirow{3}{*}{$\begin{array}{l}\text { Composite of samples A, B, and C. } \\
\text { Sample is fine to very fine sand with } \\
\text { sandy silt clumps. Sandy silt clumps } \\
\text { are somewhat rusty (perhaps due to } \\
\text { time in sampler). Other clumps are } \\
\text { dark brown. }\end{array}$} \\
\hline & B & 6/17/1999 & $?$ & 127.4 & $?$ & & $?$ & & & $\begin{array}{l}\text { Sample is suspect due to } \\
\text { ? contacting bottom }\end{array}$ & & & & & & & \\
\hline & C & 6/17/1999 & ? & 127.4 & ? & $64 \%$ & ? & ? & & $\begin{array}{l}\text { Recovered } 4.5 \text { " of sample. } \\
\text { ? Sampler bent. }\end{array}$ & 8/9/1999 & $\begin{array}{l}\text { SILTY [F-VF] } \\
\text { SAND }\end{array}$ & moist & none & $\mid 5 Y R 4 / 4$ to $5 / 4 \mid$ & olive & \\
\hline
\end{tabular}




\section{Appendix D}

Particle Size Data and Folk/Wentworth Classification 


\begin{tabular}{|c|c|c|c|c|c|c|c|c|c|c|c|c|c|c|c|c|c|}
\hline & & & & \multicolumn{2}{|c|}{ Gravel } & \multicolumn{7}{|c|}{ Sand } & & & & & \\
\hline & & & & $\begin{array}{c}\text { Fine } \\
\text { Pebble }\end{array}$ & \begin{tabular}{|c|} 
Very Fine \\
Pebble
\end{tabular} & $\begin{array}{c}\text { Very } \\
\text { Coarse }\end{array}$ & Coarse & Medium & Fine & Very Fine & $\begin{array}{l}\text { Silt \& } \\
\text { Clay }\end{array}$ & & & & & & \\
\hline & & & & 5 & 10 & 18 & 35 & 60 & 120 & 230 & Pan & & & & & & \\
\hline Borehole & $\begin{array}{c}\text { Sample } \\
\text { Type }\end{array}$ & Depth (ft) & $\begin{array}{l}\mathrm{CaC}_{3} \\
\text { wt. \% } \\
\end{array}$ & $>4 \mathrm{~mm}$ & $2-4 \mathrm{~mm}$ & $1-2 \mathrm{~mm}$ & $\begin{array}{c}0.5-1.0 \\
\mathrm{~mm}\end{array}$ & $\begin{array}{c}0.25-0.5 \\
\mathrm{~mm}\end{array}$ & $\begin{array}{c}0.125- \\
0.25 \mathrm{~mm} \\
\end{array}$ & $\begin{array}{c}0.0625- \\
0.125 \mathrm{~mm}\end{array}$ & $\begin{array}{c}<0.0625 \\
\mathrm{~mm}\end{array}$ & $\%$ Gravel & $\%$ Sand & $\% \mathrm{Mud}$ & $\begin{array}{c}\text { Sand:Mud } \\
\text { Ratio }\end{array}$ & CLASS & $\begin{array}{c}\text { Hydrogeologic } \\
\text { Unit }\end{array}$ \\
\hline 299-W22-39 & & 45 & & $0.00 \%$ & $0.00 \%$ & $0.20 \%$ & 6.53\% & $26.89 \%$ & "48.39\% & 13.93\% & $4.06 \%$ & $0.00 \%$ & 95.94\% & $4.06 \%$ & 23.7 & $\mathrm{~S}$ & H1a \\
\hline & & 50 & & $0.00 \%$ & $0.35 \%$ & $2.82 \%$ & $8.65 \%$ & $24.51 \%$ & $32.93 \%$ & $22.73 \%$ & $8.03 \%$ & $0.35 \%$ & $91.62 \%$ & $8.03 \%$ & \begin{tabular}{l|l|}
11.4 & \\
\end{tabular} & $\mathrm{~S}$ & $\mathrm{H1a}$ \\
\hline & & 55 & & $62.85 \%$ & $5.15 \%$ & $5.25 \%$ & $5.51 \%$ & $4.66 \%$ & $10.40 \%$ & $3.39 \%$ & $2.80 \%$ & $68.00 \%$ & $29.21 \%$ & $2.80 \%$ & \begin{tabular}{l|l}
10.4 \\
\end{tabular} & sG & $\mathrm{H1}$ \\
\hline & & 60 & & $0.15 \%$ & $0.24 \%$ & $0.98 \%$ & $9.27 \%$ & $47.30 \%$ & $22.80 \%$ & $10.04 \%$ & $9.21 \%$ & $0.39 \%$ & $90.39 \%$ & $9.21 \%$ & 9.8 & $\mathrm{~S}$ & $\mathrm{H} 2$ \\
\hline & & 65 & & $0.44 \%$ & $1.24 \%$ & $7.03 \%$ & $22.98 \%$ & $32.55 \%$ & $16.98 \%$ & $12.83 \%$ & $5.95 \%$ & $1.68 \%$ & $92.36 \%$ & $5.95 \%$ & 15.5 & $\mathrm{~S}$ & $\mathrm{H} 2$ \\
\hline $299-W 22-46$ & & 50 & & $0.30 \%$ & $0.34 \%$ & $0.59 \%$ & $0.92 \%$ & $23.26 \%$ & $40.84 \%$ & $27.53 \%$ & $6.21 \%$ & $0.63 \%$ & $93.15 \%$ & $6.21 \%$ & 15.0 & $\mathrm{~S}$ & H1a \\
\hline & & 55 & & $8.03 \%$ & $13.56 \%$ & $26.40 \%$ & $25.01 \%$ & $11.57 \%$ & $9.70 \%$ & $4.75 \%$ & $0.97 \%$ & $21.60 \%$ & $77.43 \%$ & $0.97 \%$ & 79.8 & $\mathrm{gS}$ & $\mathrm{H} 1$ \\
\hline & & 60 & & $95.36 \%$ & $1.91 \%$ & $1.27 \%$ & $0.43 \%$ & $0.10 \%$ & $0.80 \%$ & $0.05 \%$ & $0.08 \%$ & $97.27 \%$ & $2.64 \%$ & $0.08 \%$ & 32.0 & $\mathrm{G}$ & $\mathrm{H} 1$ \\
\hline & & 65 & & $94.51 \%$ & $2.30 \%$ & $1.81 \%$ & $0.85 \%$ & $0.22 \%$ & $0.11 \%$ & $0.08 \%$ & $0.14 \%$ & $96.80 \%$ & $3.06 \%$ & $0.14 \%$ & 22.4 & $\mathrm{G}$ & $\mathrm{H1}$ \\
\hline & & 70 & & $0.43 \%$ & $0.04 \%$ & $0.45 \%$ & $0.23 \%$ & $6.49 \%$ & $56.83 \%$ & $27.30 \%$ & $8.22 \%$ & $0.48 \%$ & $91.30 \%$ & $8.22 \%$ & \begin{tabular}{l|l}
11.1 \\
\end{tabular} & $\mathrm{~S}$ & $\mathrm{H} 2$ \\
\hline & & 75 & & $0.00 \%$ & $0.01 \%$ & $0.17 \%$ & $1.50 \%$ & $25.23 \%$ & $36.83 \%$ & $28.56 \%$ & $7.69 \%$ & $0.01 \%$ & $92.30 \%$ & $7.69 \%$ & \begin{tabular}{l|}
12.0 \\
\end{tabular} & $\mathrm{~S}$ & $\mathrm{H} 2$ \\
\hline $299-W 22-48$ & SS & 37 & & $0.20 \%$ & $0.40 \%$ & $0.60 \%$ & $3.89 \%$ & $13.83 \%$ & $43.84 \%$ & $29.54 \%$ & $7.71 \%$ & $0.60 \%$ & $91.69 \%$ & $7.71 \%$ & 11.9 & $\mathrm{~S}$ & H1a \\
\hline B8812 & SS & 39.5 & & $0.02 \%$ & $1.40 \%$ & $0.74 \%$ & $2.00 \%$ & $25.21 \%$ & $40.12 \%$ & $21.73 \%$ & $8.78 \%$ & $1.42 \%$ & $89.80 \%$ & $8.78 \%$ & 10.2 & $\mathrm{~S}$ & H1a \\
\hline & SS & $42-42.5$ & & & $63.17 \%$ & & & & & & $36.83 \%$ & $63.17 \%$ & & & & $\mathrm{sG}$ & $\mathrm{H1}$ \\
\hline & SS & 44.5 & & & $63.26 \%$ & & & & & & $36.74 \%$ & $63.26 \%$ & & & & sG & $\mathrm{H1}$ \\
\hline & SS & 47 & & $63.69 \%$ & $9.69 \%$ & $7.25 \%$ & $6.53 \%$ & $3.73 \%$ & $2.91 \%$ & $2.55 \%$ & $3.64 \%$ & $73.38 \%$ & $22.98 \%$ & $3.64 \%$ & 6.3 & $\mathrm{msG}$ & $\mathrm{H1}$ \\
\hline & SS & 50 & & $18.51 \%$ & $0.96 \%$ & $2.45 \%$ & $8.42 \%$ & $40.00 \%$ & $20.94 \%$ & $4.89 \%$ & $3.84 \%$ & $19.46 \%$ & $76.70 \%$ & $3.84 \%$ & 20.0 & $\mathrm{gS}$ & H1 \\
\hline & SS & 53.5 & & $7.32 \%$ & $13.90 \%$ & $19.31 \%$ & $34.85 \%$ & $17.87 \%$ & $4.15 \%$ & $1.53 \%$ & $1.07 \%$ & $21.22 \%$ & $77.71 \%$ & $1.07 \%$ & \begin{tabular}{l|}
72.7 \\
\end{tabular} & $\mathrm{gS}$ & $\mathrm{H1}$ \\
\hline & SS & 56 & & $4.35 \%$ & $5.10 \%$ & $16.32 \%$ & $36.19 \%$ & $24.03 \%$ & $7.90 \%$ & $3.58 \%$ & $2.52 \%$ & $9.46 \%$ & $88.02 \%$ & $2.52 \%$ & \begin{tabular}{l|l|}
34.9 \\
\end{tabular} & (g)S & $\mathrm{H} 1$ \\
\hline & SS & 57.5 & & $2.42 \%$ & $0.54 \%$ & $8.25 \%$ & $37.56 \%$ & $32.37 \%$ & $9.20 \%$ & $5.01 \%$ & $4.66 \%$ & $2.96 \%$ & $92.39 \%$ & $4.66 \%$ & \begin{tabular}{l|}
19.8 \\
\end{tabular} & $\mathrm{~S}$ & H1 \\
\hline & SS & 62 & & $0.00 \%$ & $0.29 \%$ & $13.29 \%$ & $43.59 \%$ & $25.95 \%$ & $7.55 \%$ & $4.15 \%$ & $5.19 \%$ & $0.29 \%$ & $94.52 \%$ & $5.19 \%$ & \begin{tabular}{l|l|}
18.2 \\
\end{tabular} & $\mathrm{~S}$ & $\mathrm{H} 2$ \\
\hline & SS & 64.5 & & $0.06 \%$ & $0.00 \%$ & $1.31 \%$ & $31.13 \%$ & $45.90 \%$ & $11.85 \%$ & $5.18 \%$ & $4.56 \%$ & $0.06 \%$ & $95.38 \%$ & $4.56 \%$ & 20.9 & $\mathrm{~S}$ & $\mathrm{H} 2$ \\
\hline & SS & 67 & & $0.39 \%$ & $0.59 \%$ & $8.19 \%$ & $25.40 \%$ & $42.95 \%$ & $12.43 \%$ & $7.08 \%$ & $2.95 \%$ & $0.98 \%$ & $96.06 \%$ & $2.95 \%$ & 32.5 & $\mathrm{~S}$ & $\mathrm{H} 2$ \\
\hline & SS & 69.5 & & $0.36 \%$ & $1.21 \%$ & $7.32 \%$ & $39.39 \%$ & $34.53 \%$ & $8.84 \%$ & $4.18 \%$ & $4.17 \%$ & $1.58 \%$ & $94.26 \%$ & $4.17 \%$ & 22.6 & $\mathrm{~S}$ & $\mathrm{H} 2$ \\
\hline & SS & 70 & & $0.36 \%$ & $0.61 \%$ & $8.31 \%$ & $43.13 \%$ & $32.80 \%$ & $8.73 \%$ & $3.17 \%$ & $2.89 \%$ & $0.96 \%$ & $96.15 \%$ & $2.89 \%$ & \begin{tabular}{l|}
33.3 \\
\end{tabular} & $\mathrm{~S}$ & $\mathrm{H} 2$ \\
\hline & SS & 74.5 & & $0.18 \%$ & $1.56 \%$ & $13.92 \%$ & $6.86 \%$ & $25.05 \%$ & $25.78 \%$ & $14.84 \%$ & $11.81 \%$ & $1.74 \%$ & $86.46 \%$ & $11.81 \%$ & \begin{tabular}{l|l|}
7.3 \\
\end{tabular} & (m)S & $\mathrm{H} 2$ \\
\hline & SS & 77 & & $0.00 \%$ & $0.02 \%$ & $0.15 \%$ & $0.10 \%$ & $1.56 \%$ & $48.28 \%$ & $39.67 \%$ & $10.21 \%$ & $0.02 \%$ & $89.77 \%$ & $10.21 \%$ & \begin{tabular}{l|l}
8.8 \\
\end{tabular} & $(\mathrm{~m}) \mathrm{S}$ & $\mathrm{H} 2$ \\
\hline & SS & 91.5 & & $0.09 \%$ & $0.30 \%$ & $4.40 \%$ & $24.23 \%$ & $42.87 \%$ & $15.11 \%$ & $7.99 \%$ & $5.00 \%$ & $0.39 \%$ & $94.60 \%$ & $5.00 \%$ & \begin{tabular}{l|l}
18.9 \\
\end{tabular} & $\mathrm{~S}$ & $\mathrm{H} 2$ \\
\hline
\end{tabular}




\begin{tabular}{|c|c|c|c|c|c|c|c|c|c|c|c|c|c|c|c|c|c|}
\hline & & & & \multicolumn{2}{|c|}{ Gravel } & \multicolumn{7}{|c|}{ Sand } & & & & & \\
\hline & & & & $\begin{array}{c}\text { Fine } \\
\text { Pebble }\end{array}$ & \begin{tabular}{|c|} 
Very Fine \\
Pebble
\end{tabular} & $\begin{array}{c}\text { Very } \\
\text { Coarse }\end{array}$ & Coarse & Medium & Fine & Very Fine & $\begin{array}{l}\text { Silt \& } \\
\text { Clay }\end{array}$ & & & & & & \\
\hline & & & & 5 & 10 & 18 & 35 & 60 & 120 & 230 & Pan & & & & & & \\
\hline \multirow[t]{5}{*}{ Borehole } & $\begin{array}{c}\text { Sample } \\
\text { Type } \\
\end{array}$ & Depth (ft) & $\begin{array}{l}\mathrm{CaCO}_{3} \\
\text { wt. } \% \\
\end{array}$ & $>4 \mathrm{~mm}$ & $2-4 \mathrm{~mm}$ & $1-2 \mathrm{~mm}$ & $\begin{array}{c}0.5-1.0 \\
\mathrm{~mm}\end{array}$ & $\begin{array}{c}0.25-0.5 \\
\mathrm{~mm}\end{array}$ & $\begin{array}{c}0.125- \\
0.25 \mathrm{~mm} \\
\end{array}$ & $\begin{array}{c}0.0625- \\
0.125 \mathrm{~mm}\end{array}$ & $\begin{array}{c}<0.0625 \\
\mathrm{~mm}\end{array}$ & $\%$ Gravel & $\%$ Sand & $\%$ Mud & $\begin{array}{c}\text { Sand:Mud } \\
\text { Ratio }\end{array}$ & CLASS & $\begin{array}{c}\text { Hydrogeologic } \\
\text { Unit } \\
\end{array}$ \\
\hline & SS & 101.5 & & $0.00 \%$ & $0.01 \%$ & |0.35\% & $24.14 \%$ & $47.54 \%$ & $13.83 \%$ & $12.25 \%$ & $1.88 \%$ & $0.01 \%$ & $98.11 \%$ & $1.88 \%$ & \begin{tabular}{l|l}
52.1 &
\end{tabular} & $\mathrm{SS}$ & $\mathrm{H} 2$ \\
\hline & SS & 136 & & $0.01 \%$ & $1.05 \%$ & $0.27 \%$ & $6.12 \%$ & $4.00 \%$ & $9.11 \%$ & $40.88 \%$ & $38.56 \%$ & $1.06 \%$ & $60.38 \%$ & $38.56 \%$ & 1.6 & $\mathrm{mS}$ & $\mathrm{PP}$ \\
\hline & SS & 163.5 & & $0.00 \%$ & $0.03 \%$ & $0.27 \%$ & $13.84 \%$ & $53.61 \%$ & $20.11 \%$ & $6.35 \%$ & $5.79 \%$ & $0.03 \%$ & $94.18 \%$ & $5.79 \%$ & \begin{tabular}{l|}
16.3 \\
\end{tabular} & $\mathrm{~S}$ & Rwi(e) \\
\hline & SS & 475 & & $0.00 \%$ & $0.00 \%$ & $0.28 \%$ & $181 \%$ & $1166 \%$ & $26.35^{\circ}$ & $37.76 \%$ & & $0.00 \%$ & $77.86^{\circ}$ & & & St & $\mathrm{H} 1 \mathrm{a}$ \\
\hline \multirow[t]{18}{*}{ B8814 } & SS & 47.5 & & $1.22 \%$ & $4.16 \%$ & 5.01\% & $4.66 \%$ & $10.48 \%$ & $39.04 \%$ & $27.34 \%$ & $8.09 \%$ & $5.38 \%$ & $86.53 \%$ & $8.09 \%$ & 10.7 & (g)S & H1a \\
\hline & SS & 48.5 & & $0.01 \%$ & $0.00 \%$ & $0.03 \%$ & $0.76 \%$ & $10.58 \%$ & $35.82 \%$ & $45.25 \%$ & $7.55 \%$ & $0.01 \%$ & $92.45 \%$ & $7.55 \%$ & \begin{tabular}{l|l}
12.3 \\
\end{tabular} & S & H1a \\
\hline & SS & $50-51$ & & $0.20 \%$ & $0.36 \%$ & $0.84 \%$ & $5.76 \%$ & $31.29 \%$ & $29.26 \%$ & $22.97 \%$ & $9.33 \%$ & $0.56 \%$ & $90.11 \%$ & $9.33 \%$ & 9.7 & $\mathrm{~S}$ & H1a \\
\hline & SS & 51 & & $0.01 \%$ & $0.23 \%$ & $0.64 \%$ & $3.03 \%$ & $23.34 \%$ & $29.43 \%$ & $34.38 \%$ & $8.95 \%$ & $0.23 \%$ & $90.81 \%$ & $8.95 \%$ & \begin{tabular}{l|l}
10.1 \\
\end{tabular} & $\mathrm{~S}$ & $\mathrm{H} 1 \mathrm{a}$ \\
\hline & SS & 52.5 & & $1.34 \%$ & $3.48 \%$ & $14.12 \%$ & $25.98 \%$ & $23.20 \%$ & $17.35 \%$ & $9.90 \%$ & $4.63 \%$ & $4.82 \%$ & $90.55 \%$ & $4.63 \%$ & \begin{tabular}{l|l}
19.6 \\
\end{tabular} & $\mathrm{~S}$ & H1a \\
\hline & SS & 53.5 & & $0.43 \%$ & $5.63 \%$ & $28.21 \%$ & $45.22 \%$ & $13.56 \%$ & $3.28 \%$ & $1.82 \%$ & $1.85 \%$ & $6.06 \%$ & $92.09 \%$ & $1.85 \%$ & 49.7 & (g)S & $\mathrm{H} 1$ \\
\hline & SS & $53.5-54.5$ & & $0.48 \%$ & $3.46 \%$ & $28.40 \%$ & $50.45 \%$ & $12.90 \%$ & $2.81 \%$ & $1.49 \%$ & $0.00 \%$ & $3.95 \%$ & $96.05 \%$ & $0.00 \%$ & 96052.4 & $\mathrm{~S}$ & H1 \\
\hline & SS & 56 & & $4.02 \%$ & $6.67 \%$ & $0.52 \%$ & $62.35 \%$ & $12.37 \%$ & $5.98 \%$ & $4.12 \%$ & $3.97 \%$ & $10.69 \%$ & $85.34 \%$ & $3.97 \%$ & 21.5 & $\mathrm{gS}$ & H1 \\
\hline & SS & 57 & & $1.38 \%$ & $3.17 \%$ & $24.29 \%$ & $41.55 \%$ & $16.67 \%$ & $7.49 \%$ & $3.95 \%$ & $1.50 \%$ & $4.55 \%$ & $93.95 \%$ & $1.50 \%$ & 62.5 & $\mathrm{~S}$ & $\mathrm{H} 1$ \\
\hline & SS & 58 & & $37.16 \%$ & $11.46 \%$ & $16.45 \%$ & $19.19 \%$ & $8.36 \%$ & $3.18 \%$ & $2.21 \%$ & $2.00 \%$ & $48.62 \%$ & $49.38 \%$ & $2.00 \%$ & 24.6 & $\mathrm{sG}$ & $\mathrm{H} 1$ \\
\hline & SS & 60 & & $81.68 \%$ & $4.95 \%$ & $4.36 \%$ & $3.37 \%$ & $2.14 \%$ & $1.14 \%$ & $1.04 \%$ & $1.33 \%$ & $86.63 \%$ & $12.04 \%$ & $1.33 \%$ & \begin{tabular}{l|l}
9.0 \\
\end{tabular} & $\mathrm{G}$ & $\mathrm{H1}$ \\
\hline & SS & 61 & & $33.76 \%$ & $17.15 \%$ & $14.50 \%$ & $13.81 \%$ & $9.58 \%$ & $4.50 \%$ & $3.29 \%$ & $3.42 \%$ & $50.91 \%$ & $45.67 \%$ & $3.42 \%$ & \begin{tabular}{l|l}
13.4 \\
\end{tabular} & sG & H1 \\
\hline & SS & 62.5 & & $0.07 \%$ & $0.92 \%$ & $4.81 \%$ & $26.29 \%$ & $46.08 \%$ & $13.88 \%$ & $4.93 \%$ & $3.01 \%$ & $0.99 \%$ & $96.00 \%$ & $3.01 \%$ & 31.9 & $\mathrm{~S}$ & $\mathrm{H} 2$ \\
\hline & SS & 63.5 & & $0.94 \%$ & $0.76 \%$ & $6.54 \%$ & $38.28 \%$ & $41.57 \%$ & $8.17 \%$ & $2.19 \%$ & $1.55 \%$ & $1.70 \%$ & $96.74 \%$ & $1.55 \%$ & \begin{tabular}{l|l}
62.2 \\
\end{tabular} & S & $\mathrm{H} 2$ \\
\hline & SS & 65 & & $0.00 \%$ & $0.26 \%$ & $2.44 \%$ & $21.41 \%$ & $45.41 \%$ & $18.51 \%$ & $6.74 \%$ & $5.23 \%$ & $0.26 \%$ & $94.51 \%$ & $5.23 \%$ & \begin{tabular}{l|l}
18.1 \\
\end{tabular} & $\mathrm{~S}$ & $\mathrm{H} 2$ \\
\hline & SS & 66 & & $0.30 \%$ & $1.05 \%$ & $3.15 \%$ & $14.22 \%$ & $40.04 \%$ & $29.71 \%$ & $9.56 \%$ & $1.97 \%$ & $1.34 \%$ & $96.69 \%$ & $1.97 \%$ & 49.1 & $\mathrm{~S}$ & $\mathrm{H} 2$ \\
\hline & SS & 68.5 & & $0.00 \%$ & $0.00 \%$ & \begin{tabular}{l|l|}
$0.01 \%$ \\
\end{tabular} & $0.02 \%$ & $2.58 \%$ & $52.17 \%$ & $37.44 \%$ & $\begin{array}{l}7.77 \% \\
\end{array}$ & $0.00 \%$ & $92.23 \%$ & $7.77 \%$ & \begin{tabular}{l|l}
11.9 \\
\end{tabular} & $\mathrm{~s}$ & $\mathrm{H} 2$ \\
\hline & SS & $70-71$ & & $1.00 \%$ & $1.28 \%$ & $1.04 \%$ & $1.79 \%$ & $17.03 \%$ & $51.16 \%$ & $21.19 \%$ & $5.50 \%$ & $2.28 \%$ & $92.22 \%$ & $5.50 \%$ & \begin{tabular}{l|l}
16.8 \\
\end{tabular} & $\mathrm{~S}$ & $\mathrm{H} 2$ \\
\hline \multirow[t]{7}{*}{ 299-W23-14 } & & 65 & $0.8 \%$ & $0.01 \%$ & $0.01 \%$ & $0.03 \%$ & $0.38 \%$ & $3.46 \%$ & $36.02 \%$ & $44.16 \%$ & $15.93 \%$ & $0.02 \%$ & $84.05 \%$ & $15.93 \%$ & $\begin{array}{l}5.3 \\
\end{array}$ & (m)S & $\mathrm{H1a}$ \\
\hline & & 70 & $0.9 \%$ & $0.00 \%$ & $0.06 \%$ & $0.24 \%$ & $0.51 \%$ & $10.86 \%$ & $39.81 \%$ & $38.68 \%$ & $9.84 \%$ & $0.06 \%$ & $90.10 \%$ & $9.84 \%$ & \begin{tabular}{l|l|}
9.2 \\
\end{tabular} & $\mathrm{~S}$ & $\mathrm{H1a}$ \\
\hline & & 75 & $0.5 \%$ & $0.01 \%$ & $0.13 \%$ & $1.64 \%$ & $8.88 \%$ & $40.81 \%$ & $37.75 \%$ & $8.07 \%$ & $2.71 \%$ & $0.13 \%$ & $97.15 \%$ & $2.71 \%$ & \begin{tabular}{l|l}
35.8 \\
\end{tabular} & $\mathrm{~S}$ & $\mathrm{H} 1 \mathrm{a}$ \\
\hline & & 81 & $1.0 \%$ & $0.07 \%$ & $0.26 \%$ & $1.77 \%$ & $8.97 \%$ & $12.97 \%$ & $31.29 \%$ & $21.95 \%$ & $22.74 \%$ & $0.32 \%$ & $76.94 \%$ & $22.74 \%$ & 3.4 & $\mathrm{mS}$ & $\mathrm{H} 1 \mathrm{a}$ \\
\hline & & 85 & $0.5 \%$ & $2.39 \%$ & $8.99 \%$ & $23.99 \%$ & $16.58 \%$ & \begin{tabular}{l|l|}
$8.61 \%$ \\
\end{tabular} & $16.46 \%$ & $15.76 \%$ & $7.21 \%$ & $11.38 \%$ & $81.41 \%$ & $7.21 \%$ & \begin{tabular}{l|l}
11.3 \\
\end{tabular} & $\mathrm{gS}$ & $\mathrm{H1}$ \\
\hline & & 90 & $0.3 \%$ & $18.39 \%$ & $22.33 \%$ & $33.34 \%$ & $16.41 \%$ & $5.39 \%$ & $1.89 \%$ & $1.11 \%$ & $1.13 \%$ & $40.73 \%$ & $58.15 \%$ & $1.13 \%$ & 51.7 & sG & $\mathrm{H1}$ \\
\hline & & 95 & $0.5 \%$ & $12.09 \%$ & $16.22 \%$ & $34.27 \%$ & $21.89 \%$ & $7.35 \%$ & $4.15 \%$ & $2.52 \%$ & $1.53 \%$ & $28.30 \%$ & $70.17 \%$ & $1.53 \%$ & 46.0 & $\mathrm{gS}$ & $\mathrm{H} 1$ \\
\hline
\end{tabular}




\begin{tabular}{|c|c|c|c|c|c|c|c|c|c|c|c|c|c|c|c|c|c|}
\hline & & & & \multicolumn{2}{|c|}{ Gravel } & \multicolumn{7}{|c|}{ Sand } & & & & & \\
\hline & & & & $\begin{array}{l}\text { Fine } \\
\text { Pebble }\end{array}$ & $\begin{array}{c}\text { Very Fine } \\
\text { Pebble }\end{array}$ & $\begin{array}{c}\text { Very } \\
\text { Coarse }\end{array}$ & Coarse & Medium & Fine & Very Fine & $\begin{array}{l}\text { Silt \& } \\
\text { Clay }\end{array}$ & & & & & & \\
\hline & & & & 5 & 10 & 18 & 35 & 60 & 120 & 230 & Pan & & & & & & \\
\hline Borehole & $\begin{array}{c}\text { Sample } \\
\text { Type }\end{array}$ & Depth (ft) & $\begin{array}{l}\mathrm{CaCO}_{3} \\
\text { wt. \% }\end{array}$ & $>4 \mathrm{~mm}$ & $2-4 \mathrm{~mm}$ & $1-2 \mathrm{~mm}$ & $\begin{array}{c}0.5-1.0 \\
\mathrm{~mm}\end{array}$ & $\begin{array}{c}0.25-0.5 \\
\mathrm{~mm}\end{array}$ & $\begin{array}{c}0.125- \\
0.25 \mathrm{~mm}\end{array}$ & $\begin{array}{c}0.0625- \\
0.125 \mathrm{~mm}\end{array}$ & $\begin{array}{c}<0.0625 \\
\mathrm{~mm}\end{array}$ & $\%$ Gravel & $\%$ Sand & $\%$ Mud & $\begin{array}{c}\text { Sand:Mud } \\
\text { Ratio }\end{array}$ & CLASS & $\begin{array}{c}\text { Hydrogeologic } \\
\text { Unit }\end{array}$ \\
\hline & & 101 & $0.9 \%$ & "0.06\% & 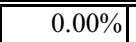 & $0.05 \%$ & $1.37 \%$ & $9.82 \%$ & \begin{tabular}{|c|}
$38.48 \%$ \\
\end{tabular} & 36.84\% & $13.39 \%$ & 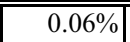 & $86.55 \%$ & $13.39 \%$ & \begin{tabular}{l|}
6.5 \\
\end{tabular} & (m)S & $\overline{\mathrm{H} 2}$ \\
\hline & & 105 & $1.7 \%$ & $0.02 \%$ & \begin{tabular}{|c|}
$0.02 \%$ \\
\end{tabular} & $0.05 \%$ & $0.37 \%$ & $9.03 \%$ & $34.81 \%$ & $17.87 \%$ & $37.84 \%$ & $0.03 \%$ & $62.13 \%$ & $37.84 \%$ & 1.6 & $\mathrm{mS}$ & $\mathrm{H} 2$ \\
\hline & & 111 & $2.0 \%$ & $0.00 \%$ & $0.01 \%$ & $0.26 \%$ & $1.00 \%$ & $4.01 \%$ & $20.35 \%$ & $55.21 \%$ & $19.15 \%$ & $0.01 \%$ & $80.84 \%$ & $19.15 \%$ & \begin{tabular}{l|}
4.2 \\
\end{tabular} & (m)S & $\mathrm{H} 2$ \\
\hline $\mid 299-W 23-15$ & & 50 & $0.6 \%$ & $0.77 \%$ & $0.61 \%$ & $1.72 \%$ & $8.48 \%$ & $49.50 \%$ & $22.83 \%$ & $9.16 \%$ & $6.94 \%$ & $1.38 \%$ & $91.68 \%$ & $6.94 \%$ & \begin{tabular}{l|l}
13.2 \\
\end{tabular} & $\mathrm{~S}$ & $\mathrm{H1a}$ \\
\hline & & 55 & $0.4 \%$ & $0.01 \%$ & $0.09 \%$ & $0.62 \%$ & $2.80 \%$ & $17.43 \%$ & $37.94 \%$ & $29.21 \%$ & $11.91 \%$ & $0.10 \%$ & $88.00 \%$ & $11.91 \%$ & 7.4 & (m)S & $\mathrm{H} 1 \mathrm{a}$ \\
\hline & & 60 & & $0.07 \%$ & \begin{tabular}{|l|}
$0.44 \%$ \\
\end{tabular} & $6.89 \%$ & $36.38 \%$ & $30.10 \%$ & $17.81 \%$ & $4.57 \%$ & $3.74 \%$ & $0.51 \%$ & $95.75 \%$ & $3.74 \%$ & 25.6 & $\mathrm{~S}$ & $\mathrm{H1}$ \\
\hline & & 65 & & $31.27 \%$ & $6.27 \%$ & $16.52 \%$ & $21.33 \%$ & $10.81 \%$ & $5.09 \%$ & $3.55 \%$ & $5.16 \%$ & $37.54 \%$ & $57.30 \%$ & $5.16 \%$ & \begin{tabular}{l|}
11.1 \\
\end{tabular} & $\mathrm{sG}$ & $\mathrm{H} 1$ \\
\hline & & 70 & & $16.44 \%$ & $7.15 \%$ & $19.46 \%$ & \begin{tabular}{l|}
$19.16 \%$ \\
\end{tabular} & $13.52 \%$ & $9.66 \%$ & $9.14 \%$ & $5.48 \%$ & $23.59 \%$ & $70.93 \%$ & $5.48 \%$ & \begin{tabular}{l|}
12.9 \\
\end{tabular} & $\mathrm{gS}$ & $\mathrm{H} 1$ \\
\hline & & 75 & $0.4 \%$ & $5.17 \%$ & $9.46 \%$ & $26.48 \%$ & $23.42 \%$ & $11.91 \%$ & $8.52 \%$ & $11.84 \%$ & $3.20 \%$ & $14.63 \%$ & $82.17 \%$ & $3.20 \%$ & 25.7 & gS & $\mathrm{H} 1$ \\
\hline & & 80 & $0.4 \%$ & $0.00 \%$ & $0.03 \%$ & $0.00 \%$ & $10.33 \%$ & $31.59 \%$ & $26.53 \%$ & $21.31 \%$ & $10.20 \%$ & $0.03 \%$ & $89.77 \%$ & $10.20 \%$ & 8.8 & (m)S & $\mathrm{H} 2$ \\
\hline & & 85 & $0.6 \%$ & $0.00 \%$ & $0.42 \%$ & $1.58 \%$ & $9.22 \%$ & $28.93 \%$ & $22.20 \%$ & $24.22 \%$ & $13.43 \%$ & $0.42 \%$ & $86.15 \%$ & $13.43 \%$ & \begin{tabular}{l|}
6.4 \\
\end{tabular} & (m)S & $\mathrm{H} 2$ \\
\hline 299-W23-19 & SS & $72-72.5$ & & $0.00 \%$ & \begin{tabular}{l|l}
$0.14 \%$ \\
\end{tabular} & $1.94 \%$ & $19.79 \%$ & $46.48 \%$ & $18.92 \%$ & $9.13 \%$ & $3.60 \%$ & $0.14 \%$ & $96.27 \%$ & $3.60 \%$ & 26.8 & S & $\mathrm{H} 1 \mathrm{a}$ \\
\hline B8809 & SS & $72.5-73$ & & $1.50 \%$ & $0.65 \%$ & $5.39 \%$ & $32.79 \%$ & $40.70 \%$ & \begin{tabular}{l|}
$12.14 \%$ \\
\end{tabular} & $4.28 \%$ & $2.54 \%$ & $2.15 \%$ & $95.31 \%$ & $2.54 \%$ & 37.5 & $\mathrm{~S}$ & H1a \\
\hline & SS & $73-73.5$ & & $1.38 \%$ & $0.61 \%$ & $5.45 \%$ & $34.81 \%$ & $32.68 \%$ & $11.49 \%$ & $8.08 \%$ & $5.49 \%$ & $1.99 \%$ & $92.52 \%$ & $5.49 \%$ & 16.8 & $\mathrm{~S}$ & $\mathrm{H} 1 \mathrm{a}$ \\
\hline & SS & $73.5-74$ & & $0.00 \%$ & $0.30 \%$ & $1.01 \%$ & $3.88 \%$ & $10.14 \%$ & $34.37 \%$ & $32.32 \%$ & $17.98 \%$ & $0.30 \%$ & $81.72 \%$ & $17.98 \%$ & 4.5 & (m)S & H1a \\
\hline & SS & $74-74.5$ & & $0.00 \%$ & $0.10 \%$ & $0.95 \%$ & $3.12 \%$ & $8.83 \%$ & $27.18 \%$ & $36.67 \%$ & $23.14 \%$ & $0.10 \%$ & $76.76 \%$ & $23.14 \%$ & 3.3 & $\mathrm{mS}$ & H1a \\
\hline & SS & $74.5-75$ & & $0.00 \%$ & $0.01 \%$ & $0.55 \%$ & $2.66 \%$ & $13.15 \%$ & $27.90 \%$ & $34.82 \%$ & $20.90 \%$ & $0.01 \%$ & $79.09 \%$ & $20.90 \%$ & 3.8 & $\mathrm{mS}$ & H1a \\
\hline & SS & $75-75.5$ & & $0.00 \%$ & $0.25 \%$ & $1.43 \%$ & $7.87 \%$ & $28.16 \%$ & $31.73 \%$ & $20.33 \%$ & $10.22 \%$ & $0.25 \%$ & $89.52 \%$ & $10.22 \%$ & 8.8 & (m)S & H1a \\
\hline & SS & $75.5-76$ & & $0.43 \%$ & $2.17 \%$ & $9.80 \%$ & $34.37 \%$ & $36.75 \%$ & $8.78 \%$ & $4.16 \%$ & $3.55 \%$ & $2.60 \%$ & $93.85 \%$ & $3.55 \%$ & 26.5 & $\mathrm{~S}$ & H1a \\
\hline & SS & $76-76.5$ & & $0.00 \%$ & \begin{tabular}{l|l|}
$0.92 \%$ \\
\end{tabular} & $6.60 \%$ & 41.05\% & $35.33 \%$ & $7.71 \%$ & $4.38 \%$ & $4.00 \%$ & $0.92 \%$ & $95.07 \%$ & $4.00 \%$ & 23.8 & $\mathrm{~S}$ & H1a \\
\hline & SS & $78.5-79$ & & $0.51 \%$ & $1.26 \%$ & $7.57 \%$ & $15.32 \%$ & $28.85 \%$ & $27.37 \%$ & $12.19 \%$ & $6.93 \%$ & $1.77 \%$ & $91.31 \%$ & $6.93 \%$ & \begin{tabular}{l|}
13.2 \\
\end{tabular} & $\mathrm{~S}$ & $\mathrm{H1}$ \\
\hline & SS & $78-78.5$ & & $15.63 \%$ & $14.24 \%$ & $20.03 \%$ & $19.98 \%$ & $12.51 \%$ & $8.10 \%$ & $5.65 \%$ & $3.85 \%$ & $29.88 \%$ & $66.28 \%$ & $3.85 \%$ & \begin{tabular}{l|}
17.2 \\
\end{tabular} & $\mathrm{gS}$ & $\mathrm{H} 1$ \\
\hline & SS & $79-79.5$ & & $0.00 \%$ & $0.70 \%$ & $3.63 \%$ & $6.62 \%$ & $11.07 \%$ & $28.16 \%$ & $32.42 \%$ & $17.40 \%$ & $0.70 \%$ & $81.90 \%$ & $17.40 \%$ & 4.7 & (m)S & $\mathrm{H} 1$ \\
\hline & SS & $79.5-80$ & & $0.00 \%$ & $2.37 \%$ & $4.13 \%$ & $5.06 \%$ & $5.74 \%$ & $32.30 \%$ & $37.05 \%$ & $13.36 \%$ & $2.37 \%$ & $84.27 \%$ & $13.36 \%$ & 6.3 & (m)S & $\mathrm{H1}$ \\
\hline & SS & $80-80.5$ & & $0.00 \%$ & $0.13 \%$ & $0.58 \%$ & $1.01 \%$ & $4.76 \%$ & $19.18 \%$ & $51.99 \%$ & $22.34 \%$ & $0.13 \%$ & $77.53 \%$ & $22.34 \%$ & 3.5 & $\mathrm{mS}$ & H1 \\
\hline & SS & $81.5-82.5$ & & $18.20 \%$ & $6.41 \%$ & $20.39 \%$ & $31.35 \%$ & $15.42 \%$ & $4.09 \%$ & $2.01 \%$ & $2.14 \%$ & $24.61 \%$ & $73.25 \%$ & $2.14 \%$ & \begin{tabular}{l|}
34.2 \\
\end{tabular} & $\mathrm{gS}$ & $\mathrm{H} 1$ \\
\hline & SS & $82.5-83.5$ & & $43.22 \%$ & $11.18 \%$ & $15.03 \%$ & $14.85 \%$ & $9.67 \%$ & $3.29 \%$ & $1.51 \%$ & $1.24 \%$ & $54.40 \%$ & $44.36 \%$ & $1.24 \%$ & 35.8 & $\mathrm{sG}$ & $\mathrm{H1}$ \\
\hline & SS & $83.5-84.5$ & & $4.76 \%$ & $9.89 \%$ & $25.35 \%$ & $32.66 \%$ & $17.65 \%$ & $4.55 \%$ & $2.42 \%$ & $2.71 \%$ & $14.65 \%$ & $82.64 \%$ & $2.71 \%$ & 30.5 & $\mathrm{gS}$ & $\mathrm{H1}$ \\
\hline & SS & $84.5-85.5$ & & $7.07 \%$ & $13.74 \%$ & $33.04 \%$ & $29.62 \%$ & $9.03 \%$ & $3.00 \%$ & $1.94 \%$ & $2.55 \%$ & $20.82 \%$ & $76.63 \%$ & $2.55 \%$ & 30.0 & $\mathrm{gS}$ & $\mathrm{H} 1$ \\
\hline
\end{tabular}




\begin{tabular}{|c|c|c|c|c|c|c|c|c|c|c|c|c|c|c|c|c|c|}
\hline & & & & \multicolumn{2}{|c|}{ Gravel } & \multicolumn{7}{|c|}{ Sand } & & & & & \\
\hline & & & & $\begin{array}{c}\text { Fine } \\
\text { Pebble }\end{array}$ & \begin{tabular}{|c|} 
Very Fine \\
Pebble
\end{tabular} & $\begin{array}{c}\text { Very } \\
\text { Coarse }\end{array}$ & Coarse & Medium & Fine & Very Fine & $\begin{array}{r}\text { Silt \& } \\
\text { Clay }\end{array}$ & & & & & & \\
\hline & & & & 5 & 10 & 18 & 35 & 60 & 120 & 230 & Pan & & & & & & \\
\hline Borehole & $\begin{array}{c}\text { Sample } \\
\text { Type }\end{array}$ & Depth (ft) & $\begin{array}{l}\mathrm{CaCO}_{3} \\
\text { wt. \% }\end{array}$ & $>4 \mathrm{~mm}$ & $2-4 \mathrm{~mm}$ & $1-2 \mathrm{~mm}$ & $\begin{array}{c}\begin{array}{c}0.5-1.0 \\
\mathrm{~mm}\end{array} \\
\end{array}$ & $\begin{array}{c}0.25-0.5 \\
\mathrm{~mm}\end{array}$ & $\begin{array}{c}0.125- \\
0.25 \mathrm{~mm} \\
\end{array}$ & \begin{tabular}{|c|}
$0.0625-$ \\
$0.125 \mathrm{~mm}$ \\
\end{tabular} & $\begin{array}{c}<0.0625 \\
\mathrm{~mm} \\
\end{array}$ & $\%$ Gravel & $\%$ Sand & $\%$ Mud & \begin{tabular}{|c|} 
Sand:Mud \\
Ratio
\end{tabular} & CLASS & $\begin{array}{c}\text { Hydrogeologic } \\
\text { Unit }\end{array}$ \\
\hline & SS & \begin{tabular}{|l|}
$85.5-86.5$ \\
\end{tabular} & & $18.29 \%$ & $11.03 \%$ & $25.59 \%$ & $29.77 \%$ & $8.69 \%$ & $2.80 \%$ & $1.69 \%$ & $2.15 \%$ & $29.32 \%$ & $68.53 \%$ & $2.15 \%$ & 31.9 & $\mathrm{gS}$ & $\overline{\mathrm{H} 1}$ \\
\hline & SS & \begin{tabular}{|l|}
$86.6-87.6$ \\
\end{tabular} & & $23.23 \%$ & 5.23\% & $11.54 \%$ & $\begin{array}{l}16.58 \% \\
\end{array}$ & \begin{tabular}{|l|l|}
$11.17 \%$ \\
\end{tabular} & $15.54 \%$ & $11.53 \%$ & $5.02 \%$ & $28.47 \%$ & $66.35 \%$ & $5.02 \%$ & 13.2 & $\mathrm{gS}$ & $\mathrm{H1}$ \\
\hline & SS & \begin{tabular}{|c|}
$87.6-88.6$ \\
\end{tabular} & & $0.00 \%$ & $\begin{array}{ll}0.11 \% \\
\end{array}$ & $0.10 \%$ & $0.56 \%$ & $2.91 \%$ & $20.35 \%$ & $56.16 \%$ & $19.80 \%$ & $0.11 \%$ & $80.08 \%$ & $19.80 \%$ & 4.0 & (m)S & $\mathrm{H} 2$ \\
\hline & SS & \begin{tabular}{|l|}
$88.6-89.6$ \\
\end{tabular} & & $0.00 \%$ & $0.02 \%$ & $0.09 \%$ & $4.73 \%$ & $48.59 \%$ & $25.40 \%$ & $14.05 \%$ & $7.13 \%$ & $0.02 \%$ & $92.86 \%$ & $7.13 \%$ & 13.0 & $\mathrm{~s}$ & $\mathrm{H} 2$ \\
\hline & SS & \begin{tabular}{|l|}
$89.6-90.6$ \\
\end{tabular} & & $0.00 \%$ & $0.03 \%$ & $0.06 \%$ & $0.90 \%$ & $21.81 \%$ & $43.78 \%$ & $23.65 \%$ & $9.76 \%$ & $0.03 \%$ & $90.21 \%$ & $9.76 \%$ & 9.2 & $\mathrm{~s}$ & $\mathrm{H} 2$ \\
\hline & SS & \begin{tabular}{|l|}
$90.6-91.6$ \\
\end{tabular} & & $0.00 \%$ & $0.00 \%$ & $0.05 \%$ & $0.33 \%$ & $32.22 \%$ & $45.68 \%$ & $13.94 \%$ & $7.78 \%$ & $0.00 \%$ & $92.22 \%$ & $7.78 \%$ & 11.9 & $\mathrm{~S}$ & $\mathrm{H} 2$ \\
\hline & SS & \begin{tabular}{|l|}
$92.5-93.5$ \\
\end{tabular} & & $2.62 \%$ & $\begin{array}{ll}0.73 \% \\
\end{array}$ & $3.29 \%$ & $5.76 \%$ & $14.05 \%$ & $30.81 \%$ & $33.81 \%$ & $8.93 \%$ & $3.35 \%$ & $87.72 \%$ & $8.93 \%$ & 9.8 & $\mathrm{~s}$ & $\mathrm{H} 2$ \\
\hline & SS & \begin{tabular}{|l|}
$93.5-94.5$ \\
\end{tabular} & & $0.00 \%$ & $\begin{array}{ll}0.45 \% \\
\end{array}$ & $1.40 \%$ & $4.94 \%$ & $16.67 \%$ & $33.04 \%$ & $32.33 \%$ & $11.17 \%$ & $0.45 \%$ & $88.38 \%$ & $11.17 \%$ & 7.9 & (m)S & $\mathrm{H} 2$ \\
\hline & SS & \begin{tabular}{|l|}
$94.5-95.5$ \\
\end{tabular} & & $0.00 \%$ & $0.00 \%$ & $0.01 \%$ & $0.29 \%$ & $1.02 \%$ & $13.13 \%$ & $63.23 \%$ & $22.32 \%$ & $0.00 \%$ & $77.68 \%$ & $22.32 \%$ & 3.5 & $\mathrm{mS}$ & $\mathrm{H} 2$ \\
\hline & SS & \begin{tabular}{|l|}
$95.6-96.6$ \\
\end{tabular} & & $13.78 \%$ & $0.22 \%$ & $0.21 \%$ & $2.74 \%$ & $25.02 \%$ & $29.20 \%$ & $21.85 \%$ & $6.79 \%$ & $13.99 \%$ & $79.03 \%$ & $6.79 \%$ & 11.6 & $\mathrm{gS}$ & $\mathrm{H} 2$ \\
\hline & SS & \begin{tabular}{|l|}
$96.6-97.6$ \\
\end{tabular} & & $0.00 \%$ & $0.00 \%$ & $0.11 \%$ & $1.74 \%$ & $21.65 \%$ & $38.58 \%$ & $30.87 \%$ & $7.04 \%$ & $0.00 \%$ & $92.96 \%$ & $7.04 \%$ & 13.2 & $\mathrm{~s}$ & $\mathrm{H} 2$ \\
\hline & SS & \begin{tabular}{|l|}
$97.6-98.6$ \\
\end{tabular} & & $0.00 \%$ & $0.01 \%$ & $0.10 \%$ & $1.53 \%$ & $22.71 \%$ & $33.59 \%$ & $34.64 \%$ & $7.43 \%$ & $0.01 \%$ & $92.56 \%$ & $7.43 \%$ & 12.5 & $\mathrm{~s}$ & $\mathrm{H} 2$ \\
\hline & SS & \begin{tabular}{|l|}
$98.6-99.6$ \\
\end{tabular} & & $0.00 \%$ & $0.02 \%$ & $0.24 \%$ & $1.20 \%$ & $21.75 \%$ & $36.34 \%$ & $32.58 \%$ & $7.88 \%$ & $0.02 \%$ & $92.10 \%$ & $7.88 \%$ & 11.7 & $\mathrm{~s}$ & $\mathrm{H} 2$ \\
\hline & SS & \begin{tabular}{|l|}
$99.6-100.6$ \\
\end{tabular} & & $0.00 \%$ & \begin{tabular}{|l|l|}
$0.04 \%$ \\
\end{tabular} & $1.18 \%$ & $2.60 \%$ & $14.90 \%$ & $28.12 \%$ & $33.08 \%$ & $20.09 \%$ & $0.04 \%$ & $79.87 \%$ & $20.09 \%$ & 4.0 & (m)S & $\mathrm{H} 2$ \\
\hline
\end{tabular}




\section{Appendix E}

Details on Mineralogy for Borehole 41-09-39 


\section{Appendix E}

\section{Details on Mineralogy for Borehole 41-09-39}

Table E.1. Semiquantitative Mineral Composition for Bulk Samples (wt\%)

\begin{tabular}{|c|c|c|c|c||}
\hline Sample ID & Quartz & K-Feldspar & Na-Feldspar & Calcite \\
\hline \hline $56-57^{\prime}$ & 50 & 5 & 50 & ND \\
\hline $74-75^{\prime}$ & 50 & 5 & 25 & ND \\
\hline $79-80^{\prime}$ & 35 & 5 & 55 & ND \\
\hline $90^{\prime}$ & 50 & 15 & 25 & $<5$ \\
\hline $108-109^{\prime}$ & 45 & 5 & 40 & $<5$ \\
\hline ND = Not detected.
\end{tabular}

Table E.2. Semiquantitative Mineral Composition for the Clay Fraction $(<2 \mu \mathrm{m})$ of Sediment Samples (wt\%)

\begin{tabular}{||c|c|c|c|c|c|c||}
\hline Sample ID & Quartz & Feldspar & Smectite & Illite & Chlorite & Kaolinite \\
\hline \hline $56-57^{\prime}$ & 5 & $<5$ & 20 & 35 & 30 & 10 \\
\hline $74-75^{\prime}$ & 10 & 10 & 5 & 30 & 20 & 10 \\
\hline $79-80^{\prime}$ & 10 & $<5$ & 10 & 20 & 15 & 5 \\
\hline $90^{\prime}$ & $<5$ & $<5$ & 15 & 20 & 10 & 10 \\
\hline $108-109^{\prime}$ & 5 & $<5$ & 20 & 20 & 10 & 10 \\
\hline \hline
\end{tabular}




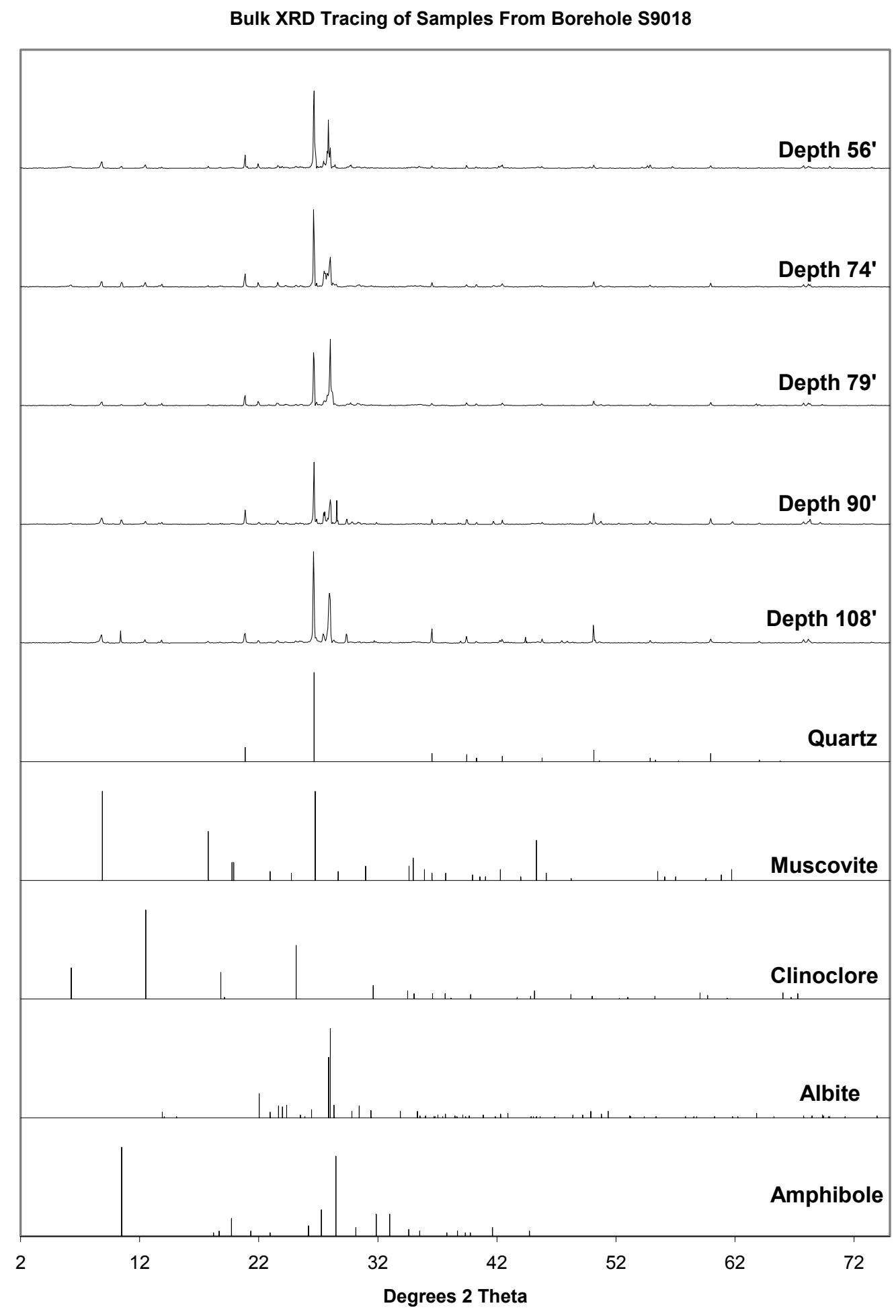

E. 2 
2CD From Depth $\sim 133 \mathrm{ft}$ bgs XRD Tracings vs Size Fraction (Sieve Size)

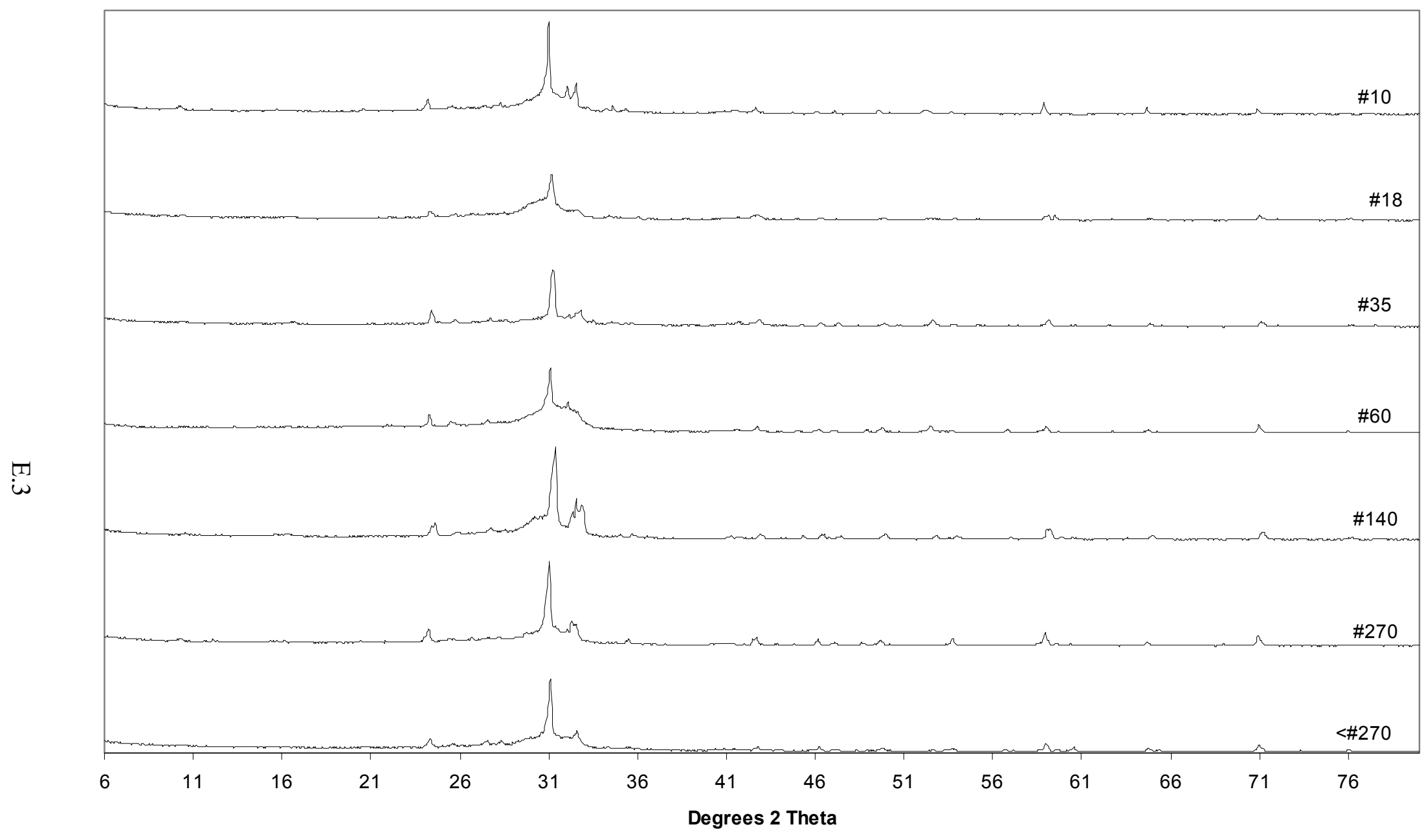


XRD of 2CD Various Size Fractions

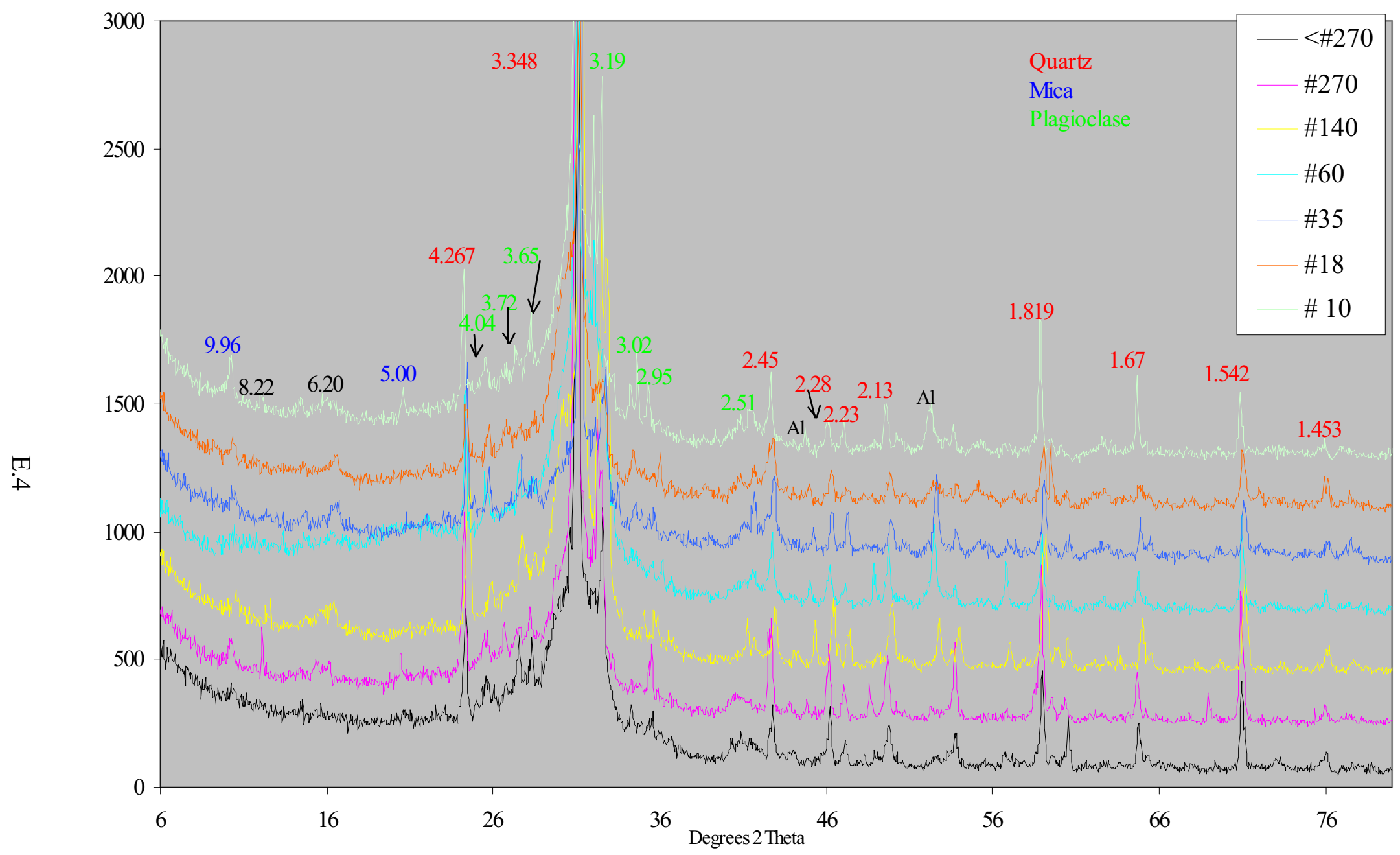


XRD Pattern of the Clay Fraction From Depth 56-57' in Borehole S9018

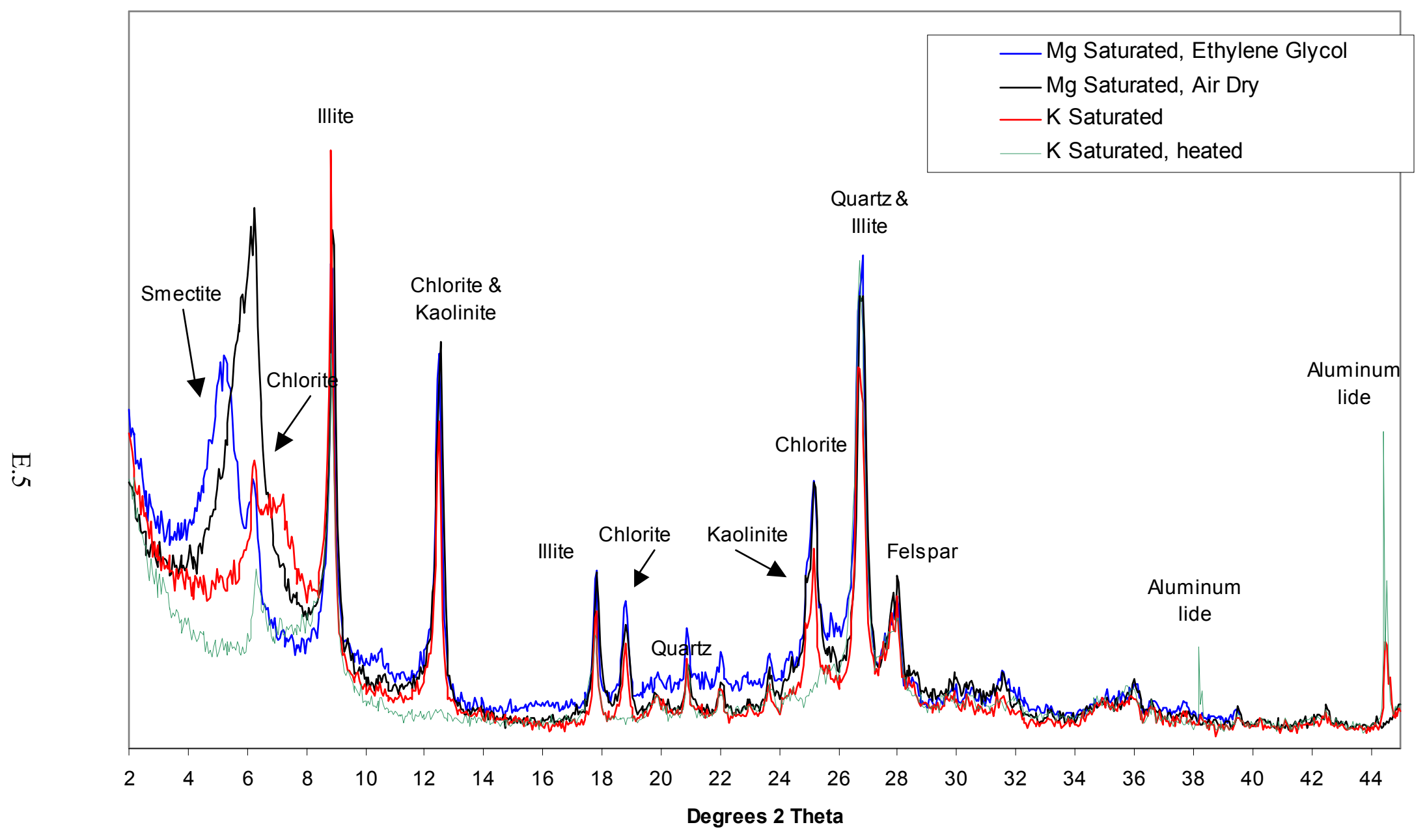


XRD Pattern of the Clay Fraction From Depth 74-75' in Borehole $\mathbf{S 9 0 1 8}$

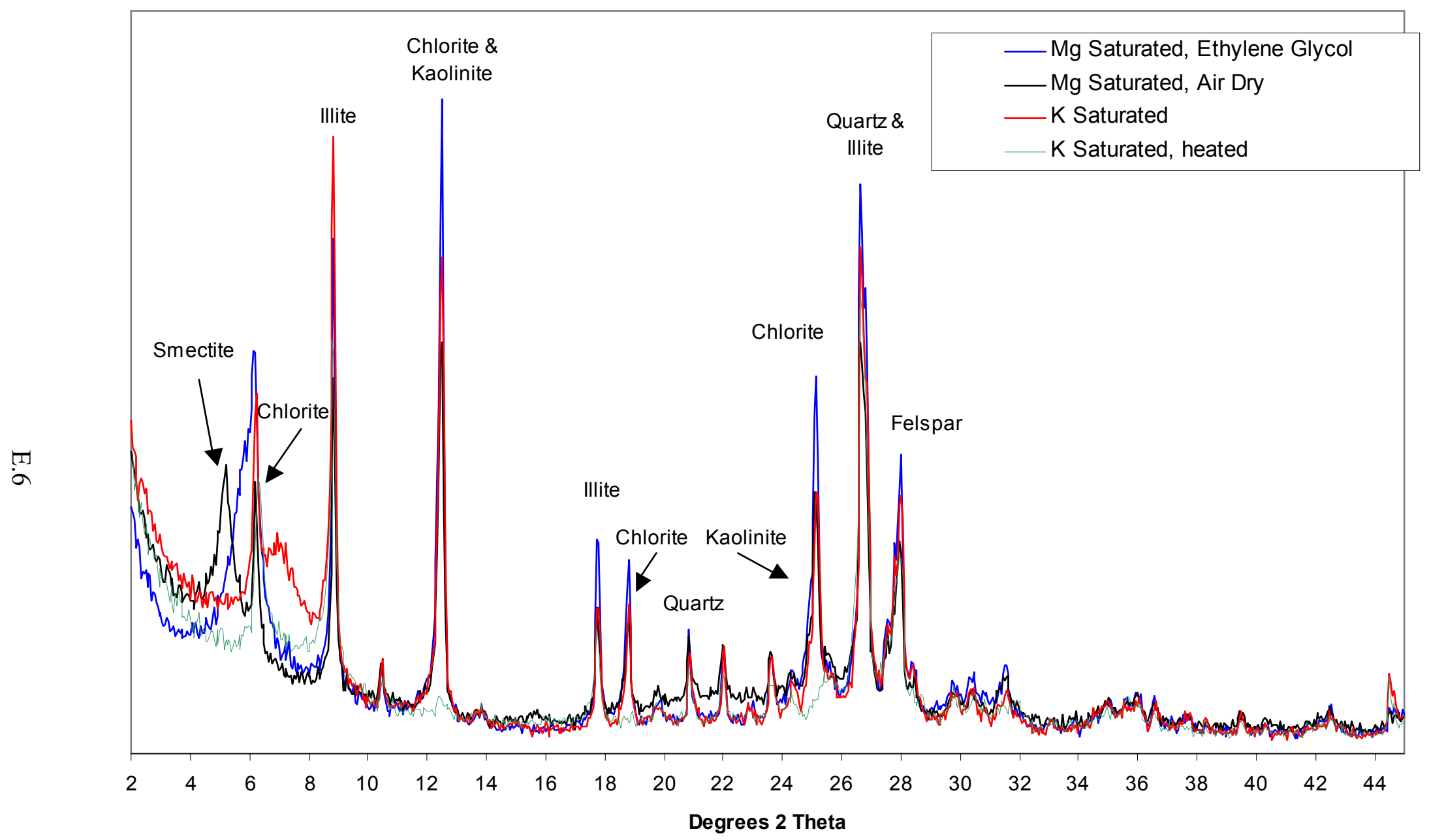


XRD Pattern of the Clay Fraction From Depth 79-80' in Borehole $\mathbf{5 9 0 1 8}$

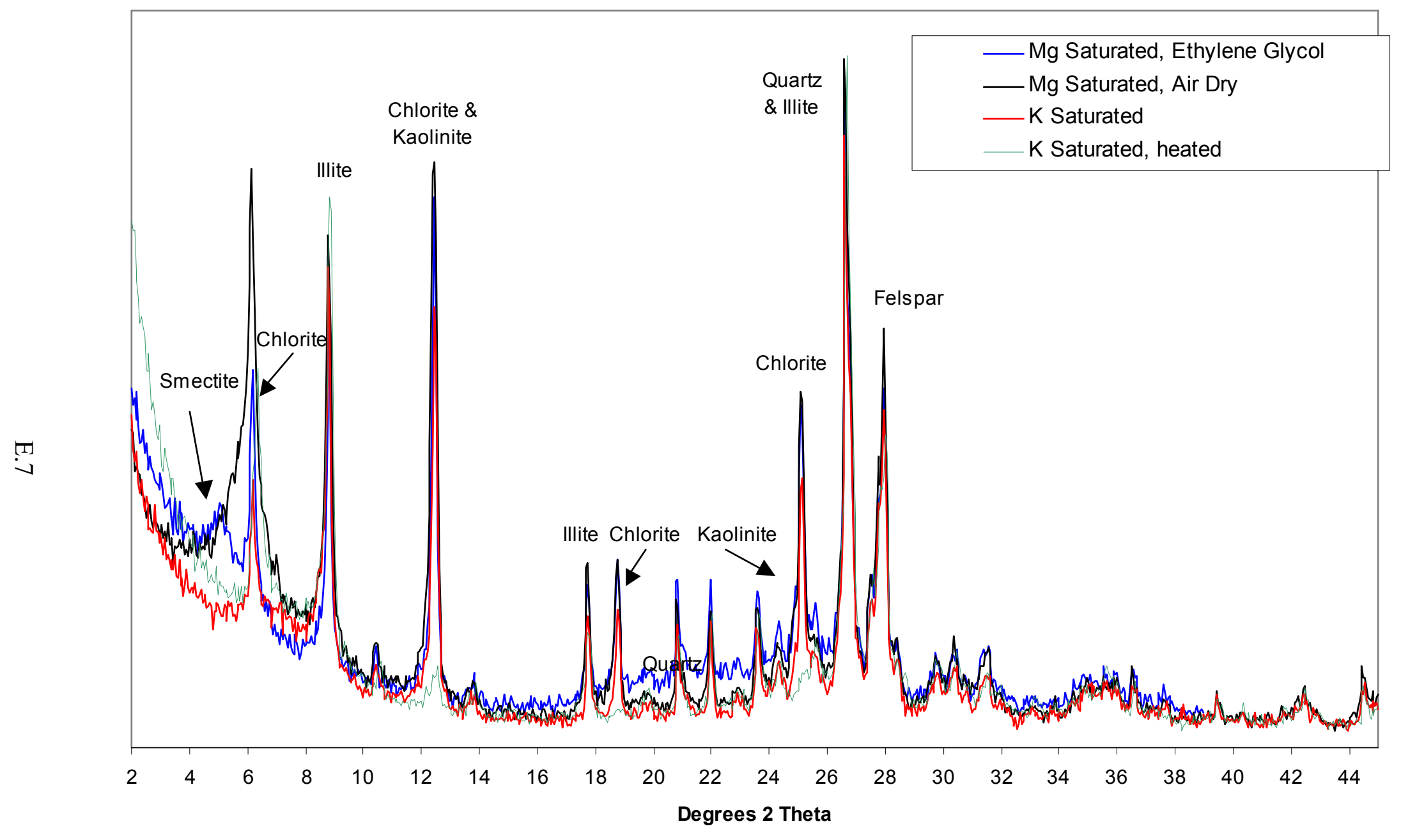


XRD Patterns of the Clay Fraction From Depth 90' in Borehole S9018

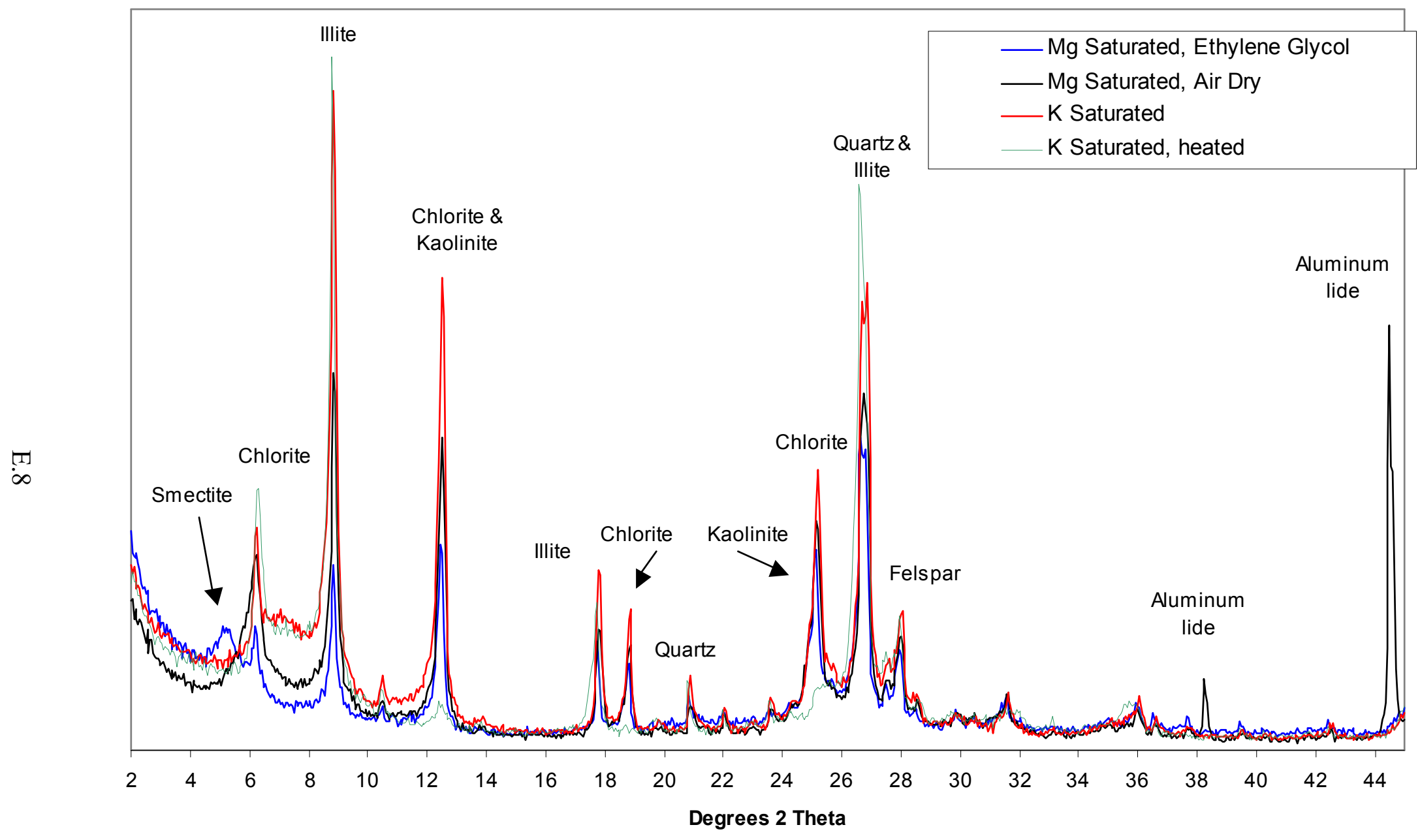


XRD Pattern of the Clay Fraction From Depth 108'-109' in Borehole S9018

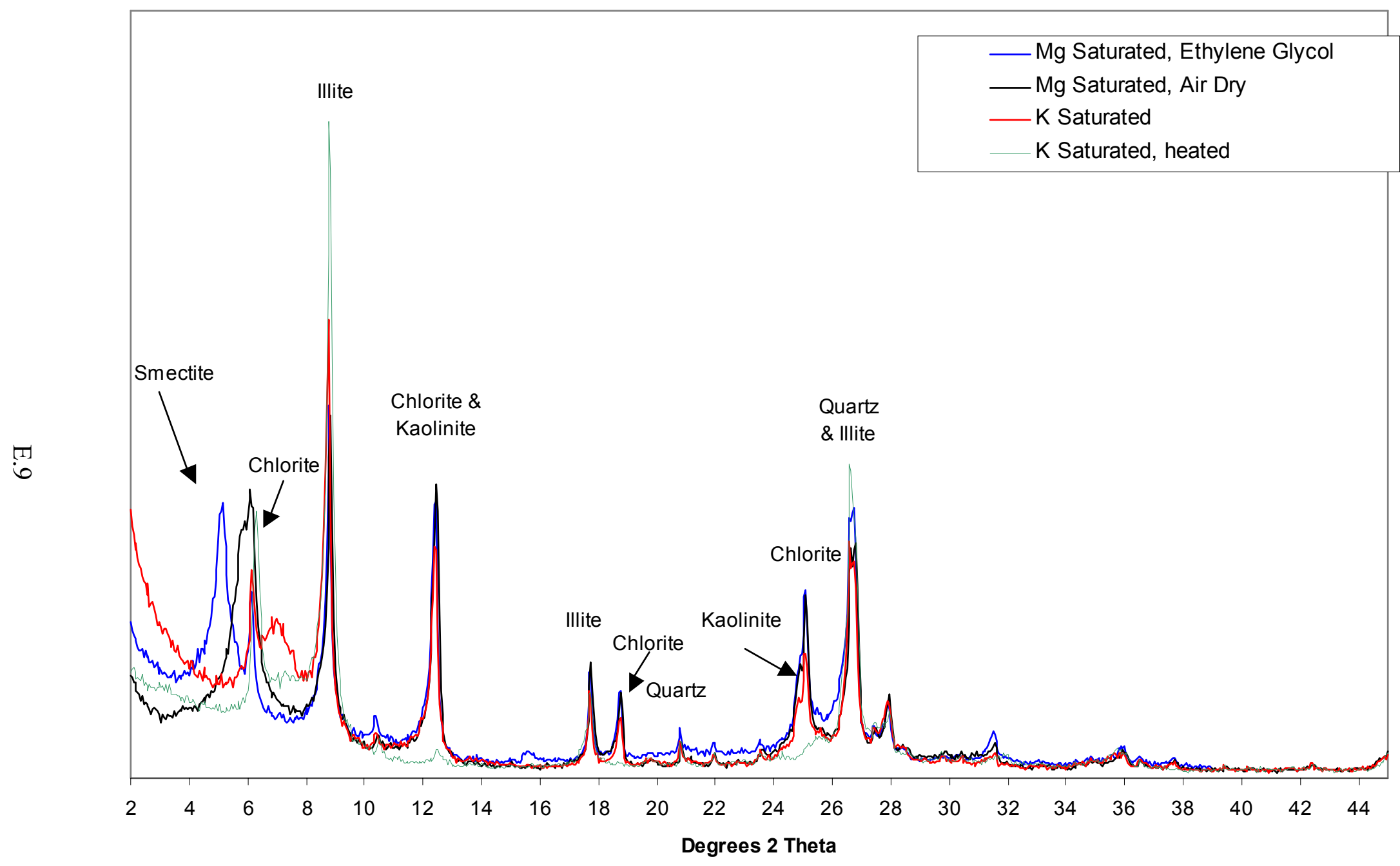


XRD Tracings of Clay Fraction From Samples 2C/D, 2A/B, and 3A/B all $\sim 133 \mathrm{ft}$ bgs

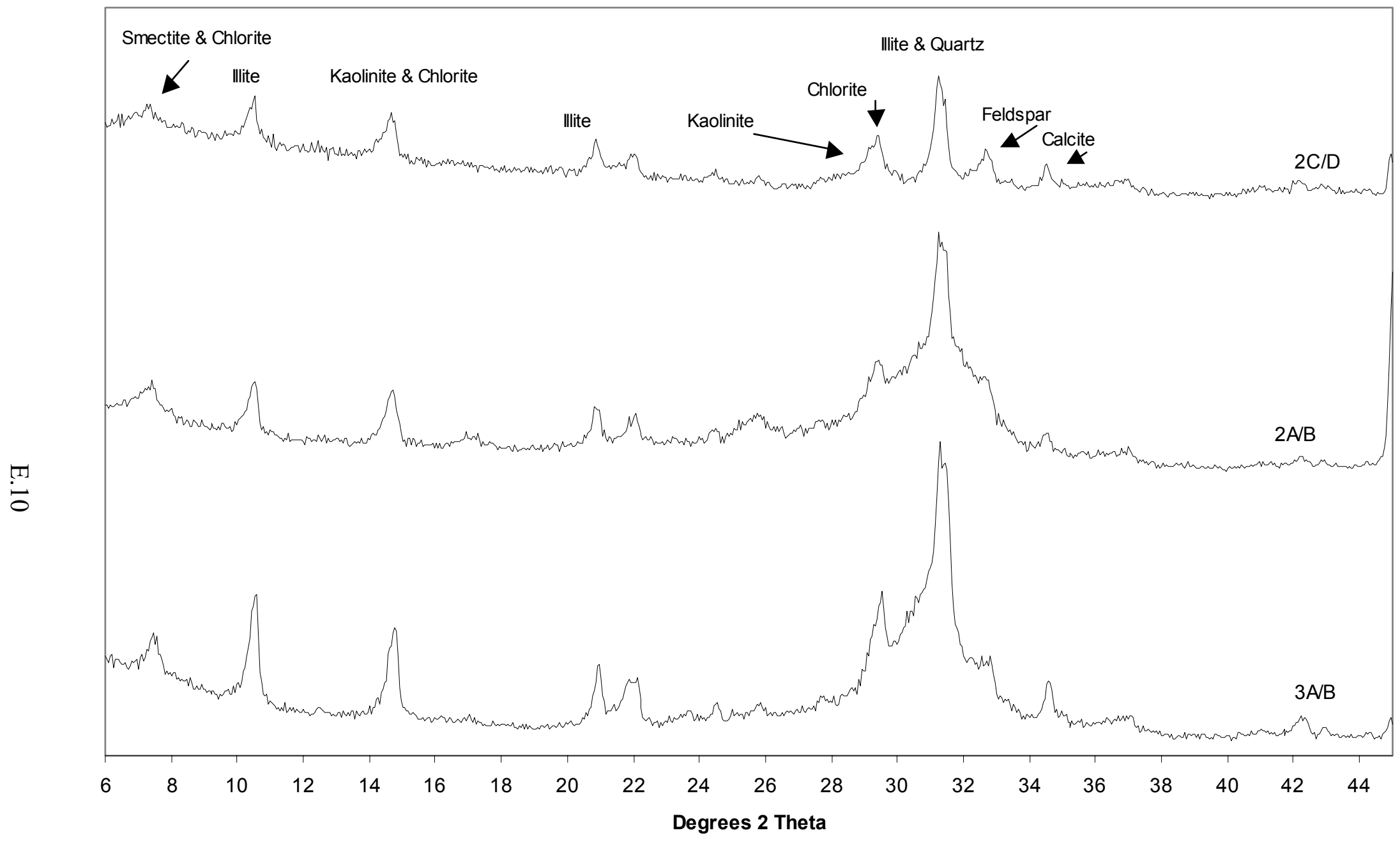


XRD Tracings of Clay Fraction From Samples 2C/D, 2A/B, and 3A/B all $\sim 133 \mathrm{ft}$ bgs

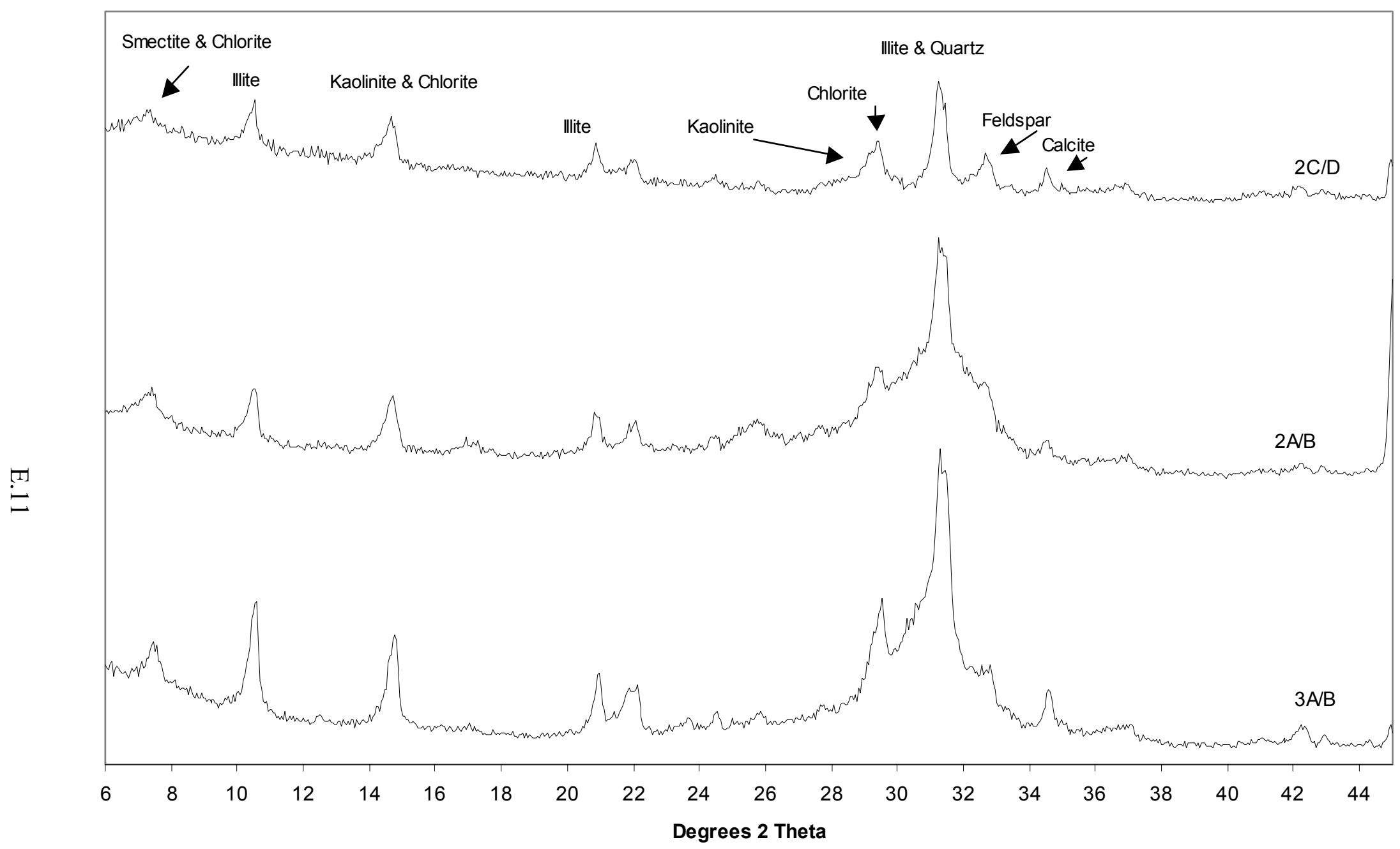


PNNL-13757-3

\section{Distribution}

No. of

Copies

ONSITE

CH2M HILL Hanford Group, Inc.

Anthony J. Knepp (10 CD)

Fredrick M. Mann

H0-22

$\mathrm{H} 0-22$

U.S. Department of Energy

Robert M. Yasek

U.S. Department of Ecology

Joseph Caggiano

H6-60

B5-18
No. of

Copies

$\underline{\text { Pacific Northwest National Laboratory }}$

R. Jeff Serne (50 CD) K6-81

Clark W. Lindenmeier P8-37

George V. Last K6-81

Bruce N. Bjornstad K6-81

Duane G. Horton K6-81

H. Todd Schaef K6-81

1 hard copy and $1 \mathrm{CD}$ except as noted.

Distr.1 